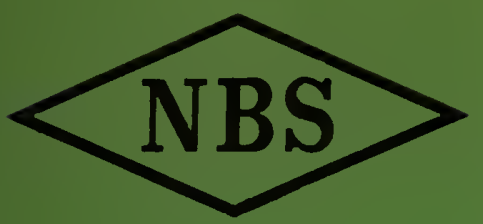

Eechnical Note

\title{
PROVISIONAL THERMODYNAMIC FUNCTIONS FOR PARA-HYDROGEN
}

HANS M. RODER AND ROBERT D. GOODWIN

U. S. DEPARTMENT OF COMMERCE NATIONAL BUREAU OF STANDARDS 


\section{THE NATIONAL BUREAU OF STANDARDS}

\section{Functions and Activities}

The functions of the National Bureau of Standards are set forth in the Act of Congress, March 3, 1901, as amended by Congress in Public Law 619, 1950. These include the development and maintenance of the national standards of measurement and the provision of means and methods for making measurements consistent with these standards; the determination of physical constants and properties of materials; the development of methods and instruments for testing materials; devices, and structures; advisory services to government agencies on scientific and technical problems; invention and development of devices to serve special needs of the Government; and the development of standard practices, codes, and specifications. The work includes basic and applied research, development, engineering, instrumentation, testing, evaluation, calibration services, and various consultation and information services. Research projects are also performed for other government agencies when the work relates to and supplements the basic program of the Bureau or when the Bureau's unique competence is required. The scope of activities is suggested by the listing of divisions and sections on the inside of the back cover.

\section{Publications}

The results of the Bureau's research are published either in the Bureau's own series of publications or in the journals of professional and scientific societies. The Bureau itself publishes three periodicals available from the Government Printing Office: The Journal of Research, published in four separate sections, presents complete scientific and technical papers; the Technical News Bulletin presents summary and preliminary reports on work in progress; and Basic Radio Propagation Predictions provides data for determining the best frequencies to use for radio communications thro ughout the world. There are also five series of nonperiodical publications: Monographs, Applied Mathematics Series, Handbooks, Miscellaneous Publications, and Technical Notes.

A complete listing of the Bureau's publications can be found in National Bureau of Standards Circular 460, Publications of the National Bureau of Standards, 1901 to June 1947 (\$1.25), and the Supplement to National Bureau of Standards Circular 460, July 1947 to June 1957 (\$1.50), and Miscellaneous Publication 240, July 1957 to June 1960 (Includes Titles of Papers Published in Outside Journals 1950 to 1959) (\$2.25); available from the Superintendent of Documents, Government Printing Office, Washington 25, D. C. 


\title{
NATIONAL BUREAU OF STANDARDS Eechnical Note
}

130

DECEMBER 1961

\section{PROVISIONAL THERMODYNAMIC FUNCTIONS FOR PARA-HYDROGEN}

\author{
Hans M. Roder and Robert D. Goodwin \\ NBS Boulder Laboratories
}

The work described in this report was supported in part by the National Aeronautics and Space Administration.

NBS Technical Notes are designed to supplement the Bureau's regular publications program. They provide a means for making available scientific data that are of transient or limited interest. Technical Notes may be listed or referred to in the open literature. They are for sale by the Office of Technical Services, U. S. Department of Commerce, Washington 25, D. C.

\section{DISTRIBUTED BY \\ UNITED STATES DEPARTMENT OF COMMERCE \\ OFFICE OF TECHNICAL SERVICES \\ WASHINGTON 25, D. C.}

Price $\$ 3.00$ 

CONTENTS

LIST OF TABLES . . . . . . . . . . . . . . III

LIST OF FIGURES. .................. III

LIST OF SYMBOLS \& UNITS ............. . IV

ABSTRACT. . . . . . . . . . . . . . . . 1

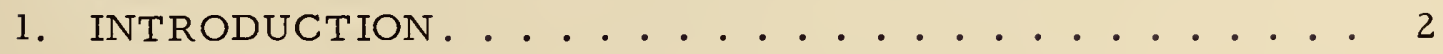

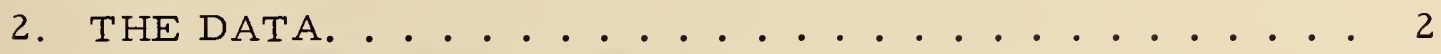

2. 1 Experimental PVT data .......... 2

2.2 "Specific heat of ideal gas" ......... 3

2.3 Vapor pressure ............. 3

2.4 Saturated liquid density .......... . . 3

2.5 Saturated vapor density ............ 4

2. 6 Heat of vaporization at the boiling point. . . . . . 4

2. 7 The specific heat of the saturated liquid . . . . . 4

2. 8 Vapor pressure vs. saturated liquid density . . . . 6

2. 9 Selected parameters........... 6

3. THE CALCULATED PVT SURFACE . . . . . . . . 7

3.1 The equation of state........... 7

3. 2 Regions of application . . . . . . . . . . 8

3.3 Deviations .............. . . . 9

4. THE DERIVED THERMODYNAMIC FUNCTIONS . . . . . . 12

4. 1 The baseline or reference values . . . . . . . 12

4. 2 Equations used in the high temperature region . . . 13

4. 3 Equations used in the compressed liquid region . . . 14

4. 4 The transition between the two regions . . . . . . 14

4. 5 Discussion of errors. . . . . . . . . . . 15

5. EXTENSION OF THE TABULATED VALUES TO $300^{\circ} \mathrm{K}$. . . 15

6. DESCRIPTION OF THE PROVISIONAL THERMODYNAMIC

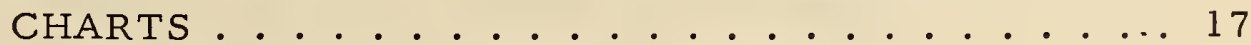

6. $1 \mathrm{~T}-\mathrm{S}$ chart, $20-100^{\circ} \mathrm{K} . \ldots . . . . . . .17$

$6.2 \mathrm{~T}-\mathrm{S}$ chart, $80-300^{\circ} \mathrm{K} . \ldots . . . . . .17$

$6.3 \mathrm{H}-\mathrm{S}$ chart, $20-60^{\circ} \mathrm{K}$............. 18

7. ACKNOWLEDGEMENTS. . . . . . . . . 18

8. REFERENCES . . . . . . . . . . . . . . 19

9. THE TABLES OF PROVISIONAL THERMODYNAMIC

FUNCTIONS FOR PARA-HYDROGEN. . . . . . 21

SUPPLEMENT A (British units) . . . . . . . . . 75 


\section{LIST OF TABLES}

Table 1 Densities of saturated para-hydrogen vapor Table 2 Adjustments in entropy and enthalpy $31-37^{\circ} \mathrm{K}$ Table 3 Adjustments in specific volume and enthalpy at $101^{\circ} \mathrm{K}$

\section{LIST OF FIGURES}

Figure 1 Schematic deviations along isotherms, pressure vs. volume

Figure 2 Schematic phase diagram, density vs. temperature Figure D-20A T-S Chart 20 to $100^{\circ} \mathrm{K} 1$ to $340 \mathrm{~atm}$ Figure D-2lA T-S Chart 80 to $300^{\circ} \mathrm{K} 1$ to $100 \mathrm{~atm}$ Figure D-22A H-S Chart 20 to $60^{\circ} \mathrm{K} \quad 1$ to $340 \mathrm{~atm}$ 


\section{LIST OF SYMBOLS AND UNITS}

$\mathrm{R}$ gas constant, $82.082 \mathrm{~cm}^{3} \mathrm{~atm} / \mathrm{g}$ mole ${ }^{\circ} \mathrm{K}$

$\mathrm{P}$ pressure atm

$P_{\text {sat }}$ vapor pressure

$P_{C}$ critical pressure, $12.770 \mathrm{~atm}$

$\mathrm{V}$ volume $\mathrm{cm}^{3} / \mathrm{g}$ mole

$\mathrm{V}_{\text {gas }}$ volume of saturated vapor, $\mathrm{cm}^{3} / \mathrm{g}$ mole

$\mathrm{V}_{\text {liq }}$ volume of saturated liquid, $\mathrm{cm}^{3} / \mathrm{g}$ mole

$\mathrm{T}$ absolute temperature, degrees Kelvin, Triple point of

$\mathrm{H}_{2} \mathrm{O} 273.16^{\circ} \mathrm{K}$

$\mathrm{T}_{\mathrm{c}}$ critical temperature, $32.984^{\circ} \mathrm{K}$

$\mathrm{C}_{\mathrm{p}}^{\mathrm{O}} \quad$ "Specific heat of ideal gas", cal $/ \mathrm{g}$ mole ${ }^{\circ} \mathrm{K}$

$\rho$ density, g mole/ $\mathrm{cm}^{3}$

$\rho_{\text {sat }}$ saturated liquid density

$\rho_{c}$ critical density, $0.0152672 \mathrm{~g} \mathrm{~mole} / \mathrm{cm}^{3}$

$\triangle \mathrm{H}_{\text {vap }}$ heat of vaporization, cal/g mole

$\mathrm{C}_{\text {sat }}$ saturated liquid heat capacity, cal/g mole ${ }^{\circ} \mathrm{K}$

$\mathrm{Z}=\mathrm{PV} / \mathrm{RT}$

$h_{T, \rho}$ enthalpy at $\mathrm{T}$ and $\rho$, cal/g or $\mathrm{j} / \mathrm{g}$

$u_{T, \rho}$ internal energy at $T$ and $\rho, c a l / g$ or $j / g$

${ }^{s}, \rho$ entropy at $T$ and $\rho, \operatorname{cal} / g^{\circ} \mathrm{K}$ or $j / g^{\circ} \mathrm{K}$ 

PROVISIONAL THERMODYNAMIC FUNCTIONS FOR

PARA-HYDROGEN

by

Hans M. Roder and Robert D. Goodwin

Cryogenic Engineering Laboratory

\section{ABSTRACT}

New PVT data recently obtained at this laboratory were used to compute tabular values of internal energy, enthalpy, and entropy of para-hydrogen. These properties, together with specific volume, are presented here as functions of temperature and pressure. The new data encompassed the temperature range $20^{\circ}$ to $100^{\circ} \mathrm{K}$ at pressures up to $340 \mathrm{~atm}$. Earlier data were used to extend the tables to $300^{\circ} \mathrm{K}$. Above $100^{\circ} \mathrm{K}$ the upper limit of pressure is $100 \mathrm{~atm}$. The information is also presented in the form of thermodynamic charts. In supplement A to this report the thermodynamic tables and charts are presented in units of: psia, degrees Rankine, BTU, pounds, and cubic feet. 


\section{INTRODUCTION}

The urgency of the need for thermodynamic functions of para-hydrogen prompts this Technical Note, and justifies the approximations used in computation of the provisional functions. The $20.3^{\circ} \mathrm{K}$ equilibrium hydrogen $(0.21 \%$ or tho $)$ is simply called parahydrogen in this report.

Subsequent sections present the data used, the equation of state which is used to represent the PVT data, the derived thermodynamic functions, the extension of these functions above $100^{\circ} \mathrm{K}$, and the resulting thermodynamic tables and charts.

More accurate computations of the thermodynamic functions are planned. When completed, they will supersede results given in the present report. Either direct numerical techniques or an improved equation of state will be employed with PVT data from Goodwin [1961, 1962] and specific heats from Younglove [1962].

In consideration of engineering requirements Supplement A of this report presents the thermodynamic tables and charts in the following units: psia, degrees Rankine, BTU, pounds, cubic feet.

\section{THE DATA}

The data needed for the calculations include experimental PVT data, the ideal specific heat, the vapor pressure as a function of temperature, the saturated liquid and vapor densities, the heat of vaporization at the normal boiling point, the specific heat of the saturated liquid, the vapor pressure as a function of saturated liquid density, and the critical point data.

\section{1 Experimental PVT Data}

Goodwin and co-workers [1961, 1962] measured 1211 PVT points for "para-hydrogen" in the ranges of 15 to $100^{\circ} \mathrm{K}$ and 2 to 350 atm. Of these, 15 experimental points represent vapor pressure determinations while 2 others fall on the melting line. For computational purposes these data were represented by an equation of state as described in section 3. 


\section{2 "Specific Heat of Ideal Gas"}

The values given by Woolley, Scott, and Brickwedde [ 1948] for para-hydrogen were approximated in the range 10 to $100^{\circ} \mathrm{K}$ by

$$
\mathrm{C}_{\mathrm{p}}{ }^{\circ}=\mathrm{A}+\mathrm{BT}+\mathrm{C \textrm {T } ^ { 2 }}+\mathrm{DT} \mathrm{T}^{3}+\mathrm{ET}^{4}+\mathrm{FT}^{5} \ldots .
$$

No corrections were made for the more recent values of the physical constants. The maximum deviation is $-.0043 \mathrm{cal} / \mathrm{mole}^{\circ} \mathrm{K}$ at $90^{\circ} \mathrm{K}$ or $.08 \%$, and the mean deviation is $.0024 \mathrm{cal} / \mathrm{mole}{ }^{\circ} \mathrm{K}$. Values of the coefficients of the power series are:

$$
\begin{aligned}
& A=+.4977816011 \times 10^{-2} \\
& B=-.3384077523 \times 10^{-3} \\
& C=+.3521443738 \times 10^{-4} \\
& D=-.1435633178 \times 10^{-6} \\
& E=+.2303247505 \times 10^{-6} \\
& F=-.1038316229 \times 10^{-8}
\end{aligned}
$$

\section{3 Vapor Pressure}

Values used are those given by Hoge and Arnold [1951], at temperatures adjusted by $-0.01^{\circ} \mathrm{K}$ to conform to the NBS 1955 scale for platinum resistance thermometers. After this change the maximum difference between Hoge and Arnold [1951] and Goodwin [1961, 1962] is .01 atm, while the mean difference for Goodwin's 15 points is less than .003 atm.

Smooth interpolation of $\mathrm{P}_{\text {sat }}, \mathrm{dP}_{\text {sat }} / \mathrm{dT}$, and of $\mathrm{d}^{2} \mathrm{P}_{\text {sat }} / \mathrm{dT}^{2}$ close to the critical temperature is required for calculation of the saturated liquid and vapor densities and of the specific heat of the saturated liquid. An interpolation polynomial was used between $30^{\circ} \mathrm{K}$ and the critical point as follows:

$$
\log _{10} \mathrm{P}_{\text {sat }}=\mathrm{A}+\mathrm{BT}+\mathrm{CT}^{2}+\mathrm{DT}^{3} \ldots \ldots
$$

with

$$
\begin{aligned}
& A=-.93945023 \times 10^{+1} \\
& B=+.82691525 \\
& C=-.23118467 \times 10^{-1} \\
& D=+.23345215 \times 10^{-3} .
\end{aligned}
$$

\section{4 Saturated Liquid Density}

The isotherms in the compressed fluid region were expressed as low-order polynomials of pressure in density. These were then iterated to yield the densities at the appropriate vapor pressures. 
The resulting densities can be expressed as a function of temperature as suggested by Hou and Martin [1959],

$$
\rho_{\text {sat }}-\rho_{c}=\sum_{n=1}^{4} A_{n}\left(T_{c}-T\right)^{n / 3} \ldots \ldots .
$$

where

$$
\begin{aligned}
& A_{1}=.62675345 \times 10^{-2} \\
& A_{2}=.14973511 \times 10^{-2} \\
& A_{3}=-.18306903 \times 10^{-3} \\
& A_{4}=-.20693181 \times 10^{-4}
\end{aligned}
$$

with results presented by Goodwin, et al., [1961]. The uncertainty of $\pm 2 \%$ in the critical density from Hoge and Lassiter [1951] implies that (3) should not be extrapolated above $32.5^{\circ} \mathrm{K}$.

\section{5 Saturated Vapor Density}

These densities were obtained in a manner similar to section 2. 4, except that experimental lines of constant volume were also intersected to the respective vapor pressures. Values used are given in Table 1 .

\subsection{The Heat of Vaporization at the Boiling Point}

If (3) is fitted to the saturated vapor data and extrapolated to the normal boiling point, one can compute $\mathrm{V}$ gas $\mathrm{V} \mathrm{V}_{\text {liq }}$ is obtained in similar manner from the saturated liquid data, while $\mathrm{dP} / \mathrm{sat}$ at the normal boiling point is interpolated from the tables by foge and Arnold [1951]. The Clapeyron equation

$$
\frac{\mathrm{dP}_{\text {sat }}}{\mathrm{dT}}=\frac{\Delta \mathrm{H}_{\text {vap }}}{\mathrm{T}\left(\mathrm{V}_{\text {gas }}-\mathrm{V}_{\text {liq }} \mathrm{r}\right.} \ldots . . .
$$

yields a value of $215.3 \mathrm{cal} / \mathrm{mole}$ in excellent agreement with the value of $214.8 \mathrm{cal} / \mathrm{mole}$ given by Johnston [ 1950]. The latter value was used in subsequent computations.

\section{7 The Specific Heat of the Saturated Liquid}

Younglove [1962] recently has measured the specific heat of the saturated liquid at this laboratory. The 33 experimental points in the temperature range of $14.8^{\circ} \mathrm{K}$ to $31.5^{\circ} \mathrm{K}$ are represented by an equation of the form

$$
C_{\text {sat }}=\frac{A T}{\left(T_{c}-T\right)^{n}}+B+C T+D T^{2}+E T^{3} \ldots .
$$


first suggested by Osborne and Van Dusen [1918]. The coefficients in (5) are:

$$
\begin{aligned}
& A=+.43272654 \\
& B=-.13333618 \times 10 \\
& C=+.36251851 \\
& D=-.12869527 \times 10^{-1} \\
& E=+.17227638 \times 10^{-3} \\
& n=0.5
\end{aligned}
$$

Table 1. Densities of Saturated Para Hydrogen Vapor

$\begin{array}{cc}\begin{array}{c}\text { Temperature } \\ { }^{\circ} \mathrm{K}\end{array} & \begin{array}{c}\text { Experimental Density } \\ \text { g mol/cm }\end{array} \\ 22.222 & .0010851 \\ 23.645 & .0014968 \\ 24.000 & .0016161 \\ 25.000 & .0019939 \\ 25.389 & .0021612 \\ 26.000 & .0024447 \\ 26.246 & .0025670 \\ 27.000 & .0029795 \\ 27.571 & .0033329 \\ 28.000 & .0036225 \\ 28.482 & .0039820 \\ 29.000 & .0044107 \\ 29.585 & .0049651 \\ 30.000 & .0054028 \\ 31.000 & .0067190 \\ 31.047 & .0067862 \\ 31.853 & .0083107 \\ 32.000 & .0087699 \\ 32.480 & .0101436 \\ 32.795 & .0118154 \\ 25 & \end{array}$


With $0.1<n<1$ the equation has the required singular point at the critical temperature, yet the integrals

$$
\int_{T}^{T}{ }^{T} C_{\text {sat }} d T \text { and } \quad \int_{T}^{T_{C}} \frac{C_{\text {sat }}}{T} d T
$$

remain finite. The behavior of (5) near the critical point is sensitive of the choice of value for $n$; Equation (5) should not be extrapolated above $32.6^{\circ} \mathrm{K}$.

\section{8 Vapor Pressure vs. Saturated Liquid Density}

For computation of changes in internal energy or enthalpy along the saturated liquid line, a relation between vapor pressure and saturated liquid density is required. The data in sections 2.3 and 2.4 above have been combined in the following expression:

$$
\begin{aligned}
P_{c}-P_{\text {sat }}=A\left(\rho_{\text {sat }}-\rho_{c}\right)^{3} & +B\left(\rho_{\text {sat }}-\rho_{c}\right)^{4}+C\left(\rho_{\text {sat }}-\rho_{c}\right)^{5}+D\left(\rho_{\text {sat }}-\rho_{c}\right)^{6} \\
& +E\left(\rho_{\text {sat }}-\rho_{c}\right)^{7} \ldots \ldots . . . . . . . . .
\end{aligned}
$$

Equation (6) is not valid for temperatures above $32.5^{\circ} \mathrm{K}$. The values of the coefficients in (6) are:

$$
\begin{aligned}
& A=+7.3123950 \times 10^{+6} \\
& B=-6.2975538 \times 10^{+8} \\
& C=+3.4607546 \times 10^{+10} \\
& D=-1.2549385 \times 10^{+12} \\
& E=+1.8502401 \times 10^{+13}
\end{aligned}
$$

\section{9 Selected Parameters}

The value of the normal boiling point temperature was selected from Hoge and Arnold [1951] to be $20.268^{\circ} \mathrm{K}$ after adjustment by $-0.01^{\circ} \mathrm{K}$ from the published value.

The critical constants were selected from Hoge and Lassiter [1951]. After adjusting the critical temperature by $-0.01^{\circ} \mathrm{K}$, the values are:

$$
\begin{array}{ll}
\text { critical temperature, } & 32.984^{\circ} \mathrm{K} ; \\
\text { critical pressure, } & 12.770 \mathrm{~atm} ; \\
\text { critical volume, } & 65.5 \mathrm{~cm}^{3} / \text { mole. }
\end{array}
$$


Experimental work is in progress for more accurate determination of the critical properties.

A value of $4.25 \mathrm{cal} / \mathrm{g}^{\circ} \mathrm{K}$ has been adopted for the entropy at the critical point. This value is thought to be most consistent with the other values tabulated. Extrapolation of (5) yields an erroneous value of $4.036 \mathrm{cal} / \mathrm{g}^{\circ} \mathrm{K}$. The most probable value is $4.17 \mathrm{cal} / \mathrm{g}^{\circ} \mathrm{K}$ obtained by integrating along the $33^{\circ} \mathrm{K}$ isotherm, using $\mathrm{S}^{*}$ as a base to a final density of $0.01555 \mathrm{~mole} / \mathrm{cm}^{3}$. This value for the density is that indicated at present by work in progress on the determination of the rectilinear diameler.

The value of $82.082 \mathrm{~cm}^{3} \mathrm{~atm} / \mathrm{mole}{ }^{\circ} \mathrm{K}$, adopted throughout the computations for the gas constant $R$, is subject to adjustment. Errors from other sources, however, are much more significant; it is felt that no useful purpose would be served by any recalculation. The molecular weight of hydrogen used in the computations is $2.01572 \mathrm{~g} / \mathrm{mole}$. Conversion of calories to joules is accomplished by:

$1 \mathrm{cal}=4.184$ joules.

\section{THE CALCULATED PVT SURFACE}

The basic approximation in these calculations is the substitution of a PVT surface, generated from an equation of state, for the actual PVT data.

\section{1 The Equation of State}

The equation of state used is an adaptation of the Benedict, Webb, Rubin [1940] equation proposed by Strobridge [1962]:

$$
P=A_{1} T \rho+A_{1} A_{2} T \rho^{2}+A_{3} \rho^{2}+\frac{* A_{4} \rho^{2}}{T}+\frac{A_{5} \rho^{2}}{T^{2}}+\frac{*{ }_{6}^{*} \rho^{2}}{T}+A_{7} A_{1} T \rho^{3}+
$$

$$
\mathrm{A}_{8} \rho^{3}+\stackrel{*}{A}_{9} \mathrm{~T} \rho^{4}+\frac{\mathrm{A}_{10} \rho^{3} \mathrm{e}^{-\mathrm{A}_{17} \rho^{2}}}{\mathrm{~T}^{2}}+\frac{\stackrel{*}{A}_{11} \rho^{3} \mathrm{e}^{-\mathrm{A}_{17} \rho^{2}}}{\mathrm{~T}^{3}}+\frac{\stackrel{*}{A}_{12} \rho^{3} \mathrm{e}^{-\mathrm{A}_{17} \rho^{2}}}{\mathrm{~T}^{4}}
$$


$\frac{+\mathrm{A}_{13} \rho^{5} \mathrm{e}^{-\mathrm{A}} 17^{\rho^{2}}}{\mathrm{~T}^{2}}+\frac{\stackrel{*}{A}_{14} \rho^{5} \mathrm{e}^{-\mathrm{A}_{17} \rho^{2}}}{\mathrm{~T}^{3}}+\frac{\mathrm{A}_{15^{*}} \rho^{5} \mathrm{e}^{-\mathrm{A}_{17} \rho^{2}}}{\mathrm{~T}^{4}}+\mathrm{A}_{16} \rho^{6} \ldots$

The starred terms are additions to the original BWR equation.

\section{2 Regions of Application}

Equation (7) first was applied to the entire set of PVT data. The coefficient $A_{17}$ was varied over a wide range of values. A final value of 0.0018 was adopted and is used in both of the following regions of application. These regions were selected to obtain the best possible fit along one side of the two phase boundary, without having to fit the other side at the same time.

(1) The High Temperature Region. The following experimental points were used to determine the coefficients of the PVT surface:

All experimental points at $\mathrm{T} \geq 33^{\circ} \mathrm{K}$;

all experimental points at $\mathrm{T} \geq 33^{\circ} \mathrm{K}$ and $\rho<\rho$;

all saturated vapor line points (these were doubly weighted).

(2) The Compressed Liquid Region. The data used to determine the coefficients include:

All experimental points with $\mathrm{T} \leq 40^{\circ} \mathrm{K}$ and $\rho>\rho$; all points on saturated liquid line (doubly weighted). 
The Coefficients for (7) as determined by least squares are:

High Temperature

$A_{1}=.8208199823 \times 10^{+2}$

$\dot{A}_{2}=.2062278898 \times 10^{+2}$

$A_{3}=-.1292792029 \times 10^{+6}$

$A_{4}=-.7237230137 \times 10^{+7}$

$A_{5}=.1159242745 \times 10^{+9}$

$A_{6}=-.1010879875 \times 10^{+11}$

$A_{7}=.3176293970 \times 10^{+3}$

$A_{8}=.2581305967 \times 10^{+7}$

$A_{9}^{8}=.2410669065 \times 10^{+6}$

$A_{10}=-.1070380625 \times 10^{+11}$

$A_{11}=.1016369054 \times 10^{+13}$

$A_{12}=-.1938431002 \times 10^{+14}$

$A_{13}=.3857308627 \times 10^{+13}$

$A_{14}=-.6757463236 \times 10^{+15}$

$A_{15}=.1462114653 \times 10^{+17}$

$A_{16}=.5254992259 \times 10^{+11}$

$A_{17}=.1800100800 \times 10^{+4}$
Compressed Liquid

$A_{1}=.8208199823 \times 10^{+2}$

$A_{2}=.6374020840 \times 10^{+2}$

$A_{3}=-.3539180407 \times 10^{+6}$

$A_{4}=-.4810952457 \times 10^{+7}$

$A_{5}=.9127883349 \times 10^{+8}$

$A_{6}=-.8816106422 \times 10^{+10}$

$A_{7}=-.1283735749 \times 10^{+4}$

$A_{8}=.8076213444 \times 10^{+7}$

$A_{9}=.1425160973 \times 10^{+7}$

$A_{10}=.6410245277 \times 10^{+10}$

$A_{11}=.1 .085162913 \times 10^{+12}$

$A_{12}=-.2930340262 \times 10^{+13}$

$A_{13}=-.5235483345 \times 10^{+13}$

$A_{14}=-.2551114380 \times 10^{+15}$

$A_{15}=.4732799310 \times 10^{+16}$

$A_{16}=.3522327774 \times 10^{+11}$

$A_{17}=.1800100800 \times 10^{+4}$

\section{3 Deviations}

In the calculation of densities or specific volumes for pressures greater than critical, the compressed liquid PVT surface of (7) was assumed to be valid for temperatures less than or equal to $32^{\circ} \mathrm{K}$, while the high temperature PVT surface was used for temperatures greater than or equal to $33^{\circ} \mathrm{K}$. 
Some continuity of these independently-defined PVT surfaces at their common boundary $\left(32-33^{\circ} \mathrm{K}\right.$, at densities $\left.>\rho_{C}\right)$ was achieved by determining the constants for the compressed liquid surface with data extending up to $40^{\circ} \mathrm{K}$ into the high temperature region. The densities were not smoothed from one surface to the other. All derived properties, however, were smoothed from one surface to the other (see sec. 4.4).

For the high temperature PVT surface the greatest deviations between the surface and the experimental data occur near the critical point. Schematic deviations from isotherms are shown greatly exaggerated in Fig. 1. Near the critical point $(\partial \mathrm{P} / \partial \rho)_{\mathrm{T}} \approx 0$. The pressures are approximated with reasonable deviations; the densities however, are subject to large deviations, i.e., up to $16 \%$. For this reason computations on isobars between 10 atm and $15 \mathrm{~atm}$ were omitted intentionally. Computations utilizing (7) in this area may be subject to serious errors. The deviations of the approximate PVT surface along the saturated vapor line are $0 \%$ deviation in density at $22^{\circ} \mathrm{K}, 0.06 \%$ at $28^{\circ} \mathrm{K}$, and $16 \%$ at $32.795^{\circ} \mathrm{K}$.

For the compressed liquid PVT surface the greatest deviations again occur along the saturated liquid line. At $20^{\circ} \mathrm{K}$ the calculated pressure is $0.18 \mathrm{~atm}$ or $20 \%$ too low. The deviation falls off to $1.5 \%$ at $22^{\circ} \mathrm{K}$ and is negligible above $24^{\circ} \mathrm{K}$.

For other regions the deviations are sufficiently small to be summarized as mean deviations:

Surface

High temperature

Compressed liquid
Density

$\pm 0.00046 \mathrm{~mole} / \mathrm{cm}^{3}$ or $\pm 0.35 \%$

$\pm 0.00027 \mathrm{~mole} / \mathrm{cm}^{3}$ or $\pm 0.14 \%$
Pressure

$\pm 0.18 \mathrm{~atm}$

or $\pm 0.3 \%$

$\pm 0.1 \mathrm{~atm}$

or $\pm 0.9 \%$ 


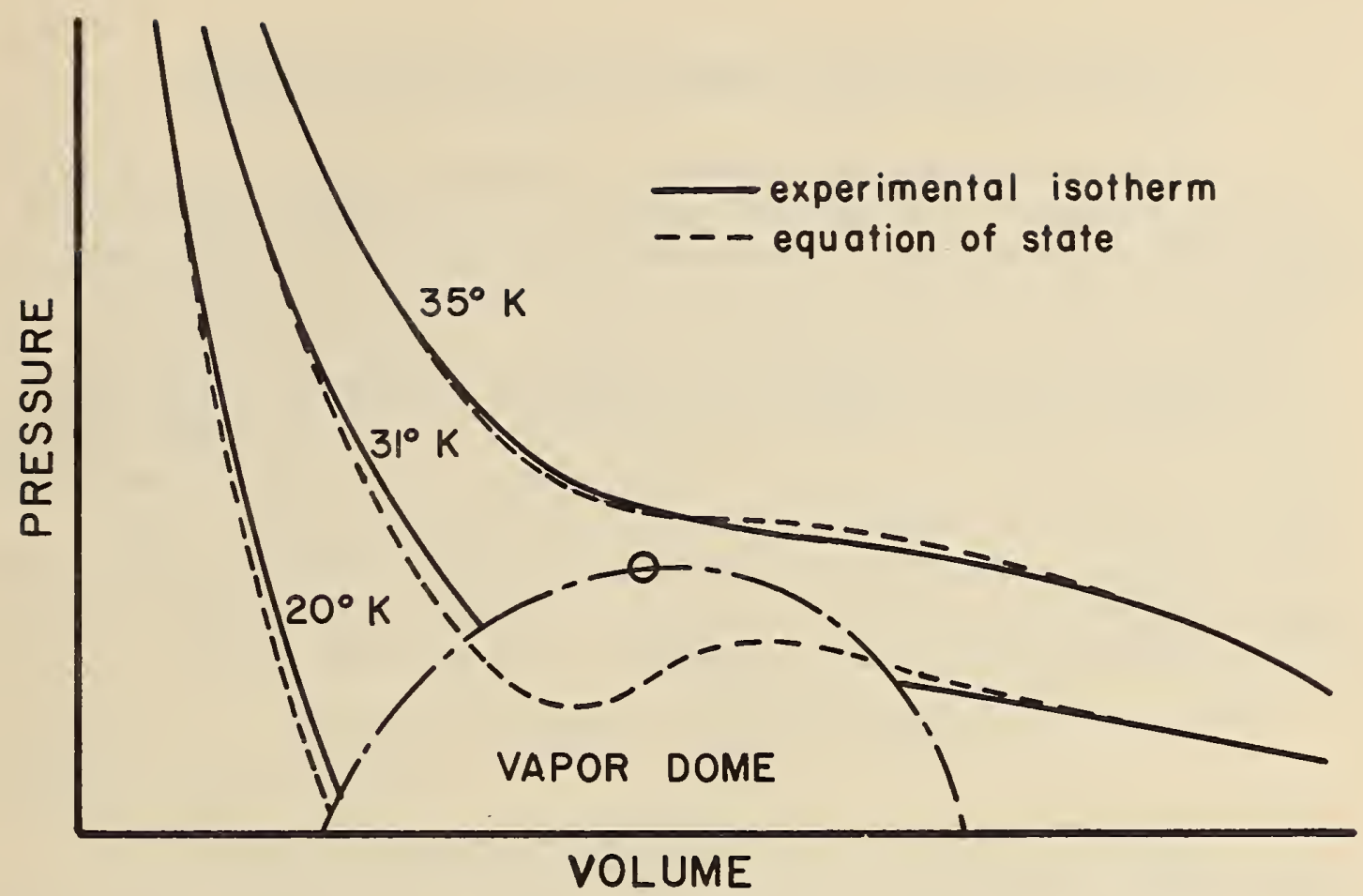

FIGURE I SCHEMATIC DEVIATIONS ALONG ISOTHERMS

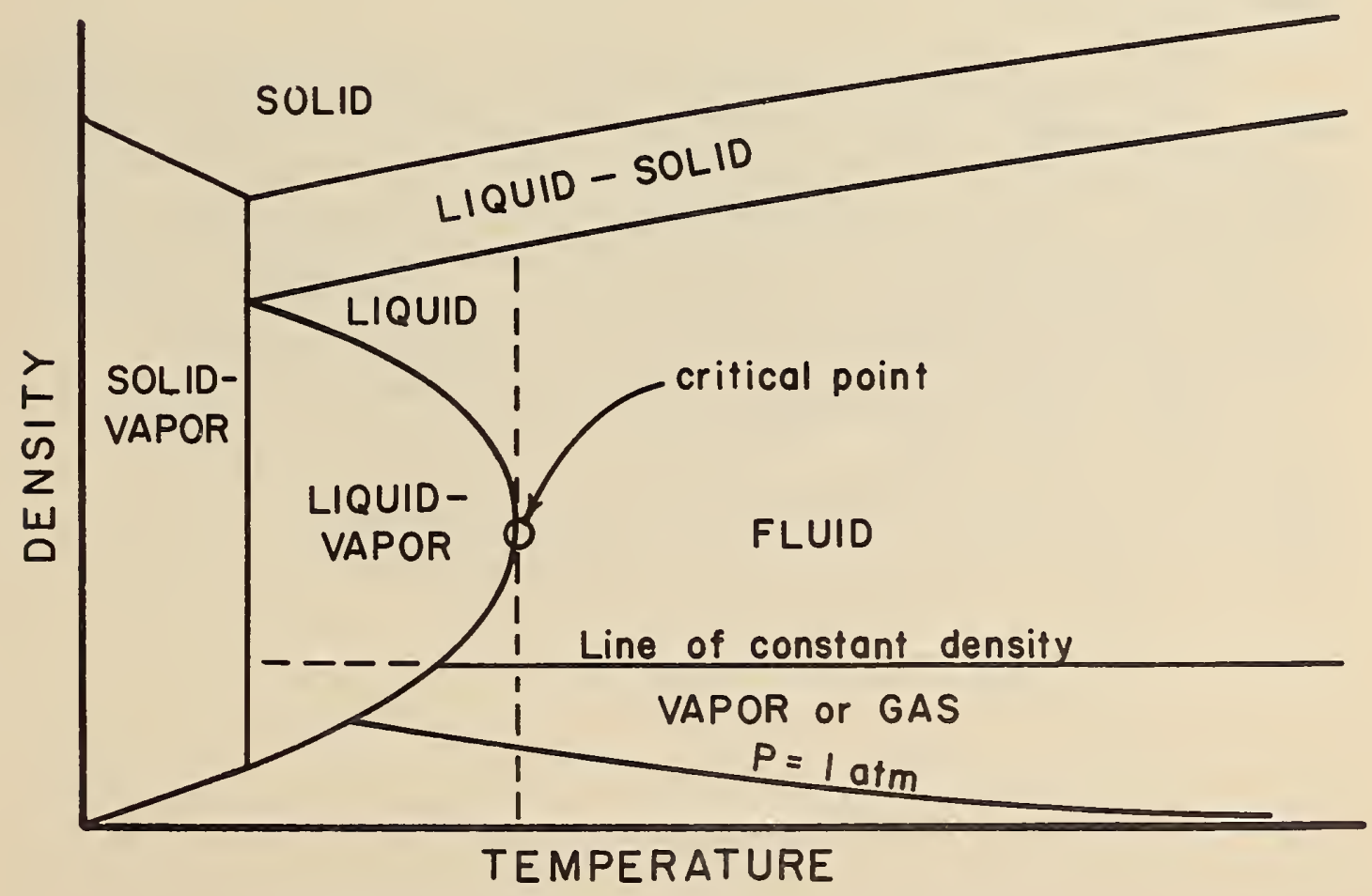

FIGURE 2 SCHEMATIC PHASE DIAGRAM 


\section{THE DERIVED THERMODYNAMIC FUNCTIONS}

The computed thermodynamic functions presented here are entropy, enthalpy, and internal energy. For practical applications pressure is required as an independent variable. For computational purposes, however, density is selected as an independent variable to obtain well-behaved functions. Computations follow horizontal or vertical paths on a density vs. temperature diagram as shown in Figure 2. PVT data are required for computation on paths of constant temperature, while specific heat data are required for computation on paths of constant density or pressure. The following sections discuss the reference values, the equations used in the high temperature and compressed liquid regions respectively, the transition between the two regions, and possible errors.

\section{1 The baseline or reference values}

Specific heat data for the ideal gas are given by Woolley, Scott, and Brickwedde [1948]. These specific heats and the thermodynamic functions derived from them constitute the reference values for computation of the thermodynamic functions in all other states of the fluid by means of PVT data.

For the compressed liquid region the following considerations apply. The vertical paths of computation with PVT data on the $\rho-T$ diagram are blocked below the critical temperature by the coexistence region. Hence a new baseline for thermodynamic functions is established along the saturated liquid line of the phase diagram (Fig. 2). Computations are carried to the co-existence region at the boiling point by means of the virial coefficients, and are carried across the co-existence region by means of the heat of vaporization (sec. 2.6). Properties of the saturated liquid at temperatures other than the boiling point are then computed by means of the specific heat of the saturated liquid (sec. 2.7), the vapor pressure (sec. 2.3), and the saturated liquid density (sec. 2.4). 
4.2 Equations used in the high temperature region

To compute enthalpy:

$\mathrm{H}_{\mathrm{T}, \rho}=\mathrm{H}_{\mathrm{T}}^{\mathrm{O}}+\int_{\mathrm{T}_{0}}^{\mathrm{T}} \mathrm{C}_{\mathrm{p}}^{\mathrm{O}} \mathrm{dT}+\mathrm{RT}(\mathrm{Z}-1)+\int_{0}^{\rho}\left[\frac{\mathrm{P}}{\rho^{2}}-\frac{\mathrm{T}}{\rho^{2}}\left(\frac{\partial \mathrm{P}}{\partial \mathrm{T}}\right)\right]_{\mathrm{T}} \mathrm{d} \rho$

where $\mathrm{H}_{\mathrm{To}}^{\mathrm{O}}$ is the molal enthalpy of the ideal gas at the normal boiling point. The symbol $\mathrm{H}_{\mathrm{To}}^{\mathrm{O}}$ is used for brevity in place of $\mathrm{H}_{20.268}^{\mathrm{O}}-\mathrm{H}_{0}^{\circ}$.

To compute entropy:

$\mathrm{S}_{\mathrm{T}, \rho}=\mathrm{S}^{*} \mathrm{To}+\int_{\mathrm{To}}^{\mathrm{T}} \mathrm{C}_{\mathrm{p}}^{\mathrm{O}} \frac{\mathrm{dT}}{\mathrm{T}}-\mathrm{R} \ln \frac{\mathrm{P}}{\mathrm{Z}}+\int_{\mathrm{O}}^{\rho}\left[\frac{\mathrm{R}}{\rho}-\frac{1}{\rho}\left(\frac{\partial \mathrm{P}}{\partial \mathrm{T}}\right){ }_{\rho}\right] \mathrm{d} \rho$

where $S^{*}$ is the molal entropy of the ideal gas at 1 atm. at the normal boiling point.

To compute internal energy:

$$
\mathrm{U}_{\mathrm{T}, \mathrm{\rho}}=\mathrm{H}_{\mathrm{T}, \rho}-\mathrm{P} \cdot \mathrm{V} \ldots . . \cdot \cdot
$$

Normally the values of enthalpy and entropy for the saturated vapor at the boiling point are calculated from (8) and (9) expressing the PVT behavior by virial coefficients. If the PVT behavior is expressed by (7) the following deviations result: $+0.201 \mathrm{cal} / \mathrm{g}$, $+0.013 \mathrm{cal} / \mathrm{g}^{\circ} \mathrm{K}$. For subsequent comparisons with more accurate values of the thermodynamic functions, it is convenient to establish a defined agreement of values for the vapor at the boiling point. For computations with (7), therefore, the following arbitrary values for the constants in (8) and (9) are selected, differing by above amounts from those of Woolley, Scott and Brickwedde [1948]

$$
\begin{aligned}
\mathrm{S}_{20.268}^{*} & =7.319 \mathrm{cal} / \mathrm{g}^{\circ} \mathrm{K} \text { and } \\
\mathrm{H}_{20.268}^{\circ} & =50.177 \mathrm{cal} / \mathrm{g} .
\end{aligned}
$$


4. 3 Equations used in the compressed liquid region

To compute enthalpy:

$\mathrm{H}_{\mathrm{T}, \rho}=\mathrm{H}_{\mathrm{T}, \rho \text { sat }}+\int_{\rho \text { sat }}^{\rho}\left[\frac{\mathrm{P}}{\rho}-\frac{\mathrm{T}}{\rho}\left(\frac{\partial \mathrm{P}}{\partial \mathrm{T}}\right)_{\rho}\right]_{\mathrm{T}} \mathrm{d} \rho+\frac{\mathrm{P}}{\rho}-\frac{\text { Psat }}{\rho \text { sat }}(1 \mathrm{l})$

To compute entropy:

$\mathrm{S}_{\mathrm{T}, \rho}=\mathrm{S}_{\mathrm{T}, \rho \text { sat }}+\int_{\rho \text { sat }}^{\rho}\left[-\frac{1}{\rho}\left(\frac{\partial \mathrm{P}}{\partial \mathrm{T}}\right)_{\rho}\right]_{\mathrm{T}} \mathrm{d} \rho \ldots . .$.

To compute internal energy, equation (10) was used.

4. 4 The transition between the two regions

The two regions were matched for all pressures greater than 15 atm by utilizing differencing techniques and the empirical relations (13) and (14)

Adjustment in $S=A_{1}+B_{1} \cdot P . \cdots . . .$.
Adjustment in $H=A_{2}+\left(B_{2} \cdot T\right) \cdot P . . . .$.

The adjustments calculated for the 31 and $32^{\circ} \mathrm{K}$ isotherms are added to the values computed from the compressed liquid PVT surface, while those for the 33 thru $37^{\circ} \mathrm{K}$ isotherms are added to the values calculated from the high temperature PVT surface. The smoothing was checked for consistency on a final plot of isobars and isotherms in enthalpy vs. entropy co-ordinates. Minima in the isotherms are in good agreement with the Joule-Thomson inversion characteristics of normal hydrogen as given by Woolley, Scott and Brickwedde [1948]. Table 2 gives the parameters used in (13) and (14)

\section{Table 2}

Adjustments in entropy and enthalpy $31-37^{\circ} \mathrm{K}$

\begin{tabular}{|c|c|c|}
\hline $\mathrm{T}^{\circ} \mathrm{K}$ & $\mathrm{A}_{1}$ & $\mathrm{~B}_{1}$ \\
\hline 31 & .00282 & $.132 \times 10^{-}$ \\
\hline 32 & .02134 & $.464 \times 10^{-5}$ \\
\hline 33 & .11576 & $4.792 \times 10^{-5}$ \\
\hline 34 & .06626 & $3.688 \times 10^{-5}$ \\
\hline 35 & .03494 & $2.964 \times 10^{-5}$ \\
\hline 36 & .01579 & $2.144 \times 10^{-}$ \\
\hline 37 & .00496 & $-\quad 1.084 \times 10^{-}$ \\
\hline
\end{tabular}

$\begin{array}{rrr}\mathrm{A}_{2} & \left(\mathrm{~B}_{2} \cdot \mathrm{T}\right) \\ .087 & -4.1 \times 10^{-5} \\ .637 & -14.8 \times 10^{-5} \\ 3.954 & -158.1 \times 10^{-5} \\ 2.284 & -125.4 \times 10^{-5} \\ 1.213 & -103.7 \times 10^{-5} \\ .554 & -77.2 \times 10^{-5} \\ .184 & -40.1 \times 10^{-5}\end{array}$


Reliable determination of uncertainties in thermodynamic networks is a difficult task. This disucssion is necessarily brief. The difference between the computed reference values at the boiling point and those computed from virial coefficients has already been stated (sec. 4.2). The adjustments between high temperature and compressed liquid regions have also been discussed (sec. 4.4).

An error of $4 \%$ in the saturated vapor volume at $10 \mathrm{~atm}$, resulting from substitution of the PVT surface of (7) for experimental data, leads directly to an error of $4 \%$ in the heat of vaporization, and thus directly to equivalent errors of $0.074 \mathrm{cal} / \mathrm{g}^{\circ} \mathrm{K}$ in the entropy and of $2.316 \mathrm{cal} / \mathrm{g}$ in. the enthalpy of the saturated vapor at $10 \mathrm{~atm}$.

In the compressed liquid region it is possible to compare the derived entropies with values computed from preliminary measurements of specific heat at the constant, triple-point density and the triple-point liquid thermal functions as established in Section 4. 1 [Younglove 1962]. Disagreement in entropy is $1.1 \%$ (equivalent to $5 \mathrm{~atm}$ on $\mathrm{T}-\mathrm{S}$ chart) at $23^{\circ} \mathrm{K}$, and is $2.7 \%$ (equivalent to $24 \mathrm{~atm}$ on $\mathrm{T}-\mathrm{S}$ chart) at $42^{\circ} \mathrm{K}$, the specific heat data in each case indicating a lower value of entropy than given here.

Along the $100^{\circ} \mathrm{K}$ is otherm the agreement with other data is exceedingly good (see sec. 5 ).

\section{EXTENSION OF THE TABULATED VALUES TO $300^{\circ} \mathrm{K}$}

The data of Goodwin $[1961,1962]$ are limited to temper atures below $100^{\circ} \mathrm{K}$. It is possible to extend the tabulations presented here to $300^{\circ} \mathrm{K}$ on isobars between $1 \mathrm{~atm}$ and $100 \mathrm{~atm}$ by using published thermal data for normal hydrogen and the inter polation and conversion procedure proposed by Dean [1961].

The values thus obtained are adjusted slightly to fit smoothly to Goodwin's [1961, 1962] data in the two independent variables, specific volume and enthalpy. Internal energy and entropy are then recalculated from the smoothed values. The adjustments in specific volume and enthalpy were made as follows. The first difference $\left(100^{\circ} \mathrm{K}-101^{\circ} \mathrm{K}\right) \mathrm{calc}$ was set equal to the average of the first differences $\left(99^{\circ} \mathrm{K}-100^{\circ} \mathrm{K}\right)$ Goodwin and $\left(101^{\circ} \mathrm{K}-102^{\circ} \mathrm{K}\right)$ Dean 
The value at $101^{\circ} \mathrm{K}$ calc was then calculated from $100^{\circ} \mathrm{K}$ Goodwin by means of this adjusted first difference. Finally the resulting differance between $101^{\circ} \mathrm{K}$ calc and $101^{\circ} \mathrm{K}$ Dean was applied to all of Dean's entries up to $300^{\circ} \mathrm{K}$. The values of these actual adjustments as weli as the percentage for all isobars at $101^{\circ} \mathrm{K}$ is given in Table 3 . Irregularities in Table 3 occur because (7) is assumed to yield smooth results, while Dean uses 3 different interpolations and changes the order of interpolation within a set of computations.

\section{Table 3}

Adjustments in specific volume and enthalpy at $101^{\circ} \mathrm{K}$

$\begin{array}{ccccc}\begin{array}{c}\text { Pressure } \\ \text { Atm }\end{array} & \begin{array}{l}\text { Delta } \mathrm{H} \\ \text { cal/g }\end{array} & \begin{array}{l}\text { Per cent } \\ \text { Delta H }\end{array} & \begin{array}{c}\text { Delta } \\ \mathrm{cm}^{3} / \mathrm{g}\end{array} & \begin{array}{l}\text { Per cent } \\ \text { Delta V }\end{array} \\ \begin{array}{c}0.2380 \\ 2.0\end{array} & 0.090 & 1.1259 & 0.027 \\ 3.0 & 0.2150 & 0.081 & 0.4475 & 0.022 \\ 4.0 & 0.1930 & 0.073 & 0.2250 & 0.016 \\ 5.0 & 0.1690 & 0.064 & 0.1180 & 0.011 \\ & 0.1480 & 0.056 & 0.0568 & 0.007 \\ 6.0 & 0.1280 & 0.048 & 0.0149 & 0.002 \\ 7.0 & 0.1060 & 0.040 & -0.0177 & -0.003 \\ 8.0 & 0.0850 & 0.032 & -0.0650 & -0.012 \\ 9.0 & 0.0660 & 0.025 & -0.1168 & -0.026 \\ 10.0 & 0.0440 & 0.017 & -0.1629 & -0.040 \\ 15.0 & -0.0560 & -0.022 & -0.2708 & -0.099 \\ 20.0 & -0.1380 & -0.053 & -0.1347 & -0.066 \\ 25.0 & -0.2080 & -0.081 & -0.0793 & -0.048 \\ 30.0 & -0.2750 & -0.108 & -0.0725 & -0.053 \\ 35.0 & -0.3540 & -0.140 & -0.0566 & -0.048 \\ 40.0 & -0.4040 & -0.160 & -0.0398 & -0.038 \\ 45.0 & -0.4200 & -0.167 & -0.0239 & -0.026 \\ 50.0 & -0.4210 & -0.168 & -0.0100 & -0.012 \\ 60.0 & -0.5290 & -0.214 & 0.0182 & 0.026 \\ 70.0 & -0.4780 & -0.195 & 0.0337 & 0.056 \\ 80.0 & -0.4720 & -0.194 & 0.0443 & 0.083 \\ 90.0 & -0.4710 & -0.195 & 0.0504 & 0.106 \\ 100.0 & -0.4540 & -0.189 & 0.0563 & 0.129\end{array}$


6. DESCRIPTION OF THE PROVISIONAL THERMODYNAMIC CHARTS

Temperature-entropy and enthalpy-entropy charts are included as Figures D-20A, D-21 A and D-22A. The intersections of lines of constant density and lines of constant enthalpy with isobars are required for accurate plots in addition to the values given in Section 9. These additional state points were obtained and utilized as outlined below.

$$
\text { 6. } 1 \mathrm{~T}-\mathrm{S} \text { chart, } 20-100^{\circ} \mathrm{K}
$$

Intersections of isobars, with lines of constant enthalpy and constant density, were computed by interative procedures using equations (7), (8), (9), (11), and (12), except in the range of temperatures from $31^{\circ}$ to $37^{\circ} \mathrm{K}$ for densities above critical density. In the latter range the values tabulated in Section 9 were used to obtain graphical interpolations.

Additional values of entropy along lines of constant density were computed at intermediate values of temperature as needed.

$$
\text { Values of } \mathrm{H}_{\mathrm{T}, \rho_{\text {sat }}} \text { and } \mathrm{S}_{\mathrm{T}}, \rho_{\text {sat }} \text {, for use in (11) and (12) at }
$$

intermediate temperatures, were determined by parabolic interpolation.

The properties for the two-phase region were determined for each isobar using the saturated liquid and vapor properties listed in Section 9.

$$
\text { 6. } 2 \mathrm{~T}-\mathrm{S} \text { chart, } 80-300^{\circ} \mathrm{K}
$$

Values in Section 9 were used for temperatures from $100^{\circ}$ to $300^{\circ} \mathrm{K}$ in the construction of this $\mathrm{T}-\mathrm{S}$ chart. The particular values used in the determination of the constant property lines were obtained by parabolic interpolation. The values from $80^{\circ}$ to $100^{\circ} \mathrm{K}$ are the same as those used on the T-S chart from $20^{\circ}$ to $' 100^{\circ} \mathrm{K}$. The constant property lines for this temperature range were determined as outlined above. 


\section{$6.3 \mathrm{H}-\mathrm{S}$ chart, $20-60^{\circ} \mathrm{K}$}

The values of the properties were calculated as outlined in section 6.1. In addition, values of enthalpy and entropy at the intersections of the constant temperature and the constant density lines were computed and plotted.

\section{ACKNOWLEDGEMENTS}

This report incorporates results of research programs in three different sections of the Cryogenic Engineering Division under R. B. Scott. The continuing experimental program under R. J. Corruccini is conducted by a group including the authors, D. E. Diller, L. A. Weber, and B. A. Younglove. A program under B. W. Birmingham for computation and correlation of thermal properties of cryogenic fluids from published data has been conducted by J. W. Dean, D. B. Mann and T. R. Strobridge. Thermal properties of hydrogen above $100^{\circ} \mathrm{K}$ were computed by Dean. Correlation of properties across the boundary at $100^{\circ} \mathrm{K}$ and computer programming for the final tabulations were performed by Mann. The several computer programs required for examination of his modified B. W. R. equation, and for its application to the computation of thermal properties, were developed by Strobridge. The cryogenic data compilation program under V. J. Johnson provided the assistance of R. D. McCarty and R. B. Stewart who programmed the interpolation procedures for use of the digital plotter in preparing the charts. The quality of these charts is due to the painstaking draftsmanship of R. D. Weekley, I. J. Ericks and T. W. Griffith, and careful checking by McCarty and Stewart. 


\section{REFEREINCES}

Benedict, M., G. B. Webb and L. C. Rubin, An empirical equation for thermodynamic properties of light hydrocarbons and their mixtures, J. Chem. Phys. 8, No. 4, 334-345 (April 1940).

Dean, J. W., A tabulation of thermodynamic properties of $n$-hydrogen from low temperatures to $300^{\circ} \mathrm{K}$ and from 1 to $100 \mathrm{~atm}$, NBS TN \#120 (PB161621), Nov., 1961.

Goodwin, R. D., Apparatus for determination of pressure-densitytemperature relations and specific heats of hydrogen to 350 atmospheres at temperatures above $10^{\circ} \mathrm{K}$, J. Research NBS 65C, (Engineering \& Instrumentation), No.4, 231-243 (Oct$\overline{\mathrm{Dec}} ., 1961)$.

Goodwin, R. D., to be published, (1962).

Goodwin, R. D., D. E. Diller, H. M. Roder and L. A. Weber, The densities of saturated liquid hydrogen, Cryogenics 2 , (2), $81-83$ (Dec., 1961).

Hoge, H. J., and R. D. Arnold, Vapor pressures of hydrogen, deuterium, and hydrogen deuteride and dew-point pressures of their mixtures, J. Research NBS 47, No.2, 63-74 (Aug. , 1951).

Hoge, H. J., and J. W. Lassiter, Critical temperatures, pressures, and volumes of hydrogen, deuterium, and hydrogen deuteride, J. Research NBS 47, No.2, 75-79 (Aug., 1951).

Hou, Y. C., and J. J. Martin, Physical and thermodynamic properties of trifluoromethane, A. I. Ch. E. Journal 5, No.1, 125-129 (Mar., 1959).

Johnston, H. L., J. T. Clarke, E. B. Rifkin and E. C. Kerr, Condensed gas calorimetry. I. Heat capacities, latent heats and entropies of pure para-hydrogen from 12.7 to $20.3^{\circ} \mathrm{K}$. Des cription of the condensed gas calorimeter in use in the cryogenic laboratory of the Ohio State University, J. Am. Chem. Soc. 72, No.9, 3933-3938 (Sept., 1950).

Osborne, N. S., and M. S. Van Dusen, Specific heat of liquid ammonia (saturation), J. Am. Chem. Soc. 40, No. 1, (Jan. , 1918). 
Strobridge, T. R., The thermodynamic properties of nitrogen from 64 to $300^{\circ} \mathrm{K}$ between 0.1 and 200 atmospheres, NBS TN \#129 (PB161630), January, 1962.

Woolley, H. W., R. B. Scott and F. G. Brickwedde, Compilation of thermal properties of hydrogen in its various isotopic and ortho-para modifications, J. Research NBS 41, No.5, 379475 (Nov., 1948).

Younglove, B. A., to be published J. Research NBS, (1962). 
9. THE TABLES OF PROVISIONAL THERMODYNAMIC FUNCTIONS FOR PARA-HYDROGEN

The following 26 pages of tables present 35 isobars, distributed as follows: 1 to $10 \mathrm{~atm}$. by $1 ; 15$ to $50 \mathrm{~atm}$. by 5; 60 to $100 \mathrm{~atm}$. by 10; and 120 to 340 atm. by 20 . Column headings also give the units. 


\begin{tabular}{|c|c|c|c|c|c|c|c|c|c|}
\hline $\begin{array}{l}\text { TEMPER- } \\
\text { ATURE } \\
\text { (K) }\end{array}$ & $\begin{array}{l}\text { SPECIFIC } \\
\text { VOLUME } \\
\text { (CC/GM) }\end{array}$ & $\begin{array}{c}\text { ENTHALPY } \\
(J / G M)\end{array}$ & $\begin{array}{l}\text { INTERNAL } \\
\text { ENERGY } \\
(J / G M)\end{array}$ & $\begin{array}{l}\text { ENTROPY } \\
(J / G M-K)\end{array}$ & $\begin{array}{l}\text { TEMPER- } \\
\text { ATURE } \\
(K)\end{array}$ & $\begin{array}{l}\text { SPEC IF IC } \\
\text { VOLUME } \\
\text { (CC/GM) }\end{array}$ & $\begin{array}{c}\text { ENTHALPY } \\
(J / G M)\end{array}$ & $\begin{array}{l}\text { INTERNAL } \\
\text { ENERGY } \\
(J / G M)\end{array}$ & $\begin{array}{l}\text { ENT ROPY } \\
(J / G M-K)\end{array}$ \\
\hline 20.00 & 14.06 & -258.23 & -259.66 & 7.889 & & & & & \\
\hline $\begin{array}{l}20.268 \\
20.268 \\
21.00 \\
22.00 \\
23.00 \\
24.00 \\
25.00 \\
26.00 \\
27.00 \\
28.00 \\
29.00 \\
30.00\end{array}$ & $\begin{array}{r}14.13 \\
746.07 \\
781.25 \\
827.95 \\
873.57 \\
918.44 \\
962.77 \\
1006.68 \\
1050.26 \\
1093.59 \\
1136.69 \\
1179.61\end{array}$ & $\begin{array}{r}-255.65 \\
190.21 \\
199.44 \\
211.50 \\
223.12 \\
234.47 \\
245.63 \\
256.64 \\
267.57 \\
278.42 \\
289.21 \\
299.96\end{array}$ & $\begin{array}{r}-257.08 \\
114.61 \\
120.28 \\
127.60 \\
134.61 \\
141.41 \\
148.07 \\
154.64 \\
161.15 \\
167.61 \\
174.04 \\
180.44\end{array}$ & $\begin{array}{l}8.017 \\
30.016 \\
30.464 \\
31.025 \\
31.542 \\
32.025 \\
32.480 \\
32.912 \\
33.325 \\
33.719 \\
34.098 \\
34.462\end{array}$ & $\begin{array}{r}91.00 \\
92.00 \\
93.00 \\
94.00 \\
95.00 \\
96.00 \\
97.00 \\
98.00 \\
99.00 \\
100.00\end{array}$ & $\begin{array}{l}3702.82 \\
3743.72 \\
3784.61 \\
3825.50 \\
3866.39 \\
3907.27 \\
3948.14 \\
3989.02 \\
4029.89 \\
4070.75\end{array}$ & $\begin{array}{r}975.55 \\
988.26 \\
1001.06 \\
1013.95 \\
1026.92 \\
1039.98 \\
1053.12 \\
1066.34 \\
1079.65 \\
1093.04\end{array}$ & $\begin{array}{l}600.36 \\
608.93 \\
617.58 \\
626.33 \\
635.16 \\
644.07 \\
653.07 \\
662.16 \\
671.33 \\
680.57\end{array}$ & $\begin{array}{l}46.586 \\
46.725 \\
46.864 \\
47.001 \\
47.139 \\
47.275 \\
47.412 \\
47.547 \\
47.682 \\
47.817\end{array}$ \\
\hline $\begin{array}{l}31.00 \\
32.00 \\
33.00 \\
34.00 \\
35.00 \\
36.00 \\
37.00 \\
38.00 \\
39.00 \\
40.00\end{array}$ & $\begin{array}{l}1222.37 \\
1264.99 \\
1307.48 \\
1349.86 \\
1392.15 \\
1434.34 \\
1476.44 \\
1518.47 \\
1560.43 \\
1602.32\end{array}$ & $\begin{array}{l}310.67 \\
321.36 \\
332.02 \\
342.66 \\
353.28 \\
363.89 \\
374.48 \\
385.05 \\
395.62 \\
406.18\end{array}$ & $\begin{array}{l}186.82 \\
193.19 \\
199.54 \\
205.89 \\
212.23 \\
218.55 \\
224.88 \\
231.20 \\
237.51 \\
243.82\end{array}$ & $\begin{array}{l}34.814 \\
35.153 \\
35.481 \\
35.799 \\
36.106 \\
36.405 \\
36.695 \\
36.977 \\
37.252 \\
37.519\end{array}$ & $\begin{array}{l}101.00 \\
102.00 \\
103.00 \\
104.00 \\
105.00 \\
106.00 \\
107.00 \\
108.00 \\
109.00 \\
110.00\end{array}$ & $\begin{array}{l}4111.60 \\
4152.44 \\
4193.27 \\
4234.09 \\
4274.92 \\
4315.74 \\
4356.55 \\
4397.37 \\
4438.18 \\
4478.99\end{array}$ & $\begin{array}{l}1106.52 \\
1120.08 \\
1133.72 \\
1147.45 \\
1161.26 \\
1175.15 \\
1189.13 \\
1203.18 \\
1217.31 \\
1231.53\end{array}$ & $\begin{array}{l}689.90 \\
699.32 \\
708.83 \\
718.42 \\
728.09 \\
737.85 \\
747.69 \\
757.61 \\
767.60 \\
777.68\end{array}$ & $\begin{array}{l}47.951 \\
48.085 \\
48.218 \\
48.350 \\
48.482 \\
48.614 \\
48.745 \\
48.876 \\
49.006 \\
49.136\end{array}$ \\
\hline $\begin{array}{l}41.00 \\
42.00 \\
43.00 \\
44.00 \\
45.00 \\
46.00 \\
47.00 \\
48.00 \\
49.00 \\
50.00\end{array}$ & $\begin{array}{l}1644.15 \\
1685.92 \\
1727.64 \\
1769.32 \\
1810.94 \\
1852.53 \\
1894.07 \\
1935.58 \\
1977.05 \\
2018.49\end{array}$ & $\begin{array}{l}416.73 \\
427.27 \\
437.81 \\
448.34 \\
458.88 \\
469.41 \\
479.95 \\
490.49 \\
501.03 \\
511.57\end{array}$ & $\begin{array}{l}250.14 \\
256.45 \\
262.76 \\
269.07 \\
275.39 \\
281.70 \\
288.03 \\
294.36 \\
300.70 \\
307.05\end{array}$ & $\begin{array}{l}37.780 \\
38.034 \\
38.282 \\
38.524 \\
38.761 \\
38.992 \\
39.219 \\
39.441 \\
39.658 \\
39.871\end{array}$ & $\begin{array}{l}111.00 \\
112.00 \\
113.00 \\
114.00 \\
115.00 \\
116.00 \\
117.00 \\
118.00 \\
119.00 \\
120.00\end{array}$ & $\begin{array}{l}4519.80 \\
4560.61 \\
4601.42 \\
4642.22 \\
4683.03 \\
4723.84 \\
4764.64 \\
4805.45 \\
4846.26 \\
4887.07\end{array}$ & $\begin{array}{l}1245.82 \\
1260.18 \\
1274.62 \\
1289.14 \\
1303.73 \\
1318.39 \\
1333.12 \\
1347.92 \\
1362.79 \\
1377.72\end{array}$ & $\begin{array}{l}787.83 \\
798.07 \\
808.37 \\
818.75 \\
829.21 \\
839.73 \\
850.33 \\
860.99 \\
871.73 \\
882.53\end{array}$ & $\begin{array}{l}49.265 \\
49.394 \\
49.523 \\
49.651 \\
49.778 \\
49.905 \\
50.031 \\
50.157 \\
50.283 \\
50.408\end{array}$ \\
\hline $\begin{array}{l}51.00 \\
52.00 \\
53.00 \\
54.00 \\
55.00 \\
56.00 \\
57.00 \\
58.00 \\
59.00 \\
60.00\end{array}$ & $\begin{array}{l}2059.90 \\
2101.28 \\
2142.63 \\
2183.96 \\
2225.26 \\
2266.54 \\
2307.80 \\
2349.03 \\
2390.25 \\
2431.44\end{array}$ & $\begin{array}{l}522.13 \\
532.70 \\
543.28 \\
553.87 \\
564.48 \\
575.10 \\
585.75 \\
596.42 \\
607.11 \\
617.83\end{array}$ & $\begin{array}{l}313.41 \\
319.79 \\
326.18 \\
332.58 \\
339.00 \\
345.45 \\
351.91 \\
358.40 \\
364.92 \\
371.46\end{array}$ & $\begin{array}{l}40.080 \\
40.285 \\
40.487 \\
40.685 \\
40.879 \\
41.071 \\
41.259 \\
41.445 \\
41.628 \\
41.808\end{array}$ & $\begin{array}{l}121.00 \\
122.00 \\
123.00 \\
124.00 \\
125.00 \\
126.00 \\
127.00 \\
128.00 \\
129.00 \\
130.00\end{array}$ & $\begin{array}{l}4927.87 \\
4968.68 \\
5009.49 \\
5050.31 \\
5091.12 \\
5131.90 \\
5172.68 \\
5213.46 \\
5254.24 \\
5295.02\end{array}$ & $\begin{array}{l}1392.71 \\
1407.76 \\
1422.88 \\
1438.05 \\
1453.28 \\
1468.58 \\
1483.92 \\
1499.33 \\
1514.78 \\
1530.29\end{array}$ & $\begin{array}{l}893.38 \\
904.30 \\
915.28 \\
926.32 \\
937.41 \\
948.57 \\
959.79 \\
971.06 \\
982.38 \\
993.76\end{array}$ & $\begin{array}{l}50.532 \\
50.656 \\
50.779 \\
50.902 \\
51.025 \\
51.146 \\
51.268 \\
51.389 \\
51.509 \\
51.629\end{array}$ \\
\hline $\begin{array}{l}61.00 \\
62.00 \\
63.00 \\
64.00 \\
65.00 \\
66.00 \\
67.00 \\
68.00 \\
69.00 \\
70.00\end{array}$ & $\begin{array}{l}2472.62 \\
2513.79 \\
2554.93 \\
2596.07 \\
2637.18 \\
2678.29 \\
2719.38 \\
2760.46 \\
2801.52 . \\
2842.58\end{array}$ & $\begin{array}{l}628.57 \\
639.35 \\
650.16 \\
661.01 \\
671.89 \\
682.82 \\
693.78 \\
704.79 \\
715.85 \\
726.96\end{array}$ & $\begin{array}{l}378.04 \\
384.64 \\
391.28 \\
397.96 \\
404.68 \\
411.44 \\
418.24 \\
425.09 \\
431.99 \\
438.94\end{array}$ & $\begin{array}{l}41.985 \\
42.161 \\
42.334 \\
42.504 \\
42.673 \\
42.840 \\
43.005 \\
43.168 \\
43.329 \\
43.489\end{array}$ & $\begin{array}{l}131.00 \\
132.00 \\
133.00 \\
134.00 \\
135.00 \\
136.00 \\
137.00 \\
138.00 \\
139.00 \\
140.00\end{array}$ & $\begin{array}{l}5335.79 \\
5376.57 \\
5417.34 \\
5458.12 \\
5498.89 \\
5539.66 \\
5580.44 \\
5621.21 \\
5661.98 \\
5702.76\end{array}$ & $\begin{array}{l}1545.85 \\
1561.46 \\
1577.11 \\
1592.81 \\
1608.56 \\
1624.35 \\
1640.18 \\
1656.05 \\
1671.96 \\
1687.91\end{array}$ & $\begin{array}{l}1005.19 \\
1016.66 \\
1028.18 \\
1039.75 \\
1051.37 \\
1063.03 \\
1074.73 \\
1086.47 \\
1098.24 \\
1110.06\end{array}$ & $\begin{array}{l}51.748 \\
51.867 \\
51.985 \\
52.102 \\
52.219 \\
52.336 \\
52.452 \\
52.567 \\
52.682 \\
52.796\end{array}$ \\
\hline $\begin{array}{l}71.00 \\
72.00 \\
73.00 \\
74.00 \\
75.00 \\
76.00 \\
77.00 \\
78.00 \\
79.00 \\
80.00\end{array}$ & $\begin{array}{l}2883.62 \\
2924.66 \\
2965.68 \\
3006.70 \\
3047.70 \\
3088.70 \\
3129.69 \\
3170.67 \\
3211.64 \\
3252.61\end{array}$ & $\begin{array}{l}738.12 \\
749.33 \\
760.61 \\
771.94 \\
783.33 \\
794.78 \\
806.31 \\
817.90 \\
829.55 \\
841.29\end{array}$ & $\begin{array}{l}445.94 \\
452.99 \\
460.11 \\
467.29 \\
474.52 \\
481.83 \\
489.19 \\
496.63 \\
504.13 \\
511.72\end{array}$ & $\begin{array}{l}43.648 \\
43.804 \\
43.960 \\
44.114 \\
44.267 \\
44.419 \\
44.569 \\
44.719 \\
44.867 \\
45.015\end{array}$ & $\begin{array}{l}141.00 \\
142.00 \\
143.00 \\
144.00 \\
145.00 \\
146.00 \\
147.00 \\
148.00 \\
149.00 \\
150.00\end{array}$ & $\begin{array}{l}5743.53 \\
5784.30 \\
5825.08 \\
5865.85 \\
5906.62 \\
5947.40 \\
5988.17 \\
6028.95 \\
6069.72 \\
6110.50\end{array}$ & $\begin{array}{l}1703.89 \\
1719.91 \\
1735.96 \\
1752.04 \\
1768.15 \\
1784.29 \\
1800.46 \\
1816.65 \\
1832.87 \\
1849.10\end{array}$ & $\begin{array}{l}1121.91 \\
1133.80 \\
1145.72 \\
1157.67 \\
1169.65 \\
1181.65 \\
1193.69 \\
1205.75 \\
1217.83 \\
1229.94\end{array}$ & $\begin{array}{l}52.910 \\
53.023 \\
53.136 \\
53.248 \\
53.360 \\
53.471 \\
53.581 \\
53.691 \\
53.800 \\
53.909\end{array}$ \\
\hline $\begin{array}{l}81.00 \\
82.00 \\
83.00 \\
84.00 \\
85.00 \\
86.00 \\
87.00 \\
88.00 \\
89.00 \\
90.00\end{array}$ & $\begin{array}{l}3293.57 \\
3334.52 \\
3375.46 \\
3416.40 \\
3457.34 \\
3498.26 \\
3539.19 \\
3580.10 \\
3621.02 \\
3661.92\end{array}$ & $\begin{array}{l}853.09 \\
864.97 \\
876.93 \\
888.97 \\
901.09 \\
913.29 \\
925.57 \\
937.94 \\
950.39 \\
962.93\end{array}$ & $\begin{array}{l}519.37 \\
527.10 \\
534.91 \\
542.80 \\
550.77 \\
558.83 \\
566.96 \\
575.18 \\
583.49 \\
591.89\end{array}$ & $\begin{array}{l}45.162 \\
45.307 \\
45.452 \\
45.597 \\
45.740 \\
45.883 \\
46.025 \\
46.166 \\
46.307 \\
46.447\end{array}$ & $\begin{array}{l}151.00 \\
152.00 \\
153.00 \\
154.00 \\
155.00 \\
156.00 \\
157.00 \\
158.00 \\
159.00 \\
160.00\end{array}$ & $\begin{array}{l}6151.25 \\
6192.01 \\
6232.77 \\
6273.52 \\
6314.28 \\
6355.03 \\
6395.79 \\
6436.54 \\
6477.30 \\
6518.05\end{array}$ & $\begin{array}{l}1865.31 \\
1881.53 \\
1897.77 \\
1914.03 \\
1930.30 \\
1946.58 \\
1962.88 \\
1979.18 \\
1995.50 \\
2011.82\end{array}$ & $\begin{array}{l}1242.02 \\
1254.11 \\
1266.22 \\
1278.35 \\
1290.49 \\
1302.64 \\
1314.80 \\
1326.98 \\
1339.17 \\
1351.36\end{array}$ & $\begin{array}{l}54.016 \\
54.123 \\
54.230 \\
54.336 \\
54.441 \\
54.546 \\
54.650 \\
54.753 \\
54.856 \\
54.959\end{array}$ \\
\hline
\end{tabular}


TEMPER- SPECIFIC ENTHALPY INTERNAL ENTROPY

ATURE VOLUME (J/GM) ENERGY (J/GM-K) (K) (CC/GM) (J/GM)
TEMPER-

(K)
SPECIFIC (CC/GM)
ENTHALPY $J / G M$

(1)

INTERNAL ENTROPY

NERGY

(J/GM]
161.00
162.00
163.00
164.00
165.00
166.00
167.00
168.00
169.00
170.00

171.00

172.00

173.00

174.00

175.00

176.00

177.00

178.00

179.00

180.00

181.00

182.00

183.00

184.00

185.00

186.00

187.00

188.00

189.00

190.00

191.00

192.00

193.00

194.00

195.00

196.00

197.00

198.00

199.00

200.00

201.00

202.00

203.00

204.00

205.00

206.00

207.00

208.00

209.00

210.00

211.00

212.00

213.00

214.00

215.00

216.00

217.00

218.00

219.00

220.00

221.00

222.00

223.00

224.00

225.00

226.00

227.00

228.00

229.00

230.00

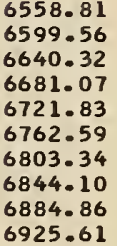

6966.36

7007.11

7047.85

7088.60

7129.34

7170.08

7210.83

7251.57

7292.31

7333.05

7373.79

7414.53
7455.27

7496.01

7536.75

7577.49

7618.23

7658.97

7699.71

7740.45

7781.19

7821.93

7862.67

7903.40

7944.14

7984.88

8025.62

8066.36

8107.09

8147.83

8188.56

8229.28

8270.01

8310.73

8351.45

8392.18

8432.90

8473.63

8514.35

8555 .08-

8595.80

8636.53

8677.26

8717.99

8758.72

8799.45

8840.18

8880.91

8921.64

8962.38

9003.12

9043.85

9084.59

9125.33

9166.07

9206.81

9247.55

9288.29

9329.03

9369.77

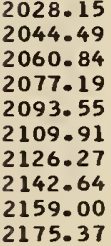

2191.73

2208.10

2224.46

2240.82

2257.18

2273.54

2289.89

2306.23

2322.57

2338.90

2355.23

2371.55

2387.86

2404.16

2420.45

2436.73

2453.00

2469.27

2485.52

2501.76

2517.98

2534.20

2550.40

2566.59

2582.77

2598.93

2615.08

2631.22

2647.34

2663.44

2679.52

2695.59

2711.64

2727.68

2743.69

2759.70

2775.69

2791.66

2807.62

2823.56

2839.48

2855.39

2871.28

2887.15

2903.01

2918.85

2934.68

2950.49

2966.28

2982.06

2997.82

3013.56

3029.29

3045.00

3060.70

3076.38

3092.04

3107.69

3123.32

3138.93
1363.57

375.78

1387.99

1400.21

1412.44

1424.67

1436.90

1449.14

1461.37

1473.61

1485.85

1498.08

1510.32

1522.55

1534.78

1547.01

1559.23

1571.45

1583.66

1595.86

1608.06

1620.25

1632.43

1644.60

1656.77

1668.92

1681.07

1693.20

1705.32

1717.43

1729.53

1741.62

1753.70

1765.76

1777.81

1789.84

1801.86

1813.87

1825.86

1837.84

1849.80

1861.73

1873.66

1885.57

1897.46

1909.34

1921.20

1933.05

1944.88

1956.69

1968.49

1992.03

2003.78

2015.51

2027.23

2038.93

2050.61

2062.27

2073.92

2085.56

2097.17

2108.77

2120.36

2131.92

2143.47

2155.01

2166.53

2178.03

2189.52

5.060

55.162
55.262

55.362

55.462

55.560

55.659

55.756

55.854

55.950

56.046 


\begin{tabular}{|c|c|c|c|c|c|c|c|c|c|}
\hline $\begin{array}{l}\text { TEMPER- } \\
\text { ATURE } \\
\text { (K) }\end{array}$ & $\begin{array}{l}\text { SPEC IF IC } \\
\text { VOLUME } \\
\text { (CC/GM) }\end{array}$ & $\begin{array}{l}\text { ENTHALPY } \\
(J / G M)\end{array}$ & $\begin{array}{l}\text { I NTERNAL } \\
\text { ENERGY } \\
\text { (J/GM) }\end{array}$ & $\begin{array}{l}\text { ENTROPY } \\
(J / G M-K)\end{array}$ & $\begin{array}{c}\text { TEMPER- } \\
\text { ATURE } \\
(K)\end{array}$ & $\begin{array}{l}\text { SPEC IF IC } \\
\text { VOLUME } \\
\text { (CC/GM) }\end{array}$ & $\begin{array}{l}\text { ENTHALPY } \\
(J / G M)\end{array}$ & $\begin{array}{l}\text { INTERNAL } \\
\text { ENERGY } \\
(J / G M)\end{array}$ & $\begin{array}{l}\text { ENTROPY } \\
(J / G M-K)\end{array}$ \\
\hline 20.00 & 14.03 & -257.27 & -260.12 & 7.866 & & & & & \\
\hline $\begin{array}{l}21.00 \\
22.00 \\
22.855 \\
22.855 \\
23.00 \\
24.00 \\
25.00 \\
26.00 \\
27.00 \\
28.00 \\
29.00 \\
30.00\end{array}$ & $\begin{array}{r}14.27 \\
14.54 \\
14.80 \\
394.76 \\
398.71 \\
425.02 \\
450.19 \\
474.57 \\
498.39 \\
521.80 \\
544.87 \\
567.69\end{array}$ & $\begin{array}{r}-247.44 \\
-236.94 \\
-227.36 \\
201.59 \\
203.66 \\
217.31 \\
230.14 \\
242.43 \\
254.36 \\
266.04 \\
277.54 \\
288.89\end{array}$ & $\begin{array}{r}-250.33 \\
-239.89 \\
-230.36 \\
121.60 \\
122.87 \\
131.18 \\
138.90 \\
146.26 \\
153.36 \\
160.30 \\
167.12 \\
173.85\end{array}$ & $\begin{array}{r}8.348 \\
8.834 \\
9.262 \\
28.037 \\
28.127 \\
28.708 \\
29.231 \\
29.714 \\
30.164 \\
30.589 \\
30.992 \\
31.377\end{array}$ & $\begin{array}{r}91.00 \\
92.00 \\
93.00 \\
94.00 \\
95.00 \\
96.00 \\
97.00 \\
98.00 \\
99.00 \\
100.00\end{array}$ & $\begin{array}{l}1850.11 \\
1870.65 \\
1891.18 \\
1911.71 \\
1932.24 \\
1952.76 \\
1973.27 \\
1993.78 \\
2014.29 \\
2034.80\end{array}$ & $\begin{array}{r}973.63 \\
986.38 \\
999.22 \\
1012.14 \\
1025.15 \\
1038.25 \\
1051.43 \\
1064.69 \\
1078.03 \\
1091.46\end{array}$ & $\begin{array}{l}598.70 \\
607.29 \\
615.97 \\
624.73 \\
633.59 \\
642.52 \\
651.55 \\
660.65 \\
669.84 \\
679.11\end{array}$ & $\begin{array}{l}43.708 \\
43.848 \\
43.986 \\
44.125 \\
44.262 \\
44.399 \\
44.536 \\
44.672 \\
44.807 \\
44.942\end{array}$ \\
\hline $\begin{array}{l}31.00 \\
32.00 \\
33.00 \\
34.00 \\
35.00 \\
36.00 \\
37.00 \\
38.00 \\
39.00 \\
40.00\end{array}$ & $\begin{array}{l}590.29 \\
612.70 \\
634.97 \\
657.09 \\
679.10 \\
701.00 \\
722.81 \\
744.52 \\
766.16 \\
787.73\end{array}$ & $\begin{array}{l}300.14 \\
311.30 \\
322.39 \\
333.42 \\
344.41 \\
355.35 \\
366.25 \\
377.13 \\
387.97 \\
398.79\end{array}$ & $\begin{array}{l}180.51 \\
187.13 \\
193.72 \\
200.26 \\
206.79 \\
213.29 \\
219.78 \\
226.25 \\
232.71 \\
239.16\end{array}$ & $\begin{array}{l}31.746 \\
32.100 \\
32.442 \\
32.771 \\
33.089 \\
33.398 \\
33.696 \\
33.986 \\
34.268 \\
34.542\end{array}$ & $\begin{array}{l}101.00 \\
102.00 \\
103.00 \\
104.00 \\
105.00 \\
106.00 \\
107.00 \\
108.00 \\
109.00 \\
110.00\end{array}$ & $\begin{array}{l}2055.29 \\
2075.78 \\
2096.25 \\
2116.73 \\
2137.20 \\
2157.67 \\
2178.14 \\
2198.60 \\
2219.07 \\
2239.53\end{array}$ & $\begin{array}{l}1104.97 \\
1118.56 \\
1132.23 \\
1145.99 \\
1159.83 \\
1173.75 \\
1187.75 \\
1201.83 \\
1215.99 \\
1230.23\end{array}$ & $\begin{array}{l}688.45 \\
697.89 \\
707.42 \\
717.02 \\
726.71 \\
736.49 \\
746.34 \\
756.27 \\
766.28 \\
776.38\end{array}$ & $\begin{array}{l}45.077 \\
45.211 \\
45.344 \\
45.477 \\
45.609 \\
45.741 \\
45.873 \\
46.004 \\
46.134 \\
46.264\end{array}$ \\
\hline $\begin{array}{l}41.00 \\
42.00 \\
43.00 \\
44.00 \\
45.00 \\
46.00 \\
47.00 \\
48.00 \\
49.00 \\
50.00\end{array}$ & $\begin{array}{r}809.23 \\
830.68 \\
852.06 \\
873.39 \\
894.68 \\
915.92 \\
937.12 \\
958.28 \\
979.40 \\
1000.49\end{array}$ & $\begin{array}{l}409.59 \\
420.37 \\
431.14 \\
441.89 \\
452.63 \\
463.35 \\
474.08 \\
484.79 \\
495.50 \\
506.21\end{array}$ & $\begin{array}{l}245.60 \\
252.04 \\
258.47 \\
264.89 \\
271.32 \\
277.74 \\
284.17 \\
290.60 \\
297.03 \\
303.47\end{array}$ & $\begin{array}{l}34.809 \\
35.069 \\
35.322 \\
35.569 \\
35.810 \\
36.046 \\
36.277 \\
36.502 \\
36.723 \\
36.939\end{array}$ & $\begin{array}{l}111.00 \\
112.00 \\
113.00 \\
114.00 \\
115.00 \\
116.00 \\
117.00 \\
118.00 \\
119.00 \\
120.00\end{array}$ & $\begin{array}{l}2259.99 \\
2280.44 \\
2300.90 \\
2321.35 \\
2341.81 \\
2362.26 \\
2382.71 \\
2403.17 \\
2423.62 \\
2444.07\end{array}$ & $\begin{array}{l}1244.54 \\
1258.93 \\
1273.40 \\
1287.94 \\
1302.55 \\
1317.23 \\
1331.98 \\
1346.80 \\
1361.69 \\
1376.65\end{array}$ & $\begin{array}{l}786.54 \\
796.79 \\
807.11 \\
817.50 \\
827.97 \\
838.51 \\
849.11 \\
859.79 \\
870.53 \\
881.34\end{array}$ & $\begin{array}{l}46.394 \\
46.523 \\
46.652 \\
46.780 \\
46.907 \\
47.034 \\
47.161 \\
47.287 \\
47.413 \\
47.538\end{array}$ \\
\hline $\begin{array}{l}51.00 \\
52.00 \\
53.00 \\
54.00 \\
55.00 \\
56.00 \\
57.00 \\
58.00 \\
59.00 \\
60.00\end{array}$ & $\begin{array}{l}1021.55 \\
1042.57 \\
1063.57 \\
1084.54 \\
1105.49 \\
1126.41 \\
1147.31 \\
1168.19 \\
1189.05 \\
1209.89\end{array}$ & $\begin{array}{l}516.93 \\
527.64 \\
538.37 \\
549.10 \\
559.84 \\
570.59 \\
581.36 \\
592.15 \\
602.96 \\
613.78\end{array}$ & $\begin{array}{l}309.91 \\
316.37 \\
322.84 \\
329.32 \\
335.81 \\
342.33 \\
348.86 \\
355.42 \\
362.00 \\
368.60\end{array}$ & $\begin{array}{l}37.152 \\
37.360 \\
37.564 \\
37.765 \\
37.962 \\
38.155 \\
38.346 \\
38.534 \\
38.718 \\
38.900\end{array}$ & $\begin{array}{l}121.00 \\
122.00 \\
123.00 \\
124.00 \\
125.00 \\
126.00 \\
127.00 \\
128.00 \\
129.00 \\
130.00\end{array}$ & $\begin{array}{l}2464.52 \\
2484.97 \\
2505.42 \\
2525.87 \\
2546.32 \\
2566.76 \\
2587.19 \\
2607.62 \\
2628.05 \\
2648.47\end{array}$ & $\begin{array}{l}1391.66 \\
1406.73 \\
1421.86 \\
1437.05 \\
1452.31 \\
1467.61 \\
1482.98 \\
1498.40 \\
1513.87 \\
1529.40\end{array}$ & $\begin{array}{l}892.21 \\
903.14 \\
914.12 \\
925.17 \\
936.28 \\
947.45 \\
958.67 \\
969.95 \\
981.28 \\
992.67\end{array}$ & $\begin{array}{l}47.663 \\
47.787 \\
47.910 \\
48.033 \\
48.156 \\
48.278 \\
48.399 \\
48.520 \\
48.640 \\
48.760\end{array}$ \\
\hline $\begin{array}{l}61.00 \\
62.00 \\
63.00 \\
64.00 \\
65.00 \\
66.00 \\
67.00 \\
68.00 \\
69.00 \\
70.00\end{array}$ & $\begin{array}{l}1230.71 \\
1251.51 \\
1272.30 \\
1293.07 \\
1313.83 \\
1334.58 \\
1355.31 \\
1376.03 \\
1396.74 \\
1417.43\end{array}$ & $\begin{array}{l}624.64 \\
635.52 \\
646.43 \\
657.37 \\
668.34 \\
679.36 \\
690.41 \\
701.51 \\
712.64 \\
723.83\end{array}$ & $\begin{array}{l}375.23 \\
381.90 \\
388.59 \\
395.33 \\
402.09 \\
408.91 \\
415.76 \\
422.65 \\
429.60 \\
436.59\end{array}$ & $\begin{array}{l}39.080 \\
39.257 \\
39.431 \\
39.604 \\
39.774 \\
39.942 \\
40.108 \\
40.272 \\
40.435 \\
40.596\end{array}$ & $\begin{array}{l}131.00 \\
132.00 \\
133.00 \\
134.00 \\
135.00 \\
136.00 \\
137.00 \\
138.00 \\
139.00 \\
140.00\end{array}$ & $\begin{array}{l}2668.90 \\
2689.32 \\
2709.75 \\
2730.17 \\
2750.59 \\
2771.01 \\
2791.43 \\
2811.85 \\
2832.27 \\
2852.69\end{array}$ & $\begin{array}{l}1544.97 \\
1560.59 \\
1576.26 \\
1591.98 \\
1607.74 \\
1623.55 \\
1639.39 \\
1655.28 \\
1671.20 \\
1687.16\end{array}$ & $\begin{array}{l}1004.10 \\
1015.59 \\
1027.12 \\
1038.70 \\
1050.32 \\
1061.99 \\
1073.69 \\
1085.44 \\
1097.23 \\
1109.05\end{array}$ & $\begin{array}{l}48.880 \\
48.998 \\
49.117 \\
49.234 \\
49.352 \\
49.468 \\
49.584 \\
49.700 \\
49.815 \\
49.929\end{array}$ \\
\hline $\begin{array}{l}71.00 \\
72.00 \\
73.00 \\
74.00 \\
75.00 \\
76.00 \\
77.00 \\
78.00 \\
79.00 \\
80.00\end{array}$ & $\begin{array}{l}1438.12 \\
1458.79 \\
1479.45 \\
1500.11 \\
1520.76 \\
1541.39 \\
1562.02 \\
1582.64 \\
1603.25 \\
1623.86\end{array}$ & $\begin{array}{l}735.07 \\
746.35 \\
757.70 \\
769.10 \\
780.55 \\
792.08 \\
803.66 \\
815.31 \\
827.03 \\
838.82\end{array}$ & $\begin{array}{l}443.63 \\
450.73 \\
457.89 \\
465.10 \\
472.38 \\
479.71 \\
487.12 \\
494.59 \\
502.13 \\
509.75\end{array}$ & $\begin{array}{l}40.755 \\
40.913 \\
41.070 \\
41.225 \\
41.379 \\
41.531 \\
41.683 \\
41.833 \\
41.982 \\
42.131\end{array}$ & $\begin{array}{l}141.00 \\
142.00 \\
143.00 \\
144.00 \\
145.00 \\
146.00 \\
147.00 \\
148.00 \\
149.00 \\
150.00\end{array}$ & $\begin{array}{l}2873.11 \\
2893.53 \\
2913.95 \\
2934.36 \\
2954.78 \\
2975.20 \\
2995.61 \\
3016.03 \\
3036.44 \\
3056.86\end{array}$ & $\begin{array}{l}1703.16 \\
1719.19 \\
1735.25 \\
1751.35 \\
1767.47 \\
1783.63 \\
1799.81 \\
1816.01 \\
1832.24 \\
1848.49\end{array}$ & $\begin{array}{l}1120.91 \\
1132.80 \\
1144.73 \\
1156.68 \\
1168.67 \\
1180.69 \\
1192.73 \\
1204.80 \\
1216.89 \\
1229.00\end{array}$ & $\begin{array}{l}50.043 \\
50.156 \\
50.269 \\
50.381 \\
50.493 \\
50.604 \\
50.714 \\
50.824 \\
50.933 \\
51.042\end{array}$ \\
\hline $\begin{array}{l}81.00 \\
82.00 \\
83.00 \\
84.00 \\
85.00 \\
86.00 \\
87.00 \\
88.00 \\
89.00 \\
90.00\end{array}$ & $\begin{array}{l}1644.46 \\
1665.05 \\
1685.64 \\
1706.22 \\
1726.79 \\
1747.36 \\
1767.92 \\
1788.47 \\
1809.03 \\
1829.57\end{array}$ & $\begin{array}{l}850.68 \\
862.62 \\
874.63 \\
886.72 \\
898.89 \\
911.13 \\
923.47 \\
935.88 \\
948.37 \\
960.96\end{array}$ & $\begin{array}{l}517.43 \\
525.20 \\
533.03 \\
540.95 \\
548.95 \\
557.03 \\
565.20 \\
573.45 \\
581.78 \\
590.20\end{array}$ & $\begin{array}{l}42.278 \\
42.424 \\
42.570 \\
42.715 \\
42.859 \\
43.002 \\
43.145 \\
43.286 \\
43.428 \\
43.568\end{array}$ & $\begin{array}{l}151.00 \\
152.00 \\
153.00 \\
154.00 \\
155.00 \\
156.00 \\
157.00 \\
158.00 \\
159.00 \\
160.00\end{array}$ & $\begin{array}{l}3077.27 \\
3097.67 \\
3118.07 \\
3138.48 \\
3158.88 \\
3179.28 \\
3199.69 \\
3220.09 \\
3240.49 \\
3260.89\end{array}$ & $\begin{array}{l}1864.71 \\
1880.95 \\
1897.20 \\
1913.47 \\
1929.75 \\
1946.05 \\
1962.35 \\
1978.67 \\
1995.00 \\
2011.34\end{array}$ & $\begin{array}{l}1241.09 \\
1253.19 \\
1265.31 \\
1277.44 \\
1289.59 \\
1301.75 \\
1313.92 \\
1326.10 \\
1338.30 \\
1350.50\end{array}$ & $\begin{array}{l}51.150 \\
51.257 \\
51.364 \\
51.470 \\
51.575 \\
51.680 \\
51.784 \\
51.888 \\
51.991 \\
52.093\end{array}$ \\
\hline
\end{tabular}


TEMPER- SPECIFIC ENTHALPY INTERNAL ENTROPY ATURE VOLUME (J/GM) ENERGY (J/GM-K) (K) (CC/GM)
(J/GM)
TEMPER- SPECIFIC ENTHALPY INTERNAL ENTROPY ATURE VOLUME (J/GM) ENERGY (J/GM-K) (K) $\quad(C C / G M) \quad(J / G M)$

\begin{tabular}{|c|c|c|c|c|c|c|c|c|c|}
\hline $\begin{array}{l}161.00 \\
162.00 \\
163.00 \\
164.00 \\
165.00 \\
166.00 \\
167.00 \\
168.00 \\
169.00 \\
170.00\end{array}$ & $\begin{array}{l}3281.29 \\
3301.69 \\
3322.09 \\
3342.50 \\
3362.90 \\
3383.30 \\
3403.70 \\
3424.10 \\
3444.50 \\
3464.90\end{array}$ & $\begin{array}{l}2027.68 \\
2044.03 \\
2060.39 \\
2076.76 \\
2093.12 \\
2109.50 \\
2125.87 \\
2142.24 \\
2158.62 \\
2175.00\end{array}$ & $\begin{array}{l}1362.71 \\
1374.93 \\
1387.15 \\
1399.38 \\
1411.61 \\
1423.85 \\
1436.09 \\
1448.33 \\
1460.58 \\
1472.82\end{array}$ & $\begin{array}{l}52.195 \\
52.296 \\
52.397 \\
52.497 \\
52.596 \\
52.695 \\
52.794 \\
52.891 \\
52.989 \\
53.085\end{array}$ & $\begin{array}{l}231.00 \\
232.00 \\
233.00 \\
234.00 \\
235.00 \\
236.00 \\
237.00 \\
238.00 \\
239.00 \\
240.00\end{array}$ & $\begin{array}{l}4708.26 \\
4728.65 \\
4749.04 \\
4769.42 \\
4789.81 \\
4810.20 \\
4830.58 \\
4850.96 \\
4871.34 \\
4891.71\end{array}$ & $\begin{array}{l}3154.61 \\
3170.20 \\
3185.78 \\
3201.34 \\
3216.88 \\
3232.41 \\
3247.92 \\
3263.41 \\
3278.89 \\
3294.35\end{array}$ & $\begin{array}{l}2200.45 \\
2211.91 \\
2223.36 \\
2234.79 \\
2246.20 \\
2257.60 \\
2268.98 \\
2280.34 \\
2291.69 \\
2303.02\end{array}$ & $\begin{array}{l}58.016 \\
58.083 \\
58.150 \\
58.217 \\
58.283 \\
58.349 \\
58.415 \\
58.480 \\
58.545 \\
58.609\end{array}$ \\
\hline $\begin{array}{l}171.00 \\
172.00 \\
173.00 \\
174.00 \\
175.00 \\
176.00 \\
177.00 \\
178.00 \\
179.00 \\
180.00\end{array}$ & $\begin{array}{l}3485.29 \\
3505.69 \\
3526.08 \\
3546.47 \\
3566.86 \\
3587.25 \\
3607.65 \\
3628.04 \\
3648.43 \\
3668.82\end{array}$ & $\begin{array}{l}2191.38 \\
2207.75 \\
2224.13 \\
2240.50 \\
2256.86 \\
2273.23 \\
2289.59 \\
2305.94 \\
2322.29 \\
2338.63\end{array}$ & $\begin{array}{l}1485.06 \\
1497.31 \\
1509.55 \\
1521.79 \\
1534.02 \\
1546.25 \\
1558.48 \\
1570.70 \\
1582.92 \\
1595.13\end{array}$ & $\begin{array}{l}53.181 \\
53.277 \\
53.372 \\
53.466 \\
53.560 \\
53.653 \\
53.746 \\
53.838 \\
53.930 \\
54.021\end{array}$ & $\begin{array}{l}241.00 \\
242.00 \\
243.00 \\
244.00 \\
245.00 \\
246.00 \\
247.00 \\
248.00 \\
249.00 \\
250.00\end{array}$ & $\begin{array}{l}4912.09 \\
4932.46 \\
4952.82 \\
4973.19 \\
4993.55 \\
5013.90 \\
5034.26 \\
5054.60 \\
5074.95 \\
5095.29\end{array}$ & $\begin{array}{l}3309.80 \\
3325.22 \\
3340.64 \\
3356.03 \\
3371.41 \\
3386.77 \\
3402.12 \\
3417.45 \\
3432.77 \\
3448.07\end{array}$ & $\begin{array}{l}2314.34 \\
2325.63 \\
2336.92 \\
2348.19 \\
2359.44 \\
2370.68 \\
2381.90 \\
2393.11 \\
2404.30 \\
2415.48\end{array}$ & $\begin{array}{l}58.674 \\
58.737 \\
58.801 \\
58.864 \\
58.927 \\
58.990 \\
59.052 \\
59.114 \\
59.176 \\
59.237\end{array}$ \\
\hline $\begin{array}{l}181.00 \\
182.00 \\
183.00 \\
184.00 \\
185.00 \\
186.00 \\
187.00 \\
188.00 \\
189.00 \\
190.00\end{array}$ & $\begin{array}{l}3689.20 \\
3709.59 \\
3729.98 \\
3750.37 \\
3770.76 \\
3791.14 \\
3811.53 \\
3831.92 \\
3852.31 \\
3872.69\end{array}$ & $\begin{array}{l}2354.97 \\
2371.29 \\
2387.61 \\
2403.92 \\
2420.22 \\
2436.51 \\
2452.79 \\
2469.06 \\
2485.32 \\
2501.57\end{array}$ & $\begin{array}{l}1607.33 \\
1619.52 \\
1631.71 \\
1643.89 \\
1656.06 \\
1668.21 \\
1680.36 \\
1692.50 \\
1704.63 \\
1716.74\end{array}$ & $\begin{array}{l}54.111 \\
54.201 \\
54.290 \\
54.379 \\
54.468 \\
54.556 \\
54.643 \\
54.730 \\
54.816 \\
54.902\end{array}$ & $\begin{array}{l}251.00 \\
252.00 \\
253.00 \\
254.00 \\
255.00 \\
256.00 \\
257.00 \\
258.00 \\
259.00 \\
260.00\end{array}$ & $\begin{array}{l}5115.62 \\
5135.96 \\
5156.28 \\
5176.61 \\
5196.93 \\
5217.25 \\
5237.56 \\
5257.87 \\
5278.18 \\
5298.48\end{array}$ & $\begin{array}{l}3463.39 \\
3478.70 \\
3493.99 \\
3509.26 \\
3524.53 \\
3539.77 \\
3555.01 \\
3570.23 \\
3585.43 \\
3600.63\end{array}$ & $\begin{array}{l}2426.68 \\
2437.87 \\
2449.04 \\
2460.20 \\
2471.34 \\
2482.47 \\
2493.59 \\
2504.69 \\
2515.78 \\
2526.86\end{array}$ & $\begin{array}{l}59.298 \\
59.359 \\
59.419 \\
59.480 \\
59.540 \\
59.599 \\
59.659 \\
59.718 \\
59.777 \\
59.835\end{array}$ \\
\hline $\begin{array}{l}191.00 \\
192.00 \\
193.00 \\
194.00 \\
195.00 \\
196.00 \\
197.00 \\
198.00 \\
199.00 \\
200.00\end{array}$ & $\begin{array}{l}3893.08 \\
3913.46 \\
3933.85 \\
3954.23 \\
3974.62 \\
3995.00 \\
4015.39 \\
4035.77 \\
4056.16 \\
4076.54\end{array}$ & $\begin{array}{l}2517.80 \\
2534.02 \\
2550.23 \\
2566.43 \\
2582.61 \\
2598.78 \\
2614.94 \\
2631.08 \\
2647.21 \\
2663.32\end{array}$ & $\begin{array}{l}1728.85 \\
1740.94 \\
1753.02 \\
1765.08 \\
1777.14 \\
1789.17 \\
1801.20 \\
1813.21 \\
1825.21 \\
1837.19\end{array}$ & $\begin{array}{l}54.987 \\
55.072 \\
55.156 \\
55.239 \\
55.323 \\
55.405 \\
55.488 \\
55.569 \\
55.651 \\
55.731\end{array}$ & $\begin{array}{l}261.00 \\
262.00 \\
263.00 \\
264.00 \\
265.00 \\
266.00 \\
267.00 \\
268.00 \\
269.00 \\
270.00\end{array}$ & $\begin{array}{l}5318.78 \\
5339.08 \\
5359.38 \\
5379.68 \\
5399.98 \\
5420.27 \\
5440.57 \\
5460.87 \\
5481.17 \\
5501.47\end{array}$ & $\begin{array}{l}3615.81 \\
3630.98 \\
3646.13 \\
3661.28 \\
3676.41 \\
3691.53 \\
3706.64 \\
3721.74 \\
3736.83 \\
3751.91\end{array}$ & $\begin{array}{l}2537.93 \\
2548.98 \\
2560.02 \\
2571.05 \\
2582.07 \\
2593.08 \\
2604.08 \\
2615.07 \\
2626.04 \\
2637.01\end{array}$ & $\begin{array}{l}59.894 \\
59.952 \\
60.009 \\
60.067 \\
60.124 \\
60.181 \\
60.238 \\
60.294 \\
60.350 \\
60.406\end{array}$ \\
\hline $\begin{array}{l}201.00 \\
202.00 \\
203.00 \\
204.00 \\
205.00 \\
206.00 \\
207.00 \\
208.00 \\
209.00 \\
210.00\end{array}$ & $\begin{array}{l}4096.92 \\
4117.29 \\
4137.67 \\
4158.05 \\
4178.42 \\
4198.80 \\
4219.17 \\
4239.55 \\
4259.92 \\
4280.30\end{array}$ & $\begin{array}{l}2679.41 \\
2695.48 \\
2711.54 \\
2727.58 \\
2743.61 \\
2759.62 \\
2775.61 \\
2791.59 \\
2807.55 \\
2823.50\end{array}$ & $\begin{array}{l}1849.15 \\
1861.09 \\
1873.02 \\
1884.93 \\
1896.83 \\
1908.71 \\
1920.58 \\
1932.42 \\
1944.26 \\
1956.08\end{array}$ & $\begin{array}{l}55.812 \\
55.891 \\
55.971 \\
56.049 \\
56.128 \\
56.206 \\
56.283 \\
56.360 \\
56.437 \\
56.513\end{array}$ & $\begin{array}{l}271.00 \\
272.00 \\
273.00 \\
274.00 \\
275.00 \\
276.00 \\
277.00 \\
278.00 \\
279.00 \\
280.00\end{array}$ & $\begin{array}{l}5521.77 \\
5542.08 \\
5562.39 \\
5582.71 \\
5603.03 \\
5623.35 \\
5643.09 \\
5664.03 \\
5684.37 \\
5704.73\end{array}$ & $\begin{array}{l}3766.98 \\
3782 \cdot 04 \\
3797 \cdot 10 \\
3812.14 \\
3827.18 \\
3842 \cdot 21 \\
3857 \cdot 23 \\
3872 \cdot 24 \\
3887 \cdot 25 \\
3902 \cdot 26\end{array}$ & $\begin{array}{l}2647.96 \\
2658.91 \\
2669.85 \\
2680.77 \\
2691.69 \\
2702.60 \\
2713.51 \\
2724.40 \\
2735.28 \\
2746.16\end{array}$ & $\begin{array}{l}60.462 \\
60.517 \\
60.573 \\
60.628 \\
60.682 \\
60.737 \\
60.791 \\
60.845 \\
60.899 \\
60.953\end{array}$ \\
\hline $\begin{array}{l}211.00 \\
212.00 \\
213.00 \\
214.00 \\
215.00 \\
216.00 \\
217.00 \\
218.00 \\
219.00 \\
220.00\end{array}$ & $\begin{array}{l}4300.67 \\
4321.05 \\
4341.42 \\
4361.80 \\
4382.18 \\
4402.55 \\
4422.93 \\
4443.31 \\
4463.69 \\
4484.06\end{array}$ & $\begin{array}{l}2839.43 \\
2855.34 \\
2871.24 \\
2887.12 \\
2902.99 \\
2918.84 \\
2934.67 \\
2950.49 \\
2966.28 \\
2982.07\end{array}$ & $\begin{array}{l}1967.88 \\
1979.66 \\
1991.43 \\
2003.18 \\
2014.92 \\
2026.64 \\
2038.34 \\
2050.02 \\
2061.69 \\
2073.35\end{array}$ & $\begin{array}{l}56.589 \\
56.664 \\
56.739 \\
56.813 \\
56.887 \\
56.960 \\
57.034 \\
57.106 \\
57.179 \\
57.251\end{array}$ & $\begin{array}{l}281.00 \\
282.00 \\
283.00 \\
284.00 \\
285.00 \\
286.00 \\
287.00 \\
288.00 \\
289.00 \\
290.00\end{array}$ & $\begin{array}{l}5725.09 \\
5745.46 \\
5765.84 \\
5786.23 \\
5806.63 \\
5827.03 \\
5847.45 \\
5867.88 \\
5888.32 \\
5908.76\end{array}$ & $\begin{array}{l}3917.25 \\
3932.24 \\
3947.23 \\
3962.21 \\
3977.18 \\
3992.15 \\
4007.12 \\
4022.08 \\
4037.03 \\
4051.98\end{array}$ & $\begin{array}{l}2757.03 \\
2767.89 \\
2778.75 \\
2789.60 \\
2800.44 \\
2811.27 \\
2822.10 \\
2832.92 \\
2843.73 \\
2854.54\end{array}$ & $\begin{array}{l}61.006 \\
61.060 \\
61.113 \\
61.166 \\
61.218 \\
61.271 \\
61.323 \\
61.375 \\
61.427 \\
61.478\end{array}$ \\
\hline $\begin{array}{l}221.00 \\
222.00 \\
223.00 \\
224.00 \\
225.00 \\
226.00 \\
227.00 \\
228.00 \\
229.00 \\
230.00\end{array}$ & $\begin{array}{l}4504.44 \\
4524.82 \\
4545.20 \\
4565.58 \\
4585.96 \\
4606.34 \\
4626.72 \\
4647.10 \\
4667.49 \\
4687.97\end{array}$ & $\begin{array}{l}2997.83 \\
3013.58 \\
3029.32 \\
3045.03 \\
3060.74 \\
3076.42 \\
3092.09 \\
3107.74 \\
3123.38 \\
3139.00\end{array}$ & $\begin{array}{l}2084 \cdot 98 \\
2096 \cdot 60 \\
2108 \cdot 21 \\
2119 \cdot 79 \\
2131 \cdot 37 \\
2142.92 \\
2154 \cdot 46 \\
2165 \cdot 98 \\
2177 \cdot 48 \\
2188.97\end{array}$ & $\begin{array}{l}57.322 \\
57.393 \\
57.464 \\
57.534 \\
57.604 \\
57.674 \\
57.743 \\
57.812 \\
57.880 \\
57.948\end{array}$ & $\begin{array}{l}291.00 \\
292.00 \\
293.00 \\
294.00 \\
295.00 \\
296.00 \\
297.00 \\
298.00 \\
299.00 \\
300.00\end{array}$ & $\begin{array}{l}5929.22 \\
5949.68 \\
5970.15 \\
5990.63 \\
6011.11 \\
6031.60 \\
6052.09 \\
6072.59 \\
6093.09 \\
6113.59\end{array}$ & $\begin{array}{l}4066.93 \\
4081.87 \\
4096.80 \\
4111.73 \\
4126.66 \\
4141.57 \\
4156.49 \\
4171.39 \\
4186.29 \\
4201.19\end{array}$ & $\begin{array}{l}2865.34 \\
2876.13 \\
2886.92 \\
2897.70 \\
2908.47 \\
2919.24 \\
2930.00 \\
2940.75 \\
2951.50 \\
2962.24\end{array}$ & $\begin{array}{l}61.530 \\
61.581 \\
61.632 \\
61.683 \\
61.734 \\
61.784 \\
61.834 \\
61.885 \\
61.934 \\
61.984\end{array}$ \\
\hline
\end{tabular}




\begin{tabular}{|c|c|c|c|c|c|c|c|c|c|}
\hline $\begin{array}{l}\text { TEMPER- } \\
\text { ATURE } \\
\text { (K) }\end{array}$ & $\begin{array}{l}\text { SPECIFIC } \\
\text { VOLUME } \\
\text { (CC/GM) }\end{array}$ & $\begin{array}{l}\text { ENTHALPY } \\
(J / G M)\end{array}$ & $\begin{array}{l}\text { INTERNAL } \\
\text { ENERGY } \\
(J / G M)\end{array}$ & $\begin{array}{l}\text { ENTROPY } \\
(\mathrm{J} / \mathrm{GM}-\mathrm{K})\end{array}$ & $\begin{array}{l}\text { TEMPER- } \\
\text { ATURE } \\
\text { (K) }\end{array}$ & $\begin{array}{l}\text { SPEC IF IC } \\
\text { VOLUME } \\
\text { (CC/GM) }\end{array}$ & $\begin{array}{c}\text { ENTHALPY } \\
(J / G M)\end{array}$ & $\begin{array}{c}\text { INTERNAL } \\
\text { ENERGY } \\
(J / G M)\end{array}$ & $\begin{array}{l}\text { ENTROPY } \\
(\mathrm{J} / \mathrm{GM}-\mathrm{K})\end{array}$ \\
\hline 20.00 & 14.01 & $-256 \cdot 31$ & -260.56 & 7.843 & & & & & \\
\hline $\begin{array}{l}21.00 \\
22.00 \\
23.00 \\
24.00 \\
24.628 \\
24.628 \\
25.00 \\
26.00 \\
27.00 \\
28.00 \\
29.00 \\
30.00\end{array}$ & $\begin{array}{l}14.24 \\
14.51 \\
14.80 \\
15.14 \\
15.37 \\
268.20 \\
275.62 \\
294.57 \\
312.49 \\
329.72 \\
346.45 \\
362.81\end{array}$ & $\begin{array}{r}-246.53 \\
-236.10 \\
-224.92 \\
-212.89 \\
-204.82 \\
205.13 \\
210.97 \\
225.62 \\
239.26 \\
252.23 \\
264.74 \\
276.93\end{array}$ & $\begin{array}{r}-250.86 \\
-240.51 \\
-229.42 \\
-217.49 \\
-209.50 \\
123.61 \\
127.18 \\
136.08 \\
144.27 \\
152.00 \\
159.43 \\
166.65\end{array}$ & $\begin{array}{r}8.323 \\
8.805 \\
9.304 \\
9.818 \\
10.148 \\
26.804 \\
27.039 \\
27.614 \\
28.129 \\
28.601 \\
29.040 \\
29.453\end{array}$ & $\begin{array}{l}91.00 \\
92.00 \\
93.00 \\
94.00 \\
95.00 \\
96.00 \\
97.00 \\
98.00 \\
99.00 \\
100.00\end{array}$ & $\begin{array}{l}1232.57 \\
1246.32 \\
1260.07 \\
1273.81 \\
1287.54 \\
1301.28 \\
1315.01 \\
1328.73 \\
1342.45 \\
1356.17\end{array}$ & $\begin{array}{r}971.71 \\
984.50 \\
997.38 \\
1010.35 \\
1023.40 \\
1036.53 \\
1049.74 \\
1063.05 \\
1076.43 \\
1089.89\end{array}$ & $\begin{array}{l}597.04 \\
605.65 \\
614.35 \\
623.14 \\
632.01 \\
640.98 \\
650.02 \\
659.14 \\
668.35 \\
677.64\end{array}$ & $\begin{array}{l}42.017 \\
42.157 \\
42.296 \\
42.435 \\
42.573 \\
42.710 \\
42.847 \\
42.984 \\
43.120 \\
43.255\end{array}$ \\
\hline $\begin{array}{l}31.00 \\
32.00 \\
33.00 \\
34.00 \\
35.00 \\
36.00 \\
37.00 \\
38.00 \\
39.00 \\
40.00\end{array}$ & $\begin{array}{l}378.87 \\
394.69 \\
410.31 \\
425.77 \\
441.09 \\
456.28 \\
471.37 \\
486.35 \\
501.25 \\
516.07\end{array}$ & $\begin{array}{l}288.88 \\
300.65 \\
312.27 \\
323.78 \\
335.19 \\
346.52 \\
357.79 \\
368.99 \\
380.14 \\
391.25\end{array}$ & $\begin{array}{l}173.72 \\
180.68 \\
187.55 \\
194.36 \\
201.11 \\
207.82 \\
214.50 \\
221.15 \\
227.78 \\
234.38\end{array}$ & $\begin{array}{l}29.845 \\
30.219 \\
30.576 \\
30.920 \\
31.251 \\
31.570 \\
31.879 \\
32.177 \\
32.467 \\
32.748\end{array}$ & $\begin{array}{l}101.00 \\
102.00 \\
103.00 \\
104.00 \\
105.00 \\
106.00 \\
107.00 \\
108.00 \\
109.00 \\
110.00\end{array}$ & $\begin{array}{l}1369.88 \\
1383.58 \\
1397.27 \\
1410.97 \\
1424.66 \\
1438.34 \\
1452.03 \\
1465.71 \\
1479.39 \\
1493.06\end{array}$ & $\begin{array}{l}1103.43 \\
1117.05 \\
1130.76 \\
1144.55 \\
1158.42 \\
1172.37 \\
1186.39 \\
1200.50 \\
1214.69 \\
1228.95\end{array}$ & $\begin{array}{l}687.01 \\
696.47 \\
706.01 \\
715.64 \\
725.34 \\
735.13 \\
745.00 \\
754.95 \\
764.98 \\
775.09\end{array}$ & $\begin{array}{l}43.390 \\
43.524 \\
43.658 \\
43.791 \\
43.924 \\
44.056 \\
44.187 \\
44.319 \\
44.449 \\
44.580\end{array}$ \\
\hline $\begin{array}{l}41.00 \\
42.00 \\
43.00 \\
44.00 \\
45.00 \\
46.00 \\
47.00 \\
48.00 \\
49.00 \\
50.00\end{array}$ & $\begin{array}{l}530.82 \\
545.51 \\
560.13 \\
574.70 \\
589.21 \\
603.68 \\
618.11 \\
632.49 \\
646.84 \\
661.15\end{array}$ & $\begin{array}{l}402.32 \\
413.35 \\
424.36 \\
435.34 \\
446.29 \\
457.22 \\
468.14 \\
479.04 \\
489.93 \\
500.81\end{array}$ & $\begin{array}{l}240.96 \\
247.54 \\
254.09 \\
260.64 \\
267.19 \\
273.72 \\
280.25 \\
286.78 \\
293.31 \\
299.84\end{array}$ & $\begin{array}{l}33.022 \\
33.288 \\
33.547 \\
33.799 \\
34.045 \\
34.285 \\
34.520 \\
34.750 \\
34.974 \\
35.194\end{array}$ & $\begin{array}{l}111.00 \\
112.00 \\
113.00 \\
114.00 \\
115.00 \\
116.00 \\
117.00 \\
118.00 \\
119.00 \\
120.00\end{array}$ & $\begin{array}{l}1506.74 \\
1520.41 \\
1534.08 \\
1547.75 \\
1561.42 \\
1575.09 \\
1588.76 \\
1602.43 \\
1616.09 \\
1629.76\end{array}$ & $\begin{array}{l}1243.29 \\
1257.71 \\
1272.20 \\
1286.76 \\
1301.39 \\
1316.10 \\
1330.87 \\
1345.71 \\
1360.62 \\
1375.59\end{array}$ & $\begin{array}{l}785.27 \\
795.53 \\
805.86 \\
816.27 \\
826.75 \\
837.30 \\
847.91 \\
858.60 \\
869.35 \\
880.17\end{array}$ & $\begin{array}{l}44.710 \\
44.839 \\
44.968 \\
45.096 \\
45.224 \\
45.351 \\
45.478 \\
45.604 \\
45.730 \\
45.855\end{array}$ \\
\hline $\begin{array}{l}51.00 \\
52.00 \\
53.00 \\
54.00 \\
55.00 \\
56.00 \\
57.00 \\
58.00 \\
59.00 \\
60.00\end{array}$ & $\begin{array}{l}675.43 \\
689.67 \\
703.89 \\
718.08 \\
732.24 \\
746.38 \\
760.50 \\
774.60 \\
788.67 \\
802.73\end{array}$ & $\begin{array}{l}511.69 \\
522.56 \\
533.43 \\
544.30 \\
555.18 \\
566.07 \\
576.96 \\
587.87 \\
598.79 \\
609.73\end{array}$ & $\begin{array}{l}306.37 \\
312.92 \\
319.47 \\
326.03 \\
332.60 \\
339.18 \\
345.79 \\
352.41 \\
359.05 \\
365.72\end{array}$ & $\begin{array}{l}35.409 \\
35.620 \\
35.828 \\
36.031 \\
36.230 \\
36.426 \\
36.619 \\
36.809 \\
36.996 \\
37.180\end{array}$ & $\begin{array}{l}121.00 \\
122.00 \\
123.00 \\
124.00 \\
125.00 \\
126.00 \\
127.00 \\
128.00 \\
129.00 \\
130.00\end{array}$ & $\begin{array}{l}1643.42 \\
1657.08 \\
1670.75 \\
1684.41 \\
1698.07 \\
1711.72 \\
1725.37 \\
1739.01 \\
1752.66 \\
1766.30\end{array}$ & $\begin{array}{l}1390.62 \\
1405.70 \\
1420.85 \\
1436.06 \\
1451.33 \\
1466.65 \\
1482.04 \\
1497.47 \\
1512.96 \\
1528.50\end{array}$ & $\begin{array}{l}891.04 \\
901.98 \\
912.97 \\
924.03 \\
935.14 \\
946.32 \\
957.55 \\
968.84 \\
980.18 \\
991.58\end{array}$ & $\begin{array}{l}45.980 \\
46.104 \\
46.228 \\
46.351 \\
46.474 \\
46.596 \\
46.717 \\
46.838 \\
46.959 \\
47.079\end{array}$ \\
\hline $\begin{array}{l}61.00 \\
62.00 \\
63.00 \\
64.00 \\
65.00 \\
66.00 \\
67.00 \\
68.00 \\
69.00 \\
70.00\end{array}$ & $\begin{array}{l}816.76 \\
830.78 \\
844.79 \\
858.77 \\
872.75 \\
886.71 \\
900.65 \\
914.58 \\
928.50 \\
942.41\end{array}$ & $\begin{array}{l}620.69 \\
631.68 \\
642.69 \\
653.72 \\
664.80 \\
675.90 \\
687.04 \\
698.22 \\
709.44 \\
720.70\end{array}$ & $\begin{array}{l}372.42 \\
379.14 \\
385.89 \\
392.68 \\
399.50 \\
406.36 \\
413.26 \\
420.21 \\
427.20 \\
434.24\end{array}$ & $\begin{array}{l}37.361 \\
37.539 \\
37.716 \\
37.889 \\
38.061 \\
38.231 \\
38.398 \\
38.564 \\
38.728 \\
38.890\end{array}$ & $\begin{array}{l}131.00 \\
132.00 \\
133.00 \\
134.00 \\
135.00 \\
136.00 \\
137.00 \\
138.00 \\
139.00 \\
140.00\end{array}$ & $\begin{array}{l}1779.94 \\
1793.58 \\
1807.22 \\
1820.86 \\
1834.50 \\
1848.13 \\
1861.77 \\
1875.40 \\
1889.04 \\
1902.67\end{array}$ & $\begin{array}{l}1544.09 \\
1559.73 \\
1575.42 \\
1591.15 \\
1606.93 \\
1622.75 \\
1638.61 \\
1654.51 \\
1670.45 \\
1686.43\end{array}$ & $\begin{array}{l}1003.02 \\
1014.52 \\
1026.06 \\
1037.64 \\
1049.28 \\
1060.95 \\
1072.67 \\
1084.42 \\
1096.22 \\
1108.05\end{array}$ & $\begin{array}{l}47.198 \\
47.317 \\
47.436 \\
47.554 \\
47.671 \\
47.788 \\
47.904 \\
48.019 \\
48.134 \\
48.249\end{array}$ \\
\hline $\begin{array}{l}71.00 \\
72.00 \\
73.00 \\
74.00 \\
75.00 \\
76.00 \\
77.00 \\
78.00 \\
79.00 \\
80.00\end{array}$ & $\begin{array}{r}956.31 \\
970.20 \\
984.08 \\
997.95 \\
1011.80 \\
1025.65 \\
1039.50 \\
1053.33 \\
1067.16 \\
1080.97\end{array}$ & $\begin{array}{l}732.02 \\
743.38 \\
754.79 \\
766.26 \\
777.79 \\
789.37 \\
801.02 \\
812.73 \\
824.51 \\
836.36\end{array}$ & $\begin{array}{l}441.32 \\
448.46 \\
455.66 \\
462.91 \\
470.23 \\
477.60 \\
485.04 \\
492.55 \\
500.13 \\
507.77\end{array}$ & $\begin{array}{l}39.050 \\
39.209 \\
39.366 \\
39.522 \\
39.677 \\
39.831 \\
39.983 \\
40.134 \\
40.284 \\
40.433\end{array}$ & $\begin{array}{l}141.00 \\
142.00 \\
143.00 \\
144.00 \\
145.00 \\
146.00 \\
147.00 \\
148.00 \\
149.00 \\
150.00\end{array}$ & $\begin{array}{l}1916.31 \\
1929.94 \\
1943.57 \\
1957.20 \\
1970.83 \\
1984.47 \\
1998.10 \\
2011.73 \\
2025.36 \\
2038.99\end{array}$ & $\begin{array}{l}1702.44 \\
1718.49 \\
1734.57 \\
1750.68 \\
1766.82 \\
1782.99 \\
1799.18 \\
1815.40 \\
1831.64 \\
1847.91\end{array}$ & $\begin{array}{l}1119.92 \\
1131.82 \\
1143.76 \\
1155.72 \\
1167.72 \\
1179.74 \\
1191.79 \\
1203.87 \\
1215.97 \\
1228.09\end{array}$ & $\begin{array}{l}48.363 \\
48.476 \\
48.589 \\
48.701 \\
48.813 \\
48.924 \\
49.035 \\
49.145 \\
49.254 \\
49.363\end{array}$ \\
\hline $\begin{array}{l}81.00 \\
82.00 \\
83.00 \\
84.00 \\
85.00 \\
86.00 \\
87.00 \\
88.00 \\
89.00 \\
90.00\end{array}$ & $\begin{array}{l}1094.79 \\
1108.59 \\
1122.39 \\
1136.18 \\
1149.97 \\
1163.75 \\
1177.52 \\
1191.29 \\
1205.06 \\
1218.82\end{array}$ & $\begin{array}{l}848.28 \\
860.27 \\
872.33 \\
884.47 \\
896.69 \\
908.99 \\
921.37 \\
933.83 \\
946.37 \\
959.00\end{array}$ & $\begin{array}{l}515.49 \\
523.28 \\
531.15 \\
539.10 \\
547.13 \\
555.24 \\
563.43 \\
571.71 \\
580.07 \\
588.51\end{array}$ & $\begin{array}{l}40.581 \\
40.728 \\
40.875 \\
41.020 \\
41.165 \\
41.308 \\
41.451 \\
41.594 \\
41.736 \\
41.877\end{array}$ & $\begin{array}{l}151.00 \\
152.00 \\
153.00 \\
154.00 \\
155.00 \\
156.00 \\
157.00 \\
158.00 \\
159.00 \\
160.00\end{array}$ & $\begin{array}{l}2052.61 \\
2066.23 \\
2079.85 \\
2093.47 \\
2107.09 \\
2120.71 \\
2134.33 \\
2147.95 \\
2161.56 \\
2175.18\end{array}$ & $\begin{array}{l}1864.14 \\
1880.39 \\
1896.65 \\
1912.93 \\
1929.23 \\
1945.53 \\
1961.85 \\
1978.18 \\
1994.52 \\
2010.87\end{array}$ & $\begin{array}{l}1240.18 \\
1252.29 \\
1264.41 \\
1276.55 \\
1288.71 \\
1300.87 \\
1313.05 \\
1325.24 \\
1337.44 \\
1349.65\end{array}$ & $\begin{array}{l}49.471 \\
49.578 \\
49.685 \\
49.791 \\
49.896 \\
50.001 \\
50.105 \\
50.209 \\
50.312 \\
50.415\end{array}$ \\
\hline
\end{tabular}


TEMPER - SPECIFIC ENTHALPY INTERNAL ENTROPY ATURE VOLUME (J/GM) ENERGY (J/GM-K) (K) (CC/GM)
$(J / G M)$ ATURE (K)
SPEC IF I

( $C \mathrm{C} / \mathrm{GM}$ )
ENTHALPY INTERNAL (J/GM)
161.00

163.00

164.00

165.00

166.00

167.00

168.00

169.00

170.00

171.00

172.00

173.00

174.00

175.00

176.00

177.00

178.00

179.00

180.00

181.00

182.00

183.00

184.00

185.00

186.00

187.00

188.00

189.00

190.00

191.00

192.00

193.00

194.00

195.00

196.00

197.00

198.00

199.00

200.00

201.00

202.00

203.00

204.00

205.00

206.00

207.00

208.00

209.00

210.00

211.00

212.00

213.00

214.00

215.00

216.00

217.00

218.00

219.00

220.00

221.00

222.00

223.00

224.00

225.00

226.00

227.00

228.00

229.00

230.00
2188.80

2202.42

2216.03

2229.65

2243.26

2256.88

2270.50

2284.11

2297.73

2311.34

2324.95

2338.56

2352.17

2365.78

2379.39

2393.00

2406.60

2420.21

2433.81

2447.42

2461.02

2474.63

2488.23

2501.83

2515.44

2529.04

2542.64

2556.24

2569.85

2583.45

2597.05

2610.65

2624.25

2637.85

2651.45

2665.05

2678.65

2692.24

2705.84

2719.44

2733.04

2746.63

2760.22

2773.81

2787.41

2801.00

2814.59

2828.18

2841.78

2855.37

2868.96

2882.55

2896.15

2909. 74

2923.33

2936.92

2950. 52

2964.11

2977.70

2991.30

$3004 \cdot 89$

3018.48

3032.08

3045.67

3059.27

3072.86

3086.45

3100.05

3113.64

3127.24
2027.22

2043.58

2059.95

2076.32

2092.70

2109.08

2125.47

2141.85

2158.24

2174.62

2191. 01

2207.39

2223.78

2240.16

2256.53

2272.91

2289.28

2305.64

2322.00

2338.35

2354.69

2371.02

2387.35

2403.67

2419.98

2436.28

452.57

2468.85

2485.11

2501.37

2517.61

2533. 84

2550.06

2566.27

2582.46

2598.63

2614.80

2630.95

2647.08

2663.20

2679.30

2695.38

2711.44

2727.49

2743.53

2759.54

2775.55

2791.53

2807.50

2823.45

2839.39

2855.31

2871.21

2887.10

2902.97

2918.83

2934.66

2950.49

2966.29

2982.08

2997.85

3013.61

3029.34

3045.07

3060.77

3076.46

3092.14

3107.79

3123.43

3139.06

1361.86

1374.09

1386.31

1398.55

1410.79

1423.03

1435.27

447.52

1459.77

1472.01

1484.26

1496.51

1508.76

1521.00

1533.24

1545.48

1557.71

1569.94

1582.16

1594.37

1606.58

1618.78

1630.97

1643.15

1655.33

1667.49

1679.65

1691.79

1703.92

1716.04

1728.15

1740.25

1752.33

1764.40

1776.46

1788.51

1800.54

1812.55

$1824: 55$

1836.54

1848.50

1860.45

1872.38

1884.30

1896.20

1908.09

1919.96

1931.81

1943.65

1955.47

1967.27

1979.06

1990.83

2002.59

2014.33

2026.05

2037.76

2049.45

2061.12 


\begin{tabular}{|c|c|c|c|c|c|c|c|c|c|}
\hline $\begin{array}{l}\text { TEMPER- } \\
\text { ATURE } \\
(K)\end{array}$ & $\begin{array}{l}\text { SPEC IF IC } \\
\text { VOLUME } \\
(C C / G M)\end{array}$ & $\begin{array}{c}\text { ENTHALPY } \\
(J / G M)\end{array}$ & $\begin{array}{l}\text { INTERNAL } \\
\text { ENERGY } \\
(J / G M)\end{array}$ & $\begin{array}{l}\text { ENTROPY } \\
(J / G M-K)\end{array}$ & $\begin{array}{c}\text { TEMPER- } \\
\text { ATURE } \\
(K)\end{array}$ & $\begin{array}{l}\text { SPEC IF IC } \\
\text { VOLUME } \\
\text { (CC/GM) }\end{array}$ & $\begin{array}{c}\text { ENTHALPY } \\
(J / G M)\end{array}$ & $\begin{array}{l}\text { INTERNAL } \\
\text { ENERGY } \\
(J / G M)\end{array}$ & $\begin{array}{l}\text { ENTROPY } \\
(J / G M-K)\end{array}$ \\
\hline 20.00 & 13.98 & -255.34 & -261.00 & 7.821 & & & & & \\
\hline $\begin{array}{l}21.00 \\
22.00 \\
23.00 \\
24.00 \\
25.00 \\
26.00 \\
26.020 \\
26.020 \\
27.00 \\
28.00 \\
29.00 \\
30.00\end{array}$ & $\begin{array}{r}14.21 \\
14.47 \\
14.76 \\
15.09 \\
15.47 \\
15.93 \\
15.93 \\
201.69 \\
217.40 \\
232.17 \\
246.13 \\
259.52\end{array}$ & $\begin{array}{r}-245.61 \\
-235.25 \\
-224.15 \\
-212.23 \\
-199.32 \\
-185.17 \\
-184.87 \\
204.93 \\
221.33 \\
236.43 \\
250.49 \\
263.87\end{array}$ & $\begin{array}{r}-251.37 \\
-241.11 \\
-230.14 \\
-218.35 \\
-205.59 \\
-191.62 \\
-191.33 \\
123.18 \\
133.22 \\
142.33 \\
150.73 \\
158.68\end{array}$ & $\begin{array}{r}8.298 \\
8.777 \\
9.273 \\
9.782 \\
10.307 \\
10.861 \\
10.872 \\
25.868 \\
26.487 \\
27.036 \\
27.530 \\
27.983\end{array}$ & $\begin{array}{r}91.00 \\
92.00 \\
93.00 \\
94.00 \\
95.00 \\
96.00 \\
97.00 \\
98.00 \\
99.00 \\
100.00\end{array}$ & $\begin{array}{r}923.82 \\
934.18 \\
944.53 \\
954.87 \\
965.22 \\
975.56 \\
985.89 \\
996.22 \\
1006.55 \\
1016.88\end{array}$ & $\begin{array}{r}969.80 \\
982.64 \\
995.56 \\
1008.56 \\
1021.65 \\
1034.82 \\
1048.08 \\
1061.41 \\
1074.82 \\
1088.32\end{array}$ & $\begin{array}{l}595.37 \\
604.01 \\
612.74 \\
621.55 \\
630.45 \\
639.4 .3 \\
648.49 \\
657.64 \\
666.87 \\
676.18\end{array}$ & $\begin{array}{l}40.812 \\
40.952 \\
41.092 \\
41.231 \\
41.370 \\
41.507 \\
41.645 \\
41.782 \\
41.918 \\
42.053\end{array}$ \\
\hline $\begin{array}{l}31.00 \\
32.00 \\
33.00 \\
34.00 \\
35.00 \\
36.00 \\
37.00 \\
38.00 \\
39.00 \\
40.00\end{array}$ & $\begin{array}{l}272.50 \\
285.16 \\
297.57 \\
309.78 \\
321.81 \\
333.70 \\
345.47 \\
357.12 \\
368.68 \\
380.15\end{array}$ & $\begin{array}{l}276.76 \\
289.32 \\
301.60 \\
313.68 \\
325.59 \\
337.38 \\
349.05 \\
360.62 \\
372.12 \\
383.54\end{array}$ & $\begin{array}{l}166.32 \\
173.74 \\
180.99 \\
188.13 \\
195.16 \\
202.12 \\
209.03 \\
215.88 \\
222.69 \\
229.47\end{array}$ & $\begin{array}{l}28.406 \\
28.805 \\
29.183 \\
29.543 \\
29.889 \\
30.221 \\
30.540 \\
30.849 \\
31.148 \\
31.437\end{array}$ & $\begin{array}{l}101.00 \\
102.00 \\
103.00 \\
104.00 \\
105.00 \\
106.00 \\
107.00 \\
108.00 \\
109.00 \\
110.00\end{array}$ & $\begin{array}{l}1027.19 \\
1037.50 \\
1047.80 \\
1058.10 \\
1068.40 \\
1078.69 \\
1088.98 \\
1099.27 \\
1109.56 \\
1119.84\end{array}$ & $\begin{array}{l}1101.89 \\
1115.54 \\
1129.28 \\
1143.10 \\
1157.00 \\
1170.97 \\
1185.03 \\
1199.17 \\
1213.38 \\
1227.67\end{array}$ & $\begin{array}{l}685.56 \\
695.03 \\
704.60 \\
714.24 \\
723.96 \\
733.77 \\
743.66 \\
753.62 \\
763.67 \\
773.79\end{array}$ & $\begin{array}{l}42.188 \\
42.323 \\
42.457 \\
42.591 \\
42.724 \\
42.856 \\
42.988 \\
43.120 \\
43.251 \\
43.381\end{array}$ \\
\hline $\begin{array}{l}41.00 \\
42.00 \\
43.00 \\
44.00 \\
45.00 \\
46.00 \\
47.00 \\
48.00 \\
49.00 \\
50.00\end{array}$ & $\begin{array}{l}391.54 \\
402.86 \\
414.11 \\
425.31 \\
436.45 \\
447.54 \\
458.59 \\
469.59 \\
480.55 \\
491.48\end{array}$ & $\begin{array}{l}394.90 \\
406.21 \\
417.47 \\
428.69 \\
439.87 \\
451.02 \\
462.14 \\
473.24 \\
484.31 \\
495.36\end{array}$ & $\begin{array}{l}236.21 \\
242.93 \\
249.63 \\
256.31 \\
262.98 \\
269.63 \\
276.27 \\
282.91 \\
289.54 \\
296.17\end{array}$ & $\begin{array}{l}31.717 \\
31.990 \\
32.255 \\
32.513 \\
32.764 \\
33.009 \\
33.248 \\
33.482 \\
33.710 \\
33.934\end{array}$ & $\begin{array}{l}111.00 \\
112.00 \\
113.00 \\
114.00 \\
115.00 \\
116.00 \\
117.00 \\
118.00 \\
119.00 \\
120.00\end{array}$ & $\begin{array}{l}1130.13 \\
1140.41 \\
1150.69 \\
1160.97 \\
1171.24 \\
1181.52 \\
1191.79 \\
1202.07 \\
1212.34 \\
1222.61\end{array}$ & $\begin{array}{l}1242.04 \\
1256.48 \\
1270.99 \\
1285.57 \\
1300.23 \\
1314.96 \\
1329.75 \\
1344.61 \\
1359.54 \\
1374.53\end{array}$ & $\begin{array}{l}783.98 \\
794.26 \\
804.60 \\
815.02 \\
825.51 \\
836.07 \\
846.70 \\
857.40 \\
868.16 \\
878.99\end{array}$ & $\begin{array}{l}43.511 \\
43.641 \\
43.770 \\
43.898 \\
44.026 \\
44.154 \\
44.281 \\
44.407 \\
44.533 \\
44.558\end{array}$ \\
\hline $\begin{array}{l}51.00 \\
52.00 \\
53.00 \\
54.00 \\
55.00 \\
56.00 \\
57.00 \\
58.00 \\
59.00 \\
60.00\end{array}$ & $\begin{array}{l}502.37 \\
513.23 \\
524.06 \\
534.86 \\
545.64 \\
556.39 \\
567.11 \\
577.82 \\
588.50 \\
599.17\end{array}$ & $\begin{array}{l}506.41 \\
517.44 \\
528.46 \\
539.48 \\
550.50 \\
561.52 \\
572.54 \\
583.58 \\
594.62 \\
605.67\end{array}$ & $\begin{array}{l}302.80 \\
309.43 \\
316.06 \\
322.70 \\
329.36 \\
336.02 \\
342.69 \\
349.38 \\
356.10 \\
362.83\end{array}$ & $\begin{array}{l}34.152 \\
34.366 \\
34.576 \\
34.782 \\
34.985 \\
35.183 \\
35.378 \\
35.570 \\
35.759 \\
35.945\end{array}$ & $\begin{array}{l}121.00 \\
122.00 \\
123.00 \\
124.00 \\
125.00 \\
126.00 \\
127.00 \\
128.00 \\
129.00 \\
130.00\end{array}$ & $\begin{array}{l}1232.89 \\
1243.16 \\
1253.43 \\
1263.70 \\
1273.97 \\
1284.22 \\
1294.48 \\
1304.73 \\
1314.98 \\
1325.24\end{array}$ & $\begin{array}{l}1389.58 \\
1404.68 \\
1419.85 \\
1435.08 \\
1450.36 \\
1465.70 \\
1481.10 \\
1496.56 \\
1512.07 \\
1527.62\end{array}$ & $\begin{array}{l}889.88 \\
900.82 \\
911.82 \\
922.89 \\
934.01 \\
945.19 \\
956.44 \\
967.74 \\
979.09 \\
990.49\end{array}$ & $\begin{array}{l}44.783 \\
44.908 \\
45.031 \\
45.155 \\
45.277 \\
45.400 \\
45.521 \\
45.643 \\
45.763 \\
45.883\end{array}$ \\
\hline $\begin{array}{l}61.00 \\
62.00 \\
63.00 \\
64.00 \\
65.00 \\
66.00 \\
67.00 \\
68.00 \\
69.00 \\
70.00\end{array}$ & $\begin{array}{l}609.81 \\
620.44 \\
631.05 \\
641.65 \\
652.23 \\
662.79 \\
673.35 \\
683.89 \\
694.41 \\
704.93\end{array}$ & $\begin{array}{l}616.74 \\
627.83 \\
638.94 \\
650.08 \\
661.24 \\
672.44 \\
683.67 \\
694.93 \\
706.23 \\
717.58\end{array}$ & $\begin{array}{l}369.59 \\
376.37 \\
383.18 \\
390.02 \\
396.90 \\
403.81 \\
410.76 \\
417.76 \\
424.79 \\
431.87\end{array}$ & $\begin{array}{l}36.128 \\
36.308 \\
36.486 \\
36.661 \\
36.834 \\
37.005 \\
37.174 \\
37.341 \\
37.506 \\
37.669\end{array}$ & $\begin{array}{l}131.00 \\
132.00 \\
133.00 \\
134.00 \\
135.00 \\
136.00 \\
137.00 \\
138.00 \\
139.00 \\
140.00\end{array}$ & $\begin{array}{l}1335.48 \\
1345.73 \\
1355.98 \\
1366.23 \\
1376.47 \\
1386.72 \\
1396.96 \\
1407.20 \\
1417.45 \\
1427.69\end{array}$ & $\begin{array}{l}1543.23 \\
1558.89 \\
1574.59 \\
1590.34 \\
1606.13 \\
1621.97 \\
1637.84 \\
1653.76 \\
1669.71 \\
1685.70\end{array}$ & $\begin{array}{l}1001.94 \\
1013.45 \\
1025.00 \\
1036.59 \\
1048.23 \\
1059.91 \\
1071.64 \\
1083.40 \\
1095.21 \\
1107.05\end{array}$ & $\begin{array}{l}46.003 \\
46.122 \\
46.241 \\
46.359 \\
46.476 \\
46.593 \\
46.709 \\
46.825 \\
46.940 \\
47.055\end{array}$ \\
\hline $\begin{array}{l}71.00 \\
72.00 \\
73.00 \\
74.00 \\
75.00 \\
76.00 \\
77.00 \\
78.00 \\
79.00 \\
80.00\end{array}$ & $\begin{array}{l}715.43 \\
725.93 \\
736.41 \\
746.89 \\
757.35 \\
767.81 \\
778.26 \\
788.70 \\
799.13 \\
809.56\end{array}$ & $\begin{array}{l}728.97 \\
740.41 \\
751.89 \\
763.43 \\
775.03 \\
786.68 \\
798.39 \\
810.16 \\
822.00 \\
833.91\end{array}$ & $\begin{array}{l}439.01 \\
446.19 \\
453.43 \\
460.72 \\
468.07 \\
475.49 \\
482.96 \\
490.51 \\
498.12 \\
505.80\end{array}$ & $\begin{array}{l}37.831 \\
37.991 \\
38.149 \\
38.306 \\
38.462 \\
38.616 \\
38.769 \\
38.921 \\
39.072 \\
39.222\end{array}$ & $\begin{array}{l}141.00 \\
142.00 \\
143.00 \\
144.00 \\
145.00 \\
146.00 \\
147.00 \\
148.00 \\
149.00 \\
150.00\end{array}$ & $\begin{array}{l}1437.93 \\
1448.17 \\
1458.41 \\
1468.65 \\
1478.88 \\
1489.12 \\
1499.36 \\
1509.60 \\
1519.83 \\
1530.07\end{array}$ & $\begin{array}{l}1701.73 \\
1717.79 \\
1733.88 \\
1750.01 \\
1766.16 \\
1782.34 \\
1798.55 \\
1814.78 \\
1831.04 \\
1847.31\end{array}$ & $\begin{array}{l}1118.92 \\
1130.83 \\
1142.78 \\
1154.75 \\
1166.75 \\
1178.78 \\
1190.84 \\
1202.92 \\
1215.03 \\
1227.16\end{array}$ & $\begin{array}{l}47.169 \\
47.282 \\
47.395 \\
47.508 \\
47.619 \\
47.731 \\
47.841 \\
47.951 \\
48.061 \\
48.170\end{array}$ \\
\hline $\begin{array}{l}81.00 \\
82.00 \\
83.00 \\
84.00 \\
85.00 \\
86.00 \\
87.00 \\
88.00 \\
89.00 \\
90.00\end{array}$ & $\begin{array}{l}819.97 \\
830.38 \\
840.79 \\
851.19 \\
861.58 \\
871.97 \\
882.35 \\
892.72 \\
903.09 \\
913.46\end{array}$ & $\begin{array}{l}845.88 \\
857.93 \\
870.05 \\
882.24 \\
894.51 \\
906.86 \\
919.28 \\
931.79 \\
944.37 \\
957.05\end{array}$ & $\begin{array}{l}513.55 \\
521.37 \\
529.28 \\
537.25 \\
545.31 \\
553.45 \\
561.67 \\
569.97 \\
578.35 \\
586.82\end{array}$ & $\begin{array}{l}39.370 \\
39.518 \\
39.665 \\
39.811 \\
39.956 \\
40.101 \\
40.244 \\
40.387 \\
40.530 \\
40.671\end{array}$ & $\begin{array}{l}151.00 \\
152.00 \\
153.00 \\
154.00 \\
155.00 \\
156.00 \\
157.00 \\
158.00 \\
159.00 \\
160.00\end{array}$ & $\begin{array}{l}1540.30 \\
1550.53 \\
1560.76 \\
1570.98 \\
1581.21 \\
1591.44 \\
1601.66 \\
1611.89 \\
1622.12 \\
1632.34\end{array}$ & $\begin{array}{l}1863.56 \\
1879.82 \\
1896.09 \\
1912.38 \\
1928.69 \\
1945.01 \\
1961.34 \\
1977.67 \\
1994.02 \\
2010.38\end{array}$ & $\begin{array}{l}1239.26 \\
1251.37 \\
1263.50 \\
1275.65 \\
1287.81 \\
1299.98 \\
1312.16 \\
1324.36 \\
1336.56 \\
1348.78\end{array}$ & $\begin{array}{l}48.278 \\
48.385 \\
48.492 \\
48.598 \\
48.703 \\
48.808 \\
48.913 \\
49.016 \\
49.119 \\
49.222\end{array}$ \\
\hline
\end{tabular}


TEMPER- SPECIFIC ENTHALPY INTERNAL ENTROPY

ATURE VOLUME (J/GM) ENERGY (J/GM-K) (K) ( $C$ C / GM)

$(J / G M)$
TEMPER-

(K)
SPEC IF IC

(CC/GM)
ENTHALPY

$J / G M$
INTERNAL ENTROPY

ENERGY

\begin{tabular}{|c|c|c|c|c|c|c|c|c|c|}
\hline $\begin{array}{l}161.00 \\
162.00 \\
163.00 \\
164.00 \\
165.00 \\
166.00 \\
167.00 \\
168.00 \\
169.00 \\
170.00\end{array}$ & $\begin{array}{l}1642.57 \\
1652.79 \\
1663.01 \\
1673.24 \\
1683.46 \\
1693.69 \\
1703.91 \\
1714.13 \\
1724.35 \\
1734.58\end{array}$ & $\begin{array}{l}2026.75 \\
2043.12 \\
2059.50 \\
2075.88 \\
2092.27 \\
2108.66 \\
2125.05 \\
2141.45 \\
2157.84 \\
2174.24\end{array}$ & $\begin{array}{l}1361.00 \\
1373.23 \\
1385.46 \\
1397.70 \\
1409.94 \\
1422.19 \\
1434.44 \\
1446.69 \\
1458.94 \\
1471.19\end{array}$ & $\begin{array}{l}49.324 \\
49.425 \\
49.526 \\
49.626 \\
49.726 \\
49.825 \\
49.923 \\
50.021 \\
50.119 \\
50.215\end{array}$ & $\begin{array}{l}231.00 \\
232.00 \\
233.00 \\
234.00 \\
235.00 \\
236.00 \\
237.00 \\
238.00 \\
239.00 \\
240.00\end{array}$ & $\begin{array}{l}2357.13 \\
2367.34 \\
2377.54 \\
2387.75 \\
2397.95 \\
2408.15 \\
2418.35 \\
2428.55 \\
2438.75 \\
2448.95\end{array}$ & $\begin{array}{l}3154.74 \\
3170.34 \\
3185.93 \\
3201.50 \\
3217.05 \\
3232.59 \\
3248.11 \\
3263.61 \\
3279.10 \\
3294.57\end{array}$ & $\begin{array}{l}2199.37 \\
2210.84 \\
2222.29 \\
2233.72 \\
2245.14 \\
2256.54 \\
2267.93 \\
2279.30 \\
2290.65 \\
2301.99\end{array}$ & $\begin{array}{l}55.151 \\
55.218 \\
55.285 \\
55.352 \\
55.418 \\
55.484 \\
55.550 \\
55.615 \\
55.680 \\
55.744\end{array}$ \\
\hline $\begin{array}{l}171.00 \\
172.00 \\
173.00 \\
174.00 \\
175.00 \\
176.00 \\
177.00 \\
178.00 \\
179.00 \\
180.00\end{array}$ & $\begin{array}{l}1744.79 \\
1755.01 \\
1765.23 \\
1775.44 \\
1785.66 \\
1795.87 \\
1806.09 \\
1816.30 \\
1826.52 \\
1836.73\end{array}$ & $\begin{array}{l}2190.63 \\
2207.03 \\
2223.42 \\
2239.81 \\
2256.20 \\
2272.58 \\
2288.96 \\
2305.33 \\
2321.69 \\
2338.05\end{array}$ & $\begin{array}{l}1483.45 \\
1495.70 \\
1507.95 \\
1520.20 \\
1532.45 \\
1544.69 \\
1556.93 \\
1569.16 \\
1581.39 \\
1593.61\end{array}$ & $\begin{array}{l}50.312 \\
50.407 \\
50.502 \\
50.597 \\
50.691 \\
50.784 \\
50.877 \\
50.969 \\
51.061 \\
51.152\end{array}$ & $\begin{array}{l}241.00 \\
242.00 \\
243.00 \\
244.00 \\
245.00 \\
246.00 \\
247.00 \\
248.00 \\
249.00 \\
250.00\end{array}$ & $\begin{array}{l}2459.14 \\
2469.34 \\
2479.53 \\
2489.72 \\
2499.91 \\
2510.10 \\
2520.28 \\
2530.47 \\
2540.65 \\
2550.83\end{array}$ & $\begin{array}{l}3310.02 \\
3325.46 \\
3340.88 \\
3356.29 \\
3371.68 \\
3387.05 \\
3402.41 \\
3417.75 \\
3433.07 \\
3448.38\end{array}$ & $\begin{array}{l}2313.31 \\
2324.61 \\
2335.90 \\
2347.18 \\
2358.44 \\
2369.68 \\
2380.91 \\
2392.12 \\
2403.32 \\
2414.51\end{array}$ & $\begin{array}{l}55.809 \\
55.873 \\
55.936 \\
55.999 \\
56.062 \\
56.125 \\
56.187 \\
56.249 \\
56.311 \\
56.372\end{array}$ \\
\hline $\begin{array}{l}181.00 \\
182.00 \\
183.00 \\
184.00 \\
185.00 \\
186.00 \\
187.00 \\
188.00 \\
189.00 \\
190.00\end{array}$ & $\begin{array}{l}1846.94 \\
1857.15 \\
1867.37 \\
1877.58 \\
1887.79 \\
1898.00 \\
1908.21 \\
1918.42 \\
1928.63 \\
1938.84\end{array}$ & $\begin{array}{l}2354.41 \\
2370.75 \\
2387.09 \\
2403.41 \\
2419.73 \\
2436.04 \\
2452.34 \\
2468.62 \\
2484.90 \\
2501.16\end{array}$ & $\begin{array}{l}1605.82 \\
1618.03 \\
1630.22 \\
1642.41 \\
1654.59 \\
1666.76 \\
1678.92 \\
1691.07 \\
1703.20 \\
1715.33\end{array}$ & $\begin{array}{l}51.242 \\
51.332 \\
51.422 \\
51.511 \\
51.599 \\
51.687 \\
51.775 \\
51.861 \\
51.948 \\
52.034\end{array}$ & $\begin{array}{l}251.00 \\
252.00 \\
253.00 \\
254.00 \\
255.00 \\
256.00 \\
257.00 \\
258.00 \\
259.00 \\
260.00\end{array}$ & $\begin{array}{l}2561.00 \\
2571.18 \\
2581.35 \\
2591.52 \\
2601.69 \\
2611.86 \\
2622.03 \\
2632.19 \\
2642.35 \\
2652.52\end{array}$ & $\begin{array}{l}3463.71 \\
3479.03 \\
3494.33 \\
3509.62 \\
3524.89 \\
3540.15 \\
3555.39 \\
3570.62 \\
3585.84 \\
3601.04\end{array}$ & $\begin{array}{l}2425.71 \\
2436.90 \\
2448.08 \\
2459.24 \\
2470.39 \\
2481.53 \\
2492.65 \\
2503.76 \\
2514.86 \\
2525.95\end{array}$ & $\begin{array}{l}56.433 \\
56.494 \\
56.555 \\
56.615 \\
56.675 \\
56.735 \\
56.794 \\
56.854 \\
56.912 \\
56.971\end{array}$ \\
\hline $\begin{array}{l}191.00 \\
192.00 \\
193.00 \\
194.00 \\
195.00 \\
196.00 \\
197.00 \\
198.00 \\
199.00 \\
200.00\end{array}$ & $\begin{array}{l}1949.04 \\
1959.25 \\
1969.46 \\
1979.67 \\
1989.87 \\
2000.08 \\
2010.29 \\
2020.49 \\
2030.70 \\
2040.91\end{array}$ & $\begin{array}{l}2517.41 \\
2533.65 \\
2549.88 \\
2566.09 \\
2582.29 \\
2598.48 \\
2614.65 \\
2630.81 \\
2646.95 \\
2663.08\end{array}$ & $\begin{array}{l}1727.45 \\
1739.55 \\
1751.64 \\
1763.71 \\
1775.77 \\
1787.82 \\
1799.86 \\
1811.88 \\
1823.89 \\
1835.88\end{array}$ & $\begin{array}{l}52.119 \\
52.204 \\
52.288 \\
52.372 \\
52.455 \\
52.538 \\
52.620 \\
52.702 \\
52.783 \\
52.864\end{array}$ & $\begin{array}{l}261.00 \\
262.00 \\
263.00 \\
264.00 \\
265.00 \\
266.00 \\
267.00 \\
268.00 \\
269.00 \\
270.00\end{array}$ & $\begin{array}{l}2662.68 \\
2672.84 \\
2682.99 \\
2693.15 \\
2703.31 \\
2713.47 \\
2723.62 \\
2733.78 \\
2743.94 \\
2754.10\end{array}$ & $\begin{array}{l}3616.23 \\
3631.41 \\
3646.57 \\
3661.73 \\
3676.87 \\
3692.00 \\
3707.12 \\
3722.23 \\
3737.33 \\
3752.41\end{array}$ & $\begin{array}{l}2537.02 \\
2548.08 \\
2559.13 \\
2570.16 \\
2581.19 \\
2592.20 \\
2603.20 \\
2614.20 \\
2625.18 \\
2636.15\end{array}$ & $\begin{array}{l}57.029 \\
57.087 \\
57.145 \\
57.203 \\
57.260 \\
57.317 \\
57.374 \\
57.430 \\
57.486 \\
57.542\end{array}$ \\
\hline $\begin{array}{l}201.00 \\
202.00 \\
203.00 \\
204.00 \\
205.00 \\
206.00 \\
207.00 \\
208.00 \\
209.00 \\
210.00\end{array}$ & $\begin{array}{l}2051.11 \\
2061.31 \\
2071.51 \\
2081.71 \\
2091.91 \\
2102.11 \\
2112.31 \\
2122.52 \\
2132.72 \\
2142.92\end{array}$ & $\begin{array}{l}2679.18 \\
2695.27 \\
2711.34 \\
2727.40 \\
2743.44 \\
2759.46 \\
2775.47 \\
2791.46 \\
2807.44 \\
2823.40\end{array}$ & $\begin{array}{l}1847.84 \\
1859.80 \\
1871.73 \\
1883.66 \\
1895.56 \\
1907.45 \\
1919.33 \\
1931.18 \\
1943.03 \\
1954.85\end{array}$ & $\begin{array}{l}52.945 \\
53.024 \\
53.104 \\
53.183 \\
53.261 \\
53.339 \\
53.417 \\
53.494 \\
53.570 \\
53.646\end{array}$ & $\begin{array}{l}271.00 \\
272.00 \\
273.00 \\
274.00 \\
275.00 \\
276.00 \\
277.00 \\
278.00 \\
279.00 \\
280.00\end{array}$ & $\begin{array}{l}2764.26 \\
2774.42 \\
2784.59 \\
2794.75 \\
2804.92 \\
2815.09 \\
2825.26 \\
2835.44 \\
2845.62 \\
2855.80\end{array}$ & $\begin{array}{l}3767.49 \\
3782.56 \\
3797.63 \\
3812.68 \\
3827.72 \\
3842.76 \\
3857.79 \\
3872.81 \\
3887.83 \\
3902.84\end{array}$ & $\begin{array}{l}2647.11 \\
2658.06 \\
2669.00 \\
2679.94 \\
2690.86 \\
2701.77 \\
2712.68 \\
2723.58 \\
2734.47 \\
2745.35\end{array}$ & $\begin{array}{l}57.598 \\
57.654 \\
57.709 \\
57.764 \\
57.819 \\
57.873 \\
57.928 \\
57.982 \\
58.036 \\
58.084\end{array}$ \\
\hline $\begin{array}{l}211.00 \\
212.00 \\
213.00 \\
214.00 \\
215.00 \\
216.00 \\
217.00 \\
218.00 \\
219.00 \\
220.00\end{array}$ & $\begin{array}{l}2153.12 \\
2163.32 \\
2173.52 \\
2183.72 \\
2193.92 \\
2204.12 \\
2214.32 \\
2224.52 \\
2234.72 \\
2244.92\end{array}$ & $\begin{array}{l}2839.34 \\
2855.27 \\
2871.18 \\
2887.07 \\
2902.95 \\
2918.81 \\
2934.65 \\
2950.48 \\
2966.29 \\
2982.09\end{array}$ & $\begin{array}{l}1966.66 \\
1978.45 \\
1990.23 \\
2001.99 \\
2013.73 \\
2025.46 \\
2037.17 \\
2048.86 \\
2060.54 \\
2072.20\end{array}$ & $\begin{array}{l}53.722 \\
53.797 \\
53.872 \\
53.947 \\
54.021 \\
54.094 \\
54.168 \\
54.240 \\
54.313 \\
54.385\end{array}$ & $\begin{array}{l}281.00 \\
282.00 \\
283.00 \\
284.00 \\
285.00 \\
286.00 \\
287.00 \\
288.00 \\
289.00 \\
290.00\end{array}$ & $\begin{array}{l}2865.99 \\
2876.18 \\
2886.37 \\
2896.57 \\
2906.77 \\
2916.98 \\
2927.19 \\
2937.41 \\
2947.63 \\
2957.86\end{array}$ & $\begin{array}{l}3917.84 \\
3932.84 \\
3947.83 \\
3962.81 \\
3977.79 \\
3992.77 \\
4007.74 \\
4022.70 \\
4037.66 \\
4052.61\end{array}$ & $\begin{array}{l}2756.22 \\
2767.09 \\
2777.95 \\
2788.80 \\
2799.65 \\
2810.48 \\
2821.31 \\
2832.14 \\
2842.95 \\
2853.76\end{array}$ & $\begin{array}{l}58.143 \\
58.196 \\
58.249 \\
58.302 \\
58.355 \\
58.407 \\
58.459 \\
58.511 \\
58.563 \\
58.615\end{array}$ \\
\hline $\begin{array}{l}221.00 \\
222.00 \\
223.00 \\
224.00 \\
225.00 \\
226.00 \\
227.00 \\
228.00 \\
229.00 \\
230.00\end{array}$ & $\begin{array}{l}2255.12 \\
2265.32 \\
2275.52 \\
2285.72 \\
2295.93 \\
2306.13 \\
2316.33 \\
2326.53 \\
2336.73 \\
2346.93\end{array}$ & $\begin{array}{l}2997.86 \\
3013.63 \\
3029.37 \\
3045.10 \\
3060.81 \\
3076.50 \\
3092.18 \\
3107.85 \\
3123.49 \\
3139.12\end{array}$ & $\begin{array}{l}2083.84 \\
2095.47 \\
2107.08 \\
2118.67 \\
2130.25 \\
2141.81 \\
2153.35 \\
2164.88 \\
2176.39 \\
2187.89\end{array}$ & $\begin{array}{l}54.456 \\
54.527 \\
54.598 \\
54.669 \\
54.739 \\
54.808 \\
54.877 \\
54.946 \\
55.015 \\
55.083\end{array}$ & $\begin{array}{l}291.00 \\
292.00 \\
293.00 \\
294.00 \\
295.00 \\
296.00 \\
297.00 \\
298.00 \\
299.00 \\
300.00\end{array}$ & $\begin{array}{l}2968.09 \\
2978.32 \\
2988.56 \\
2998.80 \\
3009.04 \\
3019.28 \\
3029.53 \\
3039.78 \\
3050.03 \\
3060.28\end{array}$ & $\begin{array}{l}4067.56 \\
4082.51 \\
4097.44 \\
4112.38 \\
4127.30 \\
4142.23 \\
4157.14 \\
4172.05 \\
4186.95 \\
4201.85\end{array}$ & $\begin{array}{l}2864.56 \\
2875.36 \\
2886.15 \\
2896.93 \\
2907.71 \\
2918.48 \\
2929.24 \\
2939.99 \\
2950.74 \\
2961.48\end{array}$ & $\begin{array}{l}58.66 \\
58.71 \\
58.76 \\
58.82 \\
58.87 \\
58.92 \\
58.97 \\
59.02 \\
59.07 \\
59.12\end{array}$ \\
\hline
\end{tabular}




\begin{tabular}{|c|c|c|c|c|c|c|c|c|c|}
\hline $\begin{array}{l}\text { TEMPER- } \\
\text { ATURE } \\
(K)\end{array}$ & $\begin{array}{l}\text { SPECIFIC } \\
\text { VOLUME } \\
\text { (CC/GM) }\end{array}$ & $\begin{array}{c}\text { ENTHALPY } \\
(J / G M)\end{array}$ & $\begin{array}{l}\text { INTERNAL } \\
\text { ENERGY } \\
(J / G M)\end{array}$ & $\begin{array}{l}\text { ENTROPY } \\
(J / G M-K)\end{array}$ & $\begin{array}{c}\text { TEMPER- } \\
\text { ATURE } \\
(K)\end{array}$ & $\begin{array}{l}\text { SPECIFIC } \\
\text { VDLUME } \\
(C C / G M)\end{array}$ & $\begin{array}{c}\text { ENTHALPY } \\
(\mathrm{J} / \mathrm{GM})\end{array}$ & $\begin{array}{l}\text { INTERNAL } \\
\text { ENERGY } \\
(\mathrm{J} / \mathrm{GM})\end{array}$ & $\begin{array}{l}\text { ENTROPY } \\
(J / G M-K)\end{array}$ \\
\hline 20.00 & 13.96 & -254.36 & $-261 \cdot 43$ & 7.799 & & & & & \\
\hline 21.00 & $14 \cdot 18$ & -244.68 & -251.87 & 8.274 & & & & & \\
\hline 22.00 & 14.44 & -234.38 & -241.70 & 8.750 & 91.00 & 738.59 & 967.90 & 593.71 & 39.873 \\
\hline 23.00 & 14.72 & -223.37 & -230.83 & 9.242 & 92.00 & 746.91 & 980.78 & 602.38 & 40.014 \\
\hline 24.00 & 15.04 & $-211 \cdot 56$ & -219.18 & 9.746 & 93.00 & 755.22 & 993.74 & 611.13 & 40.154 \\
\hline 25.00 & 15.42 & -198.79 & -206.60 & 10.265 & 94.00 & 763.53 & 1006.78 & 619.96 & 40.294 \\
\hline 26.00 & 15.85 & -184.84 & -192.87 & 10.811 & 95.00 & 771.84 & 1019.91 & 628.88 & 40.433 \\
\hline 27.00 & 16.39 & -169.36 & -177.66 & 11.395 & 96.00 & 780.14 & 1033.12 & 637.88 & 40.571 \\
\hline 27.184 & 16.49 & -166.29 & -174.64 & 11.510 & 97.00 & 788.44 & 1046.41 & 646.97 & 40.709 \\
\hline 27.184 & 160.24 & $202 \cdot 35$ & $121 \cdot 17$ & 25.087 & 98.00 & 796.74 & 1059.78 & 656.13 & 40.846 \\
\hline 28.00 & 171.83 & 217.62 & 130.57 & 25.641 & 99.00 & 805.03 & 1073.23 & 665.39 & 40.982 \\
\hline 29.00 & 184.70 & 234.20 & 140.62 & 26.223 & 100.00 & 813.31 & 1086.76 & 674.72 & 41.118 \\
\hline 30.00 & 196.67 & 249.34 & 149.70 & 26.736 & & & & & \\
\hline 31.00 & 208.03 & 263.56 & 158.17 & 27.202 & 101.00 & 821.59 & 1100.37 & 684.11 & 41.254 \\
\hline 32.00 & 218.95 & 277.14 & 166.22 & 27.634 & 102.00 & 829.86 & 1114.05 & 693.61 & 41.388 \\
\hline 33.00 & $229 \cdot 55$ & 290.27 & 173.97 & 28.038 & 103.00 & 838.13 & 1127.82 & 703.19 & 41.523 \\
\hline 34.00 & 239.88 & 303.05 & 181.52 & 28.419 & 104.00 & 846.39 & 1141.66 & 712.85 & 41.657 \\
\hline 35.00 & 250.01 & 315.57 & 188.91 & 28.782 & 105.00 & 854.65 & 1155.59 & 722.59 & 41.790 \\
\hline 36.00 & 259.96 & 327.87 & 196.17 & 29.129 & 106.00 & 862.91 & 1169.60 & 732.41 & 41.923 \\
\hline 37.00 & 269.77 & 340.01 & 203.34 & 29.461 & 107.00 & 871.17 & 1183.68 & 742.31 & 42.055 \\
\hline 38.00 & 279.45 & 352.00 & 210.43 & 29.781 & 108.00 & 879.42 & 1197.84 & 752.29 & 42.187 \\
\hline 39.00 & 289.03 & 363.88 & 217.45 & 30.090 & 109.00 & 887.67 & 1212.08 & 762.35 & 42.318 \\
\hline 40.00 & 298.51 & 375.65 & 224.42 & 30.388 & 110.00 & 895.92 & 1226.40 & 772.49 & 42.449 \\
\hline 41.00 & 307.90 & 387.33 & 231.34 & 30.676 & 111.00 & 904.17 & 1240.79 & 782.70 & 42.579 \\
\hline 42.00 & 317.21 & 398.93 & 238.22 & 30.956 & 112.00 & 912.41 & 1255.25 & 792.99 & 42.709 \\
\hline 43.00 & 326.46 & 410.47 & 245.08 & 31.227 & 113.00 & 920.66 & 1269.79 & 803.35 & 42.836 \\
\hline 44.00 & 335.64 & 421.94 & 251.90 & 31.491 & 114.00 & 928.90 & 1284.40 & 813.78 & 42.966 \\
\hline 45.00 & 344.76 & 433.37 & 258.70 & 31.748 & 115.00 & 937.14 & 1299.07 & 824.28 & 43.095 \\
\hline 46.00 & 353.84 & 444.74 & 265.48 & 31.998 & 116.00 & 945.38 & 1313.82 & 834.85 & $43 \cdot 222$ \\
\hline 47.00 & 362.86 & 456.07 & 272.24 & 32.241 & 117.00 & 953.62 & 1328.64 & 845.50 & 43.349 \\
\hline 48.00 & 371.84 & 467.37 & 278.99 & 32.479 & 118.00 & 961.86 & 1343.52 & 856.20 & 43.476 \\
\hline $\begin{array}{l}49.00 \\
50.00\end{array}$ & $\begin{array}{l}380.78 \\
389.68\end{array}$ & $\begin{array}{l}478.64 \\
489.88\end{array}$ & $\begin{array}{l}285.73 \\
292.46\end{array}$ & $\begin{array}{l}32.712 \\
32.939\end{array}$ & $\begin{array}{l}119.00 \\
120.00\end{array}$ & $\begin{array}{l}970 \cdot 10 \\
978 \cdot 34\end{array}$ & $\begin{array}{l}1358.47 \\
1373.48\end{array}$ & $\begin{array}{l}866.98 \\
877.82\end{array}$ & $\begin{array}{l}43.602 \\
43.728\end{array}$ \\
\hline 51.00 & 398.54 & 501.09 & 299.18 & 33.161 & 121.00 & 986.57 & 1388.55 & 888.71 & 43.853 \\
\hline 52.00 & 407.37 & 512.29 & 305.90 & 33.378 & 122.00 & 994.81 & 1403.67 & 899.66 & 43.977 \\
\hline 53.00 & 416.17 & 523.47 & 312.63 & 33.591 & 123.00 & 1003.04 & 1418.86 & 910.68 & 44.101 \\
\hline 54.00 & 424.94 & 534.64 & 319.36 & 33.800 & 124.00 & 1011.28 & 1434.10 & 921.75 & 44.225 \\
\hline 55.00 & 433.68 & 545.80 & 326.08 & 34.005 & 125.00 & 1019.51 & 1449.41 & 932.88 & $44 \cdot 348$ \\
\hline 56.00 & 442.40 & 556.96 & 332.82 & 34.206 & 126.00 & 1027.74 & 1464.77 & 944.08 & 44.470 \\
\hline 57.00 & 451.09 & 568.11 & 339.58 & 34.403 & 127.00 & 1035.96 & 1480.19 & 955.33 & 44.592 \\
\hline 58.00 & 459.77 & 579.27 & 346.34 & 34.597 & 128.00 & 1044.17 & 1495.66 & 966.64 & 44.713 \\
\hline 59.00 & 468.42 & 590.43 & 353.12 & 34.788 & 129.00 & 1052.39 & 1511.18 & 978.00 & 44.834 \\
\hline 60.00 & 477.05 & 601.60 & 359.92 & 34.976 & 130.00 & 1060.61 & 1526.76 & 989.41 & 44.954 \\
\hline 61.00 & 485.66 & 612.78 & 366.74 & 35.161 & 131.00 & 1068.82 & 1542.38 & 1000.87 & 45.074 \\
\hline 62.00 & 494.25 & 623.98 & 373.58 & 35.343 & 132.00 & 1077.04 & 1558.05 & 1012.38 & 193 \\
\hline 63.00 & 502.83 & 635.20 & 380.45 & 35.522 & 133.00 & 1085.25 & 1573.77 & 1023.94 & 45.312 \\
\hline 64.00 & 511.39 & 646.43 & 387.35 & 35.699 & 134.00 & 1093.46 & 1589.54 & 1035.55 & 45.430 \\
\hline 65.00 & 519.93 & 657.70 & 394.28 & 35.874 & 135.00 & 1101.67 & 1605.34 & 1047.19 & 45.548 \\
\hline 66.00 & 528.46 & 668.98 & 401.25 & 36.046 & 136.00 & 1109.88 & 1621.19 & 1058.89 & 45.665 \\
\hline 67.00 & $536 \cdot 98$ & 680.30 & 408.25 & 36.216 & 137.00 & 1118.09 & 1637.09 & 1070.62 & 45.781 \\
\hline 68.00 & 545.49 & 691.65 & 415.29 & 36.384 & 138.00 & $1126 \cdot 30$ & 1653.02 & 1082.39 & 45.897 \\
\hline 69.00 & 553.98 & 703.04 & $422 \cdot 38$ & 36.551 & 139.00 & 1134.50 & 1668.99 & 1094.20 & 46.012 \\
\hline 70.00 & $562 \cdot 46$ & $714 \cdot 46$ & 429.51 & 36.715 & 140.00 & 1142.71 & 1684.99 & 1106.05 & 46.127 \\
\hline 71.00 & 570.93 & 725.93 & 436.68 & 36.878 & 141.00 & 1150.91 & 1701.03 & 1117.94 & 46.241 \\
\hline 72.00 & 579.39 & 737.44 & 443.91 & 37.039 & 142.00 & 1159.12 & 1717.11 & 1129.85 & 46.355 \\
\hline 73.00 & 587.83 & 749.00 & 451.19 & 37.198 & 143.00 & 1167.32 & 1733.21 & 1141.80 & 46.468 \\
\hline 74.00 & 596.27 & 760.61 & 458.52 & 37.356 & 144.00 & 1175.53 & 1749.35 & 1153.78 & 46.580 \\
\hline 75.00 & $604 \cdot 70$ & 772.27 & 465.92 & 37.513 & 145.00 & 1183.73 & 1765.52 & 1165.79 & 46.692 \\
\hline 76.00 & 613.12 & 783.99 & 473.37 & 37.668 & 146.00 & 1191.93 & 1781.71 & 1177.83 & 46.803 \\
\hline 77.00 & 621.53 & 795.77 & 480.88 & 37.822 & 147.00 & 1200.13 & 1797.93 & 1189.90 & 46.914 \\
\hline 78.00 & 629.94 & 807.60 & 488.46 & 37.974 & 148.00 & 1208.33 & 1814.18 & 1201.99 & 47.024 \\
\hline 79.00 & 638.33 & 819.50 & 496.11 & 38.126 & 149.00 & 1216.53 & 1830.45 & 1214.10 & 47.134 \\
\hline 80.00 & 646.72 & 831.47 & 503.82 & 38.277 & 150.00 & 1224.73 & 1846.74 & 1226.24 & 47.243 \\
\hline 81.00 & 655.10 & 843.49 & 511.50 & $38 \cdot 426$ & 151.00 & 1232.92 & 1862.99 & 1238.34 & 47.351 \\
\hline 82.00 & 663.48 & 855.59 & 519.46 & 38.574 & 152.00 & 1241.12 & 1879.26 & 1250.47 & \\
\hline 83.00 & 671.85 & 867.77 & 527.39 & 38.722 & 153.00 & 1249.31 & 1895.55 & 1262.60 & \\
\hline 84.00 & 680.21 & 880.01 & 535.40 & 38.869 & 154.00 & 1257.50 & 1311.85 & 1274.76 & 47.671 \\
\hline 85.00 & 688.56 & 892.33 & 543.49 & 39.014 & 155.00 & 1265.70 & 1928.17 & 1286.92 & 47.777 \\
\hline 86.00 & 696.91 & 904.73 & 551.66 & 39.159 & 156.00 & 1273.89 & 1944.50 & 1299.10 & 47. $A B 2$ \\
\hline 87.00 & 705.26 & 917.20 & 559.90 & 39.304 & 157.00 & & 1960.84 & 1311.29 & 47.986 \\
\hline 88.00 & 713.60 & 929.76 & 568.23 & 39.447 & 158.00 & 1290.27 & 1977.19 & 1323.49 & 48.090 \\
\hline 89.00 & 721.93 & 942.39 & 576.64 & 39.590 & 159.00 & & 1993.55 & 1335.70 & 48.193 \\
\hline 90.00 & 730.26 & 955.10 & 585.14 & 39.732 & $160: 00$ & 1306.65 & 2009.92 & i 347.92 & 48.296 \\
\hline
\end{tabular}


TEMPER- SPECIFIC ENTHALPY INTERNAL ENTROPY ATURE VOLUME (J/GM) ENERGY (J/GM-K) (K) $(C C / G M)$
TEMPERATURE (K)
SPECIFIC (CC/GM)
ENTHALPY INTERNAL ENTROPY (J/GM) ENERGY

\begin{tabular}{|c|c|c|c|c|c|c|c|c|c|}
\hline 161.00 & 1314.84 & 2026.30 & 1360.15 & 48.398 & 231.00 & 1886.92 & 3154.82 & 2198.83 & 54.227 \\
\hline 162.00 & 1323.02 & 2042.68 & 1372.39 & 48.499 & 232.00 & 1895.09 & 3170.43 & 2210.30 & 54.295 \\
\hline 163.00 & 1331.21 & 2059.07 & 1384.63 & 48.600 & 233.00 & 1903.25 & 3186.02 & 2221.76 & 54.362 \\
\hline 164.00 & 1339.40 & 2075.46 & 1396.87 & 48.700 & 234.00 & 1911.42 & 3201.59 & 2233.19 & 54.428 \\
\hline 165.00 & 1347.59 & 2091.86 & 1409.12 & 48.800 & 235.00 & 1919.59 & 3217.15 & 2244.62 & 54.495 \\
\hline 166.00 & 1355.78 & 2108.26 & 1421.37 & 48.899 & 236.00 & 1927.75 & 3232.69 & 2256.02 & 54.561 \\
\hline 167.00 & 1363.96 & 2124.66 & 1433.63 & 48.998 & 237.00 & 1935.92 & 3248.22 & 2267.41 & 54.626 \\
\hline 168.00 & 1372.15 & 2141.07 & 1445.88 & 49.096 & 238.00 & 1944.08 & 3263.73 & 2278.78 & 54.692 \\
\hline 169.00 & 1380.34 & 2157.47 & 1458.14 & 49.193 & 239.00 & 1952.24 & 3279.22 & 2290.14 & 54.757 \\
\hline 170.00 & 1388.52 & 2173.88 & 1470.40 & 49.290 & 240.00 & 1960.40 & 3294.69 & 2301.48 & 54.821 \\
\hline 171.00 & 1396.70 & 2190.28 & 1482.66 & 49.386 & 241.00 & 1968.56 & 3310.15 & 2312.80 & 54.886 \\
\hline 172.00 & 1404.89 & 2206.69 & 1494.92 & 49.482 & 242.00 & 1976.72 & 3325.60 & 2324.11 & 54.949 \\
\hline 173.00 & 1413.07 & 2223.09 & 1507.17 & 49.577 & 243.00 & 1984.88 & 3341.02 & 2335.41 & 55.013 \\
\hline 174.00 & 1421.25 & 2239.49 & 1519.43 & 49.671 & 244.00 & 1993.03 & 3356.43 & 2346.68 & 55.076 \\
\hline 175.00 & 1429.43 & 2255.88 & 1531.68 & 49.765 & 245.00 & 2001.19 & 3371.83 & 2357.95 & 55.139 \\
\hline 176.00 & 1437.61 & 2272.27 & 1543.93 & 49.859 & 246.00 & 2009.34 & 3387.20 & 2369.19 & 55.202 \\
\hline 177.00 & 1445.79 & 2288.66 & 1556.17 & 49.952 & 247.00 & 2017.49 & 3402.57 & 2380.43 & 55.264 \\
\hline $178 \cdot 00$ & 1453.96 & 2305.04 & 1568.41 & 50.044 & 248.00 & 2025.64 & 3417.91 & 2391.64 & 55.326 \\
\hline 179.00 & 1462.14 & 2321.42 & 1580.64 & 50.136 & 249.00 & 2033.79 & 3433.24 & 2402.85 & 55.388 \\
\hline 180.00 & 1470.32 & 2337.78 & 1592.86 & 50.227 & 250.00 & 2041.94 & 3448.56 & 2414.03 & 55.449 \\
\hline 181.00 & 1478.50 & 2354.15 & 1605.08 & 50.317 & 251.00 & 2050.09 & 3463.89 & 2425.24 & 55.511 \\
\hline 182.00 & 1486.67 & 2370.50 & 1617.29 & 50.407 & 252.00 & 2058.23 & 3479.22 & 2436.44 & 55.572 \\
\hline 183.00 & 1494.85 & 2386.84 & 1629.49 & 50.497 & 253.00 & 2066.37 & 3494.52 & 2447.62 & 55.632 \\
\hline 184.00 & 1503.03 & 2403.18 & 1641.69 & 50.586 & 254.06 & 2074.51 & 3509.81 & 2458.78 & 55.692 \\
\hline 185.00 & 1511.20 & 2419.50 & 1653.87 & 50.675 & 255.00 & 2082.65 & 3525.09 & 2469.94 & 55.752 \\
\hline 186.00 & 1519.38 & 2435.82 & 1666.05 & 50.762 & 256.00 & 2090.79 & 3540.35 & 2481.08 & 55.312 \\
\hline 187.00 & 1527.55 & 2452.13 & 1678.21 & 50.850 & 257.00 & 2098.93 & 3555.60 & 2492.20 & 55.872 \\
\hline 188.00 & 1535.72 & 2468.42 & 1690.36 & 50.937 & 258.00 & 2107.06 & 3570.83 & 2503.32 & 55.931 \\
\hline 189.00 & 1543.90 & 2484.70 & 1702.50 & 51.023 & 259.00 & 2115.20 & 3586.06 & 2514.42 & 55.990 \\
\hline 190.0 .0 & 1552.07 & 2500.97 & 1714.64 & 51.109 & 260.00 & 2123.33 & 3601.26 & 2525.50 & 56.048 \\
\hline 191.00 & 1560.25 & 2517.23 & 1726.75 & 51.194 & 261.00 & 2131.46 & 3616.46 & 2536.58 & 56.107 \\
\hline 192.00 & 1568.42 & 2533.48 & 1738.86 & 51.279 & 262.00 & 2139.59 & 3631.64 & 2547.64 & 56.165 \\
\hline 193.00 & 1576.59 & 2549.72 & 1750.95 & 51.364 & 263.00 & 2147.72 & 3646.81 & 2558.69 & 56.222 \\
\hline 194.00 & 1584.76 & 2565.94 & 1763.03 & 51.447 & 264.00 & 2155.85 & 3661.97 & 2569.73 & 56.280 \\
\hline 195.00 & 1592.94 & 2582.14 & 1775.10 & 51.531 & 265.00 & 2163.98 & $3677 \cdot 12$ & 2580.76 & 56.337 \\
\hline 196.00 & 1601.11 & 2598.34 & 1787.15 & 51.614 & 266.00 & 2172.11 & 3692.25 & 2591.78 & 56.394 \\
\hline 197.00 & 1609.28 & 2614.52 & 1799.19 & 51.696 & 267.00 & 2180.24 & 3707.37 & 2602.78 & 56.451 \\
\hline 198.00 & 1617.45 & 2630.68 & 1811.22 & 51.778 & 268.00 & 2188.37 & 3722.49 & 2613.77 & 56.508 \\
\hline 199.00 & 1625.62 & 2646.83 & 1823.23 & 51.859 & 269.00 & 2196.50 & 3737.59 & 2624.76 & 56.564 \\
\hline 200.00 & 1633.79 & 2662.96 & 1835.22 & 51.940 & 270.00 & 2204.63 & 3752.68 & 2635.73 & 56.620 \\
\hline 201.00 & 1641.96 & 2679.07 & 1847.20 & 52.020 & 271.00 & 2212.76 & 3767.77 & 2646.70 & 56.676 \\
\hline 202.00 & 1650.12 & 2695.17 & 1859.15 & 52.100 & 272.00 & 2220.90 & 3782.84 & 2657.65 & .731 \\
\hline 203.00 & 1658.29 & 2711.25 & 1871.09 & 52.180 & 273.00 & 2229.03 & 3797.91 & 2668.59 & 56.786 \\
\hline 204.00 & 1666.46 & 2727.31 & 1883.02 & 52.259 & 274.00 & 2237.17 & 3812.96 & 2679.53 & 56.841 \\
\hline 205.00 & 1674.62 & 2743.36 & 1894.93 & 52.337 & 275.00 & 2245.30 & 3828.01 & 2690.45 & 56.896 \\
\hline 206.00 & 1682.79 & 2759.39 & 1906.82 & 52.415 & 276.00 & 2253.44 & 3843.05 & 2701.37 & 56.951 \\
\hline 207.00 & 1690.96 & 2775.41 & 1918.70 & 52.493 & 277.00 & 2261.58 & 3858.08 & 2712.28 & 57.005 \\
\hline 208.00 & 1699.12 & 2791.40 & 1930.56 & 52.570 & 278.00 & 2269.73 & 3873.11 & 2723.18 & 57.059 \\
\hline 209.00 & 1707.29 & 2807.39 & 1942.41 & 52.646 & 279.00 & 2277.87 & 3888.13 & 2734.07 & 57.113 \\
\hline 210.00 & 1715.45 & 2823.35 & 1954.24 & 52.723 & 280.00 & 2286.02 & 3903.14 & 2744.95 & 57.167 \\
\hline 211.00 & 1723.62 & 2839.30 & 1966.05 & 52.798 & 281.00 & 2294.17 & 3918.14 & 2755.83 & 57.220 \\
\hline 212.00 & 1731.78 & 2855.24 & 1977.85 & 52.874 & 282.00 & 2302.33 & 3933.14 & 2766.69 & 57.274 \\
\hline 213.00 & 1739.95 & 2871.15 & 1989.63 & 52.949 & 283.00 & 2310.49 & 3948.14 & 2777.56 & 57.327 \\
\hline 214.00 & 1748.11 & 2887.05 & 2001.39 & 53.023 & 284.00 & 2318.65 & 3963.12 & 2788.41 & 57.380 \\
\hline 215.00 & 1756.28 & 2902.93 & 2013.14 & 53.097 & 285.00 & 2326.81 & 3978.11 & 2799.25 & 57.432 \\
\hline 216.00 & 1764.44 & 2918.80 & 2024.87 & 53.171 & 286.00 & 2334.98 & 3993.08 & 2810.09 & 57.485 \\
\hline 217.00 & 1772.61 & 2934.65 & 2036.58 & 53.244 & 287.00 & 2343.15 & 4008.05 & 2820.92 & 57.537 \\
\hline 218.00 & 1780.77 & 2950.48 & $2048 \cdot 28$ & 53.317 & 288.00 & 2351.33 & 4023.02 & 2831.75 & 57.589 \\
\hline 219.00 & 1788.94 & 2966.30 & 2059.96 & 53.389 & 289.00 & 2359.50 & 4037.98 & 2842.56 & 57.641 \\
\hline 220.00 & 1797.10 & 2982.10 & 2071.62 & 53.461 & 290.00 & 2367.68 & 4052.94 & 2853.38 & 57.693 \\
\hline 221.00 & 1805.27 & 2997.89 & 2083.27 & 53.533 & .00 & 87 & 7.89 & 18 & .744 \\
\hline 222.00 & 1813.43 & 3013.65 & 2094.90 & 53.604 & 292.00 & 2384.06 & 4082.83 & 2874.98 & 57.795 \\
\hline 223.00 & 1821.60 & $3029 \cdot 40$ & 2106.51 & 53.675 & 293.00 & 2392.25 & 4097.77 & 2885.77 & 57.846 \\
\hline 224.00 & 1829.76 & 3045.14 & 2118.11 & 53.745 & 294.00 & 2400.44 & 4112.70 & 2896.55 & 57.897 \\
\hline 225.00 & 1837.93 & 3060.85 & 2129.69 & 53.815 & 295.00 & 2408.63 & 4127.63 & 2907.33 & 57.948 \\
\hline 226.00 & 1846.09 & $307 i .55$ & 2141.25 & 53.885 & 296.00 & 2416.83 & 4142.56 & 2918.10 & 57.998 \\
\hline 227.00 & 1854.26 & 3092.24 & 2152.80 & 53.954 & 297.00 & 2425.03 & 4157.47 & 2928.86 & 58.049 \\
\hline 228.00 & 1862.42 & 3107.91 & 2164.33 & 54.023 & 298.00 & 2433.23 & 4172.38 & 2939.62 & 58.099 \\
\hline 229.00 & 1870.59 & 3123.56 & 2175.84 & 54.091 & 299.00 & 2441.43 & 4187.29 & 2950.37 & 58.149 \\
\hline 230.00 & 1878.75 & 3139.19 & 2187.34 & 54.159 & 300.00 & 2449.63 & 4202.18 & 2961.11 & 58.199 \\
\hline
\end{tabular}




\begin{tabular}{|c|c|c|c|c|c|c|c|c|c|}
\hline $\begin{array}{c}\text { TEMPER- } \\
\text { ATURE } \\
(K)\end{array}$ & $\begin{array}{l}\text { SPECIFIC } \\
\text { VOLUME } \\
\text { (CC/GM) }\end{array}$ & $\begin{array}{c}\text { ENTHALPY } \\
(J / G M)\end{array}$ & $\begin{array}{l}\text { INTERNAL } \\
\text { ENERGY } \\
(J / G M)\end{array}$ & $\begin{array}{l}\text { ENTROPY } \\
(J / G M-K)\end{array}$ & $\begin{array}{l}\text { TEMPER- } \\
\text { ATURE } \\
(\mathrm{X})\end{array}$ & $\begin{array}{l}\text { SPECIFIC } \\
\text { VOLUME } \\
\text { (CC/GM) }\end{array}$ & $\begin{array}{c}\text { ENTHALPY } \\
(J / G M)\end{array}$ & $\begin{array}{l}\text { INTERNAL } \\
\text { ENERGY } \\
(J / G M)\end{array}$ & $\begin{array}{l}\text { ENTROPY } \\
(J / G M-K)\end{array}$ \\
\hline 20.00 & 13.93 & -253.38 & -261.85 & 7.777 & & & & & \\
\hline $\begin{array}{l}21.00 \\
22.00 \\
23.00 \\
24.00 \\
25.00 \\
26.00 \\
27.00 \\
28.00 \\
28.195 \\
28.195 \\
29.00 \\
30.00\end{array}$ & $\begin{array}{l}14.16 \\
14.40 \\
14.68 \\
15.00 \\
15.36 \\
15.78 \\
16.29 \\
16.93 \\
17.08 \\
131.72 \\
142.21 \\
153.77\end{array}$ & $\begin{array}{r}-243.76 \\
-233.51 \\
-222.58 \\
-210.87 \\
-198.23 \\
-184.48 \\
-169.28 \\
-152.09 \\
-148.41 \\
198.05 \\
214.81 \\
232.79\end{array}$ & $\begin{array}{r}-252.36 \\
-242.27 \\
-231.50 \\
-219.98 \\
-207.57 \\
-194.07 \\
-179.19 \\
-162.39 \\
-158.80 \\
117.97 \\
128.36 \\
139.31\end{array}$ & $\begin{array}{r}8.249 \\
8.723 \\
9.212 \\
9.712 \\
10.225 \\
10.764 \\
11.337 \\
11.963 \\
12.093 \\
24.401 \\
24.987 \\
25.597\end{array}$ & $\begin{array}{r}91.00 \\
92.00 \\
93.00 \\
94.00 \\
95.00 \\
96.00 \\
97.00 \\
98.00 \\
99.00 \\
100.00\end{array}$ & $\begin{array}{l}615.11 \\
622.07 \\
629.03 \\
635.98 \\
642.93 \\
649.88 \\
656.82 \\
663.75 \\
670.69 \\
677.62\end{array}$ & $\begin{array}{r}966.01 \\
978.93 \\
991.93 \\
1005.02 \\
1018.18 \\
1031.43 \\
1044.76 \\
1058.16 \\
1071.65 \\
1085.21\end{array}$ & $\begin{array}{l}592.05 \\
600.74 \\
609.51 \\
618.37 \\
627.31 \\
636.34 \\
645.44 \\
654.63 \\
663.90 \\
673.25\end{array}$ & $\begin{array}{l}39.103 \\
39.244 \\
39.385 \\
39.525 \\
39.664 \\
39.803 \\
39.941 \\
40.078 \\
40.215 \\
40.352\end{array}$ \\
\hline $\begin{array}{l}31.00 \\
32.00 \\
33.00 \\
34.00 \\
35.00 \\
36.00 \\
37.00 \\
38.00 \\
39.00 \\
40.00\end{array}$ & $\begin{array}{l}164.35 \\
174.31 \\
183.82 \\
192.99 \\
201.91 \\
210.63 \\
219.17 \\
227.57 \\
235.84 \\
244.01\end{array}$ & $\begin{array}{l}248.94 \\
263.94 \\
278.15 \\
291.81 \\
305.06 \\
317.98 \\
330.65 \\
343.12 \\
355.41 \\
367.56\end{array}$ & $\begin{array}{l}149.02 \\
157.96 \\
166.40 \\
174.48 \\
182.30 \\
189.93 \\
197.41 \\
204.77 \\
212.04 \\
219.22\end{array}$ & $\begin{array}{l}26.126 \\
26.602 \\
27.040 \\
27.448 \\
27.832 \\
28.196 \\
28.543 \\
28.876 \\
29.195 \\
29.503\end{array}$ & $\begin{array}{l}101.00 \\
102.00 \\
103.00 \\
104.00 \\
105.00 \\
106.00 \\
107.00 \\
108.00 \\
109.00 \\
110.00\end{array}$ & $\begin{array}{l}684.54 \\
691.45 \\
698.36 \\
705.27 \\
712.17 \\
719.07 \\
725.97 \\
732.87 \\
739.76 \\
746.65\end{array}$ & $\begin{array}{l}1098.85 \\
1112.57 \\
1126.37 \\
1140.24 \\
1154.20 \\
1168.23 \\
1182.34 \\
1196.53 \\
1210.80 \\
1225.14\end{array}$ & $\begin{array}{l}682.68 \\
692.19 \\
701.79 \\
711.46 \\
721.22 \\
731.06 \\
740.98 \\
750.98 \\
761.05 \\
771.20\end{array}$ & $\begin{array}{l}40.487 \\
40.622 \\
40.757 \\
40.891 \\
41.025 \\
41.158 \\
41.290 \\
41.422 \\
41.554 \\
41.685\end{array}$ \\
\hline $\begin{array}{l}41.00 \\
42.00 \\
43.00 \\
44.00 \\
45.00 \\
46.00 \\
47.00 \\
48.00 \\
49.00 \\
50.00\end{array}$ & $\begin{array}{l}252.08 \\
260.07 \\
267.99 \\
275.84 \\
283.62 \\
291.35 \\
299.03 \\
306.67 \\
314.26 \\
321.81\end{array}$ & $\begin{array}{l}379.59 \\
391.52 \\
403.35 \\
415.09 \\
426.77 \\
438.39 \\
449.94 \\
461.45 \\
472.92 \\
484.34\end{array}$ & $\begin{array}{l}226.34 \\
233.40 \\
240.43 \\
247.40 \\
254.35 \\
261.26 \\
268.15 \\
275.01 \\
281.86 \\
288.70\end{array}$ & $\begin{array}{l}29.800 \\
30.087 \\
30.365 \\
30.635 \\
30.898 \\
31.153 \\
31.402 \\
31.644 \\
31.880 \\
32.111\end{array}$ & $\begin{array}{l}111.00 \\
112.00 \\
113.00 \\
114.00 \\
115.00 \\
116.00 \\
117.00 \\
118.00 \\
119.00 \\
120.00\end{array}$ & $\begin{array}{l}753.54 \\
760.43 \\
767.32 \\
774.20 \\
781.09 \\
787.97 \\
794.85 \\
801.73 \\
808.61 \\
815.49\end{array}$ & $\begin{array}{l}1239.56 \\
1254.04 \\
1268.60 \\
1283.23 \\
1297.94 \\
1312.70 \\
1327.54 \\
1342.45 \\
1357.41 \\
1372.45\end{array}$ & $\begin{array}{l}781.43 \\
791.73 \\
802.10 \\
812.55 \\
823.06 \\
833.65 \\
844.30 \\
855.02 \\
865.80 \\
876.65\end{array}$ & $\begin{array}{l}41.815 \\
41.945 \\
42.074 \\
42.203 \\
42.332 \\
42.460 \\
42.587 \\
42.714 \\
42.840 \\
42.966\end{array}$ \\
\hline $\begin{array}{l}51.00 \\
52.00 \\
53.00 \\
54.00 \\
55.00 \\
56.00 \\
57.00 \\
58.00 \\
59.00 \\
60.00\end{array}$ & $\begin{array}{l}329.32 \\
336.80 \\
344.25 \\
351.67 \\
359.06 \\
366.42 \\
373.76 \\
381.08 \\
388.37 \\
395.65\end{array}$ & $\begin{array}{l}495.74 \\
507.10 \\
518.45 \\
529.77 \\
541.08 \\
552.38 \\
563.67 \\
574.95 \\
586.24 \\
597.53\end{array}$ & $\begin{array}{l}295.53 \\
302.35 \\
309.16 \\
315.98 \\
322.79 \\
329.61 \\
336.44 \\
343.28 \\
350.13 \\
356.99\end{array}$ & $\begin{array}{l}32.337 \\
32.558 \\
32.774 \\
32.985 \\
33.193 \\
33.396 \\
33.596 \\
33.793 \\
33.985 \\
34.175\end{array}$ & $\begin{array}{l}121.00 \\
122.00 \\
123.00 \\
124.00 \\
125.00 \\
126.00 \\
127.00 \\
128.00 \\
129.00 \\
130.00\end{array}$ & $\begin{array}{l}822.37 \\
829.25 \\
836.13 \\
843.01 \\
849.88 \\
856.75 \\
863.61 \\
870.47 \\
877.34 \\
884.19\end{array}$ & $\begin{array}{l}1387.53 \\
1402.68 \\
1417.88 \\
1433.14 \\
1448.46 \\
1463.84 \\
1479.28 \\
1494.77 \\
1510.31 \\
1525.90\end{array}$ & $\begin{array}{l}887.56 \\
898.52 \\
909.54 \\
920.62 \\
931.76 \\
942.97 \\
954.23 \\
965.55 \\
976.92 \\
988.34\end{array}$ & $\begin{array}{l}43.091 \\
43.216 \\
43.340 \\
43.464 \\
43.587 \\
43.709 \\
43.831 \\
43.953 \\
44.074 \\
44.194\end{array}$ \\
\hline $\begin{array}{l}61.00 \\
62.00 \\
63.00 \\
64.00 \\
65.00 \\
66.00 \\
67.00 \\
68.00 \\
69.00 \\
70.00\end{array}$ & $\begin{array}{l}402.90 \\
410.14 \\
417 \cdot 36 \\
424.57 \\
431.76 \\
438.93 \\
446.09 \\
453.24 \\
460.37 \\
467.49\end{array}$ & $\begin{array}{l}608.82 \\
620.13 \\
631.45 \\
642.79 \\
654.14 \\
665.52 \\
676.93 \\
688.37 \\
699.84 \\
711.35\end{array}$ & $\begin{array}{l}363.88 \\
370.79 \\
377.71 \\
384.67 \\
391.66 \\
398.68 \\
405.74 \\
412.83 \\
419.96 \\
427.14\end{array}$ & $\begin{array}{l}34.362 \\
34.546 \\
34.727 \\
34.905 \\
35.082 \\
35.255 \\
35.427 \\
35.596 \\
35.764 \\
35.929\end{array}$ & $\begin{array}{l}131.00 \\
132.00 \\
133.00 \\
134.00 \\
135.00 \\
136.00 \\
137.00 \\
138.00 \\
139.00 \\
140.00\end{array}$ & $\begin{array}{l}891.05 \\
897.91 \\
904.77 \\
911.62 \\
918.47 \\
925.33 \\
932.18 \\
939.03 \\
945.88 \\
952.73\end{array}$ & $\begin{array}{l}1541.54 \\
1557.23 \\
1572.96 \\
1588.74 \\
1604.57 \\
1620.43 \\
1636.34 \\
1652.28 \\
1668.27 \\
1684.29\end{array}$ & $\begin{array}{r}999.81 \\
1011.33 \\
1022.90 \\
1034.51 \\
1046.17 \\
1057.86 \\
1069.61 \\
1081.39 \\
1093.21 \\
1105.06\end{array}$ & $\begin{array}{l}44.314 \\
44.433 \\
44.552 \\
44.670 \\
44.788 \\
44.905 \\
45.021 \\
45.137 \\
45.253 \\
45.368\end{array}$ \\
\hline $\begin{array}{l}71.00 \\
72.00 \\
73.00 \\
74.00 \\
75.00 \\
76.00 \\
77.00 \\
78.00 \\
79.00 \\
80.00\end{array}$ & $\begin{array}{l}474.61 \\
481.71 \\
488.80 \\
495.88 \\
502.95 \\
510.01 \\
517.07 \\
524.11 \\
531.15 \\
538.18\end{array}$ & $\begin{array}{l}722.89 \\
734.48 \\
746.11 \\
757.79 \\
769.53 \\
781.31 \\
793.15 \\
805.05 \\
817.01 \\
829.03\end{array}$ & $\begin{array}{l}434.36 \\
441.63 \\
448.95 \\
456.32 \\
463.75 \\
471.24 \\
478.80 \\
486.41 \\
494.09 \\
501.84\end{array}$ & $\begin{array}{l}36.093 \\
36.255 \\
36.416 \\
36.574 \\
36.732 \\
36.888 \\
37.043 \\
37.196 \\
37.349 \\
37.500\end{array}$ & $\begin{array}{l}141.00 \\
142.00 \\
143.00 \\
144.00 \\
145.00 \\
146.00 \\
147.00 \\
148.00 \\
149.00 \\
150.00\end{array}$ & $\begin{array}{r}959.57 \\
966.42 \\
973.27 \\
980.11 \\
986.96 \\
993.80 \\
1000.65 \\
1007.49 \\
1014.33 \\
1021.17\end{array}$ & $\begin{array}{l}1700.34 \\
1716.43 \\
1732.55 \\
1748.70 \\
1764.88 \\
1781.09 \\
1797.32 \\
1813.58 \\
1829.86 \\
1846.16\end{array}$ & $\begin{array}{l}1116.95 \\
1128.88 \\
1140.84 \\
1152.82 \\
1164.84 \\
1176.89 \\
1188.96 \\
1201.06 \\
1213.18 \\
1225.32\end{array}$ & $\begin{array}{l}45.482 \\
45.595 \\
45.709 \\
45.821 \\
45.933 \\
46.045 \\
46.155 \\
46.266 \\
46.375 \\
46.484\end{array}$ \\
\hline $\begin{array}{l}81.00 \\
82.00 \\
83.00 \\
84.00 \\
85.00 \\
86.00 \\
87.00 \\
88.00 \\
89.00 \\
90.00\end{array}$ & $\begin{array}{l}545.21 \\
552.22 \\
559.23 \\
566.24 \\
573.24 \\
580.23 \\
587.22 \\
594.20 \\
601.17 \\
608.14\end{array}$ & $\begin{array}{l}841.12 \\
853.27 \\
865.50 \\
877.79 \\
890.17 \\
902.61 \\
915.13 \\
927.73 \\
940.41 \\
953.17\end{array}$ & $\begin{array}{l}509.66 \\
517.55 \\
525.51 \\
533.55 \\
541.66 \\
549.86 \\
558.14 \\
566.49 \\
574.93 \\
583.45\end{array}$ & $\begin{array}{l}37.650 \\
37.799 \\
37.947 \\
38.095 \\
38.241 \\
38.387 \\
38.531 \\
38.675 \\
38.819 \\
38.961\end{array}$ & $\begin{array}{l}151.00 \\
152.00 \\
153.00 \\
154.00 \\
155.00 \\
156.00 \\
157.00 \\
158.00 \\
159.00 \\
160.00\end{array}$ & $\begin{array}{l}1028.01 \\
1034.85 \\
1041.68 \\
1048.52 \\
1055.35 \\
1062.19 \\
1069.02 \\
1075.86 \\
1082.69 \\
1089.52\end{array}$ & $\begin{array}{l}1862.43 \\
1878.71 \\
1895.01 \\
1911.33 \\
1927.66 \\
1944.00 \\
1960.35 \\
1976.71 \\
1993.08 \\
2009.46\end{array}$ & $\begin{array}{l}1237.43 \\
1249.56 \\
1261.71 \\
1273.87 \\
1286.04 \\
1298.22 \\
1310.42 \\
1322.63 \\
1334.85 \\
1347.07\end{array}$ & $\begin{array}{l}46.592 \\
46.700 \\
46.807 \\
46.913 \\
47.019 \\
47.124 \\
47.228 \\
47.332 \\
47.435 \\
47.538\end{array}$ \\
\hline
\end{tabular}


TEMPERATUR

(K)
ENTHALPY
(J/GM)
INTERNAL ENTROPY ENERGY (J/GM-K) (J/GM)
TEMPERATUR

(K)
SPECIFIC ENTHALPY VOLUME
(J/GM)
INTERNAL ENTROPY

(J/GM)
(J/GM-K)

\begin{tabular}{|c|c|c|c|c|c|c|c|c|c|}
\hline $\begin{array}{l}161.00 \\
162.00 \\
163.00 \\
164.00 \\
165.00 \\
166.00 \\
167.00 \\
168.00 \\
169.00 \\
170.00\end{array}$ & $\begin{array}{l}1096.35 \\
1103.18 \\
1110.01 \\
1116.85 \\
1123.68 \\
1130.51 \\
1137.34 \\
1144.17 \\
1150.99 \\
1157.82\end{array}$ & $\begin{array}{l}2025.85 \\
2042.25 \\
2058.64 \\
2075.05 \\
2091.46 \\
2107.87 \\
2124.28 \\
2140.70 \\
2157.11 \\
2173.53\end{array}$ & $\begin{array}{l}1359.31 \\
1371.55 \\
1383.79 \\
1396.04 \\
1408.30 \\
1420.56 \\
1432.82 \\
1445.08 \\
1457.35 \\
1469.61\end{array}$ & $\begin{array}{l}47.640 \\
47.742 \\
47.843 \\
47.943 \\
48.043 \\
48.142 \\
48.240 \\
48.338 \\
48.436 \\
48.533\end{array}$ & $\begin{array}{l}231.00 \\
232.00 \\
233.00 \\
234.00 \\
235.00 \\
236.00 \\
237.00 \\
238.00 \\
239.00 \\
240.00\end{array}$ & $\begin{array}{l}1573.44 \\
1580.25 \\
1587.06 \\
1593.87 \\
1600.68 \\
1607.48 \\
1614.29 \\
1621.10 \\
1627.90 \\
1634.70\end{array}$ & $\begin{array}{l}3154.90 \\
3170.52 \\
3186.11 \\
3201.69 \\
3217.26 \\
3232.81 \\
3248.34 \\
3263.85 \\
3279.35 \\
3294.83\end{array}$ & $\begin{array}{l}2198.30 \\
2209.78 \\
2221.23 \\
2232.67 \\
2244.10 \\
2255.51 \\
2266.90 \\
2278.28 \\
2289.64 \\
2300.98\end{array}$ & $\begin{array}{l}53.472 \\
53.540 \\
53.607 \\
53.674 \\
53.740 \\
53.806 \\
53.872 \\
53.937 \\
54.002 \\
54.067\end{array}$ \\
\hline $\begin{array}{l}171.00 \\
172.00 \\
173.00 \\
174.00 \\
175.00 \\
176.00 \\
177.00 \\
178.00 \\
179.00 \\
180.00\end{array}$ & $\begin{array}{l}1164.65 \\
1171.47 \\
1178.30 \\
1185.12 \\
1191.94 \\
1198.77 \\
1205.59 \\
1212.41 \\
1219.23 \\
1226.05\end{array}$ & $\begin{array}{l}2189.94 \\
2206.35 \\
2222.77 \\
2239.18 \\
2255.58 \\
2271.98 \\
2288.38 \\
2304.77 \\
2321.15 \\
2337.53\end{array}$ & $\begin{array}{l}1481.87 \\
1494.14 \\
1506.40 \\
1518.66 \\
1530.92 \\
1543.17 \\
1555.42 \\
1567.66 \\
1579.90 \\
1592.13\end{array}$ & $\begin{array}{l}48.629 \\
48.725 \\
48.820 \\
48.914 \\
49.008 \\
49.102 \\
49.195 \\
49.287 \\
49.379 \\
49.470\end{array}$ & $\begin{array}{l}241.00 \\
242.00 \\
243.00 \\
244.00 \\
245.00 \\
246.00 \\
247.00 \\
248.00 \\
249.00 \\
250.00\end{array}$ & $\begin{array}{l}1641.51 \\
1648.31 \\
1655.11 \\
1661.91 \\
1668.71 \\
1675.51 \\
1682.30 \\
1689.10 \\
1695.89 \\
1702.68\end{array}$ & $\begin{array}{l}3310.29 \\
3325.74 \\
3341.17 \\
3356.59 \\
3371.99 \\
3387.37 \\
3402.74 \\
3418.09 \\
3433.42 \\
3448.74\end{array}$ & $\begin{array}{l}2312.31 \\
2323.62 \\
2334.92 \\
2346.20 \\
2357.47 \\
2368.72 \\
2379.95 \\
2391.17 \\
2402.38 \\
2413.57\end{array}$ & $\begin{array}{l}54.131 \\
54.195 \\
54.258 \\
54.322 \\
54.385 \\
54.447 \\
54.510 \\
54.572 \\
54.633 \\
54.695\end{array}$ \\
\hline $\begin{array}{l}181.00 \\
182.00 \\
183.00 \\
184.00 \\
185.00 \\
186.00 \\
187.00 \\
188.00 \\
189.00 \\
190.00\end{array}$ & $\begin{array}{l}1232.87 \\
1239.69 \\
1246.51 \\
1253.33 \\
1260.15 \\
1266.96 \\
1273.78 \\
1280.60 \\
1287.42 \\
1294.23\end{array}$ & $\begin{array}{l}2353.90 \\
2370.26 \\
2386.61 \\
2402.95 \\
2419.29 \\
2435.61 \\
2451.93 \\
2468.23 \\
2484.52 \\
2500.80\end{array}$ & $\begin{array}{l}1604.35 \\
1616.57 \\
1628.78 \\
1640.97 \\
1653.16 \\
1665.34 \\
1677.51 \\
1689.67 \\
1701.82 \\
1713.95\end{array}$ & $\begin{array}{l}49.561 \\
49.651 \\
49.741 \\
49.830 \\
49.918 \\
50.006 \\
50.094 \\
50.181 \\
50.267 \\
50.353\end{array}$ & $\begin{array}{l}251.00 \\
252.00 \\
253.00 \\
254.00 \\
255.00 \\
256.00 \\
257.00 \\
258.00 \\
259.00 \\
260.00\end{array}$ & $\begin{array}{l}1709.47 \\
1716.26 \\
1723.05 \\
1729.84 \\
1736.62 \\
1743.41 \\
1750.19 \\
1756.97 \\
1763.75 \\
1770.53\end{array}$ & $\begin{array}{l}3464.08 \\
3479.41 \\
3494.72 \\
3510.02 \\
3525.30 \\
3540.56 \\
3555.82 \\
3571.06 \\
3586.28 \\
3601.50\end{array}$ & $\begin{array}{l}2424.78 \\
2435.98 \\
2447.16 \\
2458.33 \\
2469.49 \\
2480.63 \\
2491.76 \\
2502.88 \\
2513.98 \\
2525.07\end{array}$ & $\begin{array}{l}54.756 \\
54.817 \\
54.878 \\
54.938 \\
54.998 \\
55.058 \\
55.117 \\
55.177 \\
55.235 \\
55.294\end{array}$ \\
\hline $\begin{array}{l}191.00 \\
192.00 \\
193.00 \\
194.00 \\
195.00 \\
196.00 \\
197.00 \\
198.00 \\
199.00 \\
200.00\end{array}$ & $\begin{array}{l}1301.05 \\
1307.87 \\
1314.68 \\
1321.50 \\
1328.31 \\
1335.13 \\
1341.94 \\
1348.75 \\
1355.57 \\
1362.38\end{array}$ & $\begin{array}{l}2517.07 \\
2533.32 \\
2549.56 \\
2565.79 \\
2582.01 \\
2598.21 \\
2614.39 \\
2630.57 \\
2646.72 \\
2662.86\end{array}$ & $\begin{array}{l}1726.07 \\
1738.18 \\
1750.28 \\
1762.37 \\
1774.44 \\
1786.50 \\
1798.54 \\
1810.57 \\
1822.58 \\
1834.58\end{array}$ & $\begin{array}{l}50.438 \\
50.523 \\
50.608 \\
50.691 \\
50.775 \\
50.858 \\
50.940 \\
51.022 \\
51.103 \\
51.184\end{array}$ & $\begin{array}{l}261.00 \\
262.00 \\
263.00 \\
264.00 \\
265.00 \\
266.00 \\
267.00 \\
268.00 \\
269.00 \\
270.00\end{array}$ & $\begin{array}{l}1777.31 \\
1784.09 \\
1790.87 \\
1797.65 \\
1804.43 \\
1811.21 \\
1817.98 \\
1824.76 \\
1831.54 \\
1838.32\end{array}$ & $\begin{array}{l}3616.70 \\
3631.88 \\
3647.06 \\
3662.22 \\
3677.37 \\
3692.51 \\
3707.64 \\
3722.75 \\
3737.86 \\
3752.96\end{array}$ & $\begin{array}{l}2536.15 \\
2547.21 \\
2558.27 \\
2569.31 \\
2580.34 \\
2591.36 \\
2602.36 \\
2613.36 \\
2624.35 \\
2635.32\end{array}$ & $\begin{array}{l}55.352 \\
55.410 \\
55.468 \\
55.526 \\
55.583 \\
55.640 \\
55.697 \\
55.753 \\
55.810 \\
55.866\end{array}$ \\
\hline $\begin{array}{l}201.00 \\
202.00 \\
203.00 \\
204.00 \\
205.00 \\
206.00 \\
207.00 \\
208.00 \\
209.00 \\
210.00\end{array}$ & $\begin{array}{l}1369.19 \\
1376.00 \\
1382.81 \\
1389.62 \\
1396.43 \\
1403.24 \\
1410.05 \\
1416.86 \\
1423.67 \\
1430.48\end{array}$ & $\begin{array}{l}2678.98 \\
2695.08 \\
2711.17 \\
2727.24 \\
2743.29 \\
2759.33 \\
2775.35 \\
2791.36 \\
2807.35 \\
2823.32\end{array}$ & $\begin{array}{l}1846.56 \\
1858.52 \\
1870.46 \\
1882.39 \\
1894.31 \\
1906.21 \\
1918.09 \\
1929.95 \\
1941.80 \\
1953.64\end{array}$ & $\begin{array}{l}51.265 \\
51.345 \\
51.424 \\
51.503 \\
51.581 \\
51.659 \\
51.737 \\
51.814 \\
51.891 \\
51.967\end{array}$ & $\begin{array}{l}271.00 \\
272.00 \\
273.00 \\
274.00 \\
275.00 \\
276.00 \\
277.00 \\
278.00 \\
279.00 \\
280.00\end{array}$ & $\begin{array}{l}1845.10 \\
1851.88 \\
1858.66 \\
1865.44 \\
1872.22 \\
1879.01 \\
1885.80 \\
1892.58 \\
1899.37 \\
1906.17\end{array}$ & $\begin{array}{l}3768.04 \\
3783.12 \\
3798.19 \\
3813.25 \\
3828.30 \\
3843.34 \\
3858.38 \\
3873.41 \\
3888.43 \\
3903.44\end{array}$ & $\begin{array}{l}2646.29 \\
2657.24 \\
2668.19 \\
2679.12 \\
2690.05 \\
2700.97 \\
2711.88 \\
2722.78 \\
2733.67 \\
2744.56\end{array}$ & $\begin{array}{l}55.921 \\
55.977 \\
56.032 \\
56.087 \\
56.142 \\
56.197 \\
56.251 \\
56.305 \\
56.359 \\
56.413\end{array}$ \\
\hline $\begin{array}{l}211.00 \\
212.00 \\
213.00 \\
214.00 \\
215.00 \\
216.00 \\
217.00 \\
218.00 \\
219.00 \\
220.00\end{array}$ & $\begin{array}{l}1437.29 \\
1444.10 \\
1450.90 \\
1457.71 \\
1464.52 \\
1471.33 \\
1478.14 \\
1484.94 \\
1491.75 \\
1498.56\end{array}$ & $\begin{array}{l}2839.27 \\
2855.21 \\
2871.13 \\
2887.04 \\
2902.93 \\
2918.80 \\
2934.66 \\
2950.50 \\
2966.32 \\
2982.13\end{array}$ & $\begin{array}{l}1965.45 \\
1977.25 \\
1989.03 \\
2000.80 \\
2012.55 \\
2024.28 \\
2036.00 \\
2047.70 \\
2059.39 \\
2071.05\end{array}$ & $\begin{array}{l}52.043 \\
52.118 \\
52.193 \\
52.268 \\
52.342 \\
52.415 \\
52.489 \\
52.562 \\
52.634 \\
52.706\end{array}$ & $\begin{array}{l}281.00 \\
282.00 \\
283.00 \\
284.00 \\
285.00 \\
286.00 \\
287.00 \\
288.00 \\
289.00 \\
290.00\end{array}$ & $\begin{array}{l}1912.96 \\
1919.76 \\
1926.56 \\
1933.36 \\
1940.17 \\
1946.98 \\
1953.79 \\
1960.60 \\
1967.42 \\
1974.23\end{array}$ & $\begin{array}{l}3918.45 \\
3933.45 \\
3948.45 \\
3963.44 \\
3978.42 \\
3993.40 \\
4008.37 \\
4023.34 \\
4038.30 \\
4053.26\end{array}$ & $\begin{array}{l}2755.44 \\
2766.30 \\
2777.17 \\
2788.02 \\
2798.87 \\
2809.71 \\
2820.54 \\
2831.36 \\
2842.18 \\
2852.99\end{array}$ & $\begin{array}{l}56.466 \\
56.520 \\
56.573 \\
56.626 \\
56.678 \\
56.731 \\
56.783 \\
56.835 \\
56.887 \\
56.939\end{array}$ \\
\hline $\begin{array}{l}221.00 \\
222.00 \\
223.00 \\
224.00 \\
225.00 \\
226.00 \\
227.00 \\
228.00 \\
229.00 \\
230.00\end{array}$ & $\begin{array}{l}1505.37 \\
1512.18 \\
1518.98 \\
1525.79 \\
1532.60 \\
1539.41 \\
1546.21 \\
1553.02 \\
1559.83 \\
1566.63\end{array}$ & $\begin{array}{l}2997.91 \\
3013.69 \\
3029.44 \\
3045.18 \\
3060.91 \\
3076.61 \\
3092.30 \\
3107.97 \\
3123.63 \\
3139.27\end{array}$ & $\begin{array}{l}2082.70 \\
2094.34 \\
2105.95 \\
2117.55 \\
2129.14 \\
2140.70 \\
2152.26 \\
2163.79 \\
2175.31 \\
2186.81\end{array}$ & $\begin{array}{l}52.778 \\
52.849 \\
52.920 \\
52.990 \\
53.060 \\
53.130 \\
53.199 \\
53.268 \\
53.336 \\
53.405\end{array}$ & $\begin{array}{l}291.00 \\
292.00 \\
293.00 \\
294.00 \\
295.00 \\
296.00 \\
297.00 \\
298.00 \\
299.00 \\
300.00\end{array}$ & $\begin{array}{l}1981.05 \\
1987.88 \\
1994.70 \\
2001.53 \\
2008.36 \\
2015.19 \\
2022.02 \\
2028.86 \\
2035.69 \\
2042.52\end{array}$ & $\begin{array}{l}4068.21 \\
4083.16 \\
4098.10 \\
4113.04 \\
4127.97 \\
4142.89 \\
4157.81 \\
4172.72 \\
4187.63 \\
4202.53\end{array}$ & $\begin{array}{l}2863.80 \\
2874.60 \\
2885.39 \\
2896.17 \\
2906.95 \\
2917.72 \\
2928.49 \\
2939.25 \\
2950.00 \\
2960.74\end{array}$ & $\begin{array}{l}56.990 \\
57.041 \\
57.093 \\
57.143 \\
57.194 \\
57.245 \\
57.295 \\
57.345 \\
57.395 \\
57.445\end{array}$ \\
\hline
\end{tabular}




\begin{tabular}{|c|c|c|c|c|c|c|c|c|c|}
\hline $\begin{array}{l}\text { TEMPER- } \\
\text { ATURE } \\
(K)\end{array}$ & $\begin{array}{l}\text { SPECIFIC } \\
\text { VDLUME } \\
(C C / G M)\end{array}$ & $\begin{array}{c}\text { ENTHALPY } \\
(\mathrm{J} / \mathrm{GM})\end{array}$ & $\begin{array}{l}\text { INTERNAL } \\
\text { ENERGY } \\
(J / G M)\end{array}$ & $\begin{array}{l}\text { ENTROPY } \\
(J / G M-K)\end{array}$ & $\begin{array}{l}\text { TEMPER- } \\
\text { ATURE } \\
\text { (K) }\end{array}$ & $\begin{array}{l}\text { SPECIFIC } \\
\text { VOLUME } \\
(C C / G M)\end{array}$ & $\begin{array}{c}\text { ENTHALPY } \\
(J / G M)\end{array}$ & $\begin{array}{l}\text { INTERNAL } \\
\text { ENERGY } \\
(J / G M)\end{array}$ & $\begin{array}{l}\text { ENTROPY } \\
(\mathrm{J} / \mathrm{GM}-\mathrm{K})\end{array}$ \\
\hline 20.00 & 13.91 & $-252 \cdot 40$ & -262.27 & 7.756 & & & & & \\
\hline 21.00 & 14.13 & -242.82 & -252.84 & 8.226 & & & & & \\
\hline 22.00 & $14 \cdot 37$ & -232.63 & -242.83 & 8.697 & 91.00 & 526.93 & 964.13 & 590.39 & 38.449 \\
\hline 23.00 & 14.65 & -221.77 & -232.16 & 9.182 & 92.00 & 532.92 & 977.09 & 599.11 & 38.591 \\
\hline 24.00 & 14.95 & -210.15 & -220.76 & 9.678 & 93.00 & 538.90 & 990.14 & 607.90 & 38.732 \\
\hline 25.00 & 15.31 & -197.65 & -208.51 & 10.187 & 94.00 & 544.89 & 1003.26 & 616.78 & 38.872 \\
\hline 26.00 & 15.72 & -184.08 & -195.22 & 10.718 & 95.00 & 550.87 & 1016.46 & 625.75 & 39.012 \\
\hline 27.00 & 16.20 & -169.15 & -180.64 & 11.281 & 96.00 & 556.84 & 1029.75 & 634.79 & 39.151 \\
\hline 28.00 & 16.81 & -152.39 & -164.32 & 11.892 & 97.00 & 562.81 & 1043.11 & 643.92 & 39.289 \\
\hline 29.00 & 17.62 & -132.84 & -145.33 & 12.575 & 98.00 & 568.78 & 1056.55 & 653.13 & 39.427 \\
\hline 29.094 & 17.73 & -130.79 & -143.36 & 12.647 & 99.00 & 574.74 & 1070.07 & 662.42 & 39.565 \\
\hline 29.094 & 110.76 & 192.30 & 113.74 & 23.773 & 100.00 & 580.70 & 1083.67 & 671.79 & 39.701 \\
\hline 30.00 & 121.83 & 213.17 & 126.76 & 24.480 & & & & & \\
\hline 31.00 & 132.34 & $232 \cdot 38$ & 138.50 & 25.110 & 101.00 & 586.66 & 1097.34 & 681.23 & 39.837 \\
\hline 32.00 & 141.87 & 249.39 & 148.77 & 25.650 & 102.00 & 592.60 & 1111.08 & 690.76 & 39.973 \\
\hline 33.00 & 150.76 & 265.06 & 158.13 & 26.132 & 103.00 & 598.54 & 1124.91 & 700.37 & 40.108 \\
\hline 34.00 & 159.22 & 279.84 & 166.91 & 26.574 & 104.00 & 604.48 & 1138.82 & 710.07 & 40.242 \\
\hline 35.00 & 167.34 & 293.98 & 175.28 & 26.983 & 105.00 & 610.41 & 1152.80 & 719.84 & 40.376 \\
\hline 36.00 & 175.22 & 307.64 & 183.36 & 27.368 & 106.00 & 616.34 & 1166.87 & 729.70 & 40.509 \\
\hline 37.00 & 182.89 & 320.93 & 191.21 & 27.733 & 107.00 & 622.27 & 1181.01 & 739.63 & 40.642 \\
\hline 38.00 & 190.40 & 333.94 & 198.89 & 28.079 & 108.00 & 628.20 & 1195.22 & 749.65 & 40.774 \\
\hline 39.00 & 197.77 & 346.71 & 206.43 & $28: 411$ & 109.00 & 634.12 & 1209.51 & 759.74 & 40.906 \\
\hline 40.00 & 205.02 & 359.28 & 213.87 & 28.729 & 110.00 & 640.04 & 1223.88 & 769.90 & 41.037 \\
\hline 41.00 & 212.17 & 371.69 & 221.21 & 29.036 & 111.00 & 645.96 & 1238.32 & 780.14 & 41.168 \\
\hline 42.00 & 219.22 & 383.96 & 228.47 & 29.331 & 112.00 & 651.88 & 1252.83 & 790.46 & 41.298 \\
\hline 43.00 & 226.19 & 396.10 & 235.67 & 29.617 & 113.00 & 657.80 & 1267.42 & 800.85 & 41.428 \\
\hline 44.00 & 233.10 & 408.15 & 242.81 & 29.894 & 114.00 & 663.71 & 1282.07 & 811.30 & 41.557 \\
\hline 45.00 & 239.93 & 420.09 & 249.91 & 30.162 & 115.00 & 669.63 & 1296.80 & 821.83 & 41.685 \\
\hline 46.00 & 246.71 & 431.96 & 256.97 & 30.423 & 116.00 & 675.54 & 1311.59 & 832.43 & 41.813 \\
\hline 47.00 & 253.44 & 443.75 & 263.99 & 30.677 & 117.00 & 681.45 & 1326.45 & 843.09 & 41.941 \\
\hline 48.00 & 260.12 & 455.48 & 270.99 & 30.924 & 118.00 & 687.37 & 1341.37 & 853.83 & 42.068 \\
\hline 49.00 & 266.75 & 467.15 & 277.96 & 31.165 & 119.00 & 693.28 & 1356.36 & 864.62 & 42.194 \\
\hline 50.00 & 273.34 & 478.78 & 284.90 & 31.399 & 120.00 & $699 \cdot 19$ & 1371.41 & 875.48 & 42.320 \\
\hline 51.00 & 279.89 & 490.35 & 291.83 & 31.629 & 121.00 & 705.09 & 1386.51 & 886.40 & 42.446 \\
\hline 52.00 & 286.41 & 501.90 & 298.75 & 31.853 & 122.00 & 711.00 & 1401.68 & 897.37 & 42.570 \\
\hline 53.00 & 292.89 & 513.40 & 305.67 & 32.072 & 123.00 & 716.91 & 1416.90 & 908.40 & 42.695 \\
\hline 54.00 & 299.34 & 524.89 & 312.57 & 32.287 & 124.00 & 722.82 & 1432.18 & 919.49 & 42.818 \\
\hline 55.00 & 305.77 & 536.34 & 319.47 & $32 \cdot 497$ & 125.00 & 728.72 & 1447.52 & 930.64 & 42.942 \\
\hline 56.00 & 312.17 & 547.79 & 326.37 & 32.703 & 126.00 & 734.62 & 1462.92 & 941.86 & 43.064 \\
\hline 57.00 & 318.54 & 559.21 & 333.28 & 32.905 & 127.00 & 740.51 & 1478.37 & 953.13 & 43.186 \\
\hline 58.00 & 324.89 & 570.63 & 340.19 & 33.104 & 128.00 & 746.40 & 1493.88 & 964.46 & 43.308 \\
\hline 59.00 & 331.22 & 582.04 & 347.12 & 33.299 & 129.00 & $752 \cdot 30$ & 1509.43 & 975.83 & 43.429 \\
\hline 60.00 & 337.52 & 593.45 & 354.05 & 33.491 & 130.00 & 758.18 & 1525.04 & 987.26 & 43.550 \\
\hline 61.00 & 343.81 & 604.86 & 361.00 & 33.679 & 131.00 & 764.07 & 1540.70 & 998.74 & 43.670 \\
\hline 62.00 & 350.08 & 616.27 & 367.97 & 33.865 & 132.00 & 769.96 & 1556.40 & 1010.27 & 43.789 \\
\hline 63.00 & 356.33 & 627.70 & 374.97 & 34.048 & 133.00 & 775.85 & 1572.15 & 1021.85 & 43.908 \\
\hline 64.00 & $362 \cdot 57$ & 639.14 & 381.98 & 34.228 & 134.00 & 781.73 & 1587.95 & 1033.47 & 44.026 \\
\hline 65.00 & 368.79 & 650.60 & 389.02 & 34.405 & 135.00 & 787.62 & 1603.79 & 1045.13 & 44.144 \\
\hline 66.00 & 374.99 & 662.07 & $396 \cdot 10$ & 34.581 & 136.00 & 793.50 & 1619.67 & 1056.84 & 44.261 \\
\hline 67.00 & 381.18 & 673.57 & 403.21 & 34.754 & 137.00 & 799.38 & 1635.59 & 1068.59 & 44. 378 \\
\hline 68.00 & 387.36 & 685.10 & $410 \cdot 35$ & 34.924 & 138.00 & 805.26 & 1651.55 & $1080 \cdot 38$ & 44.494 \\
\hline 69.00 & 393.53 & 696.65 & 417.53 & 35.093 & 139.00 & 811.14 & 1667.55 & 1092.21 & 44.609 \\
\hline 70.00 & 399.68 & 708.24 & 424.76 & 35.260 & 140.00 & 817.02 & 1683.58 & 1104.07 & 44.724 \\
\hline 71.00 & 405.82 & 719.86 & 432.02 & 35.425 & 141.00 & 822.90 & 1699.65 & 1115.97 & 44.839 \\
\hline 72.00 & 411.95 & 731.53 & 439.34 & 35.588 & 142.00 & $828: 78$ & 1715.75 & 1127.90 & 44.953 \\
\hline 73.00 & 418.07 & 743.23 & 446.70 & 35.749 & 143.00 & 834.66 & 1731.88 & 1139.87 & 45.066 \\
\hline 74.00 & 424.18 & 754.98 & 454.12 & 35.909 & 144.00 & 840.53 & 1748.05 & 1151.86 & 45.178 \\
\hline 75.00 & 430.29 & 766.78 & 461.59 & 36.068 & 145.00 & 846.41 & 1764.24 & 1163.89 & 45.290 \\
\hline 76.00 & 436.38 & 778.63 & 469.12 & 36.224 & 146.00 & 852.28 & 1780.46 & 1175.94 & 45.402 \\
\hline 77.00 & 442.46 & 790.54 & 476.71 & 36.380 & 147.00 & 858.16 & 1796.71 & 1188.02 & 45.513 \\
\hline 78.00 & 448.54 & 802.50 & 484.36 & 36.534 & 148.00 & 864.03 & 1812.98 & 1200.13 & 45.623 \\
\hline 79.00 & 454.61 & 814.52 & 492.08 & 36.688 & 149.00 & 869.91 & 1829.27 & 1212.25 & 45.733 \\
\hline 80.00 & 460.67 & 826.60 & 499.86 & 36.839 & 150.00 & 875.78 & 1845.59 & 2224.40 & 45.842 \\
\hline 81.00 & 466.72 & 838.75 & 507.71 & 36.990 & 151.00 & 881.65 & 1861.87 & 1236.52 & 45.950 \\
\hline 82.00 & 472.77 & 850.96 & 515.63 & 37.140 & 152.00 & 887.51 & 1878.17 & 1248.66 & 46.058 \\
\hline 83.00 & 478.81 & 863.23 & 523.63 & 37.289 & 153.00 & 893.38 & 1894.48 & 1260.81 & 46.165 \\
\hline 84.00 & 484.84 & 875.59 & 531.70 & 37.437 & 154.00 & 899.25 & 1910.81 & 1272.97 & 46.271 \\
\hline 85.00 & 490.87 & 888.01 & 539.84 & 37.584 & 155.00 & 905.11 & 1927.15 & 1285.15 & 46.377 \\
\hline 86.00 & 496.89 & 900.50 & 548.07 & 37.730 & 156.00 & 910.98 & 1943.50 & 1297.35 & 46.482 \\
\hline 87.00 & 502.91 & 913.07 & 556.37 & 37.875 & 157.00 & 916.84 & 1959.86 & $\lcm{309.55}$ & 46.587 \\
\hline 88.00 & 508.92 & 925.72 & 564.75 & 38.020 & 158.00 & 922.71 & 1976.23 & 1321.76 & 46.691 \\
\hline 89.00 & 514.93 & 938.44 & 573.22 & 38.164 & 159.00 & 928.57 & 1992.62 & 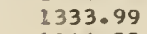 & 46.794 \\
\hline & 520.93 & 951.24 & 581.76 & 38.307 & 160.00 & 934.43 & 2009.01 & 1346.22 & \\
\hline
\end{tabular}


TEMPER- SPECIFIC ENTHALPY INTERNAL ENTROPY ATURE VOLUME (J/GM) ENERGY (J/GM-K) (K) $(\mathrm{CC} / \mathrm{GM})$ ( J/GM)
TEMPERATUR

(K)
SPECIFI
VOLUME

(CC/GM)
161.00

162.00

163.00

164.00

165.00

166.00

167.00

168.00

169.00

170.00

171.00

172.00

173.00

174.00

175.00

176.00

177.00

178.00

179.00

180.00

181.00

182.00

183.00

184.00

185.00

186.00

187.00

188.00

189.00

190.00

191.00

192.00

193.00

194.00

195.00

196.00

197.00

198.00

199.00

200.00

201.00

202.00

203.00

204.00

205.00

206.00

207.00

208.00

209.00

210.00

211.00

212.00

213.00

214.00

215.00

216.00

217.00

218.00

219.00

220.00

221.00

222.00

223.00

224.00

225.00

226.00

227.00

228.00

229.00

230.00

$\begin{array}{lll}940.29 & 2025.41 & 1358.46 \\ 946.16 & 2041.81 & 1370.71 \\ 952.02 & 2058.22 & 1382.96 \\ 957.88 & 2074.64 & 1395.22 \\ 963.74 & 2091.05 & 1407.48 \\ 969.60 & 2107.48 & 1419.74 \\ 975.46 & 2123.90 & 1432.01 \\ 981.32 & 2140.32 & 1444.28 \\ 987.18 & 2156.75 & 1456.55 \\ 993.04 & 2173.17 & 1468.82\end{array}$

998.89

1004.75

1010.60

1016.46

1022.31

1028.17

1034.02

1039.87

1045.72

1051.57

1057.42

1063.27

1069.12

1074.97

1080.82

1086.67

1092.52

1098.37

1104.22

1110.06

1115.91

1121.76

1127.60

1133.45

1139.30

1145.14

1150.99

1156.83

1162.68

1168.52

1174.36

1180.20

1186.04

1191.89

1197.73

1203.57

1209.41

1215.25

1221.09

1226.93

1232.77

1238.60

1244.44

1250.28

1256.12

1261.96

1267.80

1273.64

1279.48

1285.32

1291.15

1296.99

1302.83

1308.67

1314.51

1320.34

1326.18

1332.02

1337.86

1343.69
2189.60

2206.02

222.44

2238.86

2255.28

271.69

2288.09

2304.49

2320.88

2337.27

2353.64

2370.01

2386.38

2402.73

2419.07

2435.40

2451.72

2468.03

2484.33

2500.62

2516.90

2533.16

2549.41

2565.64

2581.86

598.07

2614.26

2630.44

646.61

2662.75

2678.88

2694.99

2711.08

2727.16

2743.22

2759.26

2775.29

2791.30

2807.30

2823.28

2839.24

2855.18

2871.11

2887.02

2902.92

2918.80

2934.66

2950.51

2966.33

2982.15

2997.94

3013.72

3029.48

3045.23

3060.95

3076.67

3092.36

3108.04

3123.70

3139.35
1481.09

1493.36

1505.63

1517.89

1530.16

1542.41

1554.67

1566.91

1579.16

1591.39

1603.62

1615.84

1628.05

1640.25

1652.45

1664.63

1676.81

1688.97

1701.12

1713.26

1725.39

1737.50

1749.60

1761.69

1773.77

1785.83

1797.88

1809.91

(821.93

1833.93

1845.91

1857.88

1869.83

1881.76

1893.68

1905.58

1917.47

1929.33

1941.19

1953.02

1964.85

1976.65

1988.44

2000.21

2011.96

2023.70

2035.42

2047.12

2058.81

2070.48

2082.13

2093.77

2105.39

2116.99

2128.58

2140.15

2151.71

2163.25

2174.77

2186.27

46.999

47.100

47.201

47.302

47.402

47.501

47.599

47.698

47.795

47.892

47.988

48.084

48.179

48.274

48.368

48.461

48.554

48.647

48.739

48.830

48.921

49.011 


\begin{tabular}{|c|c|c|c|c|c|c|c|c|c|}
\hline $\begin{array}{c}\text { TEHPER- } \\
\text { ATURE } \\
\text { (K) }\end{array}$ & $\begin{array}{l}\text { SPECIFIC } \\
\text { VOLUME } \\
\text { (CC/GM) }\end{array}$ & $\begin{array}{c}\text { ENTHALPY } \\
(\mathrm{J} / \mathrm{GM})\end{array}$ & $\begin{array}{l}\text { INTERNAL } \\
\text { ENERGY } \\
(J / G M)\end{array}$ & $\begin{array}{l}\text { ENTROPY } \\
(J / G M-K)\end{array}$ & $\begin{array}{c}\text { TEMPER- } \\
\text { ATURE } \\
(K)\end{array}$ & $\begin{array}{l}\text { SPECIFIC } \\
\text { VOLUME } \\
\text { (CC/GM) }\end{array}$ & $\begin{array}{c}\text { ENTHALPY } \\
(J / G M)\end{array}$ & $\begin{array}{l}\text { INTERNAL } \\
\text { ENERGY } \\
\text { (J/GM) }\end{array}$ & $\begin{array}{l}\text { ENTROPY } \\
(J / G M-K)\end{array}$ \\
\hline 20.00 & 13.88 & $-251 \cdot 42$ & -262.67 & 7.735 & & & & & \\
\hline $\begin{array}{l}21.00 \\
22.00 \\
23.00 \\
24.00 \\
25.00 \\
26.00 \\
27.00 \\
28.00 \\
29.00 \\
29.907 \\
29.907 \\
30.00\end{array}$ & $\begin{array}{l}14.10 \\
14.34 \\
14.61 \\
14.91 \\
15.25 \\
15.65 \\
16.12 \\
16.70 \\
17.45 \\
18.45 \\
94.60 \\
95.83\end{array}$ & $\begin{array}{r}-241.88 \\
-231.75 \\
-220.96 \\
-209.43 \\
-197.05 \\
-183.64 \\
-168.95 \\
-152.59 \\
-133.75 \\
-113.03 \\
185.22 \\
187.97\end{array}$ & $\begin{array}{r}-253.31 \\
-243.37 \\
-232.80 \\
-221.52 \\
-209.41 \\
-196.32 \\
-182.02 \\
-166.12 \\
-147.89 \\
-127.98 \\
108.54 \\
110.29\end{array}$ & $\begin{array}{r}8.202 \\
8.671 \\
9.153 \\
9.645 \\
10.149 \\
10.674 \\
11.227 \\
11.824 \\
12.482 \\
13.187 \\
23.181 \\
23.273\end{array}$ & $\begin{array}{r}91.00 \\
92.00 \\
93.00 \\
94.00 \\
95.00 \\
96.00 \\
97.00 \\
98.00 \\
99.00 \\
100.00\end{array}$ & $\begin{array}{l}460.80 \\
466.06 \\
471.32 \\
476.58 \\
481.83 \\
487.07 \\
492.32 \\
497.56 \\
502.79 \\
508.03\end{array}$ & $\begin{array}{r}962.26 \\
975.26 \\
988.34 \\
1001.51 \\
1014.75 \\
1028.07 \\
1041.47 \\
1054.95 \\
1068.51 \\
1082.14\end{array}$ & $\begin{array}{l}588.73 \\
597.47 \\
606.29 \\
615.19 \\
624.18 \\
633.25 \\
642.40 \\
651.63 \\
660.94 \\
670.33\end{array}$ & $\begin{array}{l}37.880 \\
38.022 \\
38.164 \\
38.305 \\
38.445 \\
38.584 \\
38.723 \\
38.861 \\
38.999 \\
39.136\end{array}$ \\
\hline $\begin{array}{l}31.00 \\
32.00 \\
33.00 \\
34.00 \\
35.00 \\
36.00 \\
37.00 \\
38.00 \\
39.00 \\
40.00\end{array}$ & $\begin{array}{l}107.28 \\
116.89 \\
125.54 \\
133.58 \\
141.20 \\
148.50 \\
155.56 \\
162.43 \\
169.15 \\
175.72\end{array}$ & $\begin{array}{l}212.88 \\
233.04 \\
250.76 \\
266.99 \\
282.24 \\
296.79 \\
310.82 \\
324.44 \\
337.75 \\
350.78\end{array}$ & $\begin{array}{l}125.92 \\
138.28 \\
148.99 \\
158.71 \\
167.79 \\
176.42 \\
184.72 \\
192.77 \\
200.64 \\
208.34\end{array}$ & $\begin{array}{l}24.091 \\
24.731 \\
25.276 \\
25.761 \\
26.203 \\
26.613 \\
26.997 \\
27.361 \\
27.706 \\
28.036\end{array}$ & $\begin{array}{l}101.00 \\
102.00 \\
103.00 \\
104.00 \\
105.00 \\
106.00 \\
107.00 \\
108.00 \\
109.00 \\
110.00\end{array}$ & $\begin{array}{l}513.25 \\
518.47 \\
523.69 \\
528.90 \\
534.11 \\
539.32 \\
544.52 \\
549.72 \\
554.92 \\
560.12\end{array}$ & $\begin{array}{l}1095.84 \\
1109.62 \\
1123.47 \\
1137.41 \\
1151.42 \\
1165.52 \\
1179.68 \\
1193.93 \\
1208.25 \\
1222.64\end{array}$ & $\begin{array}{l}679.78 \\
689.33 \\
698.96 \\
708.67 \\
718.46 \\
728.33 \\
738.28 \\
748.31 \\
758.41 \\
768.59\end{array}$ & $\begin{array}{l}39.272 \\
39.408 \\
39.543 \\
39.678 \\
39.812 \\
39.946 \\
40.079 \\
40.211 \\
40.343 \\
40.475\end{array}$ \\
\hline $\begin{array}{l}41.00 \\
42.00 \\
43.00 \\
44.00 \\
45.00 \\
46.00 \\
47.00 \\
48.00 \\
49.00 \\
50.00\end{array}$ & $\begin{array}{l}182.19 \\
188.55 \\
194.83 \\
201.03 \\
207.16 \\
213.23 \\
219.24 \\
225.20 \\
231.11 \\
236.99\end{array}$ & $\begin{array}{l}363.61 \\
376.25 \\
388.74 \\
401.09 \\
413.32 \\
425.45 \\
437.49 \\
449.45 \\
461.34 \\
473.16\end{array}$ & $\begin{array}{l}215.93 \\
223.41 \\
230.81 \\
238.13 \\
245.40 \\
252.61 \\
259.77 \\
266.90 \\
273.99 \\
281.06\end{array}$ & $\begin{array}{l}28.353 \\
28.658 \\
28.951 \\
29.235 \\
29.510 \\
29.777 \\
30.036 \\
30.288 \\
30.533 \\
30.772\end{array}$ & $\begin{array}{l}111.00 \\
112.00 \\
113.00 \\
114.00 \\
115.00 \\
116.00 \\
117.00 \\
118.00 \\
119.00 \\
120.00\end{array}$ & $\begin{array}{l}565.31 \\
570.50 \\
575.69 \\
580.88 \\
586.06 \\
591.25 \\
596.43 \\
601.61 \\
606.79 \\
611.97\end{array}$ & $\begin{array}{l}1237.10 \\
1251.64 \\
1266.25 \\
1280.92 \\
1295.67 \\
1310.48 \\
1325.36 \\
1340.31 \\
1355.31 \\
1370.39\end{array}$ & $\begin{array}{l}778.85 \\
789.18 \\
799.58 \\
810.05 \\
820.59 \\
831.20 \\
841.88 \\
852.63 \\
863.44 \\
874.31\end{array}$ & $\begin{array}{l}40.605 \\
40.736 \\
40.866 \\
40.995 \\
41.124 \\
41.252 \\
41.380 \\
41.507 \\
41.634 \\
41.760\end{array}$ \\
\hline $\begin{array}{l}51.00 \\
52.00 \\
53.00 \\
54.00 \\
55.00 \\
56.00 \\
57.00 \\
58.00 \\
59.00 \\
60.00\end{array}$ & $\begin{array}{l}242.82 \\
248.61 \\
254.38 \\
260.11 \\
265.81 \\
271.48 \\
277.13 \\
282.76 \\
288.36 \\
293.94\end{array}$ & $\begin{array}{l}484.93 \\
496.65 \\
508.34 \\
519.97 \\
531.59 \\
543.18 \\
554.74 \\
566.29 \\
577.83 \\
589.36\end{array}$ & $\begin{array}{l}288.10 \\
295.13 \\
302.14 \\
309.13 \\
316.13 \\
323.11 \\
330.10 \\
337.09 \\
344.09 \\
351.10\end{array}$ & $\begin{array}{l}31.005 \\
31.232 \\
31.455 \\
31.672 \\
31.886 \\
32.094 \\
32.299 \\
32.500 \\
32.697 \\
32.891\end{array}$ & $\begin{array}{l}121.00 \\
122.00 \\
123.00 \\
124.00 \\
125.00 \\
126.00 \\
127.00 \\
128.00 \\
129.00 \\
130.00\end{array}$ & $\begin{array}{l}617.14 \\
622.32 \\
627.49 \\
632.66 \\
637.84 \\
643.00 \\
648.16 \\
653.32 \\
658.49 \\
663.64\end{array}$ & $\begin{array}{l}1385.51 \\
1400.69 \\
1415.93 \\
1431.23 \\
1446.58 \\
1462.00 \\
1477.47 \\
1492.99 \\
1508.56 \\
1524.19\end{array}$ & $\begin{array}{l}885.24 \\
896.23 \\
907.27 \\
918.38 \\
929.54 \\
940.77 \\
952.05 \\
963.39 \\
974.78 \\
986.22\end{array}$ & $\begin{array}{l}41.885 \\
42.010 \\
42.135 \\
42.258 \\
42.382 \\
42.505 \\
42.627 \\
42.749 \\
42.870 \\
42.990\end{array}$ \\
\hline $\begin{array}{l}61.00 \\
62.00 \\
63.00 \\
64.00 \\
65.00 \\
66.00 \\
67.00 \\
68.00 \\
69.00 \\
70.00\end{array}$ & $\begin{array}{l}299.50 \\
305.05 \\
310.57 \\
316.08 \\
321.57 \\
327.05 \\
332.51 \\
337.97 \\
343.40 \\
348.83\end{array}$ & $\begin{array}{l}600.89 \\
612.42 \\
623.95 \\
635.50 \\
647.05 \\
658.62 \\
670.21 \\
681.82 \\
693.46 \\
705.13\end{array}$ & $\begin{array}{l}358.11 \\
365.15 \\
372.20 \\
379.28 \\
386.38 \\
393.51 \\
400.67 \\
407.87 \\
415.10 \\
422.37\end{array}$ & $\begin{array}{l}33.082 \\
33.269 \\
33.454 \\
33.635 \\
33.814 \\
33.991 \\
34.165 \\
34.337 \\
34.507 \\
34.675\end{array}$ & $\begin{array}{l}131.00 \\
132.00 \\
133.00 \\
134.00 \\
135.00 \\
136.00 \\
137.00 \\
138.00 \\
139.00 \\
140.00\end{array}$ & $\begin{array}{l}668.80 \\
673.96 \\
679.12 \\
684.27 \\
689.43 \\
694.58 \\
699.74 \\
704.89 \\
710.05 \\
715.20\end{array}$ & $\begin{array}{l}1539.86 \\
1555.58 \\
1571.34 \\
1587.16 \\
1603.01 \\
1618.90 \\
1634.84 \\
1650.81 \\
1666.83 \\
1682.88\end{array}$ & $\begin{array}{r}997.71 \\
1009.25 \\
1020.84 \\
1032.47 \\
1044.14 \\
1055.86 \\
1067.62 \\
1079.41 \\
1091.25 \\
1103.12\end{array}$ & $\begin{array}{l}43.111 \\
43.230 \\
43.349 \\
43.467 \\
43.585 \\
43.703 \\
43.819 \\
43.936 \\
44.051 \\
44.166\end{array}$ \\
\hline $\begin{array}{l}71.00 \\
72.00 \\
73.00 \\
74.00 \\
75.00 \\
76.00 \\
77.00 \\
78.00 \\
79.00 \\
80.00\end{array}$ & $\begin{array}{l}354.25 \\
359.65 \\
365.04 \\
370.43 \\
375.80 \\
381.17 \\
386.52 \\
391.87 \\
397.21 \\
402.55\end{array}$ & $\begin{array}{l}716.84 \\
728.58 \\
740.36 \\
752.18 \\
764.05 \\
775.97 \\
787.94 \\
799.96 \\
812.04 \\
824.18\end{array}$ & $\begin{array}{l}429.69 \\
437.05 \\
444.45 \\
451.91 \\
459.43 \\
466.99 \\
474.62 \\
482.31 \\
490.06 \\
497.88\end{array}$ & $\begin{array}{l}34.841 \\
35.006 \\
35.168 \\
35.329 \\
35.488 \\
35.646 \\
35.802 \\
35.958 \\
36.112 \\
36.264\end{array}$ & $\begin{array}{l}141.00 \\
142.00 \\
143.00 \\
144.00 \\
145.00 \\
146.00 \\
147.00 \\
148.00 \\
149.00 \\
150.00\end{array}$ & $\begin{array}{l}720.35 \\
725.50 \\
730.66 \\
735.81 \\
740.96 \\
746.11 \\
751.26 \\
756.41 \\
761.56 \\
766.71\end{array}$ & $\begin{array}{l}1698.96 \\
1715.07 \\
1731.22 \\
1747.40 \\
1763.61 \\
1779.84 \\
1796.10 \\
1812.38 \\
1828.69 \\
1845.02\end{array}$ & $\begin{array}{l}1115.03 \\
1126.96 \\
1138.94 \\
1150.94 \\
1162.97 \\
1175.03 \\
1187.11 \\
1199.22 \\
1211.35 \\
1223.51\end{array}$ & $\begin{array}{l}44.281 \\
44.395 \\
44.508 \\
44.621 \\
44.733 \\
44.844 \\
44.955 \\
45.066 \\
45.176 \\
45.285\end{array}$ \\
\hline $\begin{array}{l}81.00 \\
82.00 \\
83.00 \\
84.00 \\
85.00 \\
86.00 \\
87.00 \\
88.00 \\
89.00 \\
90.00\end{array}$ & $\begin{array}{l}407.87 \\
413.19 \\
418.50 \\
423.81 \\
429.11 \\
434.40 \\
439.69 \\
444.98 \\
450.26 \\
455.53\end{array}$ & $\begin{array}{l}836.39 \\
848.65 \\
860.98 \\
873.38 \\
885.86 \\
898.40 \\
911.02 \\
923.71 \\
936.48 \\
949.33\end{array}$ & $\begin{array}{l}505.77 \\
513.72 \\
521.74 \\
529.85 \\
538.02 \\
546.28 \\
554.61 \\
563.02 \\
571.51 \\
580.08\end{array}$ & $\begin{array}{l}36.416 \\
36.566 \\
36.716 \\
36.864 \\
37.012 \\
37.159 \\
37.305 \\
37.450 \\
37.594 \\
37.737\end{array}$ & $\begin{array}{l}151.00 \\
152.00 \\
153.00 \\
154.00 \\
155.00 \\
156.00 \\
157.00 \\
158.00 \\
159.00 \\
160.00\end{array}$ & $\begin{array}{l}771.85 \\
777.00 \\
782.14 \\
787.28 \\
792.42 \\
797.55 \\
802.69 \\
807.83 \\
812.97 \\
818.10\end{array}$ & $\begin{array}{l}1861.31 \\
1877.62 \\
1893.95 \\
1910.29 \\
1926.64 \\
1943.00 \\
1959.38 \\
1975.76 \\
1992.16 \\
2008.56\end{array}$ & $\begin{array}{l}1235.63 \\
1247.77 \\
1259.93 \\
1272.10 \\
1284.29 \\
1296.49 \\
1308.70 \\
1320.92 \\
1333.15 \\
1345.39\end{array}$ & $\begin{array}{l}45.393 \\
45.501 \\
45.608 \\
45.714 \\
45.820 \\
45.925 \\
46.030 \\
46.134 \\
46.237 \\
46.340\end{array}$ \\
\hline
\end{tabular}


TEMPER- SPECIFIC ENTHALPY INTERNAL ENTROPY ATURE VOLUME (J/GM) ENERGY (J/GM-K)

(K)

(CC/GM)

( J/GM)
TEMPERATUR

(K)
SPECIFIC

(CC/GM)
ENTHALPY

$(J / G M)$

ENERGY
ENTROPY

$(J / G M-K)$

\begin{tabular}{|c|c|c|c|c|c|c|c|c|c|}
\hline $\begin{array}{l}161.00 \\
162.00 \\
163.00 \\
164.00 \\
165.00 \\
166.00 \\
167.00 \\
168.00 \\
169.00 \\
170.00\end{array}$ & $\begin{array}{l}823.24 \\
828.37 \\
833.51 \\
838.64 \\
843.77 \\
848.91 \\
854.04 \\
859.17 \\
864.30 \\
869.43\end{array}$ & $\begin{array}{l}2024.97 \\
2041.38 \\
2057.80 \\
2074.23 \\
2090.66 \\
2107.09 \\
2123.53 \\
2139.96 \\
2156.40 \\
2172.83\end{array}$ & $\begin{array}{l}1357.63 \\
1369.89 \\
1382.15 \\
1394.41 \\
1406.68 \\
1418.95 \\
1431.22 \\
1443.50 \\
1455.78 \\
1468.05\end{array}$ & $\begin{array}{l}46.442 \\
46.544 \\
46.645 \\
46.746 \\
46.846 \\
46.945 \\
47.043 \\
47.142 \\
47.239 \\
47.336\end{array}$ & $\begin{array}{l}231.00 \\
232.00 \\
233.00 \\
234.00 \\
235.00 \\
236.00 \\
237.00 \\
238.00 \\
239.00 \\
240.00\end{array}$ & $\begin{array}{l}1181.58 \\
1186.69 \\
1191.80 \\
1196.91 \\
1202.02 \\
1207.13 \\
1212.24 \\
1217.35 \\
1222.46 \\
1227.56\end{array}$ & $\begin{array}{l}3155.06 \\
3170.69 \\
3186.29 \\
3201.88 \\
3217.46 \\
3233.02 \\
3248.56 \\
3264.08 \\
3279.59 \\
3295.08\end{array}$ & $\begin{array}{l}2197.25 \\
2208.73 \\
2220.19 \\
2231.64 \\
2243.07 \\
2254.49 \\
2265.89 \\
2277.27 \\
2288.64 \\
2299.99\end{array}$ & $\begin{array}{l}52.280 \\
52.348 \\
52.415 \\
52.481 \\
52.548 \\
52.614 \\
52.680 \\
52.745 \\
52.810 \\
52.875\end{array}$ \\
\hline $\begin{array}{l}171.00 \\
172.00 \\
173.00 \\
174.00 \\
175.00 \\
176.00 \\
177.00 \\
178.00 \\
179.00 \\
180.00\end{array}$ & $\begin{array}{l}874.56 \\
879.69 \\
884.81 \\
889.94 \\
895.07 \\
900.19 \\
905.32 \\
910.44 \\
915.57 \\
920.69\end{array}$ & $\begin{array}{l}2189.27 \\
2205.70 \\
2222.13 \\
2238.56 \\
2254.98 \\
2271.40 \\
2287.81 \\
2304.22 \\
2320.62 \\
2337.02\end{array}$ & $\begin{array}{l}1480.33 \\
1492.61 \\
1504.88 \\
1517.15 \\
1529.42 \\
1541.68 \\
1553.94 \\
1566.20 \\
1578.44 \\
1590.68\end{array}$ & $\begin{array}{l}47.433 \\
47.528 \\
47.624 \\
47.718 \\
47.812 \\
47.906 \\
47.999 \\
48.091 \\
48.183 \\
48.275\end{array}$ & $\begin{array}{l}241.00 \\
242.00 \\
243.00 \\
244.00 \\
245.00 \\
246.00 \\
247.00 \\
248.00 \\
249.00 \\
250.00\end{array}$ & $\begin{array}{l}1232.67 \\
1237.78 \\
1242.88 \\
1247.99 \\
1253.09 \\
1258.19 \\
1263.29 \\
1268.39 \\
1273.49 \\
1278.59\end{array}$ & $\begin{array}{l}3310.55 \\
3326.01 \\
3341.45 \\
3356.88 \\
3372.28 \\
3387.68 \\
3403.05 \\
3418.41 \\
3433.76 \\
3449.09\end{array}$ & $\begin{array}{l}2311.32 \\
2322.64 \\
2333.94 \\
2345.23 \\
2356.50 \\
2367.76 \\
2379.00 \\
2390.23 \\
2401.44 \\
2412.64\end{array}$ & $\begin{array}{l}52.93 \\
53.00 \\
53.06 \\
53.13 \\
53.19 \\
53.25 \\
53.31 \\
53.38 \\
53.44 \\
53.50\end{array}$ \\
\hline $\begin{array}{l}181.00 \\
182.00 \\
183.00 \\
184.00 \\
185.00 \\
186.00 \\
187.00 \\
188.00 \\
189.00 \\
190.00\end{array}$ & $\begin{array}{l}925.82 \\
930.94 \\
936.06 \\
941.19 \\
946.31 \\
951.43 \\
956.55 \\
961.67 \\
966.79 \\
971.91\end{array}$ & $\begin{array}{l}2353 \cdot 40 \\
2369.78 \\
2386.15 \\
2402.51 \\
2418.86 \\
2435.20 \\
2451.53 \\
2467.85 \\
2484.16 \\
2500.45\end{array}$ & $\begin{array}{l}1602.92 \\
1615.14 \\
1627.36 \\
1639.57 \\
1651.77 \\
1663.95 \\
1676.13 \\
1688.30 \\
1700.46 \\
1712.60\end{array}$ & $\begin{array}{l}48.365 \\
48.456 \\
48.545 \\
48.634 \\
48.723 \\
48.811 \\
48.899 \\
48.986 \\
49.072 \\
49.158\end{array}$ & $\begin{array}{l}251.00 \\
252.00 \\
253.00 \\
254.00 \\
255.00 \\
256.00 \\
257.00 \\
258.00 \\
259.00 \\
260.00\end{array}$ & $\begin{array}{l}1283.69 \\
1288.79 \\
1293.88 \\
1298.98 \\
1304.07 \\
1309.16 \\
1314.25 \\
1319.34 \\
1324.44 \\
1329.52\end{array}$ & $\begin{array}{l}3464.44 \\
3479.78 \\
3495.10 \\
3510.40 \\
3525.69 \\
3540.97 \\
3556.23 \\
3571.48 \\
3586.72 \\
3601.94\end{array}$ & $\begin{array}{l}2423.86 \\
2435.06 \\
2446.25 \\
2457.42 \\
2468.59 \\
2479.73 \\
2490.87 \\
2501.99 \\
2513.10 \\
2524.20\end{array}$ & $\begin{array}{l}53.56 \\
53.62 \\
53.68 \\
53.74 \\
53.80 \\
53.86 \\
53.92 \\
53.98 \\
54.04 \\
54.10\end{array}$ \\
\hline $\begin{array}{l}191.00 \\
192.00 \\
193.00 \\
194.00 \\
195.00 \\
196.00 \\
197.00 \\
198.00 \\
199.00 \\
200.00\end{array}$ & $\begin{array}{r}977.03 \\
982.15 \\
987.27 \\
992.39 \\
997.51 \\
1002.63 \\
1007.75 \\
1012.87 \\
1017.98 \\
1023.10\end{array}$ & $\begin{array}{l}2516.74 \\
2533.01 \\
2549.26 \\
2565.50 \\
2581.73 \\
2597.95 \\
2614.15 \\
2630.33 \\
2646.50 \\
2662.66\end{array}$ & $\begin{array}{l}1724.73 \\
1736.85 \\
1748.96 \\
1761.05 \\
1773.13 \\
1785.19 \\
1797.25 \\
1809.28 \\
1821.30 \\
1833.31\end{array}$ & $\begin{array}{l}49.244 \\
49.329 \\
49.413 \\
49.497 \\
49.581 \\
49.663 \\
49.746 \\
49.828 \\
49.909 \\
49.990\end{array}$ & $\begin{array}{l}261.00 \\
262.00 \\
263.00 \\
264.00 \\
265.00 \\
266.00 \\
267.00 \\
268.00 \\
269.00 \\
270.00\end{array}$ & $\begin{array}{l}1334.61 \\
1339.70 \\
1344.79 \\
1349.88 \\
1354.97 \\
1360.05 \\
1365.14 \\
1370.23 \\
1375.32 \\
1380.40\end{array}$ & $\begin{array}{l}3617.15 \\
3632.34 \\
3647.53 \\
3662.70 \\
3677.86 \\
3693.00 \\
3708.14 \\
3723.27 \\
3738.38 \\
3753.48\end{array}$ & $\begin{array}{l}2535.28 \\
2546.35 \\
2557.41 \\
2568.46 \\
2579.49 \\
2590.51 \\
2601.53 \\
2612.53 \\
2623.52 \\
2634.50\end{array}$ & $\begin{array}{l}54.16 \\
54.21 \\
54.27 \\
54.33 \\
54.392 \\
54.44 \\
54.506 \\
54.563 \\
54.61 \\
54.675\end{array}$ \\
\hline $\begin{array}{l}201.00 \\
202.00 \\
203.00 \\
204.00 \\
205.00 \\
206.00 \\
207.00 \\
208.00 \\
209.00 \\
210.00\end{array}$ & $\begin{array}{l}1028.22 \\
1033.33 \\
1038.44 \\
1043.56 \\
1048.67 \\
1053.79 \\
1058.90 \\
1064.01 \\
1069.13 \\
1074.24\end{array}$ & $\begin{array}{l}2678.79 \\
2694.90 \\
2711.00 \\
2727.09 \\
2743.15 \\
2759.20 \\
2775.24 \\
2791.26 \\
2807.26 \\
2823.24\end{array}$ & $\begin{array}{l}1845.30 \\
1857.26 \\
1869.22 \\
1881.16 \\
1893.08 \\
1904.98 \\
1916.87 \\
1928.74 \\
1940.60 \\
1952.44\end{array}$ & $\begin{array}{l}50.071 \\
50.151 \\
50.230 \\
50.309 \\
50.388 \\
50.466 \\
50.544 \\
50.621 \\
50.698 \\
50.774\end{array}$ & $\begin{array}{l}271.00 \\
272.00 \\
273.00 \\
274.00 \\
275.00 \\
276.00 \\
277.00 \\
278.00 \\
279.00 \\
280.00\end{array}$ & $\begin{array}{l}1385.49 \\
1390.58 \\
1395.67 \\
1400.76 \\
1405.85 \\
1410.94 \\
1416.04 \\
1421.13 \\
1426.23 \\
1431.32\end{array}$ & $\begin{array}{l}3768.58 \\
3783.66 \\
3798.74 \\
3813.81 \\
3828.87 \\
3843.92 \\
3858.96 \\
3873.99 \\
3889.02 \\
3904.04\end{array}$ & $\begin{array}{l}2645.47 \\
2656.43 \\
2667.38 \\
2678.32 \\
2689.25 \\
2700.18 \\
2711.09 \\
2721.99 \\
2732.89 \\
2743.78\end{array}$ & $\begin{array}{l}54.731 \\
54.786 \\
54.842 \\
54.897 \\
54.952 \\
55.006 \\
55.061 \\
55.115 \\
55.169 \\
55.222\end{array}$ \\
\hline $\begin{array}{l}211.00 \\
212.00 \\
213.00 \\
214.00 \\
215.00 \\
216.00 \\
21.7 .00 \\
218.00 \\
219.00 \\
220.00\end{array}$ & $\begin{array}{l}1079.35 \\
1084.46 \\
1089.58 \\
1094.69 \\
1099.80 \\
1104.91 \\
1110.02 \\
1115.14 \\
1120.25 \\
1125.36\end{array}$ & $\begin{array}{l}2839.21 \\
2855.16 \\
2871 \cdot 10 \\
2887.01 \\
2902.91 \\
2918.80 \\
2934.67 \\
2950.52 \\
2966.35 \\
2982.17\end{array}$ & $\begin{array}{l}1964.26 \\
1976.07 \\
1987.86 \\
1999.64 \\
2011.39 \\
2023.13 \\
2034.86 \\
2046.56 \\
2058.25 \\
2069.93\end{array}$ & $\begin{array}{l}50.850 \\
50.925 \\
51.000 \\
51.075 \\
51.149 \\
51.222 \\
51.296 \\
51.369 \\
51.441 \\
51.513\end{array}$ & $\begin{array}{l}281.00 \\
282.00 \\
283.00 \\
284.00 \\
285.00 \\
286.00 \\
287.00 \\
288.00 \\
289.00 \\
290.00\end{array}$ & $\begin{array}{l}1436.42 \\
1441.52 \\
1446.63 \\
1451.73 \\
1456.84 \\
1461.94 \\
1467.05 \\
1472.17 \\
1477.28 \\
1482.39\end{array}$ & $\begin{array}{l}3919.06 \\
3934.06 \\
3949.06 \\
3964.06 \\
3979.05 \\
3994.03 \\
4009.01 \\
4023.98 \\
4038.95 \\
4053.91\end{array}$ & $\begin{array}{l}2754.66 \\
2765.53 \\
2776.40 \\
2787.26 \\
2798.11 \\
2808.95 \\
2819.78 \\
2830.61 \\
2841.43 \\
2852.25\end{array}$ & $\begin{array}{l}55.276 \\
55.329 \\
55.382 \\
55.435 \\
55.488 \\
55.540 \\
55.593 \\
55.645 \\
55.697 \\
55.748\end{array}$ \\
\hline $\begin{array}{l}221.00 \\
222.00 \\
223.00 \\
224.00 \\
225.00 \\
226.00 \\
227.00 \\
228.00 \\
229.00\end{array}$ & $\begin{array}{l}1130.47 \\
1135.58 \\
1140.69 \\
1145.80 \\
1150.91 \\
1156.02 \\
1161.14 \\
1166.25 \\
1171.36 \\
1176.47\end{array}$ & $\begin{array}{l}2997.97 \\
3013.75 \\
3029.52 \\
3045.27 \\
3061.00 \\
3076.72 \\
3092.42 \\
3108.10 \\
3123.77 \\
3139.42\end{array}$ & $\begin{array}{l}2081 \cdot 59 \\
2093.23 \\
2104.85 \\
2116.46 \\
2128.05 \\
2139.62 \\
2151 \cdot 18 \\
2162.72 \\
2174.24 \\
2185.75\end{array}$ & $\begin{array}{l}51.585 \\
51.656 \\
51.727 \\
51.797 \\
51.868 \\
51.937 \\
52.007 \\
52.075 \\
52.144 \\
52.212\end{array}$ & $\begin{array}{l}291.00 \\
292.00 \\
293.00 \\
294.00 \\
295.00 \\
296.00 \\
297.00 \\
298.00 \\
299.00 \\
300.00\end{array}$ & $\begin{array}{l}1487.51 \\
1492.63 \\
1497: 75 \\
1502.87 \\
1508.00 \\
1513.12 \\
1518.24 \\
1523.37 \\
1528.50 \\
1533.62\end{array}$ & $\begin{array}{l}4068.87 \\
4083.82 \\
4098.76 \\
4113.70 \\
4128.63 \\
4143.56 \\
4158.48 \\
4173.40 \\
4188.31 \\
4203.21\end{array}$ & $\begin{array}{l}2863.06 \\
2873.86 \\
2834.65 \\
2895.44 \\
2906.22 \\
2916.99 \\
2927.76 \\
2938.52 \\
2949.28 \\
2960.02\end{array}$ & $\begin{array}{l}55.800 \\
55.851 \\
55.902 \\
55.953 \\
56.004 \\
56.054 \\
56.105 \\
56.155 \\
56.205 \\
56.255\end{array}$ \\
\hline
\end{tabular}


9.00 ATMOSPHERE ISD8AR

\begin{tabular}{|c|c|c|c|c|c|c|c|c|c|}
\hline $\begin{array}{c}\text { TEMPER- } \\
\text { ATURE } \\
(K)\end{array}$ & $\begin{array}{l}\text { SPECIFIC } \\
\text { VOLUME } \\
\text { (CC/GM) }\end{array}$ & $\begin{array}{c}\text { ENTHALPY } \\
(J / G M)\end{array}$ & $\begin{array}{l}\text { INTERNAL } \\
\text { ENERGY } \\
(J / G M)\end{array}$ & $\begin{array}{l}\text { ENTROPY } \\
(J / G M-K)\end{array}$ & $\begin{array}{c}\text { TEMPER- } \\
\text { ATURE } \\
(K)\end{array}$ & $\begin{array}{l}\text { SPECIF IC } \\
\text { VOLUME } \\
\text { (CC/GM) }\end{array}$ & $\begin{array}{c}\text { ENTHALPY } \\
(J / G M)\end{array}$ & $\begin{array}{l}\text { INTERNAL } \\
\text { ENERGY } \\
(J / G M)\end{array}$ & $\begin{array}{l}\text { ENTROPY } \\
(J / G M-K)\end{array}$ \\
\hline 20.00 & 13.86 & -250.43 & -263.06 & 7.714 & & & & & \\
\hline $\begin{array}{l}21.00 \\
22.00 \\
23.00 \\
24.00 \\
25.00 \\
26.00 \\
27.00 \\
28.00 \\
29.00 \\
30.00\end{array}$ & $\begin{array}{l}14.07 \\
14.31 \\
14.57 \\
14.87 \\
15.20 \\
15.59 \\
16.04 \\
16.59 \\
17.29 \\
18.27\end{array}$ & $\begin{array}{l}-240.94 \\
-230.86 \\
-220.14 \\
-208.69 \\
-196.42 \\
-183.17 \\
-168.70 \\
-152.68 \\
-134.45 \\
-112.65\end{array}$ & $\begin{array}{l}-253.77 \\
-243.91 \\
-233.43 \\
-222.25 \\
-210.29 \\
-197.38 \\
-183.33 \\
-167.82 \\
-150.22 \\
-129.30\end{array}$ & $\begin{array}{r}8.179 \\
8.645 \\
9.124 \\
9.613 \\
10.112 \\
10.631 \\
11.176 \\
11.760 \\
12.397 \\
13.137\end{array}$ & $\begin{array}{r}91.00 \\
92.00 \\
93.00 \\
94.00 \\
95.00 \\
96.00 \\
97.00 \\
98.00 \\
99.00 \\
100.00\end{array}$ & $\begin{array}{l}409.37 \\
414.07 \\
418.77 \\
423.45 \\
428.14 \\
432.82 \\
437.50 \\
442.17 \\
446.84 \\
451.51\end{array}$ & $\begin{array}{r}960.39 \\
973.44 \\
986.56 \\
999.77 \\
1013.05 \\
1026.41 \\
1039.85 \\
1053.36 \\
1066.95 \\
1080.62\end{array}$ & $\begin{array}{l}587.07 \\
595.84 \\
604.68 \\
613.61 \\
622.62 \\
631.71 \\
640.88 \\
650.14 \\
659.47 \\
668.88\end{array}$ & $\begin{array}{l}37.376 \\
37.519 \\
37.661 \\
37.802 \\
37.943 \\
38.083 \\
38.222 \\
38.360 \\
38.498 \\
38.636\end{array}$ \\
\hline $\begin{array}{l}30.650 \\
30.650 \\
31.00 \\
32.00 \\
33.00 \\
34.00 \\
35.00 \\
36.00 \\
37.00 \\
38.00 \\
39.00 \\
40.00\end{array}$ & $\begin{array}{r}19.29 \\
81.66 \\
86.14 \\
96.64 \\
105.43 \\
113.32 \\
120.64 \\
127.56 \\
134.19 \\
140.59 \\
146.82 \\
152.89\end{array}$ & $\begin{array}{l}-94.75 \\
176.79 \\
188.23 \\
214.03 \\
234.82 \\
253.05 \\
269.73 \\
285.37 \\
300.26 \\
314.59 \\
328.50 \\
342.06\end{array}$ & $\begin{array}{r}-112.34 \\
102.32 \\
109.67 \\
125.90 \\
138.67 \\
149.72 \\
159.72 \\
169.04 \\
177.89 \\
186.38 \\
194.61 \\
202.64\end{array}$ & $\begin{array}{l}13.726 \\
22.609 \\
22.980 \\
23.800 \\
24.440 \\
24.984 \\
25.468 \\
25.908 \\
26.316 \\
26.699 \\
27.060 \\
27.403\end{array}$ & $\begin{array}{l}101.00 \\
102.00 \\
103.00 \\
104.00 \\
105.00 \\
106.00 \\
107.00 \\
108.00 \\
109.00 \\
110.00\end{array}$ & $\begin{array}{l}456.17 \\
460.83 \\
465.48 \\
470.13 \\
474.78 \\
479.43 \\
484.07 \\
488.71 \\
493.34 \\
497.98\end{array}$ & $\begin{array}{l}1094.36 \\
1108.17 \\
1122.05 \\
1136.02 \\
1150.07 \\
1164.19 \\
1178.38 \\
1192.65 \\
1207.00 \\
1221.42\end{array}$ & $\begin{array}{l}678.35 \\
687.91 \\
697.56 \\
707.28 \\
717.09 \\
726.97 \\
736.93 \\
746.98 \\
757.09 \\
767.29\end{array}$ & $\begin{array}{l}38.772 \\
38.909 \\
39.044 \\
39.179 \\
39.313 \\
39.447 \\
39.580 \\
39.713 \\
39.845 \\
39.977\end{array}$ \\
\hline $\begin{array}{l}41.00 \\
42.00 \\
43.00 \\
44.00 \\
45.00 \\
46.00 \\
47.00 \\
48.00 \\
49.00 \\
50.00\end{array}$ & $\begin{array}{l}158.83 \\
164.67 \\
170.41 \\
176.07 \\
181.65 \\
187.18 \\
192.64 \\
198.04 \\
203.40 \\
208.72\end{array}$ & $\begin{array}{l}355.34 \\
368.39 \\
381.24 \\
393.92 \\
406.45 \\
418.86 \\
431.16 \\
443.36 \\
455.47 \\
467.51\end{array}$ & $\begin{array}{l}210.50 \\
218.22 \\
225.84 \\
233.36 \\
240.80 \\
248.17 \\
255.49 \\
262.76 \\
269.99 \\
277.17\end{array}$ & $\begin{array}{l}27.731 \\
28.045 \\
28.348 \\
28.639 \\
28.921 \\
29.194 \\
29.458 \\
29.715 \\
29.965 \\
30.208\end{array}$ & $\begin{array}{l}111.00 \\
112.00 \\
113.00 \\
114.00 \\
115.00 \\
116.00 \\
117.00 \\
118.00 \\
119.00 \\
120.00\end{array}$ & $\begin{array}{l}502.60 \\
507.23 \\
511.85 \\
516.47 \\
521.09 \\
525.71 \\
530.32 \\
534.93 \\
539.53 \\
544.14\end{array}$ & $\begin{array}{l}1235.91 \\
1250.47 \\
1265.10 \\
1279.80 \\
1294.57 \\
1309.40 \\
1324.30 \\
1339.26 \\
1354.29 \\
1369.38\end{array}$ & $\begin{array}{l}777.56 \\
787.90 \\
798.31 \\
808.80 \\
819.36 \\
829.98 \\
840.68 \\
851.44 \\
862.26 \\
873.15\end{array}$ & $\begin{array}{l}40.108 \\
40.239 \\
40.369 \\
40.498 \\
40.627 \\
40.756 \\
40.884 \\
41.011 \\
41.138 \\
41.264\end{array}$ \\
\hline $\begin{array}{l}51.00 \\
52.00 \\
53.00 \\
54.00 \\
55.00 \\
56.00 \\
57.00 \\
58.00 \\
59.00 \\
60.00\end{array}$ & $\begin{array}{l}213.99 \\
219.23 \\
224.43 \\
229.60 \\
234.74 \\
239.85 \\
244.94 \\
250.00 \\
255.04 \\
260.06\end{array}$ & $\begin{array}{l}479.47 \\
491.38 \\
503.24 \\
515.05 \\
526.82 \\
538.56 \\
550.26 \\
561.95 \\
573.62 \\
585.27\end{array}$ & $\begin{array}{l}284.33 \\
291.46 \\
298.57 \\
305.67 \\
312.75 \\
319.83 \\
326.90 \\
333.97 \\
341.04 \\
348.12\end{array}$ & $\begin{array}{l}30.445 \\
30.676 \\
30.902 \\
31.123 \\
31.339 \\
31.550 \\
31.758 \\
31.961 \\
32.160 \\
32.356\end{array}$ & $\begin{array}{l}121.00 \\
122.00 \\
123.00 \\
124.00 \\
125.00 \\
126.00 \\
127.00 \\
128.00 \\
129.00 \\
130.00\end{array}$ & $\begin{array}{l}548.74 \\
553.34 \\
557.94 \\
562.54 \\
567.14 \\
571.73 \\
576.32 \\
580.91 \\
585.50 \\
590.09\end{array}$ & $\begin{array}{l}1384.52 \\
1399.72 \\
1414.98 \\
1430.29 \\
1445.66 \\
1461.09 \\
1476.58 \\
1492.12 \\
1507.71 \\
1523.35\end{array}$ & $\begin{array}{l}884.10 \\
895.10 \\
906.16 \\
917.28 \\
928.46 \\
939.71 \\
951.00 \\
962.36 \\
973.76 \\
985.21\end{array}$ & $\begin{array}{l}41.390 \\
41.515 \\
41.639 \\
41.763 \\
41.887 \\
42.010 \\
42.132 \\
42.254 \\
42.375 \\
42.496\end{array}$ \\
\hline $\begin{array}{l}61.00 \\
62.00 \\
63.00 \\
64.00 \\
65.00 \\
66.00 \\
67.00 \\
68.00 \\
69.00 \\
70.00\end{array}$ & $\begin{array}{l}265.05 \\
270.03 \\
274.99 \\
279.94 \\
284.86 \\
289.78 \\
294.68 \\
299.56 \\
304.43 \\
309.29\end{array}$ & $\begin{array}{l}596.92 \\
608.56 \\
620.20 \\
631.85 \\
643.50 \\
655.17 \\
666.85 \\
678.56 \\
690.28 \\
702.04\end{array}$ & $\begin{array}{l}355.21 \\
362.31 \\
369.43 \\
376.57 \\
383.73 \\
390.92 \\
398.13 \\
405.38 \\
412.66 \\
419.99\end{array}$ & $\begin{array}{l}32.549 \\
32.738 \\
32.924 \\
33.108 \\
33.288 \\
33.467 \\
33.642 \\
33.816 \\
33.987 \\
34.156\end{array}$ & $\begin{array}{l}131.00 \\
132.00 \\
133.00 \\
134.00 \\
135.00 \\
136.00 \\
137.00 \\
138.00 \\
139.00 \\
140.00\end{array}$ & $\begin{array}{l}594.68 \\
599.27 \\
603.86 \\
608.45 \\
613.03 \\
617.62 \\
622.21 \\
626.80 \\
631.39 \\
635.98\end{array}$ & $\begin{array}{l}1539.04 \\
1554.77 \\
1570.55 \\
1586.38 \\
1602.25 \\
1618.16 \\
1634.11 \\
1650.10 \\
1666.12 \\
1682.19\end{array}$ & $\begin{array}{r}996.72 \\
1008.27 \\
1019.87 \\
1031.51 \\
1043.19 \\
1054.92 \\
1066.68 \\
1078.49 \\
1090.33 \\
1102.21\end{array}$ & $\begin{array}{l}42.616 \\
42.736 \\
42.855 \\
42.974 \\
43.092 \\
43.209 \\
43.326 \\
43.442 \\
43.558 \\
43.673\end{array}$ \\
\hline $\begin{array}{l}71.00 \\
72.00 \\
73.00 \\
74.00 \\
75.00 \\
76.00 \\
77.00 \\
78.00 \\
79.00 \\
80.00\end{array}$ & $\begin{array}{l}314.14 \\
318.98 \\
323.81 \\
328.63 \\
333.44 \\
338.23 \\
343.03 \\
347.81 \\
352.58 \\
357.35\end{array}$ & $\begin{array}{l}713.82 \\
725.64 \\
737.49 \\
749.39 \\
761.32 \\
773.31 \\
785.35 \\
797.43 \\
809.57 \\
821.77\end{array}$ & $\begin{array}{l}427.35 \\
434.75 \\
442.20 \\
449.70 \\
457.26 \\
464.87 \\
472.53 \\
480.26 \\
488.04 \\
495.90\end{array}$ & $\begin{array}{l}34.323 \\
34.488 \\
34.652 \\
34.814 \\
34.974 \\
35.133 \\
35.290 \\
35.446 \\
35.601 \\
35.754\end{array}$ & $\begin{array}{l}141.00 \\
142.00 \\
143.00 \\
144.00 \\
145.00 \\
146.00 \\
147.00 \\
148.00 \\
149.00 \\
150.00\end{array}$ & $\begin{array}{l}640.57 \\
645.15 \\
649.74 \\
654.33 \\
658.92 \\
663.51 \\
668.10 \\
672.69 \\
677.28 \\
681.87\end{array}$ & $\begin{array}{l}1698.28 \\
1714.41 \\
1730.58 \\
1746.77 \\
1762.99 \\
1779.24 \\
1795.51 \\
1811.81 \\
1828.13 \\
1844.47\end{array}$ & $\begin{array}{l}1114.12 \\
1126.07 \\
1138.04 \\
1150.05 \\
1162.08 \\
1174.14 \\
1186.23 \\
1198.34 \\
1210.48 \\
1222.64\end{array}$ & $\begin{array}{l}43.788 \\
43.902 \\
44.015 \\
44.128 \\
44.240 \\
44.352 \\
44.463 \\
44.573 \\
44.683 \\
44.793\end{array}$ \\
\hline $\begin{array}{l}81.00 \\
82.00 \\
83.00 \\
84.00 \\
85.00 \\
86.00 \\
87.00 \\
88.00 \\
89.00 \\
90.00\end{array}$ & $\begin{array}{l}362.11 \\
366.86 \\
371.61 \\
376.35 \\
381.08 \\
385.81 \\
390.53 \\
395.25 \\
399.96 \\
404.67\end{array}$ & $\begin{array}{l}834.03 \\
846.36 \\
858.74 \\
871.20 \\
883.72 \\
896.31 \\
908.98 \\
921.72 \\
934.53 \\
947.42\end{array}$ & $\begin{array}{l}503.82 \\
511.80 \\
519.86 \\
527.99 \\
536.20 \\
544.48 \\
552.84 \\
561.28 \\
569.80 \\
578.40\end{array}$ & $\begin{array}{l}35.906 \\
36.058 \\
36.208 \\
36.357 \\
36.505 \\
36.652 \\
36.799 \\
36.944 \\
37.089 \\
37.233\end{array}$ & $\begin{array}{l}151.00 \\
152.00 \\
153.00 \\
154.00 \\
155.00 \\
156.00 \\
157.00 \\
158.00 \\
159.00 \\
160.00\end{array}$ & $\begin{array}{l}686.45 \\
691.03 \\
695.60 \\
700.18 \\
704.75 \\
709.33 \\
713.90 \\
718.47 \\
723.04 \\
727.61\end{array}$ & $\begin{array}{l}1860.77 \\
1877.09 \\
1893.43 \\
1909.78 \\
1926.15 \\
1942.52 \\
1958.91 \\
1975.30 \\
1991.71 \\
2008.12\end{array}$ & $\begin{array}{l}1234.77 \\
1246.91 \\
1259.08 \\
1271.25 \\
1283.45 \\
1295.65 \\
1307.87 \\
1320.09 \\
1332.33 \\
1344.58\end{array}$ & $\begin{array}{l}44.901 \\
45.009 \\
45.116 \\
45.222 \\
45.328 \\
45.434 \\
45.538 \\
45.642 \\
45.746 \\
45.849\end{array}$ \\
\hline
\end{tabular}


TEMPER- SPECIFIC ENTHALPY INTERNAL ENTROPY ATURE VOLUME (J/GM) ENERGY (J/GM-K) (K) (CC/GM)
(J/GM)
TEMPER- SPECIFIC ENTHALPY INTERNAL ENTROPY $\begin{array}{clll}\text { ATURE } & \text { VOLUME } & \text { (J/GM) } & \text { ENERGY } \\ \text { (K) } & \text { (CC/GM) } & & \end{array}$

\begin{tabular}{|c|c|c|c|c|c|c|c|c|c|}
\hline $\begin{array}{l}161.00 \\
162.00 \\
163.00 \\
164.00 \\
165.00 \\
166.00 \\
167.00 \\
168.00 \\
169.00 \\
170.00\end{array}$ & $\begin{array}{l}732.18 \\
736.75 \\
741.32 \\
745.89 \\
750.46 \\
755.02 \\
759.59 \\
764.15 \\
768.72 \\
773.28\end{array}$ & $\begin{array}{l}2024.54 \\
2040.97 \\
2057.40 \\
2073.84 \\
2090.28 \\
2106.72 \\
2123.17 \\
2139.61 \\
2156.06 \\
2172.50\end{array}$ & $\begin{array}{l}1356.83 \\
1369.09 \\
1381.36 \\
1393.63 \\
1405.90 \\
1418.18 \\
1430.46 \\
1442.74 \\
1455.03 \\
1467.31\end{array}$ & $\begin{array}{l}45.951 \\
46.053 \\
46.154 \\
46.255 \\
46.354 \\
46.454 \\
46.553 \\
46.651 \\
46.748 \\
46.845\end{array}$ & $\begin{array}{l}231.00 \\
232.00 \\
233.00 \\
234.00 \\
235.00 \\
236.00 \\
237.00 \\
238.00 \\
239.00 \\
240.00\end{array}$ & $\begin{array}{l}1050.93 \\
1055.48 \\
1060.02 \\
1064.57 \\
1069.11 \\
1073.66 \\
1078.20 \\
1082.74 \\
1087.29 \\
1091.83\end{array}$ & $\begin{array}{l}3155.16 \\
3170.79 \\
3186.40 \\
3201.99 \\
3217.57 \\
3233.13 \\
3248.68 \\
3264.21 \\
3279.72 \\
3295.22\end{array}$ & $\begin{array}{l}2196.76 \\
2208.24 \\
2219.71 \\
2231.16 \\
2242.59 \\
2254.01 \\
2265.41 \\
2276.80 \\
2288.17 \\
2299.52\end{array}$ & $\begin{array}{l}51.792 \\
51.859 \\
51.926 \\
51.993 \\
52.059 \\
52.125 \\
52.191 \\
52.257 \\
52.322 \\
52.386\end{array}$ \\
\hline $\begin{array}{l}171.00 \\
172.00 \\
173.00 \\
174.00 \\
175.00 \\
176.00 \\
177.00 \\
178.00 \\
179.00 \\
180.00\end{array}$ & $\begin{array}{l}777.84 \\
782.40 \\
786.97 \\
791.53 \\
796.09 \\
800.65 \\
805.21 \\
809.77 \\
814.33 \\
818.88\end{array}$ & $\begin{array}{l}2188.95 \\
2205.39 \\
2221.83 \\
2238.27 \\
2254.70 \\
2271.13 \\
2287.55 \\
2303.97 \\
2320.38 \\
2336.78\end{array}$ & $\begin{array}{l}1479.59 \\
1491.88 \\
1504.16 \\
1516.43 \\
1528.71 \\
1540.98 \\
1553.24 \\
1565.50 \\
1577.75 \\
1590.00\end{array}$ & $\begin{array}{l}46.942 \\
47.038 \\
47.133 \\
47.228 \\
47.322 \\
47.416 \\
47.509 \\
47.601 \\
47.693 \\
47.784\end{array}$ & $\begin{array}{l}241.00 \\
242.00 \\
243.00 \\
244.00 \\
245.00 \\
246.00 \\
247.00 \\
248.00 \\
249.00 \\
250.00\end{array}$ & $\begin{array}{l}1096.37 \\
1100.91 \\
1105.45 \\
1109.99 \\
1114.53 \\
1119.06 \\
1123.60 \\
1128.14 \\
1132.67 \\
1137.20\end{array}$ & $\begin{array}{l}3310.70 \\
3326.16 \\
3341.60 \\
3357.03 \\
3372.45 \\
3387.85 \\
3403.23 \\
3418.59 \\
3433.94 \\
3449.28\end{array}$ & $\begin{array}{l}2310.86 \\
2322.18 \\
2333.49 \\
2344.78 \\
2356.06 \\
2367.32 \\
2378.56 \\
2389.79 \\
2401.01 \\
2412.21\end{array}$ & $\begin{array}{l}52.451 \\
52.515 \\
52.578 \\
52.642 \\
52.705 \\
52.768 \\
52.830 \\
52.892 \\
52.954 \\
53.015\end{array}$ \\
\hline $\begin{array}{l}181.00 \\
182.00 \\
183.00 \\
184.00 \\
185.00 \\
186.00 \\
187.00 \\
188.00 \\
189.00 \\
190.00\end{array}$ & $\begin{array}{l}823.44 \\
828.00 \\
832.56 \\
837.11 \\
841.67 \\
846.23 \\
850.78 \\
855.34 \\
859.89 \\
864.45\end{array}$ & $\begin{array}{l}2353.17 \\
2369.56 \\
2385.94 \\
2402.31 \\
2418.67 \\
2435.01 \\
2451.35 \\
2467.68 \\
2483.99 \\
2500.29\end{array}$ & $\begin{array}{l}1602.24 \\
1614.47 \\
1626.69 \\
1638.90 \\
1651.11 \\
1663.30 \\
1675.48 \\
1687.65 \\
1699.81 \\
1711.96\end{array}$ & $\begin{array}{l}47.875 \\
47.966 \\
48.055 \\
48.144 \\
48.233 \\
48.321 \\
48.409 \\
48.496 \\
48.582 \\
48.668\end{array}$ & $\begin{array}{l}251.00 \\
252.00 \\
253.00 \\
254.00 \\
255.00 \\
256.00 \\
257.00 \\
258.00 \\
259.00 \\
260.00\end{array}$ & $\begin{array}{l}1141.74 \\
1146.27 \\
1150.80 \\
1155.33 \\
1159.86 \\
1164.39 \\
1168.92 \\
1173.44 \\
1177.97 \\
1182.50\end{array}$ & $\begin{array}{l}3464.63 \\
3479.97 \\
3495.30 \\
3510.61 \\
3525.90 \\
3541.19 \\
3556.45 \\
3571.71 \\
3586.95 \\
3602.17\end{array}$ & $\begin{array}{l}2423.43 \\
2434.63 \\
2445.83 \\
2457.01 \\
2468.17 \\
2479.32 \\
2490.46 \\
2501.59 \\
2512.70 \\
2523.80\end{array}$ & $\begin{array}{l}53.077 \\
53.138 \\
53.198 \\
53.259 \\
53.319 \\
53.379 \\
53.438 \\
53.497 \\
53.556 \\
53.615\end{array}$ \\
\hline $\begin{array}{l}191.00 \\
192.00 \\
193.00 \\
194.00 \\
195.00 \\
196.00 \\
197.00 \\
198.00 \\
199.00 \\
200.00\end{array}$ & $\begin{array}{l}869.00 \\
873.56 \\
878.11 \\
882.67 \\
887.22 \\
891.77 \\
896.33 \\
900.88 \\
905.43 \\
909.98\end{array}$ & $\begin{array}{l}2516.58 \\
2532.86 \\
2549.13 \\
2565.37 \\
2581.61 \\
2597.83 \\
2614.04 \\
2630.23 \\
2646.41 \\
2662.57\end{array}$ & $\begin{array}{l}1724.10 \\
1736.22 \\
1748.33 \\
1760.43 \\
1772.51 \\
1784.58 \\
1796.64 \\
1808.68 \\
1820.70 \\
1832.71\end{array}$ & $\begin{array}{l}48.754 \\
48.839 \\
48.923 \\
49.007 \\
49.091 \\
49.174 \\
49.256 \\
49.338 \\
49.420 \\
49.501\end{array}$ & $\begin{array}{l}261.00 \\
262.00 \\
263.00 \\
264.00 \\
265.00 \\
266.00 \\
267.00 \\
268.00 \\
269.00 \\
270.00\end{array}$ & $\begin{array}{l}1187.02 \\
1191.55 \\
1196.07 \\
1200.60 \\
1205.12 \\
1209.64 \\
1214.17 \\
1218.69 \\
1223.22 \\
1227.74\end{array}$ & $\begin{array}{l}3617.39 \\
3632.59 \\
3647.77 \\
3662.95 \\
3678.11 \\
3693.27 \\
3708.41 \\
3723.54 \\
3738.65 \\
3753.76\end{array}$ & $\begin{array}{l}2534.88 \\
2545.96 \\
2557.02 \\
2568.07 \\
2579.11 \\
2590.13 \\
2601.15 \\
2612.15 \\
2623.14 \\
2634.13\end{array}$ & $\begin{array}{l}53.673 \\
53.731 \\
53.789 \\
53.847 \\
53.904 \\
53.961 \\
54.018 \\
54.075 \\
54.131 \\
54.187\end{array}$ \\
\hline $\begin{array}{l}201.00 \\
202.00 \\
203.00 \\
204.00 \\
205.00 \\
206.00 \\
207.00 \\
208.00 \\
209.00 \\
210.00\end{array}$ & $\begin{array}{l}914.53 \\
919.08 \\
923.63 \\
928.18 \\
932.73 \\
937.28 \\
941.83 \\
946.37 \\
950.92 \\
955.47\end{array}$ & $\begin{array}{l}2678.71 \\
2694.83 \\
2710.94 \\
2727.02 \\
2743.10 \\
2759.16 \\
2775.20 \\
2791.22 \\
2807.23 \\
2823.22\end{array}$ & $\begin{array}{l}1844.70 \\
1856.67 \\
1868.63 \\
1880.57 \\
1892.50 \\
1904.41 \\
1916.30 \\
1928.17 \\
1940.03 \\
1951.88\end{array}$ & $\begin{array}{l}49.581 \\
49.661 \\
49.741 \\
49.820 \\
49.899 \\
49.977 \\
50.054 \\
50.132 \\
50.208 \\
50.285\end{array}$ & $\begin{array}{l}271.00 \\
272.00 \\
273.00 \\
274.00 \\
275.00 \\
276.00 \\
277.00 \\
278.00 \\
279.00 \\
280.00\end{array}$ & $\begin{array}{l}1232.26 \\
1236.79 \\
1241.31 \\
1245.84 \\
1250.37 \\
1254.90 \\
1259.42 \\
1263.95 \\
1268.49 \\
1273.02\end{array}$ & $\begin{array}{l}3768.86 \\
3783.95 \\
3799.03 \\
3814.10 \\
3829.16 \\
3844.22 \\
3859.26 \\
3874.30 \\
3889.33 \\
3904.36\end{array}$ & $\begin{array}{l}2645.10 \\
2656.06 \\
2667.01 \\
2677.96 \\
2688.89 \\
2699.82 \\
2710.73 \\
2721.64 \\
2732.54 \\
2743.43\end{array}$ & $\begin{array}{l}54.243 \\
54.298 \\
54.354 \\
54.409 \\
54.464 \\
54.518 \\
54.573 \\
54.627 \\
54.681 \\
54.735\end{array}$ \\
\hline $\begin{array}{l}211.00 \\
212.00 \\
213.00 \\
214.00 \\
215.00 \\
216.00 \\
217.00 \\
218.00 \\
219.00 \\
220.00\end{array}$ & $\begin{array}{r}960.02 \\
964.56 \\
969.11 \\
973.66 \\
978.20 \\
982.75 \\
987.30 \\
991.84 \\
996.39 \\
1000.94\end{array}$ & $\begin{array}{l}2839.19 \\
2855.15 \\
2871.09 \\
2887.01 \\
2902.92 \\
2918.81 \\
2934.68 \\
2950.54 \\
2966.38 \\
2982.20\end{array}$ & $\begin{array}{l}1963.71 \\
1975.52 \\
1987.31 \\
1999.09 \\
2010.85 \\
2022.59 \\
2034.32 \\
2046.03 \\
2057.72 \\
2069.40\end{array}$ & $\begin{array}{l}50.361 \\
50.436 \\
50.511 \\
50.586 \\
50.660 \\
50.734 \\
50.807 \\
50.880 \\
50.952 \\
51.024\end{array}$ & $\begin{array}{l}281.00 \\
282.00 \\
283.00 \\
284.00 \\
285.00 \\
286.00 \\
287.00 \\
288.00 \\
289.00 \\
290.00\end{array}$ & $\begin{array}{l}1277.55 \\
1282.09 \\
1286.62 \\
1291.16 \\
1295.70 \\
1300.24 \\
1304.79 \\
1309.33 \\
1313.88 \\
1318.42\end{array}$ & $\begin{array}{l}3919.37 \\
3934.38 \\
3949.39 \\
3964.38 \\
3979.38 \\
3994.36 \\
4009.34 \\
4024.32 \\
4039.29 \\
4054.25\end{array}$ & $\begin{array}{l}2754.31 \\
2765.19 \\
2776.05 \\
2786.91 \\
2797.76 \\
2808.61 \\
2819.44 \\
2830.27 \\
2841.10 \\
2851.91\end{array}$ & $\begin{array}{l}54.788 \\
54.842 \\
54.895 \\
54.948 \\
55.000 \\
55.053 \\
55.105 \\
55.157 \\
55.209 \\
55.261\end{array}$ \\
\hline $\begin{array}{l}221.00 \\
222.00 \\
223.00 \\
224.00 \\
225.00 \\
226.00 \\
227.00 \\
228.00 \\
229.00 \\
230.00\end{array}$ & $\begin{array}{l}1005.48 \\
1010.03 \\
1014.57 \\
1019.12 \\
1023.66 \\
1028.21 \\
1032.75 \\
1037.30 \\
1041.84 \\
1046.39\end{array}$ & $\begin{array}{l}2998.01 \\
3013.80 \\
3029.57 \\
3045.32 \\
3061.06 \\
3076.79 \\
3092.49 \\
3108.18 \\
3123.85 \\
3139.51\end{array}$ & $\begin{array}{l}2081.06 \\
2092.70 \\
2104.33 \\
2115.94 \\
2127.53 \\
2139.11 \\
2150.67 \\
2162.22 \\
2173.75 \\
2185.26\end{array}$ & $\begin{array}{l}51.096 \\
51.167 \\
51.238 \\
51.309 \\
51.379 \\
51.449 \\
51.518 \\
51.587 \\
51.655 \\
51.724\end{array}$ & $\begin{array}{l}291.00 \\
292.00 \\
293.00 \\
294.00 \\
295.00 \\
296.00 \\
297.00 \\
290.00 \\
299.00 \\
300.00\end{array}$ & $\begin{array}{r}1322.97 \\
1327.52 \\
1332.08 \\
.1336 .63 \\
1341.18 \\
1345.74 \\
1350.29 \\
1354.05 \\
1359.41 \\
1363.06\end{array}$ & $\begin{array}{l}4069.21 \\
4084.16 \\
4099.11 \\
4114.05 \\
4128.98 \\
4143.81 \\
4158.83 \\
4173.75 \\
4188.66 \\
4203.56\end{array}$ & $\begin{array}{l}2862.72 \\
2873.53 \\
2884.32 \\
2895.11 \\
2905.09 \\
2916.67 \\
2927.43 \\
2938.20 \\
2948.95 \\
2959.70\end{array}$ & $\begin{array}{l}55.312 \\
55.364 \\
55.415 \\
55.466 \\
55.516 \\
55.567 \\
55.617 \\
55.667 \\
55.717 \\
55.767\end{array}$ \\
\hline
\end{tabular}




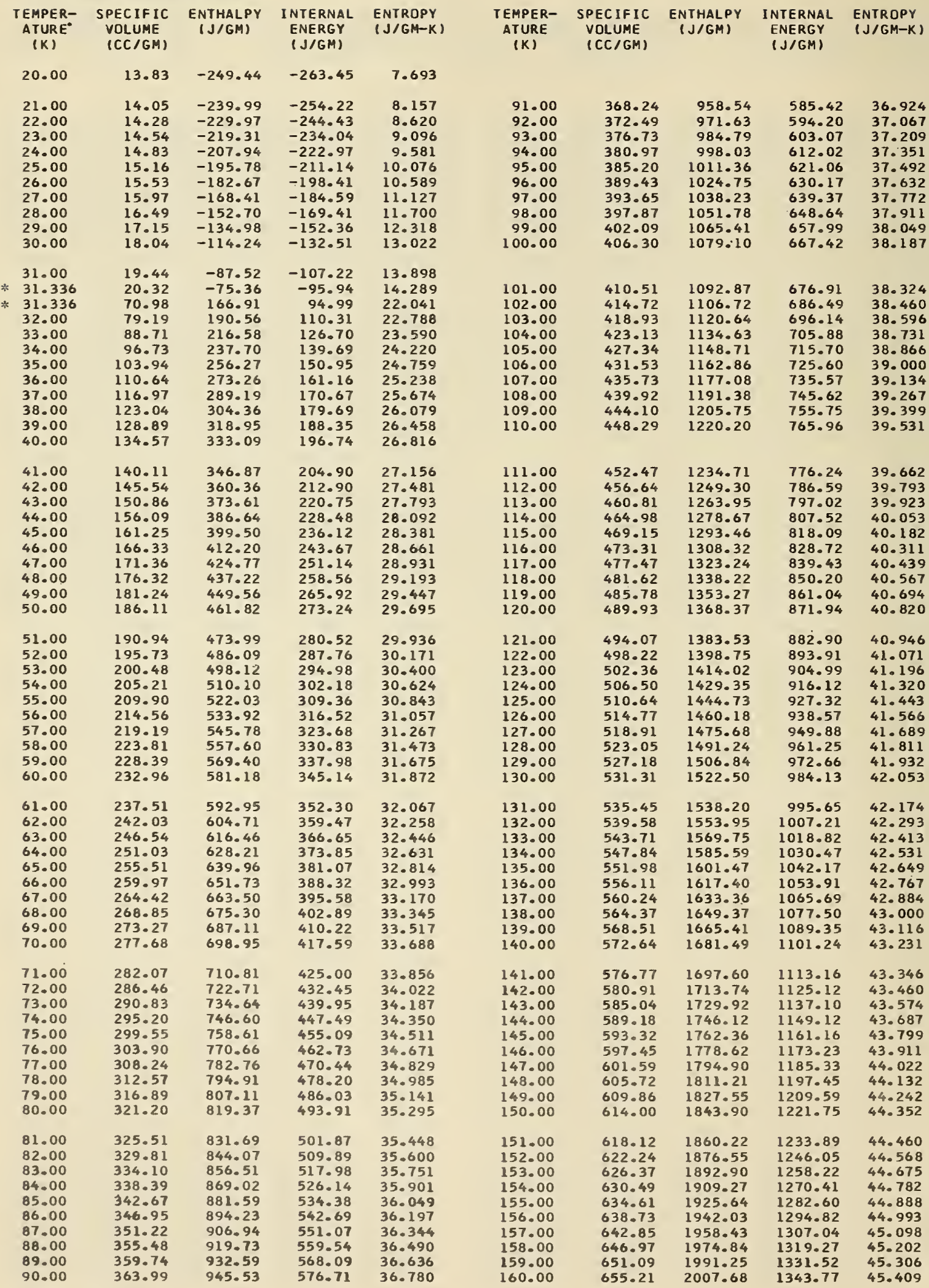


TEMPER- SPECIFIC ENTHALPY INTERNAL ENTROPY

(J/GM) ENERGY (J/GM-K) (CC/GM)

(J/GM)

(

TEMPER-

ATURE

(K)
SPECIFIC

(CC/GM)
ENTHALPY
INTERNAL ENTROPY

ENERGY

\begin{tabular}{|c|c|c|c|c|c|c|c|c|c|}
\hline $\begin{array}{l}161.00 \\
162.00 \\
163.00 \\
164.00 \\
165.00 \\
166.00 \\
167.00 \\
168.00 \\
169.00 \\
170.00\end{array}$ & $\begin{array}{l}659.33 \\
663.44 \\
667.56 \\
671.67 \\
675.79 \\
679.90 \\
684.02 \\
688.13 \\
692.24 \\
696.35\end{array}$ & $\begin{array}{l}2024.11 \\
2040.55 \\
2056.99 \\
2073.44 \\
2089.89 \\
2106.34 \\
2122.80 \\
2139.25 \\
2155.71 \\
2172.16\end{array}$ & $\begin{array}{l}1356.03 \\
1368.30 \\
1380.57 \\
1392.85 \\
1405.13 \\
1417.41 \\
1429.70 \\
1441.98 \\
1454.27 \\
1466.56\end{array}$ & $\begin{array}{l}45.511 \\
45.613 \\
45.714 \\
45.815 \\
45.915 \\
46.014 \\
46.113 \\
46.207 \\
46.301 \\
46.398\end{array}$ & $\begin{array}{l}231.00 \\
232.00 \\
233.00 \\
234.00 \\
235.00 \\
236.00 \\
237.00 \\
238.00 \\
239.00 \\
240.00\end{array}$ & $\begin{array}{l}946.41 \\
950.50 \\
954.60 \\
958.69 \\
962.78 \\
966.87 \\
970.96 \\
975.05 \\
979.14 \\
983.23\end{array}$ & $\begin{array}{l}3155.23 \\
3170.86 \\
3186.48 \\
3202.07 \\
3217.65 \\
3233.21 \\
3248.76 \\
3264.29 \\
3279.81 \\
3295.31\end{array}$ & $\begin{array}{l}2196.26 \\
2207.74 \\
2219.21 \\
2230.66 \\
2242.09 \\
2253.51 \\
2264.91 \\
2276.30 \\
2287.67 \\
2299.03\end{array}$ & $\begin{array}{l}51.354 \\
51.421 \\
51.488 \\
51.555 \\
51.622 \\
51.688 \\
51.753 \\
51.819 \\
51.884 \\
51.949\end{array}$ \\
\hline $\begin{array}{l}171.00 \\
172.00 \\
173.00 \\
174.00 \\
175.00 \\
176.00 \\
177.00 \\
178.00 \\
179.00 \\
180.00\end{array}$ & $\begin{array}{l}700.47 \\
704.58 \\
708.69 \\
712.80 \\
716.91 \\
721.01 \\
725.12 \\
729.23 \\
733.34 \\
737.44\end{array}$ & $\begin{array}{l}2188.62 \\
2205.07 \\
2221.52 \\
2237.97 \\
2254.41 \\
2270.84 \\
2287.28 \\
2303.70 \\
2320.12 \\
2336.53\end{array}$ & $\begin{array}{l}1478.85 \\
1491.14 \\
1503.42 \\
1515.71 \\
1527.98 \\
1540.26 \\
1552.53 \\
1564.79 \\
1577.05 \\
1589.30\end{array}$ & $\begin{array}{l}46.495 \\
46.592 \\
46.688 \\
46.783 \\
46.878 \\
46.972 \\
47.066 \\
47.159 \\
47.251 \\
47.343\end{array}$ & $\begin{array}{l}241.00 \\
242.00 \\
243.00 \\
244.00 \\
245.00 \\
246.00 \\
247.00 \\
248.00 \\
249.00 \\
250.00\end{array}$ & $\begin{array}{r}987.32 \\
991.40 \\
995.49 \\
999.58 \\
1003.66 \\
1007.75 \\
1011.84 \\
1015.92 \\
1020.01 \\
1024.09\end{array}$ & $\begin{array}{l}3310.79 \\
3326.26 \\
3341.71 \\
3357.15 \\
3372.57 \\
3387.98 \\
3403.37 \\
3418.75 \\
3434.11 \\
3449.45\end{array}$ & $\begin{array}{l}2310.37 \\
2321.70 \\
2333.01 \\
2344.30 \\
2355.58 \\
2366.85 \\
2378.10 \\
2389.34 \\
2400.56 \\
2411.77\end{array}$ & $\begin{array}{l}52.013 \\
52.077 \\
52.141 \\
52.204 \\
52.267 \\
52.330 \\
52.392 \\
52.455 \\
52.516 \\
52.578\end{array}$ \\
\hline $\begin{array}{l}181.00 \\
182.00 \\
183.00 \\
184.00 \\
185.00 \\
186.00 \\
187.00 \\
188.00 \\
189.00 \\
190.00\end{array}$ & $\begin{array}{l}741.55 \\
745.65 \\
749.76 \\
753.86 \\
757.97 \\
762.07 \\
766.17 \\
770.28 \\
774.38 \\
778.48\end{array}$ & $\begin{array}{l}2352.94 \\
2369.33 \\
2385.72 \\
2402.09 \\
2418.46 \\
2434.82 \\
2451.16 \\
2467.50 \\
2483.82 \\
2500.13\end{array}$ & $\begin{array}{l}1601.54 \\
1613.78 \\
1626.00 \\
1638.22 \\
1650.43 \\
1662.63 \\
1674.82 \\
1686.99 \\
1699.16 \\
1711.31\end{array}$ & $\begin{array}{l}47.435 \\
47.525 \\
47.616 \\
47.705 \\
47.794 \\
47.882 \\
47.970 \\
48.057 \\
48.143 \\
48.230\end{array}$ & $\begin{array}{l}251.00 \\
252.00 \\
253.00 \\
254.00 \\
255.00 \\
256.00 \\
257.00 \\
258.00 \\
259.00 \\
260.00\end{array}$ & $\begin{array}{l}1028.17 \\
1032.25 \\
1036.34 \\
1040.42 \\
1044.50 \\
1048.58 \\
1052.66 \\
1056.73 \\
1060.81 \\
1064.89\end{array}$ & $\begin{array}{l}3464.82 \\
3480.17 \\
3495.51 \\
3510.83 \\
3526.14 \\
3541.44 \\
3556.71 \\
3571.98 \\
3587.23 \\
3602.47\end{array}$ & $\begin{array}{l}2423.00 \\
2434.21 \\
2445.41 \\
2456.60 \\
2467.78 \\
2478.94 \\
2490.08 \\
2501.22 \\
2512.34 \\
2523.44\end{array}$ & $\begin{array}{l}52.639 \\
52.700 \\
52.761 \\
52.821 \\
52.882 \\
52.941 \\
53.001 \\
53.060 \\
53.119 \\
53.178\end{array}$ \\
\hline $\begin{array}{l}191.00 \\
192.00 \\
193.00 \\
194.00 \\
195.00 \\
196.00 \\
197.00 \\
198.00 \\
199.00 \\
200.00\end{array}$ & $\begin{array}{l}782.58 \\
786.69 \\
790.79 \\
794.89 \\
798.99 \\
803.09 \\
807.19 \\
811.29 \\
815.39 \\
819.49\end{array}$ & $\begin{array}{l}2516.43 \\
2532.71 \\
2548.98 \\
2565.24 \\
2581.48 \\
2597.71 \\
2613.92 \\
2630.12 \\
2646.31 \\
2662.47\end{array}$ & $\begin{array}{l}1723.45 \\
1735.58 \\
1747.69 \\
1759.80 \\
1771.88 \\
1783.96 \\
1796.02 \\
1808.06 \\
1820.09 \\
1832.11\end{array}$ & $\begin{array}{l}48.315 \\
48.400 \\
48.485 \\
48.569 \\
48.652 \\
48.735 \\
48.818 \\
48.900 \\
48.981 \\
49.062\end{array}$ & $\begin{array}{l}261.00 \\
262.00 \\
263.00 \\
264.00 \\
265.00 \\
266.00 \\
267.00 \\
268.00 \\
269.00 \\
270.00\end{array}$ & $\begin{array}{l}1068.97 \\
1073.04 \\
1077.12 \\
1081.19 \\
1085.26 \\
1089.34 \\
1093.41 \\
1097.49 \\
1101.56 \\
1105.63\end{array}$ & $\begin{array}{l}3617.69 \\
3632.90 \\
3648.10 \\
3663.28 \\
3678.45 \\
3693.61 \\
3708.76 \\
3723.89 \\
3739.01 \\
3754.12\end{array}$ & $\begin{array}{l}2534.54 \\
2545.62 \\
2556.68 \\
2567.74 \\
2578.78 \\
2589.81 \\
2600.83 \\
2611.83 \\
2622.83 \\
2633.81\end{array}$ & $\begin{array}{l}53.237 \\
53.295 \\
53.353 \\
53.410 \\
53.468 \\
53.525 \\
53.581 \\
53.638 \\
53.694 \\
53.750\end{array}$ \\
\hline $\begin{array}{l}201.00 \\
202.00 \\
203.00 \\
204.00 \\
205.00 \\
206.00 \\
207.00 \\
208.00 \\
209.00 \\
210.00\end{array}$ & $\begin{array}{l}823.58 \\
827.68 \\
831.78 \\
835.87 \\
839.97 \\
844.07 \\
848.16 \\
852.26 \\
856.35 \\
860.45\end{array}$ & $\begin{array}{l}2678.62 \\
2694.75 \\
2710.86 \\
2726.96 \\
2743.03 \\
2759.10 \\
2775.15 \\
2791.18 \\
2807.19 \\
2823.19\end{array}$ & $\begin{array}{l}1844.10 \\
1856.08 \\
1868.04 \\
1879.98 \\
1891.91 \\
1903.82 \\
1915.72 \\
1927.60 \\
1939.47 \\
1951.31\end{array}$ & $\begin{array}{l}49.143 \\
49.223 \\
49.302 \\
49.382 \\
49.460 \\
49.538 \\
49.616 \\
49.693 \\
49.770 \\
49.846\end{array}$ & $\begin{array}{l}271.00 \\
272.00 \\
273.00 \\
274.00 \\
275.00 \\
276.00 \\
277.00 \\
278.00 \\
279.00 \\
280.00\end{array}$ & $\begin{array}{l}1109.70 \\
1113.78 \\
1117.85 \\
1121.92 \\
1126.00 \\
1130.07 \\
1134.14 \\
1138.22 \\
1142.29 \\
1146.37\end{array}$ & $\begin{array}{l}3769.22 \\
3784.31 \\
3799.38 \\
3814.45 \\
3829.51 \\
3844.55 \\
3859.59 \\
3874.62 \\
3889.64 \\
3904.66\end{array}$ & $\begin{array}{l}2644.78 \\
2655.74 \\
2666.69 \\
2677.63 \\
2688.56 \\
2699.48 \\
2710.39 \\
2721.29 \\
2732.18 \\
2743.07\end{array}$ & $\begin{array}{l}53.806 \\
53.862 \\
53.917 \\
53.972 \\
54.027 \\
54.082 \\
54.136 \\
54.190 \\
54.244 \\
54.298\end{array}$ \\
\hline $\begin{array}{l}211.00 \\
212.00 \\
213.00 \\
214.00 \\
215.00 \\
216.00 \\
217.00 \\
218.00 \\
219.00 \\
220.00\end{array}$ & $\begin{array}{l}864.54 \\
868.64 \\
872.73 \\
876.83 \\
880.92 \\
885.02 \\
889.11 \\
893.20 \\
897.30 \\
901.39\end{array}$ & $\begin{array}{l}2839.17 \\
2855.13 \\
2871.08 \\
2887.01 \\
2902.92 \\
2918.81 \\
2934.69 \\
2950.55 \\
2966.40 \\
2982.23\end{array}$ & $\begin{array}{l}1963.14 \\
1974.96 \\
1986.76 \\
1998.54 \\
2010.30 \\
2022.05 \\
2033.78 \\
2045.49 \\
2057.19 \\
2068.87\end{array}$ & $\begin{array}{l}49.922 \\
49.998 \\
50.073 \\
50.148 \\
50.222 \\
50.295 \\
50.369 \\
50.442 \\
50.514 \\
50.586\end{array}$ & $\begin{array}{l}281.00 \\
282.00 \\
283.00 \\
284.00 \\
285.00 \\
286.00 \\
287.00 \\
288.00 \\
289.00 \\
290.00\end{array}$ & $\begin{array}{l}1150.45 \\
1154.53 \\
1158.61 \\
1162.69 \\
1166.77 \\
1170.85 \\
1174.94 \\
1179.03 \\
1183.12 \\
1187.21\end{array}$ & $\begin{array}{l}3919.66 \\
3934.66 \\
3949.66 \\
3964.64 \\
3979.62 \\
3994.60 \\
4009.57 \\
4024.54 \\
4039.50 \\
4054.46\end{array}$ & $\begin{array}{l}2753.94 \\
2764.81 \\
2775.67 \\
2786.52 \\
2797.36 \\
2808.20 \\
2819.03 \\
2829.86 \\
2840.68 \\
2851.49\end{array}$ & $\begin{array}{l}54.351 \\
54.405 \\
54.458 \\
54.511 \\
54.563 \\
54.616 \\
54.668 \\
54.720 \\
54.772 \\
54.824\end{array}$ \\
\hline $\begin{array}{l}221.00 \\
222.00 \\
223.00 \\
224.00 \\
225.00 \\
226.00 \\
227.00 \\
228.00 \\
229.00 \\
230.00\end{array}$ & $\begin{array}{l}905.48 \\
909.58 \\
913.67 \\
917.76 \\
921.86 \\
925.95 \\
930.04 \\
934.14 \\
938.23 \\
942.32\end{array}$ & $\begin{array}{l}2998.04 \\
3013.83 \\
3029.61 \\
3045.37 \\
3061.12 \\
3076.84 \\
3092.56 \\
3108.25 \\
3123.93 \\
3139.59\end{array}$ & $\begin{array}{l}2089.53 \\
2092.18 \\
2103.81 \\
2115.42 \\
2127.02 \\
2138.60 \\
2150.16 \\
2161.71 \\
2173.24 \\
2184.76\end{array}$ & $\begin{array}{l}50.658 \\
50.729 \\
50.800 \\
50.871 \\
50.941 \\
51.011 \\
51.080 \\
51.149 \\
51.218 \\
51.286\end{array}$ & $\begin{array}{l}291.00 \\
292.00 \\
293.00 \\
294.00 \\
295.00 \\
296.00 \\
297.00 \\
298.00 \\
299.00 \\
300.00\end{array}$ & $\begin{array}{l}1191.30 \\
1195.40 \\
1199.49 \\
1203.59 \\
1207.69 \\
1211.80 \\
1215.90 \\
1220.01 \\
1224.12 \\
1228.23\end{array}$ & $\begin{array}{l}4069.42 \\
4084.37 \\
4099.32 \\
4114.27 \\
4129.22 \\
4144.16 \\
4159.10 \\
4174.04 \\
4188.97 \\
4203.91\end{array}$ & $\begin{array}{l}2862.30 \\
2873.10 \\
2883.90 \\
2894.70 \\
2905.49 \\
2916.27 \\
2927.05 \\
2937.83 \\
2948.60 \\
2959.37\end{array}$ & $\begin{array}{l}54.875 \\
54.926 \\
54.977 \\
55.028 \\
55.079 \\
55.130 \\
55.180 \\
55.230 \\
55.280 \\
55.330\end{array}$ \\
\hline
\end{tabular}


TEMPER- SPECIFIC ENTHALPY INTERNAL ENTROPY ATURE VOLUME (J/GM) ENERGY (J/GM-K) (K) (CC/GM)
(J/GM)

$(J / G M-K)$ ATURE

(K)
SPECIFIC

(CC/GH)
ENTHALPY

(J/GM)

(1)

INTERNAL ENTROPY

ENERGY (J/GM-K) (J/GM)

\begin{tabular}{|c|c|c|c|c|c|c|c|c|c|}
\hline 20.00 & 13.72 & $-244 \cdot 44$ & -265.29 & 7.594 & & & & & \\
\hline $\begin{array}{l}21.00 \\
22.00 \\
23.00 \\
24.00 \\
25.00 \\
26.00 \\
27.00 \\
28.00 \\
29.00 \\
30.00\end{array}$ & $\begin{array}{l}13.92 \\
14.14 \\
14.37 \\
14.64 \\
14.93 \\
15.26 \\
15.64 \\
16.08 \\
16.59 \\
17.22\end{array}$ & $\begin{array}{l}-235.18 \\
-225.40 \\
-215.03 \\
-204.03 \\
-192.33 \\
-179.82 \\
-166.39 \\
-151.85 \\
-135.95 \\
-118.32\end{array}$ & $\begin{array}{l}-256.34 \\
-246.89 \\
-236.88 \\
-226.28 \\
-215.02 \\
-203.02 \\
-190.16 \\
-176.29 \\
-161.16 \\
-144.49\end{array}$ & $\begin{array}{r}8.048 \\
8.501 \\
8.964 \\
9.434 \\
9.909 \\
10.399 \\
10.905 \\
11.436 \\
11.991 \\
12.589\end{array}$ & $\begin{array}{r}91.00 \\
92.00 \\
93.00 \\
94.00 \\
95.00 \\
96.00 \\
97.00 \\
98.00 \\
99.00 \\
100.00\end{array}$ & $\begin{array}{l}244.94 \\
247.82 \\
250.70 \\
253.58 \\
256.45 \\
259.32 \\
262.18 \\
265.04 \\
267.90 \\
270.75\end{array}$ & $\begin{array}{r}949.42 \\
962.72 \\
976.09 \\
989.53 \\
1003.04 \\
1016.63 \\
1030.28 \\
1044.01 \\
1057.81 \\
1071.68\end{array}$ & $\begin{array}{l}577.15 \\
586.06 \\
595.05 \\
604.12 \\
613.27 \\
622.50 \\
631.80 \\
641.18 \\
650.64 \\
660.17\end{array}$ & $\begin{array}{l}35.163 \\
35.308 \\
35.453 \\
35.597 \\
35.740 \\
35.882 \\
36.024 \\
36.164 \\
36.304 \\
36.444\end{array}$ \\
\hline $\begin{array}{l}31.00 \\
32.00 \\
33.00 \\
34.00 \\
35.00 \\
36.00 \\
37.00 \\
38.00 \\
39.00 \\
40.00\end{array}$ & $\begin{array}{l}18.04 \\
19.19 \\
20.73 \\
25.16 \\
49.07 \\
57.35 \\
63.78 \\
69.36 \\
74.44 \\
79.17\end{array}$ & $\begin{array}{r}-98.09 \\
-73.71 \\
-45.01 \\
13.55 \\
163.89 \\
198.35 \\
223.80 \\
245.60 \\
265.63 \\
284.02\end{array}$ & $\begin{array}{r}-125.51 \\
-102.87 \\
-76.52 \\
-24.70 \\
89.31 \\
111.18 \\
126.86 \\
140.18 \\
152.49 \\
163.69\end{array}$ & $\begin{array}{l}13.252 \\
14.050 \\
14.923 \\
16.668 \\
21.050 \\
22.019 \\
22.714 \\
23.296 \\
23.816 \\
24.281\end{array}$ & $\begin{array}{l}101.00 \\
102.00 \\
103.00 \\
104.00 \\
105.00 \\
106.00 \\
107.00 \\
108.00 \\
109.00 \\
110.00\end{array}$ & $\begin{array}{l}273.61 \\
276.47 \\
279.33 \\
282.19 \\
285.04 \\
287.89 \\
290.73 \\
293.57 \\
296.40 \\
299.23\end{array}$ & $\begin{array}{l}1085.61 \\
1099.61 \\
1113.68 \\
1127.83 \\
1142.04 \\
1156.33 \\
1170.69 \\
1185.12 \\
1199.62 \\
1214.19\end{array}$ & $\begin{array}{l}669.75 \\
679.40 \\
689.12 \\
698.92 \\
708.81 \\
718.77 \\
728.81 \\
738.92 \\
749.11 \\
759.38\end{array}$ & $\begin{array}{l}36.582 \\
36.720 \\
36.858 \\
36.994 \\
37.130 \\
37.266 \\
37.401 \\
37.535 \\
37.668 \\
37.801\end{array}$ \\
\hline $\begin{array}{l}41.00 \\
42.00 \\
43.00 \\
44.00 \\
45.00 \\
46.00 \\
47.00 \\
48.00 \\
49.00 \\
50.00\end{array}$ & $\begin{array}{r}83.66 \\
87.95 \\
92.09 \\
96.10 \\
100.00 \\
103.82 \\
107.56 \\
111.22 \\
114.83 \\
118.38\end{array}$ & $\begin{array}{l}301.26 \\
317.64 \\
333.36 \\
348.56 \\
363.34 \\
377.76 \\
391.88 \\
405.74 \\
419.39 \\
432.84\end{array}$ & $\begin{array}{l}174.11 \\
183.97 \\
193.40 \\
202.51 \\
211.35 \\
219.97 \\
228.41 \\
236.70 \\
244.86 \\
252.91\end{array}$ & $\begin{array}{l}24.707 \\
25.102 \\
25.472 \\
25.821 \\
26.154 \\
26.470 \\
26.774 \\
27.066 \\
27.347 \\
27.619\end{array}$ & $\begin{array}{l}111.00 \\
112.00 \\
113.00 \\
114.00 \\
115.00 \\
116.00 \\
117.00 \\
118.00 \\
119.00 \\
120.00\end{array}$ & $\begin{array}{l}302.05 \\
304.87 \\
307.69 \\
310.49 \\
313.29 \\
316.09 \\
318.88 \\
321.67 \\
324.45 \\
327.23\end{array}$ & $\begin{array}{l}1228.82 \\
1243.53 \\
1258.29 \\
1273.13 \\
1288.02 \\
1302.98 \\
1318.01 \\
1333.09 \\
1348.23 \\
1363.43\end{array}$ & $\begin{array}{l}769.73 \\
780.15 \\
790.64 \\
801.20 \\
811.84 \\
822.55 \\
833.33 \\
844.18 \\
855.09 \\
866.07\end{array}$ & $\begin{array}{l}37.934 \\
38.066 \\
38.197 \\
38.328 \\
38.458 \\
38.587 \\
38.716 \\
38.845 \\
38.973 \\
39.100\end{array}$ \\
\hline $\begin{array}{l}51.00 \\
52.00 \\
53.00 \\
54.00 \\
55.00 \\
56.00 \\
57.00 \\
58.00 \\
59.00 \\
60.00\end{array}$ & $\begin{array}{l}121.88 \\
125.34 \\
128.76 \\
132.14 \\
135.49 \\
138.81 \\
142.09 \\
145.35 \\
148.59 \\
151.80\end{array}$ & $\begin{array}{l}446.12 \\
459.26 \\
472.26 \\
485.16 \\
497.94 \\
510.64 \\
523.26 \\
535.80 \\
548.29 \\
560.72\end{array}$ & $\begin{array}{l}260.88 \\
268.76 \\
276.57 \\
284.32 \\
292.02 \\
299.67 \\
307.29 \\
314.88 \\
322.45 \\
330.00\end{array}$ & $\begin{array}{l}27.882 \\
28.137 \\
28.385 \\
28.626 \\
28.861 \\
29.089 \\
29.313 \\
29.531 \\
29.744 \\
29.953\end{array}$ & $\begin{array}{l}121.00 \\
122.00 \\
123.00 \\
124.00 \\
125.00 \\
126.00 \\
127.00 \\
128.00 \\
129.00 \\
130.00\end{array}$ & $\begin{array}{l}330.00 \\
332.77 \\
335.53 \\
338.30 \\
341.05 \\
343.82 \\
346.59 \\
349.36 \\
352.12 \\
354.89\end{array}$ & $\begin{array}{l}1378.68 \\
1393.99 \\
1409.35 \\
1424.77 \\
1440.23 \\
1455.77 \\
1471.35 \\
1486.99 \\
1502.68 \\
1518.41\end{array}$ & $\begin{array}{l}877.11 \\
888.21 \\
899.37 \\
910.58 \\
921.86 \\
933.19 \\
944.57 \\
956.00 \\
967.48 \\
979.01\end{array}$ & $\begin{array}{l}39.226 \\
39.352 \\
39.478 \\
39.602 \\
39.727 \\
39.850 \\
39.974 \\
40.096 \\
40.218 \\
40.340\end{array}$ \\
\hline $\begin{array}{l}61.00 \\
62.00 \\
63.00 \\
64.00 \\
65.00 \\
66.00 \\
67.00 \\
68.00 \\
69.00 \\
70.00\end{array}$ & $\begin{array}{l}154.99 \\
158.17 \\
161.32 \\
164.45 \\
167.57 \\
170.67 \\
173.76 \\
176.84 \\
179.90 \\
182.95\end{array}$ & $\begin{array}{l}573.11 \\
585.46 \\
597.78 \\
610.07 \\
622.34 \\
634.61 \\
646.86 \\
659.11 \\
671.36 \\
683.62\end{array}$ & $\begin{array}{l}337.54 \\
345.07 \\
352.59 \\
360.13 \\
367.66 \\
375.20 \\
382.76 \\
390.34 \\
397.94 \\
405.57\end{array}$ & $\begin{array}{l}30.158 \\
30.359 \\
30.556 \\
30.750 \\
30.940 \\
31.127 \\
31.311 \\
31.493 \\
31.672 \\
31.848\end{array}$ & $\begin{array}{l}131.00 \\
132.00 \\
133.00 \\
134.00 \\
135.00 \\
136.00 \\
137.00 \\
138.00 \\
139.00 \\
140.00\end{array}$ & $\begin{array}{l}357.65 \\
360.42 \\
363.19 \\
365.95 \\
368.72 \\
371.49 \\
374.27 \\
377.04 \\
379.81 \\
382.59\end{array}$ & $\begin{array}{l}1534.19 \\
1550.02 \\
1565.90 \\
1581.81 \\
1597.77 \\
1613.77 \\
1629.81 \\
1645.88 \\
1661.99 \\
1678.14\end{array}$ & $\begin{array}{r}990.59 \\
1002.22 \\
1013.88 \\
1025.59 \\
1037.34 \\
1049.13 \\
1060.95 \\
1072.81 \\
1084.71 \\
1096.64\end{array}$ & $\begin{array}{l}40.461 \\
40.581 \\
40.701 \\
40.820 \\
40.939 \\
41.057 \\
41.174 \\
41.291 \\
41.408 \\
41.523\end{array}$ \\
\hline $\begin{array}{l}71.00 \\
72.00 \\
73.00 \\
74.00 \\
75.00 \\
76.00 \\
77.00 \\
78.00 \\
79.00 \\
80.00\end{array}$ & $\begin{array}{l}185.98 \\
189.01 \\
192.02 \\
195.03 \\
198.02 \\
201.01 \\
203.98 \\
206.95 \\
209.91 \\
212.87\end{array}$ & $\begin{array}{l}695.89 \\
708.18 \\
720.48 \\
732.82 \\
745.17 \\
757.57 \\
769.99 \\
782.46 \\
794.97 \\
807.53\end{array}$ & $\begin{array}{l}413.22 \\
420.91 \\
428.63 \\
436.40 \\
444.21 \\
452.06 \\
459.96 \\
467.92 \\
475.93 \\
484.00\end{array}$ & $\begin{array}{l}32.022 \\
32.194 \\
32.364 \\
32.532 \\
32.697 \\
32.862 \\
33.024 \\
33.185 \\
33.344 \\
33.502\end{array}$ & $\begin{array}{l}141.00 \\
142.00 \\
143.00 \\
144.00 \\
145.00 \\
146.00 \\
147.00 \\
148.00 \\
149.00 \\
150.00\end{array}$ & $\begin{array}{l}385.37 \\
388.15 \\
390.93 \\
393.71 \\
396.50 \\
399.29 \\
402.07 \\
404.66 \\
407.65 \\
410.44\end{array}$ & $\begin{array}{l}1694.32 \\
1710.54 \\
1726.78 \\
1743.05 \\
1759.35 \\
1775.68 \\
1792.03 \\
1808.41 \\
1824.81 \\
1841.23\end{array}$ & $\begin{array}{l}1108.59 \\
1120.58 \\
1132.60 \\
1144.64 \\
1156.71 \\
1168.80 \\
1180.92 \\
1193.06 \\
1205.22 \\
1217.40\end{array}$ & $\begin{array}{l}41.639 \\
41.753 \\
41.867 \\
41.981 \\
42.093 \\
42.206 \\
42.317 \\
42.428 \\
42.539 \\
42.648\end{array}$ \\
\hline $\begin{array}{l}81.00 \\
82.00 \\
83.00 \\
84.00 \\
85.00 \\
86.00 \\
87.00 \\
88.00 \\
89.00 \\
90.00\end{array}$ & $\begin{array}{l}215.81 \\
218.75 \\
221.69 \\
224.61 \\
227.53 \\
230.45 \\
233.36 \\
236.26 \\
239.16 \\
242.05\end{array}$ & $\begin{array}{l}820.13 \\
832.79 \\
845.50 \\
858.27 \\
871.10 \\
883.99 \\
896.94 \\
909.96 \\
923.04 \\
936.20\end{array}$ & $\begin{array}{l}492.12 \\
500.31 \\
508.57 \\
516.89 \\
525.28 \\
533.74 \\
542.27 \\
550.87 \\
559.56 \\
568.31\end{array}$ & $\begin{array}{l}33.659 \\
33.814 \\
33.968 \\
34.121 \\
34.273 \\
34.424 \\
34.573 \\
34.722 \\
34.870 \\
35.017\end{array}$ & $\begin{array}{l}151.00 \\
152.00 \\
153.00 \\
154.00 \\
155.00 \\
156.00 \\
157.00 \\
158.00 \\
159.00 \\
160.00\end{array}$ & $\begin{array}{l}413.21 \\
415.97 \\
418.74 \\
421.51 \\
424.27 \\
427.04 \\
429.80 \\
432.56 \\
435.32 \\
438.08\end{array}$ & $\begin{array}{l}1857.60 \\
1874.00 \\
1890.40 \\
1906.83 \\
1923.26 \\
1939.70 \\
1956.16 \\
1972.62 \\
1989.09 \\
2005.57\end{array}$ & $\begin{array}{l}1229.57 \\
1241.75 \\
1253.95 \\
1266.17 \\
1278.40 \\
1290.64 \\
1302.90 \\
1315.16 \\
1327.44 \\
1339.72\end{array}$ & $\begin{array}{l}42.757 \\
42.866 \\
42.973 \\
43.080 \\
43.186 \\
43.292 \\
43.397 \\
43.502 \\
43.606 \\
43.709\end{array}$ \\
\hline
\end{tabular}


TEMPER- SPECIFIC ENTHALPY INTERNAL ENTROPY ATURE VOLUME (J/GM) ENERGY (J/GM-K) (K) (CC/GM) ( $J / G M)$
TEMPERATURE

(K)
SPECIFIC (CC/GM)
ENTHALPY INTERNAL ENTROPY

(J/GM) ENERGY
$(J / G M-K)$

\begin{tabular}{|c|c|c|c|c|c|c|c|c|c|}
\hline $\begin{array}{l}161.00 \\
162.00 \\
163.00 \\
164.00 \\
165.00 \\
166.00 \\
167.00 \\
168.00 \\
169.00 \\
170.00\end{array}$ & $\begin{array}{l}440.84 \\
443.60 \\
446.36 \\
449.12 \\
451.87 \\
454.63 \\
457.38 \\
460.13 \\
462.89 \\
465.64\end{array}$ & $\begin{array}{l}2022.05 \\
2038.54 \\
2055.04 \\
2071.54 \\
2088.04 \\
2104.54 \\
2121.04 \\
2137.55 \\
2154.05 \\
2170.55\end{array}$ & $\begin{array}{l}1352.01 \\
1364.31 \\
1376.61 \\
1388.92 \\
1401.23 \\
1413.55 \\
1425.86 \\
1438.18 \\
1450.50 \\
1462.83\end{array}$ & $\begin{array}{l}43.812 \\
43.914 \\
44.015 \\
44.116 \\
44.217 \\
44.316 \\
44.415 \\
44.514 \\
44.612 \\
44.709\end{array}$ & $\begin{array}{l}231.00 \\
232.00 \\
233.00 \\
234.00 \\
235.00 \\
236.00 \\
237.00 \\
238.00 \\
239.00 \\
240.00\end{array}$ & $\begin{array}{l}632.89 \\
635.63 \\
638.36 \\
641.09 \\
643.83 \\
646.56 \\
649.29 \\
652.02 \\
654.75 \\
657.49\end{array}$ & $\begin{array}{l}3155.71 \\
3171.36 \\
3187.00 \\
3202.62 \\
3218.22 \\
3233.81 \\
3249.38 \\
3264.94 \\
3280.48 \\
3296.00\end{array}$ & $\begin{array}{l}2193.77 \\
2205.26 \\
2216.75 \\
2228.21 \\
2239.66 \\
2251.10 \\
2262.52 \\
2273.92 \\
2285.31 \\
2296.68\end{array}$ & $\begin{array}{l}49.668 \\
49.736 \\
49.803 \\
49.870 \\
49.937 \\
50.003 \\
50.069 \\
50.134 \\
50.199 \\
50.264\end{array}$ \\
\hline $\begin{array}{l}171.00 \\
172.00 \\
173.00 \\
174.00 \\
175.00 \\
176.00 \\
177.00 \\
178.00 \\
179.00 \\
180.00\end{array}$ & $\begin{array}{l}468.39 \\
471.14 \\
473.90 \\
476.65 \\
479.40 \\
482.15 \\
484.90 \\
487.65 \\
490.40 \\
493.15\end{array}$ & $\begin{array}{l}2187.06 \\
2203.56 \\
2220.05 \\
2236.55 \\
2253.03 \\
2269.52 \\
2285.99 \\
2302.46 \\
2318.93 \\
2335.38\end{array}$ & $\begin{array}{l}1475.14 \\
1487.46 \\
1499.77 \\
1512.08 \\
1524.39 \\
1536.69 \\
1548.99 \\
1561.28 \\
1573.56 \\
1585.84\end{array}$ & $\begin{array}{l}44.806 \\
44.902 \\
44.998 \\
45.093 \\
45.187 \\
45.281 \\
45.375 \\
45.468 \\
45.560 \\
45.651\end{array}$ & $\begin{array}{l}241.00 \\
242.00 \\
243.00 \\
244.00 \\
245.00 \\
246.00 \\
247.00 \\
248.00 \\
249.00 \\
250.00\end{array}$ & $\begin{array}{l}660.22 \\
662.95 \\
665.68 \\
668.41 \\
671.14 \\
673.87 \\
676.60 \\
679.32 \\
682.05 \\
684.78\end{array}$ & $\begin{array}{l}3311.51 \\
3327.00 \\
3342.48 \\
3357.94 \\
3373.39 \\
3388.82 \\
3404.24 \\
3419.64 \\
3435.02 \\
3450.39\end{array}$ & $\begin{array}{l}2308.04 \\
2319.38 \\
2330.71 \\
2342.02 \\
2353.32 \\
2364.60 \\
2375.87 \\
2387.12 \\
2398.36 \\
2409.58\end{array}$ & $\begin{array}{l}50.329 \\
50.393 \\
50.457 \\
50.520 \\
50.583 \\
50.646 \\
50.709 \\
50.771 \\
50.833 \\
50.894\end{array}$ \\
\hline $\begin{array}{l}181.00 \\
182.00 \\
183.00 \\
184.00 \\
185.00 \\
186.00 \\
187.00 \\
188.00 \\
189.00 \\
190.00\end{array}$ & $\begin{array}{l}495.90 \\
498.64 \\
501.39 \\
504.14 \\
506.88 \\
509.63 \\
512.38 \\
515.12 \\
517.87 \\
520.61\end{array}$ & $\begin{array}{l}2351.83 \\
2368.26 \\
2384.69 \\
2401.11 \\
2417.52 \\
2433.91 \\
2450.30 \\
2466.67 \\
2483.03 \\
2499.38\end{array}$ & $\begin{array}{l}1598.11 \\
1610.37 \\
1622.62 \\
1634.86 \\
1647.10 \\
1659.32 \\
1671.53 \\
1683.73 \\
1695.92 \\
1708.10\end{array}$ & $\begin{array}{l}45.743 \\
45.833 \\
45.923 \\
46.013 \\
46.102 \\
46.190 \\
46.278 \\
46.365 \\
46.452 \\
46.538\end{array}$ & $\begin{array}{l}251.00 \\
252.00 \\
253.00 \\
254.00 \\
255.00 \\
256.00 \\
257.00 \\
258.00 \\
259.00 \\
260.00\end{array}$ & $\begin{array}{l}687.51 \\
690.23 \\
692.96 \\
695.69 \\
698.41 \\
701.14 \\
703.86 \\
706.58 \\
709.31 \\
712.03\end{array}$ & $\begin{array}{l}3465.78 \\
3481.16 \\
3496.52 \\
3511.87 \\
3527.20 \\
3542.51 \\
3557.82 \\
3573.11 \\
3588.38 \\
3603.64\end{array}$ & $\begin{array}{l}2420.83 \\
2432.06 \\
2443.28 \\
2454.48 \\
2465.67 \\
2476.85 \\
2488.01 \\
2499.16 \\
2510.29 \\
2521.42\end{array}$ & $\begin{array}{l}50.956 \\
51.017 \\
51.078 \\
51.138 \\
51.199 \\
51.258 \\
51.318 \\
51.377 \\
51.437 \\
51.495\end{array}$ \\
\hline $\begin{array}{l}191.00 \\
192.00 \\
193.00 \\
194.00 \\
195.00 \\
196.00 \\
197.00 \\
198.00 \\
199.00 \\
200.00\end{array}$ & $\begin{array}{l}523.36 \\
526.10 \\
528.85 \\
531.59 \\
534.33 \\
537.08 \\
539.82 \\
542.56 \\
545.30 \\
548.04\end{array}$ & $\begin{array}{l}2515.72 \\
2532.04 \\
2548.35 \\
2564.64 \\
2580.92 \\
2597.18 \\
2613.43 \\
2629.67 \\
2645.88 \\
2662.09\end{array}$ & $\begin{array}{l}1720.26 \\
1732.41 \\
1744.55 \\
1756.67 \\
1768.78 \\
1780.87 \\
1792.95 \\
1805.02 \\
1817.07 \\
1829.10\end{array}$ & $\begin{array}{l}46.624 \\
46.709 \\
46.794 \\
46.878 \\
46.962 \\
47.045 \\
47.128 \\
47.210 \\
47.292 \\
47.373\end{array}$ & $\begin{array}{l}261.00 \\
262.00 \\
263.00 \\
264.00 \\
265.00 \\
266.00 \\
267.00 \\
268.00 \\
269.00 \\
270.00\end{array}$ & $\begin{array}{l}714.75 \\
717.48 \\
720.20 \\
722.92 \\
725.64 \\
728.36 \\
731.08 \\
733.80 \\
736.52 \\
739.24\end{array}$ & $\begin{array}{l}3618.89 \\
3634.12 \\
3649.34 \\
3664.54 \\
3679.74 \\
3694.91 \\
3710.08 \\
3725.23 \\
3740.38 \\
3755.51\end{array}$ & $\begin{array}{l}2532.52 \\
2543.62 \\
2554.70 \\
2565.77 \\
2576.82 \\
2587.87 \\
2598.90 \\
2609.92 \\
2620.92 \\
2631.92\end{array}$ & $\begin{array}{l}51.554 \\
51.612 \\
51.670 \\
51.728 \\
51.785 \\
51.842 \\
51.899 \\
51.956 \\
52.012 \\
52.069\end{array}$ \\
\hline $\begin{array}{l}201.00 \\
202.00 \\
203.00 \\
204.00 \\
205.00 \\
206.00 \\
207.00 \\
208.00 \\
209.00 \\
210.00\end{array}$ & $\begin{array}{l}550.78 \\
553.52 \\
556.26 \\
559.00 \\
561.74 \\
564.48 \\
567.22 \\
569.96 \\
572.70 \\
575.44\end{array}$ & $\begin{array}{l}2678.26 \\
2694.42 \\
2710.57 \\
2726.69 \\
2742.80 \\
2758.90 \\
2774.98 \\
2791.04 \\
2807.08 \\
2823.10\end{array}$ & $\begin{array}{l}1841.12 \\
1853.11 \\
1865.09 \\
1877.06 \\
1889.00 \\
1900.93 \\
1912.85 \\
1924.75 \\
1936.63 \\
1948.49\end{array}$ & $\begin{array}{l}47.453 \\
47.534 \\
47.613 \\
47.693 \\
47.771 \\
47.850 \\
47.928 \\
48.005 \\
48.082 \\
48.158\end{array}$ & $\begin{array}{l}271.00 \\
272.00 \\
273.00 \\
274.00 \\
275.00 \\
276.00 \\
277.00 \\
278.00 \\
279.00 \\
280.00\end{array}$ & $\begin{array}{l}741.97 \\
744.69 \\
747.41 \\
750.13 \\
752.85 \\
755.57 \\
758.29 \\
761.01 \\
763.74 \\
766.46\end{array}$ & $\begin{array}{l}3770.62 \\
3785.73 \\
3800.82 \\
3815.91 \\
3830.98 \\
3846.05 \\
3861.10 \\
3876.15 \\
3891.18 \\
3906.21\end{array}$ & $\begin{array}{l}2642.90 \\
2653.87 \\
2664.83 \\
2675.78 \\
2686.72 \\
2697.65 \\
2708.56 \\
2719.47 \\
2730.37 \\
2741.26\end{array}$ & $\begin{array}{l}52.124 \\
52.180 \\
52.235 \\
52.291 \\
52.346 \\
52.400 \\
52.455 \\
52.509 \\
52.563 \\
52.617\end{array}$ \\
\hline $\begin{array}{l}211.00 \\
212.00 \\
213.00 \\
214.00 \\
215.00 \\
216.00 \\
217.00 \\
218.00 \\
219.00 \\
220.00\end{array}$ & $\begin{array}{l}578.17 \\
580.91 \\
583.65 \\
586.39 \\
589.12 \\
591.86 \\
594.60 \\
597.33 \\
600.07 \\
602.81\end{array}$ & $\begin{array}{l}2839.11 \\
2855.11 \\
2871.08 \\
2887.04 \\
2902.98 \\
2918.90 \\
2934.81 \\
2950.70 \\
2966.57 \\
2982.42\end{array}$ & $\begin{array}{l}1960.34 \\
1972.17 \\
1983.98 \\
1995.78 \\
2007.56 \\
2019.32 \\
2031.07 \\
2042.80 \\
2054.51 \\
2066.21\end{array}$ & $\begin{array}{l}48.234 \\
48.310 \\
48.385 \\
48.460 \\
48.534 \\
48.608 \\
48.682 \\
48.755 \\
48.827 \\
48.900\end{array}$ & $\begin{array}{l}281.00 \\
282.00 \\
283.00 \\
284.00 \\
285.00 \\
286.00 \\
287.00 \\
288.00 \\
289.00 \\
290.00\end{array}$ & $\begin{array}{l}769.18 \\
771.91 \\
774.63 \\
777.35 \\
780.08 \\
782.81 \\
785.53 \\
788.26 \\
790.99 \\
793.72\end{array}$ & $\begin{array}{l}3921.23 \\
3936.25 \\
3951.25 \\
3966.26 \\
3981 \cdot 25 \\
3996.24 \\
4011.22 \\
4026.20 \\
4041.17 \\
4056.14\end{array}$ & $\begin{array}{l}2752.14 \\
2763.02 \\
2773.88 \\
2784.74 \\
2795.59 \\
2806.44 \\
2817.28 \\
2828.11 \\
2838.93 \\
2849.75\end{array}$ & $\begin{array}{l}52.670 \\
52.724 \\
52.777 \\
52.830 \\
52.882 \\
52.935 \\
52.987 \\
53.039 \\
53.091 \\
53.143\end{array}$ \\
\hline $\begin{array}{l}221.00 \\
222.00 \\
223.00 \\
224.00 \\
225.00 \\
226.00 \\
227.00 \\
228.00 \\
229.00 \\
230.00\end{array}$ & $\begin{array}{l}605.54 \\
608 \cdot 28 \\
611.01 \\
613.75 \\
616.48 \\
619.22 \\
621.95 \\
624.69 \\
627.42 \\
630.16\end{array}$ & $\begin{array}{l}2998.26 \\
3014.08 \\
3029.88 \\
3045.67 \\
3061.44 \\
3077.19 \\
3092.93 \\
3108.65 \\
3124.35 \\
3140.04\end{array}$ & $\begin{array}{l}2077.89 \\
2089.55 \\
2101.19 \\
2112.82 \\
2124.44 \\
2136.03 \\
2147.61 \\
2159.17 \\
2170.72 \\
2182.25\end{array}$ & $\begin{array}{l}48.971 \\
49.043 \\
49.114 \\
49.184 \\
49.255 \\
49.325 \\
49.394 \\
49.463 \\
49.532 \\
49.600\end{array}$ & $\begin{array}{l}291.00 \\
292.00 \\
293.00 \\
294.00 \\
295.00 \\
296.00 \\
297.00 \\
298.00 \\
299.00 \\
300.00\end{array}$ & $\begin{array}{l}796.45 \\
799.19 \\
801.92 \\
804.65 \\
807.39 \\
810.12 \\
812.86 \\
815.60 \\
818.33 \\
821.07\end{array}$ & $\begin{array}{l}4071.11 \\
4086.07 \\
4101.03 \\
4115.99 \\
4130.95 \\
4145.90 \\
4160.85 \\
4175.80 \\
4190.74 \\
4205.68\end{array}$ & $\begin{array}{l}2860.57 \\
2871 \cdot 38 \\
2882 \cdot 19 \\
2892 \cdot 99 \\
2903.79 \\
2914.58 \\
2925.37 \\
2936.16 \\
2946.94 \\
2957.72\end{array}$ & $\begin{array}{l}53.194 \\
53.246 \\
53.297 \\
53.348 \\
53.399 \\
53.449 \\
53.500 \\
53.550 \\
53.600 \\
53.650\end{array}$ \\
\hline
\end{tabular}




\begin{tabular}{|c|c|c|c|c|c|c|c|c|c|}
\hline $\begin{array}{l}\text { EMPER- } \\
\text { ATURE } \\
(K)\end{array}$ & $\begin{array}{l}\text { SPECIF IC } \\
\text { VOLUME } \\
(C C / G M)\end{array}$ & $\begin{array}{l}\text { ENTHALPY } \\
\text { (J/GM) }\end{array}$ & $\begin{array}{l}\text { INTERNAL } \\
\text { ENERGY } \\
(J / G M)\end{array}$ & $\begin{array}{l}\text { ENTROPY } \\
(J / G H-K)\end{array}$ & $\begin{array}{c}\text { TEMPER- } \\
\text { ATURE } \\
\text { (K) }\end{array}$ & $\begin{array}{l}\text { SPECIFIC } \\
\text { VOLUME } \\
\text { (CC/GM) }\end{array}$ & $\begin{array}{c}\text { ENTHALPY } \\
\text { (J/GM) }\end{array}$ & $\begin{array}{l}\text { INTERNAL } \\
\text { ENERGY } \\
(J / G M)\end{array}$ & $\begin{array}{l}\text { ENTROPY } \\
(J / G M-K)\end{array}$ \\
\hline
\end{tabular}

\begin{tabular}{|c|c|c|c|c|c|c|c|c|c|}
\hline 20.00 & 13.62 & -239.38 & -266.97 & 7.501 & & & & & \\
\hline $\begin{array}{l}21.00 \\
22.00 \\
23.00 \\
24.00 \\
25.00 \\
26.00 \\
27.00 \\
28.00 \\
29.00 \\
30.00\end{array}$ & $\begin{array}{l}13.80 \\
14.01 \\
14.23 \\
14.47 \\
14.74 \\
15.04 \\
15.37 \\
15.75 \\
16.18 \\
16.68\end{array}$ & $\begin{array}{l}-230.30 \\
-220.71 \\
-210.59 \\
-199.89 \\
-188.56 \\
-176.53 \\
-163.69 \\
-149.96 \\
-135.17 \\
-119.17\end{array}$ & $\begin{array}{l}-258.27 \\
-249.10 \\
-239.43 \\
-229.22 \\
-218.43 \\
-207.00 \\
-194.84 \\
-181.87 \\
-167.95 \\
-152.98\end{array}$ & $\begin{array}{r}7.947 \\
8.390 \\
8.842 \\
9.299 \\
9.759 \\
10.231 \\
10.714 \\
11.215 \\
11.732 \\
12.275\end{array}$ & $\begin{array}{r}91.00 \\
92.00 \\
93.00 \\
94.00 \\
95.00 \\
96.00 \\
97.00 \\
98.00 \\
99.00 \\
100.00\end{array}$ & $\begin{array}{l}183.40 \\
185.60 \\
187.80 \\
189.99 \\
192.18 \\
194.37 \\
196.55 \\
198.73 \\
200.90 \\
203.07\end{array}$ & $\begin{array}{r}940.58 \\
954.08 \\
967.65 \\
981.29 \\
994.99 \\
1008.76 \\
1022.59 \\
1036.50 \\
1050.46 \\
1064.50\end{array}$ & $\begin{array}{l}568.92 \\
577.97 \\
587.08 \\
596.27 \\
605.53 \\
614.87 \\
624.29 \\
633.78 \\
643.34 \\
652.97\end{array}$ & $\begin{array}{l}33.890 \\
34.038 \\
34.184 \\
34.330 \\
34.475 \\
34.619 \\
34.763 \\
34.005 \\
35.047 \\
35.188\end{array}$ \\
\hline $\begin{array}{l}31.00 \\
32.00 \\
33.00 \\
34.00 \\
35.00 \\
36.00 \\
37.00 \\
38.00 \\
39.00 \\
40.00\end{array}$ & $\begin{array}{l}17.29 \\
18.04 \\
19.02 \\
20.34 \\
22.43 \\
26.67 \\
34.40 \\
41.10 \\
46.46 \\
51.08\end{array}$ & $\begin{array}{r}-101.52 \\
-81.79 \\
-60.20 \\
-33.29 \\
1.62 \\
54.16 \\
121.04 \\
166.67 \\
199.62 \\
226.29\end{array}$ & $\begin{array}{r}-136.56 \\
-118.36 \\
-98.74 \\
-74.51 \\
-43.84 \\
.11 \\
51.34 \\
83.38 \\
105.47 \\
122.78\end{array}$ & $\begin{array}{l}12.853 \\
13.482 \\
14.159 \\
14.964 \\
15.974 \\
17.450 \\
19.281 \\
20.499 \\
21.355 \\
22.030\end{array}$ & $\begin{array}{l}101.00 \\
102.00 \\
103.00 \\
104.00 \\
105.00 \\
106.00 \\
107.00 \\
108.00 \\
109.00 \\
110.00\end{array}$ & $\begin{array}{l}205.24 \\
207.40 \\
209.56 \\
211.72 \\
213.87 \\
216.02 \\
218.17 \\
220.31 \\
222.46 \\
224.59\end{array}$ & $\begin{array}{l}1078.59 \\
1092.74 \\
1106.95 \\
1121.24 \\
1135.59 \\
1150.02 \\
1164.51 \\
1179.06 \\
1193.69 \\
1208.38\end{array}$ & $\begin{array}{l}662.66 \\
672.42 \\
682.26 \\
692.18 \\
702.17 \\
712.23 \\
722.37 \\
732.58 \\
742.87 \\
753.22\end{array}$ & $\begin{array}{l}35.328 \\
35.468 \\
35.606 \\
35.744 \\
35.882 \\
36.019 \\
36.155 \\
36.290 \\
36.425 \\
36.559\end{array}$ \\
\hline $\begin{array}{l}41.00 \\
42.00 \\
43.00 \\
44.00 \\
45.00 \\
46.00 \\
47.00 \\
48.00 \\
49.00 \\
50.00\end{array}$ & $\begin{array}{l}55.24 \\
59.10 \\
62.73 \\
66.19 \\
69.51 \\
72.72 \\
75.83 \\
78.86 \\
81.82 \\
84.72\end{array}$ & $\begin{array}{l}249.43 \\
270.31 \\
289.62 \\
307.78 \\
325.06 \\
341.65 \\
357.67 \\
373.23 \\
388.40 \\
403.23\end{array}$ & $\begin{array}{l}137.48 \\
150.54 \\
162.49 \\
173.64 \\
184.20 \\
194.28 \\
204.00 \\
213.42 \\
222.58 \\
231.54\end{array}$ & $\begin{array}{l}22.602 \\
23.105 \\
23.559 \\
23.977 \\
24.365 \\
24.730 \\
25.075 \\
25.402 \\
25.715 \\
26.015\end{array}$ & $\begin{array}{l}111.00 \\
112.00 \\
113.00 \\
114.00 \\
115.00 \\
116.00 \\
117.00 \\
118.00 \\
119.00 \\
120.00\end{array}$ & $\begin{array}{l}226.73 \\
228.86 \\
230.99 \\
233.12 \\
235.25 \\
237.37 \\
239.49 \\
241.61 \\
243.73 \\
245.85\end{array}$ & $\begin{array}{l}1223.13 \\
1237.95 \\
1252.83 \\
1267.78 \\
1282.78 \\
1297.85 \\
1312.98 \\
1328.16 \\
1343.41 \\
1358.71\end{array}$ & $\begin{array}{l}763.65 \\
774.15 \\
784.71 \\
795.34 \\
806.04 \\
816.80 \\
827.63 \\
838.52 \\
849.47 \\
860.48\end{array}$ & $\begin{array}{l}36.692 \\
36.825 \\
36.958 \\
37.089 \\
37.220 \\
37.351 \\
37.481 \\
37.610 \\
37.739 \\
37.867\end{array}$ \\
\hline $\begin{array}{l}51.00 \\
52.00 \\
53.00 \\
54.00 \\
55.00 \\
56.00 \\
57.00 \\
58.00 \\
59.00 \\
60.00\end{array}$ & $\begin{array}{r}87.57 \\
90.36 \\
93.11 \\
95.82 \\
98.50 \\
101.14 \\
103.75 \\
106.33 \\
108.89 \\
111.42\end{array}$ & $\begin{array}{l}417.78 \\
432.07 \\
446.15 \\
460.03 \\
473.75 \\
487.31 \\
500.75 \\
514.06 \\
527.27 \\
540.38\end{array}$ & $\begin{array}{l}240.32 \\
248.96 \\
257.46 \\
265.85 \\
274.14 \\
282.36 \\
290.50 \\
298.57 \\
306.60 \\
314.59\end{array}$ & $\begin{array}{l}26.303 \\
26.580 \\
26.848 \\
27.108 \\
27.360 \\
27.604 \\
27.842 \\
28.073 \\
28.299 \\
28.520\end{array}$ & $\begin{array}{l}121.00 \\
122.00 \\
123.00 \\
124.00 \\
125.00 \\
126.00 \\
127.00 \\
128.00 \\
129.00 \\
130.00\end{array}$ & $\begin{array}{l}247.96 \\
250.08 \\
252.19 \\
254.30 \\
256.41 \\
258.52 \\
260.62 \\
262.72 \\
264.82 \\
266.92\end{array}$ & $\begin{array}{l}1374.05 \\
1389.45 \\
1404.91 \\
1420.42 \\
1435.98 \\
1451.60 \\
1467.27 \\
1482.99 \\
1498.76 \\
1514.57\end{array}$ & $\begin{array}{l}871.54 \\
882.66 \\
893.83 \\
905.06 \\
916.34 \\
927.70 \\
939.11 \\
950.57 \\
962.08 \\
973.64\end{array}$ & $\begin{array}{l}37.994 \\
38.121 \\
38.247 \\
38.372 \\
38.497 \\
38.622 \\
38.746 \\
38.869 \\
38.992 \\
39.114\end{array}$ \\
\hline $\begin{array}{l}61.00 \\
62.00 \\
63.00 \\
64.00 \\
65.00 \\
66.00 \\
67.00 \\
68.00 \\
69.00 \\
70.00\end{array}$ & $\begin{array}{l}113.94 \\
116.43 \\
118.90 \\
121.35 \\
123.79 \\
126.21 \\
128.61 \\
131.00 \\
133.38 \\
135.75\end{array}$ & $\begin{array}{l}553.42 \\
566.39 \\
579.30 \\
592.14 \\
604.95 \\
617.72 \\
630.46 \\
643.18 \\
655.88 \\
668.56\end{array}$ & $\begin{array}{l}322.54 \\
330.45 \\
338.35 \\
346.23 \\
354.10 \\
361.97 \\
369.83 \\
377.70 \\
385.58 \\
393.48\end{array}$ & $\begin{array}{l}28.735 \\
28.946 \\
29.152 \\
29.355 \\
29.553 \\
29.748 \\
29.940 \\
30.128 \\
30.314 \\
30.496\end{array}$ & $\begin{array}{l}131.00 \\
132.00 \\
133.00 \\
134.00 \\
135.00 \\
136.00 \\
137.00 \\
138.00 \\
139.00 \\
140.00\end{array}$ & $\begin{array}{l}269.02 \\
271.12 \\
273.22 \\
275.32 \\
277.42 \\
279.52 \\
281.61 \\
283.71 \\
285.81 \\
287.91\end{array}$ & $\begin{array}{l}1530.43 \\
1546.34 \\
1562.29 \\
1578.28 \\
1594.31 \\
1610.38 \\
1626.49 \\
1642.64 \\
1658.82 \\
1675.04\end{array}$ & $\begin{array}{r}985.24 \\
996.90 \\
1008.59 \\
1020.33 \\
1032.11 \\
1043.93 \\
1055.79 \\
1067.68 \\
1079.61 \\
1091.58\end{array}$ & $\begin{array}{l}39.235 \\
39.356 \\
39.477 \\
39.597 \\
39.716 \\
39.834 \\
39.952 \\
40.070 \\
40.187 \\
40.303\end{array}$ \\
\hline $\begin{array}{l}71.00 \\
72.00 \\
73.00 \\
74.00 \\
75.00 \\
76.00 \\
77.00 \\
78.00 \\
79.00 \\
80.00\end{array}$ & $\begin{array}{l}138.10 \\
140.44 \\
142.77 \\
145.09 \\
147.41 \\
149.71 \\
152.00 \\
154.29 \\
156.57 \\
158.84\end{array}$ & $\begin{array}{l}681.24 \\
693.93 \\
706.61 \\
719.31 \\
732.02 \\
744.76 \\
757.51 \\
770.30 \\
783.12 \\
795.97\end{array}$ & $\begin{array}{l}401.39 \\
409.32 \\
417.29 \\
425.28 \\
433.31 \\
441.37 \\
449.48 \\
457.64 \\
465.84 \\
474.09\end{array}$ & $\begin{array}{l}30.676 \\
30.853 \\
31.028 \\
31.201 \\
31.372 \\
31.541 \\
31.707 \\
31.872 \\
32.036 \\
32.197\end{array}$ & $\begin{array}{l}141.00 \\
142.00 \\
143.00 \\
144.00 \\
145.00 \\
146.00 \\
147.00 \\
148.00 \\
149.00 \\
150.00\end{array}$ & $\begin{array}{l}290.01 \\
292.10 \\
294.20 \\
296.30 \\
298.40 \\
300.50 \\
302.59 \\
304.69 \\
306.79 \\
308.89\end{array}$ & $\begin{array}{l}1691.29 \\
1707.56 \\
1723.87 \\
1740.21 \\
1756.58 \\
1772.97 \\
1789.38 \\
1805.81 \\
1822.27 \\
1838.75\end{array}$ & $\begin{array}{l}1103.57 \\
1115.60 \\
1127.66 \\
1139.74 \\
1151.86 \\
1163.99 \\
1176.15 \\
1188.34 \\
1200.55 \\
1212.77\end{array}$ & $\begin{array}{l}40.419 \\
40.534 \\
40.648 \\
40.762 \\
40.875 \\
40.983 \\
41.100 \\
41.211 \\
41.322 \\
41.432\end{array}$ \\
\hline $\begin{array}{l}81.00 \\
82.00 \\
83.00 \\
84.00 \\
85.00 \\
86.00 \\
87.00 \\
88.00 \\
89.00 \\
90.00\end{array}$ & $\begin{array}{l}161.10 \\
163.36 \\
165.61 \\
167.85 \\
170.09 \\
172.32 \\
174.54 \\
176.77 \\
178.98 \\
181.19\end{array}$ & $\begin{array}{l}808.86 \\
821.80 \\
834.77 \\
847.80 \\
860.88 \\
874.02 \\
887.21 \\
900.46 \\
913.77 \\
927.15\end{array}$ & $\begin{array}{l}482.39 \\
490.75 \\
499.18 \\
507.66 \\
516.21 \\
524.82 \\
533.50 \\
542.25 \\
551.07 \\
559.96\end{array}$ & $\begin{array}{l}32.357 \\
32.516 \\
32.673 \\
32.829 \\
32.984 \\
33.138 \\
33.290 \\
33.442 \\
33.592 \\
33.742\end{array}$ & $\begin{array}{l}151.00 \\
152.00 \\
153.00 \\
154.00 \\
155.00 \\
156.00 \\
157.00 \\
158.00 \\
159.00 \\
160.00\end{array}$ & $\begin{array}{l}310.97 \\
313.06 \\
315.15 \\
317.23 \\
319.32 \\
321.40 \\
323.48 \\
325.57 \\
327.65 \\
329.73\end{array}$ & $\begin{array}{l}1855.18 \\
1871.63 \\
1888.10 \\
1904.57 \\
1921.06 \\
1937.55 \\
1954.06 \\
1970.58 \\
1987.10 \\
2003.63\end{array}$ & $\begin{array}{l}1224.98 \\
1237.20 \\
1249.43 \\
1261.68 \\
1273.94 \\
1286.22 \\
1298.50 \\
1310.80 \\
1323.10 \\
1335.41\end{array}$ & $\begin{array}{l}41.541 \\
41.650 \\
41.758 \\
41.865 \\
41.972 \\
42.078 \\
42.184 \\
42.288 \\
42.393 \\
42.496\end{array}$ \\
\hline
\end{tabular}


TEMPER- SPECIFIC ENTHALPY INTERNAL ENTROPY ATURE VOLUME (J/GM) ENERGY (J/GM-K) (K) (CC/GM)
( $J / G M)$ ATURE (K)
SPECIFIC (CC/GM)
ENTHALP

( $J / G M)$

(1)

INTERNAL ENTROPY (J/GM)

161.00
162.00
163.00
164.00
165.00
166.00
167.00
168.00
169.00
170.00
171.00
172.00
173.00
174.00
175.00
176.00
177.00
178.00
179.00
180.00

181.00 182.00

183.00

184.00

185.00

186.00

187.00

188.00

189.00

190.00

191.00

192.00

193.00

194.00

195.00

196.00

197.00

198.00

199.00

200.00

201.00

202.00

203.00

204.00

205.00

206.00

207.00

208.00

209.00

210.00

211.00

212.00

213.00

214.00

215.00

216.00

217.00

218.00

219.00

220.00

221.00

222.00

223.00

224.00

225.00

226.00

227.00

228.00

229.00

230.00

331.81
333.89
335.97
338.04
340.12
342.20
344.27
346.35
348.42
350.50

2020.16

1347.73

1384.72

1397.06

1409.40

352.57

354.65

356.72

358.79

360.86

362.93

365.00

367.07

369.14

371.21

373.28

375.35

377.42

379.49

381.55

383.62

385.69

387.75

389.82

391.89

393.95

396.02

398.08

400.15

402.21

404.28

406.34

408.40

10.47

412.53

414.59

416.66

418.72

420.78

422.84

424.90

426.96

429.02

$431: 08$

433.14

435.20

437.26

439.31

441.37

443.43

445.49

447.55

449.61

451.66

453.72

455.78

457.83

459.89

461.95

464.00

466.06

468.11

470.17

472.23

474.28
1421.75

1434.09

1446.43

1458.78

119.44

2135.99

2152.53

2185.63

2202.17

1483.47

1483.47

1508.14

1520.48

1532.81

251.78

2268.31

1557.45

$2334.34 \quad 1582.06$

$2350.83 \quad 1594.36$

$2367.31 \quad 1606.64$

2400.24

2416.68

2433.12

2449.55

2465.96

2482.36

2498.75

2515.12

2531.48

2547.82

2564.15

2580.47

2596.77

2613.05

2629.32

645.58

2661.81

2678.02

2694.22

2710.39

2726.55

2742.70

2758.82

2774.93

2791.02

2823.15

2855.21

2871.21

2887.20

2903.17

2919.12

2935.05

2950.97

2966.87

2982.75

2998.61

3014.46

3030.29

3046.10

3061.90

3077.68

3093.44

3109.18

3124.91

3140.62
1618.92

1631.19

1643.45

1655.69

1667.93

1680.15

1692.37

1704.57

1716.75

1728.93

1741.09

1753.23

1765.36

1777.48

1789.58

1801.67

1813.74

1825.80

1837.83

1849.84

1861.84

1873.83

1885.79

1897.74

1909.68

1921.59

1933.49

1945.37

1957.24

1969.09

1980.92

1992.73

2004.53

2016.31

2028.07

2039.82

2051.55

2063.26

2074.96

2086.64

2098. 30

2109.94

2121.57

2133.18

2144.78

2156.36

2167.92

2179.47
2284.83

2383.78

2807.10

2839.19

42.599

42.702

42.804

42.905

43.005

43.105

43.205

4.304

43.402

43.499

43.596

43.693

43.789

43.884

43.979

44.073

44.167

44.260

44.352

44.444

44.535

44.626

44.716

44.806 


\begin{tabular}{|c|c|c|c|c|c|c|c|c|c|}
\hline $\begin{array}{l}\text { TEMPER- } \\
\text { ATURE } \\
\text { (K) }\end{array}$ & $\begin{array}{l}\text { SPEC IFIC } \\
\text { VOLUME } \\
\text { (CC/GM) }\end{array}$ & $\begin{array}{c}\text { ENTHALPY } \\
(J / G N)\end{array}$ & $\begin{array}{l}\text { INTERNAL } \\
\text { ENERGY } \\
(\mathrm{J} / \mathrm{GM})\end{array}$ & $\begin{array}{l}\text { ENTRDPY } \\
(J / G M-K)\end{array}$ & $\begin{array}{c}\text { TEMPER- } \\
\text { ATURE } \\
\text { (K) }\end{array}$ & $\begin{array}{l}\text { SPECIFIC } \\
\text { VOLUME } \\
\text { (CC/GM) }\end{array}$ & $\begin{array}{c}\text { ENTHALPY } \\
(J / G M)\end{array}$ & $\begin{array}{l}\text { INTERNAL } \\
\text { ENERGY } \\
\text { (J/GM) }\end{array}$ & $\begin{array}{l}\text { ENTROPY } \\
(J / G M-K)\end{array}$ \\
\hline
\end{tabular}

\begin{tabular}{|c|c|c|c|c|c|c|c|c|c|}
\hline 20.00 & 13.52 & -234.26 & -268.51 & 7.413 & & & & & \\
\hline $\begin{array}{l}21.00 \\
22.00 \\
23.00 \\
24.00 \\
25.00 \\
26.00 \\
27.00 \\
28.00 \\
29.00 \\
30.00\end{array}$ & $\begin{array}{l}13.69 \\
13.89 \\
14.09 \\
14.32 \\
14.57 \\
14.84 \\
15.14 \\
15.47 \\
15.85 \\
16.27\end{array}$ & $\begin{array}{l}-225.34 \\
-215.94 \\
-206.03 \\
-195.59 \\
-184.56 \\
-172.90 \\
-160.54 \\
-147.40 \\
-133.38 \\
-118.43\end{array}$ & $\begin{array}{l}-260.03 \\
-251.11 \\
-241.73 \\
-231.86 \\
-221.45 \\
-210.48 \\
-198.88 \\
-186.59 \\
-173.52 \\
-159.66\end{array}$ & $\begin{array}{r}7.851 \\
8.286 \\
8.728 \\
9.174 \\
9.623 \\
10.079 \\
10.545 \\
11.025 \\
11.514 \\
12.021\end{array}$ & $\begin{array}{r}91.00 \\
92.00 \\
93.00 \\
94.00 \\
95.00 \\
96.00 \\
97.00 \\
98.00 \\
99.00 \\
100.00\end{array}$ & $\begin{array}{l}146.56 \\
148.36 \\
150.14 \\
151.92 \\
153.70 \\
155.48 \\
157.25 \\
159.02 \\
160.78 \\
162.54\end{array}$ & $\begin{array}{r}932.02 \\
945.73 \\
959.49 \\
973.32 \\
987.21 \\
1001.16 \\
1015.17 \\
1029.24 \\
1043.38 \\
1057.58\end{array}$ & $\begin{array}{l}560.76 \\
569.93 \\
579.17 \\
588.48 \\
597.86 . \\
607.31 \\
616.84 \\
626.44 \\
636.10 \\
645.84\end{array}$ & $\begin{array}{l}32.885 \\
33.035 \\
33.184 \\
33.332 \\
33.479 \\
33.625 \\
33.770 \\
33.914 \\
34.058 \\
34.200\end{array}$ \\
\hline $\begin{array}{l}31.00 \\
32.00 \\
33.00 \\
34.00 \\
35.00 \\
36.00 \\
37.00 \\
38.00 \\
39.00 \\
40.00\end{array}$ & $\begin{array}{l}16.77 \\
17.35 \\
18.10 \\
18.96 \\
20.06 \\
21.56 \\
23.72 \\
26.85 \\
30.81 \\
34.90\end{array}$ & $\begin{array}{r}-102.26 \\
-84.69 \\
-66.00 \\
-44.41 \\
-19.83 \\
8.73 \\
42.83 \\
82.99 \\
124.84 \\
161.66\end{array}$ & $\begin{array}{r}-144.73 \\
-128.64 \\
-111.86 \\
-92.44 \\
-70.66 \\
-45.89 \\
-17.25 \\
14.97 \\
46.79 \\
73.26\end{array}$ & $\begin{array}{l}12.552 \\
13.112 \\
13.699 \\
14.345 \\
15.056 \\
15.858 \\
16.790 \\
17.860 \\
18.947 \\
19.880\end{array}$ & $\begin{array}{l}101.00 \\
102.00 \\
103.00 \\
104.00 \\
105.00 \\
106.00 \\
107.00 \\
108.00 \\
109.00 \\
110.00\end{array}$ & $\begin{array}{l}164.30 \\
166.04 \\
167.79 \\
169.53 \\
171.26 \\
173.00 \\
174.73 \\
176.46 \\
178.19 \\
179.92\end{array}$ & $\begin{array}{l}1071.82 \\
1086.11 \\
1100.47 \\
1114.90 \\
1129.39 \\
1143.94 \\
1158.56 \\
1173.25 \\
1188.00 \\
1202.81\end{array}$ & $\begin{array}{l}655.63 \\
665.49 \\
675.44 \\
685.45 \\
695.54 \\
705.70 \\
715.93 \\
726.23 \\
736.60 \\
747.04\end{array}$ & $\begin{array}{l}34.342 \\
34.483 \\
34.623 \\
34.762 \\
34.901 \\
35.039 \\
35.176 \\
35.313 \\
35.449 \\
35.584\end{array}$ \\
\hline $\begin{array}{l}41.00 \\
42.00 \\
43.00 \\
44.00 \\
45.00 \\
46.00 \\
47.00 \\
48.00 \\
49.00 \\
50.00\end{array}$ & $\begin{array}{l}38.72 \\
42.25 \\
45.54 \\
48.64 \\
51.58 \\
54.41 \\
57.13 \\
59.76 \\
62.32 \\
64.81\end{array}$ & $\begin{array}{l}192.73 \\
219.56 \\
243.45 \\
265.27 \\
285.55 \\
304.67 \\
322.86 \\
340.31 \\
357.16 \\
373.49\end{array}$ & $\begin{array}{r}94.65 \\
112.53 \\
128.10 \\
142.06 \\
154.88 \\
166.85 \\
178.15 \\
188.93 \\
199.30 \\
209.32\end{array}$ & $\begin{array}{l}20.647 \\
21.294 \\
21.856 \\
22.358 \\
22.814 \\
23.234 \\
23.625 \\
23.993 \\
24.340 \\
24.670\end{array}$ & $\begin{array}{l}111.00 \\
112.00 \\
113.00 \\
114.00 \\
115.00 \\
116.00 \\
117.00 \\
118.00 \\
119.00 \\
120.00\end{array}$ & $\begin{array}{l}181.64 \\
183.36 \\
185.09 \\
186.81 \\
188.53 \\
190.25 \\
191.96 \\
193.68 \\
195.40 \\
197.12\end{array}$ & $\begin{array}{l}1217.68 \\
1232.61 \\
1247.61 \\
1262.66 \\
1277.78 \\
1292.95 \\
1308.18 \\
1323.47 \\
1338.82 \\
1354.21\end{array}$ & $\begin{array}{l}757.55 \\
768.12 \\
778.75 \\
789.45 \\
800.20 \\
811.02 \\
821.90 \\
832.84 \\
843.83 \\
854.88\end{array}$ & $\begin{array}{l}35.719 \\
35.853 \\
35.986 \\
36.119 \\
36.251 \\
36.382 \\
36.513 \\
36.643 \\
36.772 \\
36.901\end{array}$ \\
\hline $\begin{array}{l}51.00 \\
52.00 \\
53.00 \\
54.00 \\
55.00 \\
56.00 \\
57.00 \\
58.00 \\
59.00 \\
60.00\end{array}$ & $\begin{array}{l}67.25 \\
69.63 \\
71.97 \\
74.27 \\
76.53 \\
78.76 \\
80.96 \\
83.13 \\
85.27 \\
87.39\end{array}$ & $\begin{array}{l}389.40 \\
404.93 \\
420.14 \\
435.07 \\
449.75 \\
464.22 \\
478.49 \\
492.59 \\
506.55 \\
520.36\end{array}$ & $\begin{array}{l}219.05 \\
228.54 \\
237.82 \\
246.93 \\
255.88 \\
264.70 \\
273.41 \\
282.02 \\
290.54 \\
298.99\end{array}$ & $\begin{array}{l}24.985 \\
25.287 \\
25.576 \\
25.855 \\
26.125 \\
26.386 \\
26.638 \\
26.883 \\
27.122 \\
27.354\end{array}$ & $\begin{array}{l}121.00 \\
122.00 \\
123.00 \\
124.00 \\
125.00 \\
126.00 \\
127.00 \\
128.00 \\
129.00 \\
130.00\end{array}$ & $\begin{array}{l}198.83 \\
200.55 \\
202.27 \\
203.98 \\
205.70 \\
207.40 \\
209.10 \\
210.80 \\
212.50 \\
214.19\end{array}$ & $\begin{array}{l}1369.66 \\
1385.15 \\
1400.70 \\
1416.30 \\
1431.95 \\
1447.65 \\
1463.41 \\
1479.21 \\
1495.06 \\
1510.95\end{array}$ & $\begin{array}{l}865.98 \\
877.12 \\
888.32 \\
899.57 \\
910.87 \\
922.27 \\
933.71 \\
945.21 \\
956.76 \\
968.35\end{array}$ & $\begin{array}{l}37.029 \\
37.157 \\
37.284 \\
37.410 \\
37.536 \\
37.661 \\
37.785 \\
37.909 \\
38.033 \\
38.155\end{array}$ \\
\hline $\begin{array}{l}61.00 \\
62.00 \\
63.00 \\
64.00 \\
65.00 \\
66.00 \\
67.00 \\
68.00 \\
69.00 \\
70.00\end{array}$ & $\begin{array}{r}89.49 \\
91.57 \\
93.62 \\
95.66 \\
97.68 \\
99.69 \\
101.68 \\
103.66 \\
105.62 \\
107.57\end{array}$ & $\begin{array}{l}534.06 \\
547.65 \\
561.15 \\
574.56 \\
587.91 \\
601.19 \\
614.42 \\
627.60 \\
640.75 \\
653.86\end{array}$ & $\begin{array}{l}307.37 \\
315.71 \\
324.00 \\
332.25 \\
340.47 \\
348.67 \\
356.85 \\
365.02 \\
373.20 \\
381.37\end{array}$ & $\begin{array}{l}27.581 \\
27.802 \\
28.018 \\
28.229 \\
28.436 \\
28.639 \\
28.837 \\
29.033 \\
29.225 \\
29.413\end{array}$ & $\begin{array}{l}131.00 \\
132.00 \\
133.00 \\
134.00 \\
135.00 \\
136.00 \\
137.00 \\
138.00 \\
139.00 \\
140.00\end{array}$ & $\begin{array}{l}215.89 \\
217.59 \\
219.28 \\
220.97 \\
222.66 \\
224.35 \\
226.04 \\
227.73 \\
229.42 \\
231.11\end{array}$ & $\begin{array}{l}1526.89 \\
1542.87 \\
1558.89 \\
1574.96 \\
1591.06 \\
1607.20 \\
1623.38 \\
1639.59 \\
1655.84 \\
1672.12\end{array}$ & $\begin{array}{r}980.00 \\
991.68 \\
1003.42 \\
1015.19 \\
1027.01 \\
1038.87 \\
1050.76 \\
1062.70 \\
1074.67 \\
1086.67\end{array}$ & $\begin{array}{l}38.278 \\
38.399 \\
38.520 \\
38.640 \\
38.760 \\
38.879 \\
38.998 \\
39.116 \\
39.233 \\
39.350\end{array}$ \\
\hline $\begin{array}{l}71.00 \\
72.00 \\
73.00 \\
74.00 \\
75.00 \\
76.00 \\
77.00 \\
78.00 \\
79.00 \\
80.00\end{array}$ & $\begin{array}{l}109.51 \\
111.44 \\
113.36 \\
115.27 \\
117.16 \\
119.05 \\
120.94 \\
122.81 \\
124.67 \\
126.53\end{array}$ & $\begin{array}{l}666.95 \\
680.03 \\
693.10 \\
706.16 \\
719.23 \\
732.30 \\
745.38 \\
758.48 \\
771.60 \\
784.74\end{array}$ & $\begin{array}{l}389.55 \\
397.74 \\
405.95 \\
414.18 \\
422.43 \\
430.71 \\
439.03 \\
447.39 \\
455.78 \\
464.22\end{array}$ & $\begin{array}{l}29.599 \\
29.782 \\
29.962 \\
30.140 \\
30.315 \\
30.488 \\
30.659 \\
30.828 \\
30.996 \\
31.161\end{array}$ & $\begin{array}{l}141.00 \\
142.00 \\
143.00 \\
144.00 \\
145.00 \\
146.00 \\
147.00 \\
148.00 \\
149.00 \\
150.00\end{array}$ & $\begin{array}{l}232.80 \\
234.49 \\
236.17 \\
237.86 \\
239.54 \\
241.23 \\
242.91 \\
244.60 \\
246.28 \\
247.96\end{array}$ & $\begin{array}{l}1688.44 \\
1704.78 \\
1721.15 \\
1737.55 \\
1753.97 \\
1770.42 \\
1786.89 \\
1803.39 \\
1819.90 \\
1836.43\end{array}$ & $\begin{array}{l}1098.71 \\
1110.78 \\
1122.88 \\
1135.01 \\
1147.16 \\
1159.35 \\
1171.55 \\
1183.78 \\
1196.03 \\
1208.30\end{array}$ & $\begin{array}{l}39.466 \\
39.581 \\
39.696 \\
39.810 \\
39.924 \\
40.037 \\
40.150 \\
40.261 \\
40.373 \\
40.483\end{array}$ \\
\hline $\begin{array}{l}81.00 \\
82.00 \\
83.00 \\
84.00 \\
85.00 \\
86.00 \\
87.00 \\
88.00 \\
89.00 \\
90.00\end{array}$ & $\begin{array}{l}128.38 \\
130.23 \\
132.06 \\
133.90 \\
135.72 \\
137.54 \\
139.36 \\
141.17 \\
142.97 \\
144.77\end{array}$ & $\begin{array}{l}797.92 \\
811.12 \\
824.37 \\
837.65 \\
850.98 \\
864.36 \\
877.79 \\
891.26 \\
904.79 \\
918.38\end{array}$ & $\begin{array}{l}472.71 \\
481.24 \\
489.84 \\
498.48 \\
507.19 \\
515.95 \\
524.78 \\
533.67 \\
542.64 \\
551.66\end{array}$ & $\begin{array}{l}31.325 \\
31.487 \\
31.647 \\
31.806 \\
31.964 \\
32.121 \\
32.276 \\
32.430 \\
32.583 \\
32.734\end{array}$ & $\begin{array}{l}151.00 \\
152.00 \\
153.00 \\
154.00 \\
155.00 \\
156.00 \\
157.00 \\
158.00 \\
159.00 \\
160.00\end{array}$ & $\begin{array}{l}249.64 \\
251.32 \\
252.99 \\
254.67 \\
256.34 \\
258.02 \\
259.69 \\
261.37 \\
263.04 \\
264.71\end{array}$ & $\begin{array}{l}1852.92 \\
1869.43 \\
1885.94 \\
1902.47 \\
1919.01 \\
1935.56 \\
1952.12 \\
1968.68 \\
1985.26 \\
2001.83\end{array}$ & $\begin{array}{l}1220.54 \\
1232.80 \\
1245.07 \\
1257.35 \\
1269.65 \\
1281.95 \\
1294.27 \\
1306.59 \\
1318.93 \\
1331.27\end{array}$ & $\begin{array}{l}40.593 \\
40.702 \\
40.810 \\
40.918 \\
41.025 \\
41.131 \\
41.237 \\
41.342 \\
41.447 \\
41.551\end{array}$ \\
\hline
\end{tabular}


TEMPER- SPECIFIC ENTHALPY INTERNAL ENTROPY ATURE VOLUME (J/GM) ENERGY (J/GM-K) (K) ( $C$ C $/ G M$ ) $(J / G M)$ ATURE

(K)
SPECIFIC

(CC/GM)
$(J / G M)$

(1)

INTERNAL ENTROPY ENERGY

\begin{tabular}{|c|c|c|c|c|c|c|c|c|c|}
\hline $\begin{array}{l}161.00 \\
162.00 \\
163.00 \\
164.00 \\
165.00 \\
166.00 \\
167.00 \\
168.00 \\
169.00 \\
170.00\end{array}$ & $\begin{array}{l}266.38 \\
268.05 \\
269.73 \\
271.40 \\
273.07 \\
274.74 \\
276.41 \\
278.08 \\
279.74 \\
281.41\end{array}$ & $\begin{array}{l}2018.42 \\
2035.00 \\
2051.60 \\
2068.19 \\
2084.78 \\
2101.38 \\
2117.97 \\
2134.57 \\
2151.16 \\
2167.76\end{array}$ & $\begin{array}{l}1343.62 \\
1355.97 \\
1368.33 \\
1380.69 \\
1393.05 \\
1405.42 \\
1417.78 \\
1430.15 \\
1442.52 \\
1454.88\end{array}$ & $\begin{array}{l}41.654 \\
41.757 \\
41.859 \\
41.960 \\
42.061 \\
42.161 \\
42.261 \\
42.360 \\
42.459 \\
42.557\end{array}$ & $\begin{array}{l}231.00 \\
232.00 \\
233.00 \\
234.00 \\
235.00 \\
236.00 \\
237.00 \\
238.00 \\
239.00 \\
240.00\end{array}$ & $\begin{array}{l}382.39 \\
384.03 \\
385.68 \\
387.33 \\
388.97 \\
390.62 \\
392.26 \\
393.91 \\
395.56 \\
397.20\end{array}$ & $\begin{array}{l}3157.05 \\
3172.75 \\
3188.44 \\
3204.11 \\
3219.76 \\
3235.40 \\
3251.02 \\
3266.62 \\
3282.21 \\
3297.78\end{array}$ & $\begin{array}{l}2188.39 \\
2199.92 \\
2211.44 \\
2222.94 \\
2234.42 \\
2245.89 \\
2257.34 \\
2268.77 \\
2280.19 \\
2291.60\end{array}$ & $\begin{array}{l}47.537 \\
47.604 \\
47.672 \\
47.739 \\
47.806 \\
47.872 \\
47.938 \\
48.004 \\
48.069 \\
48.134\end{array}$ \\
\hline $\begin{array}{l}171.00 \\
172.00 \\
173.00 \\
174.00 \\
175.00 \\
176.00 \\
177.00 \\
178.00 \\
179.00 \\
180.00\end{array}$ & $\begin{array}{l}283.08 \\
284.74 \\
286.41 \\
288.07 \\
289.74 \\
291.40 \\
293.06 \\
294.73 \\
296.39 \\
298.05\end{array}$ & $\begin{array}{l}2184.35 \\
2200.94 \\
2217.52 \\
2234.10 \\
2250.67 \\
2267.24 \\
2283.80 \\
2300.36 \\
2316.90 \\
2333.44\end{array}$ & $\begin{array}{l}1467.25 \\
1479.62 \\
1491.99 \\
1504.35 \\
1516.71 \\
1529.07 \\
1541.41 \\
1553.76 \\
1566.09 \\
1578.42\end{array}$ & $\begin{array}{l}42.654 \\
42.751 \\
42.847 \\
42.942 \\
43.037 \\
43.132 \\
43.226 \\
43.319 \\
43.411 \\
43.504\end{array}$ & $\begin{array}{l}241.00 \\
242.00 \\
243.00 \\
244.00 \\
245.00 \\
246.00 \\
247.00 \\
248.00 \\
249.00 \\
250.00\end{array}$ & $\begin{array}{l}398.84 \\
400.49 \\
402.13 \\
403.78 \\
405.42 \\
407.06 \\
408.71 \\
410.35 \\
411.99 \\
413.63\end{array}$ & $\begin{array}{l}3313.34 \\
3328.88 \\
3344.40 \\
3359.91 \\
3375.40 \\
3390.88 \\
3406.34 \\
3421.78 \\
3437.21 \\
3452.63\end{array}$ & $\begin{array}{l}2302.99 \\
2314.36 \\
2325.72 \\
2337.06 \\
2348.39 \\
2359.70 \\
2371.00 \\
2382.29 \\
2393.56 \\
2404.81\end{array}$ & $\begin{array}{l}48.199 \\
48.263 \\
48.327 \\
48.391 \\
48.454 \\
48.517 \\
48.580 \\
48.643 \\
48.705 \\
48.766\end{array}$ \\
\hline $\begin{array}{l}181.00 \\
182.00 \\
183.00 \\
184.00 \\
185.00 \\
186.00 \\
187.00 \\
188.00 \\
189.00 \\
190.00\end{array}$ & $\begin{array}{l}299.71 \\
301.37 \\
303.03 \\
304.69 \\
306.35 \\
308.01 \\
309.67 \\
311.33 \\
312.99 \\
314.65\end{array}$ & $\begin{array}{l}2349.97 \\
2366.49 \\
2383.00 \\
2399.50 \\
2415.98 \\
2432.46 \\
2448.92 \\
2465.37 \\
2481.81 \\
2498.24\end{array}$ & $\begin{array}{l}1590.74 \\
1603.05 \\
1615.35 \\
1627.65 \\
1639.93 \\
1652.20 \\
1664.46 \\
1676.71 \\
1688.95 \\
1701.17\end{array}$ & $\begin{array}{l}43.595 \\
43.686 \\
43.777 \\
43.867 \\
43.956 \\
44.045 \\
44.133 \\
44.221 \\
44.308 \\
44.395\end{array}$ & $\begin{array}{l}251.00 \\
252.00 \\
253.00 \\
254.00 \\
255.00 \\
256.00 \\
257.00 \\
258.00 \\
259.00 \\
260.00\end{array}$ & $\begin{array}{l}415.28 \\
416.92 \\
418.56 \\
420.20 \\
421.84 \\
423.48 \\
425.12 \\
426.76 \\
428.40 \\
430.04\end{array}$ & $\begin{array}{l}3468.07 \\
3483.49 \\
3498.89 \\
3514.28 \\
3529.66 \\
3545.02 \\
3560.36 \\
3575.70 \\
3591.01 \\
3606.31\end{array}$ & $\begin{array}{l}2416.09 \\
2427.35 \\
2438.60 \\
2449.83 \\
2461.05 \\
2472.26 \\
2483.45 \\
2494.62 \\
2505.78 \\
2516.93\end{array}$ & $\begin{array}{l}48.828 \\
48.889 \\
48.950 \\
49.011 \\
49.071 \\
49.132 \\
49.191 \\
49.251 \\
49.310 \\
49.369\end{array}$ \\
\hline $\begin{array}{l}191.00 \\
192.00 \\
193.00 \\
194.00 \\
195.00 \\
196.00 \\
197.00 \\
198.00 \\
199.00 \\
200.00\end{array}$ & $\begin{array}{l}316.31 \\
317.96 \\
319.62 \\
321.28 \\
322.93 \\
324.59 \\
326.25 \\
327.90 \\
329.56 \\
331.21\end{array}$ & $\begin{array}{l}2514.65 \\
2531.04 \\
2547.42 \\
2563.79 \\
2580.14 \\
2596.48 \\
2612.80 \\
2629.10 \\
2645.39 \\
2661.66\end{array}$ & $\begin{array}{l}1713.38 \\
1725.58 \\
1737.76 \\
1749.93 \\
1762.09 \\
1774.23 \\
1786.35 \\
1798.46 \\
1810.55 \\
1822.63\end{array}$ & $\begin{array}{l}44.481 \\
44.566 \\
44.651 \\
44.736 \\
44.820 \\
44.904 \\
44.987 \\
45.069 \\
45.151 \\
45.233\end{array}$ & $\begin{array}{l}261.00 \\
262.00 \\
263.00 \\
264.00 \\
265.00 \\
266.00 \\
267.00 \\
268.00 \\
269.00 \\
270.00\end{array}$ & $\begin{array}{l}431.68 \\
433.32 \\
434.96 \\
436.60 \\
438.24 \\
439.88 \\
441.52 \\
443.16 \\
444.79 \\
446.43\end{array}$ & $\begin{array}{l}3621.60 \\
3636.88 \\
3652.14 \\
3667.38 \\
3682.61 \\
3697.83 \\
3713.04 \\
3728.23 \\
3743.41 \\
3758.57\end{array}$ & $\begin{array}{l}2528.07 \\
2539.19 \\
2550.29 \\
2561.39 \\
2572.47 \\
2583.53 \\
2594.59 \\
2605.63 \\
2616.66 \\
2627.67\end{array}$ & $\begin{array}{l}49.428 \\
49.486 \\
49.544 \\
49.602 \\
49.660 \\
49.717 \\
49.774 \\
49.831 \\
49.888 \\
49.944\end{array}$ \\
\hline $\begin{array}{l}201.00 \\
202.00 \\
203.00 \\
204.00 \\
205.00 \\
206.00 \\
207.00 \\
208.00 \\
209.00 \\
210.00\end{array}$ & $\begin{array}{l}332.87 \\
334.52 \\
336.18 \\
337.83 \\
339.48 \\
341.14 \\
342.79 \\
344.44 \\
346.09 \\
347.75\end{array}$ & $\begin{array}{l}2677.90 \\
2694.13 \\
2710.34 \\
2726.53 \\
2742.70 \\
2758.86 \\
2775.00 \\
2791.12 \\
2807.23 \\
2823.31\end{array}$ & $\begin{array}{l}1834.68 \\
1846.72 \\
1858.74 \\
1870.74 \\
1882.73 \\
1894.70 \\
1906.65 \\
1918.59 \\
1930.51 \\
1942.41\end{array}$ & $\begin{array}{l}45.314 \\
45.394 \\
45.474 \\
45.554 \\
45.633 \\
45.712 \\
45.790 \\
45.868 \\
45.945 \\
46.022\end{array}$ & $\begin{array}{l}271.00 \\
272.00 \\
273.00 \\
274.00 \\
275.00 \\
276.00 \\
277.00 \\
278.00 \\
279.00 \\
280.00\end{array}$ & $\begin{array}{l}448.07 \\
449.71 \\
451.35 \\
452.99 \\
454.63 \\
456.26 \\
457.90 \\
459.54 \\
461.18 \\
462.82\end{array}$ & $\begin{array}{l}3773.73 \\
3788.87 \\
3804.00 \\
3819.12 \\
3834.22 \\
3849.32 \\
3864.41 \\
3879.48 \\
3894.55 \\
3909.61\end{array}$ & $\begin{array}{l}2638.67 \\
2649.67 \\
2660.64 \\
2671.61 \\
2682.57 \\
2693.51 \\
2704.45 \\
2715.37 \\
2726.29 \\
2737.20\end{array}$ & $\begin{array}{l}50.000 \\
50.056 \\
50.111 \\
50.166 \\
50.221 \\
50.276 \\
50.331 \\
50.385 \\
50.439 \\
50.493\end{array}$ \\
\hline $\begin{array}{l}211.00 \\
212.00 \\
213.00 \\
214.00 \\
215.00 \\
216.00 \\
217.00 \\
218.00 \\
219.00 \\
220.00\end{array}$ & $\begin{array}{l}349.40 \\
351.05 \\
352.70 \\
354.35 \\
356.00 \\
357.65 \\
359.30 \\
360.95 \\
362.60 \\
364.25\end{array}$ & $\begin{array}{l}2839.38 \\
2855.43 \\
2871.46 \\
2887.48 \\
2903.48 \\
2919.46 \\
2935.42 \\
2951.36 \\
2967.29 \\
2983.20\end{array}$ & $\begin{array}{l}1954.29 \\
1966.16 \\
1978.01 \\
1989.84 \\
2001.66 \\
2013.45 \\
2025.23 \\
2037.00 \\
2048.74 \\
2060.47\end{array}$ & $\begin{array}{l}46.098 \\
46.174 \\
46.249 \\
46.324 \\
46.399 \\
46.473 \\
46.547 \\
46.620 \\
46.693 \\
46.765\end{array}$ & $\begin{array}{l}281.00 \\
282.00 \\
283.00 \\
284.00 \\
285.00 \\
286.00 \\
287.00 \\
288.00 \\
289.00 \\
290.00\end{array}$ & $\begin{array}{l}464.46 \\
466.10 \\
467.74 \\
469.38 \\
471.02 \\
472.66 \\
474.30 \\
475.94 \\
477.58 \\
479.22\end{array}$ & $\begin{array}{l}3924.66 \\
3939.70 \\
3954.73 \\
3969.76 \\
3984.77 \\
3999.79 \\
4014.79 \\
4029.79 \\
4044.79 \\
4059.78\end{array}$ & $\begin{array}{l}2748.09 \\
2758.98 \\
2769.86 \\
2780.73 \\
2791.60 \\
2802.45 \\
2813.31 \\
2824.15 \\
2834.99 \\
2845.82\end{array}$ & $\begin{array}{l}50.547 \\
50.600 \\
50.653 \\
50.706 \\
50.759 \\
50.812 \\
50.864 \\
50.916 \\
50.968 \\
51.020\end{array}$ \\
\hline $\begin{array}{l}221.00 \\
222.00 \\
223.00 \\
224.00 \\
225.00 \\
226.00 \\
227.00 \\
228.00 \\
229.00 \\
230.00\end{array}$ & $\begin{array}{l}365.90 \\
367.55 \\
369.20 \\
370.85 \\
372.50 \\
374.15 \\
375.80 \\
377.44 \\
379.09 \\
380.74\end{array}$ & $\begin{array}{l}2999.09 \\
3014.96 \\
3030.82 \\
3046.66 \\
3062.48 \\
3078.28 \\
3094.07 \\
3109.84 \\
3125.59 \\
3141.33\end{array}$ & $\begin{array}{l}2072.19 \\
2083.88 \\
2095.56 \\
2107.22 \\
2118.87 \\
2130.50 \\
2142.11 \\
2153.70 \\
2165.28 \\
2176.85\end{array}$ & $\begin{array}{l}46.838 \\
46.909 \\
46.980 \\
47.051 \\
47.122 \\
47.192 \\
47.262 \\
47.331 \\
47.400 \\
47.468\end{array}$ & $\begin{array}{l}291.00 \\
292.00 \\
293.00 \\
294.00 \\
295.00 \\
296.00 \\
297.00 \\
298.00 \\
299.00 \\
300.00\end{array}$ & $\begin{array}{l}480.86 \\
482.50 \\
484.14 \\
485.79 \\
487.43 \\
489.07 \\
490.71 \\
492.36 \\
494.00 \\
495.64\end{array}$ & $\begin{array}{l}4074.77 \\
4089.75 \\
4104.73 \\
4119.70 \\
4134.68 \\
4149.65 \\
4164.61 \\
4179.58 \\
4194.54 \\
4209.49\end{array}$ & $\begin{array}{l}2856.65 \\
2867.48 \\
2878.30 \\
2889.11 \\
2899.93 \\
2910.74 \\
2921.54 \\
2932.34 \\
2943.14 \\
2953.94\end{array}$ & $\begin{array}{l}51.072 \\
51.123 \\
51.174 \\
51.225 \\
51.276 \\
51.327 \\
51.377 \\
51.428 \\
51.478 \\
51.528\end{array}$ \\
\hline
\end{tabular}


SPECIFIC ENTHALPY

VOLUME

(J/GM)

INTERNAL ENTROPY

ENERGY (J/GM-K)

(J/GM) ATURE

(K)

SPECIFIC

ENTHALPY

INTERNAL ENTROPY

(1)

20.00

13.43

$-229.11$

$-269.92$

7.329

21.00

13.59

$-220.32$

$-261.64$

13.77

22.00
23.00

24.00

25.00

26.00

27.00

28.00

29.00

30.00

31.00
32.00

33.00

34.00

35.00

36.00

37.00

38.00

39.00

40.00

41.00

42.00

43.00

44.00

45.00

46.00

47.00

48.00

49.00

50.00

51.00

53.00

54.00

55.00

56.00

57.00

58.00

59.00

60.00

61.00

62.00

63.00

64.00

65.00

66.00

67.00

68.00

69.00

70.00

71.00

73.00

74.00

75.00

76.00

77.00

78.00

79.00

80.00

14.18

14.41

14.66

14.93

15.57

15.94

$-201.36$

$-191.14$

$-180.37$

$-169.02$

$-157.03$

$-144.36$

$-130.93$

16.37

$-116.73$

16.85

$-101.54$

17.47

18.12

18. 90

19.86

21.05

22.58

4.51

29.53

32.30

35.05

40.28

42.76

45.15

47.46

49.71

51.90

54.03

56.12

58.17

60.17

62.14

64.08

65.99

67.87

69.73

71.57

73.38

75.17

76.94

78.70

80.44

82.16

83.87

85.57

87.25

88.92

90.58

92.23

93.87

95.50

97.12

98.73

100.33

101.93

103.51

81.00

106.67

82.00

83.00

84.00

85.00

86.00

87.00

88.00

89.00

90.00

106.67

109.79

111.35

112.90

114.44

115.98

117.51

119.04

120.56
$-211.08$

$-252.95$

$-243.82$

$-234.24$

$-224.17$

$-213.58$

$-202.43$

$-190.68$

$-178.26$

$-165.20$

$-151.29$

$-136.51$

$-68.29$

$-49.22$

$-28.35$

$-5.50$

19.69

47.56

78.32

142.11

171.79

198.99

223.91

246.95

268.47

288.77

308.08

326.58

344.40

361.64

378.40

394.73

410.70

426.34

441.70

456.80

471.68

486.37

500.88

515.22

529.43

543.51

557.48

571.35

585.14

598.84

612.48

626.07

639.60

653.10

666.56

680.00

693.42

706.82

720.23

733.63

747.04

760.45

773.88

787.34 800.81

814.32

827.85

841.42

855.03

868.69

882.38

896.13

909.93

28.74

52.33

73.60
92.45

109.28

124.51

138.50

151.54

163.81

175.48

186.64

197.40

207.81

217.92

227.79

237.43

246.90

256.20

265. 36

274.40

283.33

292.17

300.93

309.62

318.26

326.84

335.39

343.90

352.38

360.85

369.30

377.75

386.20

394.66

403.13

411.61

420.12

428.65

437.20

445.80

454.42

463.09

471.80

480.57
7.761

8.188

8.622

9.059

9.497

9.941

10.393

10.855

11.324

11.806

12.304

12.822

$\begin{array}{ll}-121.39 & 13.357\end{array}$

$-104.30 \quad 13.928$

$\begin{array}{ll}-85.81 & 14.532\end{array}$

15.173

$\begin{array}{ll}-44.31 & 15.860\end{array}$

$-21.07 \quad 16.603$

$3.80 \quad 17.402$ 
TEMPER- SPECIFIC ENTHALPY INTERNAL ENTROPY ATURE VOLUME (K) (CC/GM) (J/GM)
TEMPERATURE (K) VOLUME (CC/GM) (J)
INTERNAL ENTROPY

ENERGY (J/GM)

\begin{tabular}{|c|c|c|c|c|c|c|c|c|c|}
\hline $\begin{array}{l}161.00 \\
162.00 \\
163.00 \\
164.00 \\
165.00 \\
166.00 \\
167.00 \\
168.00 \\
169.00 \\
170.00\end{array}$ & $\begin{array}{l}222.77 \\
224.17 \\
225.57 \\
226.96 \\
228.36 \\
229.76 \\
231.16 \\
232.55 \\
233.95 \\
235.35\end{array}$ & $\begin{array}{l}2016.82 \\
2033.45 \\
2050.09 \\
2066.73 \\
2083.38 \\
2100.02 \\
2116.66 \\
2133.30 \\
2149.94 \\
2166.58\end{array}$ & $\begin{array}{l}1339.64 \\
1352.03 \\
1364.41 \\
1376.80 \\
1389.20 \\
1401.59 \\
1413.98 \\
1426.38 \\
1438.77 \\
1451.17\end{array}$ & $\begin{array}{l}40.878 \\
40.981 \\
41.083 \\
41.185 \\
41.286 \\
41.387 \\
41.487 \\
41.586 \\
41.685 \\
41.783\end{array}$ & $\begin{array}{l}231.00 \\
232.00 \\
233.00 \\
234.00 \\
235.00 \\
236.00 \\
237.00 \\
238.00 \\
239.00 \\
240.00\end{array}$ & $\begin{array}{l}319.73 \\
321.11 \\
322.48 \\
323.86 \\
325.23 \\
326.61 \\
327.98 \\
329.35 \\
330.73 \\
332.10\end{array}$ & $\begin{array}{l}3157.85 \\
3173.58 \\
3189.29 \\
3204.98 \\
3220.66 \\
3236.32 \\
3251.96 \\
3267.59 \\
3283.20 \\
3298.80\end{array}$ & $\begin{array}{l}2185.92 \\
2197.47 \\
2209.00 \\
2220.51 \\
2232.01 \\
2243.49 \\
2254.96 \\
2266.41 \\
2277.85 \\
2289.27\end{array}$ & $\begin{array}{l}46.773 \\
46.841 \\
46.909 \\
46.976 \\
47.043 \\
47.109 \\
47.175 \\
47.241 \\
47.307 \\
47.372\end{array}$ \\
\hline $\begin{array}{l}171.00 \\
172.00 \\
173.00 \\
174.00 \\
175.00 \\
176.00 \\
177.00 \\
178.00 \\
179.00 \\
180.00\end{array}$ & $\begin{array}{l}236.74 \\
238.13 \\
239.53 \\
240.92 \\
242.31 \\
243.70 \\
245.09 \\
246.48 \\
247.87 \\
249.26\end{array}$ & $\begin{array}{l}2183.21 \\
2199.84 \\
2216.47 \\
2233.09 \\
2249.71 \\
2266.32 \\
2282.92 \\
2299.51 \\
2316.10 \\
2332.68\end{array}$ & $\begin{array}{l}1463.56 \\
1475.96 \\
1488.35 \\
1500.74 \\
1513.13 \\
1525.51 \\
1537.88 \\
1550.25 \\
1562.61 \\
1574.96\end{array}$ & $\begin{array}{l}41.881 \\
41.978 \\
42.074 \\
42.170 \\
42.265 \\
42.360 \\
42.454 \\
42.547 \\
42.640 \\
42.732\end{array}$ & $\begin{array}{l}241.00 \\
242.00 \\
243.00 \\
244.00 \\
245.00 \\
246.00 \\
247.00 \\
248.00 \\
249.00 \\
250.00\end{array}$ & $\begin{array}{l}333.47 \\
334.85 \\
336.22 \\
337.59 \\
338.96 \\
340.33 \\
341.71 \\
343.08 \\
344.45 \\
345.82\end{array}$ & $\begin{array}{l}3314.38 \\
3329.94 \\
3345.49 \\
3361.02 \\
3376.53 \\
3392.03 \\
3407.52 \\
3422.98 \\
3438.44 \\
3453.87\end{array}$ & $\begin{array}{l}2300.68 \\
2312.07 \\
2323.44 \\
2334.80 \\
2346.14 \\
2357.47 \\
2368.79 \\
2380.09 \\
2391.37 \\
2402.64\end{array}$ & $\begin{array}{l}47.437 \\
47.501 \\
47.565 \\
47.629 \\
47.692 \\
47.755 \\
47.818 \\
47.881 \\
47.943 \\
48.005\end{array}$ \\
\hline $\begin{array}{l}181.00 \\
182.00 \\
183.00 \\
184.00 \\
185.00 \\
186.00 \\
187.00 \\
188.00 \\
189.00 \\
190.00\end{array}$ & $\begin{array}{l}250.65 \\
252.04 \\
253.43 \\
254.82 \\
256.21 \\
257.59 \\
258.98 \\
260.37 \\
261.75 \\
263.14\end{array}$ & $\begin{array}{l}2349.24 \\
2365.80 \\
2382.35 \\
2398.88 \\
2415.41 \\
2431.92 \\
2448.42 \\
2464.90 \\
2481.38 \\
2497.84\end{array}$ & $\begin{array}{l}1587.30 \\
1599.64 \\
1611.97 \\
1624.28 \\
1636.59 \\
1648.88 \\
1661.16 \\
1673.43 \\
1685.69 \\
1697.94\end{array}$ & $\begin{array}{l}42.824 \\
42.915 \\
43.006 \\
43.096 \\
43.186 \\
43.275 \\
43.363 \\
43.451 \\
43.539 \\
43.625\end{array}$ & $\begin{array}{l}251.00 \\
252.00 \\
253.00 \\
254.00 \\
255.00 \\
256.00 \\
257.00 \\
258.00 \\
259.00 \\
260.00\end{array}$ & $\begin{array}{l}347.19 \\
348.56 \\
349.93 \\
351.30 \\
352.67 \\
354.04 \\
355.41 \\
356.78 \\
358.15 \\
359.52\end{array}$ & $\begin{array}{l}3469.33 \\
3484.77 \\
3500 \cdot 20 \\
3515.61 \\
3531.00 \\
3546.38 \\
3561.75 \\
3577.10 \\
3592.44 \\
3607.76\end{array}$ & $\begin{array}{l}2413.93 \\
2425.21 \\
2436.47 \\
2447.72 \\
2458.95 \\
2470.17 \\
2481.37 \\
2492.56 \\
2503.73 \\
2514.89\end{array}$ & $\begin{array}{l}48.067 \\
48.128 \\
48.189 \\
48.250 \\
48.310 \\
48.370 \\
48.430 \\
48.490 \\
48.549 \\
48.608\end{array}$ \\
\hline $\begin{array}{l}191.00 \\
192.00 \\
193.00 \\
194.00 \\
195.00 \\
196.00 \\
197.00 \\
198.00 \\
199.00 \\
200.00\end{array}$ & $\begin{array}{l}264.53 \\
265.91 \\
267.30 \\
268.68 \\
270.07 \\
271.45 \\
272.83 \\
274.22 \\
275.60 \\
276.98\end{array}$ & $\begin{array}{l}2514.28 \\
2530.71 \\
2547.12 \\
2563.52 \\
2579.91 \\
2596.28 \\
2612.63 \\
2628.96 \\
2645.28 \\
2661.58\end{array}$ & $\begin{array}{l}1710.17 \\
1722.39 \\
1734.59 \\
1746.78 \\
1758.95 \\
1771.11 \\
1783.26 \\
1795.39 \\
1807.50 \\
1819.60\end{array}$ & $\begin{array}{l}43.712 \\
43.798 \\
43.883 \\
43.968 \\
44.052 \\
44.135 \\
44.219 \\
44.301 \\
44.384 \\
44.465\end{array}$ & $\begin{array}{l}261.00 \\
262.00 \\
263.00 \\
264.00 \\
265.00 \\
266.00 \\
267.00 \\
268.00 \\
269.00 \\
270.00\end{array}$ & $\begin{array}{l}360.88 \\
362.25 \\
363.62 \\
364.99 \\
366.36 \\
367.72 \\
369.09 \\
370.46 \\
371.83 \\
373.20\end{array}$ & $\begin{array}{l}3623.06 \\
3638.36 \\
3653.63 \\
3668.90 \\
3684.15 \\
3699.38 \\
3714.60 \\
3729.81 \\
3745.01 \\
3760.19\end{array}$ & $\begin{array}{l}2526.04 \\
2537.17 \\
2548.29 \\
2559.39 \\
2570.48 \\
2581.56 \\
2592.63 \\
2603.68 \\
2614.71 \\
2625.74\end{array}$ & $\begin{array}{l}48.667 \\
48.726 \\
48.784 \\
48.842 \\
48.899 \\
48.957 \\
49.014 \\
49.071 \\
49.127 \\
49.184\end{array}$ \\
\hline $\begin{array}{l}201.00 \\
202.00 \\
203.00 \\
204.00 \\
205.00 \\
206.00 \\
207.00 \\
208.00 \\
209.00 \\
210.00\end{array}$ & $\begin{array}{l}278.37 \\
279.75 \\
281.13 \\
282.51 \\
283.89 \\
285.28 \\
286.66 \\
288.04 \\
289.42 \\
290.80\end{array}$ & $\begin{array}{l}2677.86 \\
2694 \cdot 12 \\
2710.36 \\
2726.58 \\
2742.78 \\
2758.97 \\
2775.14 \\
2791.29 \\
2807.42 \\
2823.54\end{array}$ & $\begin{array}{l}1831.67 \\
1843.72 \\
1855.76 \\
1867.79 \\
1879.79 \\
1891.78 \\
1903.75 \\
1915.71 \\
1927.64 \\
1939.56\end{array}$ & $\begin{array}{l}44.547 \\
44.627 \\
44.707 \\
44.787 \\
44.866 \\
44.945 \\
45.023 \\
45.101 \\
45.179 \\
45.256\end{array}$ & $\begin{array}{l}271.00 \\
272.00 \\
273.00 \\
274.00 \\
275.00 \\
276.00 \\
277.00 \\
278.00 \\
279.00 \\
280.00\end{array}$ & $\begin{array}{l}374.56 \\
375.93 \\
377.30 \\
378.67 \\
380.03 \\
381.40 \\
382.77 \\
384.14 \\
385.51 \\
386.87\end{array}$ & $\begin{array}{l}3775.36 \\
3790.52 \\
3805.66 \\
3820.80 \\
3835.92 \\
3851.03 \\
3866.13 \\
3881.22 \\
3896.31 \\
3911.38\end{array}$ & $\begin{array}{l}2636.75 \\
2647.75 \\
2658.74 \\
2669.71 \\
2680.68 \\
2691.63 \\
2702.58 \\
2713.51 \\
2724.43 \\
2735.34\end{array}$ & $\begin{array}{l}49.240 \\
49.296 \\
49.351 \\
49.406 \\
49.462 \\
49.516 \\
49.571 \\
49.625 \\
49.680 \\
49.734\end{array}$ \\
\hline $\begin{array}{l}211.00 \\
212.00 \\
213.00 \\
214.00 \\
215.00 \\
216.00 \\
217.00 \\
218.00 \\
219.00 \\
220.00\end{array}$ & $\begin{array}{l}292.18 \\
293.56 \\
294.94 \\
296.32 \\
297.70 \\
299.07 \\
300.45 \\
301.83 \\
303.21 \\
304.59\end{array}$ & $\begin{array}{l}2839.64 \\
2855.72 \\
2871.78 \\
2887.82 \\
2903.85 \\
2919.86 \\
2935.84 \\
2951.82 \\
2967.77 \\
2983.71\end{array}$ & $\begin{array}{l}1951.47 \\
1963.35 \\
1975.22 \\
1987.07 \\
1998.90 \\
2010.72 \\
2022.52 \\
2034.30 \\
2046.06 \\
2057.81\end{array}$ & $\begin{array}{r}45.332 \\
45.408 \\
-45.484 \\
45.559 \\
45.634 \\
45.708 \\
45.782 \\
45.855 \\
45.928 \\
46.001\end{array}$ & $\begin{array}{l}281.00 \\
282.00 \\
283.00 \\
284.00 \\
285.00 \\
286.00 \\
287.00 \\
288.00 \\
289.00 \\
290.00\end{array}$ & $\begin{array}{l}388.24 \\
389.61 \\
390.98 \\
392.35 \\
393.72 \\
395.08 \\
396.45 \\
397.82 \\
399.19 \\
400.56\end{array}$ & $\begin{array}{l}3926.44 \\
3941.49 \\
3956.54 \\
3971.58 \\
3986.61 \\
4001.63 \\
4016.65 \\
4031.66 \\
4046.67 \\
4061.67\end{array}$ & $\begin{array}{l}2746.25 \\
2757.14 \\
2768.03 \\
2778.91 \\
2789.78 \\
2800.64 \\
2811.50 \\
2822.35 \\
2833.20 \\
2844.04\end{array}$ & $\begin{array}{l}49.787 \\
49.841 \\
49.89 \\
49.94 \\
50.001 \\
50.052 \\
50.10 \\
50.15 \\
50.20 \\
50.26\end{array}$ \\
\hline $\begin{array}{l}221.00 \\
222.00 \\
223.00 \\
224.00 \\
225.00 \\
226.00 \\
227.00 \\
228.00 \\
229.00 \\
230.00\end{array}$ & $\begin{array}{l}305 \cdot 97 \\
307 \cdot 34 \\
308 \cdot 72 \\
310.10 \\
311 \cdot 47 \\
312.85 \\
314.23 \\
315.60 \\
316 \cdot 98 \\
318.36\end{array}$ & $\begin{array}{l}2999.63 \\
3015.53 \\
3031.41 \\
3047.27 \\
3063.12 \\
3078.95 \\
3094.77 \\
3110.56 \\
3126.34 \\
3142.10\end{array}$ & $\begin{array}{l}2069.54 \\
2081.25 \\
2092.95 \\
2104.63 \\
2116.29 \\
2127.94 \\
2139.57 \\
2151.18 \\
2162.78 \\
2174.36\end{array}$ & $\begin{array}{l}46.073 \\
46.145 \\
46.216 \\
46.287 \\
46.358 \\
46.428 \\
46.498 \\
46.567 \\
46.636 \\
46.705\end{array}$ & $\begin{array}{l}291.00 \\
292.00 \\
293.00 \\
294.00 \\
295.00 \\
296.00 \\
297.00 \\
298.00 \\
299.00 \\
300.00\end{array}$ & $\begin{array}{l}401.93 \\
403.30 \\
404.67 \\
406.03 \\
407.40 \\
408.77 \\
410.14 \\
411.51 \\
412.88 \\
414.25\end{array}$ & $\begin{array}{l}4076.67 \\
4091.66 \\
4106.65 \\
4121.64 \\
4136.62 \\
4151.60 \\
4166.58 \\
4181.55 \\
4196.52 \\
4211.48\end{array}$ & $\begin{array}{l}2854.88 \\
2865.71 \\
2876.54 \\
2887.36 \\
2898.18 \\
2909.00 \\
2919.82 \\
2930.63 \\
2941.44 \\
2952.25\end{array}$ & $\begin{array}{l}50.31 \\
50.36 \\
50.41 \\
50.46 \\
50.51 \\
50.56 \\
50.61 \\
50.66 \\
50.71 \\
50.76\end{array}$ \\
\hline
\end{tabular}




\begin{tabular}{|c|c|c|c|c|c|c|c|c|}
\hline $\begin{array}{l}\text { TEMPER- } \\
\text { ATURE } \\
(K)\end{array}$ & $\begin{array}{l}\text { SPECIFIC } \\
\text { VOLUME } \\
\text { (CC/GM) }\end{array}$ & $\begin{array}{l}\text { ENTHALPY } \\
(\mathrm{J} / \mathrm{GM})\end{array}$ & $\begin{array}{l}\text { INTERNAL } \\
\text { ENERGY } \\
\text { (J/GM) }\end{array}$ & $\begin{array}{l}\text { ENTROPY } \\
(\mathrm{J} / \mathrm{GM}-\mathrm{K})\end{array}$ & $\begin{array}{l}\text { TEMPER- } \\
\text { ATURE } \\
(K)\end{array}$ & $\begin{array}{l}\text { SPECIFIC } \\
\text { VOLUME } \\
\text { (CC/GM) }\end{array}$ & $\begin{array}{l}\text { ENTHALPY } \\
\text { (J/GM) }\end{array}$ & $\begin{array}{cl}\text { INTERNAL } & \text { ENTROPY } \\
\text { ENERGY } & (J / G M-X) \\
\text { (J/GM) } & \end{array}$ \\
\hline
\end{tabular}

\begin{tabular}{|c|c|c|c|c|c|c|c|c|c|}
\hline 20.00 & $13 \cdot 34$ & -223.92 & -271.23 & 7.250 & & & & & \\
\hline $\begin{array}{l}21.00 \\
22.00 \\
23.00 \\
24.00 \\
25.00 \\
26.00 \\
27.00 \\
28.00 \\
29.00 \\
30.00\end{array}$ & $\begin{array}{l}13.50 \\
13.67 \\
13.85 \\
14.05 \\
14.27 \\
14.50 \\
14.75 \\
15.03 \\
15.33 \\
15.66\end{array}$ & $\begin{array}{l}-215.26 \\
-206.16 \\
-196.61 \\
-186.58 \\
-176.03 \\
-164.95 \\
-153.28 \\
-140.99 \\
-128.02 \\
-114.39\end{array}$ & $\begin{array}{l}-263.13 \\
-254.64 \\
-245.74 \\
-236.41 \\
-226.63 \\
-216.37 \\
-205.60 \\
-194.29 \\
-182.39 \\
-169.94\end{array}$ & $\begin{array}{r}7.675 \\
8.095 \\
8.523 \\
8.951 \\
9.379 \\
9.813 \\
10.253 \\
10.702 \\
11.154 \\
11.617\end{array}$ & $\begin{array}{r}91.00 \\
92.00 \\
93.00 \\
94.00 \\
95.00 \\
96.00 \\
97.00 \\
98.00 \\
99.00 \\
100.00\end{array}$ & $\begin{array}{l}104.66 \\
105.98 \\
107.29 \\
108.60 \\
109.91 \\
111.21 \\
112.51 \\
113.80 \\
115.09 \\
116.38\end{array}$ & $\begin{array}{r}915.85 \\
929.94 \\
944.08 \\
958.27 \\
972.51 \\
986.81 \\
1001.16 \\
1015.57 \\
1030.02 \\
1044.53\end{array}$ & $\begin{array}{l}544.67 \\
554.09 \\
563.58 \\
573.13 \\
582.74 \\
592.43 \\
602.17 \\
611.98 \\
621.86 \\
631.80\end{array}$ & $\begin{array}{l}31.335 \\
31.489 \\
31.642 \\
31.794 \\
31.945 \\
32.094 \\
32.243 \\
32.391 \\
32.537 \\
32.683\end{array}$ \\
\hline $\begin{array}{l}31.00 \\
32.00 \\
33.00 \\
34.00 \\
35.00 \\
36.00 \\
37.00 \\
38.00 \\
39.00 \\
40.00\end{array}$ & $\begin{array}{l}16.04 \\
16.45 \\
16.98 \\
17.52 \\
18.13 \\
18.85 \\
19.69 \\
20.70 \\
21.91 \\
23.34\end{array}$ & $\begin{array}{r}-99.91 \\
-84.59 \\
-68.70 \\
-51.16 \\
-32.32 \\
-12.21 \\
9.28 \\
32.24 \\
56.95 \\
82.81\end{array}$ & $\begin{array}{r}-156.78 \\
-142.93 \\
-128.93 \\
-113.27 \\
-96.62 \\
-79.05 \\
-60.56 \\
-41.17 \\
-20.74 \\
.04\end{array}$ & $\begin{array}{l}12.092 \\
12.581 \\
13.080 \\
13.605 \\
14.150 \\
14.715 \\
15.301 \\
15.913 \\
16.555 \\
17.209\end{array}$ & $\begin{array}{l}101.00 \\
102.00 \\
103.00 \\
104.00 \\
105.00 \\
106.00 \\
107.00 \\
108.00 \\
109.00 \\
110.00\end{array}$ & $\begin{array}{l}117.66 \\
118.94 \\
120.21 \\
121.49 \\
122.75 \\
124.02 \\
125.28 \\
126.54 \\
127.80 \\
129.06\end{array}$ & $\begin{array}{l}1059.10 \\
1073.74 \\
1088.44 \\
1103.20 \\
1118.01 \\
1132.87 \\
1147.79 \\
1162.76 \\
1177.78 \\
1192.85\end{array}$ & $\begin{array}{l}641.81 \\
651.92 \\
662.10 \\
672.35 \\
682.67 \\
693.04 \\
703.48 \\
713.98 \\
724.54 \\
735.16\end{array}$ & $\begin{array}{l}32.828 \\
32.972 \\
33.116 \\
33.258 \\
33.400 \\
33.541 \\
33.681 \\
33.820 \\
33.959 \\
34.096\end{array}$ \\
\hline $\begin{array}{l}41.00 \\
42.00 \\
43.00 \\
44.00 \\
45.00 \\
46.00 \\
47.00 \\
48.00 \\
49.00 \\
50.00\end{array}$ & $\begin{array}{l}25.01 \\
26.88 \\
28.89 \\
30.97 \\
33.07 \\
35.15 \\
37.20 \\
39.20 \\
41.17 \\
43.09\end{array}$ & $\begin{array}{l}109.55 \\
136.54 \\
163.03 \\
188.49 \\
212.67 \\
235.58 \\
257.33 \\
278.05 \\
297.88 \\
316.94\end{array}$ & $\begin{array}{r}20.87 \\
41.21 \\
60.57 \\
78.65 \\
95.40 \\
110.93 \\
125.41 \\
139.01 \\
151.88 \\
164.14\end{array}$ & $\begin{array}{l}17.869 \\
18.520 \\
19.143 \\
19.728 \\
20.272 \\
20.776 \\
21.243 \\
21.680 \\
22.088 \\
22.474\end{array}$ & $\begin{array}{l}111.00 \\
112.00 \\
113.00 \\
114.00 \\
115.00 \\
116.00 \\
-117.00 \\
118.00 \\
119.00 \\
120.00\end{array}$ & $\begin{array}{l}130.31 \\
131.56 \\
132.82 \\
134.07 \\
135.32 \\
136.57 \\
137.81 \\
139.06 \\
140.31 \\
141.56\end{array}$ & $\begin{array}{l}1207.97 \\
1223.13 \\
1238.35 \\
1253.60 \\
1268.91 \\
1284.25 \\
1299.64 \\
1315.08 \\
1330.56 \\
1346.08\end{array}$ & $\begin{array}{l}745.82 \\
756.55 \\
767.32 \\
778.14 \\
789.01 \\
799.93 \\
810.89 \\
821.90 \\
832.96 \\
844.06\end{array}$ & $\begin{array}{l}34.233 \\
3.0369 \\
34.504 \\
34.639 \\
34.773 \\
34.905 \\
35.038 \\
35.169 \\
35.300 \\
35.429\end{array}$ \\
\hline $\begin{array}{l}51.00 \\
52.00 \\
53.00 \\
54.00 \\
55.00 \\
56.00 \\
57.00 \\
58.00 \\
59.00 \\
60.00\end{array}$ & $\begin{array}{l}44.97 \\
46.81 \\
48.61 \\
50.39 \\
52.13 \\
53.84 \\
55.53 \\
57.19 \\
58.83 \\
60.45\end{array}$ & $\begin{array}{l}335.35 \\
353.18 \\
370.52 \\
387.43 \\
403.95 \\
420.13 \\
436.01 \\
451.62 \\
466.99 \\
482.15\end{array}$ & $\begin{array}{l}175.88 \\
187.18 \\
198.12 \\
208.74 \\
219.08 \\
229.18 \\
239.08 \\
248.79 \\
258.35 \\
267.76\end{array}$ & $\begin{array}{l}22.838 \\
23.184 \\
23.515 \\
23.831 \\
24.134 \\
24.425 \\
24.707 \\
24.978 \\
25.241 \\
25.496\end{array}$ & $\begin{array}{l}121.00 \\
122.00 \\
123.00 \\
124.00 \\
125.00 \\
126.00 \\
127.00 \\
128.00 \\
129.00 \\
130.00\end{array}$ & $\begin{array}{l}142.80 \\
144.05 \\
145.30 \\
146.54 \\
147.79 \\
149.02 \\
150.25 \\
151.48 \\
152.71 \\
153.94\end{array}$ & $\begin{array}{l}1361.63 \\
1377.23 \\
1392.87 \\
1408.56 \\
1424.28 \\
1440.12 \\
1456.00 \\
1471.93 \\
1487.91 \\
1503.93\end{array}$ & $\begin{array}{l}855.19 \\
866.36 \\
877.58 \\
888.85 \\
900.16 \\
911.62 \\
923.14 \\
934.71 \\
946.32 \\
957.99\end{array}$ & $\begin{array}{l}35.559 \\
35.687 \\
35.815 \\
35.942 \\
36.068 \\
36.194 \\
36.320 \\
36.445 \\
36.569 \\
36.693\end{array}$ \\
\hline $\begin{array}{l}61.00 \\
62.00 \\
63.00 \\
64.00 \\
65.00 \\
66.00 \\
67.00 \\
68.00 \\
69.00 \\
70.00\end{array}$ & $\begin{array}{l}62.05 \\
63.63 \\
65.19 \\
66.74 \\
68.27 \\
69.79 \\
71.29 \\
72.78 \\
74.26 \\
75.72\end{array}$ & $\begin{array}{l}497.11 \\
511.90 \\
526.54 \\
541.04 \\
555.42 \\
569.68 \\
583.85 \\
597.93 \\
611.94 \\
625.88\end{array}$ & $\begin{array}{l}277.06 \\
286.25 \\
295.34 \\
304.36 \\
313.31 \\
322.20 \\
331.03 \\
339.83 \\
348.60 \\
357.33\end{array}$ & $\begin{array}{l}25.743 \\
25.984 \\
26.218 \\
26.446 \\
26.669 \\
26.887 \\
27.100 \\
27.308 \\
27.513 \\
27.713\end{array}$ & $\begin{array}{l}131.00 \\
132.00 \\
133.00 \\
134.00 \\
135.00 \\
136.00 \\
137.00 \\
138.00 \\
139.00 \\
140.00\end{array}$ & $\begin{array}{l}155.17 \\
156.39 \\
157.62 \\
158.84 \\
160.06 \\
161.29 \\
162.51 \\
163.73 \\
164.95 \\
166.17\end{array}$ & $\begin{array}{l}1519.99 \\
1536.10 \\
1552.25 \\
1568.44 \\
1584.68 \\
1600.95 \\
1617.26 \\
1633.61 \\
1649.99 \\
1666.41\end{array}$ & $\begin{array}{r}969.70 \\
981.46 \\
993.27 \\
1005.12 \\
1017.02 \\
1028.95 \\
1040.93 \\
1052.94 \\
1065.00 \\
1077.09\end{array}$ & $\begin{array}{l}36.816 \\
36.938 \\
37.060 \\
37.181 \\
37.302 \\
37.422 \\
37.542 \\
37.661 \\
37.779 \\
37.897\end{array}$ \\
\hline $\begin{array}{l}71.00 \\
72.00 \\
73.00 \\
74.00 \\
75.00 \\
76.00 \\
77.00 \\
78.00 \\
79.00 \\
80.00\end{array}$ & $\begin{array}{l}77.18 \\
78.62 \\
80.06 \\
81.48 \\
82.90 \\
84.31 \\
85.71 \\
87.10 \\
88.49 \\
89.87\end{array}$ & $\begin{array}{l}639.76 \\
653.59 \\
667.39 \\
681.15 \\
694.89 \\
708.61 \\
722.32 \\
736.02 \\
749.72 \\
763.43\end{array}$ & $\begin{array}{l}366.06 \\
374.77 \\
383.47 \\
392.18 \\
400.89 \\
409.61 \\
418.35 \\
427.12 \\
435.91 \\
444.72\end{array}$ & $\begin{array}{l}27.910 \\
28.104 \\
28.294 \\
28.481 \\
28.666 \\
28.848 \\
29.027 \\
29.204 \\
29.378 \\
29.551\end{array}$ & $\begin{array}{l}141.00 \\
142.00 \\
143.00 \\
144.00 \\
145.00 \\
146.00 \\
147.00 \\
148.00 \\
149.00 \\
150.00\end{array}$ & $\begin{array}{l}167.39 \\
168.61 \\
169.83 \\
171.05 \\
172.27 \\
173.49 \\
174.71 \\
175.92 \\
177.14 \\
178.35\end{array}$ & $\begin{array}{l}1682.86 \\
1699.34 \\
1715.86 \\
1732.40 \\
1748.96 \\
1765.55 \\
1782.17 \\
1798.80 \\
1815.46 \\
1832.13\end{array}$ & $\begin{array}{l}1089.21 \\
1101.37 \\
1113.55 \\
1125.77 \\
1138.01 \\
1150.29 \\
1162.58 \\
1174.90 \\
1187.24 \\
1199.60\end{array}$ & $\begin{array}{l}38.014 \\
38.130 \\
38.246 \\
38.361 \\
38.476 \\
38.590 \\
38.703 \\
38.816 \\
38.928 \\
39.040\end{array}$ \\
\hline $\begin{array}{l}81.00 \\
82.00 \\
83.00 \\
84.00 \\
85.00 \\
86.00 \\
87.00 \\
88.00 \\
89.00 \\
90.00\end{array}$ & $\begin{array}{r}91.24 \\
92.61 \\
93.97 \\
95.32 \\
96.67 \\
98.02 \\
99.36 \\
100.69 \\
102.02 \\
103.34\end{array}$ & $\begin{array}{l}777.15 \\
790.88 \\
804.64 \\
818.42 \\
832.23 \\
846.07 \\
859.94 \\
873.85 \\
887.81 \\
901.80\end{array}$ & $\begin{array}{l}453.57 \\
462.46 \\
471.39 \\
480.37 \\
489.39 \\
498.47 \\
507.59 \\
516.77 \\
526.01 \\
535.31\end{array}$ & $\begin{array}{l}29.721 \\
29.889 \\
30.056 \\
30.221 \\
30.385 \\
30.546 \\
30.707 \\
30.866 \\
31.024 \\
31.180\end{array}$ & $\begin{array}{l}151.00 \\
152.00 \\
153.00 \\
154.00 \\
155.00 \\
156.00 \\
157.00 \\
158.00 \\
159.00 \\
160.00\end{array}$ & $\begin{array}{l}179.57 \\
180.78 \\
181.99 \\
183.19 \\
184.40 \\
185.61 \\
186.82 \\
188.02 \\
189.23 \\
190.44\end{array}$ & $\begin{array}{l}1848.74 \\
1865.35 \\
1881.98 \\
1898.62 \\
1915.27 \\
1931.93 \\
1948.59 \\
1965.26 \\
1981.94 \\
1998.62\end{array}$ & $\begin{array}{l}1211.91 \\
1224.24 \\
1236.58 \\
1248.93 \\
1261.29 \\
1273.67 \\
1286.05 \\
1298.44 \\
1310.84 \\
1323.24\end{array}$ & $\begin{array}{l}39.150 \\
39.260 \\
39.369 \\
39.477 \\
39.585 \\
39.692 \\
39.799 \\
39.904 \\
40.010 \\
40.114\end{array}$ \\
\hline
\end{tabular}


TEMPER- SPECIFIC ENTHALPY INTERNAL ENTROPY

(J/GM) ENERGY (J/GM-K) (K) (CC/GM)
(J/GM) $\begin{array}{ccccc}\text { TEMPER- } & \text { SPECIFIC } & \text { ENTHALPY } & \text { INTERNAL } & \text { ENTROPY } \\ \text { ATURE } & \text { VOLUME } & \text { (J/GM) } & \text { ENERGY } & \text { (J/GM-K) } \\ \text { (K) } & \text { (CC/GM) } & & \text { (J/GM) } & \end{array}$

\begin{tabular}{|c|c|c|c|c|c|c|c|c|c|}
\hline $\begin{array}{l}161.00 \\
162.00 \\
163.00 \\
164.00 \\
165.00 \\
166.00 \\
167.00 \\
168.00 \\
169.00 \\
170.00\end{array}$ & $\begin{array}{l}191.64 \\
192.85 \\
194.05 \\
195.25 \\
196.46 \\
197.66 \\
198.86 \\
200.07 \\
201.27 \\
202.47\end{array}$ & $\begin{array}{l}2015.30 \\
2031.99 \\
2048.67 \\
2065.36 \\
2082.05 \\
2098.73 \\
2115.42 \\
2132.10 \\
2148.78 \\
2165.45\end{array}$ & $\begin{array}{l}1335.65 \\
1348.06 \\
1360.48 \\
1372.90 \\
1385.31 \\
1397.73 \\
1410.16 \\
1422.57 \\
1434.99 \\
1447.41\end{array}$ & $\begin{array}{l}40.218 \\
40.321 \\
40.424 \\
40.526 \\
40.628 \\
40.728 \\
40.829 \\
40.928 \\
41.027 \\
41.126\end{array}$ & $\begin{array}{l}231.00 \\
232.00 \\
233.00 \\
234.00 \\
235.00 \\
236.00 \\
237.00 \\
238.00 \\
239.00 \\
240.00\end{array}$ & $\begin{array}{l}275.00 \\
276.18 \\
277.36 \\
278.54 \\
279.72 \\
280.90 \\
282.08 \\
283.26 \\
284.44 \\
285.62\end{array}$ & $\begin{array}{l}3158.65 \\
3174.41 \\
3190.14 \\
3205.86 \\
3221.57 \\
3237.25 \\
3252.92 \\
3268.57 \\
3284.20 \\
3299.82\end{array}$ & $\begin{array}{l}2183.39 \\
2194.95 \\
2206.50 \\
2218.03 \\
2229.55 \\
2241.05 \\
2252.53 \\
2264.00 \\
2275.45 \\
2286.89\end{array}$ & $\begin{array}{l}46.126 \\
46.194 \\
46.261 \\
46.329 \\
46.396 \\
46.462 \\
46.528 \\
46.594 \\
46.660 \\
46.725\end{array}$ \\
\hline $\begin{array}{l}171.00 \\
172.00 \\
173.00 \\
174.00 \\
175.00 \\
176.00 \\
177.00 \\
178.00 \\
179.00 \\
180.00\end{array}$ & $\begin{array}{l}203.67 \\
204.87 \\
206.06 \\
207.26 \\
208.46 \\
209.66 \\
210.85 \\
212.05 \\
213.24 \\
214.44\end{array}$ & $\begin{array}{l}2182.13 \\
2198.80 \\
2215.47 \\
2232.13 \\
2248.79 \\
2265.43 \\
2282.07 \\
2298.70 \\
2315.33 \\
2331.94\end{array}$ & $\begin{array}{l}1459.83 \\
1472.25 \\
1484.67 \\
1497.08 \\
1509.49 \\
1521.89 \\
1534.29 \\
1546.68 \\
1559.06 \\
1571.43\end{array}$ & $\begin{array}{l}41.223 \\
41.321 \\
41.417 \\
41.513 \\
41.609 \\
41.704 \\
41.798 \\
41.892 \\
41.985 \\
42.077\end{array}$ & $\begin{array}{l}241.00 \\
242.00 \\
243.00 \\
244.00 \\
245.00 \\
246.00 \\
247.00 \\
248.00 \\
249.00 \\
250.00\end{array}$ & $\begin{array}{l}286.80 \\
287.98 \\
289.15 \\
290.33 \\
291.51 \\
292.69 \\
293.87 \\
295.04 \\
296.22 \\
297.40\end{array}$ & $\begin{array}{l}3315.42 \\
3331.01 \\
3346.58 \\
3362.13 \\
3377.67 \\
3393.19 \\
3408.69 \\
3424.18 \\
3439.65 \\
3455.11\end{array}$ & $\begin{array}{l}2298.31 \\
2309.71 \\
2321.10 \\
2332.48 \\
2343.83 \\
2355.18 \\
2366.51 \\
2377.82 \\
2389.12 \\
2400.40\end{array}$ & $\begin{array}{l}46.790 \\
46.855 \\
46.919 \\
46.983 \\
47.046 \\
47.109 \\
47.172 \\
47.235 \\
47.297 \\
47.359\end{array}$ \\
\hline $\begin{array}{l}181.00 \\
182.00 \\
183.00 \\
184.00 \\
185.00 \\
186.00 \\
187.00 \\
188.00 \\
189.00 \\
190.00\end{array}$ & $\begin{array}{l}215.63 \\
216.83 \\
218.02 \\
219.22 \\
220.41 \\
221.60 \\
222.80 \\
223.99 \\
225.18 \\
226.37\end{array}$ & $\begin{array}{l}2348.54 \\
2365.13 \\
2381.71 \\
2398.28 \\
2414.84 \\
2431.38 \\
2447.91 \\
2464.43 \\
2480.94 \\
2497.43\end{array}$ & $\begin{array}{l}1583.80 \\
1596.15 \\
1608.50 \\
1620.84 \\
1633.16 \\
1645.47 \\
1657.78 \\
1670.06 \\
1682.34 \\
1694.61\end{array}$ & $\begin{array}{l}42.169 \\
42.261 \\
42.352 \\
42.442 \\
42.532 \\
42.621 \\
42.709 \\
42.797 \\
42.885 \\
42.972\end{array}$ & $\begin{array}{l}251.00 \\
252.00 \\
253.00 \\
254.00 \\
255.00 \\
256.00 \\
257.00 \\
258.00 \\
259.00 \\
260.00\end{array}$ & $\begin{array}{l}298.57 \\
299.75 \\
300.93 \\
302.10 \\
303.28 \\
304.45 \\
305.63 \\
306.80 \\
307.98 \\
309.15\end{array}$ & $\begin{array}{l}3470.58 \\
3486.04 \\
3501.49 \\
3516.92 \\
3532.33 \\
3547.73 \\
3563.11 \\
3578.48 \\
3593.84 \\
3609.18\end{array}$ & $\begin{array}{l}2411.70 \\
2422.99 \\
2434.26 \\
2445.52 \\
2456.77 \\
2468.00 \\
2479.21 \\
2490.41 \\
2501.60 \\
2512.77\end{array}$ & $\begin{array}{l}47.421 \\
47.482 \\
47.543 \\
47.604 \\
47.665 \\
47.725 \\
47.785 \\
47.845 \\
47.904 \\
47.963\end{array}$ \\
\hline $\begin{array}{l}191.00 \\
192.00 \\
193.00 \\
194.00 \\
195.00 \\
196.00 \\
197.00 \\
198.00 \\
199.00 \\
200.00\end{array}$ & $\begin{array}{l}227.56 \\
228.75 \\
229.95 \\
231.14 \\
232.33 \\
233.52 \\
234.71 \\
235.90 \\
237.08 \\
238.27\end{array}$ & $\begin{array}{l}2513.90 \\
2530.36 \\
2546.81 \\
2563.24 \\
2579.65 \\
2596.05 \\
2612.43 \\
2628.80 \\
2645.15 \\
2661.48\end{array}$ & $\begin{array}{l}1706.86 \\
1719.09 \\
1731.31 \\
1743.52 \\
1755.72 \\
1767.89 \\
1780.06 \\
1792.20 \\
1804.33 \\
1816.45\end{array}$ & $\begin{array}{l}43.059 \\
43.145 \\
43.230 \\
43.315 \\
43.399 \\
43.483 \\
43.566 \\
43.649 \\
43.732 \\
43.814\end{array}$ & $\begin{array}{l}261.00 \\
262.00 \\
263.00 \\
264.00 \\
265.00 \\
266.00 \\
267.00 \\
268.00 \\
269.00 \\
270.00\end{array}$ & $\begin{array}{l}310.33 \\
311.50 \\
312.68 \\
313.85 \\
315.03 \\
316.20 \\
317.38 \\
318.55 \\
319.73 \\
320.90\end{array}$ & $\begin{array}{l}3624.50 \\
3639.81 \\
3655.10 \\
3670.38 \\
3685.65 \\
3700.90 \\
3716.14 \\
3731.37 \\
3746.58 \\
3761.78\end{array}$ & $\begin{array}{l}2523.92 \\
2535.07 \\
2546.20 \\
2557.31 \\
2568.41 \\
2579.50 \\
2590.57 \\
2601.63 \\
2612.68 \\
2623.72\end{array}$ & $\begin{array}{l}48.022 \\
48.081 \\
48.139 \\
48.197 \\
48.255 \\
48.312 \\
48.369 \\
48.426 \\
48.483 \\
48.539\end{array}$ \\
\hline $\begin{array}{l}201.00 \\
202.00 \\
203.00 \\
204.00 \\
205.00 \\
206.00 \\
207.00 \\
208.00 \\
209.00 \\
210.00\end{array}$ & $\begin{array}{l}239.46 \\
240.65 \\
241.84 \\
243.02 \\
244.21 \\
245.40 \\
246.58 \\
247.77 \\
248.96 \\
250.14\end{array}$ & $\begin{array}{l}2677.78 \\
2694.07 \\
2710.34 \\
2726.60 \\
2742.83 \\
2759.05 \\
2775.25 \\
2791.43 \\
2807.60 \\
2823.74\end{array}$ & $\begin{array}{l}1828.54 \\
1840.62 \\
1852.68 \\
1864.72 \\
1876.75 \\
1888.75 \\
1900.75 \\
1912.72 \\
1924.68 \\
1936.62\end{array}$ & $\begin{array}{l}43.895 \\
43.976 \\
44.056 \\
44.136 \\
44.215 \\
44.294 \\
44.373 \\
44.451 \\
44.528 \\
44.605\end{array}$ & $\begin{array}{l}271.00 \\
272.00 \\
273.00 \\
274.00 \\
275.00 \\
276.00 \\
277.00 \\
278.00 \\
279.00 \\
280.00\end{array}$ & $\begin{array}{l}322.07 \\
323.25 \\
324.42 \\
325.60 \\
326.77 \\
327.95 \\
329.12 \\
330.29 \\
331.47 \\
332.64\end{array}$ & $\begin{array}{l}3776.96 \\
3792.14 \\
3807.30 \\
3822.45 \\
3837.59 \\
3852.72 \\
3867.83 \\
3882.94 \\
3898.03 \\
3913.12\end{array}$ & $\begin{array}{l}2634.74 \\
2645.75 \\
2656.75 \\
2667.73 \\
2678.71 \\
2689.67 \\
2700.62 \\
2711.56 \\
2722.49 \\
2733.42\end{array}$ & $\begin{array}{l}48.595 \\
48.651 \\
48.707 \\
48.762 \\
48.817 \\
48.872 \\
48.927 \\
48.982 \\
49.036 \\
49.090\end{array}$ \\
\hline $\begin{array}{l}211.00 \\
212.00 \\
213.00 \\
214.00 \\
215.00 \\
216.00 \\
217.00 \\
218.00 \\
219.00 \\
220.00\end{array}$ & $\begin{array}{l}251.33 \\
252.51 \\
253.70 \\
254.88 \\
256.07 \\
257.25 \\
258.44 \\
259.62 \\
260.81 \\
261.99\end{array}$ & $\begin{array}{l}2839.87 \\
2855.98 \\
2872.07 \\
2888.15 \\
2904.20 \\
2920.24 \\
2936.26 \\
2952.26 \\
2968.24 \\
2984.21\end{array}$ & $\begin{array}{l}1948.54 \\
1960.45 \\
1972.33 \\
1984.21 \\
1996.06 \\
2007.90 \\
2019.71 \\
2031.52 \\
2043.30 \\
2055.07\end{array}$ & $\begin{array}{l}44.682 \\
44.758 \\
44.834 \\
44.909 \\
44.984 \\
45.058 \\
45.132 \\
45.206 \\
45.279 \\
45.352\end{array}$ & $\begin{array}{l}281.00 \\
282.00 \\
283.00 \\
284.00 \\
285.00 \\
286.00 \\
287.00 \\
288.00 \\
289.00 \\
290.00\end{array}$ & $\begin{array}{l}333.82 \\
334.99 \\
336.16 \\
337.34 \\
338.51 \\
339.69 \\
340.86 \\
342.04 \\
343.21 \\
344.39\end{array}$ & $\begin{array}{l}3928.20 \\
3943.27 \\
3958.33 \\
3973.38 \\
3988.43 \\
4003.46 \\
4018.50 \\
4033.52 \\
4048.54 \\
4063.55\end{array}$ & $\begin{array}{l}2744.33 \\
2755.23 \\
2766.13 \\
2777.02 \\
2787.90 \\
2798.77 \\
2809.64 \\
2820.50 \\
2831.35 \\
2842.20\end{array}$ & $\begin{array}{l}49.143 \\
49.197 \\
49.250 \\
49.303 \\
49.356 \\
49.409 \\
49.461 \\
49.514 \\
49.566 \\
49.618\end{array}$ \\
\hline $\begin{array}{l}221.00 \\
222.00 \\
223.00 \\
224.00 \\
225.00 \\
226.00 \\
227.00 \\
228.00 \\
229.00 \\
230.00\end{array}$ & $\begin{array}{l}263.17 \\
264.36 \\
265.54 \\
266.72 \\
267.91 \\
269.09 \\
270.27 \\
271.45 \\
272.63 \\
273.82\end{array}$ & $\begin{array}{l}3000.15 \\
3016.08 \\
3032.00 \\
3047.89 \\
3063.77 \\
3079.62 \\
3095.47 \\
3111.29 \\
3127.09 \\
3142.88\end{array}$ & $\begin{array}{l}2066.82 \\
2078.55 \\
2090.27 \\
2101.97 \\
2113.65 \\
2125.31 \\
2136.96 \\
2148.59 \\
2160.21 \\
2171.80\end{array}$ & $\begin{array}{l}45.424 \\
45.496 \\
45.568 \\
45.639 \\
45.709 \\
45.780 \\
45.850 \\
45.919 \\
45.988 \\
46.057\end{array}$ & $\begin{array}{l}291.00 \\
292.00 \\
293.00 \\
294.00 \\
295.00 \\
296.00 \\
297.00 \\
298.00 \\
299.00 \\
300.00\end{array}$ & $\begin{array}{l}345.56 \\
346.73 \\
347.91 \\
349.08 \\
350.26 \\
351.43 \\
352.60 \\
353.78 \\
354.95 \\
356.12\end{array}$ & $\begin{array}{l}4078.56 \\
4093.57 \\
4108.57 \\
4123.56 \\
4138.55 \\
4153.54 \\
4168.53 \\
4183.51 \\
4198.48 \\
4213.45\end{array}$ & $\begin{array}{l}2853.05 \\
2863.89 \\
2874.72 \\
2885.55 \\
2896.38 \\
2907.21 \\
2918.03 \\
2928.85 \\
2939.66 \\
2950.48\end{array}$ & $\begin{array}{l}49.669 \\
49.721 \\
49.772 \\
49.823 \\
49.874 \\
49.925 \\
49.975 \\
50.026 \\
50.076 \\
50.126\end{array}$ \\
\hline
\end{tabular}




\begin{tabular}{|c|c|c|c|c|c|c|c|c|c|}
\hline $\begin{array}{l}\text { EMPER- } \\
\text { ATURE } \\
\text { (K) }\end{array}$ & $\begin{array}{l}\text { SPEC IF IC } \\
\text { VOLUME } \\
\text { (CC/GM) }\end{array}$ & $\begin{array}{c}\text { ENTHALPY } \\
(\mathrm{J} / \mathrm{GM})\end{array}$ & $\begin{array}{c}\text { INTERNAL } \\
\text { ENERGY } \\
(J / G M)\end{array}$ & $\begin{array}{l}\text { ENTROPY } \\
(J / G M-K)\end{array}$ & $\begin{array}{c}\text { TEMPER- } \\
\text { ATURE } \\
(K)\end{array}$ & $\begin{array}{c}\text { SPECIF IC } \\
\text { VOLUME } \\
\text { (CC/GM) }\end{array}$ & $\begin{array}{c}\text { ENTHALPY } \\
(J / G M)\end{array}$ & $\begin{array}{c}\text { INTERNAL } \\
\text { ENERGY } \\
(J / G M)\end{array}$ & $\begin{array}{l}\text { ENTROPY } \\
(J / G M-K)\end{array}$ \\
\hline
\end{tabular}

\begin{tabular}{|c|c|c|c|c|c|c|c|c|c|}
\hline 20.00 & 13.26 & -218.71 & $-272 \cdot 44$ & 7.174 & & & & & \\
\hline $\begin{array}{l}21.00 \\
22.00 \\
23.00 \\
24.00 \\
25.00 \\
26.00 \\
27.00 \\
28.00 \\
29.00 \\
30.00\end{array}$ & $\begin{array}{l}13.41 \\
13.57 \\
13.75 \\
13.93 \\
14.14 \\
14.35 \\
14.59 \\
14.84 \\
15.12 \\
15.42\end{array}$ & $\begin{array}{l}-210.16 \\
-201.19 \\
-191.79 \\
-181.92 \\
-171.58 \\
-160.72 \\
-149.32 \\
-137.35 \\
-124.75 \\
-111.58\end{array}$ & $\begin{array}{l}-264.50 \\
-256.20 \\
-247.50 \\
-238.40 \\
-228.88 \\
-218.90 \\
-208.46 \\
-197.51 \\
-186.04 \\
-174.09\end{array}$ & $\begin{array}{r}7.593 \\
8.008 \\
8.428 \\
8.850 \\
9.270 \\
9.695 \\
10.125 \\
10.562 \\
11.001 \\
11.448\end{array}$ & $\begin{array}{r}91.00 \\
92.00 \\
93.00 \\
94.00 \\
95.00 \\
96.00 \\
97.00 \\
98.00 \\
99.00 \\
100.00\end{array}$ & $\begin{array}{r}91.66 \\
92.82 \\
93.99 \\
95.14 \\
96.30 \\
97.45 \\
98.60 \\
99.75 \\
100.89 \\
102.03\end{array}$ & $\begin{array}{r}908.25 \\
922.53 \\
936.85 \\
951.21 \\
965.63 \\
980.09 \\
994.60 \\
1009.16 \\
1023.77 \\
1038.42\end{array}$ & $\begin{array}{l}536.77 \\
546.32 \\
555.93 \\
565.60 \\
575.33 \\
585.12 \\
594.97 \\
604.89 \\
614.87 \\
624.91\end{array}$ & $\begin{array}{l}30.707 \\
30.863 \\
31.018 \\
31.171 \\
31.324 \\
31.475 \\
31.626 \\
31.775 \\
31.923 \\
32.071\end{array}$ \\
\hline $\begin{array}{l}31.00 \\
32.00 \\
33.00 \\
34.00 \\
35.00 \\
36.00 \\
37.00 \\
38.00 \\
39.00 \\
40.00\end{array}$ & $\begin{array}{l}15.76 \\
16.12 \\
16.59 \\
17.04 \\
17.56 \\
18.14 \\
18.80 \\
19.56 \\
20.44 \\
21.45\end{array}$ & $\begin{array}{r}-97.66 \\
-83.02 \\
-67.93 \\
-51.44 \\
-33.93 \\
-15.48 \\
3.92 \\
24.27 \\
45.79 \\
67.99\end{array}$ & $\begin{array}{r}-161.52 \\
-148.36 \\
-135.18 \\
-120.53 \\
-105.09 \\
-88.99 \\
-72.28 \\
-55.02 \\
-37.05 \\
-18.94\end{array}$ & $\begin{array}{l}11.905 \\
12.372 \\
12.846 \\
13.340 \\
13.846 \\
14.364 \\
14.893 \\
15.435 \\
15.994 \\
16.556\end{array}$ & $\begin{array}{l}101.00 \\
102.00 \\
103.00 \\
104.00 \\
105.00 \\
106.00 \\
107.00 \\
108.00 \\
109.00 \\
110.00\end{array}$ & $\begin{array}{l}103.16 \\
104.29 \\
105.42 \\
106.54 \\
107.66 \\
108.78 \\
109.89 \\
111.01 \\
112.12 \\
113.23\end{array}$ & $\begin{array}{l}1053.14 \\
1067.91 \\
1082.74 \\
1097.62 \\
1112.55 \\
1127.54 \\
1142.57 \\
1157.66 \\
1172.79 \\
1187.96\end{array}$ & $\begin{array}{l}635.01 \\
645.21 \\
655.48 \\
665.81 \\
676.20 \\
686.65 \\
697.17 \\
707.74 \\
718.36 \\
729.05\end{array}$ & $\begin{array}{l}32.217 \\
32.362 \\
32.507 \\
32.651 \\
32.794 \\
32.936 \\
33.077 \\
33.217 \\
33.357 \\
33.495\end{array}$ \\
\hline $\begin{array}{l}41.00 \\
42.00 \\
43.00 \\
44.00 \\
45.00 \\
46.00 \\
47.00 \\
48.00 \\
49.00 \\
50.00\end{array}$ & $\begin{array}{l}22.60 \\
23.90 \\
25.34 \\
26.89 \\
28.51 \\
30.18 \\
31.87 \\
33.56 \\
35.24 \\
36.91\end{array}$ & $\begin{array}{r}90.92 \\
114.46 \\
138.31 \\
162.13 \\
185.57 \\
208.38 \\
230.45 \\
251.75 \\
272.29 \\
292.14\end{array}$ & $\begin{array}{r}-.69 \\
17.58 \\
35.61 \\
53.15 \\
70.01 \\
86.05 \\
101.27 \\
115.72 \\
129.45 \\
142.55\end{array}$ & $\begin{array}{l}17.122 \\
17.689 \\
18.251 \\
18.798 \\
19.325 \\
19.826 \\
20.301 \\
20.750 \\
21.173 \\
21.574\end{array}$ & $\begin{array}{l}111.00 \\
112.00 \\
113.00 \\
114.00 \\
115.00 \\
116.00 \\
117.00 \\
118.00 \\
119.00 \\
120.00\end{array}$ & $\begin{array}{l}114.33 \\
115.44 \\
116.54 \\
117.65 \\
118.75 \\
119.85 \\
120.95 \\
122.05 \\
123.15 \\
124.25\end{array}$ & $\begin{array}{l}1203.19 \\
1218.45 \\
1233.77 \\
1249.12 \\
1264.52 \\
1279.96 \\
1295.44 \\
1310.96 \\
1326.53 \\
1342.13\end{array}$ & $\begin{array}{l}739.78 \\
750.56 \\
761.40 \\
772.28 \\
783.21 \\
794.18 \\
805.20 \\
816.27 \\
827.38 \\
838.53\end{array}$ & $\begin{array}{l}33.633 \\
33.770 \\
33.906 \\
34.041 \\
34.176 \\
34.310 \\
34.443 \\
34.575 \\
34.706 \\
34.837\end{array}$ \\
\hline $\begin{array}{l}51.00 \\
52.00 \\
53.00 \\
54.00 \\
55.00 \\
56.00 \\
57.00 \\
58.00 \\
59.00 \\
60.00\end{array}$ & $\begin{array}{l}38.55 \\
40.17 \\
41.76 \\
43.33 \\
44.88 \\
46.41 \\
47.91 \\
49.39 \\
50.86 \\
52.30\end{array}$ & $\begin{array}{l}311.35 \\
329.99 \\
348.11 \\
365.77 \\
383.02 \\
399.90 \\
416.45 \\
432.70 \\
448.68 \\
464.42\end{array}$ & $\begin{array}{l}155.11 \\
167.18 \\
178.85 \\
190.14 \\
201.12 \\
211.82 \\
222.27 \\
232.51 \\
242.56 \\
252.45\end{array}$ & $\begin{array}{l}21.955 \\
22.316 \\
22.662 \\
22.992 \\
23.308 \\
23.612 \\
23.905 \\
24.188 \\
24.461 \\
24.726\end{array}$ & $\begin{array}{l}121.00 \\
122.00 \\
123.00 \\
124.00 \\
125.00 \\
126.00 \\
127.00 \\
128.00 \\
129.00 \\
130.00\end{array}$ & $\begin{array}{l}125.35 \\
126.45 \\
127.55 \\
128.64 \\
129.74 \\
130.83 \\
131.91 \\
132.99 \\
134.08 \\
135.16\end{array}$ & $\begin{array}{l}1357.77 \\
1373.45 \\
1389.17 \\
1404.93 \\
1420.74 \\
1436.65 \\
1452.61 \\
1468.61 \\
1484.66 \\
1500.76\end{array}$ & $\begin{array}{l}849.71 \\
860.94 \\
872.21 \\
883.52 \\
894.88 \\
906.39 \\
917.96 \\
929.57 \\
941.24 \\
952.95\end{array}$ & $\begin{array}{l}34.966 \\
35.095 \\
35.224 \\
35.351 \\
35.478 \\
35.605 \\
35.731 \\
35.857 \\
35.982 \\
36.106\end{array}$ \\
\hline $\begin{array}{l}61.00 \\
62.00 \\
63.00 \\
64.00 \\
65.00 \\
66.00 \\
67.00 \\
68.00 \\
69.00 \\
70.00\end{array}$ & $\begin{array}{l}53.73 \\
55.14 \\
56.53 \\
57.92 \\
59.28 \\
60.64 \\
1.98 \\
63.31 \\
64.63 \\
65.93\end{array}$ & $\begin{array}{l}479.94 \\
495.26 \\
510.41 \\
525.40 \\
540.24 \\
554.95 \\
569.54 \\
584.04 \\
598.44 \\
612.76\end{array}$ & $\begin{array}{l}262.18 \\
271.78 \\
281.27 \\
290.66 \\
299.96 \\
309.19 \\
318.35 \\
327.45 \\
336.51 \\
345.53\end{array}$ & $\begin{array}{l}24.982 \\
25.232 \\
25.474 \\
25.710 \\
25.940 \\
26.165 \\
26.384 \\
26.599 \\
26.809 \\
27.015\end{array}$ & $\begin{array}{l}131.00 \\
132.00 \\
133.00 \\
134.00 \\
135.00 \\
136.00 \\
137.00 \\
138.00 \\
139.00 \\
140.00\end{array}$ & $\begin{array}{l}136.24 \\
137.32 \\
138.39 \\
139.47 \\
140.55 \\
141.62 \\
142.70 \\
143.77 \\
144.85 \\
145.92\end{array}$ & $\begin{array}{l}1516.90 \\
1533.08 \\
1549.30 \\
1565.56 \\
1581.87 \\
1598.21 \\
1614.59 \\
1631.00 \\
1647.46 \\
1663.94\end{array}$ & $\begin{array}{r}964.72 \\
976.52 \\
988.38 \\
1000.27 \\
1012.21 \\
1024.19 \\
1036.21 \\
1048.27 \\
1060.37 \\
1072.50\end{array}$ & $\begin{array}{l}36.230 \\
36.353 \\
36.475 \\
36.597 \\
36.718 \\
36.839 \\
36.959 \\
37.078 \\
37.197 \\
37.315\end{array}$ \\
\hline $\begin{array}{l}71.00 \\
72.00 \\
73.00 \\
74.00 \\
75.00 \\
76.00 \\
77.00 \\
78.00 \\
79.00 \\
80.00\end{array}$ & $\begin{array}{l}67.23 \\
68.52 \\
69.80 \\
71.07 \\
72.33 \\
73.58 \\
74.83 \\
76.07 \\
77.30 \\
78.53\end{array}$ & $\begin{array}{l}627.00 \\
641.19 \\
655.32 \\
669.41 \\
683.46 \\
697.49 \\
711.49 \\
725.47 \\
739.45 \\
753.42\end{array}$ & $\begin{array}{l}354.51 \\
363.48 \\
372.43 \\
381.37 \\
390.31 \\
399.25 \\
408.20 \\
417.16 \\
426.14 \\
435.14\end{array}$ & $\begin{array}{l}27.217 \\
27.416 \\
27.610 \\
27.802 \\
27.991 \\
28.177 \\
28.360 \\
28.540 \\
28.718 \\
28.894\end{array}$ & $\begin{array}{l}141.00 \\
142.00 \\
143.00 \\
144.00 \\
145.00 \\
146.00 \\
147.00 \\
148.00 \\
149.00 \\
150.00\end{array}$ & $\begin{array}{l}147.00 \\
148.07 \\
149.14 \\
150.22 \\
151.29 \\
152.36 \\
153.43 \\
154.50 \\
155.57 \\
156.64\end{array}$ & $\begin{array}{l}1680.46 \\
1697.01 \\
1713.58 \\
1730.19 \\
1746.82 \\
1763.47 \\
1780.14 \\
1796.84 \\
1813.55 \\
1830.28\end{array}$ & $\begin{array}{l}1084.67 \\
1096.86 \\
1109.09 \\
1121.35 \\
1133.63 \\
1145.94 \\
1158.27 \\
1170.63 \\
1183.00 \\
1195.40\end{array}$ & $\begin{array}{l}37.433 \\
37.550 \\
37.666 \\
37.782 \\
37.897 \\
38.011 \\
38.125 \\
38.238 \\
38.351 \\
38.463\end{array}$ \\
\hline $\begin{array}{l}81.00 \\
82.00 \\
83.00 \\
84.00 \\
85.00 \\
86.00 \\
87.00 \\
88.00 \\
89.00 \\
90.00\end{array}$ & $\begin{array}{l}79.75 \\
80.96 \\
82.17 \\
83.37 \\
84.57 \\
85.76 \\
86.95 \\
88.13 \\
89.31 \\
90.49\end{array}$ & $\begin{array}{l}767.40 \\
781.38 \\
795.37 \\
809.39 \\
823.42 \\
837.47 \\
851.56 \\
865.68 \\
879.83 \\
894.02\end{array}$ & $\begin{array}{l}444.18 \\
453.24 \\
462.34 \\
471.47 \\
480.65 \\
489.88 \\
499.15 \\
508.47 \\
517.85 \\
527.28\end{array}$ & $\begin{array}{l}29.067 \\
29.239 \\
29.409 \\
29.576 \\
29.743 \\
29.907 \\
30.070 \\
30.231 \\
30.391 \\
30.550\end{array}$ & $\begin{array}{l}151.00 \\
152.00 \\
153.00 \\
154.00 \\
155.00 \\
156.00 \\
157.00 \\
158.00 \\
159.00 \\
160.00\end{array}$ & $\begin{array}{l}157.71 \\
158.77 \\
159.83 \\
160.90 \\
161.96 \\
163.02 \\
164.08 \\
165.14 \\
166.20 \\
167.26\end{array}$ & $\begin{array}{l}1846.95 \\
1863.62 \\
1880.30 \\
1896.99 \\
1913.69 \\
1930.40 \\
1947.11 \\
1963.83 \\
1980.55 \\
1997.28\end{array}$ & $\begin{array}{l}1207.75 \\
1220.11 \\
1232.48 \\
1244.86 \\
1257.26 \\
1269.66 \\
1282.08 \\
1294.50 \\
1306.92 \\
1319.36\end{array}$ & $\begin{array}{l}38.573 \\
38.683 \\
38.793 \\
38.902 \\
39.010 \\
39.117 \\
39.224 \\
39.330 \\
39.436 \\
39.540\end{array}$ \\
\hline
\end{tabular}


40.00 ATMOSPHERE I SO8AR

TEMPER- SPECIFIC ENTHALPY INTERNAL ENTROPY ATURE VOLUME (J/GM) ENERGY (K) (CC/GM)
(ENERGY
TEMPERATURE (K)
SPECIFIC VOLUME (CC/GM)
ENTHALPY I J/GM

M)

ENTROPY

ENERGY (J/GM-K) $(J / G M)$
161.00

162.00

163.00

164.00

165.00

166.00

167.00

168.00

169.00

170.00

171.00

172.00

173.00

174.00

175.00

176.00

177.00

178.00

179.00

180.00

181.00

182.00

183.00

184.00

185.00

186.00

187.00

188.00

189.00

190.00

191.00

192.00

193.00

194.00

195.00

196.00

197.00

198.00

199.00

200.00

201.00

202.00

203.00

204.00

205. 00

206.00

207.00

208.00

209.00

210.00

211.00

212.00

213.00

214.00

215.00

216.00

217.00

218.00

219.00

220.00

221.00

222.00

223.00

224.00

225.00

226.00

227.00

228.00

229.00

230.00
168.32

169.38

170.44

171.49

172.55

173.61

174.66

175.72

176.77

177.83

178.88

179.93

180.99

182.04

83.09

84.14

185.19

186.24

187.29

188.34

189.39

190.44

191.48

192.53

193.58

194.63

195.67

196.72

197.77

198.81

199.86

200.90

201.95

202.99

204.04

205.08

206.12

207. 17

208.21

209.25

210.30

211.34

212.38

213.42

214.46

215.50

216.54

217.58

218.63

219.67

220.71

221.74

222.78

.

224.86

225.90

226.94

227.98

229.02

230.05

231.09

232.13

233.17

234.20

235.24

236.28

237.31

238.35

239.39

240.42
2014.01

2030.74

47.47

2064.20

2080.93

2097.65

2114.38

2131.10

2147.82

2164.54

2181.26

197.97

2214.67

2231.37

2248.07

2264.75

2281.43

2298.10

2314.76

2331.41

2348.04

2364.67

2381.29

2397.89

2414.49

2431.07

2447.63

2464.18

2480.72

2497.25

2513.76

2530.25

2546.73

2563.19

2579.64

2596.07

2612.48

2628.88

2645.26

2661.62

2677.96

2694.27

2710.57

2726.86

2743.12

2759.37

2775.60

2791.80

2808.00

2824.17

2840.32

2856.46

2872.58

2888.68

2904.76

2920.82

2936.87

2952.89

2968.90

2984.89

3000.87

3016.82

3032.76

3048.67

3064.58

3080.46

3096.32

3112.17

3128.00

3143.81

1331.79

1344.23

1369.12

1381.56

1394.01

1406.46

1418.90

1431.34

1443.78

1456.23

468.67

1481.12

1493.55

505.98

1518.41

1530.83

543.24

1555.65

1568.05

1580.43

1592.81

1605.18

1617.54

1629.89

1642.22

1654.55

1666.86

1679.16

1691.44

1703.71

1715.97

1728.21

1740.44

1752.66

1764.86

1777.04

1789.21

1801.36

1813.49

1825.60

1837.70

1849.78

1861.84

1873.88

1885.91

1897.92

1909.91

1921.88

1933.84

1945.78

1957.71 
45.00 ATMOSPHERE ISOBAR

\begin{tabular}{|c|c|c|c|c|c|c|c|c|c|}
\hline $\begin{array}{l}\text { TEMPER- } \\
\text { ATURE } \\
\text { (K) }\end{array}$ & $\begin{array}{l}\text { SPECIF IC } \\
\text { VOLUME } \\
\text { (CC/GM) }\end{array}$ & $\begin{array}{l}\text { ENTHALPY } \\
\text { (J/GM) }\end{array}$ & $\begin{array}{l}\text { INTERNAL } \\
\text { ENERGY } \\
\text { (J/GM) }\end{array}$ & $\begin{array}{l}\text { ENTROPY } \\
(J / G M-K)\end{array}$ & $\begin{array}{l}\text { TEMPER- } \\
\text { ATURE } \\
(\mathrm{K})\end{array}$ & $\begin{array}{l}\text { SPECIFIC } \\
\text { VOLUME } \\
\text { (CC/GM) }\end{array}$ & $\begin{array}{l}\text { ENTHALPY } \\
\text { (J/GM) }\end{array}$ & $\begin{array}{l}\text { INTERNAL } \\
\text { ENERGY } \\
(\mathrm{J} / \mathrm{GM})\end{array}$ & $\begin{array}{l}\text { ENTROPY } \\
\text { (J/GM-K) }\end{array}$ \\
\hline
\end{tabular}




TEMPER- SPECIFIC ENTHALPY INTERNAL ENTROPY ATURE VOLUME (J/GM) ENERGY (J/GM-K) (K) (CC/GM)
(J/GM)
TEMPERATURE

(K)
SPECIFIC ENTHALPY

(CC/GM)
(J/GM) ENERGY

ENTROPY

(J/GM)
161.00

162.00

163.00

164.00

165.00

166.00

167.00

168.00

169.00

170.00

171.00

172.00

173.00

174.00

175.00

176.00

177.00

178.00

179.00

180.00

181.00

182.00

183.00

184.00

185.00

186.00

187.00

188.00

189.00

190.00

191.00

192.00

193.00

194.00

195.00

196.00

197.00

198.00

199.00

200.00

201.00

202.00

203.00

204.00

205.00

206.00

207.00

208.00

209.00

210.00

211.00

212.00

213.00

214.00

215.00

216.00

217.00

218.00

219.00

220.00

221.00

222.00

223.00

224.00

225.00

226.00

227.00

228.00

229.00

230.00

150.20
151.14
152.08
153.03
153.97
154.91
155.86
156.80
157.74
158.68

2012.91

2063.24

2080.01

2096. 78

2113.55

2130.32

2147.08

159.62

160.56

161.50

162.43

163.37

164.31

165.25

166.18

167.12

168.05

168.99

169.92

170.86

171.79

172.72

173.66

174.59

175.52

176.46

177.39

178.32

179.25

180.18

181.11

182.04

182.97

183.91

184.84

185.76

186.69

2180.60

2197.36

2214.11

2230.85

2247.59

2264.31

2281.03

2297.74

314.44

2331.13

2347.81

2364.48

2381.14

2397.78

2414.41

2431.03

2447.64

2464.23

2480. 81

2497. 37

2513.91

2530.44

2546.96

2563.46

2579.94

2596.40

2612. 85

2629.28

2645.69

2662. 09

187.62

188.55

189.48

190.41

191.34

192.26

193.19

194.12

195.04

2678.45

2694. 80

2711.12

2727.43

2743. 72

2759.99

2776.25

2792.48

2808.69

2824. 89

196.90

2841.07

2857. 23

2873. 37

2889.49

2905.59

2921.67

2937.74

2953.78

2969. 81

2985. 82

205.22

3001.81

3017.79

3033.74

3049.68

3065.60

3081.50

3097.39

3113.25

211.69

212.61

213.53

3129.10

214.46

1452.78

1465.26

1477.73

1490.19

1502.65

1515.11

1527.56

1540.00

1552.43

1564.86

1577.27

1589.68

1602.07

1614.46

1626.83

1639.20

1651.55

1663.89

1676.21

1688.52

1700.82

1713.10

1725.37

1737.62

1749.86

1762.08

1774.29

1786.48

1798.65

1810.81

1822.94

1835.05

1847.14

1859.22

1871.28

1883.32

1895.35

1907.36

1919.34

1931.32

1943.27

1955.20

1967.12

1979.02

$1328.06 \quad 39.137$

$1353.00 \quad 39.344$

$365.47 \quad 39.447$

$\begin{array}{ll}377.95 & 39.549\end{array}$

$1390.42 \quad 39.650$

$1402.89 \quad 39.751$

$1415.37 \quad 39.851$

$1427.84 \quad 39.950$

$1440.31 \quad 40.049$

40.148

40.245

40.342

40.439

40.535

40.630

40.725

40.819

40.912

41.005

41.098

41.190

41.281

41.372

41.462

41.551

41.640

41.729

41.817

41.904

41.991

42.077

42.163

42.248

42.333

42.417

42.501

42.584

42.667

42.749 
$\begin{array}{ccccc}\text { TEMPER- } & \text { SPECIFIC } & \text { ENTHALPY } & \text { INTERNAL } & \text { ENTROPY } \\ \text { ATURE } & \text { VOLUME } & \text { (J/GM) } & \text { ENERGY } & \text { (J/GM-K) }\end{array}$ (K) (CC/GM) (J/GM) $\begin{array}{ccccc}\text { TEMPER- } & \text { SPECIFIC } & \text { ENTHALPY } & \text { INTERNAL } & \text { ENTROPY } \\ \text { ATURE } & \text { VOLUME } & \text { (J/GM) } & \text { ENERGY } & \text { (J/GM-K) } \\ \text { (K) } & \text { (CC/GM) } & & \begin{array}{c}\text { (J/GM) } \\ \text { (JC/G }\end{array} & \end{array}$

$\begin{array}{rrr}91.00 & 73.58 & 894.1 \\ 92.00 & 74.54 & 908.7 \\ 93.00 & 75.48 & 923.3 \\ 94.00 & 76.43 & 938.0 \\ 95.00 & 77.37 & 952.8 \\ 96.00 & 78.31 & 967.5 \\ 97.00 & 79.25 & 982.3 \\ 98.00 & 80.18 & 997.2 \\ 99.00 & 81.11 & 1012.1 \\ 100.00 & 82.04 & 1027.0\end{array}$

17.79

54.41

40.00

18.72

20.06

42.00

43.00

44.00

45.00
46.00

47.00

48.00

49.00

50.00

51.00

53.00

54.00

55.00

56.00

57.00

58.00

59.00

60.00

61.00

62.00

63.00

64.00

65.00

66.00

68.00

69.00

70.00

20.84

21.69

22.61

23.61

24.66

26.92

28.10

29.31

73.16

92.31
111.89

131.85

152.09

172.49

192.90

213.21

233. 31

30.52

272.60

31.75

34.19

35.41

36.62

37.82

39.01

40.19

41.36

42.52

43.67

44.81

45.93

47.05

48.16

50.35

51.43

52.51

291.73

310.50

328.91

346.97

364.70

382.12

399.25

416.11

432.71

449.09

465.24

481.20

496.98

512.59

528.05

543.36

558.56

573.64

53.57

71.00

73.00

74.00

75.00

76.00

77.00

78.00

79.00

80.00

81.00

82.00

83.00

84.00

85.00

86.00

87.00

88.00

89.00

90.00

54.63

603.50

618.31

633.04

56.72

57.76

58.79

59.81

60.83

62.84

63.84

64.84

65.82

66.81

67.79

68.77

69.74

70.70

$7 ! .67$

72.63
647.71

662.32

676.89

691.42

705.91

720.38

734.84

749.28

763.71

778.15

92.59

807.04

821.49

835.97

850.47

864.99

879.54
101.00

102.00

103.00

104.00

105.00

106.00

107.00

108.00

109.00

82.96

83.89

84.80

85.72

86.63

87.54

88.45

89.36

90.26

91.17

111.00

112.00

113.00

114.00

115.00

116.00

117.00

118.00

119.00

120.00

121.00

117.97

130.90

143.47

155.69

167.59

179.20

190.54

201.63

212.51

223.18

233.68

244.02
254.20

264.27

274.21

284.05

293.80

303.47

313.07

322.61

332.10

341.54

350.96

360.35

369.71

379.07

388.41

397.76

407.11

416.47

425.85

435.24

444.66

454.11

463.60

473.11

482.67

492.26

501.91

511.60

92.07

92.97
93.86

94.76

95.65

96.55

97.44

98.33

99.22

100.11

101.00

101.89

123.00

124.00

125.00

126.00

127.00

128.00

129.00

130.00
20. 517

888

21.246
21.590

21.921

22. 241

22.549

22.847

23.135

23.414

23.685

23. 948

24.203

24.452

24.694

24.930

25.160

25.385

25.605

25.821

26. 032

26.239

26.442

26.642

26. 838

27.031

27.221

27.408

27.592

27.774

27.953

28.130

28.305

28.478

28.649

28.986

.

29.316

29.478
1042.03

1057.04

1072.09

1087.19

1102.34

1117.54

1132.79

1148.08

1163.42

1178.81 
TEMPER- SPECIFIC ENTHALPY INTERNAL ENTROPY ATURE VOLUME (J/GM) ENERGY (J/GM-K) (K)
(J/GM) $\begin{array}{ccccc}\text { TEMPER- } & \text { SPECIFIC } & \text { ENTHALPY } & \text { INTERNAL } & \text { ENTROPY } \\ \text { ATURE } & \text { VOLUME } & (J / G M) & \text { ENERGY } & \text { (J/GM-K) } \\ (K) & (C C / G M) & & \text { (J/GM) } & \end{array}$

\begin{tabular}{|c|c|c|c|c|c|c|c|c|c|}
\hline $\begin{array}{l}161.00 \\
162.00 \\
163.00 \\
164.00 \\
165.00 \\
166.00 \\
167.00 \\
168.00 \\
169.00 \\
170.00\end{array}$ & $\begin{array}{l}135.71 \\
136.56 \\
137.42 \\
138.27 \\
139.12 \\
139.97 \\
140.82 \\
141.67 \\
142.52 \\
143.37\end{array}$ & $\begin{array}{l}2011.90 \\
2028.73 \\
2045.55 \\
2062.38 \\
2079.20 \\
2096.03 \\
2112.85 \\
2129.67 \\
2146.49 \\
2163.31\end{array}$ & $\begin{array}{l}1324.34 \\
1336.84 \\
1349.35 \\
1361.86 \\
1374.37 \\
1386.88 \\
1399.40 \\
1411.91 \\
1424.42 \\
1436.93\end{array}$ & $\begin{array}{l}38.682 \\
38.786 \\
38.890 \\
38.992 \\
39.095 \\
39.196 \\
39.297 \\
39.398 \\
39.498 \\
39.597\end{array}$ & $\begin{array}{l}231.00 \\
232.00 \\
233.00 \\
234.00 \\
235.00 \\
236.00 \\
237.00 \\
238.00 \\
239.00 \\
240.00\end{array}$ & $\begin{array}{l}194.52 \\
195.35 \\
196.18 \\
197.01 \\
197.84 \\
198.67 \\
199.50 \\
200.33 \\
201.16 \\
201.99\end{array}$ & $\begin{array}{l}3161.99 \\
3177.79 \\
3193.58 \\
3209.36 \\
3225.12 \\
3240.86 \\
3256.59 \\
3272.30 \\
3288.00 \\
3303.68\end{array}$ & $\begin{array}{l}2176.47 \\
2188.06 \\
2199.64 \\
2211.21 \\
2222.76 \\
2234.30 \\
2245.82 \\
2257.33 \\
2268.82 \\
2280.30\end{array}$ & $\begin{array}{l}44.625 \\
44.694 \\
44.762 \\
44.829 \\
44.896 \\
44.963 \\
45.030 \\
45.096 \\
45.162 \\
45.227\end{array}$ \\
\hline $\begin{array}{l}171.00 \\
172.00 \\
173.00 \\
174.00 \\
175.00 \\
176.00 \\
177.00 \\
178.00 \\
179.00 \\
180.00\end{array}$ & $\begin{array}{l}144.22 \\
145.07 \\
145.91 \\
146.76 \\
147.61 \\
148.45 \\
149.30 \\
150.14 \\
150.99 \\
151.83\end{array}$ & $\begin{array}{l}2180.13 \\
2196.94 \\
2213.75 \\
2230.55 \\
2247.35 \\
2264.13 \\
2280.91 \\
2297.68 \\
2314.44 \\
2331.19\end{array}$ & $\begin{array}{l}1449.45 \\
1461.97 \\
1474.49 \\
1487.00 \\
1499.51 \\
1512.01 \\
1524.50 \\
1536.99 \\
1549.47 \\
1561.95\end{array}$ & $\begin{array}{l}39.696 \\
39.794 \\
39.891 \\
39.988 \\
40.084 \\
40.180 \\
40.275 \\
40.369 \\
40.463 \\
40.556\end{array}$ & $\begin{array}{l}241.00 \\
242.00 \\
243.00 \\
244.00 \\
245.00 \\
246.00 \\
247.00 \\
248.00 \\
249.00 \\
250.00\end{array}$ & $\begin{array}{l}202.82 \\
203.65 \\
204.48 \\
205.31 \\
206.14 \\
206.97 \\
207.80 \\
208.62 \\
209.45 \\
210.28\end{array}$ & $\begin{array}{l}3319.35 \\
3335.00 \\
3350.64 \\
3366.26 \\
3381.86 \\
3397.45 \\
3413.03 \\
3428.59 \\
3444.13 \\
3459.66\end{array}$ & $\begin{array}{l}2291.77 \\
2303.22 \\
2314.66 \\
2326.08 \\
2337.49 \\
2348.88 \\
2360.26 \\
2371.62 \\
2382.97 \\
2394.31\end{array}$ & $\begin{array}{l}45.292 \\
45.357 \\
45.422 \\
45.486 \\
45.550 \\
45.613 \\
45.676 \\
45.739 \\
45.802 \\
45.864\end{array}$ \\
\hline $\begin{array}{l}181.00 \\
182.00 \\
183.00 \\
184.00 \\
185.00 \\
186.00 \\
187.00 \\
188.00 \\
189.00 \\
190.00\end{array}$ & $\begin{array}{l}152.68 \\
153.52 \\
154.36 \\
155.21 \\
156.05 \\
156.89 \\
157.73 \\
158.58 \\
159.42 \\
160.26\end{array}$ & $\begin{array}{l}2347.93 \\
2364.66 \\
2381.38 \\
2398.08 \\
2414.77 \\
2431.44 \\
2448.10 \\
2464.75 \\
2481.38 \\
2497.99\end{array}$ & $\begin{array}{l}1574.41 \\
1586.87 \\
1599.31 \\
1611.74 \\
1624.16 \\
1636.57 \\
1648.96 \\
1661.34 \\
1673.71 \\
1686.06\end{array}$ & $\begin{array}{l}40.649 \\
40.741 \\
40.833 \\
40.924 \\
41.014 \\
41.104 \\
41.194 \\
41.282 \\
41.371 \\
41.458\end{array}$ & $\begin{array}{l}251.00 \\
252.00 \\
253.00 \\
254.00 \\
255.00 \\
256.00 \\
257.00 \\
258.00 \\
259.00 \\
260.00\end{array}$ & $\begin{array}{l}211.11 \\
211.93 \\
212.76 \\
213.59 \\
214.41 \\
215.24 \\
216.07 \\
216.89 \\
217.72 \\
218.55\end{array}$ & $\begin{array}{l}3475.21 \\
3490.74 \\
3506.26 \\
3521.76 \\
3537.24 \\
3552.71 \\
3568.16 \\
3583.60 \\
3599.02 \\
3614.42\end{array}$ & $\begin{array}{l}2405.66 \\
2417.00 \\
2428.33 \\
2439.64 \\
2450.94 \\
2462.22 \\
2473.48 \\
2484.73 \\
2495.96 \\
2507.18\end{array}$ & $\begin{array}{l}45.926 \\
45.988 \\
46.049 \\
46.110 \\
46.171 \\
46.232 \\
46.292 \\
46.352 \\
46.411 \\
46.471\end{array}$ \\
\hline $\begin{array}{l}191.00 \\
192.00 \\
193.00 \\
194.00 \\
195.00 \\
196.00 \\
197.00 \\
198.00 \\
199.00 \\
200.00\end{array}$ & $\begin{array}{l}161.10 \\
161.94 \\
162.78 \\
163.62 \\
164.46 \\
165.30 \\
166.14 \\
166.98 \\
167.82 \\
168.65\end{array}$ & $\begin{array}{l}2514.58 \\
2531.16 \\
2547.72 \\
2564.27 \\
2580.79 \\
2597.30 \\
2613.78 \\
2630.25 \\
2646.69 \\
2663.12\end{array}$ & $\begin{array}{l}1698.40 \\
1710.72 \\
1723.02 \\
1735.31 \\
1747.57 \\
1759.83 \\
1772.06 \\
1784.28 \\
1796.47 \\
1808.65\end{array}$ & $\begin{array}{l}41.545 \\
41.632 \\
41.718 \\
41.804 \\
41.889 \\
41.973 \\
42.057 \\
42.140 \\
42.223 \\
42.305\end{array}$ & $\begin{array}{l}261.00 \\
262.00 \\
263.00 \\
264.00 \\
265.00 \\
266.00 \\
267.00 \\
268.00 \\
269.00 \\
270.00\end{array}$ & $\begin{array}{l}219.37 \\
220.20 \\
221.02 \\
221.85 \\
222.68 \\
223.50 \\
224.33 \\
225.15 \\
225.98 \\
226.80\end{array}$ & $\begin{array}{l}3629.81 \\
3645.18 \\
3660.53 \\
3675.87 \\
3691.19 \\
3706.50 \\
3721.79 \\
3737.06 \\
3752.32 \\
3767.56\end{array}$ & $\begin{array}{l}2518.38 \\
2529.57 \\
2540.74 \\
2551.89 \\
2563.03 \\
2574.15 \\
2585.26 \\
2596.35 \\
2607.43 \\
2618.49\end{array}$ & $\begin{array}{l}46.530 \\
46.589 \\
46.647 \\
46.705 \\
46.763 \\
46.821 \\
46.878 \\
46.935 \\
46.992 \\
47.049\end{array}$ \\
\hline $\begin{array}{l}201.00 \\
202.00 \\
203.00 \\
204.00 \\
205.00 \\
206.00 \\
207.00 \\
208.00 \\
209.00 \\
210.00\end{array}$ & $\begin{array}{l}169.49 \\
170.33 \\
171.17 \\
172.00 \\
172.84 \\
173.68 \\
174.51 \\
175.35 \\
176.19 \\
177.02\end{array}$ & $\begin{array}{l}2679.51 \\
2695.87 \\
2712.22 \\
2728.54 \\
2744.85 \\
2761.13 \\
2777.39 \\
2793.64 \\
2809.86 \\
2826.06\end{array}$ & $\begin{array}{l}1820.79 \\
1832.92 \\
1845.02 \\
1857.10 \\
1869.17 \\
1881.21 \\
1893.24 \\
1905.25 \\
1917.24 \\
1929.20\end{array}$ & $\begin{array}{l}42.387 \\
42.468 \\
42.549 \\
42.629 \\
42.709 \\
42.788 \\
42.867 \\
42.945 \\
43.023 \\
43.100\end{array}$ & $\begin{array}{l}271.00 \\
272.00 \\
273.00 \\
274.00 \\
275.00 \\
276.00 \\
277.00 \\
278.00 \\
279.00 \\
280.00\end{array}$ & $\begin{array}{l}227.63 \\
228.45 \\
229.28 \\
230.10 \\
230.93 \\
231.75 \\
232.58 \\
233.40 \\
234.23 \\
235.05\end{array}$ & $\begin{array}{l}3782.79 \\
3798.00 \\
3813.20 \\
3828.38 \\
3843.55 \\
3858.71 \\
3873.85 \\
3888.98 \\
3904.10 \\
3919.21\end{array}$ & $\begin{array}{l}2629.53 \\
2640.57 \\
2651.58 \\
2662.59 \\
2673.57 \\
2684.55 \\
2695.52 \\
2706.47 \\
2717.41 \\
2728.34\end{array}$ & $\begin{array}{l}47.105 \\
47.161 \\
47.217 \\
47.272 \\
47.328 \\
47.383 \\
47.437 \\
47.492 \\
47.546 \\
47.600\end{array}$ \\
\hline $\begin{array}{l}211.00 \\
212.00 \\
213.00 \\
214.00 \\
215.00 \\
216.00 \\
217.00 \\
218.00 \\
219.00 \\
220.00\end{array}$ & $\begin{array}{l}177.86 \\
178.69 \\
179.53 \\
180.36 \\
181.20 \\
182.03 \\
182.86 \\
183.70 \\
184.53 \\
185.37\end{array}$ & $\begin{array}{l}2842.24 \\
2858.41 \\
2874.55 \\
2890.67 \\
2906.78 \\
2922.86 \\
2938.93 \\
2954.98 \\
2971.01 \\
2987.02\end{array}$ & $\begin{array}{l}1941.15 \\
1953.09 \\
1965.00 \\
1976.89 \\
1988.77 \\
2000.63 \\
2012.47 \\
2024.29 \\
2036.09 \\
2047.88\end{array}$ & $\begin{array}{l}43.177 \\
43.254 \\
43.330 \\
43.405 \\
43.480 \\
43.555 \\
43.629 \\
43.703 \\
43.776 \\
43.849\end{array}$ & $\begin{array}{l}281.00 \\
282.00 \\
283.00 \\
284.00 \\
285.00 \\
286.00 \\
287.00 \\
288.00 \\
289.00 \\
290.00\end{array}$ & $\begin{array}{l}235.88 \\
236.70 \\
237.53 \\
238.35 \\
239.18 \\
240.00 \\
240.83 \\
241.65 \\
242.48 \\
243.30\end{array}$ & $\begin{array}{l}3934.31 \\
3949.40 \\
3964.48 \\
3979.56 \\
3994.62 \\
4009.68 \\
4024.74 \\
4039.79 \\
4054.83 \\
4069.87\end{array}$ & $\begin{array}{l}2739.26 \\
2750.17 \\
2761.07 \\
2771.97 \\
2782.86 \\
2793.74 \\
2804.62 \\
2815.49 \\
2826.36 \\
2837.22\end{array}$ & $\begin{array}{l}47.654 \\
47.708 \\
47.761 \\
47.814 \\
47.867 \\
47.920 \\
47.973 \\
48.025 \\
48.077 \\
48.129\end{array}$ \\
\hline $\begin{array}{l}221.00 \\
222.00 \\
223.00 \\
224.00 \\
225.00 \\
226.00 \\
227.00 \\
228.00 \\
229.00 \\
230.00\end{array}$ & $\begin{array}{l}186.20 \\
187.03 \\
187.87 \\
188.70 \\
189.53 \\
190.36 \\
191.19 \\
192.03 \\
192.86 \\
193.69\end{array}$ & $\begin{array}{l}3003.01 \\
3018.98 \\
3034.94 \\
3050.88 \\
3066.80 \\
3082.71 \\
3098.60 \\
3114.47 \\
3130.32 \\
3146.16\end{array}$ & $\begin{array}{l}2059.65 \\
2071841 \\
2083.14 \\
2094.86 \\
2106.57 \\
2118.26 \\
2129.93 \\
2141.59 \\
2153.23 \\
2164.85\end{array}$ & $\begin{array}{l}43.922 \\
43.994 \\
44.066 \\
44.137 \\
44.208 \\
44.278 \\
44.349 \\
44.418 \\
44.488 \\
44.557\end{array}$ & $\begin{array}{l}291.00 \\
292.00 \\
293.00 \\
294.00 \\
295.00 \\
296.00 \\
297.00 \\
298.00 \\
299.00 \\
300.00\end{array}$ & $\begin{array}{l}244.12 \\
244.95 \\
245.77 \\
246.59 \\
247.42 \\
248.24 \\
249.06 \\
249.89 \\
250.71 \\
251.53\end{array}$ & $\begin{array}{l}4084.91 \\
4099.95 \\
4114.99 \\
4130.02 \\
4145.06 \\
4160.09 \\
4175.13 \\
4190.17 \\
4205.20 \\
4220.24\end{array}$ & $\begin{array}{l}2848.09 \\
2858.95 \\
2869.82 \\
2880.68 \\
2891.54 \\
2902.41 \\
2913.28 \\
2924.15 \\
2935.02 \\
2945.89\end{array}$ & $\begin{array}{l}48.181 \\
48.232 \\
48.284 \\
48.335 \\
48.386 \\
48.437 \\
48.488 \\
48.538 \\
48.589 \\
48.639\end{array}$ \\
\hline
\end{tabular}




\begin{tabular}{|c|c|c|c|c|c|c|c|c|c|}
\hline $\begin{array}{l}\text { TEMPER- } \\
\text { ATURE } \\
\text { (K) }\end{array}$ & $\begin{array}{l}\text { SPECIFIC } \\
\text { VOLUME } \\
\text { (CC/GM) }\end{array}$ & $\begin{array}{l}\text { ENTHALPY } \\
(\mathrm{J} / \mathrm{GM})\end{array}$ & $\begin{array}{l}\text { I NTERNAL } \\
\text { ENERGY } \\
\text { (J/GM) }\end{array}$ & $\begin{array}{l}\text { ENTROPY } \\
(\mathrm{J} / \mathrm{GM}-\mathrm{K})\end{array}$ & $\begin{array}{l}\text { TEMPER- } \\
\text { ATURE } \\
\text { (K) }\end{array}$ & $\begin{array}{l}\text { SPECIFIC } \\
\text { VOLUME } \\
\text { (CC/GM) }\end{array}$ & $\begin{array}{l}\text { ENTHALPY } \\
\text { (J/GM) }\end{array}$ & $\begin{array}{l}\text { INTERNAL } \\
\text { ENERGY } \\
(\mathrm{J} / \mathrm{GM})\end{array}$ & $\begin{array}{l}\text { ENTROPY } \\
\text { (J/GM-K) }\end{array}$ \\
\hline
\end{tabular}

\begin{tabular}{|c|c|c|c|c|c|c|c|c|c|}
\hline 20.00 & 12.96 & -197.66 & -276.47 & 6.898 & & & & & \\
\hline $\begin{array}{l}21.00 \\
22.00 \\
23.00 \\
24.00 \\
25.00 \\
26.00 \\
27.00 \\
28.00 \\
29.00 \\
30.00\end{array}$ & $\begin{array}{l}13.09 \\
13.23 \\
13.38 \\
13.53 \\
13.70 \\
13.87 \\
14.06 \\
14.26 \\
14.47 \\
14.69\end{array}$ & $\begin{array}{r}-189.48 \\
-180.93 \\
-172.00 \\
-162.67 \\
-152.93 \\
-142.76 \\
-132.13 \\
-121.05 \\
-109.49 \\
-97.50\end{array}$ & $\begin{array}{l}-269.08 \\
-261.37 \\
-253.33 \\
-244.95 \\
-236.21 \\
-227.10 \\
-217.61 \\
-207.73 \\
-197.45 \\
-186.83\end{array}$ & $\begin{array}{l}7.300 \\
7.695 \\
8.094 \\
8.493 \\
8.888 \\
9.287 \\
9.687 \\
10.092 \\
10.495 \\
10.902\end{array}$ & $\begin{array}{r}91.00 \\
92.00 \\
93.00 \\
94.00 \\
95.00 \\
96.00 \\
97.00 \\
98.00 \\
99.00 \\
100.00\end{array}$ & $\begin{array}{l}61.68 \\
62.49 \\
63.29 \\
64.09 \\
64.89 \\
65.68 \\
66.47 \\
67.26 \\
68.05 \\
68.83\end{array}$ & $\begin{array}{r}881.45 \\
896.37 \\
911.31 \\
926.29 \\
941.30 \\
956.34 \\
971.42 \\
986.53 \\
1001.68 \\
1016.87\end{array}$ & $\begin{array}{l}506.46 \\
516.48 \\
526.54 \\
536.66 \\
546.82 \\
557.03 \\
567.30 \\
577.61 \\
587.98 \\
598.40\end{array}$ & $\begin{array}{l}28.751 \\
28.914 \\
29.076 \\
29.236 \\
29.395 \\
29.552 \\
29.708 \\
29.864 \\
30.017 \\
30.170\end{array}$ \\
\hline $\begin{array}{l}31.00 \\
32.00 \\
33.00 \\
34.00 \\
35.00 \\
36.00 \\
37.00 \\
38.00 \\
39.00 \\
40.00\end{array}$ & $\begin{array}{l}14.93 \\
15.19 \\
15.51 \\
15.80 \\
16.12 \\
16.46 \\
16.84 \\
17.24 \\
17.68 \\
18.15\end{array}$ & $\begin{array}{r}-84.98 \\
-71.99 \\
-58.68 \\
-44.49 \\
-29.68 \\
-14.39 \\
1.30 \\
17.31 \\
33.77 \\
50.22\end{array}$ & $\begin{array}{r}-175.77 \\
-164.34 \\
-152.95 \\
-140.56 \\
-127.68 \\
-114.49 \\
-101.05 \\
-87.50 \\
-73.68 \\
-60.11\end{array}$ & $\begin{array}{l}11.313 \\
11.728 \\
12.143 \\
12.569 \\
12.997 \\
13.426 \\
13.853 \\
14.280 \\
14.708 \\
15.124\end{array}$ & $\begin{array}{l}101.00 \\
102.00 \\
103.00 \\
104.00 \\
105.00 \\
106.00 \\
107.00 \\
108.00 \\
109.00 \\
110.00\end{array}$ & $\begin{array}{l}69.61 \\
70.39 \\
71.17 \\
71.94 \\
72.72 \\
73.49 \\
74.25 \\
75.02 \\
75.79 \\
76.55\end{array}$ & $\begin{array}{l}1032.10 \\
1047.38 \\
1062.70 \\
1078.07 \\
1093.48 \\
1108.93 \\
1124.41 \\
1139.94 \\
1155.50 \\
1171.10\end{array}$ & $\begin{array}{l}608.87 \\
619.41 \\
630.02 \\
640.68 \\
651.39 \\
662.15 \\
672.97 \\
683.84 \\
694.75 \\
705.71\end{array}$ & $\begin{array}{l}30.322 \\
30.472 \\
30.622 \\
30.770 \\
30.917 \\
31.064 \\
31.209 \\
31.354 \\
31.497 \\
31.640\end{array}$ \\
\hline $\begin{array}{l}41.00 \\
42.00 \\
43.00 \\
44.00 \\
45.00 \\
46.00 \\
47.00 \\
48.00 \\
49.00 \\
50.00\end{array}$ & $\begin{array}{l}18.66 \\
19.21 \\
19.81 \\
20.45 \\
21.13 \\
21.86 \\
22.63 \\
23.44 \\
24.28 \\
25.15\end{array}$ & $\begin{array}{r}66.81 \\
83.63 \\
100.74 \\
118.19 \\
135.95 \\
154.00 \\
172.30 \\
190.77 \\
209.35 \\
227.97\end{array}$ & $\begin{array}{r}-46.63 \\
-33.17 \\
-19.67 \\
-6.12 \\
7.48 \\
21.11 \\
34.72 \\
48.28 \\
61.74 \\
75.06\end{array}$ & $\begin{array}{l}15.533 \\
15.939 \\
16.341 \\
16.742 \\
17.142 \\
17.538 \\
17.932 \\
18.321 \\
18.704 \\
19.080\end{array}$ & $\begin{array}{l}111.00 \\
112.00 \\
113.00 \\
114.00 \\
115.00 \\
116.00 \\
117.00 \\
118.00 \\
119.00 \\
120.00\end{array}$ & $\begin{array}{l}77.31 \\
78.07 \\
78.83 \\
79.58 \\
80.34 \\
81.09 \\
81.84 \\
82.60 \\
83.35 \\
84.09\end{array}$ & $\begin{array}{l}1186.73 \\
1202.40 \\
1218.10 \\
1233.84 \\
1249.61 \\
1265.40 \\
1281.24 \\
1297.10 \\
1312.99 \\
1328.92\end{array}$ & $\begin{array}{l}716.72 \\
727.77 \\
738.87 \\
750.00 \\
761.18 \\
772.40 \\
783.65 \\
794.95 \\
806.28 \\
817.66\end{array}$ & $\begin{array}{l}31.781 \\
31.922 \\
32.061 \\
32.200 \\
32.338 \\
32.474 \\
32.610 \\
32.745 \\
32.879 \\
33.013\end{array}$ \\
\hline $\begin{array}{l}51.00 \\
52.00 \\
53.00 \\
54.00 \\
55.00 \\
56.00 \\
57.00 \\
58.00 \\
59.00 \\
60.00\end{array}$ & $\begin{array}{l}26.05 \\
26.97 \\
27.90 \\
28.84 \\
29.79 \\
30.75 \\
31.71 \\
32.66 \\
33.62 \\
34.58\end{array}$ & $\begin{array}{l}246.55 \\
265.06 \\
283.43 \\
301.65 \\
319.69 \\
337.52 \\
355.15 \\
372.57 \\
389.78 \\
406.79\end{array}$ & $\begin{array}{r}88.19 \\
101.12 \\
113.83 \\
126.32 \\
138.57 \\
150.59 \\
162.39 \\
173.98 \\
185.37 \\
196.57\end{array}$ & $\begin{array}{l}19.448 \\
19.807 \\
20.157 \\
20.498 \\
20.829 \\
21.150 \\
21.462 \\
21.765 \\
22.059 \\
22.345\end{array}$ & $\begin{array}{l}121.00 \\
122.00 \\
123.00 \\
124.00 \\
125.00 \\
126.00 \\
127.00 \\
128.00 \\
129.00 \\
130.00\end{array}$ & $\begin{array}{l}84.84 \\
85.59 \\
86.34 \\
87.08 \\
87.82 \\
88.56 \\
89.30 \\
90.04 \\
90.78 \\
91.51\end{array}$ & $\begin{array}{l}1344.87 \\
1360.85 \\
1376.86 \\
1392.91 \\
1408.99 \\
1425.19 \\
1441.42 \\
1457.69 \\
1474.00 \\
1490.35\end{array}$ & $\begin{array}{l}829.06 \\
840.50 \\
851.97 \\
863.49 \\
875.05 \\
886.75 \\
898.49 \\
910.28 \\
922.11 \\
933.99\end{array}$ & $\begin{array}{l}33.145 \\
33.276 \\
33.407 \\
33.537 \\
33.666 \\
33.795 \\
33.924 \\
34.051 \\
34.178 \\
34.304\end{array}$ \\
\hline $\begin{array}{l}61.00 \\
62.00 \\
63.00 \\
64.00 \\
65.00 \\
66.00 \\
67.00 \\
68.00 \\
69.00 \\
70.00\end{array}$ & $\begin{array}{l}35.53 \\
36.48 \\
37.42 \\
38.36 \\
39.29 \\
40.21 \\
41.13 \\
42.05 \\
42.96 \\
43.86\end{array}$ & $\begin{array}{l}423.60 \\
440.22 \\
456.66 \\
472.93 \\
489.05 \\
505.02 \\
520.85 \\
536.54 \\
552.12 \\
567.60\end{array}$ & $\begin{array}{l}207.60 \\
218.46 \\
229.18 \\
239.75 \\
250.20 \\
260.54 \\
270.77 \\
280.91 \\
290.97 \\
300.96\end{array}$ & $\begin{array}{l}22.623 \\
22.893 \\
23.157 \\
23.413 \\
23.663 \\
23.906 \\
24.144 \\
24.377 \\
24.605 \\
24.827\end{array}$ & $\begin{array}{l}131.00 \\
132.00 \\
133.00 \\
134.00 \\
135.00 \\
136.00 \\
137.00 \\
138.00 \\
139.00 \\
140.00\end{array}$ & $\begin{array}{l}92.25 \\
92.98 \\
93.71 \\
94.45 \\
95.18 \\
95.91 \\
96.64 \\
97.37 \\
98.10 \\
98.83\end{array}$ & $\begin{array}{l}1506.74 \\
1523.16 \\
1539.62 \\
1556.12 \\
1572.65 \\
1589.21 \\
1605.81 \\
1622.44 \\
1639.10 \\
1655.79\end{array}$ & $\begin{array}{r}945.91 \\
957.87 \\
969.87 \\
981.91 \\
993.99 \\
1006.11 \\
1018.26 \\
1030.45 \\
1042.67 \\
1054.92\end{array}$ & $\begin{array}{l}34.430 \\
34.555 \\
34.679 \\
34.803 \\
34.926 \\
35.048 \\
35.169 \\
35.290 \\
35.411 \\
35.530\end{array}$ \\
\hline $\begin{array}{l}71.00 \\
72.00 \\
73.00 \\
74.00 \\
75.00 \\
76.00 \\
77.00 \\
78.00 \\
79.00 \\
80.00\end{array}$ & $\begin{array}{l}44.76 \\
45.65 \\
46.53 \\
47.41 \\
48.29 \\
49.16 \\
50.02 \\
50.88 \\
51.74 \\
52.59\end{array}$ & $\begin{array}{l}582.97 \\
598.26 \\
613.46 \\
628.59 \\
643.66 \\
658.67 \\
673.63 \\
688.56 \\
703.44 \\
718.30\end{array}$ & $\begin{array}{l}310.88 \\
320.74 \\
330.56 \\
340.34 \\
350.09 \\
359.81 \\
369.52 \\
379.21 \\
388.90 \\
398.58\end{array}$ & $\begin{array}{l}25.045 \\
25.259 \\
25.469 \\
25.675 \\
25.877 \\
26.076 \\
26.271 \\
26.464 \\
26.653 \\
26.840\end{array}$ & $\begin{array}{l}141.00 \\
142.00 \\
143.00 \\
144.00 \\
145.00 \\
146.00 \\
147.00 \\
148.00 \\
149.00 \\
150.00\end{array}$ & $\begin{array}{r}99.56 \\
100.29 \\
101.02 \\
101.75 \\
102.48 \\
103.21 \\
103.93 \\
104.66 \\
105.39 \\
106.11\end{array}$ & $\begin{array}{l}1672.51 \\
1689.26 \\
1706.04 \\
1722.84 \\
1739.66 \\
1756.50 \\
1773.37 \\
1790.25 \\
1807.15 \\
1824.07\end{array}$ & $\begin{array}{l}1067.21 \\
1079.52 \\
1091.87 \\
1104.24 \\
1116.63 \\
1129.05 \\
1141.49 \\
1153.96 \\
1166.44 \\
1178.94\end{array}$ & $\begin{array}{l}35.649 \\
35.768 \\
35.885 \\
36.003 \\
36.119 \\
36.235 \\
36.350 \\
36.464 \\
36.578 \\
36.691\end{array}$ \\
\hline $\begin{array}{l}81.00 \\
82.00 \\
83.00 \\
84.00 \\
85.00 \\
86.00 \\
87.00 \\
88.00 \\
89.00 \\
90.00\end{array}$ & $\begin{array}{l}53.44 \\
54.28 \\
55.11 \\
55.95 \\
56.78 \\
57.60 \\
58.43 \\
59.25 \\
60.06 \\
60.87\end{array}$ & $\begin{array}{l}733.14 \\
747.96 \\
762.77 \\
777.58 \\
792.38 \\
807.19 \\
822.01 \\
836.84 \\
851.69 \\
866.56\end{array}$ & $\begin{array}{l}408.28 \\
417.98 \\
427.70 \\
437.44 \\
447.20 \\
456.98 \\
466.80 \\
476.66 \\
486.55 \\
496.48\end{array}$ & $\begin{array}{l}27.025 \\
27.207 \\
27.386 \\
27.563 \\
27.739 \\
27.912 \\
28.083 \\
28.253 \\
28.420 \\
28.587\end{array}$ & $\begin{array}{l}151.00 \\
152.00 \\
153.00 \\
154.00 \\
155.00 \\
156.00 \\
157.00 \\
158.00 \\
159.00 \\
160.00\end{array}$ & $\begin{array}{l}106.83 \\
107.55 \\
108.27 \\
108.99 \\
109.71 \\
110.43 \\
111.15 \\
111.87 \\
112.58 \\
113.30\end{array}$ & $\begin{array}{l}1840.92 \\
1857.78 \\
1874.64 \\
1891.52 \\
1908.40 \\
1925.30 \\
1942.20 \\
1959.10 \\
1976.01 \\
1992.92\end{array}$ & $\begin{array}{l}1191.40 \\
1203.88 \\
1216.37 \\
1228.87 \\
1241.38 \\
1253.91 \\
1266.44 \\
1278.98 \\
1291.53 \\
1304.09\end{array}$ & $\begin{array}{l}36.803 \\
36.914 \\
37.025 \\
37.135 \\
37.244 \\
37.353 \\
37.461 \\
37.568 \\
37.675 \\
37.781\end{array}$ \\
\hline
\end{tabular}


TEMPER- SPECIFIC ENTHALPY INTERNAL ENTROPY ATURE VOLUME (J/GM) ENERGY (J/GM-K) (K) ( $C$ C / $/ \mathrm{GM}$ ) (J/GM)

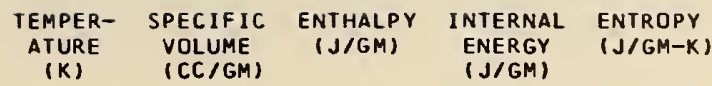

\begin{tabular}{|c|c|c|c|c|c|c|c|c|c|}
\hline $\begin{array}{l}161.00 \\
162.00 \\
163.00 \\
164.00 \\
165.00 \\
166.00 \\
167.00 \\
168.00 \\
169.00 \\
170.00\end{array}$ & $\begin{array}{l}114.02 \\
114.73 \\
115.45 \\
116.16 \\
116.88 \\
117.59 \\
118.31 \\
119.02 \\
119.73 \\
120.44\end{array}$ & $\begin{array}{l}2009.84 \\
2026.76 \\
2043.68 \\
2060.61 \\
2077.53 \\
2094.46 \\
2111.38 \\
2128.30 \\
2145.22 \\
2162.14\end{array}$ & $\begin{array}{l}1316.65 \\
1329.22 \\
1341.80 \\
1354.37 \\
1366.96 \\
1379.54 \\
1392.12 \\
1404.71 \\
1417.30 \\
1429.88\end{array}$ & $\begin{array}{l}37.886 \\
37.991 \\
38.095 \\
38.199 \\
38.302 \\
38.404 \\
38.506 \\
38.607 \\
38.707 \\
38.807\end{array}$ & $\begin{array}{l}231.00 \\
232.00 \\
233.00 \\
234.00 \\
235.00 \\
236.00 \\
237.00 \\
238.00 \\
239.00 \\
240.00\end{array}$ & $\begin{array}{l}163.26 \\
163.95 \\
164.64 \\
165.34 \\
166.03 \\
166.73 \\
167.42 \\
168.12 \\
168.81 \\
169.50\end{array}$ & $\begin{array}{l}3164.16 \\
3179.99 \\
3195.80 \\
3211.60 \\
3227.39 \\
3243.16 \\
3258.92 \\
3274.67 \\
3290.41 \\
3306.13\end{array}$ & $\begin{array}{l}2171.63 \\
2183.23 \\
2194.82 \\
2206.40 \\
2217.96 \\
2229.52 \\
2241.06 \\
2252.59 \\
2264.11 \\
2275.62\end{array}$ & $\begin{array}{l}43.853 \\
43.922 \\
43.990 \\
44.057 \\
44.125 \\
44.192 \\
44.258 \\
44.325 \\
44.391 \\
44.456\end{array}$ \\
\hline $\begin{array}{l}171.00 \\
172.00 \\
173.00 \\
174.00 \\
175.00 \\
176.00 \\
177.00 \\
178.00 \\
179.00 \\
180.00\end{array}$ & $\begin{array}{l}121.15 \\
121.87 \\
122.58 \\
123.28 \\
123.99 \\
124.70 \\
125.41 \\
126.12 \\
126.83 \\
127.53\end{array}$ & $\begin{array}{l}2179.07 \\
2195.99 \\
2212.91 \\
2229.82 \\
2246.73 \\
2263.63 \\
2280.53 \\
2297.41 \\
2314.29 \\
2331.15\end{array}$ & $\begin{array}{l}1442.49 \\
1455.09 \\
1467.69 \\
1480.29 \\
1492.89 \\
1505.48 \\
1518.07 \\
1530.65 \\
1543.22 \\
1555.78\end{array}$ & $\begin{array}{l}38.906 \\
39.005 \\
39.103 \\
39.200 \\
39.297 \\
39.394 \\
39.489 \\
39.584 \\
39.679 \\
39.773\end{array}$ & $\begin{array}{l}241.00 \\
242.00 \\
243.00 \\
244.00 \\
245.00 \\
246.00 \\
247.00 \\
248.00 \\
249.00 \\
250.00\end{array}$ & $\begin{array}{l}170.20 \\
170.89 \\
171.58 \\
172.27 \\
172.97 \\
173.66 \\
174.35 \\
175.04 \\
175.73 \\
176.43\end{array}$ & $\begin{array}{l}3321.84 \\
3337.54 \\
3353.22 \\
3368.89 \\
3384.54 \\
3400.19 \\
3415.81 \\
3431.43 \\
3447.03 \\
3462.61\end{array}$ & $\begin{array}{l}2287.11 \\
2298.59 \\
2310.07 \\
2321.52 \\
2332.97 \\
2344.40 \\
2355.83 \\
2367.23 \\
2378.63 \\
2390.01\end{array}$ & $\begin{array}{l}44.522 \\
44.587 \\
44.651 \\
44.716 \\
44.780 \\
44.843 \\
44.907 \\
44.970 \\
45.033 \\
45.095\end{array}$ \\
\hline $\begin{array}{l}181.00 \\
182.00 \\
183.00 \\
184.00 \\
185.00 \\
186.00 \\
187.00 \\
188.00 \\
189.00 \\
190.00\end{array}$ & $\begin{array}{l}128.24 \\
128.95 \\
129.65 \\
130.36 \\
131.07 \\
131.77 \\
132.48 \\
133.18 \\
133.89 \\
134.59\end{array}$ & $\begin{array}{l}2348.00 \\
2364.84 \\
2381.67 \\
2398.48 \\
2415.27 \\
2432.05 \\
2448.82 \\
2465.56 \\
2482.29 \\
2499.00\end{array}$ & $\begin{array}{l}1568.34 \\
1580.88 \\
1593.41 \\
1605.93 \\
1618.43 \\
1630.93 \\
1643.40 \\
1655.86 \\
1668.31 \\
1680.74\end{array}$ & $\begin{array}{l}39.866 \\
39.959 \\
40.051 \\
40.143 \\
40.234 \\
40.324 \\
40.414 \\
40.504 \\
40.592 \\
40.680\end{array}$ & $\begin{array}{l}251.00 \\
252.00 \\
253.00 \\
254.00 \\
255.00 \\
256.00 \\
257.00 \\
258.00 \\
259.00 \\
260.00\end{array}$ & $\begin{array}{l}177.12 \\
177.81 \\
178.50 \\
179.19 \\
179.88 \\
180.57 \\
181.26 \\
181.95 \\
182.64 \\
183.33\end{array}$ & $\begin{array}{l}3478.22 \\
3493.81 \\
3509.38 \\
3524.94 \\
3540.48 \\
3556.00 \\
3571.51 \\
3587.00 \\
3602.47 \\
3617.92\end{array}$ & $\begin{array}{l}2401.41 \\
2412.80 \\
2424.17 \\
2435.52 \\
2446.86 \\
2458.19 \\
2469.50 \\
2480.79 \\
2492.06 \\
2503.32\end{array}$ & $\begin{array}{l}45.157 \\
45.219 \\
45.281 \\
45.342 \\
45.403 \\
45.464 \\
45.525 \\
45.585 \\
45.645 \\
45.704\end{array}$ \\
\hline $\begin{array}{l}191.00 \\
192.00 \\
193.00 \\
194.00 \\
195.00 \\
196.00 \\
197.00 \\
198.00 \\
199.00 \\
200.00\end{array}$ & $\begin{array}{l}135.29 \\
136.00 \\
136.70 \\
137.40 \\
138.11 \\
138.81 \\
139.51 \\
140.22 \\
140.92 \\
141.62\end{array}$ & $\begin{array}{l}2515.69 \\
2532.36 \\
2549.01 \\
2565.63 \\
2582.24 \\
2598.82 \\
2615.38 \\
2631.91 \\
2648.42 \\
2664.91\end{array}$ & $\begin{array}{l}1693.15 \\
1705.54 \\
1717.91 \\
1730.26 \\
1742.59 \\
1754.90 \\
1767.19 \\
1779.45 \\
1791.69 \\
1803.91\end{array}$ & $\begin{array}{l}40.768 \\
40.855 \\
40.942 \\
41.028 \\
41.113 \\
41.198 \\
41.282 \\
41.366 \\
41.449 \\
41.532\end{array}$ & $\begin{array}{l}261.00 \\
262.00 \\
263.00 \\
264.00 \\
265.00 \\
266.00 \\
267.00 \\
268.00 \\
269.00 \\
270.00\end{array}$ & $\begin{array}{l}184.02 \\
184.71 \\
185.40 \\
186.09 \\
186.78 \\
187.47 \\
188.16 \\
188.85 \\
189.54 \\
190.23\end{array}$ & $\begin{array}{l}3633.35 \\
3648.77 \\
3664.17 \\
3679.54 \\
3694.90 \\
3710.24 \\
3725.56 \\
3740.86 \\
3756.15 \\
3771.41\end{array}$ & $\begin{array}{l}2514.56 \\
2525.78 \\
2536.98 \\
2548.16 \\
2559.33 \\
2570.47 \\
2581.60 \\
2592.71 \\
2603.80 \\
2614.87\end{array}$ & $\begin{array}{l}45.763 \\
45.822 \\
45.881 \\
45.939 \\
45.997 \\
46.055 \\
46.113 \\
46.170 \\
46.227 \\
46.283\end{array}$ \\
\hline $\begin{array}{l}201.00 \\
202.00 \\
203.00 \\
204.00 \\
205.00 \\
206.00 \\
207.00 \\
208.00 \\
209.00 \\
210.00\end{array}$ & $\begin{array}{l}142.32 \\
143.02 \\
143.72 \\
144.42 \\
145.12 \\
145.82 \\
146.52 \\
147.22 \\
147.92 \\
148.62\end{array}$ & $\begin{array}{l}2681.34 \\
2697.74 \\
2714.12 \\
2730.48 \\
2746.81 \\
2763.11 \\
2779.40 \\
2795.66 \\
2811.89 \\
2828.11\end{array}$ & $\begin{array}{l}1816.08 \\
1828.22 \\
1840.34 \\
1852.44 \\
1864.51 \\
1876.56 \\
1888.59 \\
1900.60 \\
1912.59 \\
1924.55\end{array}$ & $\begin{array}{l}41.613 \\
41.695 \\
41.776 \\
41.856 \\
41.936 \\
42.015 \\
42.094 \\
42.173 \\
42.250 \\
42.328\end{array}$ & $\begin{array}{l}271.00 \\
272.00 \\
273.00 \\
274.00 \\
275.00 \\
276.00 \\
277.00 \\
278.00 \\
279.00 \\
280.00\end{array}$ & $\begin{array}{l}190.92 \\
191.61 \\
192.30 \\
192.99 \\
193.68 \\
194.37 \\
195.06 \\
195.74 \\
196.43 \\
197.12\end{array}$ & $\begin{array}{l}3786.66 \\
3801.88 \\
3817.09 \\
3832.29 \\
3847.46 \\
3862.63 \\
3877.77 \\
3892.91 \\
3908.03 \\
3923.13\end{array}$ & $\begin{array}{l}2625.93 \\
2636.96 \\
2647.98 \\
2658.99 \\
2669.97 \\
2680.95 \\
2691.90 \\
2702.85 \\
2713.78 \\
2724.70\end{array}$ & $\begin{array}{l}46.340 \\
46.396 \\
46.452 \\
46.507 \\
46.563 \\
46.618 \\
46.672 \\
46.727 \\
46.781 \\
46.835\end{array}$ \\
\hline $\begin{array}{l}211.00 \\
212.00 \\
213.00 \\
214.00 \\
215.00 \\
216.00 \\
217.00 \\
218.00 \\
219.00 \\
220.00\end{array}$ & $\begin{array}{l}149.32 \\
150.02 \\
150.72 \\
151.41 \\
152.11 \\
152.81 \\
153.51 \\
154.21 \\
154.90 \\
155.60\end{array}$ & $\begin{array}{l}2844.30 \\
2860.47 \\
2876.62 \\
2892.75 \\
2908.85 \\
2924.94 \\
2941.01 \\
2957.06 \\
2973.09 \\
2989.10\end{array}$ & $\begin{array}{l}1936.49 \\
1948.42 \\
1960.32 \\
1972.20 \\
1984.06 \\
1995.91 \\
2007.73 \\
2019.54 \\
2031.33 \\
2043.11\end{array}$ & $\begin{array}{l}42.405 \\
42.481 \\
42.557 \\
42.633 \\
42.708 \\
42.782 \\
42.857 \\
42.930 \\
43.004 \\
43.077\end{array}$ & $\begin{array}{l}281.00 \\
282.00 \\
283.00 \\
284.00 \\
285.00 \\
286.00 \\
287.00 \\
288.00 \\
289.00 \\
290.00\end{array}$ & $\begin{array}{l}197.81 \\
398.50 \\
199.19 \\
199.88 \\
200.57 \\
201.25 \\
201.94 \\
202.63 \\
203.32 \\
204.01\end{array}$ & $\begin{array}{l}3938.23 \\
3953.32 \\
3968.40 \\
3983.47 \\
3998.53 \\
4013.59 \\
4028.65 \\
4043.71 \\
4058.76 \\
4073.81\end{array}$ & $\begin{array}{l}2735.61 \\
2746.51 \\
2757.40 \\
2768.28 \\
2779.16 \\
2790.03 \\
2800.91 \\
2811.78 \\
2822.65 \\
2833.52\end{array}$ & $\begin{array}{l}46.889 \\
46.943 \\
46.996 \\
47.049 \\
47.102 \\
47.155 \\
47.207 \\
47.260 \\
47.312 \\
47.364\end{array}$ \\
\hline $\begin{array}{l}221.00 \\
222.00 \\
223.00 \\
224.00 \\
225.00 \\
226.00 \\
227.00 \\
228.00 \\
229.00 \\
230.00\end{array}$ & $\begin{array}{l}156.30 \\
156.99 \\
157.69 \\
158.39 \\
159.08 \\
159.78 \\
160.47 \\
161.17 \\
161.87 \\
162.56\end{array}$ & $\begin{array}{l}3005.09 \\
3021.07 \\
3037.03 \\
3052.98 \\
3068.90 \\
3084.82 \\
3100.72 \\
3116.60 \\
3132.47 \\
3148.32\end{array}$ & $\begin{array}{l}2054.86 \\
2066.61 \\
2078.33 \\
2090.04 \\
2101.74 \\
2113.42 \\
2125.09 \\
2136.74 \\
2148.38 \\
2160.01\end{array}$ & $\begin{array}{l}43.149 \\
43.221 \\
43.293 \\
43.365 \\
43.435 \\
43.506 \\
43.576 \\
43.646 \\
43.715 \\
43.785\end{array}$ & $\begin{array}{l}291.00 \\
292.00 \\
293.00 \\
294.00 \\
295.00 \\
296.00 \\
297.00 \\
298.00 \\
299.00 \\
300.00\end{array}$ & $\begin{array}{l}204.69 \\
205.38 \\
206.07 \\
206.76 \\
207.44 \\
208.13 \\
208.82 \\
209.50 \\
210.19 \\
210.88\end{array}$ & $\begin{array}{l}4088.87 \\
4103.93 \\
4119.00 \\
4134.07 \\
4149.14 \\
4164.22 \\
4179.31 \\
4194.41 \\
4209.52 \\
4224.63\end{array}$ & $\begin{array}{l}2844.40 \\
2855.28 \\
2866.16 \\
2877.05 \\
2887.95 \\
2898.86 \\
2909.77 \\
2920.70 \\
2931.63 \\
2942.58\end{array}$ & $\begin{array}{l}47.416 \\
47.468 \\
47.519 \\
47.570 \\
47.622 \\
47.673 \\
47.724 \\
47.774 \\
47.825 \\
47.875\end{array}$ \\
\hline
\end{tabular}


$\begin{array}{ccccc}\text { TEMPER- } & \text { SPECIFIC } & \text { ENTHALPY } & \text { INTERNAL } & \text { ENTROPY } \\ \text { ATURE } & \text { VOLUME } & \text { (J/GM) } & \text { ENERGY } & \text { (J/GM-K) } \\ \text { (K) } & \text { (CC/GM) } & & & \end{array}$ (X)
ENERGY
TEMPERATURE

(K)
SPECIFIC ENTHALPY (CC/GM)

(J/GM)
INTERNAL ENTROPY (J/GM)

\begin{tabular}{|c|c|c|c|c|c|c|c|c|c|}
\hline 20.00 & 12.83 & -187.07 & -278.10 & 6.774 & & & & & \\
\hline $\begin{array}{l}21.00 \\
22.00 \\
23.00 \\
24.00 \\
25.00 \\
26.00 \\
27.00 \\
28.00 \\
29.00 \\
30.00\end{array}$ & $\begin{array}{l}12.96 \\
13.08 \\
13.22 \\
13.36 \\
13.51 \\
13.68 \\
13.84 \\
14.02 \\
14.21 \\
14.42\end{array}$ & $\begin{array}{l}-179.03 \\
-170.65 \\
-161.90 \\
-152.78 \\
-143.27 \\
-133.35 \\
-123.01 \\
-112.23 \\
-101.02 \\
-89.42\end{array}$ & $\begin{array}{l}-270.93 \\
-263.45 \\
-255.67 \\
-247.57 \\
-239.13 \\
-230.35 \\
-221.20 \\
-211.71 \\
-201.84 \\
-191.67\end{array}$ & $\begin{array}{r}7.169 \\
7.556 \\
7.947 \\
8.337 \\
8.724 \\
9.112 \\
9.502 \\
9.895 \\
10.286 \\
10.680\end{array}$ & $\begin{array}{r}91.00 \\
92.00 \\
93.00 \\
94.00 \\
95.00 \\
96.00 \\
97.00 \\
98.00 \\
99.00 \\
100.00\end{array}$ & $\begin{array}{l}53.30 \\
54.00 \\
54.69 \\
55.39 \\
56.08 \\
56.77 \\
57.45 \\
58.13 \\
58.82 \\
59.50\end{array}$ & $\begin{array}{r}870.25 \\
885.43 \\
900.63 \\
915.86 \\
931.11 \\
946.40 \\
961.71 \\
977.06 \\
992.43 \\
1007.84\end{array}$ & $\begin{array}{l}492.20 \\
502.43 \\
512.70 \\
523.02 \\
533.37 \\
543.77 \\
554.23 \\
564.72 \\
575.26 \\
585.85\end{array}$ & $\begin{array}{l}27.990 \\
28.156 \\
28.321 \\
28.484 \\
28.645 \\
28.805 \\
28.964 \\
29.121 \\
29.277 \\
29.432\end{array}$ \\
\hline $\begin{array}{l}31.00 \\
32.00 \\
33.00 \\
34.00 \\
35.00 \\
36.00 \\
37.00 \\
38.00 \\
39.00 \\
40.00\end{array}$ & $\begin{array}{l}14.63 \\
14.86 \\
15.13 \\
15.38 \\
15.65 \\
15.94 \\
16.25 \\
16.58 \\
16.94 \\
17.31\end{array}$ & $\begin{array}{r}-77.34 \\
-64.85 \\
-52.04 \\
-38.51 \\
-24.42 \\
-9.94 \\
4.86 \\
19.88 \\
35.23 \\
50.45\end{array}$ & $\begin{array}{l}-181.10 \\
-170.22 \\
-159.32 \\
-147.59 \\
-135.43 \\
-123.00 \\
-110.40 \\
-97.73 \\
-84.90 \\
-72.35\end{array}$ & $\begin{array}{l}11.076 \\
11.475 \\
11.875 \\
12.280 \\
12.688 \\
13.094 \\
13.497 \\
13.897 \\
14.296 \\
14.681\end{array}$ & $\begin{array}{l}101.00 \\
102.00 \\
103.00 \\
104.00 \\
105.00 \\
106.00 \\
107.00 \\
108.00 \\
109.00 \\
110.00\end{array}$ & $\begin{array}{l}60.17 \\
60.85 \\
61.52 \\
62.20 \\
62.87 \\
63.54 \\
64.20 \\
64.87 \\
65.53 \\
66.20\end{array}$ & $\begin{array}{l}1023.26 \\
1038.69 \\
1054.16 \\
1069.68 \\
1085.24 \\
1100.84 \\
1116.48 \\
1132.17 \\
1147.89 \\
1163.65\end{array}$ & $\begin{array}{l}596.45 \\
607.09 \\
617.78 \\
628.53 \\
639.33 \\
650.18 \\
661.09 \\
672.05 \\
683.06 \\
694.12\end{array}$ & $\begin{array}{l}29.585 \\
29.737 \\
29.888 \\
30.038 \\
30.187 \\
30.335 \\
30.482 \\
30.628 \\
30.773 \\
30.917\end{array}$ \\
\hline $\begin{array}{l}41.00 \\
42.00 \\
43.00 \\
44.00 \\
45.00 \\
46.00 \\
47.00 \\
48.00 \\
49.00 \\
50.00\end{array}$ & $\begin{array}{l}17.72 \\
18.15 \\
18.61 \\
19.10 \\
19.62 \\
20.17 \\
20.74 \\
21.35 \\
21.99 \\
22.64\end{array}$ & $\begin{array}{r}65.70 \\
81.08 \\
96.67 \\
112.51 \\
128.62 \\
145.03 \\
161.69 \\
178.61 \\
195.74 \\
213.04\end{array}$ & $\begin{array}{r}-59.97 \\
-47.65 \\
-35.33 \\
-22.95 \\
-10.51 \\
2.00 \\
14.56 \\
27.18 \\
39.81 \\
52.42\end{array}$ & $\begin{array}{l}15.058 \\
15.428 \\
15.795 \\
16.159 \\
16.521 \\
16.882 \\
17.240 \\
17.596 \\
17.950 \\
18.299\end{array}$ & $\begin{array}{l}111.00 \\
112.00 \\
113.00 \\
114.00 \\
115.00 \\
116.00 \\
117.00 \\
118.00 \\
119.00 \\
120.00\end{array}$ & $\begin{array}{l}66.86 \\
67.52 \\
68.17 \\
68.83 \\
69.48 \\
70.13 \\
70.78 \\
71.43 \\
72.08 \\
72.73\end{array}$ & $\begin{array}{l}1179.44 \\
1195.28 \\
1211.15 \\
1227.05 \\
1242.99 \\
1258.97 \\
1274.98 \\
1291.02 \\
1307.10 \\
1323.21\end{array}$ & $\begin{array}{l}705.24 \\
716.40 \\
727.61 \\
738.86 \\
750.17 \\
761.52 \\
772.91 \\
784.35 \\
795.84 \\
807.37\end{array}$ & $\begin{array}{l}31.060 \\
31.202 \\
31.343 \\
31.483 \\
31.622 \\
31.760 \\
31.898 \\
32.034 \\
32.170 \\
32.305\end{array}$ \\
\hline $\begin{array}{l}51.00 \\
52.00 \\
53.00 \\
54.00 \\
55.00 \\
56.00 \\
57.00 \\
58.00 \\
59.00 \\
60.00\end{array}$ & $\begin{array}{l}23.33 \\
24.03 \\
24.75 \\
25.49 \\
26.24 \\
27.01 \\
27.78 \\
28.55 \\
29.33 \\
30.12\end{array}$ & $\begin{array}{l}230.46 \\
247.96 \\
265.50 \\
283.05 \\
300.55 \\
317.99 \\
335.35 \\
352.60 \\
369.74 \\
386.75\end{array}$ & $\begin{array}{r}65.00 \\
77.51 \\
89.93 \\
102.23 \\
114.41 \\
126.45 \\
138.35 \\
150.09 \\
161.69 \\
173.14\end{array}$ & $\begin{array}{l}18.644 \\
18.984 \\
19.318 \\
19.646 \\
19.967 \\
20.281 \\
20.589 \\
20.889 \\
21.182 \\
21.468\end{array}$ & $\begin{array}{l}121.00 \\
122.00 \\
123.00 \\
124.00 \\
125.00 \\
126.00 \\
127.00 \\
128.00 \\
129.00 \\
130.00\end{array}$ & $\begin{array}{l}73.37 \\
74.01 \\
74.65 \\
75.29 \\
75.93 \\
76.57 \\
77.21 \\
77.85 \\
78.48 \\
79.12\end{array}$ & $\begin{array}{l}1339.34 \\
1355.50 \\
1371.70 \\
1387.93 \\
1404.19 \\
1420.55 \\
1436.95 \\
1453.38 \\
1469.85 \\
1486.35\end{array}$ & $\begin{array}{l}818.93 \\
830.53 \\
842.18 \\
853.87 \\
865.61 \\
877.43 \\
889.30 \\
901.21 \\
913.17 \\
925.16\end{array}$ & $\begin{array}{l}32.439 \\
32.572 \\
32.704 \\
32.835 \\
32.966 \\
33.096 \\
33.226 \\
33.355 \\
33.483 \\
33.610\end{array}$ \\
\hline $\begin{array}{l}61.00 \\
62.00 \\
63.00 \\
64.00 \\
65.00 \\
66.00 \\
67.00 \\
68.00 \\
69.00 \\
70.00\end{array}$ & $\begin{array}{l}30.90 \\
31.69 \\
32.48 \\
33.26 \\
34.05 \\
34.83 \\
35.61 \\
36.39 \\
37.16 \\
37.93\end{array}$ & $\begin{array}{l}403.63 \\
420.38 \\
437.00 \\
453.48 \\
469.84 \\
486.07 \\
502.18 \\
518.18 \\
534.08 \\
549.87\end{array}$ & $\begin{array}{l}184.44 \\
195.61 \\
206.64 \\
217.56 \\
228.35 \\
239.03 \\
249.62 \\
260.11 \\
270.52 \\
280.85\end{array}$ & $\begin{array}{l}21.747 \\
22.019 \\
22.285 \\
22.544 \\
22.798 \\
23.046 \\
23.288 \\
23.525 \\
23.757 \\
23.985\end{array}$ & $\begin{array}{l}131.00 \\
132.00 \\
133.00 \\
134.00 \\
135.00 \\
136.00 \\
137.00 \\
138.00 \\
139.00 \\
140.00\end{array}$ & $\begin{array}{l}79.75 \\
80.39 \\
81.02 \\
81.66 \\
82.29 \\
82.92 \\
83.55 \\
84.18 \\
84.81 \\
85.44\end{array}$ & $\begin{array}{l}1502.89 \\
1519.46 \\
1536.06 \\
1552.69 \\
1569.36 \\
1586.05 \\
1602.77 \\
1619.53 \\
1636.31 \\
1653.11\end{array}$ & $\begin{array}{r}937.19 \\
949.26 \\
961.37 \\
973.51 \\
985.69 \\
997.90 \\
1010.15 \\
1022.42 \\
1034.73 \\
1047.06\end{array}$ & $\begin{array}{l}33.737 \\
33.863 \\
33.988 \\
34.113 \\
34.237 \\
34.360 \\
34.483 \\
34.605 \\
34.726 \\
34.846\end{array}$ \\
\hline $\begin{array}{l}71.00 \\
72.00 \\
73.00 \\
74.00 \\
75.00 \\
76.00 \\
77.00 \\
78.00 \\
79.00 \\
80.00\end{array}$ & $\begin{array}{l}38.70 \\
39.46 \\
40.22 \\
40.98 \\
41.73 \\
42.48 \\
43.22 \\
43.96 \\
44.70 \\
45.43\end{array}$ & $\begin{array}{l}565.58 \\
581.20 \\
596.73 \\
612.20 \\
627.60 \\
642.95 \\
658.24 \\
673.49 \\
688.71 \\
703.89\end{array}$ & $\begin{array}{l}291.11 \\
301.31 \\
311.47 \\
321.57 \\
331.64 \\
341.68 \\
351.69 \\
361.68 \\
371.66 \\
381.64\end{array}$ & $\begin{array}{l}24.207 \\
24.426 \\
24.640 \\
24.850 \\
25.057 \\
25.261 \\
25.460 \\
25.657 \\
25.851 \\
26.042\end{array}$ & $\begin{array}{l}141.00 \\
142.00 \\
143.00 \\
144.00 \\
145.00 \\
146.00 \\
147.00 \\
148.00 \\
149.00 \\
150.00\end{array}$ & $\begin{array}{l}86.07 \\
86.70 \\
87.33 \\
87.96 \\
88.59 \\
89.22 \\
89.85 \\
90.48 \\
91.10 \\
91.73\end{array}$ & $\begin{array}{l}1669.94 \\
1686.80 \\
1703.67 \\
1720.57 \\
1737.49 \\
1754.43 \\
1771.38 \\
1738.35 \\
1805.34 \\
1822.34\end{array}$ & $\begin{array}{l}1059.42 \\
1071.81 \\
1084.22 \\
1096.66 \\
1109.11 \\
1121.60 \\
1134.10 \\
1146.62 \\
1159.15 \\
1171.71\end{array}$ & $\begin{array}{l}34.966 \\
35.085 \\
35.203 \\
35.321 \\
35.438 \\
35.555 \\
35.670 \\
35.786 \\
35.900 \\
36.014\end{array}$ \\
\hline $\begin{array}{l}81.00 \\
82.00 \\
83.00 \\
84.00 \\
85.00 \\
86.00 \\
87.00 \\
88.00 \\
89.00 \\
90.00\end{array}$ & $\begin{array}{l}46.17 \\
46.89 \\
47.62 \\
48.34 \\
49.06 \\
49.77 \\
50.48 \\
51.19 \\
51.90 \\
52.60\end{array}$ & $\begin{array}{l}719.04 \\
734.17 \\
749.30 \\
764.40 \\
779.50 \\
794.61 \\
809.71 \\
824.83 \\
839.95 \\
855.09\end{array}$ & $\begin{array}{l}391.61 \\
401.58 \\
411.56 \\
421.55 \\
431.57 \\
441.60 \\
451.66 \\
461.74 \\
471.86 \\
482.01\end{array}$ & $\begin{array}{l}26.230 \\
26.416 \\
26.599 \\
26.780 \\
26.959 \\
27.135 \\
27.310 \\
27.483 \\
27.654 \\
27.823\end{array}$ & $\begin{array}{l}151.00 \\
152.00 \\
153.00 \\
154.00 \\
155.00 \\
156.00 \\
157.00 \\
158.00 \\
159.00 \\
160.00\end{array}$ & $\begin{array}{l}92.35 \\
92.97 \\
93.60 \\
94.22 \\
94.84 \\
95.46 \\
96.08 \\
96.70 \\
97.31 \\
97.93\end{array}$ & $\begin{array}{l}1839.29 \\
1856.24 \\
1873.21 \\
1890.18 \\
1907.16 \\
1924.14 \\
1941.13 \\
1958.12 \\
1975.12 \\
1992.12\end{array}$ & $\begin{array}{l}1184.24 \\
1196.78 \\
1209.34 \\
1221.90 \\
1234.48 \\
1247.07 \\
1259.66 \\
1272.26 \\
1284.87 \\
1297.49\end{array}$ & $\begin{array}{l}36.126 \\
36.238 \\
36.349 \\
36.460 \\
36.570 \\
36.679 \\
36.788 \\
36.896 \\
37.003 \\
37.109\end{array}$ \\
\hline
\end{tabular}


70.00 ATMOSPHERE ISOBAR

TEMPER- SPECIFIC ENTHALPY INTERNAL ENTROPY ATURE VOLUME (J/GM) ENERGY (J/GM-K) (K) (CC/GH) (J/GM)

UNGM-K
TEMPERATURE (K) VOLUME (CC/GM)
ENTHALPY

J/GM
INTERNAL ENTROPY (J/GM)

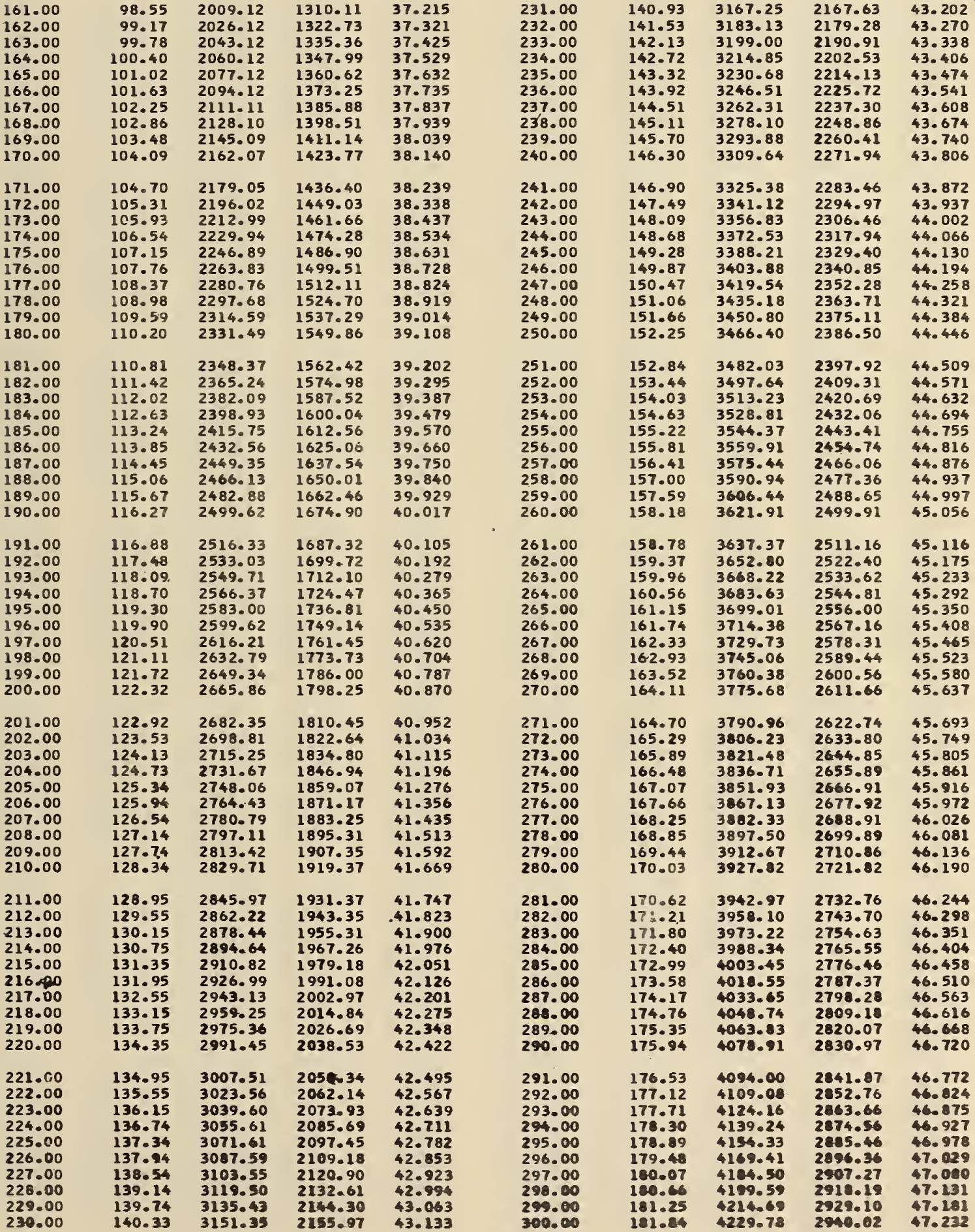


TEMPER- SPECIFIC ENTHALPY INTERNAL ENTROPY ATURE VOLUME (J/GM) ENERGY (J/GM-K) (K) (CC/GM)
$(J / G M)$
TEMPERATURE

(K)
SPECIFIC

VOLUME

(CC/GM)
ENTHALPY

(J/GM)

INTERNAL ENTROPY

ENERGY (J/GM-K)

\begin{tabular}{|c|c|c|c|c|c|c|c|c|c|}
\hline 20.00 & 12.72 & $-176 \cdot 44$ & -279.52 & 6.658 & & & & & \\
\hline $\begin{array}{l}21.00 \\
22.00 \\
23.00 \\
24.00 \\
25.00 \\
26.00 \\
27.00 \\
28.00 \\
29.00 \\
30.00\end{array}$ & $\begin{array}{l}12.83 \\
12.95 \\
13.08 \\
13.21 \\
13.35 \\
13.50 \\
13.65 \\
13.82 \\
13.99 \\
14.17\end{array}$ & $\begin{array}{r}-168.54 \\
-160.31 \\
-151.72 \\
-142.78 \\
-133.46 \\
-123.75 \\
-113.64 \\
-103.13 \\
-92.20 \\
-80.92\end{array}$ & $\begin{array}{l}-272.53 \\
-265.27 \\
-257.72 \\
-249.86 \\
-241.68 \\
-233.17 \\
-224.33 \\
-215.15 \\
-205.62 \\
-195.82\end{array}$ & $\begin{array}{r}7.047 \\
7.427 \\
7.811 \\
8.193 \\
8.572 \\
8.952 \\
9.333 \\
9.717 \\
10.097 \\
10.480\end{array}$ & $\begin{array}{r}91.00 \\
92.00 \\
93.00 \\
94.00 \\
95.00 \\
96.00 \\
97.00 \\
98.00 \\
99.00 \\
100.00\end{array}$ & $\begin{array}{l}47.11 \\
47.73 \\
48.34 \\
48.95 \\
49.56 \\
50.16 \\
50.77 \\
51.37 \\
51.97 \\
52.57\end{array}$ & $\begin{array}{l}860.52 \\
875.92 \\
891.33 \\
906.77 \\
922.24 \\
937.73 \\
953.24 \\
968.78 \\
984.36 \\
999.96\end{array}$ & $\begin{array}{l}478.62 \\
489.04 \\
499.49 \\
509.99 \\
520.52 \\
531.10 \\
541.71 \\
552.37 \\
563.08 \\
573.82\end{array}$ & $\begin{array}{l}27.326 \\
27.494 \\
27.661 \\
27.826 \\
27.990 \\
28.152 \\
28.313 \\
28.472 \\
28.630 \\
28.787\end{array}$ \\
\hline $\begin{array}{l}31.00 \\
32.00 \\
33.00 \\
34.00 \\
35.00 \\
36.00 \\
37.00 \\
38.00 \\
39.00 \\
40.00\end{array}$ & $\begin{array}{l}14.37 \\
14.57 \\
14.81 \\
15.03 \\
15.27 \\
15.52 \\
15.79 \\
16.07 \\
16.37 \\
16.69\end{array}$ & $\begin{array}{r}-69.19 \\
-57.10 \\
-44.65 \\
-31.62 \\
-18.08 \\
-4.20 \\
9.95 \\
24.26 \\
38.82 \\
53.20\end{array}$ & $\begin{array}{r}-185.65 \\
-175.20 \\
-164.66 \\
-153.44 \\
-141.84 \\
-130.00 \\
-118.01 \\
-106.00 \\
-93.86 \\
-82.06\end{array}$ & $\begin{array}{l}10.865 \\
11.252 \\
11.639 \\
12.030 \\
12.422 \\
12.811 \\
13.196 \\
13.577 \\
13.956 \\
14.319\end{array}$ & $\begin{array}{l}101.00 \\
102.00 \\
103.00 \\
104.00 \\
105.00 \\
106.00 \\
107.00 \\
108.00 \\
109.00 \\
110.00\end{array}$ & $\begin{array}{l}53.17 \\
53.77 \\
54.36 \\
54.95 \\
55.55 \\
56.14 \\
56.73 \\
57.32 \\
57.90 \\
58.49\end{array}$ & $\begin{array}{l}1015.58 \\
1031.21 \\
1046.89 \\
1062.60 \\
1078.36 \\
1094.15 \\
1109.98 \\
1125.85 \\
1141.75 \\
1157.68\end{array}$ & $\begin{array}{l}584.58 \\
595.38 \\
606.23 \\
617.13 \\
628.08 \\
639.08 \\
650.12 \\
661.22 \\
672.36 \\
683.55\end{array}$ & $\begin{array}{l}28.942 \\
29.097 \\
29.249 \\
29.401 \\
29.552 \\
29.702 \\
29.850 \\
29.998 \\
30.145 \\
30.290\end{array}$ \\
\hline $\begin{array}{l}41.00 \\
42.00 \\
43.00 \\
44.00 \\
45.00 \\
46.00 \\
47.00 \\
48.00 \\
49.00 \\
50.00\end{array}$ & $\begin{array}{l}17.02 \\
17.38 \\
17.76 \\
18.15 \\
18.57 \\
19.01 \\
19.47 \\
19.95 \\
20.46 \\
20.98\end{array}$ & $\begin{array}{r}67.53 \\
81.93 \\
96.47 \\
111.21 \\
126.19 \\
141.42 \\
156.90 \\
172.64 \\
188.61 \\
204.80\end{array}$ & $\begin{array}{r}-70.46 \\
-58.95 \\
-47.45 \\
-35.93 \\
-24.34 \\
-12.67 \\
-.92 \\
10.91 \\
22.80 \\
34.75\end{array}$ & $\begin{array}{l}14.673 \\
15.020 \\
15.362 \\
15.701 \\
16.038 \\
16.373 \\
16.706 \\
17.037 \\
17.366 \\
17.693\end{array}$ & $\begin{array}{l}111.00 \\
112.00 \\
113.00 \\
114.00 \\
115.00 \\
116.00 \\
117.00 \\
118.00 \\
119.00 \\
120.00\end{array}$ & $\begin{array}{l}59.07 \\
59.66 \\
60.24 \\
60.81 \\
61.39 \\
61.97 \\
62.54 \\
63.11 \\
63.68 \\
64.25\end{array}$ & $\begin{array}{l}1173.64 \\
1189.64 \\
1205.66 \\
1221.71 \\
1237.79 \\
1253.90 \\
1270.03 \\
1286.19 \\
1302.38 \\
1318.59\end{array}$ & $\begin{array}{l}694.78 \\
706.06 \\
717.38 \\
728.74 \\
740.14 \\
751.59 \\
763.08 \\
774.61 \\
786.18 \\
797.79\end{array}$ & $\begin{array}{l}30.434 \\
30.578 \\
30.720 \\
30.862 \\
31.002 \\
31.142 \\
31.280 \\
31.418 \\
31.554 \\
31.690\end{array}$ \\
\hline $\begin{array}{l}51.00 \\
52.00 \\
53.00 \\
54.00 \\
55.00 \\
56.00 \\
57.00 \\
58.00 \\
59.00 \\
60.00\end{array}$ & $\begin{array}{l}21.52 \\
22.08 \\
22.66 \\
23.25 \\
23.86 \\
24.48 \\
25.10 \\
25.74 \\
26.39 \\
27.04\end{array}$ & $\begin{array}{l}221.18 \\
237.71 \\
254.37 \\
271.11 \\
287.93 \\
304.78 \\
321.63 \\
338.47 \\
355.28 \\
372.04\end{array}$ & $\begin{array}{r}46.73 \\
58.71 \\
70.69 \\
82.64 \\
94.54 \\
106.38 \\
118.14 \\
129.81 \\
141.39 \\
152.88\end{array}$ & $\begin{array}{l}18.018 \\
18.339 \\
18.656 \\
18.969 \\
19.277 \\
19.581 \\
19.879 \\
20.172 \\
20.460 \\
20.741\end{array}$ & $\begin{array}{l}121.00 \\
122.00 \\
123.00 \\
124.00 \\
125.00 \\
126.00 \\
127.00 \\
128.00 \\
129.00 \\
130.00\end{array}$ & $\begin{array}{l}64.81 \\
65.38 \\
65.94 \\
66.50 \\
67.06 \\
67.63 \\
68.19 \\
68.75 \\
69.31 \\
69.87\end{array}$ & $\begin{array}{l}1334.82 \\
1351.08 \\
1367.36 \\
1383.67 \\
1400.02 \\
1416.49 \\
1433.00 \\
1449.54 \\
1466.12 \\
1482.73\end{array}$ & $\begin{array}{l}809.43 \\
821.11 \\
832.83 \\
844.60 \\
856.40 \\
868.31 \\
880.25 \\
892.24 \\
904.27 \\
916.34\end{array}$ & $\begin{array}{l}31.825 \\
31.958 \\
32.091 \\
32.223 \\
32.355 \\
32.486 \\
32.617 \\
32.746 \\
32.875 \\
33.004\end{array}$ \\
\hline $\begin{array}{l}61.00 \\
62.00 \\
63.00 \\
64.00 \\
65.00 \\
66.00 \\
67.00 \\
68.00 \\
69.00 \\
70.00\end{array}$ & $\begin{array}{l}27.69 \\
28.35 \\
29.02 \\
29.68 \\
30.35 \\
31.01 \\
31.68 \\
32.34 \\
33.01 \\
33.67\end{array}$ & $\begin{array}{l}388.74 \\
405.37 \\
421.92 \\
438.38 \\
454.77 \\
471.06 \\
487.27 \\
503.39 \\
519.43 \\
535.39\end{array}$ & $\begin{array}{l}164.26 \\
175.54 \\
186.72 \\
197.80 \\
208.79 \\
219.68 \\
230.48 \\
241.21 \\
251.86 \\
262.43\end{array}$ & $\begin{array}{l}21.017 \\
21.288 \\
21.552 \\
21.812 \\
22.066 \\
22.315 \\
22.558 \\
22.797 \\
23.031 \\
23.261\end{array}$ & $\begin{array}{l}131.00 \\
132.00 \\
133.00 \\
134.00 \\
135.00 \\
136.00 \\
137.00 \\
138.00 \\
139.00 \\
140.00\end{array}$ & $\begin{array}{l}70.43 \\
70.99 \\
71.55 \\
72.10 \\
72.66 \\
73.22 \\
73.77 \\
74.33 \\
74.89 \\
75.44\end{array}$ & $\begin{array}{l}1499.37 \\
1516.05 \\
1532.76 \\
1549.50 \\
1566.27 \\
1583.07 \\
1599.91 \\
1616.76 \\
1633.65 \\
1650.56\end{array}$ & $\begin{array}{r}928.45 \\
940.60 \\
952.78 \\
965.00 \\
977.26 \\
989.55 \\
1001.87 \\
1014.22 \\
1026.61 \\
1039.02\end{array}$ & $\begin{array}{l}33.131 \\
33.258 \\
33.384 \\
33.509 \\
33.634 \\
33.758 \\
33.881 \\
34.004 \\
34.126 \\
34.247\end{array}$ \\
\hline $\begin{array}{l}71.00 \\
72.00 \\
73.00 \\
74.00 \\
75.00 \\
76.00 \\
77.00 \\
78.00 \\
79.00 \\
80.00\end{array}$ & $\begin{array}{l}34.34 \\
35.00 \\
35.66 \\
36.31 \\
36.97 \\
37.62 \\
38.27 \\
38.92 \\
39.56 \\
40.20\end{array}$ & $\begin{array}{l}551.28 \\
567.09 \\
582.83 \\
598.51 \\
614.14 \\
629.71 \\
645.24 \\
660.73 \\
676.18 \\
691.60\end{array}$ & $\begin{array}{l}272.95 \\
283.40 \\
293.81 \\
304.16 \\
314.49 \\
324.77 \\
335.03 \\
345.27 \\
355.49 \\
365.70\end{array}$ & $\begin{array}{l}23.486 \\
23.707 \\
23.925 \\
24.138 \\
24.348 \\
24.554 \\
24.757 \\
24.957 \\
25.154 \\
25.348\end{array}$ & $\begin{array}{l}141.00 \\
142.00 \\
143.00 \\
144.00 \\
145.00 \\
146.00 \\
147.00 \\
148.00 \\
149.00 \\
150.00\end{array}$ & $\begin{array}{l}76.00 \\
76.55 \\
77.10 \\
77.66 \\
78.21 \\
78.76 \\
79.32 \\
79.87 \\
80.42 \\
80.97\end{array}$ & $\begin{array}{l}1667.50 \\
1684.46 \\
1701.45 \\
1718.45 \\
1735.48 \\
1752.52 \\
1769.58 \\
1786.66 \\
1803.75 \\
1820.85\end{array}$ & $\begin{array}{l}1051.46 \\
1063.93 \\
1076.42 \\
1088.94 \\
1101.48 \\
1114.04 \\
1126.62 \\
1139.22 \\
1151.84 \\
1164.47\end{array}$ & $\begin{array}{l}34.368 \\
34.488 \\
34.607 \\
34.725 \\
34.843 \\
34.960 \\
35.077 \\
35.192 \\
35.308 \\
35.422\end{array}$ \\
\hline $\begin{array}{l}81.00 \\
82.00 \\
83.00 \\
84.00 \\
85.00 \\
86.00 \\
87.00 \\
88.00 \\
89.00 \\
90.00\end{array}$ & $\begin{array}{l}40.84 \\
41.48 \\
42.12 \\
42.75 \\
43.38 \\
44.01 \\
44.63 \\
45.26 \\
45.88 \\
46.50\end{array}$ & $\begin{array}{l}706.99 \\
722.36 \\
737.72 \\
753.07 \\
768.41 \\
783.74 \\
799.08 \\
814.42 \\
829.78 \\
845.14\end{array}$ & $\begin{array}{l}375.91 \\
386.11 \\
396.32 \\
406.54 \\
416.77 \\
427.01 \\
437.28 \\
447.58 \\
457.89 \\
468.24\end{array}$ & $\begin{array}{l}25.539 \\
25.727 \\
25.914 \\
26.097 \\
26.279 \\
26.458 \\
26.636 \\
26.811 \\
26.984 \\
27.156\end{array}$ & $\begin{array}{l}151.00 \\
152.00 \\
153.00 \\
154.00 \\
155.00 \\
156.00 \\
157.00 \\
158.00 \\
159.00 \\
160.00\end{array}$ & $\begin{array}{l}81.52 \\
82.07 \\
82.62 \\
83.16 \\
83.71 \\
84.25 \\
84.80 \\
85.34 \\
85.89 \\
86.43\end{array}$ & $\begin{array}{l}1837.90 \\
1854.95 \\
1872.01 \\
1889.08 \\
1906.15 \\
1923.23 \\
1940.30 \\
1957.39 \\
1974.47 \\
1991.55\end{array}$ & $\begin{array}{l}1177.07 \\
1189.69 \\
1202.32 \\
1214.95 \\
1227.60 \\
1240.25 \\
1252.91 \\
1265.58 \\
1278.25 \\
1290.92\end{array}$ & $\begin{array}{l}35.535 \\
35.648 \\
35.760 \\
35.871 \\
35.981 \\
36.091 \\
36.200 \\
36.309 \\
36.417 \\
36.524\end{array}$ \\
\hline
\end{tabular}


TEMPER- SPECIFIC ENTHALPY INTERNAL ENTROPY ATURE VOLUME (J/GM) ENERGY (J/GM-K) $(K)$ ( CC /GM) (J/GM) ATURE

(K)
SPECIFIC ENTHALPY VOLUME $(C C / G M)$
(J/GM)

INTERNAL (J/GM)

\begin{tabular}{|c|c|c|c|c|c|c|c|c|c|}
\hline $\begin{array}{l}161.00 \\
162.00 \\
163.00 \\
164.00 \\
165.00 \\
166.00 \\
167.00 \\
168.00 \\
169.00 \\
170.00\end{array}$ & $\begin{array}{l}86.97 \\
87.52 \\
88.06 \\
88.60 \\
89.14 \\
89.68 \\
90.22 \\
90.77 \\
91.31 \\
91.84\end{array}$ & $\begin{array}{l}2008.63 \\
2025.71 \\
2042.79 \\
2059.86 \\
2076.93 \\
2094.00 \\
2111.06 \\
2128.11 \\
2145.16 \\
2162.20\end{array}$ & $\begin{array}{l}1303.60 \\
1316.28 \\
1328.96 \\
1341.64 \\
1354.32 \\
1367.00 \\
1379.68 \\
1392.35 \\
1405.02 \\
1417.68\end{array}$ & $\begin{array}{l}36.630 \\
36.736 \\
36.841 \\
36.945 \\
37.049 \\
37.152 \\
37.255 \\
37.356 \\
37.458 \\
37.558\end{array}$ & $\begin{array}{l}231.00 \\
232.00 \\
233.00 \\
234.00 \\
235.00 \\
236.00 \\
237.00 \\
238.00 \\
239.00 \\
240.00\end{array}$ & $\begin{array}{l}124.20 \\
124.72 \\
125.24 \\
125.77 \\
126.29 \\
126.82 \\
127.34 \\
127.86 \\
128.38 \\
128.91\end{array}$ & $\begin{array}{l}3170.35 \\
3186.29 \\
3202.21 \\
3218.12 \\
3234.01 \\
3249.88 \\
3265.73 \\
3281.56 \\
3297.37 \\
3313.17\end{array}$ & $\begin{array}{l}2163.58 \\
2175.28 \\
2186.95 \\
2198.61 \\
2210.25 \\
2221.88 \\
2233.49 \\
2245.08 \\
2256.66 \\
2268.22\end{array}$ & $\begin{array}{l}42.634 \\
42.703 \\
42.772 \\
42.840 \\
42.908 \\
42.975 \\
43.042 \\
43.109 \\
43.175 \\
43.241\end{array}$ \\
\hline $\begin{array}{l}171.00 \\
172.00 \\
173.00 \\
174.00 \\
175.00 \\
176.00 \\
177.00 \\
178.00 \\
179.00 \\
180.00\end{array}$ & $\begin{array}{l}92.38 \\
92.92 \\
93.46 \\
94.00 \\
94.53 \\
95.07 \\
95.61 \\
96.14 \\
96.68 \\
97.22\end{array}$ & $\begin{array}{l}2179.22 \\
2196.24 \\
2213.24 \\
2230.23 \\
2247.22 \\
2264.18 \\
2281.14 \\
2298.08 \\
2315.01 \\
2331.93\end{array}$ & $\begin{array}{l}1430.34 \\
1442.99 \\
1455.63 \\
1468.27 \\
1480.90 \\
1493.51 \\
1506.12 \\
1518.72 \\
1531.31 \\
1543.88\end{array}$ & $\begin{array}{l}37.658 \\
37.757 \\
37.856 \\
37.954 \\
38.051 \\
38.148 \\
38.244 \\
38.339 \\
38.434 \\
38.528\end{array}$ & $\begin{array}{l}241.00 \\
242.00 \\
243.00 \\
244.00 \\
245.00 \\
246.00 \\
247.00 \\
248.00 \\
249.00 \\
250.00\end{array}$ & $\begin{array}{l}129.43 \\
129.95 \\
130.47 \\
131.00 \\
131.52 \\
132.04 \\
132.56 \\
133.08 \\
133.60 \\
134.13\end{array}$ & $\begin{array}{l}3328.95 \\
3344.71 \\
3360.45 \\
3376.18 \\
3391.88 \\
3407.57 \\
3423.25 \\
3438.90 \\
3454.54 \\
3470.16\end{array}$ & $\begin{array}{l}2279.76 \\
2291.28 \\
2302.79 \\
2314.29 \\
2325.76 \\
2337.23 \\
2348.67 \\
2360.10 \\
2371.51 \\
2382.91\end{array}$ & $\begin{array}{l}43.307 \\
43.372 \\
43.437 \\
43.501 \\
43.566 \\
43.630 \\
43.693 \\
43.756 \\
43.819 \\
43.882\end{array}$ \\
\hline $\begin{array}{l}181.00 \\
182.00 \\
183.00 \\
184.00 \\
185.00 \\
186.00 \\
187.00 \\
188.00 \\
189.00 \\
190.00\end{array}$ & $\begin{array}{r}97.75 \\
98.28 \\
98.82 \\
99.35 \\
99.89 \\
100.42 \\
100.95 \\
101.49 \\
102.02 \\
102.55\end{array}$ & $\begin{array}{l}2348.83 \\
2365.71 \\
2382.58 \\
2399.44 \\
2416.27 \\
2433.09 \\
2449.90 \\
2466.68 \\
2483.45 \\
2500.20\end{array}$ & $\begin{array}{l}1556.45 \\
1569.00 \\
1581.53 \\
1594.06 \\
1606.57 \\
1619.06 \\
1631.54 \\
1644.01 \\
1656.46 \\
1668.89\end{array}$ & $\begin{array}{l}38.622 \\
38.715 \\
38.807 \\
38.899 \\
38.991 \\
39.081 \\
39.171 \\
39.261 \\
39.350 \\
39.438\end{array}$ & $\begin{array}{l}251.00 \\
252.00 \\
253.00 \\
254.00 \\
255.00 \\
256.00 \\
257.00 \\
258.00 \\
259.00 \\
260.00\end{array}$ & $\begin{array}{l}134.65 \\
135.17 \\
135.69 \\
136.21 \\
136.73 \\
137.25 \\
137.77 \\
138.29 \\
138.81 \\
139.33\end{array}$ & $\begin{array}{l}3485.80 \\
3501.42 \\
3517.03 \\
3532.62 \\
3548.19 \\
3563.74 \\
3579.28 \\
3594.80 \\
3610.31 \\
3625.80\end{array}$ & $\begin{array}{l}2394.32 \\
2405.72 \\
2417.11 \\
2428.48 \\
2439.83 \\
2451.17 \\
2462.49 \\
2473.79 \\
2485.08 \\
2496.36\end{array}$ & $\begin{array}{l}43.944 \\
44.006 \\
44.068 \\
44.130 \\
44.191 \\
44.252 \\
44.312 \\
44.373 \\
44.433 \\
44.492\end{array}$ \\
\hline $\begin{array}{l}191.00 \\
192.00 \\
193.00 \\
194.00 \\
195.00 \\
196.00 \\
197.00 \\
198.00 \\
199.00 \\
200.00\end{array}$ & $\begin{array}{l}103.08 \\
103.62 \\
104.15 \\
104.68 \\
105.21 \\
105.74 \\
106.27 \\
106.80 \\
107.33 \\
107.86\end{array}$ & $\begin{array}{l}2516.93 \\
2533.65 \\
2550.34 \\
2567.02 \\
2583.68 \\
2600.32 \\
2616.94 \\
2633.54 \\
2650.12 \\
2666.68\end{array}$ & $\begin{array}{l}1681.31 \\
1693.71 \\
1706.10 \\
1718.47 \\
1730.82 \\
1743.16 \\
1755.47 \\
1767.78 \\
1780.06 \\
1792.32\end{array}$ & $\begin{array}{l}39.526 \\
39.613 \\
39.700 \\
39.786 \\
39.872 \\
39.957 \\
40.042 \\
40.126 \\
40.209 \\
40.292\end{array}$ & $\begin{array}{l}261.00 \\
262.00 \\
263.00 \\
264.00 \\
265.00 \\
266.00 \\
267.00 \\
268.00 \\
269.00 \\
270.00\end{array}$ & $\begin{array}{l}139.85 \\
140.37 \\
140.89 \\
141.41 \\
141.93 \\
142.45 \\
142.97 \\
143.49 \\
144.01 \\
144.52\end{array}$ & $\begin{array}{l}3641.27 \\
3656.72 \\
3672.16 \\
3687.59 \\
3703.00 \\
3718.39 \\
3733.77 \\
3749.13 \\
3764.48 \\
3779.82\end{array}$ & $\begin{array}{l}2507.62 \\
2518.86 \\
2530.09 \\
2541.30 \\
2552.50 \\
2563.68 \\
2574.85 \\
2586.00 \\
2597.14 \\
2608.27\end{array}$ & $\begin{array}{l}44.552 \\
44.611 \\
44.670 \\
44.728 \\
44.786 \\
44.844 \\
44.902 \\
44.960 \\
45.017 \\
45.074\end{array}$ \\
\hline $\begin{array}{l}201.00 \\
202.00 \\
203.00 \\
204.00 \\
205.00 \\
206.00 \\
207.00 \\
208.00 \\
209.00 \\
210.00\end{array}$ & $\begin{array}{l}108.39 \\
108.92 \\
109.45 \\
109.98 \\
110.51 \\
111.04 \\
111.57 \\
112.99 \\
112.62 \\
113.15\end{array}$ & $\begin{array}{l}2683.22 \\
2699.74 \\
2716.24 \\
2732.72 \\
2749.18 \\
2765.63 \\
2782.05 \\
2798.45 \\
2814.83 \\
2831.19\end{array}$ & $\begin{array}{l}1804.57 \\
1816.80 \\
1829.01 \\
1841.20 \\
1853.37 \\
1865.53 \\
1877.67 \\
1889.79 \\
1901.89 \\
1913.97\end{array}$ & $\begin{array}{l}40.375 \\
40.457 \\
40.538 \\
40.619 \\
40.700 \\
40.780 \\
40.859 \\
40.938 \\
41.017 \\
41.095\end{array}$ & $\begin{array}{l}271.00 \\
272.00 \\
273.00 \\
274.00 \\
275.00 \\
276.00 \\
277.00 \\
278.00 \\
279.00 \\
280.00\end{array}$ & $\begin{array}{l}145.04 \\
145.56 \\
146.08 \\
146.60 \\
147.12 \\
147.64 \\
148.16 \\
148.68 \\
149.20 \\
149.71\end{array}$ & $\begin{array}{l}3795.14 \\
3810.44 \\
3825.74 \\
3841.01 \\
3856.28 \\
3871.53 \\
3886.78 \\
3902.01 \\
3917.22 \\
3932.43\end{array}$ & $\begin{array}{l}2619.38 \\
2630.48 \\
2641.56 \\
2652.64 \\
2663.70 \\
2674.74 \\
2685.78 \\
2696.80 \\
2707.81 \\
2718.82\end{array}$ & $\begin{array}{l}45.130 \\
45.18 \\
45.243 \\
45.29 \\
45.35 \\
45.410 \\
45.46 \\
45.520 \\
45.57 \\
45.62\end{array}$ \\
\hline $\begin{array}{l}211.00 \\
212.00 \\
213.00 \\
214.00 \\
215.00 \\
216.00 \\
217.00 \\
218.00 \\
219.00 \\
220.00\end{array}$ & $\begin{array}{l}113.68 \\
114.21 \\
114.73 \\
115.26 \\
115.79 \\
116.31 \\
116.84 \\
117.37 \\
117.89 \\
118.42\end{array}$ & $\begin{array}{l}2847.54 \\
2863.86 \\
2880.17 \\
2896.45 \\
2912.71 \\
2928.96 \\
2945.19 \\
2961.39 \\
2977.58 \\
2993.75\end{array}$ & $\begin{array}{l}1926.04 \\
1938.08 \\
1950.11 \\
1962.12 \\
1974.12 \\
1986.09 \\
1998.05 \\
2009.99 \\
2021.91 \\
2033.81\end{array}$ & $\begin{array}{r}.41 .172 \\
41.250 \\
41.326 \\
41.403 \\
41.479 \\
41.554 \\
41.629 \\
41.703 \\
41.777 \\
41.851\end{array}$ & $\begin{array}{l}281.00 \\
282.00 \\
283.00 \\
284.00 \\
285.00 \\
286.00 \\
287.00 \\
288.00 \\
289.00 \\
290.00\end{array}$ & $\begin{array}{l}150.23 \\
150.75 \\
151.27 \\
151.79 \\
152.31 \\
152.82 \\
153.34 \\
153.86 \\
154.38 \\
154.90\end{array}$ & $\begin{array}{l}3947.63 \\
3962.81 \\
3977.99 \\
3993.16 \\
4008.31 \\
4023.46 \\
4038.60 \\
4053.74 \\
4068.86 \\
4083.98\end{array}$ & $\begin{array}{l}2729.81 \\
2740.79 \\
2751.76 \\
2762.73 \\
2773.68 \\
2784.63 \\
2795.57 \\
2806.51 \\
2817.44 \\
2828.36\end{array}$ & $\begin{array}{l}45.68 \\
45.73 \\
45.79 \\
45.84 \\
45.89 \\
45.95 \\
46.00 \\
46.05 \\
46.10 \\
46.16\end{array}$ \\
\hline $\begin{array}{l}221.00 \\
222.00 \\
223.00 \\
224.00 \\
225.00 \\
226.00 \\
227.00 \\
228.00 \\
229.00 \\
230.00\end{array}$ & $\begin{array}{l}118.95 \\
119.47 \\
120.00 \\
120.52 \\
121.05 \\
121.57 \\
122.10 \\
122.62 \\
123.15 \\
123.67\end{array}$ & $\begin{array}{l}3009.90 \\
3026.03 \\
3042.14 \\
3058.23 \\
3074.31 \\
3090.36 \\
3106.39 \\
3122.41 \\
3138.41 \\
3154.39\end{array}$ & $\begin{array}{l}2045.70 \\
2057.56 \\
2069.41 \\
2081.25 \\
2093.06 \\
2104.86 \\
2116.64 \\
2128.40 \\
2140.14 \\
2151.87\end{array}$ & $\begin{array}{l}41.924 \\
41.997 \\
42.070 \\
42.142 \\
42.213 \\
42.284 \\
42.355 \\
42.426 \\
42.496 \\
42.565\end{array}$ & $\begin{array}{l}291.00 \\
292.00 \\
293.00 \\
294.00 \\
295.00 \\
296.00 \\
297.00 \\
298.00 \\
299.00 \\
300.00\end{array}$ & $\begin{array}{l}155.41 \\
155.93 \\
156.45 \\
156.96 \\
157.48 \\
158.00 \\
158.51 \\
159.03 \\
159.55 \\
160.06\end{array}$ & $\begin{array}{l}4099.09 \\
4114.20 \\
4129.30 \\
4144.39 \\
4159.48 \\
4174.56 \\
4189.64 \\
4204.71 \\
4219.78 \\
4234.84\end{array}$ & $\begin{array}{l}2839.27 \\
2850.19 \\
2861.09 \\
2872.00 \\
2882.90 \\
2893.79 \\
2904.69 \\
2915.58 \\
2926.46 \\
2937.35\end{array}$ & $\begin{array}{l}46.21 \\
46.26 \\
46.31 \\
46.36 \\
46.41 \\
46.47 \\
46.52 \\
46.57 \\
46.62 \\
46.67\end{array}$ \\
\hline
\end{tabular}


TEMPER- SPECIFIC ENTHALPY INTERNAL ENTROPY ATURE YOLUME (J/GM) ENERGY (J/GM-K) (K) $\quad(\mathrm{CC} / \mathrm{GM})$ (J/GM) ENERGY ATURE (K)
SPECIFIC ENTHALPY VOLUME (CC/GM)
$(J / G M)$

INTERNAL (J/GM)
ENTROPY $(J / G M-K)$

\begin{tabular}{|c|c|c|c|c|c|c|c|c|c|}
\hline 20.00 & 12.60 & -165.80 & -280.75 & 6.549 & & & & & \\
\hline $\begin{array}{l}21.00 \\
22.00 \\
23.00 \\
24.00 \\
25.00 \\
26.00 \\
27.00 \\
28.00 \\
29.00 \\
30.00\end{array}$ & $\begin{array}{l}12.71 \\
12.83 \\
12.94 \\
13.07 \\
13.20 \\
13.34 \\
13.48 \\
13.63 \\
13.79 \\
13.96\end{array}$ & $\begin{array}{l}-158.01 \\
-149.91 \\
-141.47 \\
-132.69 \\
-123.54 \\
-114.02 \\
-104.11 \\
-93.82 \\
-83.13 \\
-72.11\end{array}$ & $\begin{array}{l}-273.94 \\
-266.87 \\
-259.51 \\
-251.87 \\
-243.91 \\
-235.65 \\
-227.06 \\
-218.15 \\
-208.92 \\
-199.43\end{array}$ & $\begin{array}{r}6.932 \\
7.306 \\
7.683 \\
8.059 \\
8.430 \\
8.803 \\
9.177 \\
9.553 \\
9.925 \\
10.299\end{array}$ & $\begin{array}{r}91.00 \\
92.00 \\
93.00 \\
94.00 \\
95.00 \\
96.00 \\
97.00 \\
98.00 \\
99.00 \\
100.00\end{array}$ & $\begin{array}{l}42.38 \\
42.93 \\
43.47 \\
44.02 \\
44.56 \\
45.10 \\
45.64 \\
46.18 \\
46.71 \\
47.25\end{array}$ & $\begin{array}{l}852.22 \\
867.79 \\
883.38 \\
899.00 \\
914.63 \\
930.29 \\
945.98 \\
961.69 \\
977.43 \\
993.19\end{array}$ & $\begin{array}{l}465.74 \\
476.32 \\
486.94 \\
497.59 \\
508.28 \\
519.01 \\
529.78 \\
540.59 \\
551.44 \\
562.33\end{array}$ & $\begin{array}{l}26.738 \\
26.908 \\
27.076 \\
27.243 \\
27.409 \\
27.573 \\
27.735 \\
27.897 \\
28.056 \\
28.215\end{array}$ \\
\hline $\begin{array}{l}31.00 \\
32.00 \\
33.00 \\
34.00 \\
35.00 \\
36.00 \\
37.00 \\
38.00 \\
39.00 \\
40.00\end{array}$ & $\begin{array}{l}14.14 \\
14.32 \\
14.53 \\
14.73 \\
14.94 \\
15.17 \\
15.40 \\
15.65 \\
15.91 \\
16.19\end{array}$ & $\begin{array}{r}-60.67 \\
-48.90 \\
-36.73 \\
-24.10 \\
-11.00 \\
2.41 \\
16.06 \\
29.84 \\
43.81 \\
57.55\end{array}$ & $\begin{array}{l}-189.59 \\
-179.50 \\
-169.24 \\
-158.44 \\
-147.27 \\
-135.90 \\
-124.40 \\
-112.88 \\
-101.30 \\
-90.07\end{array}$ & $\begin{array}{l}10.674 \\
11.051 \\
11.429 \\
11.808 \\
12.187 \\
12.563 \\
12.934 \\
13.301 \\
13.664 \\
14.012\end{array}$ & $\begin{array}{l}101.00 \\
102.00 \\
103.00 \\
104.00 \\
105.00 \\
106.00 \\
107.00 \\
108.00 \\
109.00 \\
110.00\end{array}$ & $\begin{array}{l}47.78 \\
48.31 \\
48.85 \\
49.38 \\
49.91 \\
50.43 \\
50.96 \\
51.49 \\
52.01 \\
52.53\end{array}$ & $\begin{array}{l}1008.93 \\
1024.64 \\
1040.39 \\
1056.18 \\
1072.02 \\
1087.89 \\
1103.80 \\
1119.75 \\
1135.75 \\
1151.78\end{array}$ & $\begin{array}{l}573.19 \\
584.04 \\
594.94 \\
605.89 \\
616.90 \\
627.95 \\
639.06 \\
650.22 \\
661.43 \\
672.69\end{array}$ & $\begin{array}{l}28.371 \\
28.526 \\
28.680 \\
28.832 \\
28.984 \\
29.134 \\
29.284 \\
29.432 \\
29.580 \\
29.726\end{array}$ \\
\hline $\begin{array}{l}41.00 \\
42.00 \\
43.00 \\
44.00 \\
45.00 \\
46.00 \\
47.00 \\
48.00 \\
49.00 \\
50.00\end{array}$ & $\begin{array}{l}16.48 \\
16.78 \\
17.10 \\
17.44 \\
17.79 \\
18.15 \\
18.54 \\
18.94 \\
19.35 \\
19.78\end{array}$ & $\begin{array}{r}71.20 \\
84.88 \\
98.66 \\
112.60 \\
126.74 \\
141.10 \\
155.71 \\
170.55 \\
185.63 \\
200.93\end{array}$ & $\begin{array}{r}-79.05 \\
-68.15 \\
-57.28 \\
-46.40 \\
-35.46 \\
-24.43 \\
-13.33 \\
-2.12 \\
9.17 \\
20.54\end{array}$ & $\begin{array}{l}14.349 \\
14.679 \\
15.003 \\
15.323 \\
15.641 \\
15.957 \\
16.271 \\
16.583 \\
16.894 \\
17.204\end{array}$ & $\begin{array}{l}111.00 \\
112.00 \\
113.00 \\
114.00 \\
115.00 \\
116.00 \\
117.00 \\
118.00 \\
119.00 \\
120.00\end{array}$ & $\begin{array}{l}53.06 \\
53.58 \\
54.10 \\
54.61 \\
55.13 \\
55.64 \\
56.15 \\
56.67 \\
57.18 \\
57.68\end{array}$ & $\begin{array}{l}1167.86 \\
1183.97 \\
1200.13 \\
1216.32 \\
1232.55 \\
1248.82 \\
1265.13 \\
1281.47 \\
1297.85 \\
1314.26\end{array}$ & $\begin{array}{l}684.01 \\
695.38 \\
706.81 \\
718.28 \\
729.81 \\
741.39 \\
753.02 \\
764.71 \\
776.44 \\
788.22\end{array}$ & $\begin{array}{l}29.872 \\
30.016 \\
30.160 \\
30.302 \\
30.444 \\
30.585 \\
30.725 \\
30.864 \\
31.002 \\
31.140\end{array}$ \\
\hline $\begin{array}{l}51.00 \\
52.00 \\
53.00 \\
54.00 \\
55.00 \\
56.00 \\
57.00 \\
58.00 \\
59.00 \\
60.00\end{array}$ & $\begin{array}{l}20.23 \\
20.69 \\
21.17 \\
21.66 \\
22.16 \\
22.67 \\
23.19 \\
23.73 \\
24.27 \\
24.81\end{array}$ & $\begin{array}{l}216.45 \\
232.15 \\
248.01 \\
264.02 \\
280.15 \\
296.37 \\
312.67 \\
329.00 \\
345.38 \\
361.76\end{array}$ & $\begin{array}{r}31.97 \\
43.46 \\
54.99 \\
66.54 \\
78.10 \\
89.64 \\
101.16 \\
112.65 \\
124.09 \\
135.48\end{array}$ & $\begin{array}{l}17.511 \\
17.816 \\
18.118 \\
18.417 \\
18.713 \\
19.005 \\
19.294 \\
19.578 \\
19.858 \\
20.133\end{array}$ & $\begin{array}{l}121.00 \\
122.00 \\
123.00 \\
124.00 \\
125.00 \\
126.00 \\
127.00 \\
128.00 \\
129.00 \\
130.00\end{array}$ & $\begin{array}{l}58.19 \\
58.69 \\
59.20 \\
59.70 \\
60.20 \\
60.70 \\
61.21 \\
61.71 \\
62.21 \\
62.71\end{array}$ & $\begin{array}{l}1330.70 \\
1347.17 \\
1363.67 \\
1380.20 \\
1396.77 \\
1413.39 \\
1430.04 \\
1446.72 \\
1463.43 \\
1480.17\end{array}$ & $\begin{array}{l}800.04 \\
811.91 \\
823.82 \\
835.77 \\
847.77 \\
859.79 \\
871.85 \\
883.95 \\
896.08 \\
908.25\end{array}$ & $\begin{array}{l}31.276 \\
31.412 \\
31.546 \\
31.680 \\
31.813 \\
31.946 \\
32.077 \\
32.208 \\
32.338 \\
32.467\end{array}$ \\
\hline $\begin{array}{l}61.00 \\
62.00 \\
63.00 \\
64.00 \\
65.00 \\
66.00 \\
67.00 \\
68.00 \\
69.00 \\
70.00\end{array}$ & $\begin{array}{l}25.37 \\
25.93 \\
26.49 \\
27.06 \\
27.63 \\
28.20 \\
28.78 \\
29.35 \\
29.93 \\
30.51\end{array}$ & $\begin{array}{l}378.14 \\
394.51 \\
410.84 \\
427.15 \\
443.41 \\
459.62 \\
475.78 \\
491.89 \\
507.94 \\
523.94\end{array}$ & $\begin{array}{l}146.80 \\
158.07 \\
169.26 \\
180.39 \\
191.44 \\
202.43 \\
213.35 \\
224.20 \\
234.99 \\
245.73\end{array}$ & $\begin{array}{l}20.404 \\
20.670 \\
20.931 \\
21.188 \\
21.440 \\
21.688 \\
21.931 \\
22.169 \\
22.404 \\
22.634\end{array}$ & $\begin{array}{l}131.00 \\
132.00 \\
133.00 \\
134.00 \\
135.00 \\
136.00 \\
137.00 \\
138.00 \\
139.00 \\
140.00\end{array}$ & $\begin{array}{l}63.21 \\
63.71 \\
64.21 \\
64.71 \\
65.21 \\
65.71 \\
66.21 \\
66.70 \\
67.20 \\
67.69\end{array}$ & $\begin{array}{l}1496.94 \\
1513.73 \\
1530.55 \\
1547.40 \\
1564.27 \\
1581.17 \\
1598.09 \\
1615.03 \\
1631.99 \\
1648.97\end{array}$ & $\begin{array}{r}920.46 \\
932.69 \\
944.96 \\
957.26 \\
969.59 \\
981.94 \\
994.33 \\
1006.74 \\
1019.16 \\
1031.64\end{array}$ & $\begin{array}{l}32.596 \\
32.724 \\
32.851 \\
32.977 \\
33.102 \\
33.227 \\
33.351 \\
33.474 \\
33.596 \\
33.710\end{array}$ \\
\hline $\begin{array}{l}71.00 \\
72.00 \\
73.00 \\
74.00 \\
75.00 \\
76.00 \\
77.00 \\
78.00 \\
79.00 \\
80.00\end{array}$ & $\begin{array}{l}31.09 \\
31.66 \\
32.24 \\
32.81 \\
33.39 \\
33.96 \\
34.54 \\
35.11 \\
35.68 \\
36.24\end{array}$ & $\begin{array}{l}539.88 \\
555.77 \\
571.61 \\
587.40 \\
603.15 \\
618.86 \\
634.53 \\
650.16 \\
665.77 \\
681.36\end{array}$ & $\begin{array}{l}256.40 \\
267.03 \\
277.61 \\
288.16 \\
298.66 \\
309.14 \\
319.59 \\
330.02 \\
340.43 \\
350.83\end{array}$ & $\begin{array}{l}22.860 \\
23.082 \\
23.301 \\
23.516 \\
23.727 \\
23.935 \\
24.140 \\
24.342 \\
24.541 \\
24.737\end{array}$ & $\begin{array}{l}141.00 \\
142.00 \\
143.00 \\
144.00 \\
145.00 \\
146.00 \\
147.00 \\
148.00 \\
149.00 \\
150.00\end{array}$ & $\begin{array}{l}68.19 \\
68.68 \\
69.18 \\
69.67 \\
70.17 \\
70.66 \\
71.15 \\
72.64 \\
72.14 \\
72.63\end{array}$ & $\begin{array}{l}1665.98 \\
1683.00 \\
1700.04 \\
1717.10 \\
1734.17 \\
1751.26 \\
1768.37 \\
1705.49 \\
1802.62 \\
1019.77\end{array}$ & $\begin{array}{l}1044.13 \\
1056.63 \\
1069.17 \\
1081.72 \\
1094.29 \\
1104.96 \\
1119.49 \\
1132.12 \\
1144.77 \\
1157.43\end{array}$ & $\begin{array}{l}33.829 \\
33.960 \\
34.079 \\
34.198 \\
34.316 \\
34.434 \\
34.550 \\
34.564 \\
34.722 \\
34.896\end{array}$ \\
\hline $\begin{array}{l}81.00 \\
82.00 \\
83.00 \\
84.00 \\
85.00 \\
86.00 \\
87.00 \\
88.00 \\
89.00 \\
90.00\end{array}$ & $\begin{array}{l}36.81 \\
37.38 \\
37.94 \\
38.50 \\
39.06 \\
39.62 \\
40.17 \\
40.73 \\
41.28 \\
41.83\end{array}$ & $\begin{array}{l}696.92 \\
712.46 \\
727.99 \\
743.51 \\
759.03 \\
774.55 \\
790.06 \\
805.59 \\
821.12 \\
836.66\end{array}$ & $\begin{array}{l}361.23 \\
371.62 \\
382.02 \\
392.43 \\
402.84 \\
413.27 \\
423.71 \\
434.18 \\
444.67 \\
455.19\end{array}$ & $\begin{array}{l}24.930 \\
25.121 \\
25.309 \\
25.495 \\
25.678 \\
25.860 \\
26.039 \\
26.217 \\
26.392 \\
26.566\end{array}$ & $\begin{array}{l}151.00 \\
152.00 \\
153.00 \\
154.00 \\
155.00 \\
156.00 \\
157.00 \\
158.00 \\
159.00 \\
160.00\end{array}$ & $\begin{array}{l}73.12 \\
73.61 \\
74.10 \\
74.58 \\
75.07 \\
75.56 \\
76.05 \\
76.53 \\
77.02 \\
77.50\end{array}$ & $\begin{array}{l}1836.06 \\
1854.01 \\
1871.15 \\
1808.29 \\
1905.44 \\
1922.59 \\
1939.74 \\
1956.90 \\
1974.06 \\
1991.22\end{array}$ & $\begin{array}{l}1170.00 \\
1102.75 \\
1195.43 \\
1208.12 \\
1220.82 \\
1233.53 \\
1246.24 \\
1258.96 \\
1271.69 \\
1204.42\end{array}$ & $\begin{array}{l}35.010 \\
35.123 \\
35.236 \\
35.347 \\
35.450 \\
35.569 \\
35.678 \\
35.787 \\
35.095 \\
36.903\end{array}$ \\
\hline
\end{tabular}


TEMPER- SPECIFIC ENTHALPY INTERNAL ENTROPY ATURE VOLUME (J/GM) ENERGY (J/GM-K) (K) (CC/GM)
(J/GM)

\begin{tabular}{|c|c|c|c|}
\hline $\begin{array}{l}\text { TEMPER- } \\
\text { ATURE } \\
\text { (K) }\end{array}$ & $\begin{array}{l}\text { SPECIFIC } \\
\text { VOLUME } \\
\text { (CC/GM) }\end{array}$ & & \\
\hline
\end{tabular}

\begin{tabular}{|c|c|c|c|c|c|c|c|c|c|}
\hline $\begin{array}{l}161.00 \\
162.00 \\
163.00 \\
164.00 \\
165.00 \\
166.00 \\
167.00 \\
168.00 \\
169.00 \\
170.00\end{array}$ & $\begin{array}{l}77.99 \\
78.47 \\
78.96 \\
79.44 \\
79.93 \\
80.41 \\
80.89 \\
81.38 \\
81.86 \\
82.34\end{array}$ & $\begin{array}{l}2008.38 \\
2025.54 \\
2042.69 \\
2059.85 \\
2077.00 \\
2094.14 \\
2111.28 \\
2128.41 \\
2145.53 \\
2162.65\end{array}$ & $\begin{array}{l}1297.16 \\
1309.89 \\
1322.63 \\
1335.37 \\
1348.11 \\
1360.84 \\
1373.58 \\
1386.31 \\
1399.03 \\
1411.75\end{array}$ & $\begin{array}{l}36.110 \\
36.216 \\
36.322 \\
36.427 \\
36.531 \\
36.635 \\
36.737 \\
36.840 \\
36.941 \\
37.042\end{array}$ & $\begin{array}{l}231.00 \\
232.00 \\
233.00 \\
234.00 \\
235.00 \\
236.00 \\
237.00 \\
238.00 \\
239.00 \\
240.00\end{array}$ & $\begin{array}{l}111.19 \\
111.66 \\
112.12 \\
112.59 \\
113.06 \\
113.52 \\
113.99 \\
114.45 \\
114.92 \\
115.39\end{array}$ & $\begin{array}{l}3173.62 \\
3189.60 \\
3205.56 \\
3221.50 \\
32.37 .42 \\
3253.32 \\
3269.21 \\
3285.07 \\
3300.92 \\
3316.75\end{array}$ & $\begin{array}{l}2159.63 \\
2171.35 \\
2183.06 \\
2194.74 \\
2206.41 \\
2218.06 \\
2229.69 \\
2241.31 \\
2252.91 \\
2264.49\end{array}$ & $\begin{array}{l}42.133 \\
42.202 \\
42.271 \\
42.339 \\
42.407 \\
42.474 \\
42.541 \\
42.608 \\
42.675 \\
42.741\end{array}$ \\
\hline $\begin{array}{l}171.00 \\
172.00 \\
173.00 \\
174.00 \\
175.00 \\
176.00 \\
177.00 \\
178.00 \\
179.00 \\
180.00\end{array}$ & $\begin{array}{l}82.82 \\
83.30 \\
83.78 \\
84.26 \\
84.74 \\
85.22 \\
85.70 \\
86.18 \\
86.66 \\
87.13\end{array}$ & $\begin{array}{l}2179.74 \\
2196.82 \\
2213.89 \\
2230.94 \\
2247.99 \\
2265.02 \\
2282.03 \\
2299.03 \\
2316.02 \\
2332.99\end{array}$ & $\begin{array}{l}1424.46 \\
1437.16 \\
1449.84 \\
1462.52 \\
1475.19 \\
1487.85 \\
1500.50 \\
1513.14 \\
1525.77 \\
1538.38\end{array}$ & $\begin{array}{l}37.143 \\
37.242 \\
37.341 \\
37.439 \\
37.537 \\
37.634 \\
37.731 \\
37.826 \\
37.921 \\
38.016\end{array}$ & $\begin{array}{l}241.00 \\
242.00 \\
243.00 \\
244.00 \\
245.00 \\
246.00 \\
247.00 \\
248.00 \\
249.00 \\
250.00\end{array}$ & $\begin{array}{l}115.85 \\
116.32 \\
116.78 \\
117.25 \\
117.71 \\
118.18 \\
118.64 \\
119.11 \\
119.57 \\
120.03\end{array}$ & $\begin{array}{l}3332.56 \\
3348.35 \\
3364.13 \\
3379.88 \\
3395.62 \\
3411.34 \\
3427.04 \\
3442.73 \\
3458.39 \\
3474.04\end{array}$ & $\begin{array}{l}2276.06 \\
2287.60 \\
2299.14 \\
2310.65 \\
2322.15 \\
2333.63 \\
2345.10 \\
2356.55 \\
2367.98 \\
2379.40\end{array}$ & $\begin{array}{l}42.807 \\
42.872 \\
42.937 \\
43.002 \\
43.066 \\
43.130 \\
43.194 \\
43.257 \\
43.320 \\
43.383\end{array}$ \\
\hline $\begin{array}{l}181.00 \\
182.00 \\
183.00 \\
184.00 \\
185.00 \\
186.00 \\
187.00 \\
188.00 \\
189.00 \\
190.00\end{array}$ & $\begin{array}{l}87.61 \\
88.09 \\
88.56 \\
89.04 \\
89.52 \\
89.99 \\
90.47 \\
90.94 \\
91.42 \\
91.89\end{array}$ & $\begin{array}{l}2349.94 \\
2366.88 \\
2383.80 \\
2400.71 \\
2417.59 \\
2434.46 \\
2451.31 \\
2468.15 \\
2484.96 \\
2501.75\end{array}$ & $\begin{array}{l}1550.98 \\
1563.57 \\
1576.14 \\
1588.70 \\
1601.24 \\
1613.77 \\
1626.28 \\
1638.78 \\
1651.26 \\
1663.73\end{array}$ & $\begin{array}{l}38.110 \\
38.203 \\
38.296 \\
38.388 \\
38.480 \\
38.571 \\
38.661 \\
38.751 \\
38.840 \\
38.928\end{array}$ & $\begin{array}{l}251.00 \\
252.00 \\
253.00 \\
254.00 \\
255.00 \\
256.00 \\
257.00 \\
258.00 \\
259.00 \\
260.00\end{array}$ & $\begin{array}{l}120.50 \\
120.96 \\
121.43 \\
121.89 \\
122.35 \\
122.82 \\
123.28 \\
123.74 \\
124.21 \\
124.67\end{array}$ & $\begin{array}{l}3489.71 \\
3505.36 \\
3520.99 \\
3536.61 \\
3552.21 \\
3567.79 \\
3583.35 \\
3598.90 \\
3614.43 \\
3629.95\end{array}$ & $\begin{array}{l}2390.83 \\
2402.25 \\
2413.66 \\
2425.04 \\
2436.42 \\
2447.77 \\
2459.11 \\
2470.44 \\
2481.75 \\
2493.04\end{array}$ & $\begin{array}{l}43.445 \\
43.508 \\
43.570 \\
43.631 \\
43.693 \\
43.753 \\
43.814 \\
43.875 \\
43.935 \\
43.994\end{array}$ \\
\hline $\begin{array}{l}191.00 \\
192.00 \\
193.00 \\
194.00 \\
195.00 \\
196.00 \\
197.00 \\
198.00 \\
199.00 \\
200.00\end{array}$ & $\begin{array}{l}92.37 \\
92.84 \\
93.32 \\
93.79 \\
94.26 \\
94.74 \\
95.21 \\
95.68 \\
96.16 \\
96.63\end{array}$ & $\begin{array}{l}2518.53 \\
2535.29 \\
2552.03 \\
2568.75 \\
2585.45 \\
2602.13 \\
2618.79 \\
2635.43 \\
2652.05 \\
2668.65\end{array}$ & $\begin{array}{l}1676.18 \\
1688.61 \\
1701.02 \\
1713.42 \\
1725.80 \\
1738.17 \\
1750.51 \\
1762.84 \\
1775.15 \\
1787.45\end{array}$ & $\begin{array}{l}39.017 \\
39.104 \\
39.191 \\
39.277 \\
39.363 \\
39.449 \\
39.533 \\
39.618 \\
39.701 \\
39.785\end{array}$ & $\begin{array}{l}261.00 \\
262.00 \\
263.00 \\
264.00 \\
265.00 \\
266.00 \\
267.00 \\
268.00 \\
269.00 \\
270.00\end{array}$ & $\begin{array}{l}125.13 \\
125.59 \\
126.06 \\
126.52 \\
126.98 \\
127.44 \\
127.91 \\
128.37 \\
128.83 \\
129.29\end{array}$ & $\begin{array}{l}3645.45 \\
3660.93 \\
3676.40 \\
3691.85 \\
3707.28 \\
3722.70 \\
3738.11 \\
3753.50 \\
3768.87 \\
3784.24\end{array}$ & $\begin{array}{l}2504.32 \\
2515.58 \\
2526.83 \\
2538.06 \\
2549.27 \\
2560.48 \\
2571.66 \\
2582.84 \\
2593.99 \\
2605.14\end{array}$ & $\begin{array}{l}44.054 \\
44.113 \\
44.172 \\
44.231 \\
44.289 \\
44.347 \\
44.405 \\
44.462 \\
44.520 \\
44.577\end{array}$ \\
\hline $\begin{array}{l}201.00 \\
202.00 \\
203.00 \\
204.00 \\
205.00 \\
206.00 \\
207.00 \\
208.00 \\
209.00 \\
210.00\end{array}$ & $\begin{array}{r}97.10 \\
97.57 \\
98.05 \\
98.52 \\
98.99 \\
99.46 \\
99.93 \\
100.40 \\
100.87 \\
101.34\end{array}$ & $\begin{array}{l}2685.24 \\
2701.80 \\
2718.35 \\
2734.87 \\
2751.37 \\
2767.86 \\
2784.33 \\
2800.77 \\
2817.20 \\
2833.60\end{array}$ & $\begin{array}{l}1799.72 \\
1811.98 \\
1824.22 \\
1836.45 \\
1848.65 \\
1860.84 \\
1873.01 \\
1885.16 \\
1897.29 \\
1909.41\end{array}$ & $\begin{array}{l}39.867 \\
39.950 \\
40.031 \\
40.112 \\
40.193 \\
40.273 \\
40.353 \\
40.432 \\
40.511 \\
40.589\end{array}$ & $\begin{array}{l}271.00 \\
272.00 \\
273.00 \\
274.00 \\
275.00 \\
276.00 \\
277.00 \\
278.00 \\
279.00 \\
280.00\end{array}$ & $\begin{array}{l}129.76 \\
130.22 \\
130.68 \\
131.14 \\
131.61 \\
132.07 \\
132.53 \\
132.99 \\
133.45 \\
133.92\end{array}$ & $\begin{array}{l}3799.58 \\
3814.92 \\
3830.24 \\
3845.54 \\
3860.84 \\
3876.12 \\
3891.38 \\
3906.64 \\
3921.89 \\
3937.12\end{array}$ & $\begin{array}{l}2616.27 \\
2627.39 \\
2638.49 \\
2649.58 \\
2660.66 \\
2671.73 \\
2682.78 \\
2693.82 \\
2704.86 \\
2715.83\end{array}$ & $\begin{array}{l}44.633 \\
44.690 \\
44.746 \\
44.802 \\
44.858 \\
44.913 \\
44.969 \\
45.023 \\
45.078 \\
45.133\end{array}$ \\
\hline $\begin{array}{l}211.00 \\
212.00 \\
213.00 \\
214.00 \\
215.00 \\
216.00 \\
217.00 \\
218.00 \\
219.00 \\
220.00\end{array}$ & $\begin{array}{l}101.81 \\
102.28 \\
102.75 \\
103.22 \\
103.69 \\
104.16 \\
104.63 \\
105.10 \\
105.57 \\
106.04\end{array}$ & $\begin{array}{l}2849.99 \\
2866.36 \\
2882.70 \\
2899.03 \\
2915.34 \\
2931.63 \\
2947.89 \\
2964.14 \\
2980.37 \\
2996.58\end{array}$ & $\begin{array}{l}1921.50 \\
1933.58 \\
1945.64 \\
1957.68 \\
1969.70 \\
1981.71 \\
1993.70 \\
2005.67 \\
2017.62 \\
2029.55\end{array}$ & $\begin{array}{l}40.667 \\
40.745 \\
40.822 \\
40.898 \\
40.974 \\
41.050 \\
41.125 \\
41.200 \\
41.274 \\
41.348\end{array}$ & $\begin{array}{l}281.00 \\
282.00 \\
283.00 \\
284.00 \\
285.00 \\
286.00 \\
287.00 \\
288.00 \\
289.00 \\
290.00\end{array}$ & $\begin{array}{l}134.38 \\
134.84 \\
135.30 \\
135.76 \\
136.22 \\
136.69 \\
137.15 \\
137.61 \\
138.07 \\
138.53\end{array}$ & $\begin{array}{l}3952.34 \\
3967.56 \\
3982.76 \\
3997.95 \\
4013.14 \\
4028.31 \\
4043.48 \\
4058.63 \\
4073.78 \\
4088.92\end{array}$ & $\begin{array}{l}2726.89 \\
2737.89 \\
2748.88 \\
2759.86 \\
2770.84 \\
2781.81 \\
2792.76 \\
2803.72 \\
2814.66 \\
2825.60\end{array}$ & $\begin{array}{l}45.187 \\
45.241 \\
45.295 \\
45.348 \\
45.402 \\
45.455 \\
45.508 \\
45.561 \\
45.613 \\
45.665\end{array}$ \\
\hline $\begin{array}{l}221.00 \\
222.00 \\
223.00 \\
224.00 \\
225.00 \\
226.00 \\
227.00 \\
228.00 \\
229.00 \\
230.00\end{array}$ & $\begin{array}{l}106 \cdot 51 \\
106.98 \\
107.45 \\
107.91 \\
108 \cdot 38 \\
108.85 \\
109.32 \\
109 \cdot 79 \\
110.25 \\
110.72\end{array}$ & $\begin{array}{l}3012.77 \\
3028.94 \\
3045.10 \\
3061.23 \\
3077.34 \\
3093.44 \\
3109.51 \\
3125.57 \\
3141.60 \\
3157.62\end{array}$ & $\begin{array}{l}2041.46 \\
2053 \cdot 36 \\
2065.24 \\
2077 \cdot 10 \\
2088.95 \\
2100.77 \\
2112.58 \\
2124.37 \\
2136.14 \\
2147.90\end{array}$ & $\begin{array}{l}41.421 \\
41.494 \\
41.567 \\
41.639 \\
41.7 .11 \\
41.782 \\
41.853 \\
41.924 \\
41.994 \\
42.064\end{array}$ & $\begin{array}{l}291.00 \\
292.00 \\
293.00 \\
294.00 \\
295.00 \\
296.00 \\
297.00 \\
298.00 \\
299.00 \\
300.00\end{array}$ & $\begin{array}{l}138.99 \\
139.45 \\
139.91 \\
140.37 \\
140.83 \\
141.29 \\
141.75 \\
142.21 \\
142.67 \\
143.13\end{array}$ & $\begin{array}{l}4104.06 \\
4119.18 \\
4134.30 \\
4149.42 \\
4164.53 \\
4179.63 \\
4194.72 \\
4209.81 \\
4224.89 \\
4239.97\end{array}$ & $\begin{array}{l}2836.53 \\
2847.46 \\
2858.38 \\
2869.30 \\
2880.21 \\
2891.12 \\
2902.03 \\
2912.93 \\
2923.83 \\
2934.73\end{array}$ & $\begin{array}{l}45.718 \\
45.769 \\
45.821 \\
45.873 \\
45.924 \\
45.975 \\
46.026 \\
46.077 \\
46.127 \\
46.178\end{array}$ \\
\hline
\end{tabular}



$\begin{array}{ccccc}\text { TEMPER- } & \begin{array}{c}\text { SPECIFIC } \\ \text { VOTURE }\end{array} & \begin{array}{c}\text { ENTHALPY } \\ \text { (J/GM) }\end{array} & \begin{array}{c}\text { INTERNAL } \\ \text { ENERGY }\end{array} & \begin{array}{c}\text { ENTROPY } \\ \text { (J/GM-K) }\end{array}\end{array}$ (K)

$(J / G M)$ ATUR

(K)
SPECIFIC VOLUME
ENTHALPY

(J/GM)

\section{ENERGY}

(J/GM)
ENTROPY

(J/GM-K)

\begin{tabular}{|c|c|c|c|c|c|c|c|c|c|}
\hline 20.00 & 12.50 & -155.16 & -281.83 & 6.445 & & & & & \\
\hline $\begin{array}{l}21.00 \\
22.00 \\
23.00 \\
24.00 \\
25.00 \\
26.00 \\
27.00 \\
28.00 \\
29.00 \\
30.00\end{array}$ & $\begin{array}{l}12.60 \\
12.71 \\
12.82 \\
12.94 \\
13.06 \\
13.19 \\
13.33 \\
13.47 \\
13.62 \\
13.77\end{array}$ & $\begin{array}{r}-147.47 \\
-139.49 \\
-131.17 \\
-122.53 \\
-113.53 \\
-104.17 \\
-94.44 \\
-84.34 \\
-73.86 \\
-63.07\end{array}$ & $\begin{array}{l}-275.17 \\
-268.27 \\
-261.09 \\
-253.64 \\
-245.89 \\
-237.84 \\
-229.48 \\
-220.81 \\
-211.82 \\
-202.59\end{array}$ & $\begin{array}{r}6.823 \\
7.192 \\
7.564 \\
7.933 \\
8.299 \\
8.665 \\
9.032 \\
9.401 \\
9.766 \\
10.133\end{array}$ & $\begin{array}{r}91.00 \\
92.00 \\
93.00 \\
94.00 \\
95.00 \\
96.00 \\
97.00 \\
98.00 \\
99.00 \\
100.00\end{array}$ & $\begin{array}{l}38.66 \\
39.15 \\
39.64 \\
40.13 \\
40.62 \\
41.11 \\
41.59 \\
42.08 \\
42.56 \\
43.04\end{array}$ & $\begin{array}{l}845.28 \\
860.99 \\
876.72 \\
892.48 \\
908.25 \\
924.05 \\
939.88 \\
955.73 \\
971.60 \\
987.50\end{array}$ & $\begin{array}{l}453.57 \\
464.30 \\
475.06 \\
485.85 \\
496.67 \\
507.54 \\
518.44 \\
529.38 \\
540.36 \\
551.38\end{array}$ & $\begin{array}{l}26.211 \\
26.383 \\
26.553 \\
26.721 \\
26.888 \\
27.054 \\
27.218 \\
27.380 \\
27.541 \\
27.701\end{array}$ \\
\hline $\begin{array}{l}31.00 \\
32.00 \\
33.00 \\
34.00 \\
35.00 \\
36.00 \\
37.00 \\
38.00 \\
39.00 \\
40.00\end{array}$ & $\begin{array}{l}13.93 \\
14.10 \\
14.29 \\
14.47 \\
14.66 \\
14.87 \\
15.08 \\
15.30 \\
15.53 \\
15.78\end{array}$ & $\begin{array}{r}-51.87 \\
-40.37 \\
-28.42 \\
-16.12 \\
-3.38 \\
9.65 \\
22.91 \\
36.26 \\
49.76 \\
63.01\end{array}$ & $\begin{array}{r}-193.04 \\
-183.25 \\
-173.21 \\
-162.76 \\
-151.97 \\
-140.97 \\
-129.86 \\
-118.76 \\
-107.61 \\
-96.83\end{array}$ & $\begin{array}{l}10.500 \\
10.868 \\
11.238 \\
11.607 \\
11.976 \\
12.341 \\
12.702 \\
13.058 \\
13.409 \\
13.744\end{array}$ & $\begin{array}{l}101.00 \\
102.00 \\
103.00 \\
104.00 \\
105.00 \\
106.00 \\
107.00 \\
108.00 \\
109.00 \\
110.00\end{array}$ & $\begin{array}{l}43.52 \\
44.00 \\
44.48 \\
44.96 \\
45.44 \\
45.91 \\
46.39 \\
46.86 \\
47.33 \\
47.80\end{array}$ & $\begin{array}{l}1003.37 \\
1019.21 \\
1035.09 \\
1051.01 \\
1066.97 \\
1082.98 \\
1099.02 \\
1115.10 \\
1131.23 \\
1147.39\end{array}$ & $\begin{array}{l}562.36 \\
573.34 \\
584.37 \\
595.45 \\
606.58 \\
617.77 \\
629.00 \\
640.28 \\
651.62 \\
663.00\end{array}$ & $\begin{array}{l}27.859 \\
28.015 \\
28.170 \\
28.324 \\
28.477 \\
28.628 \\
28.779 \\
28.929 \\
29.077 \\
29.225\end{array}$ \\
\hline $\begin{array}{l}41.00 \\
42.00 \\
43.00 \\
44.00 \\
45.00 \\
46.00 \\
47.00 \\
48.00 \\
49.00 \\
50.00\end{array}$ & $\begin{array}{l}16.03 \\
16.30 \\
16.57 \\
16.86 \\
17.17 \\
17.48 \\
17.81 \\
18.15 \\
18.51 \\
18.87\end{array}$ & $\begin{array}{r}76.13 \\
89.25 \\
102.45 \\
115.77 \\
129.27 \\
142.97 \\
156.89 \\
171.04 \\
185.42 \\
200.02\end{array}$ & $\begin{array}{r}-86.29 \\
-75.87 \\
-65.50 \\
-55.11 \\
-44.68 \\
-34.17 \\
-23.58 \\
-12.88 \\
-2.09 \\
8.79\end{array}$ & $\begin{array}{l}14.068 \\
14.384 \\
14.694 \\
15.001 \\
15.304 \\
15.605 \\
15.905 \\
16.203 \\
16.499 \\
16.794\end{array}$ & $\begin{array}{l}111.00 \\
112.00 \\
113.00 \\
114.00 \\
115.00 \\
116.00 \\
117.00 \\
118.00 \\
119.00 \\
120.00\end{array}$ & $\begin{array}{l}48.28 \\
48.74 \\
49.21 \\
49.68 \\
50.15 \\
50.61 \\
51.08 \\
51.54 \\
52.00 \\
52.46\end{array}$ & $\begin{array}{l}1163.60 \\
1179.84 \\
1196.12 \\
1212.44 \\
1228.79 \\
1245.18 \\
1261.60 \\
1278.05 \\
1294.54 \\
1311.06\end{array}$ & $\begin{array}{l}674.44 \\
685.92 \\
697.45 \\
709.03 \\
720.66 \\
732.33 \\
744.06 \\
755.82 \\
767.63 \\
779.49\end{array}$ & $\begin{array}{l}29.372 \\
29.517 \\
29.662 \\
29.806 \\
29.949 \\
30.090 \\
30.231 \\
30.371 \\
30.511 \\
30.649\end{array}$ \\
\hline $\begin{array}{l}51.00 \\
52.00 \\
53.00 \\
54.00 \\
55.00 \\
56.00 \\
57.00 \\
58.00 \\
59.00 \\
60.00\end{array}$ & $\begin{array}{l}19.25 \\
19.64 \\
20.05 \\
20.46 \\
20.88 \\
21.32 \\
21.76 \\
22.22 \\
22.68 \\
23.14\end{array}$ & $\begin{array}{l}214.84 \\
229.85 \\
245.04 \\
260.41 \\
275.92 \\
291.56 \\
307.31 \\
323.15 \\
339.06 \\
355.03\end{array}$ & $\begin{array}{r}19.77 \\
30.82 \\
41.94 \\
53.11 \\
64.32 \\
75.55 \\
86.80 \\
98.05 \\
109.29 \\
120.52\end{array}$ & $\begin{array}{l}17.087 \\
17.379 \\
17.668 \\
17.956 \\
18.240 \\
18.522 \\
18.801 \\
19.076 \\
19.348 \\
19.617\end{array}$ & $\begin{array}{l}121.00 \\
122.00 \\
123.00 \\
124.00 \\
125.00 \\
126.00 \\
127.00 \\
128.00 \\
129.00 \\
130.00\end{array}$ & $\begin{array}{l}52.92 \\
53.38 \\
53.84 \\
54.29 \\
54.75 \\
55.20 \\
55.66 \\
56.11 \\
56.57 \\
57.02\end{array}$ & $\begin{array}{l}1327.60 \\
1344.18 \\
1360.78 \\
1377.41 \\
1394.07 \\
1410.79 \\
1427.54 \\
1444.32 \\
1461.13 \\
1477.96\end{array}$ & $\begin{array}{l}791.38 \\
803.31 \\
815.28 \\
827.29 \\
839.34 \\
851.43 \\
863.56 \\
875.73 \\
887.93 \\
900.16\end{array}$ & $\begin{array}{l}30.786 \\
30.922 \\
31.058 \\
31.193 \\
31.326 \\
31.460 \\
31.592 \\
31.724 \\
31.855 \\
31.985\end{array}$ \\
\hline $\begin{array}{l}61.00 \\
62.00 \\
63.00 \\
64.00 \\
65.00 \\
66.00 \\
67.00 \\
68.00 \\
69.00 \\
70.00\end{array}$ & $\begin{array}{l}23.62 \\
24.10 \\
24.59 \\
25.08 \\
25.57 \\
26.07 \\
26.57 \\
27.07 \\
27.58 \\
28.08\end{array}$ & $\begin{array}{l}371.04 \\
387.07 \\
403.13 \\
419.18 \\
435.23 \\
451.27 \\
467.29 \\
483.29 \\
499.26 \\
515.20\end{array}$ & $\begin{array}{l}131.71 \\
142.88 \\
154.01 \\
165.09 \\
176.14 \\
187.13 \\
198.08 \\
208.98 \\
219.84 \\
230.66\end{array}$ & $\begin{array}{l}19.881 \\
20.142 \\
20.399 \\
20.652 \\
20.901 \\
21.145 \\
21.386 \\
21.623 \\
21.856 \\
22.086\end{array}$ & $\begin{array}{l}131.00 \\
132.00 \\
133.00 \\
134.00 \\
135.00 \\
136.00 \\
137.00 \\
138.00 \\
139.00 \\
140.00\end{array}$ & $\begin{array}{l}57.48 \\
57.93 \\
58.38 \\
58.83 \\
59.28 \\
59.73 \\
60.18 \\
60.63 \\
61.08 \\
61.53\end{array}$ & $\begin{array}{r}1494.82 \\
1511.71 \\
1528.62 \\
1545.55 \\
1562.51 \\
1579.49 \\
1596.49 \\
1613.51 \\
1630.55 \\
1647.62\end{array}$ & $\begin{array}{r}912.43 \\
924.73 \\
937.06 \\
949.42 \\
961.81 \\
974.23 \\
986.68 \\
999.15 \\
1011.65 \\
1024.17\end{array}$ & $\begin{array}{l}32.114 \\
32.242 \\
32.370 \\
32.497 \\
32.623 \\
32.748 \\
32.873 \\
32.996 \\
33.119 \\
33.242\end{array}$ \\
\hline $\begin{array}{l}71.00 \\
72.00 \\
73.00 \\
74.00 \\
75.00 \\
76.00 \\
77.00 \\
78.00 \\
79.00 \\
80.00\end{array}$ & $\begin{array}{l}28.59 \\
29.10 \\
29.61 \\
30.12 \\
30.62 \\
31.13 \\
31.64 \\
32.15 \\
32.66 \\
33.16\end{array}$ & $\begin{array}{l}531.11 \\
546.99 \\
562.84 \\
578.66 \\
594.45 \\
610.21 \\
625.95 \\
641.67 \\
657.36 \\
673.04\end{array}$ & $\begin{array}{l}241.43 \\
252.16 \\
262.86 \\
273.52 \\
284.15 \\
294.76 \\
305.35 \\
315.92 \\
326.48 \\
337.03\end{array}$ & $\begin{array}{l}22.312 \\
22.534 \\
22.752 \\
22.967 \\
23.179 \\
23.388 \\
23.594 \\
23.797 \\
23.997 \\
24.194\end{array}$ & $\begin{array}{l}141.00 \\
142.00 \\
143.00 \\
144.00 \\
145.00 \\
146.00 \\
147.00 \\
148.00 \\
149.00 \\
150.00\end{array}$ & $\begin{array}{l}61.97 \\
62.42 \\
62.87 \\
63.31 \\
63.76 \\
64.20 \\
64.65 \\
65.09 \\
65.53 \\
65.98\end{array}$ & $\begin{array}{l}1664.70 \\
1681.79 \\
1698.91 \\
1716.04 \\
1733.19 \\
1750.35 \\
1767.53 \\
1784.72 \\
1801.93 \\
1819.15\end{array}$ & $\begin{array}{l}1036.72 \\
1049.30 \\
1061.89 \\
1074.51 \\
1087.15 \\
1099.81 \\
1112.49 \\
1125.18 \\
1137.90 \\
1150.63\end{array}$ & $\begin{array}{l}33.363 \\
33.484 \\
33.604 \\
33.724 \\
33.842 \\
33.960 \\
34.077 \\
34.194 \\
34.310 \\
34.425\end{array}$ \\
\hline $\begin{array}{l}81.00 \\
82.00 \\
83.00 \\
84.00 \\
85.00 \\
86.00 \\
87.00 \\
86.00 \\
89.00 \\
90.00\end{array}$ & $\begin{array}{l}33.67 \\
34.17 \\
34.67 \\
35.18 \\
35.68 \\
36.18 \\
36.68 \\
37.17 \\
37.67 \\
38.16\end{array}$ & $\begin{array}{l}688.71 \\
704.36 \\
720.01 \\
735.65 \\
751.29 \\
766.94 \\
782.58 \\
798.24 \\
813.91 \\
829.59\end{array}$ & $\begin{array}{l}347.58 \\
358.12 \\
368.67 \\
379.22 \\
389.79 \\
400.36 \\
410.96 \\
421.58 \\
432.22 \\
442.88\end{array}$ & $\begin{array}{l}24.388 \\
24.581 \\
24.770 \\
24.958 \\
25.143 \\
25.326 \\
25.507 \\
25.685 \\
25.863 \\
26.038\end{array}$ & $\begin{array}{l}151.00 \\
152.00 \\
153.00 \\
154.00 \\
155.00 \\
156.00 \\
157.00 \\
158.00 \\
159.00 \\
160.00\end{array}$ & $\begin{array}{l}66.42 \\
66.86 \\
67.30 \\
67.74 \\
68.18 \\
68.62 \\
69.06 \\
69.50 \\
69.94 \\
70.38\end{array}$ & $\begin{array}{l}1836.34 \\
1853.55 \\
1870.76 \\
1887.98 \\
1905.20 \\
1922.43 \\
1939.66 \\
1956.89 \\
1974.13 \\
1991.37\end{array}$ & $\begin{array}{l}1163.34 \\
1176.07 \\
1188.81 \\
1201.56 \\
1214.32 \\
1227.08 \\
1239.86 \\
1252.64 \\
1265.42 \\
1278.21\end{array}$ & $\begin{array}{l}34.539 \\
34.653 \\
34.766 \\
34.878 \\
34.989 \\
35.100 \\
35.210 \\
35.320 \\
35.428 \\
35.537\end{array}$ \\
\hline
\end{tabular}


TEMPER- SPECIFIC ENTHALPY INTERNAL ENTROPY ATURE VOLUME (J/GM) ENERGY (J/GM-K) (K) (CC/GM)
$(J / G M)$ TEMPER-
ATURE
(K)
SPECIFIC ENTHALPY VOLUME

$(J / G M)$
INTERNAL ENTROPY
ENERGY (J/GM-K)

(J/GM)

\begin{tabular}{|c|c|c|c|c|c|c|c|c|c|}
\hline $\begin{array}{l}161.00 \\
162.00 \\
163.00 \\
164.00 \\
165.00 \\
166.00 \\
167.00 \\
168.00 \\
169.00 \\
170.00\end{array}$ & $\begin{array}{l}70.82 \\
71.26 \\
71.70 \\
72.13 \\
72.57 \\
73.01 \\
73.44 \\
73.88 \\
74.32 \\
74.75\end{array}$ & $\begin{array}{l}2008.60 \\
2025.84 \\
2043.07 \\
2060.29 \\
2077.52 \\
2094.74 \\
2111.95 \\
2129.15 \\
2146.35 \\
2163.53\end{array}$ & $\begin{array}{l}1291.00 \\
1303.80 \\
1316.59 \\
1329.38 \\
1342.17 \\
1354.96 \\
1367.75 \\
1380.53 \\
1393.31 \\
1406.08\end{array}$ & $\begin{array}{l}35.644 \\
35.751 \\
35.857 \\
35.962 \\
36.067 \\
36.171 \\
36.274 \\
36.377 \\
36.479 \\
36.580\end{array}$ & $\begin{array}{l}231.00 \\
232.00 \\
233.00 \\
234.00 \\
235.00 \\
236.00 \\
237.00 \\
238.00 \\
239.00 \\
240.00\end{array}$ & $\begin{array}{l}100.79 \\
101.21 \\
101.63 \\
102.05 \\
102.48 \\
102.90 \\
103.32 \\
103.74 \\
104.16 \\
104.58\end{array}$ & $\begin{array}{l}3177.17 \\
3193.18 \\
3209.17 \\
3225.15 \\
3241.10 \\
3257.03 \\
3272.94 \\
3288.84 \\
3304.71 \\
3320.57\end{array}$ & $\begin{array}{l}2155.88 \\
2167.62 \\
2179.35 \\
2191.05 \\
2202.74 \\
2214.41 \\
2226.07 \\
2237.70 \\
2249.32 \\
2260.92\end{array}$ & $\begin{array}{l}41.685 \\
41.754 \\
41.822 \\
41.891 \\
41.959 \\
42.027 \\
42.094 \\
42.161 \\
42.227 \\
42.294\end{array}$ \\
\hline $\begin{array}{l}171.00 \\
172.00 \\
173.00 \\
174.00 \\
175.00 \\
176.00 \\
177.00 \\
178.00 \\
179.00 \\
180.00\end{array}$ & $\begin{array}{l}75.19 \\
75.62 \\
76.06 \\
76.49 \\
76.92 \\
77.36 \\
77.79 \\
78.22 \\
78.65 \\
79.08\end{array}$ & $\begin{array}{l}2180.69 \\
2197.83 \\
2214.96 \\
2232.08 \\
2249.18 \\
2266.27 \\
2283.34 \\
2300.40 \\
2317.44 \\
2334.46\end{array}$ & $\begin{array}{l}1418.83 \\
1431.57 \\
1444.30 \\
1457.03 \\
1469.74 \\
1482.44 \\
1495.13 \\
1507.81 \\
1520.47 \\
1533.13\end{array}$ & $\begin{array}{l}36.681 \\
36.781 \\
36.880 \\
36.979 \\
37.077 \\
37.174 \\
37.271 \\
37.367 \\
37.463 \\
37.557\end{array}$ & $\begin{array}{l}241.00 \\
242.00 \\
243.00 \\
244.00 \\
245.00 \\
246.00 \\
247.00 \\
248.00 \\
249.00 \\
250.00\end{array}$ & $\begin{array}{l}105.00 \\
105.42 \\
105.84 \\
106.25 \\
106.67 \\
107.09 \\
107.51 \\
107.93 \\
108.35 \\
108.77\end{array}$ & $\begin{array}{l}3336.41 \\
3352.23 \\
3368.03 \\
3383.81 \\
3399.57 \\
3415.32 \\
3431.05 \\
3446.75 \\
3462.45 \\
3478.12\end{array}$ & $\begin{array}{l}2272.51 \\
2284.07 \\
2295.62 \\
2307.16 \\
2318.67 \\
2330.17 \\
2341.66 \\
2353.12 \\
2364.57 \\
2376.01\end{array}$ & $\begin{array}{l}42.359 \\
42.425 \\
42.490 \\
42.555 \\
42.619 \\
42.683 \\
42.747 \\
42.811 \\
42.874 \\
42.937\end{array}$ \\
\hline $\begin{array}{l}181.00 \\
182.00 \\
183.00 \\
184.00 \\
185.00 \\
186.00 \\
187.00 \\
188.00 \\
189.00 \\
190.00\end{array}$ & $\begin{array}{l}79.52 \\
79.95 \\
80.38 \\
80.81 \\
81.24 \\
81.67 \\
82.10 \\
82.52 \\
82.95 \\
83.38\end{array}$ & $\begin{array}{l}2351.47 \\
2368.46 \\
2385.43 \\
2402.38 \\
2419.32 \\
2436.23 \\
2453.13 \\
2470.01 \\
2486.87 \\
2503.70\end{array}$ & $\begin{array}{l}1545.76 \\
1558.39 \\
1570.99 \\
1583.59 \\
1596.17 \\
1608.73 \\
1621.27 \\
1633.80 \\
1646.32 \\
1658.82\end{array}$ & $\begin{array}{l}37.652 \\
37.745 \\
37.838 \\
37.931 \\
38.022 \\
38.114 \\
38.204 \\
38.294 \\
38.384 \\
38.472\end{array}$ & $\begin{array}{l}251.00 \\
252.00 \\
253.00 \\
254.00 \\
255.00 \\
256.00 \\
257.00 \\
258.00 \\
259.00 \\
260.00\end{array}$ & $\begin{array}{l}109.19 \\
109.60 \\
110.02 \\
110.44 \\
110.86 \\
111.27 \\
111.69 \\
112.11 \\
112.53 \\
112.94\end{array}$ & $\begin{array}{l}3493.81 \\
3509.48 \\
3525.14 \\
3540.78 \\
3556.40 \\
3572.01 \\
3587.60 \\
3603.17 \\
3618.72 \\
3634.26\end{array}$ & $\begin{array}{l}2387.46 \\
2398.90 \\
2410.32 \\
2421.72 \\
2433.11 \\
2444.48 \\
2455.84 \\
2467.18 \\
2478.51 \\
2489.82\end{array}$ & $\begin{array}{l}42.999 \\
43.062 \\
43.124 \\
43.185 \\
43.247 \\
43.308 \\
43.369 \\
43.429 \\
43.489 \\
43.549\end{array}$ \\
\hline $\begin{array}{l}191.00 \\
192.00 \\
193.00 \\
194.00 \\
195.00 \\
196.00 \\
197.00 \\
198.00 \\
199.00 \\
200.00\end{array}$ & $\begin{array}{l}83.81 \\
84.24 \\
84.67 \\
85.09 \\
85.52 \\
85.95 \\
86.37 \\
86.80 \\
87.23 \\
87.65\end{array}$ & $\begin{array}{l}2520.52 \\
2537.32 \\
2554.10 \\
2570.86 \\
2587.60 \\
2604.33 \\
2621.03 \\
2637.71 \\
2654.37 \\
2671.01\end{array}$ & $\begin{array}{l}1671.30 \\
1683.76 \\
1696.20 \\
1708.63 \\
1721.04 \\
1733.44 \\
1745.81 \\
1758.17 \\
1770.51 \\
1782.84\end{array}$ & $\begin{array}{l}38.561 \\
38.648 \\
38.736 \\
38.822 \\
38.908 \\
38.994 \\
39.079 \\
39.163 \\
39.247 \\
39.331\end{array}$ & $\begin{array}{l}261.00 \\
262.00 \\
263.00 \\
264.00 \\
265.00 \\
266.00 \\
267.00 \\
268.00 \\
269.00 \\
270.00\end{array}$ & $\begin{array}{l}113.36 \\
113.78 \\
114.20 \\
114.61 \\
115.03 \\
115.45 \\
115.86 \\
116.28 \\
116.70 \\
117.11\end{array}$ & $\begin{array}{l}3649.79 \\
3665.30 \\
3680.79 \\
3696.26 \\
3711.72 \\
3727.17 \\
3742.60 \\
3758.02 \\
3773.42 \\
3788.81\end{array}$ & $\begin{array}{l}2501.12 \\
2512.40 \\
2523.66 \\
2534.91 \\
2546.15 \\
2557.37 \\
2568.58 \\
2579.77 \\
2590.95 \\
2602.11\end{array}$ & $\begin{array}{l}43.609 \\
43.668 \\
43.727 \\
43.786 \\
43.844 \\
43.902 \\
43.960 \\
44.018 \\
44.075 \\
44.132\end{array}$ \\
\hline $\begin{array}{l}201.00 \\
202.00 \\
203.00 \\
204.00 \\
205.00 \\
206.00 \\
207.00 \\
208.00 \\
209.00 \\
210.00\end{array}$ & $\begin{array}{l}88.08 \\
88.51 \\
88.93 \\
89.36 \\
89.78 \\
90.21 \\
90.63 \\
91.06 \\
91.48 \\
91.91\end{array}$ & $\begin{array}{l}2687.64 \\
2704.24 \\
2720.83 \\
2737.40 \\
2753.94 \\
2770.47 \\
2786.98 \\
2803.46 \\
2819.93 \\
2836.38\end{array}$ & $\begin{array}{l}1795.14 \\
1807.43 \\
1819.71 \\
1831.96 \\
1844.20 \\
1856.41 \\
1868.61 \\
1880.79 \\
1892.96 \\
1905.10\end{array}$ & $\begin{array}{l}39.414 \\
39.496 \\
39.578 \\
39.659 \\
39.740 \\
39.821 \\
39.901 \\
39.980 \\
40.059 \\
40.138\end{array}$ & $\begin{array}{l}271.00 \\
272.00 \\
273.00 \\
274.00 \\
275.00 \\
276.00 \\
277.00 \\
278.00 \\
279.00 \\
280.00\end{array}$ & $\begin{array}{l}117.53 \\
117.95 \\
118.37 \\
118.78 \\
119.20 \\
119.62 \\
120.03 \\
120.45 \\
120.87 \\
121.28\end{array}$ & $\begin{array}{l}3804.19 \\
3819.55 \\
3834.89 \\
3850.23 \\
3865.55 \\
3880.86 \\
3896.16 \\
3911.45 \\
3926.72 \\
3941.98\end{array}$ & $\begin{array}{l}2613.26 \\
2624.40 \\
2635.53 \\
2646.64 \\
2657.74 \\
2668.82 \\
2679.90 \\
2690.96 \\
2702.02 \\
2713.06\end{array}$ & $\begin{array}{l}44.189 \\
44.246 \\
44.302 \\
44.358 \\
44.414 \\
44.470 \\
44.525 \\
44.580 \\
44.635 \\
44.689\end{array}$ \\
\hline $\begin{array}{l}211.00 \\
212.00 \\
213.00 \\
214.00 \\
215.00 \\
216.00 \\
217.00 \\
218.00 \\
219.00 \\
220.00\end{array}$ & $\begin{array}{l}92.33 \\
92.76 \\
93.18 \\
93.60 \\
94.03 \\
94.45 \\
94.88 \\
95.30 \\
95.72 \\
96.15\end{array}$ & $\begin{array}{l}2852.81 \\
2869.21 \\
2885.60 \\
2901.97 \\
2918.31 \\
2934.64 \\
2950.95 \\
2967.24 \\
2983.51 \\
2999.75\end{array}$ & $\begin{array}{l}1917.23 \\
1929.33 \\
1941.42 \\
1953.49 \\
1965.54 \\
1977.58 \\
1989.59 \\
2001.59 \\
2013.57 \\
2025.53\end{array}$ & $\begin{array}{l}40.216 \\
40.293 \\
40.370 \\
40.447 \\
40.523 \\
40.599 \\
40.674 \\
40.749 \\
40.824 \\
40.898\end{array}$ & $\begin{array}{l}281.00 \\
282.00 \\
283.00 \\
284.00 \\
285.00 \\
286.00 \\
287.00 \\
288.00 \\
289.00 \\
290.00\end{array}$ & $\begin{array}{l}121.70 \\
122.12 \\
122.53 \\
122.95 \\
123.36 \\
123.78 \\
124.20 \\
124.61 \\
125.03 \\
125.44\end{array}$ & $\begin{array}{l}3957.23 \\
3972.48 \\
3987.71 \\
4002.93 \\
4018.14 \\
4033.34 \\
4048.53 \\
4063.71 \\
4078.89 \\
4094.05\end{array}$ & $\begin{array}{l}2724.09 \\
2735.11 \\
2746.12 \\
2757.13 \\
2768.12 \\
2779.11 \\
2790.08 \\
2801.05 \\
2812.01 \\
2822.97\end{array}$ & $\begin{array}{l}44.744 \\
44.798 \\
44.852 \\
44.906 \\
44.959 \\
45.012 \\
45.065 \\
45.118 \\
45.171 \\
45.223\end{array}$ \\
\hline $\begin{array}{l}221.00 \\
222.00 \\
223.00 \\
224.00 \\
225.00 \\
226.00 \\
227.00 \\
228.00 \\
229.00 \\
230.00\end{array}$ & $\begin{array}{r}96.57 \\
96.99 \\
97.41 \\
97.84 \\
98.26 \\
98.68 \\
99.10 \\
99.53 \\
99.95 \\
100.37\end{array}$ & $\begin{array}{l}3015.98 \\
3032.19 \\
3048.38 \\
3064.55 \\
3080.69 \\
3096.82 \\
3112.93 \\
3129.02 \\
3145.09 \\
3161.14\end{array}$ & $\begin{array}{l}2037.47 \\
2049.39 \\
2061.30 \\
2073.18 \\
2085.05 \\
2096.90 \\
2108.73 \\
2120.55 \\
2132.34 \\
2144.12\end{array}$ & $\begin{array}{l}40.971 \\
41.044 \\
41.117 \\
41.189 \\
41.261 \\
41.333 \\
41.404 \\
41.475 \\
41.545 \\
41.615\end{array}$ & $\begin{array}{l}291.00 \\
292.00 \\
293.00 \\
294.00 \\
295.00 \\
296.00 \\
297.00 \\
298.00 \\
299.00 \\
300.00\end{array}$ & $\begin{array}{l}125.86 \\
126.27 \\
126.69 \\
127.10 \\
127.52 \\
127.93 \\
128.34 \\
128.76 \\
129.17 \\
129.58\end{array}$ & $\begin{array}{l}4109.21 \\
4124.36 \\
4139.50 \\
4154.63 \\
4169.76 \\
4184.87 \\
4199.98 \\
4215.08 \\
4230.18 \\
4245.27\end{array}$ & $\begin{array}{l}2833.92 \\
2844.86 \\
2855.80 \\
2866.73 \\
2877.66 \\
2888.58 \\
2899.50 \\
2910.41 \\
2921.32 \\
2932.22\end{array}$ & $\begin{array}{l}45.275 \\
45.327 \\
45.379 \\
45.431 \\
45.482 \\
45.533 \\
45.584 \\
45.635 \\
45.685 \\
45.736\end{array}$ \\
\hline
\end{tabular}




\begin{tabular}{|c|c|c|c|c|c|c|c|c|c|}
\hline $\begin{array}{l}\text { IEMPER- } \\
\text { ATURE } \\
\text { (K) }\end{array}$ & $\begin{array}{l}\text { SPECIFIC } \\
\text { VOLUME } \\
\text { (CC/GM) }\end{array}$ & $\begin{array}{c}\text { EN THALPY } \\
(\mathrm{J} / \mathrm{GM})\end{array}$ & $\begin{array}{l}\text { INTERNAL } \\
\text { ENERGY } \\
\text { (J/GM) }\end{array}$ & $\begin{array}{l}\text { ENTROPY } \\
(\mathrm{J} / \mathrm{GM}-\mathrm{K})\end{array}$ & $\begin{array}{l}\text { TEMPER- } \\
\text { ATURE } \\
\text { (K) }\end{array}$ & $\begin{array}{l}\text { SPECIFIC } \\
\text { VOLUME } \\
\text { (CC/GM) }\end{array}$ & $\begin{array}{c}\text { ENTHALPY } \\
(\mathrm{J} / \mathrm{GM})\end{array}$ & $\begin{array}{c}\text { INT ERNAL } \\
\text { ENERGY } \\
(J / G M)\end{array}$ & $\begin{array}{l}\text { ENTROPY } \\
(J / G M-K)\end{array}$ \\
\hline
\end{tabular}

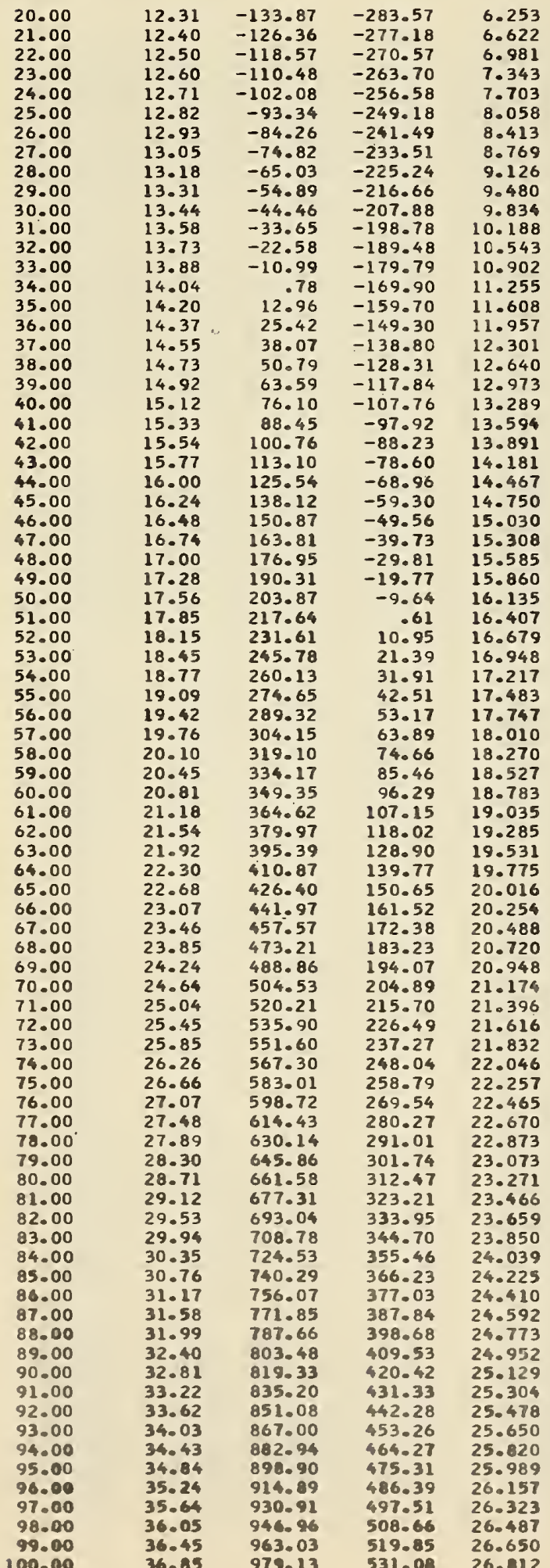

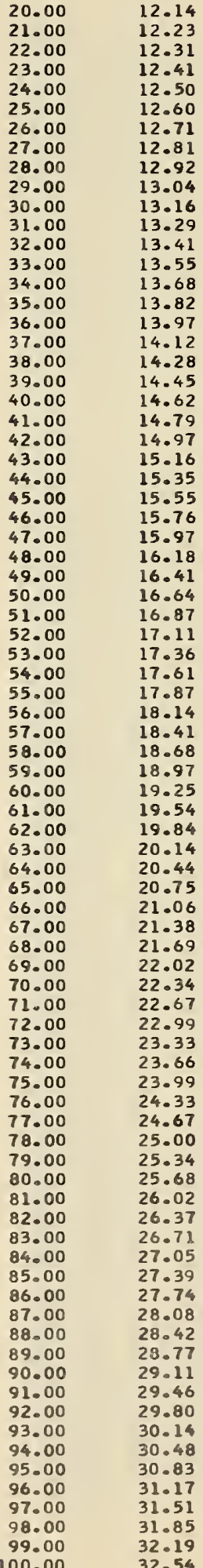

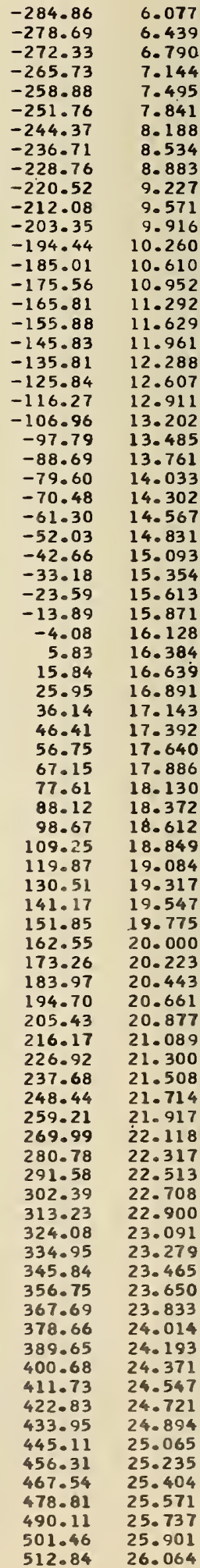


TURE

SPECIFIC

ENTHALPY

I NTERNAL

ENTROPY (CC/GM)

21.00

22.00

24.00

26.00

27.00

28.00

29.00

30.00

31.00

32.00

34.00

35.00

36.00

37.00

38.00

39.00

40.00

41.00

42.00

43.00

44.00

45.00

46.00

47.00

48.00

49.00

0.00

51.00

52.00

3.00

54.00

55.00

56.00

57.00

58.00

59.00

60.00

61.00

62.00

63.00

64.00

65.00

66.00

67.00

68.00

69.00

70.00

71.00

72.00

73.00

74.00

75.00

76.00

77.00

8.00

79.00

80.00

82.00

83.00

84.00

85.00

86.00

87.00

88.00

89.00

90.00

91.00

92.00

93.00

94.00

95.00

96.00

77.00

98.00

99.00

100.00
11.99

12.07

12.15

12.23

12.32

12.41

12.51

12.60

$-91.42$

-84.18
-76.70

$-68.95$

$-60.92$

$-52.57$

-43.91
-34.91

$-25.59$

12.81

12.92

13.03

13.15

13.26

13.51

13.64

13.77

13.91

$\$ 4.06$

14.21

14.36

14.52

14.68

14.84

15.02

15.37

15.56

15.75

15.94

16.14
16.34

16.55

16.76

16.98

17.20

17.42

17.65

17.89

18.12

18.37

18.61

18.86

19.11

19.37

19.63

19.89

20.15

20.42

20.69

20.96

21.24

21.52

21.80

22.08

22.36

22.93

23.22

23.50

23.79

24.08

24.66

24. 96

25.25

25.54

25. 84

26.13

26.43

26. 72

27.01

27.31

27.60

27. 90

28.20

28.49

28.79

29.08

$-15.93$

$-6.02$

14.70

25.77

36.83

48.25

59.94

83.73

95.64

107.21

18.60

129.90

141.21

52.57

164.04

175.65

87.4

199.35

211.48

23.79

236.28

248.97

261.84

274. 89

288. 12

301.51

315.06

328.76

342.61

356.59

370.70

384.93

399.27

413.72

428.27

442.91

457.64

472.44

487.32

502.27

517.29

532.36

547.50

562.69

577.93

593.22

608.55

623.94

639.37

654.83

670.35

685.90

701.49

717.13

732.80

748.52

764.27

780.07

795.91

811.78

827.70

843.66

859.66

875.69

891.77

907.89

924.05

940.24

956.47

972.74
(J/GM)

$-285.77$

$-279.80$

$-273.64$

$-267.27$

$-260.65$

$-253.78$

-246.65
-239.25

$-231.57$

$-223.61$

$-215.46$

7.03

-198.43
-189.23

$-180.15$

$-170.77$

$-161.20$

$-151.51$

$-141.85$

$-132.28$

$-114.20$

$-105.45$

$-96.76$

$-88.09$

$-79.39$

$-70.63$

$-61.79$

-52.84
-43.79

$-34.62$

$-25.34$

$-15.94$

$-6.43$

3.18
12.89

22. 70

32.59

42.58

52.63

62.76

72.96

B3. 21

93.52

103.88

114.27

124.72

135.19

145.70

156.24

166.81

177.41

188.02

198.67

209. 33

220.02

230.73

241.46

252.22

263.00 


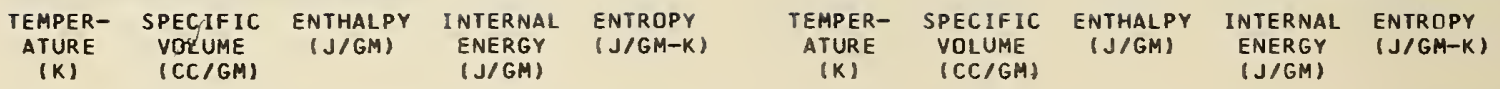

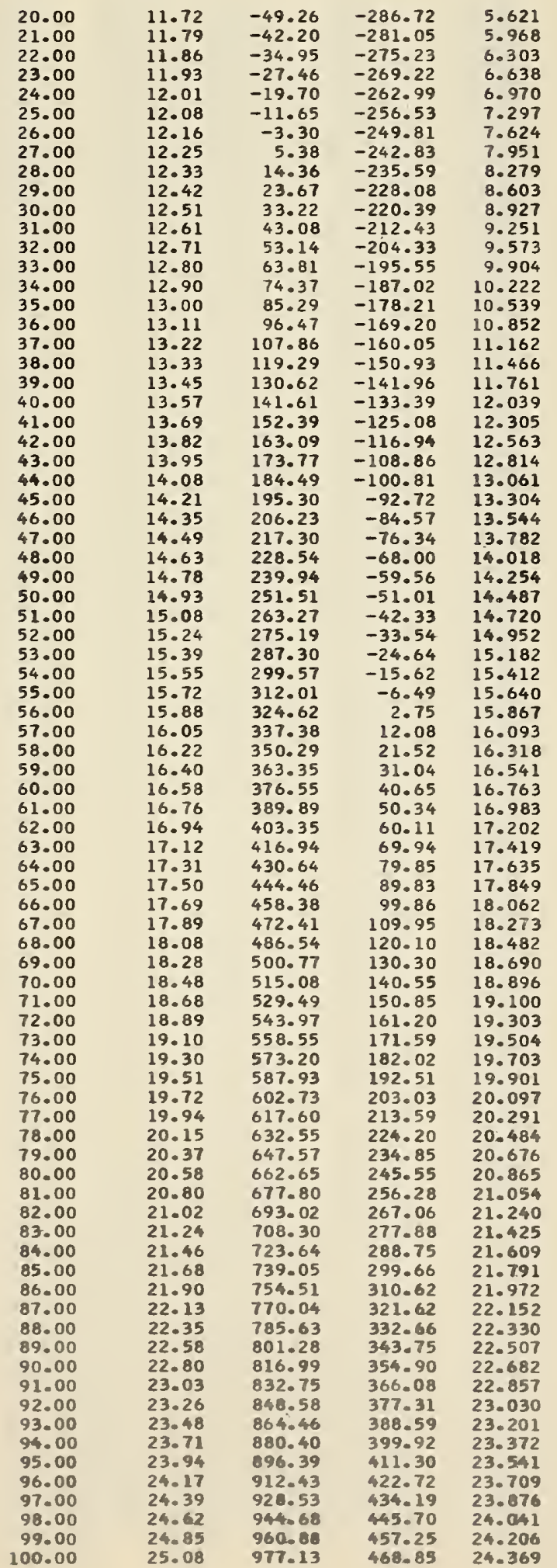

TEMPER20.00

$\begin{array}{lll}20.00 & 11.60 & -2 \\ 21.00 & 11.66 & -2 \\ 22.00 & 11.73 & -1 \\ 23.00 & 11.80 & -6 \\ 24.00 & 11.87 & \\ 25.00 & 11.94 & \\ 26.00 & 12.02 & 17 \\ 27.00 & 12.09 & \\ 28.00 & 12.17 & 3 \\ 29.00 & 12.26 & 43 \\ 30.00 & 12.34 & 52 \\ 31.00 & 12.43 & 62 \\ 32.00 & 12.52 & \end{array}$

32.00

33.00

34.00

35.00

36.00

37.00

38.00

39.00

40.00

41.00

42.00
43.00

44.00

45.00

46.00

47.00

48.00

49.00

50.00

51.00

52.00

53.00

54.00
55.00

56.00

57.00

58.00

59.00

60.00

61.00

62.00

63.00

64.00

65.00

66.00

67.00

68.00

69.00

70.00

71.00

72.00

73.00

74.00

75.00

76.00

77.00

78.00

79.00

80.00

81.00

82.00

83.00

84.00

85.00

86.00

87.00

88.00

89.00

90.00

91.00

92.00

93.00

94.00

95.00

96.00

97.00

98.00

99.00

100.00
12.52

12.60

12.79

12.89

12.99

13.10

13.20

13.31

13.43

13.54
13.66

13.78

13.90

14.02

14.15

14.28

14.41

14.54

14.68

14.82

14.96

15.10
15.25

15.40

15.55

15.70

15.85

16.01

16.17

16.33

16.50

16.66

16.83

17.00

17.17

17.35

17.52

17.70

18.06

18.24

18.42

18.61

18.80

18.98

19.17

19.36

19.55

19.75

19.94

20.14

20.33

20.53

20.73

20.93

21.13

21.33

21.53

21.73

21.93

22.13

22.33

22.54

22.74

22.95

23.15

23.36

23.56

-28.31
-21.32
-14.16
-6.77
.87
8.79
17.02
25.56
34.41
43.58
52.97
62.68
72.58
83.05
93.42
104.15
115.14
126.34
137.58
148.70
159.46
170.03
180.49
190.94

201.43

211.99

222.68

233.50

244.48

255.62

266.93

278.41

290.06

301.89

313.88

326.04

338.36

350.83

363.45

376.22

389.13

402.17

415.34

428.64

442.06

455.59

469.23

482.98

496.84

510.80

524.85

539.00

553.23

567.56

581.97

596.47

611.05

625.71

640.45

655.26

670.16

685.12

700.16

715.27

730.46

745.71

761.04

776.43

791.89

807.42

823.01

838.67

854.40

870.19

886.04

901.95

917.93

933.96

950.04

966.19

982.38
$-286.83$

$-281.28$

$-269.75$

$-263.68$

$-257.39$

$-250.84$

$-244.04$

$-236.98$

$-229.66$

$-222.15$

$-214.38$

$-206.48$

$-197.91$

$-189.61$

-181.03
-172.24

$-163.30$

$-154.38$

$-145.65$

$-137.31$

$-129.24$

$-121.33$

$-113.49$

$-105.66$

$-97.81$

$-89.89$

$-81.89$

$-73.79$

$-65.58$

$-57.26$

-48.81
-40.25

$-31.57$

$-22.78$

$-13.87$

$-4.85$

4.26
13.49

22.80

32.20

41.69

51.26

60.91

70.63

80.42

90.28

100.21

110.19

120.24

130.34

140.50

150.71

160.98

171.29

181.66

192.08

202.55

213.07

223.63

234.25

244. 91

255.63

266.40

277.21

288.07

298.99

309.95

320.97

332.04

343.16

354.33

365.56

376.83

388.16

399.54

410.97

422.44

433.97

445.54

457.16
5.488

5.831

6.161

6.492

6.819

7.141

7.463

7.785

8.108

8.427

8.746

.065 
$\begin{array}{lcccc}\text { EMPER- } & \text { SPECIFIC } & \text { ENTHALPY } & \text { INTERNAL } & \text { ENTROPY } \\ \text { ATURE } & \text { VOLUME } & \text { (J/GM) } & \text { ENERGY } & \text { (J/GM-K) }\end{array}$ (K) (CC/GM) (J/GM) ATURE VOLUME (K) (CC/GM)
INTHALPY
(J/GM) (J/GM)

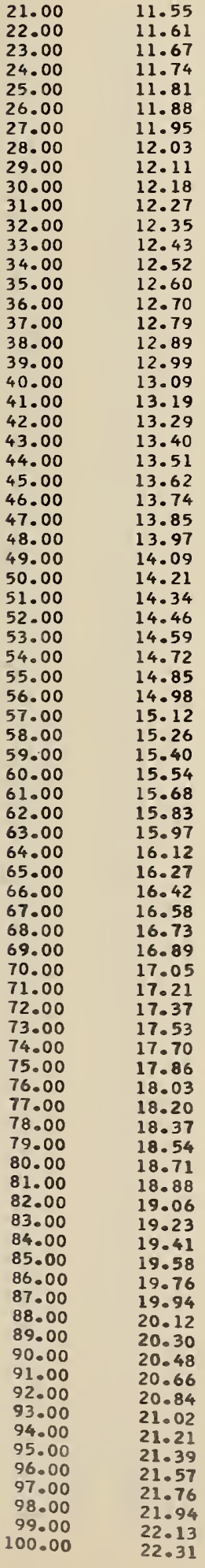

$\begin{array}{rll}-.51 & -281.30 & 5.702 \\ 6.56 & -275.75 & 6.029 \\ 13.85 & -270.03 & 6.355 \\ 21.39 & -264.12 & 6.678 \\ 29.21 & -257.97 & 6.995 \\ 37.32 & -251.58 & 7.312\end{array}$

$\begin{array}{ll}21.00 & 11.44 \\ 22.00 & 11.50 \\ 23.00 & 11.56 \\ 24.00 & 11.62 \\ 25.00 & 11.69 \\ 26.00 & 11.75 \\ 27.00 & 11.82 \\ 28.00 & 11.89 \\ 29.00 & 11.97 \\ 30.00 & 12.04 \\ 31.00 & 12.12 \\ 32.00 & 12.19\end{array}$

$92.09-208.22$

$102.38-199.88$

$112.59-191.77$

$123.14 \quad-183.39$

$133.96-174.79$

$145.01-166.03$

$\begin{array}{ll}156.10 & -157.28 \\ 167.03 & -148.76\end{array}$

$\begin{array}{ll}167.03 & -148.76 \\ 177.61 & -140.63\end{array}$

$187.98-132.76$

$198.25-125.05$

208.50 - 117.41

$218.79-109.79$

229.15

239.63

250.24

261.00

271.93

283.01

294.27

305.70

317.29

329.05

340.97

353.05

365.28

377.66

390.18

402.84

415.64

428.57

441.62

454.79

468.08

481.48

494.99

508.61

522.33

536.15

550.07

564.09

578.20

592.40

606.68

621.06

635.52

650.06

664.69

679.40

694.19

709.06

724.01

739.04

754.14

769.32

784.58

799.91

815.31

830.78

846.33

861.95

877.64

893.39

909.21

925.10

941.05

957.06

973.14

989.27
$-102.13$

-94.42
-86.62

$-78.71$

$-70.70$

-62.58
-54.33

$-45.96$

$-37.47$

$-28.87$

$-20.15$

-11.33
-2.39

6.64

15.77

25. 00

34.31

43.71
53.19

62.75

72.38

82. 09

91.86

101.70

111.60

121.57

131.60

141.69

151.83

162.03

172.29

182.61

192.97

203.40

213.88

224.41

235.00

245.65

256.35

267.10

277.91

288.77

299.69

310.67

321.70
332.79

332.79
343.93

355.13

366.39

377.70

389.07

400.48

411.96

423.48

435.06

446.68
7.630

7.949

8.263

8.892

9.204

9.525

9.832

10.138

10.442

10.742

11.038

11.321

11.589

11.845

12.093

12.334

12.571

12. 804

13.034

13.262

13.489

13.714

13.938

14.161

14.383

14.603

14.823

5.042

15.260

15.476

15.691

15.905

16. 118

16.330
16.540

16.540
16.749

16.956

17.162

17.367

17.570

17.772

17.972

18.171

18.368

18.564

18.759

18.952

19.144

19.334

19.523

19.711

19.897

20.082

20. 266

20.449

20.630

20.810

20.989

21.166

21.342

21.518

21.692

21.865

22.036

22.207

22. 377

22. 545

22.713

22.879

23. 044

23.209

23. 372

23.534
33.00

34.00

35.00

36.00

37.00

38.00

39.00

40.00

41.00

42.00

43.00

44.00
45.00

46.00

47.00

48.00

49.00

50.00

51.00

52.00

53.00

54.00

55.00

56.00
57.00

58.00

59.00

60.00

61.00

62.00

63.00

64.00

65.00

66.00

67.00

68.00

69.00

70.00

71.00

72.00

73.00

74.00

75.00

76.00

77.00

78.00

79.00

80.00

81.00

82.00

83.00

84.00

85.00

86. 00

87.00

88.00

89.00

90.00

91.00

92.00

93.00

94.00

95.00

96.00

97.00

98.00

99.00

100.00

20.20
27.21
34.42
41.87
49.58
57.59
65.90
74.52
83.44
92.59
102.04
111.66
121.75
131.82
$-275.70$

$-270.10$

$-264.32$

$-258.31$

$-252.06$ 
TEMPER- SPECIFIC ENTHALPY INTERNAL ENTROPY ATURE VOLUME (J/GM) ENERGY (J/GM-K) (K) (CC/GM)
(J/GM)
TEMPER-

ATURE

(K)
SPECIFIC (CC/GM)
ENTHALPY INTERNAL ENTROPY

( $J / G M)$ ENERGY
( J/GM-K)

\begin{tabular}{|c|c|c|c|c|}
\hline 22.00 & 11.39 & 47.79 & -275.47 & 5.784 \\
\hline 23.00 & 11.45 & 54.92 & -269.98 & 6.104 \\
\hline 24.00 & 11.51 & 62.28 & -264.32 & 6.418 \\
\hline 25.00 & 11.57 & 69.90 & -258.43 & 6.728 \\
\hline 26.00 & 11.64 & 77.81 & -252.32 & 7.037 \\
\hline 27.00 & 11.70 & 86.03 & -245.95 & 7.347 \\
\hline 28.00 & 11.77 & 94.54 & -239.33 & 7.658 \\
\hline 29.00 & 11.84 & 103.36 & -232.45 & 7.965 \\
\hline 30.00 & 11.91 & 112.41 & -225.39 & 8.272 \\
\hline 31.00 & 11.98 & 121.75 & -218.10 & 8.579 \\
\hline 32.00 & 12.05 & 131.24 & -210.68 & 8.883 \\
\hline 33.00 & 12.12 & 141.16 & -202.81 & 9.193 \\
\hline 34.00 & 12.20 & $151 \cdot 10$ & -195.03 & 9.492 \\
\hline 35.00 & 12.28 & 161.36 & -186.99 & 9.790 \\
\hline 36.00 & 12.36 & 171.92 & -178.72 & 10.086 \\
\hline 37.00 & 12.44 & 182.72 & -170.25 & 10.380 \\
\hline 38.00 & 12.53 & 193.56 & -161.79 & 10.669 \\
\hline 39.00 & 12.61 & 204.18 & -153.61 & 10.945 \\
\hline 40.00 & 12.70 & 214.45 & -145.83 & 11.205 \\
\hline 41.00 & 12.79 & 224.51 & $-138 \cdot 30$ & 11.453 \\
\hline 42.00 & 12.88 & 234.47 & -130.93 & 11.693 \\
\hline 43.00 & 12.97 & 244.40 & -123.62 & 11.927 \\
\hline 44.00 & 13.07 & 254.37 & $-116 \cdot 32$ & 12.156 \\
\hline 45.00 & 13.16 & 264.41 & -109.00 & $12 \cdot 382$ \\
\hline 46.00 & 13.26 & 274.56 & -101.61 & 12.605 \\
\hline 47.00 & 13.36 & 284.84 & -94.13 & 12.826 \\
\hline 48.00 & 13.46 & 295.26 & -86.55 & 13.045 \\
\hline 49.00 & 13.56 & 305.85 & -78.86 & 13.263 \\
\hline 50.00 & 13.66 & 316.59 & -71.06 & 13.480 \\
\hline 51.00 & 13.77 & 327.49 & -63.13 & 13.696 \\
\hline 52.00 & 13.87 & 338.56 & -55.07 & 13.911 \\
\hline 53.00 & 13.98 & 349.79 & -46.90 & 14.125 \\
\hline 54.00 & 14.09 & 361.18 & -38.61 & 14.338 \\
\hline 55.00 & 14.20 & 372.72 & -30.20 & 14.550 \\
\hline 56.00 & 14.31 & 384.43 & -21.68 & 14.761 \\
\hline 57.00 & 14.43 & 396.28 & -13.05 & 14.970 \\
\hline 58.00 & 14.54 & 408.27 & -4.32 & 15.179 \\
\hline 59.00 & 14.66 & 420.41 & 4.51 & 15.387 \\
\hline 60.00 & 14.78 & 432.68 & 13.45 & 15.593 \\
\hline 61.00 & 14.90 & 445.09 & 22.47 & 15.798 \\
\hline 62.00 & 15.02 & 457.62 & 31.59 & 16.002 \\
\hline 63.00 & 15.14 & 470.28 & 40.79 & 16.204 \\
\hline 64.00 & 15.26 & 483.06 & 50.07 & 16.405 \\
\hline 65.00 & 15.39 & 495.95 & 59.43 & 16.605 \\
\hline 66.00 & 15.51 & 508.97 & 68.87 & 16.804 \\
\hline 67.00 & 15.64 & 522.09 & 78.39 & 17.001 \\
\hline 68.00 & 15.77 & 535.32 & 87.97 & 17.197 \\
\hline 69.00 & 15.90 & 548.65 & 97.63 & 17.392 \\
\hline 70.00 & 16.03 & 562.09 & 107.36 & 17.585 \\
\hline 71.00 & 16.16 & 575.63 & 117.16 & 17.777 \\
\hline 72.00 & 16.29 & 589.27 & 127.02 & 17.968 \\
\hline 73.00 & 16.43 & 603.01 & 136.95 & 18.158 \\
\hline 74.00 & 16.56 & 616.84 & 146.94 & 18.346 \\
\hline 75.00 & 16.70 & 630.77 & 157.00 & 18.533 \\
\hline 76.00 & 16.84 & 644.79 & 167.12 & 18.719 \\
\hline 77.00 & 16.98 & 658.91 & 177.30 & 18.903 \\
\hline 78.00 & 17.11 & 673.11 & 187.54 & 19.086 \\
\hline 79.00 & 17.26 & 687.41 & 197.85 & 19.269 \\
\hline 80.00 & 17.40 & 701.79 & 208.23 & 19.449 \\
\hline 81.00 & 17.54 & 716.27 & 218.66 & 19.629 \\
\hline 82.00 & 17.68 & 730.83 & 229.16 & 19.808 \\
\hline 83.00 & 17.83 & 745.48 & 239.72 & 19.986 \\
\hline 84.00 & 17.97 & 760.21 & 250.34 & 20.162 \\
\hline 85.00 & 18.12 & 775.03 & 261.02 & 20.337 \\
\hline 86.00 & 18.26 & 789.94 & 271.77 & 20.512 \\
\hline 87.00 & 18.41 & 804.93 & 282.58 & 20.685 \\
\hline 88.00 & 18.56 & 820.00 & 293.46 & 20.857 \\
\hline 89.00 & 18.71 & 835.16 & 304.40 & 21.028 \\
\hline 90.00 & 18.86 & 850.39 & 315.40 & 21.199 \\
\hline 91.00 & 19.01 & 865.71 & 326.46 & 21.368 \\
\hline 92.00 & 19.16 & 881.11 & 337.59 & 21.536 \\
\hline 93.00 & 19.31 & 096.58 & 348.78 & 21.704 \\
\hline 94.00 & 19.46 & 912.14 & 360.03 & 21.870 \\
\hline 95.00 & 19.61 & 927.76 & 371.34 & 22.035 \\
\hline 96.00 & 19.76 & 943.47 & 382.71 & 22.200 \\
\hline 97.00 & 19.92 & 959.24 & 394.15 & 22.363 \\
\hline 98.00 & 20.07 & 975.09 & 405.63 & 22.526 \\
\hline 99.00 & 20.23 & 991.00 & 417.17 & 22.687 \\
\hline 100.00 & 20.38 & 1006.98 & 428.77 & 22.848 \\
\hline
\end{tabular}

22.00

23.00

24.00

25.00

26.00

27.00

28.00

29.00

30.00

32.00

33.00

34.00

35.00

36.00

37.00

38.00

39.00

40.00

41.00

42.00

43.00

44.00

45.00

46.00

47.00

48.00

49.00

50.00

51.00

52.00

53.00

54.00

55.00

56.00

57.00

58.00

59.00

60.00

61.00

62.00

63.00

64.00

65.00

66.00

67.00

68.00

69.00

70.00

71.00

72.00

73.00

74.00

75.00

76.00

77.00

78.00

79.00

80.00

81.00

82.00

83.00

84.00

85.00

86.00

87.00

88.00

89.00

90.00

91.00

92.00

93.00

94.00

95.00

96.00

97.00

98.00

99.00

100.00

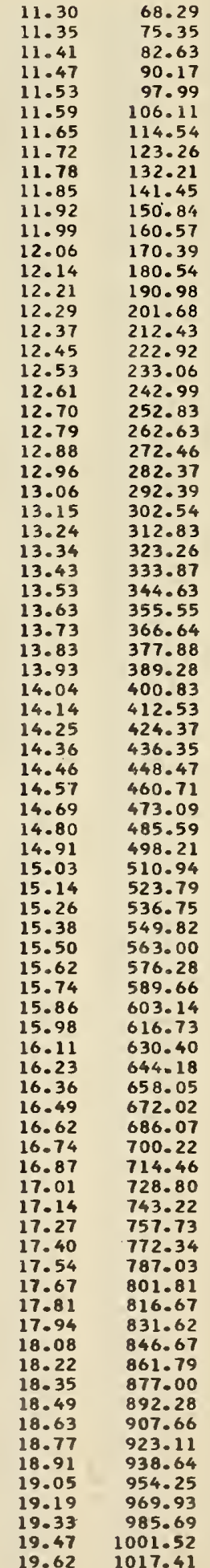

$-275.08$ -269.70
-264.14

$-258.37$

$-252.37$

$-246.13$

$-239.63$

$-232.88$

$-225.94$

$-218.77$

$-211.50$

$-203.87$

$-196.24$

$-188.34$

$-180.21$

$-171.87$

$-163.52$

$-155.49$

$-147.85$

$-140.47$

$-133.23$

$-126.07$

$-118.91$

$-111.72$

$-104.47$

$-97.13$

$-89.68$

$-82.13$

$-74.45$

$-66.66$

$-58.73$

$-50.69$

$-42.53$

$-34.25$

$-25.86$

$-17.35$

$-8.74$

8.78

8.78
17.68

26.67

35.76

44.92

54.17

63.50

72.91

91.94

101.56

111.26

121.02

130.85

140.75

150.71

160.75

170.84

181.00

191.23

201.53

211.89

222.31

232.80

243.36

253.98

264. 67

275.43

286. 25

297.14

308.09

319.11

330.19

341.34

352.55

363.83

375.17

386.56

398.02

409.54

421.12
5.671

5.987

6.299

6.605

6.911

7.525

7.828

8.132

8.435

8.736

9.041

9.337

9.631

9.924

10.215

10.502

10.774

11.031

11.276

11.513

11. 744

11.970

12.193

12.413

12.631

12. 848

13.063

13.277

13.490

13. 702

13.913

14.123

14.333

14.541

14. 748

14. 954

15.159

15.362

15.565

15. 766

15. 966

16. 165

16.362

16.558

16.753

16. 947

17.139

17.330

17.520

17. 709

17.896

18.082

18.267

18.451

18.633

18.815

18. 995

19.174

19.352

19.529

19.705

19.880

20.054

20.227

20.398

20.569

20. 739

20. 908

21.076

21.243

21.409

21.575

21.739

21.903

22.065

22.227

22.387 
ENTHALPY INTERNAL ENTROPY (K) $\quad$ (CC/GM) (J/GM)
TEMPERATURE

(K)
SPECIFIC ENTHALPY VOLUME (CC/GM)

(J/GM)
INTERNAL ENTROPY ENERGY (J/GM)
( $J / G M-K)$
11.26
11.31
11.37
11.42
11.48
11.54
11.60
11.67
11.73
11.80
11.86
11.93
12.00
12.07
12.15
12.22
12.30
12.38
12.46
12.54
12.62
12.70
12.79
12.87
12.96
13.05
13.13
13.22
13.32

13.22
13.32

13.41

13.50

13.60

13.69

13.79

13.89

13.99

14.09

14.19

14.29

14.39

14.50

14.60

14.71

14.82

14.92

15.03

15.14

15.25

15.48

15.59

5.71

15.83

15.94

16.18

16.30

16.42

16.54

16.66

16.78

16.90

17.03

17.15

17.28

17.40

17.53

17.66

17.78
17.91

18.04

18.17

18.30

18.43

18.56

18.69

18.82

18. 95

95.70
102.91
110.38
118.12
126.16
134.49
143.13
151.99
161.14
170.42
179.97
189.69
199.73

$-269.26$

$-263.81$

$-258.15$

$-252.26$

$-246.12$

$-239.74$

$-233.10$

$-226.28$

$-219.23$

$-212.08$

$-204.71$

$-197.21$

$-189.44$

$-181.43$

$-173.20$

$-164.96$

$-157.07$

$-149.56$

$-142.31$

$-135.20$

$-128.16$

$-121.13$

$-114.06$

$-106.93$

1012.89

1028.71
$-99.71$

$-92.39$

$-77.40$

$-69.71$

$-61.91$

$-53.99$

$-45.94$

$-37.77$

$-29.49$

$-21.10$

$-12.61$

-4.00
4.69

13.49

22. 38

31.35

40.41

49.56
58.79

58.79
68.09

77.47

86.93

96.46

106. 06

115.73

125.47

135.28

145.16

155.11

165.13

175.21

185.36

195.59

205. 87

216.23

226.66

237.14

247.71

258.34

269.04

279.80

290.64

301.54

312.51

323.55

334.65

345.82

357.06

368.36

379.73

391.16

402.65

6.185

.488

6.790

093

7.698

.000

8.598

896

9.771

10.060

10.344

10.613

0.867

11.110

11. 344

572

11.795

12.015

12.233

12.449

12.663

12.876

13.087

13. 298

13.507

13.716

13.924

14.131

14.336

14.541

14.745

14.947

15.149

15.349

15.548

15.745

15.942

16.137

16.331

16.524

16.715

16.906

17.095

17.283

17.469

17.655

17.839

18.022
18.204

18.384

18.564

18.743

18.920

19.097

19.272

19.446

19.620

19.792

19.964

20.134

20.304

20.473

20.640

20.807

20.973

21.138

21.302

21.466

21.628

21.790

21.951

22.110

414.13

23.00
24.00
25.00
26.00

11.17
11.22
11.27
11.33
11.38
11.44
11.50
11.56
11.62
11.68
11.75
11.81
11.88
11.95
11.02

12.02

12.09

12.16

12.23

12.31
12.39

12.46

12.54

12.62

12.70

12.78

12.87

12.95

13.03

13.12

13.21

13.29

13.38

13.47

13.56

13.66

13.84

13.94

14.03

14.13

14.23

14.42

14.52

14.62

14.73

14.83

14.93

15.04

15.14

15.25

15.35

15.46

15.57

15.68

15.79

15.90

16.01

16.12

16.24

16.35

16.47

16.58

16.70

16.81

16.93

17.05

17.16

17.28

17.40

17.52

17.64

17.76

17.88

18.00 



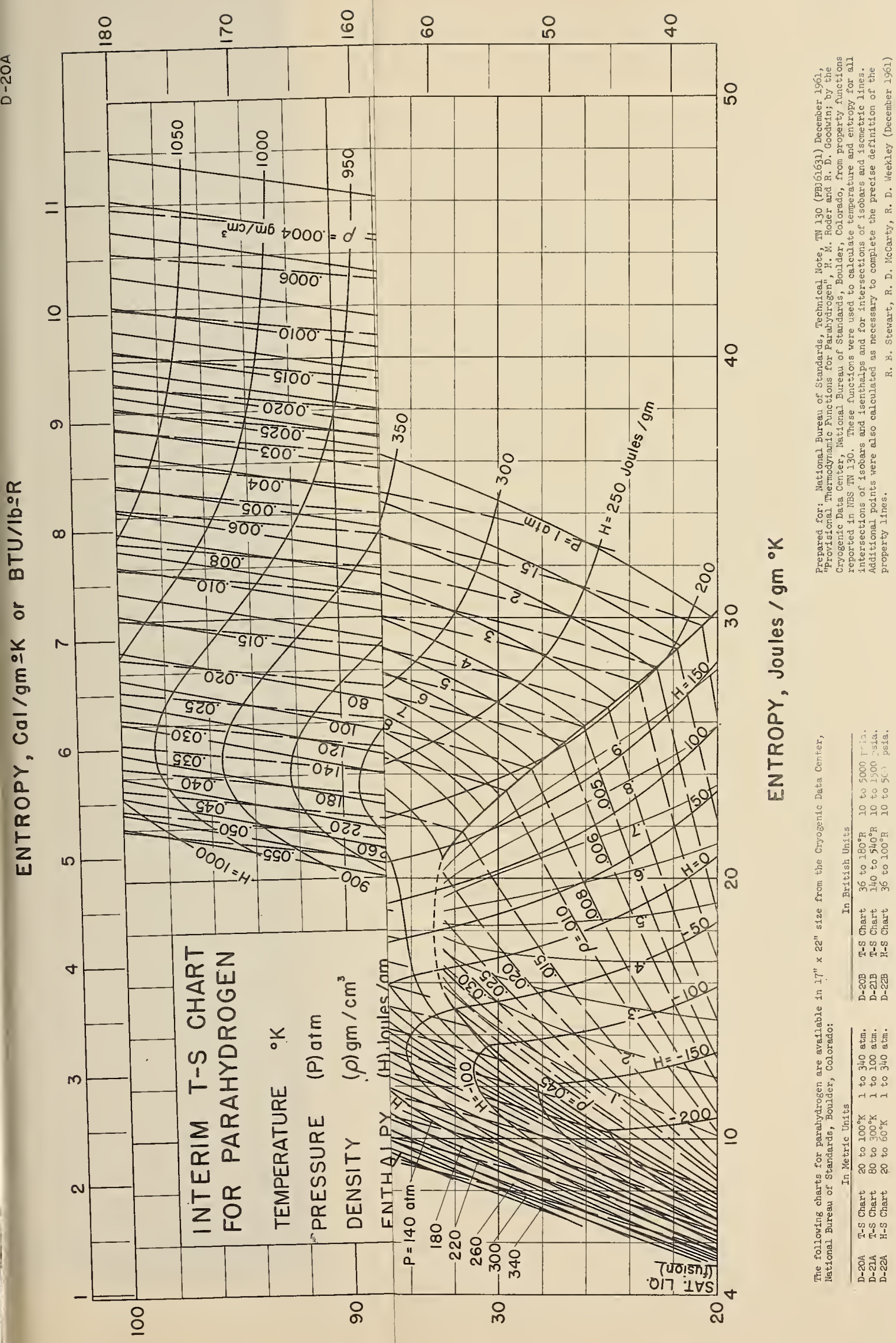





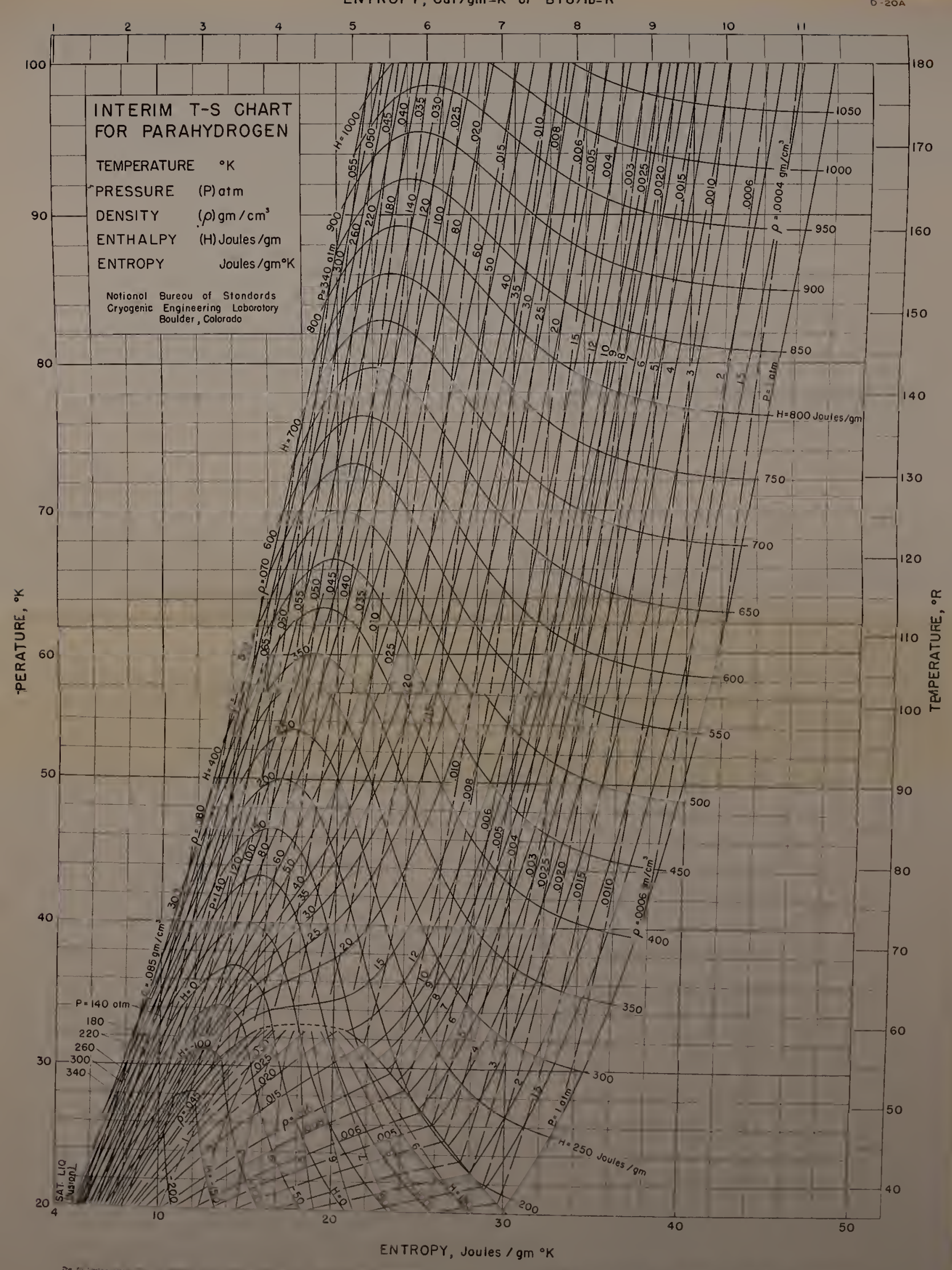






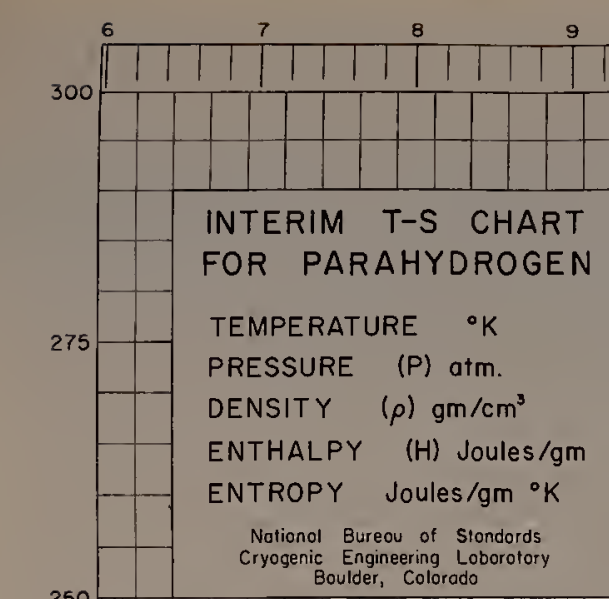

Boulder, Colorado

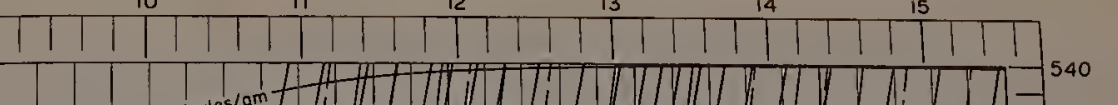

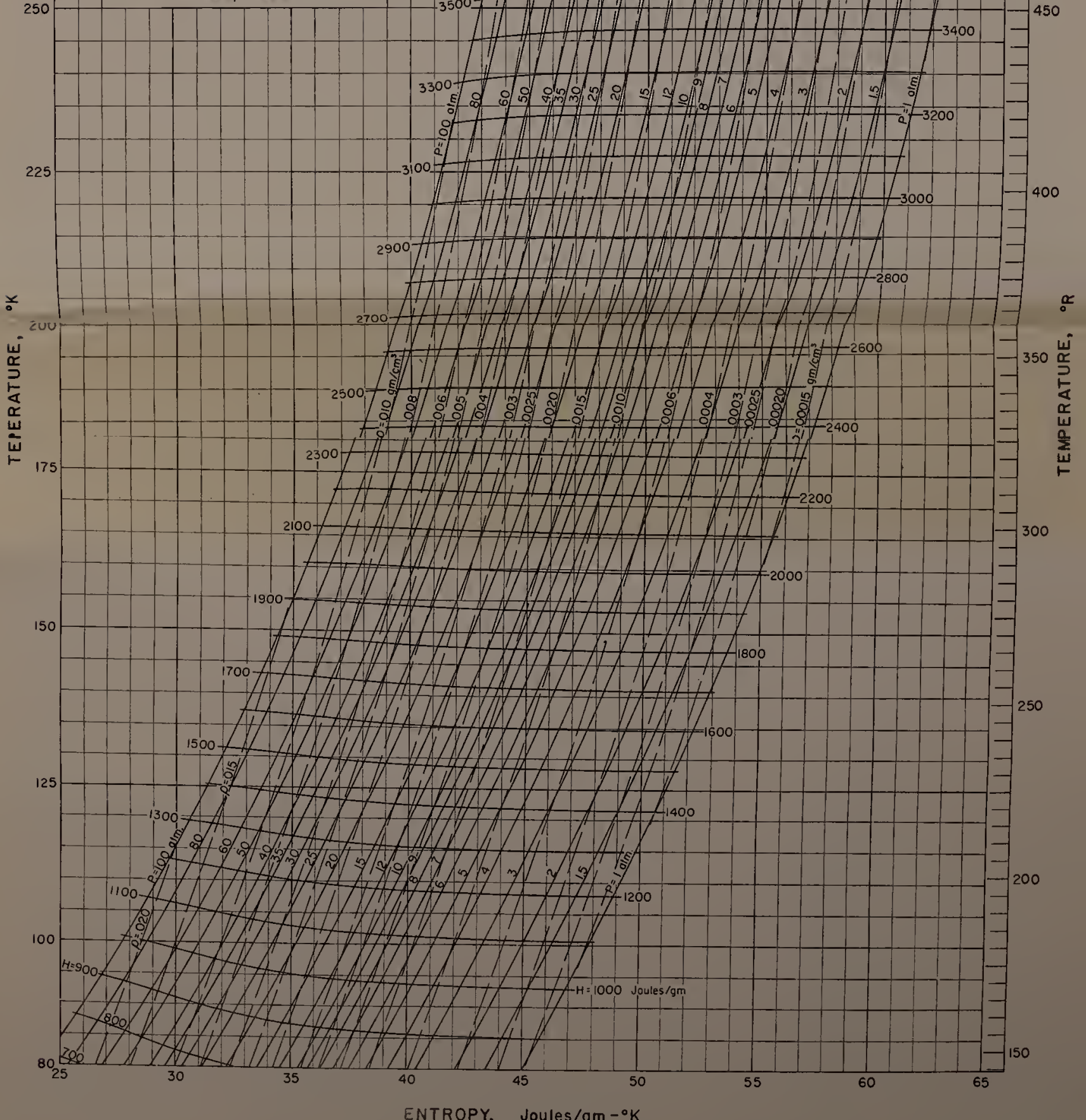

ENTROPY, Joules $/ \mathrm{gm}-{ }^{\circ} \mathrm{K}$ 



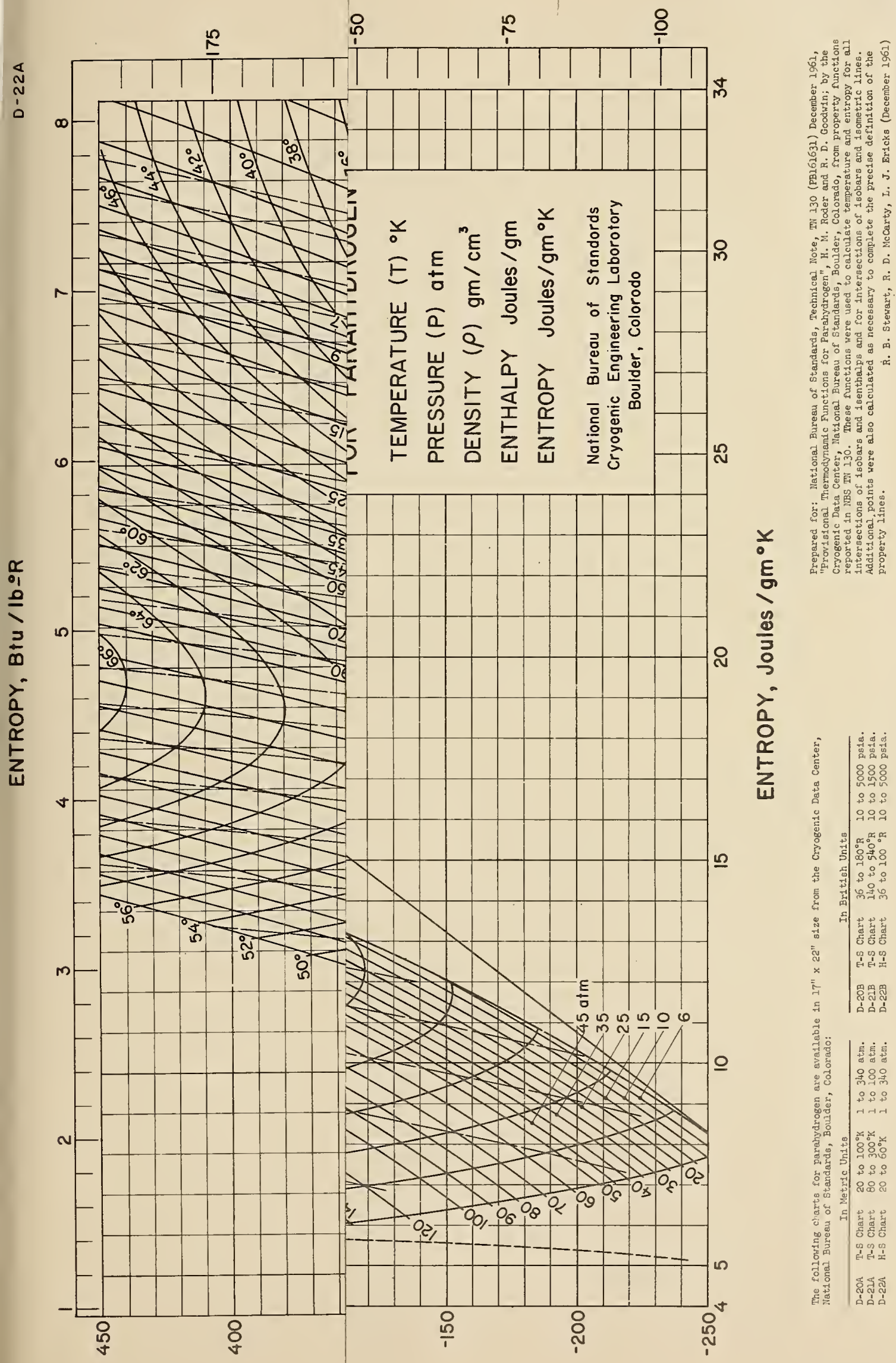





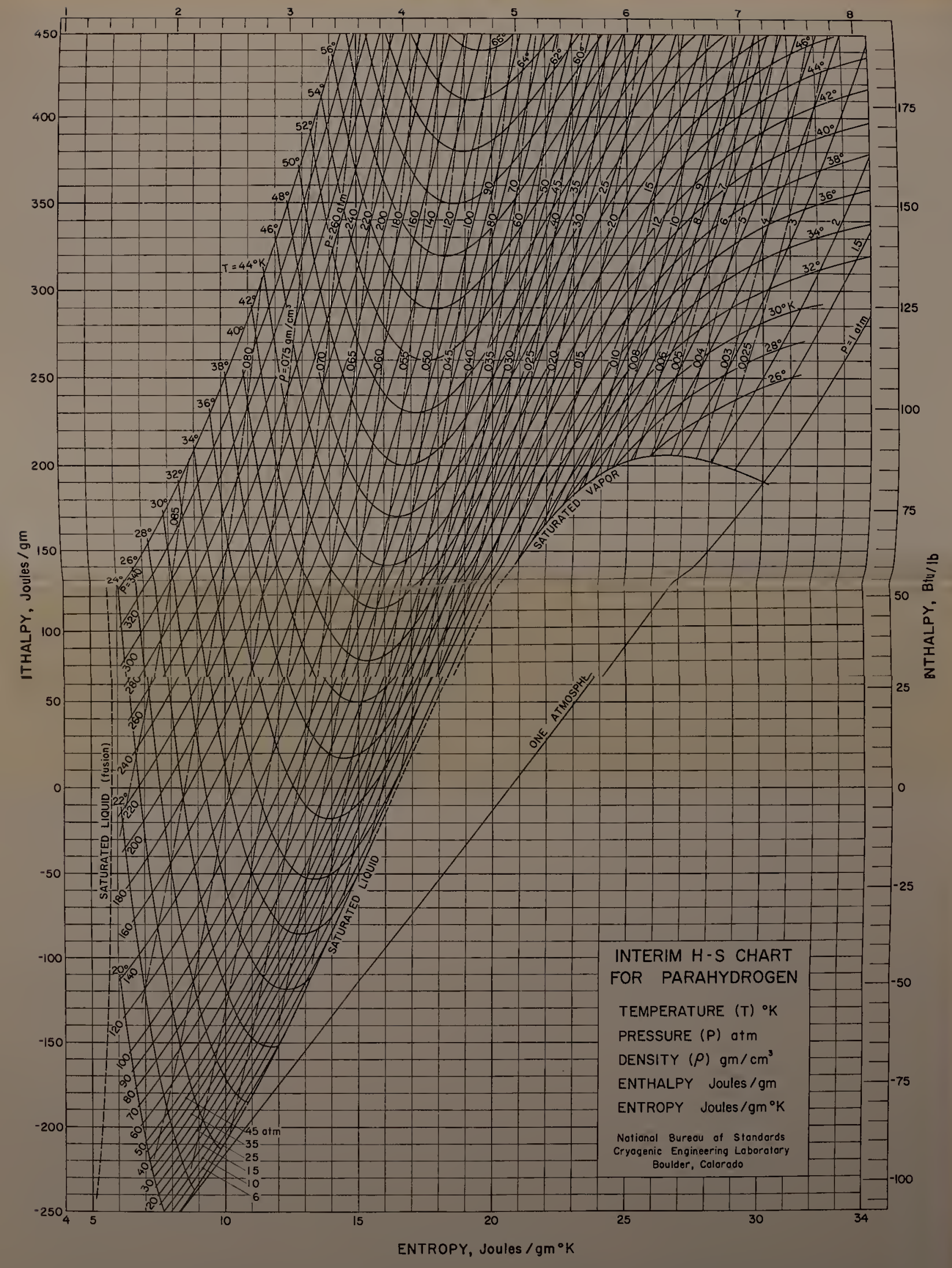



SUPPLEMENT A (British units)

\section{CONTENTS}

Preface

Conversion factors used

Table 2A Adjustments in entropy and enthalpy $56-66^{\circ} \mathrm{R}$ Table $3 \mathrm{~A}$ Adjustments in specific volume and enthalpy at $182^{\circ} \mathrm{R}$

The tables of provisional thermodynamic functions for parahydrogen (British units)

Figure D-20B T-S Chart $36-180^{\circ} \mathrm{R} \quad 10-5000$ psia

Figure D-21B T-S Chart $140-540^{\circ} \mathrm{R} 10-1500$ psia

Figure D-22B H-S Chart $36-100^{\circ} \mathrm{R} \quad 10-5000$ psia 


\section{Preface}

At the request of the sponsor the tables and charts were recomputed in units commonly used in engineering applications. Changes in existing computer programs were held to a minimum. If the input to all programs is in those fractional metric units which correspond to the desired output, the entire computational procedure remains exactly as described in the Technical Note. Conversion of units is accomplished after all computations are completed and just prior to print-out. Conversion factors used and tables of adjustments are given below.

Conversion factors used

$\begin{array}{ccc}\text { degree K } & \text { times } 1.8 & \text { equals degree R } \\ \mathrm{atm} & 14.696 & \mathrm{psia} \\ \mathrm{g} / \mathrm{cm}^{3} & 62.428227 & \text { pounds } / \mathrm{cu} \mathrm{ft} \\ \mathrm{cal} / \mathrm{g} & 1.798823 & \mathrm{BTU} / \mathrm{pound} \\ \mathrm{cal} / \mathrm{g}{ }^{\circ} \mathrm{K} & 0.999346 & \mathrm{BTU} / \text { pound }^{\circ} \mathrm{R}\end{array}$

Table 2A

Adjustments in entropy and enthalpy $56-66^{\circ} \mathrm{R}$

In entropy

$\mathrm{T}^{\circ} \mathrm{R}$

56

58

60

62

64

66

$\mathrm{A}_{1}$
$0.00261+0.0160 \times 10^{-5}$

$0.03426+0.0187 \times 10^{-5}$

$0.09432-0.2125 \times 10^{-5}$

$0.0474-0.1093 \times 10^{-5}$

$0.0201-0.0429 \times 10^{-5}$

$0.00566-0.0080 \times 10^{-5}$
In enthalpy

$\mathrm{A}_{2}$

$\left(\mathrm{B}_{2} \cdot \mathrm{T}\right)$

$.1342+.896 \times 10^{-5}$

$1.929+1.082 \times 10^{-5}$

$5.919-12.75 \times 10^{-5}$

$3.021-6.777 \times 10^{-5}$

$1.262-2.746 \times 10^{-5}$

$.346-.528 \times 10^{-5}$ 
Table 3A

Adjustments in specific volume and enthalpy at $182^{\circ} \mathrm{R}$

$\begin{array}{cc}\begin{array}{c}\text { Pressure } \\ \text { psia }\end{array} & \begin{array}{c}\text { Delta } \\ \text { BTU/po }\end{array} \\ 10 . & .439 \\ 15 . & .428 \\ 20 . & .415 \\ 30 . & .385 \\ 40 . & .358 \\ 50 . & .330 \\ 60 . & .304 \\ 70 . & .278 \\ 80 . & .252 \\ 90 . & .226 \\ 100 . & .202 \\ 120 . & .151 \\ 140 . & .102 \\ 160 . & .048 \\ 180 . & -.002 \\ 200 . & -.049 \\ 250 . & -.159 \\ 300 . & -.257 \\ 350 . & -.340 \\ 400 . & -.419 \\ 450 . & -.504 \\ 500 . & -.596 \\ 600 . & -.720 \\ 700 . & -.741 \\ 800 . & -.820 \\ 900 . & -.916 \\ 1000 . & -.837 \\ 1250 . & -.828 \\ 1500 . & -.831 \\ & \end{array}$

Delta V
$\mathrm{Cu} \mathrm{Ft/pound}$

.02279

.01748

.01213

.00687

.00428

.00274

.00176

.00106

.00056

.00015

$-.00021$

$-.00118$

$-.00222$

$-.00309$

$-.00374$

$-.00417$

$-.00337$

$-.00200$

$-.00135$

$-.00125$

$-.00115$

$-.00097$

$-.00062$

$-.00027$

$+.00004$

.00033

.00051

.00078

.00091 
TEMPER- SPECIFIC ENTHALPY INTERNAL ENTROPY ATURE VOLUME (BTU/LB) ENERGY (BTU/L8-R) (R) (CU FT/L8)
(BTU/L8)
IEMPER- SPECIFIC ENTHALPY

ATURE VOLUME (8TU/LB) (R) (CU FT/L8)
INTERNAL ENTROPY

ENERGY (8TU/L8-R) (BTU/LB)

\section{* 34.260 \\ $+34.260$ \\ 36.00 \\ 38.00 \\ 40.00}

42.00

44.00

46.00

48.00

50.00

52.00

54.00

56.00

58.00

60.00

62.00

64.00

66.00

68.00

70.00

72.00

74.00

76.00

78.00

80.00

82.00

84.00

86.00

88.00

90.00

92.00

94.00

96.00

98.00

100.00

102.00

104.00

106.00

108.00

110.00

112.00

114.00

116.00

118.00

120.00

.2221
16.8879
17.9374
19.1125
20.2660

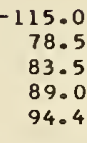

21.4049

22.5337

23.6547

24.7699

25.8803

26.9868

28.0899

29.1902

30.2879

31.3833

32.4768

33.5684

34.6584

35.7468

36.8339

37.9197

39.0043

40.0878

41.1704

42.2520

43.3328

44.4127

45.4919

46.5704

47.6483

48.7256

49.8023

50.8784

51.9541

53.0293

54.1041

55.1784

56.2523

57.3259

58.3992

59.4721

60.5447

61.6170

62.6890

63.7608
99.72

104.92

110.07

115.18

120.28

125.35

130.41

135.46

140.51

145.54

150.57

155.59

160.60

165.61

170.62

175.63

180.63

185.63

190.63

195.64

200.64

205.64

210.65

215.66

220.67

225.69

230.72

235.75

240.80

245.85

250.91

255.99

261.07

266.18

271.29

276.43

281.58

286.76

291.95

297.17

\section{$-115.85$}

47.26

50.35

53.72

56.95

60.11

63.22

66.29

69.35

72.38

75.41

78.43

81.45

84.46

87.46

90.47

93.47

96.47

99.46

102.46

105.46

108.45

111.45

114.45

117.45

120.45

123.46

126.46

129.48

132.50

135.53

138.56

141.60

144.65

147.72

150.79

153.88

156.98

160.09

163.23

166.38

169.54

172.73

175.95

179.18
1.777

7.424

7.568

7.717

7. 855

7.984

8.104

8.219

8.328

8.432

8.531

8.627

8.719

8.807

8.892

8.975

9.055

9.132

9.207

9.279

9.350

9.418

9.485

9.550

9.613

9.675

9.735

9.794

9.852

9.908

9.963

10.017

10.070

10.122

10.173

10.223

10.273

10.321

10.369

10.416

10.462

10.508

10.553

10.597

10.641

122.00
124.00
126.00
128.00
130.00
132.00
134.00
136.00
138.0
140.0

142.00

144.00

146.00

148.00

150.00

152.00

154.00

156.00

158.00

160.00

162.00

164.00

166.00

168.00

170.00

172.00

174.00

176.00

178.00

180.00

182.00

186.00

188.00

190.00

192.00

194.00

196.00

198.00

200.00

202.00

204.00

206.00

208.00

210.00

212.00

214.00

216.00

218.00

220.00

222.00

224.00

226.00

228.00

230.00

232.00

234.00

236.00

238.00 
TEMPER - SPECIFIC ENTHALPY INTERNAL ENTROPY ATURE VOLUME (8TU/L8) ENERGY (8TU/LB-R) (R) (CU FT/LB) (8TU/L8)

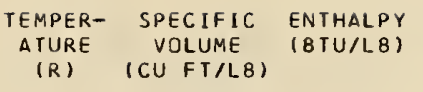

INTERNAL ENTROPY (BTU/L8)

\begin{tabular}{|c|c|c|c|c|c|c|c|c|c|}
\hline $\begin{array}{l}262.00 \\
264.00 \\
266.00 \\
268.00 \\
270.00 \\
272.00 \\
274.00 \\
276.00 \\
278.00 \\
280.00\end{array}$ & $\begin{array}{l}139.5574 \\
140.6232 \\
141.6891 \\
142.7549 \\
143.8208 \\
144.8866 \\
145.9524 \\
147.0181 \\
148.0839 \\
149.1496\end{array}$ & $\begin{array}{l}764.12 \\
771.84 \\
779.57 \\
787.31 \\
795.06 \\
802.80 \\
810.55 \\
818.31 \\
826.08 \\
833.85\end{array}$ & $\begin{array}{l}505.87 \\
511.61 \\
517.37 \\
523.14 \\
528.92 \\
534.69 \\
540.46 \\
546.25 \\
552.04 \\
557.84\end{array}$ & $\begin{array}{l}13.140 \\
13.169 \\
13.198 \\
13.227 \\
13.256 \\
13.284 \\
13.313 \\
13.341 \\
13.369 \\
13.397\end{array}$ & $\begin{array}{l}402.00 \\
404.00 \\
406.00 \\
408.00 \\
410.00 \\
412.00 \\
414.00 \\
416.00 \\
418.00 \\
420.00\end{array}$ & $\begin{array}{l}214.1317 \\
215.1970 \\
216.2624 \\
217.3278 \\
218.3932 \\
219.4586 \\
220.5241 \\
221.5901 \\
222.6560 \\
223.7219\end{array}$ & $\begin{array}{l}1304.63 \\
1312.13 \\
1319.62 \\
1327.11 \\
1334.58 \\
1342.05 \\
1349.51 \\
1356.96 \\
1364.41 \\
1371.84\end{array}$ & $\begin{array}{l}908.37 \\
913.90 \\
919.42 \\
924.94 \\
930.44 \\
935.93 \\
941.42 \\
946.90 \\
952.37 \\
957.84\end{array}$ & $\begin{array}{l}14.794 \\
14.813 \\
14.831 \\
14.850 \\
14.868 \\
14.886 \\
14.904 \\
14.922 \\
14.940 \\
14.958\end{array}$ \\
\hline $\begin{array}{l}282.00 \\
284.00 \\
286.00 \\
288.00 \\
290.00 \\
292.00 \\
294.00 \\
296.00 \\
298.00 \\
300.00\end{array}$ & $\begin{array}{l}150.2153 \\
151.2809 \\
152.3466 \\
153.4122 \\
154.4778 \\
155.5434 \\
156.6089 \\
157.6745 \\
158.7400 \\
159.8055\end{array}$ & $\begin{array}{l}841.63 \\
849.42 \\
857.21 \\
865.00 \\
872.80 \\
880.61 \\
888.42 \\
896.23 \\
904.04 \\
911.86\end{array}$ & $\begin{array}{l}563.65 \\
569.47 \\
575.29 \\
581.11 \\
586.94 \\
592.77 \\
598.61 \\
604.45 \\
610.29 \\
616.13\end{array}$ & $\begin{array}{l}13.425 \\
13.452 \\
13.479 \\
13.507 \\
13.534 \\
13.560 \\
13.587 \\
13.614 \\
13.640 \\
13.666\end{array}$ & $\begin{array}{l}422.00 \\
424.00 \\
426.00 \\
428.00 \\
430.00 \\
432.00 \\
434.00 \\
436.00 \\
438.00 \\
440.00\end{array}$ & $\begin{array}{l}224.7877 \\
225.8535 \\
226.9192 \\
227.9848 \\
229.0502 \\
230.1155 \\
231.1807 \\
232.2457 \\
233.3105 \\
234.3751\end{array}$ & $\begin{array}{l}1379.27 \\
1386.69 \\
1394.10 \\
1401.50 \\
1408.89 \\
1416.27 \\
1423.64 \\
1431.01 \\
1438.37 \\
1445.71\end{array}$ & $\begin{array}{r}963.29 \\
968.74 \\
974.17 \\
979.60 \\
985.02 \\
990.43 \\
995.84 \\
1001.23 \\
1006.62 \\
1012.00\end{array}$ & $\begin{array}{l}14.975 \\
14.993 \\
15.010 \\
15.028 \\
15.045 \\
15.062 \\
15.079 \\
15.096 \\
15.113 \\
15.130\end{array}$ \\
\hline $\begin{array}{l}302.00 \\
304.00 \\
306.00 \\
308.00 \\
310.00 \\
312.00 \\
314.00 \\
316.00 \\
318.00 \\
320.00\end{array}$ & $\begin{array}{l}160.8710 \\
161.9364 \\
163.0019 \\
164.0673 \\
165.1327 \\
166.1981 \\
167.2635 \\
168.3289 \\
169.3942 \\
170.4596\end{array}$ & $\begin{array}{l}919.67 \\
927.49 \\
935.31 \\
943.12 \\
950.94 \\
958.75 \\
966.57 \\
974.38 \\
982.19 \\
990.00\end{array}$ & $\begin{array}{l}621.98 \\
627.82 \\
633.67 \\
639.51 \\
645.36 \\
651.20 \\
657.04 \\
662.88 \\
668.72 \\
674.56\end{array}$ & $\begin{array}{l}13.692 \\
13.718 \\
13.743 \\
13.769 \\
13.794 \\
13.819 \\
13.844 \\
13.869 \\
13.894 \\
13.918\end{array}$ & $\begin{array}{l}442.00 \\
444.00 \\
446.00 \\
448.00 \\
450.00 \\
452.00 \\
454.00 \\
456.00 \\
458.00 \\
460.00\end{array}$ & $\begin{array}{l}235.4395 \\
236.5037 \\
237.5677 \\
238.6314 \\
239.6950 \\
240.7582 \\
241.8213 \\
242.8841 \\
243.9467 \\
245.0091\end{array}$ & $\begin{array}{l}1453.05 \\
1460.39 \\
1467.71 \\
1475.02 \\
1482.33 \\
1489.65 \\
1496.95 \\
1504.25 \\
1511.54 \\
1518.83\end{array}$ & $\begin{array}{l}1017.37 \\
1022.73 \\
1028.08 \\
1033.43 \\
1038.77 \\
1044.12 \\
1049.46 \\
1054.79 \\
1060.11 \\
1065.43\end{array}$ & $\begin{array}{l}15.146 \\
15.163 \\
15.179 \\
15.196 \\
15.212 \\
15.228 \\
15.244 \\
15.260 \\
15.276 \\
15.292\end{array}$ \\
\hline $\begin{array}{l}322.00 \\
324.00 \\
326.00 \\
328.00 \\
330.00 \\
332.00 \\
334.00 \\
336.00 \\
338.00 \\
340.00\end{array}$ & $\begin{array}{l}171.5249 \\
172.5903 \\
173.6556 \\
174.7210 \\
175.7863 \\
176.8516 \\
177.9170 \\
178.9823 \\
180.0476 \\
181.1130\end{array}$ & $\begin{array}{r}997.80 \\
1005.60 \\
1013.40 \\
1021.19 \\
1028.98 \\
1036.76 \\
1044.54 \\
1052.32 \\
1060.09 \\
1067.85\end{array}$ & $\begin{array}{l}680.39 \\
686.22 \\
692.04 \\
697.87 \\
703.68 \\
709.50 \\
715.30 \\
721.11 \\
726.90 \\
732.69\end{array}$ & $\begin{array}{l}13.942 \\
13.967 \\
13.991 \\
14.014 \\
14.038 \\
14.062 \\
14.085 \\
14.108 \\
14.131 \\
14.154\end{array}$ & $\begin{array}{l}462.00 \\
464.00 \\
466.00 \\
468.00 \\
470.00 \\
472.00 \\
474.00 \\
476.00 \\
478.00 \\
480.00\end{array}$ & $\begin{array}{l}246.0713 \\
247.1333 \\
248.1951 \\
249.2568 \\
250.3184 \\
251.3798 \\
252.4411 \\
253.5024 \\
254.5637 \\
255.6249\end{array}$ & $\begin{array}{l}1526.11 \\
1533.37 \\
1540.64 \\
1547.89 \\
1555.14 \\
1562.38 \\
1569.62 \\
1576.85 \\
1584.07 \\
1591.29\end{array}$ & $\begin{array}{l}1070.74 \\
1076.05 \\
1081.34 \\
1086.64 \\
1091.92 \\
1097.20 \\
1102.47 \\
1107.73 \\
1112.99 \\
1118.25\end{array}$ & $\begin{array}{l}15.308 \\
15.324 \\
15.339 \\
15.355 \\
15.370 \\
15.386 \\
15.401 \\
15.416 \\
15.431 \\
15.446\end{array}$ \\
\hline $\begin{array}{l}342.00 \\
344.00 \\
346.00 \\
348.00 \\
350.00 \\
352.00 \\
354.00 \\
356.00 \\
358.00 \\
360.00\end{array}$ & $\begin{array}{l}182.1783 \\
183.2436 \\
184.3089 \\
185.3743 \\
186.4396 \\
187.5049 \\
188.5702 \\
189.6355 \\
190.7008 \\
191.7661\end{array}$ & $\begin{array}{l}1075.61 \\
1083.36 \\
1091.10 \\
1098.84 \\
1106.57 \\
1114.29 \\
1122.01 \\
1129.71 \\
1137.41 \\
1145.11\end{array}$ & $\begin{array}{l}738.48 \\
744.26 \\
750.03 \\
755.80 \\
761.55 \\
767.31 \\
773.05 \\
778.79 \\
784.52 \\
790.24\end{array}$ & $\begin{array}{l}14.177 \\
14.200 \\
14.222 \\
14.244 \\
14.266 \\
14.288 \\
14.310 \\
14.332 \\
14.354 \\
14.375\end{array}$ & $\begin{array}{l}482.00 \\
484.00 \\
486.00 \\
488.00 \\
490.00 \\
492.00 \\
494.00 \\
496.00 \\
498.00 \\
500.00\end{array}$ & $\begin{array}{l}256.6862 \\
257.7476 \\
258.8091 \\
259.8708 \\
260.9326 \\
261.9947 \\
263.0570 \\
264.1197 \\
265.1827 \\
266.2460\end{array}$ & $\begin{array}{l}1598.50 \\
1605.71 \\
1612.91 \\
1620.11 \\
1627.30 \\
1634.49 \\
1641.67 \\
1648.85 \\
1656.02 \\
1663.19\end{array}$ & $\begin{array}{l}1123.50 \\
1128.74 \\
1133.98 \\
1139.21 \\
1144.43 \\
1149.66 \\
1154.87 \\
1160.09 \\
1165.29 \\
1170.50\end{array}$ & $\begin{array}{l}15.461 \\
15.476 \\
15.491 \\
15.506 \\
15.521 \\
15.535 \\
15.550 \\
15.564 \\
15.579 \\
15.593\end{array}$ \\
\hline $\begin{array}{l}362.00 \\
364.00 \\
366.00 \\
368.00 \\
370.00 \\
372.00 \\
374.00 \\
376.00 \\
378.00 \\
380.00\end{array}$ & $\begin{array}{l}192.8310 \\
193.8960 \\
194.9609 \\
196.0258 \\
197.0907 \\
198.1556 \\
199.2205 \\
200.2854 \\
201.3504 \\
202.4153\end{array}$ & $\begin{array}{l}1152.79 \\
1160.46 \\
1168.12 \\
1175.78 \\
1183.43 \\
1191.07 \\
1198.70 \\
1206.32 \\
1213.94 \\
1221.54\end{array}$ & $\begin{array}{l}795.95 \\
801.65 \\
807.34 \\
813.03 \\
818.71 \\
824.38 \\
830.04 \\
835.69 \\
841.33 \\
846.97\end{array}$ & $\begin{array}{l}14.396 \\
14.417 \\
14.438 \\
14.459 \\
14.480 \\
14.501 \\
14.521 \\
14.541 \\
14.562 \\
14.582\end{array}$ & $\begin{array}{l}502.00 \\
504.00 \\
506.00 \\
508.00 \\
510.00 \\
512.00 \\
514.00 \\
516.00 \\
518.00 \\
520.00\end{array}$ & $\begin{array}{l}267.3098 \\
268.3741 \\
269.4388 \\
270.5040 \\
271.5698 \\
272.6361 \\
273.7029 \\
274.7704 \\
275.8384 \\
276.9069\end{array}$ & $\begin{array}{l}1670.36 \\
1677.53 \\
1684.69 \\
1691.85 \\
1699.00 \\
1706.16 \\
1713.31 \\
1720.46 \\
1727.60 \\
1734.74\end{array}$ & $\begin{array}{l}1175.70 \\
1180.89 \\
1186.09 \\
1191.27 \\
1196.46 \\
1201.64 \\
1206.81 \\
1211.99 \\
1217.15 \\
1222.32\end{array}$ & $\begin{array}{l}15.607 \\
15.622 \\
15.636 \\
15.650 \\
15.664 \\
15.678 \\
15.692 \\
15.706 \\
15.720 \\
15.733\end{array}$ \\
\hline $\begin{array}{l}382.00 \\
384.00 \\
386.00 \\
388.00 \\
390.00 \\
392.00 \\
394.00 \\
396.00 \\
398.00 \\
400.00\end{array}$ & $\begin{array}{l}203.4803 \\
204.5453 \\
205.6103 \\
206.6754 \\
207.7405 \\
208.8056 \\
209.8707 \\
210.9359 \\
212.0012 \\
213.0664\end{array}$ & $\begin{array}{l}1229.14 \\
1236.73 \\
1244.30 \\
1251.88 \\
1259.44 \\
1266.99 \\
1274.54 \\
1282.07 \\
1289.60 \\
1297.12\end{array}$ & $\begin{array}{l}852.59 \\
858.21 \\
863.82 \\
869.42 \\
875.01 \\
880.59 \\
886.16 \\
891.73 \\
897.29 \\
902.83\end{array}$ & $\begin{array}{l}14.602 \\
14.621 \\
14.641 \\
14.661 \\
14.680 \\
14.699 \\
14.719 \\
14.738 \\
14.757 \\
14.775\end{array}$ & $\begin{array}{l}522.00 \\
524.00 \\
526.00 \\
528.00 \\
530.00 \\
532.00 \\
534.00 \\
536.00 \\
538.00 \\
540.00\end{array}$ & $\begin{array}{l}277.9760 \\
279.0456 \\
280.1158 \\
281.1863 \\
282.2573 \\
283.3287 \\
284.4004 \\
285.4722 \\
286.5443 \\
287.6163\end{array}$ & $\begin{array}{l}1741.88 \\
1749.02 \\
1756.16 \\
1763.29 \\
1770.42 \\
1777.55 \\
1784.67 \\
1791.79 \\
1798.91 \\
1806.02\end{array}$ & $\begin{array}{l}1227.48 \\
1232.64 \\
1237.79 \\
1242.95 \\
1248.09 \\
1253.24 \\
1258.38 \\
1263.52 \\
1268.65 \\
1273.78\end{array}$ & $\begin{array}{l}15.747 \\
15.761 \\
15.774 \\
15.788 \\
15.801 \\
15.815 \\
15.828 \\
15.841 \\
15.855 \\
15.868\end{array}$ \\
\hline
\end{tabular}


TEMPER- SPECIFIC ENTHALPY INTERNAL ENTROPY ATURE VOLUME (8TU/L8) ENERGY (8TU/L8-R) (R) $\quad(C \cup F T / L 8)$
(8TU/L8)
TEMPER- SPECIFIC ENTHALPY INTERNAL ENTROPY ATURE VOLUME (8TU/L8) ENERGY (8TU/L8-R) $\begin{array}{ll}\text { (R) }(C U F T / L B) & (8 T U / L 8)\end{array}$

\begin{tabular}{|c|c|c|c|c|c|c|c|c|c|}
\hline & & & & & $\begin{array}{l}122.00 \\
124.00 \\
126.00 \\
128.00 \\
130.00 \\
132.00 \\
134.00 \\
136.00 \\
138.00 \\
140.00\end{array}$ & $\begin{array}{l}43.1759 \\
43.8922 \\
44.6082 \\
45.3240 \\
46.0397 \\
46.7551 \\
47.4703 \\
48.1854 \\
48.9003 \\
49.6150\end{array}$ & $\begin{array}{l}301.93 \\
307.21 \\
312.51 \\
317.85 \\
323.21 \\
328.60 \\
334.03 \\
339.48 \\
344.98 \\
350.50\end{array}$ & $\begin{array}{l}182.08 \\
185.37 \\
188.69 \\
192.04 \\
195.41 \\
198.82 \\
202.26 \\
205.73 \\
209.24 \\
212.78\end{array}$ & $\begin{array}{l}10.282 \\
10.325 \\
10.367 \\
10.409 \\
10.451 \\
10.492 \\
10.533 \\
10.573 \\
10.613 \\
10.653\end{array}$ \\
\hline & & & & & $\begin{array}{l}142.00 \\
144.00 \\
146.00 \\
148.00 \\
150.00 \\
152.00 \\
154.00 \\
156.00 \\
158.00 \\
160.00\end{array}$ & $\begin{array}{l}50.3296 \\
51.0440 \\
51.7583 \\
52.4725 \\
53.1865 \\
53.9005 \\
54.6143 \\
55.3280 \\
56.0415 \\
56.7550\end{array}$ & $\begin{array}{l}356.07 \\
361.67 \\
367.31 \\
373.00 \\
378.72 \\
384.48 \\
390.29 \\
396.15 \\
402.04 \\
407.98\end{array}$ & $\begin{array}{l}216.36 \\
219.98 \\
223.64 \\
227.34 \\
231.08 \\
234.87 \\
238.70 \\
242.57 \\
246.48 \\
250.44\end{array}$ & $\begin{array}{l}10.692 \\
10.731 \\
10.770 \\
10.809 \\
10.847 \\
10.886 \\
10.924 \\
10.961 \\
10.999 \\
11.036\end{array}$ \\
\hline $\begin{array}{l}36.00 \\
* 36.603 \\
* 36.603 \\
38.00 \\
40.00\end{array}$ & $\begin{array}{r}.2252 \\
.2266 \\
11.7294 \\
12.3162 \\
13.1300\end{array}$ & $\begin{array}{r}-111.01 \\
-109.62 \\
81.93 \\
86.14 \\
91.89\end{array}$ & $\begin{array}{r} \\
-111.64 \\
-110.89 \\
49.37 \\
51.96 \\
55.44\end{array}$ & $\begin{array}{l} \\
1.885 \\
1.923 \\
7.155 \\
7.268 \\
7.416\end{array}$ & $\begin{array}{l}162.00 \\
164.00 \\
166.00 \\
168.00 \\
170.00 \\
172.00 \\
174.00 \\
176.00 \\
178.00 \\
180.00\end{array}$ & $\begin{array}{l}57.4684 \\
58.1817 \\
58.8949 \\
59.6080 \\
60.3210 \\
61.0340 \\
61.7469 \\
62.4597 \\
63.1724 \\
63.8850\end{array}$ & $\begin{array}{l}413.97 \\
420.01 \\
426.09 \\
432.21 \\
438.38 \\
444.60 \\
450.86 \\
457.17 \\
463.52 \\
469.92\end{array}$ & $\begin{array}{l}254.45 \\
258.51 \\
262.61 \\
266.75 \\
270.94 \\
275.18 \\
279.47 \\
283.80 \\
288.17 \\
292.59\end{array}$ & $\begin{array}{l}11.074 \\
11.111 \\
11.147 \\
11.184 \\
11.221 \\
11.257 \\
11.293 \\
11.329 \\
11.365 \\
11.401\end{array}$ \\
\hline $\begin{array}{l}42.00 \\
44.00 \\
46.00 \\
48.00 \\
50.00 \\
52.00 \\
54.00 \\
56.00 \\
58.00 \\
60.00\end{array}$ & $\begin{array}{l}13.9239 \\
14.7041 \\
15.4747 \\
16.2381 \\
16.9959 \\
17.7491 \\
18.4985 \\
19.2448 \\
19.9883 \\
20.7294\end{array}$ & $\begin{array}{r}97.42 \\
102.81 \\
108.12 \\
113.36 \\
118.56 \\
123.72 \\
128.87 \\
133.99 \\
139.10 \\
144.19\end{array}$ & $\begin{array}{l}58.77 \\
62.00 \\
65.16 \\
68.29 \\
71.38 \\
74.46 \\
77.52 \\
80.57 \\
83.61 \\
86.65\end{array}$ & $\begin{array}{l}7.551 \\
7.676 \\
7.794 \\
7.906 \\
8.012 \\
8.113 \\
8.210 \\
8.303 \\
8.393 \\
8.479\end{array}$ & $\begin{array}{l}182.00 \\
184.00 \\
186.00 \\
188.00 \\
190.00 \\
192.00 \\
194.00 \\
196.00 \\
198.00 \\
200.00\end{array}$ & $\begin{array}{l}64.5975 \\
65.3099 \\
66.0221 \\
66.7343 \\
67.4465 \\
68.1585 \\
68.8705 \\
69.5824 \\
70.2943 \\
71.0061\end{array}$ & $\begin{array}{l}476.36 \\
482.84 \\
489.36 \\
495.94 \\
502.55 \\
509.21 \\
515.91 \\
522.65 \\
529.44 \\
536.27\end{array}$ & $\begin{array}{l}297.05 \\
301.55 \\
306.10 \\
310.69 \\
315.33 \\
320.01 \\
324.74 \\
329.51 \\
334.32 \\
339.17\end{array}$ & $\begin{array}{l}11.436 \\
11.472 \\
11.507 \\
11.542 \\
11.577 \\
11.612 \\
11.647 \\
11.681 \\
11.716 \\
11.750\end{array}$ \\
\hline $\begin{array}{l}62.00 \\
64.00 \\
66.00 \\
68.00 \\
70.00 \\
72.00 \\
74.00 \\
76.00 \\
78.00 \\
80.00\end{array}$ & $\begin{array}{l}21.4684 \\
22.2054 \\
22.9407 \\
23.6744 \\
24.4067 \\
25.1377 \\
25.8675 \\
26.5962 \\
27.3238 \\
28.0505\end{array}$ & $\begin{array}{l}149.27 \\
154.34 \\
159.41 \\
164.47 \\
169.52 \\
174.56 \\
179.60 \\
184.64 \\
189.68 \\
194.71\end{array}$ & $\begin{array}{r}89.68 \\
92.71 \\
95.73 \\
98.75 \\
101.77 \\
104.79 \\
107.80 \\
110.82 \\
113.83 \\
116.85\end{array}$ & $\begin{array}{l}8.562 \\
8.643 \\
8.721 \\
8.796 \\
8.870 \\
8.941 \\
9.010 \\
9.077 \\
9.142 \\
9.206\end{array}$ & $\begin{array}{l}202.00 \\
204.00 \\
206.00 \\
208.00 \\
210.00 \\
212.00 \\
214.00 \\
216.00 \\
218.00 \\
220.00\end{array}$ & $\begin{array}{l}71.7178 \\
72.4295 \\
73.1411 \\
73.8527 \\
74.5642 \\
75.2757 \\
75.9871 \\
76.6985 \\
77.4099 \\
78.1212\end{array}$ & $\begin{array}{l}543.14 \\
550.05 \\
557.00 \\
563.98 \\
571.01 \\
578.07 \\
585.17 \\
592.30 \\
599.47 \\
606.67\end{array}$ & $\begin{array}{l}344.06 \\
349.00 \\
353.97 \\
358.98 \\
364.03 \\
369.12 \\
374.25 \\
379.41 \\
384.59 \\
389.82\end{array}$ & $\begin{array}{l}11.784 \\
11.818 \\
11.852 \\
11.886 \\
11.920 \\
11.953 \\
11.986 \\
12.020 \\
12.053 \\
12.085\end{array}$ \\
\hline $\begin{array}{r}82.00 \\
84.00 \\
86.00 \\
88.00 \\
90.00 \\
92.00 \\
94.00 \\
96.00 \\
98.00 \\
100.00\end{array}$ & $\begin{array}{l}28.7764 \\
29.5014 \\
30.2256 \\
30.9492 \\
31.6721 \\
32.3944 \\
33.1161 \\
33.8372 \\
34.5579 \\
35.2781\end{array}$ & $\begin{array}{l}199.75 \\
204.78 \\
209.82 \\
214.85 \\
219.89 \\
224.94 \\
229.99 \\
235.04 \\
240.11 \\
245.18\end{array}$ & $\begin{array}{l}119.87 \\
122.89 \\
125.92 \\
128.94 \\
131.98 \\
135.02 \\
138.06 \\
141.12 \\
144.18 \\
147.26\end{array}$ & $\begin{array}{l}9.268 \\
9.329 \\
9.388 \\
9.446 \\
9.503 \\
9.558 \\
9.612 \\
9.666 \\
9.718 \\
9.769\end{array}$ & $\begin{array}{l}222.00 \\
224.00 \\
226.00 \\
228.00 \\
230.00 \\
232.00 \\
234.00 \\
236.00 \\
238.00 \\
240.00\end{array}$ & $\begin{array}{l}78.8325 \\
79.5437 \\
80.2550 \\
80.9662 \\
81.6774 \\
82.3886 \\
83.0997 \\
83.8108 \\
84.5220 \\
85.2330\end{array}$ & $\begin{array}{l}613.90 \\
621.16 \\
628.45 \\
635.77 \\
643.12 \\
650.51 \\
657.91 \\
665.35 \\
672.81 \\
680.29\end{array}$ & $\begin{array}{l}395.07 \\
400.36 \\
405.68 \\
411.03 \\
416.41 \\
421.81 \\
427.25 \\
432.71 \\
438.19 \\
443.70\end{array}$ & $\begin{array}{l}12.118 \\
12.151 \\
12.183 \\
12.215 \\
12.248 \\
12.279 \\
12.311 \\
12.343 \\
12.374 \\
12.406\end{array}$ \\
\hline $\begin{array}{l}102.00 \\
104.00 \\
106.00 \\
108.00 \\
110.00 \\
112.00 \\
114.00 \\
116.00 \\
118.00 \\
120.00\end{array}$ & $\begin{array}{l}35.9979 \\
36.7172 \\
37.4361 \\
38.1547 \\
38.8729 \\
39.5908 \\
40.3084 \\
41.0257 \\
41.7427 \\
42.4594\end{array}$ & $\begin{array}{l}250.26 \\
255.36 \\
260.47 \\
265.59 \\
270.72 \\
275.87 \\
281.04 \\
286.23 \\
291.44 \\
296.67\end{array}$ & $\begin{array}{l}150.34 \\
153.44 \\
156.55 \\
159.68 \\
162.82 \\
165.98 \\
169.16 \\
172.35 \\
175.57 \\
178.81\end{array}$ & $\begin{array}{l}9.819 \\
9.869 \\
9.917 \\
9.965 \\
10.012 \\
10.059 \\
10.105 \\
10.150 \\
10.194 \\
10.238\end{array}$ & $\begin{array}{l}242.00 \\
244.00 \\
246.00 \\
248.00 \\
250.00 \\
252.00 \\
254.00 \\
256.00 \\
258.00 \\
260.00\end{array}$ & $\begin{array}{l}85.9441 \\
86.6552 \\
87.3662 \\
88.0773 \\
88.7883 \\
89.4993 \\
90.2103 \\
90.9212 \\
91.6322 \\
92.3431\end{array}$ & $\begin{array}{l}687.80 \\
695.34 \\
702.89 \\
710.47 \\
718.06 \\
725.68 \\
733.32 \\
740.97 \\
748.64 \\
756.33\end{array}$ & $\begin{array}{l}449.24 \\
454.80 \\
460.38 \\
465.98 \\
471.61 \\
477.25 \\
482.91 \\
488.59 \\
494.29 \\
500.00\end{array}$ & $\begin{array}{l}12.437 \\
12.468 \\
12.499 \\
12.529 \\
12.560 \\
12.590 \\
12.620 \\
12.650 \\
12.680 \\
12.710\end{array}$ \\
\hline
\end{tabular}


TEMPER- SPECIFIC ENTHALPY INTERNAL ENTROPY ATURE VOLUME (BTU/LB) ENERGY (BTU/L8-R) (R) (CU FT/LB)
( $8 \mathrm{TU} / L$ S) $\begin{array}{ccc}\text { TEMPER- } & \text { SPECIFIC } & \text { ENTHALPY } \\ \text { ATURE } & \text { VOLUME } & \text { (8TU/L8) } \\ \text { (R) } & (C U F T / L B) & \end{array}$
INTERNAL ENTROPY

ENERGY (8TU/L8-R)

(8TU/L8)

\begin{tabular}{|c|c|c|c|c|c|c|c|c|c|}
\hline $\begin{array}{l}262.00 \\
264.00 \\
266.00 \\
268.00 \\
270.00 \\
272.00 \\
274.00 \\
276.00 \\
278.00 \\
280.00\end{array}$ & $\begin{array}{l}93.0541 \\
93.7650 \\
94.4758 \\
95.1867 \\
95.8975 \\
96.6084 \\
97.3192 \\
98.0300 \\
98.7407 \\
99.4515\end{array}$ & $\begin{array}{l}764.03 \\
771.75 \\
779.48 \\
787.22 \\
794.98 \\
802.72 \\
810.47 \\
818.23 \\
826.00 \\
833.77\end{array}$ & $\begin{array}{l}505.73 \\
511.48 \\
517.23 \\
523.00 \\
528.79 \\
534.56 \\
540.33 \\
546.12 \\
551.92 \\
557.72\end{array}$ & $\begin{array}{l}12.739 \\
12.769 \\
12.798 \\
12.827 \\
12.856 \\
12.884 \\
12.913 \\
12.941 \\
12.969 \\
12.997\end{array}$ & $\begin{array}{l}402.00 \\
404.00 \\
406.00 \\
408.00 \\
410.00 \\
412.00 \\
414.00 \\
416.00 \\
418.00 \\
420.00\end{array}$ & $\begin{array}{l}142.7858 \\
143.4961 \\
144.2065 \\
144.9168 \\
145.6272 \\
146.3377 \\
147.0481 \\
147.7589 \\
148.4696 \\
149.1803\end{array}$ & $\begin{array}{l}1304.63 \\
1312.14 \\
1319.63 \\
1327.12 \\
1334.59 \\
1342.06 \\
1349.52 \\
1356.97 \\
1364.42 \\
1371.86\end{array}$ & $\begin{array}{l}908.29 \\
913.82 \\
919.34 \\
924.86 \\
930.36 \\
935.86 \\
941.34 \\
946.83 \\
952.30 \\
957.76\end{array}$ & $\begin{array}{l}14.394 \\
14.413 \\
14.432 \\
14.450 \\
14.468 \\
14.486 \\
14.504 \\
14.522 \\
14.540 \\
14.558\end{array}$ \\
\hline $\begin{array}{l}282.00 \\
284.00 \\
286.00 \\
288.00 \\
290.00 \\
292.00 \\
294.00 \\
296.00 \\
298.00 \\
300.00\end{array}$ & $\begin{array}{l}100.1622 \\
100.8729 \\
101.5836 \\
102.2943 \\
103.0050 \\
103.7156 \\
104.4262 \\
105.1369 \\
105.8475 \\
106.5580\end{array}$ & $\begin{array}{l}841.56 \\
849.34 \\
857.14 \\
864.93 \\
872.74 \\
880.54 \\
888.35 \\
896.17 \\
903.98 \\
911.80\end{array}$ & $\begin{array}{l}563.53 \\
569.34 \\
575.16 \\
380.99 \\
586.82 \\
592.65 \\
598.49 \\
604.33 \\
610.17 \\
616.01\end{array}$ & $\begin{array}{l}13.025 \\
13.052 \\
13.079 \\
13.107 \\
13.134 \\
13.160 \\
13.187 \\
13.214 \\
13.240 \\
13.266\end{array}$ & $\begin{array}{l}422.00 \\
424.00 \\
426.00 \\
428.00 \\
430.00 \\
432.00 \\
434.00 \\
436.00 \\
438.00 \\
440.00\end{array}$ & $\begin{array}{l}149.8910 \\
150.6017 \\
151.3123 \\
152.0228 \\
152.7332 \\
153.4435 \\
154.1537 \\
154.8638 \\
155.5738 \\
156.2836\end{array}$ & $\begin{array}{l}1379.28 \\
1386.70 \\
1394.11 \\
1401.51 \\
1408.91 \\
1416.29 \\
1423.67 \\
1431.03 \\
1438.39 \\
1445.74\end{array}$ & $\begin{array}{r}963.22 \\
968.66 \\
974.10 \\
979.53 \\
984.95 \\
990.36 \\
995.77 \\
1001.16 \\
1006.55 \\
1011.93\end{array}$ & $\begin{array}{l}14.576 \\
14.593 \\
14.611 \\
14.628 \\
14.645 \\
14.662 \\
14.679 \\
14.696 \\
14.713 \\
14.730\end{array}$ \\
\hline $\begin{array}{l}302.00 \\
304.00 \\
306.00 \\
308.00 \\
310.00 \\
312.00 \\
314.00 \\
316.00 \\
318.00 \\
320.00\end{array}$ & $\begin{array}{l}107.2686 \\
107.9792 \\
108.6897 \\
109.4002 \\
110.1108 \\
110.8213 \\
111.5318 \\
112.2423 \\
112.9528 \\
113.6633\end{array}$ & $\begin{array}{l}919.61 \\
927.43 \\
935.25 \\
943.07 \\
950.89 \\
958.70 \\
966.52 \\
974.33 \\
982.14 \\
989.95\end{array}$ & $\begin{array}{l}621.86 \\
627.70 \\
633.55 \\
639.40 \\
645.24 \\
651.09 \\
656.93 \\
662.77 \\
668.61 \\
674.45\end{array}$ & $\begin{array}{l}13.292 \\
13.318 \\
13.343 \\
13.369 \\
13.394 \\
13.419 \\
13.444 \\
13.469 \\
13.494 \\
13.518\end{array}$ & $\begin{array}{l}442.00 \\
444.00 \\
446.00 \\
448.00 \\
450.00 \\
452.00 \\
454.00 \\
456.00 \\
458.00 \\
460.00\end{array}$ & $\begin{array}{l}156.9933 \\
157.7029 \\
158.4123 \\
159.1216 \\
159.8307 \\
160.5396 \\
161.2484 \\
161.9570 \\
162.6655 \\
163.3738\end{array}$ & $\begin{array}{l}1453.08 \\
1460.41 \\
1467.74 \\
1475.05 \\
1482.36 \\
1489.67 \\
1496.98 \\
1504.28 \\
1511.58 \\
1518.86\end{array}$ & $\begin{array}{l}1017.30 \\
1022.66 \\
1028.02 \\
1033.36 \\
1038.70 \\
1044.05 \\
1049.39 \\
1054.72 \\
1060.05 \\
1065.37\end{array}$ & $\begin{array}{l}14.746 \\
14.763 \\
14.779 \\
14.796 \\
14.812 \\
14.828 \\
14.844 \\
14.861 \\
14.876 \\
14.892\end{array}$ \\
\hline $\begin{array}{l}322.00 \\
324.00 \\
326.00 \\
328.00 \\
330.00 \\
332.00 \\
334.00 \\
336.00 \\
338.00 \\
340.00\end{array}$ & $\begin{array}{l}114.3737 \\
115.0842 \\
115.7947 \\
116.5051 \\
117.2156 \\
117.9261 \\
118.6365 \\
119.3470 \\
120.0574 \\
120.7679\end{array}$ & $\begin{array}{r}997.76 \\
1005.56 \\
1013.36 \\
1021.15 \\
1028.94 \\
1036.73 \\
1044.51 \\
1052.28 \\
1060.05 \\
1067.82\end{array}$ & $\begin{array}{l}680.28 \\
686.11 \\
691.94 \\
697.76 \\
703.58 \\
709.39 \\
715.20 \\
721.00 \\
726.80 \\
732.59\end{array}$ & $\begin{array}{l}13.543 \\
13.567 \\
13.591 \\
13.615 \\
13.638 \\
13.662 \\
13.685 \\
13.708 \\
13.731 \\
13.754\end{array}$ & $\begin{array}{l}462.00 \\
464.00 \\
466.00 \\
468.00 \\
470.00 \\
472.00 \\
474.00 \\
476.00 \\
478.00 \\
480.00\end{array}$ & $\begin{array}{l}164.0820 \\
164.7901 \\
165.4980 \\
166.2058 \\
166.9136 \\
167.6213 \\
168.3289 \\
169.0364 \\
169.7440 \\
170.4516\end{array}$ & $\begin{array}{l}1526.14 \\
1533.41 \\
1540.67 \\
1547.93 \\
1555.18 \\
1562.42 \\
1569.66 \\
1576.89 \\
1584.11 \\
1591.33\end{array}$ & $\begin{array}{l}1070.68 \\
1075.98 \\
1081.28 \\
1086.57 \\
1091.86 \\
1097.14 \\
1102.41 \\
1107.68 \\
1112.94 \\
1118.19\end{array}$ & $\begin{array}{l}14.908 \\
14.924 \\
14.939 \\
14.955 \\
14.970 \\
14.986 \\
15.001 \\
15.016 \\
15.031 \\
15.047\end{array}$ \\
\hline $\begin{array}{l}342.00 \\
344.00 \\
346.00 \\
348.00 \\
350.00 \\
352.00 \\
354.00 \\
356.00 \\
358.00 \\
360.00\end{array}$ & $\begin{array}{l}121.4783 \\
122.1888 \\
122.8992 \\
123.6096 \\
124.3201 \\
125.0305 \\
125.7409 \\
126.4513 \\
127.1617 \\
127.8721\end{array}$ & $\begin{array}{l}1075.58 \\
1083.33 \\
1091.07 \\
1098.81 \\
1106.54 \\
1114.27 \\
1121.98 \\
1129.69 \\
1137.40 \\
1145.09\end{array}$ & $\begin{array}{l}738.38 \\
744.16 \\
749.93 \\
755.70 \\
761.46 \\
767.21 \\
772.95 \\
778.69 \\
784.42 \\
790.14\end{array}$ & $\begin{array}{l}13.777 \\
13.800 \\
13.822 \\
13.844 \\
13.867 \\
13.889 \\
13.910 \\
13.932 \\
13.954 \\
13.975\end{array}$ & $\begin{array}{l}482.00 \\
484.00 \\
486.00 \\
488.00 \\
490.00 \\
492.00 \\
494.00 \\
496.00 \\
498.00 \\
500.00\end{array}$ & $\begin{array}{l}171.1592 \\
171.8668 \\
172.5745 \\
173.2824 \\
173.9903 \\
174.6985 \\
175.4068 \\
176.1153 \\
176.8240 \\
177.5331\end{array}$ & $\begin{array}{l}1598.54 \\
1605.75 \\
1612.95 \\
1620.15 \\
1627.34 \\
1634.53 \\
1641.71 \\
1648.89 \\
1656.07 \\
1663.24\end{array}$ & $\begin{array}{l}1123.44 \\
1128.68 \\
1133.92 \\
1139.15 \\
1144.38 \\
1149.60 \\
1154.82 \\
1160.03 \\
1165.24 \\
1170.44\end{array}$ & $\begin{array}{l}15.062 \\
15.076 \\
15.091 \\
15.106 \\
15.121 \\
15.135 \\
15.150 \\
15.165 \\
15.179 \\
15.193\end{array}$ \\
\hline $\begin{array}{l}362.00 \\
364.00 \\
366.00 \\
368.00 \\
370.00 \\
372.00 \\
374.00 \\
376.00 \\
378.00 \\
380.00\end{array}$ & $\begin{array}{l}128.5823 \\
129.2924 \\
130.0025 \\
130.7126 \\
131.4227 \\
132.1328 \\
132.8429 \\
133.5530 \\
134.02632 \\
134.9733\end{array}$ & $\begin{array}{l}1152.77 \\
1160.45 \\
1168.11 \\
1175.77 \\
1183.42 \\
1191.06 \\
1198.69 \\
1206.31 \\
1213.93 \\
1221.54\end{array}$ & $\begin{array}{l}795.85 \\
801.56 \\
807.25 \\
812.94 \\
818.62 \\
824.28 \\
829.95 \\
835.60 \\
841.24 \\
846.88\end{array}$ & $\begin{array}{l}13.996 \\
14.018 \\
14.039 \\
14.059 \\
14.080 \\
14.101 \\
14.121 \\
14.142 \\
14.162 \\
14.182\end{array}$ & $\begin{array}{l}502.00 \\
504.00 \\
506.00 \\
508.00 \\
510.00 \\
512.00 \\
514.00 \\
516.00 \\
518.00 \\
520.00\end{array}$ & $\begin{array}{l}178.2424 \\
178.9520 \\
179.6619 \\
180.3722 \\
181.0828 \\
181.7939 \\
182.5053 \\
183.2170 \\
183.9292 \\
184.6418\end{array}$ & $\begin{array}{l}1670.41 \\
1677.57 \\
1684.73 \\
1691.89 \\
1699.05 \\
1706.20 \\
1713.35 \\
1720.50 \\
1727.65 \\
1734.79\end{array}$ & $\begin{array}{l}1175.64 \\
1180.84 \\
1186.03 \\
1191.22 \\
1196.40 \\
1201.58 \\
1206.76 \\
1211.93 \\
1217.10 \\
1222.26\end{array}$ & $\begin{array}{l}15.208 \\
15.222 \\
15.236 \\
15.250 \\
15.264 \\
15.278 \\
15.292 \\
15.306 \\
15.320 \\
15.334\end{array}$ \\
\hline $\begin{array}{l}382.00 \\
384.00 \\
386.00 \\
388.00 \\
390.00 \\
392.00 \\
394.00 \\
396.00 \\
398.00 \\
400.00\end{array}$ & $\begin{array}{l}135.6834 \\
136.3936 \\
137.1037 \\
137.8139 \\
138.5241 \\
139.2343 \\
139.9446 \\
140.6548 \\
141.3651 \\
142.0754\end{array}$ & $\begin{array}{l}1229.13 \\
1236.72 \\
1244.30 \\
1251.87 \\
1259.44 \\
1266.99 \\
1274.54 \\
1282.08 \\
1289.60 \\
1297.12\end{array}$ & $\begin{array}{l}852.50 \\
858.12 \\
863.73 \\
869.33 \\
874.92 \\
880.51 \\
886.08 \\
891.65 \\
897.20 \\
902.75\end{array}$ & $\begin{array}{l}14.202 \\
14.222 \\
14.241 \\
14.261 \\
14.280 \\
14.300 \\
14.319 \\
14.338 \\
14.357 \\
14.376\end{array}$ & $\begin{array}{l}522.00 \\
524.00 \\
526.00 \\
528.00 \\
530.00 \\
532.00 \\
534.00 \\
536.00 \\
538.00 \\
540.00\end{array}$ & $\begin{array}{l}185.3547 \\
186.0680 \\
186.7816 \\
187.4955 \\
188.2097 \\
188.9241 \\
189.6388 \\
190.3536 \\
191.0684 \\
191.7834\end{array}$ & $\begin{array}{l}1741.93 \\
1749.07 \\
1756.21 \\
1763.34 \\
1770.47 \\
1777.60 \\
1784.72 \\
1791.84 \\
1798.96 \\
1806.07\end{array}$ & $\begin{array}{l}1227.43 \\
1232.58 \\
1237.74 \\
1242.89 \\
1248.04 \\
1253.18 \\
1258.32 \\
1263.46 \\
1268.59 \\
1273.72\end{array}$ & $\begin{array}{l}15.347 \\
15.361 \\
15.375 \\
15.388 \\
15.402 \\
15.415 \\
15.428 \\
15.442 \\
15.455 \\
15.468\end{array}$ \\
\hline
\end{tabular}


TEMPER- SPECIFIC ENTHALPY INTERNAL ENTROPY

ATURE VOLUME (8TU/LB) ENERGY (8TU/L8-R) (R) $\quad(C U F T / L 8 \mid$

\section{(BTU/LB)}

TEMPER- SPECIFIC ENTHALPY

ATURE

(R)
VDLUME (CU FT/L8)
INTERNAL ENTROPY

ENERGY (GTU/L8-R) $(8 \mathrm{TU} / \mathrm{L} 8)$

$\begin{array}{lr}36.00 & .2251 \\ 38.00 & .2294 \\ \% 38.436 & .2305 \\ \% 38.436 & 9.0293 \\ 40.00 & 9.5393 \\ & \\ 42.00 & 10.1676 \\ 44.00 & 10.7781 \\ 46.00 & 11.3764 \\ 48.00 & 11.9659 \\ 50.00 & 12.5487 \\ 52.00 & 13.1264 \\ 54.00 & 13.6998 \\ 56.00 & 14.2697 \\ 58.00 & 14.8366 \\ 60.00 & 15.4008 \\ & \\ 62.00 & 15.9628 \\ 64.00 & 16.5228 \\ 66.00 & 17.0810 \\ 68.00 & 17.6375 \\ 70.00 & 18.1925 \\ 72.00 & 18.7462 \\ 74.00 & 19.2987 \\ 76.00 & 19.8500 \\ 78.00 & 20.4003 \\ 80.00 & 20.9496 \\ & \\ 82.00 & 21.4980 \\ 84.00 & 22.0456 \\ 86.00 & 22.5924 \\ 88.00 & 23.1385 \\ 90.00 & 23.6839 \\ 92.00 & 24.2288 \\ 94.00 & 24.7730 \\ 96.00 & 25.3167 \\ 98.00 & 25.8599 \\ 100.00 & 26.4026 \\ & \\ 102.00 & 26.9448 \\ 104.00 & 27.4867 \\ 106.00 & 28.0281 \\ 108.00 & 28.5692 \\ 110.00 & 29.1099 \\ 112.00 & 29.6503 \\ 114.00 & 30.1904 \\ 116.00 & 30.7302 \\ 118.00 & 31.2697 \\ 120.00 & 31.8089\end{array}$
$-110.87$
$-106.14$
$-105.06$
84.17
89.04
94.92
100.57
106.07
111.46
116.78
122.05
127.28
132.48
137.66
142.82

147.96

153.09

158.20

163.31

168.40

173.49

178.57

183.65

188.72

193.79

198.85

203.92

208.98

214.04

219.11

224.18

229.25

234.33

239.42

244.51

249.62

254.73

259.86

264.99

270.15

275.32

280.50

285.71

290.93

296.18
$-111.71$

$-106.99$

$-106.78$

50.75

53.73

57.29

60.68

63.96

67.17

70.34

73.47

76.58

79.67

82.75

85.82

88.88

91.93

94.98

98.03

101.07

104.11

107.15

110.18

116.25

119.29

122.32

125.36

128.41

131.46

134.51

137.57

140.64

143.71

146.80

149.89

153.00

156.12

159.26

162.41

165.58

168.77

171.97

175.20

178.45
1.883

2.011

2.039

6.962

7.086

7.230

7.361

7.483

7.598

7.707

7.810

7.909

8.003

8.094

8.182

8.266

8.347

8.426

8.502

8.576

8.648

8.717

8.785

8.851

8.915

8.978

9.039

9.098

9.156

9.213

9.269

9.324

9.377

9.430

9.481

9.532

9.581

9.630

9.678

9.725

9.772

9.818

9.863

9.908

9.952

32.3479
32.8866
33.4252
33.9635
34.5016
35.0395
35.5772
36.1148
36.6521
37.1894
37.7264
38.2633
38.8001
39.3368
39.8733
40.4097
40.9460
41.4822
42.0182
42.5542

301.44

306.74

312.06

317.40

322.78

328.18

333.62

339.08

344.59

138.00

140.00

142.00

144.00

146.00

148.00

150.00

152.00

154.00

156.00

158.00

160.00

162.00

164.00

166.00

168.00

170.00

172.00

174.00

176.00

178.00

180.00

182.00

184.00

186.00

188.00

190.00

192.00

194.00

196.00

198.00

200.00

202.00

204.00

206.00

208.00

210.00

212.00

214.00

216.00

218.00

220.00

222.00

02.00

226.00

228.00

230.00

232.00

234.00

236.00

238.00

240.00

242.00

244.00

246.00

248.00

250.00

252.00

254.00

256.00

258.00

260.00
43.0901

43.6259

44.1615

44.6971

45.2327

45.7681

46.3034

46.8387

47.3739

47. 9091

48.4441

48.9789

49.5137

50.0484

50.5831

51.1177

51.6522

52.1866

52.7210

53.2554

53.7896

54.3239

54.8580

55.3921

55.9262

56.4602

56.9942

57.5282

58.0621

58.5959

59.1298

59.6636

60.1974

60.7311

61.2649

61.7986

62.3323

62.8660

63.3996

63.9333

64.4669

65.0005

65.5341

66.0677

66.6013

67.1348

67.6683

68.2019

68.7354

69.2689

181.72

185.02

188.35

191.70

195.08

198.50 
TEMPER- SPECIFIC ENTHALPY INTERNAL ENTROPY ATURE VOLUME (BTU/LO) ENERGY (BTU/LB-R) (R) (CUFT/LB) (BTU/LQ) $\begin{array}{ccc}\text { TEMPER- } & \text { SPECIFIC } & \text { ENTHALPY } \\ \text { ATURE } & \text { VOLUME } & \text { (8TULL8) } \\ \text { (R) } & \text { (CU FT/L8) } & \end{array}$
INTERNAL ENTROPY

ENERGY (BTU/LB-R)

$(B T U / L 8)$

\begin{tabular}{|c|c|c|c|c|c|c|c|c|c|}
\hline $\begin{array}{l}262.00 \\
264.00 \\
266.00 \\
268.00 \\
270.00 \\
272.00 \\
274.00 \\
276.00 \\
278.00 \\
280.00\end{array}$ & $\begin{array}{l}69.8023 \\
70.3358 \\
70.8692 \\
71.4027 \\
71.9361 \\
72.4694 \\
73.0028 \\
73.5362 \\
74.0695 \\
74.6028\end{array}$ & $\begin{array}{l}763.93 \\
771.65 \\
779.39 \\
787.13 \\
794.89 \\
802.63 \\
810.39 \\
818.15 \\
825.92 \\
833.70\end{array}$ & $\begin{array}{l}505.59 \\
511.34 \\
517.10 \\
522.87 \\
528.65 \\
534.42 \\
540.20 \\
545.99 \\
551.78 \\
557.59\end{array}$ & $\begin{array}{l}12.455 \\
12.485 \\
12.514 \\
12.543 \\
12.572 \\
12.600 \\
12.629 \\
12.657 \\
12.685 \\
12.713\end{array}$ & $\begin{array}{l}402.00 \\
404.00 \\
406.00 \\
408.00 \\
410.00 \\
412.00 \\
414.00 \\
416.00 \\
418.00 \\
420.00\end{array}$ & $\begin{array}{l}107.1129 \\
107.6458 \\
108.1787 \\
108.7116 \\
109.2445 \\
109.7775 \\
110.3104 \\
110.8436 \\
111.3767 \\
111.9098\end{array}$ & $\begin{array}{l}1304.64 \\
1312.14 \\
1319.64 \\
1327.12 \\
1334.60 \\
1342.07 \\
1349.53 \\
1356.98 \\
1364.43 \\
1371.87\end{array}$ & $\begin{array}{l}908.21 \\
913.74 \\
919.26 \\
924.78 \\
930.28 \\
935.78 \\
941.26 \\
946.75 \\
952.22 \\
957.68\end{array}$ & $\begin{array}{l}14.111 \\
14.129 \\
14.148 \\
14.166 \\
14.184 \\
14.203 \\
14.221 \\
14.239 \\
14.257 \\
14.274\end{array}$ \\
\hline $\begin{array}{l}282.00 \\
284.00 \\
286.00 \\
288.00 \\
290.00 \\
292.00 \\
294.00 \\
296.00 \\
298.00 \\
300.00\end{array}$ & $\begin{array}{l}75.1361 \\
75.6694 \\
76.2027 \\
76.7359 \\
77.2692 \\
77.8024 \\
78.3356 \\
78.8687 \\
79.4019 \\
79.9351\end{array}$ & $\begin{array}{l}841.48 \\
849.27 \\
857.06 \\
864.86 \\
872.67 \\
880.48 \\
888.29 \\
896.10 \\
903.92 \\
911.74\end{array}$ & $\begin{array}{l}563.40 \\
569.21 \\
575.03 \\
580.86 \\
586.69 \\
592.53 \\
598.36 \\
604.20 \\
610.05 \\
615.89\end{array}$ & $\begin{array}{l}12.741 \\
12.768 \\
12.795 \\
12.823 \\
12.850 \\
12.876 \\
12.903 \\
12.930 \\
12.956 \\
12.982\end{array}$ & $\begin{array}{l}422.00 \\
424.00 \\
426.00 \\
428.00 \\
430.00 \\
432.00 \\
434.00 \\
436.00 \\
438.00 \\
440.00\end{array}$ & $\begin{array}{l}112.4429 \\
112.9760 \\
113.5090 \\
114.0419 \\
114.5748 \\
115.1076 \\
115.6404 \\
116.1730 \\
116.7056 \\
117.2380\end{array}$ & $\begin{array}{l}1379.30 \\
1386.72 \\
1394.13 \\
1401.53 \\
1408.92 \\
1416.31 \\
1423.68 \\
1431.05 \\
1438.41 \\
1445.76\end{array}$ & $\begin{array}{r}963.14 \\
968.59 \\
974.02 \\
979.45 \\
984.88 \\
990.29 \\
995.69 \\
1001.09 \\
1006.48 \\
1011.85\end{array}$ & $\begin{array}{l}14.292 \\
14.309 \\
14.327 \\
14.344 \\
14.361 \\
14.379 \\
14.396 \\
14.413 \\
14.429 \\
14.446\end{array}$ \\
\hline $\begin{array}{l}302.00 \\
304.00 \\
306.00 \\
308.00 \\
310.00 \\
312.00 \\
314.00 \\
316.00 \\
318.00 \\
320.00\end{array}$ & $\begin{array}{l}80.4682 \\
81.0013 \\
81.5344 \\
82.0675 \\
82.6006 \\
83.1336 \\
83.6667 \\
84.1997 \\
84.7328 \\
85.2658\end{array}$ & $\begin{array}{l}919.56 \\
927.38 \\
935.20 \\
943.02 \\
950.84 \\
958.65 \\
966.47 \\
974.29 \\
982.10 \\
989.91\end{array}$ & $\begin{array}{l}621.74 \\
627.59 \\
633.43 \\
639.28 \\
645.13 \\
650.97 \\
656.82 \\
662.66 \\
668.50 \\
674.34\end{array}$ & $\begin{array}{l}13.008 \\
13.034 \\
13.060 \\
13.085 \\
13.110 \\
13.135 \\
13.160 \\
13.185 \\
13.210 \\
13.234\end{array}$ & $\begin{array}{l}442.00 \\
444.00 \\
446.00 \\
448.00 \\
450.00 \\
452.00 \\
454.00 \\
456.00 \\
458.00 \\
460.00\end{array}$ & $\begin{array}{l}117.7704 \\
118.3026 \\
118.8347 \\
119.3667 \\
119.8986 \\
120.4304 \\
120.9621 \\
121.4936 \\
122.0250 \\
122.5563\end{array}$ & $\begin{array}{l}1453.10 \\
1460.43 \\
1467.76 \\
1475.07 \\
1482.38 \\
1489.70 \\
1497.01 \\
1504.31 \\
1511.60 \\
1518.89\end{array}$ & $\begin{array}{l}1017.23 \\
1022.59 \\
1027.94 \\
1033.29 \\
1038.63 \\
1043.98 \\
1049.32 \\
1054.65 \\
1059.98 \\
1065.30\end{array}$ & $\begin{array}{l}14.463 \\
14.479 \\
14.496 \\
14.512 \\
14.528 \\
14.545 \\
14.561 \\
14.577 \\
14.593 \\
14.609\end{array}$ \\
\hline $\begin{array}{l}322.00 \\
324.00 \\
326.00 \\
328.00 \\
330.00 \\
332.00 \\
334.00 \\
336.00 \\
338.00 \\
340.00\end{array}$ & $\begin{array}{l}85.7988 \\
86.3318 \\
86.8648 \\
87.3978 \\
87.9308 \\
88.4638 \\
88.9967 \\
89.5297 \\
90.0627 \\
90.5956\end{array}$ & $\begin{array}{r}997.72 \\
1005.52 \\
1013.32 \\
1021.12 \\
1028.91 \\
1036.69 \\
1044.48 \\
1052.25 \\
1060.02 \\
1067.79\end{array}$ & $\begin{array}{l}680.17 \\
686.00 \\
691.83 \\
697.65 \\
703.47 \\
709.28 \\
715.09 \\
720.90 \\
726.70 \\
732.49\end{array}$ & $\begin{array}{l}13.259 \\
13.283 \\
13.307 \\
13.331 \\
13.354 \\
13.378 \\
13.401 \\
13.424 \\
13.448 \\
13.470\end{array}$ & $\begin{array}{l}462.00 \\
464.00 \\
466.00 \\
468.00 \\
470.00 \\
472.00 \\
474.00 \\
476.00 \\
478.00 \\
480.00\end{array}$ & $\begin{array}{l}123.0876 \\
123.6187 \\
124.1497 \\
124.6807 \\
125.2116 \\
125.7424 \\
126.2732 \\
126.8039 \\
127.3347 \\
127.8654\end{array}$ & $\begin{array}{l}1526.17 \\
1533.44 \\
1540.70 \\
1547.96 \\
1555.21 \\
1562.45 \\
1569.69 \\
1576.92 \\
1584.14 \\
1591.36\end{array}$ & $\begin{array}{l}1070.61 \\
1075.92 \\
1081.22 \\
1086.51 \\
1091.79 \\
1097.07 \\
1102.34 \\
1107.61 \\
1112.87 \\
1118.13\end{array}$ & $\begin{array}{l}14.624 \\
14.640 \\
14.656 \\
14.671 \\
14.687 \\
14.702 \\
14.717 \\
14.733 \\
14.748 \\
14.763\end{array}$ \\
\hline $\begin{array}{l}342.00 \\
344.00 \\
346.00 \\
348.00 \\
350.00 \\
352.00 \\
354.00 \\
356.00 \\
358.00 \\
360.00\end{array}$ & $\begin{array}{l}91.1286 \\
91.6615 \\
92.1945 \\
92.7274 \\
93.2603 \\
93.7933 \\
94.3262 \\
94.8591 \\
95.3920 \\
95.9249\end{array}$ & $\begin{array}{l}1075.55 \\
1083.30 \\
1091.05 \\
1098.79 \\
11106.52 \\
11114.24 \\
11121.96 \\
11129.67 \\
11137.38 \\
11145.07\end{array}$ & $\begin{array}{l}738.28 \\
744.06 \\
749.83 \\
755.60 \\
761.36 \\
767.11 \\
772.86 \\
778.59 \\
784.33 \\
790.05\end{array}$ & $\begin{array}{l}13.493 \\
13.516 \\
13.538 \\
13.561 \\
13.583 \\
13.605 \\
13.627 \\
13.648 \\
13.670 \\
13.691\end{array}$ & $\begin{array}{l}482.00 \\
484.00 \\
486.00 \\
488.00 \\
490.00 \\
492.00 \\
494.00 \\
496.00 \\
498.00 \\
500.00\end{array}$ & $\begin{array}{l}128.3962 \\
128.9270 \\
129.4579 \\
129.9888 \\
130.5199 \\
131.0510 \\
131.5823 \\
132.1138 \\
132.6454 \\
133.1772\end{array}$ & $\begin{array}{l}1598.58 \\
1605.78 \\
1612.99 \\
1620.18 \\
1627.38 \\
1634.57 \\
1641.75 \\
1648.93 \\
1656.11 \\
1663.28\end{array}$ & $\begin{array}{l}1123.37 \\
1128.62 \\
11133.86 \\
1139.09 \\
1144.32 \\
11149.54 \\
11154.76 \\
1159.97 \\
11165.18 \\
11170.38\end{array}$ & $\begin{array}{l}14.778 \\
14.793 \\
14.808 \\
14.822 \\
14.837 \\
14.852 \\
14.866 \\
14.881 \\
14.895 \\
14.910\end{array}$ \\
\hline $\begin{array}{l}362.00 \\
364.00 \\
366.00 \\
368.00 \\
370.00 \\
372.00 \\
374.00 \\
376.00 \\
378.00 \\
380.00\end{array}$ & $\begin{array}{r}96.4577 \\
96.9904 \\
97.5231 \\
98.0558 \\
98.5885 \\
99.1212 \\
99.6540 \\
100.1867 \\
100.7194 \\
101.2521\end{array}$ & $\begin{array}{l}1152.75 \\
1160.43 \\
1168.10 \\
1175.75 \\
1183.40 \\
1191.05 \\
1198.68 \\
1206.30 \\
1213.92 \\
1221.53\end{array}$ & $\begin{array}{l}795.76 \\
801.46 \\
807.16 \\
812.84 \\
818.52 \\
824.19 \\
829.85 \\
835.51 \\
841.15 \\
846.79\end{array}$ & $\begin{array}{l}13.713 \\
13.734 \\
13.755 \\
13.776 \\
13.796 \\
13.817 \\
13.837 \\
13.858 \\
13.878 \\
13.898\end{array}$ & $\begin{array}{l}502.00 \\
504.00 \\
506.00 \\
508.00 \\
510.00 \\
512.00 \\
514.00 \\
516.00 \\
518.00 \\
520.00\end{array}$ & $\begin{array}{l}133.7092 \\
134.2415 \\
134.7740 \\
135.3067 \\
135.8397 \\
136.3730 \\
136.9065 \\
137.4404 \\
137.9745 \\
138.5089\end{array}$ & $\begin{array}{l}1670.45 \\
1677.61 \\
1684.78 \\
1691.94 \\
1699.09 \\
1706.25 \\
1713.40 \\
1720.55 \\
1727.69 \\
1734.84\end{array}$ & $\begin{array}{l}1175.58 \\
1180.78 \\
1185.97 \\
1191.16 \\
1196.34 \\
1201.52 \\
1206.70 \\
1211.87 \\
1217.04 \\
1222.21\end{array}$ & $\begin{array}{l}14.924 \\
14.938 \\
14.952 \\
14.967 \\
14.981 \\
14.995 \\
15.009 \\
15.022 \\
15.036 \\
15.050\end{array}$ \\
\hline $\begin{array}{l}382.00 \\
384.00 \\
386.00 \\
388.00 \\
390.00 \\
392.00 \\
394.00 \\
396.00 \\
398.00 \\
400.00\end{array}$ & $\begin{array}{l}101.7849 \\
102.3176 \\
102.8504 \\
103.3831 \\
103.9159 \\
104.4487 \\
104.9815 \\
105.5144 \\
106.0472 \\
106.5801\end{array}$ & $\begin{array}{l}1229.13 \\
1236.72 \\
1244.30 \\
1251.87 \\
1259.44 \\
1266.99 \\
1274.54 \\
1282.08 \\
1289.61 \\
1297.13\end{array}$ & $\begin{array}{l}852.42 \\
858.03 \\
863.64 \\
869.24 \\
874.84 \\
880.42 \\
886.00 \\
891.56 \\
897.12 \\
902.67\end{array}$ & $\begin{array}{l}13.918 \\
13.938 \\
13.957 \\
13.977 \\
13.996 \\
14.016 \\
14.035 \\
14.054 \\
14.073 \\
14.092\end{array}$ & $\begin{array}{l}522.00 \\
524.00 \\
526.00 \\
528.00 \\
530.00 \\
532.00 \\
534.00 \\
536.00 \\
538.00 \\
540.00\end{array}$ & $\begin{array}{l}139.0435 \\
139.5784 \\
140.1136 \\
140.6490 \\
141.1846 \\
141.7204 \\
142.2563 \\
142.7923 \\
143.3284 \\
143.8645\end{array}$ & $\begin{array}{l}1741.98 \\
1749.12 \\
1756.25 \\
1763.39 \\
1770.52 \\
1777.64 \\
1784.77 \\
1791.89 \\
1799.01 \\
1806.12\end{array}$ & $\begin{array}{l}1227.37 \\
1232.53 \\
1237.68 \\
1242.84 \\
1247.98 \\
1253.13 \\
1258.27 \\
1263.41 \\
1268.54 \\
1273.67\end{array}$ & $\begin{array}{l}15.064 \\
15.077 \\
15.091 \\
15.105 \\
15.118 \\
15.131 \\
15.145 \\
15.158 \\
15.171 \\
15.185\end{array}$ \\
\hline
\end{tabular}


TEMPER- SPECIFIC ENTHALPY INTERNAL ENTROPY ATURE VOLUME (BTU/L8) ENERGY (8TU/L8-R)

(R) (CU FT/LB)
(BTU/LB)
TEMPER- SPECIFIC ENTHALPY INTERNAL ENTROPY ATURE VOLUME (BTU/L8) ENERGY (8TU/L8-R) (R) (CU FT/L8)
(8TU/L8)

$\begin{array}{lrrrrl}36.00 & .2248 & -110.59 & -111.84 & 1.879 & 170.00 \\ 38.00 & .2291 & -105.88 & -107.15 & 2.006 & 174.00 \\ 40.00 & .2339 & -100.81 & -102.10 & 2.136 & 176.00 \\ * 41.291 & .2374 & -97.32 & -99.97 & 2.222 & 178.00 \\ * 41.291 & 6.2031 & 86.78 & 52.34 & 6.682 & 180.00 \\ 42.00 & 6.3707 & 89.18 & 53.82 & 6.740 & 182.00 \\ 44.00 & 6.8244 & 95.59 & 57.70 & 6.889 & 184.00 \\ 46.00 & 7.2584 & 101.61 & 61.32 & 7.023 & 186.00 \\ 48.00 & 7.6792 & 107.40 & 64.77 & 7.146 & 188.00 \\ 50.00 & 8.0906 & 113.04 & 68.12 & 7.261 & 190.00 \\ 52.00 & 8.4952 & 118.56 & 71.39 & 7.369 & 192.00 \\ 54.00 & 8.8944 & 124.00 & 74.62 & 7.472 & 194.00 \\ 56.00 & 9.2892 & 129.38 & 77.81 & 7.570 & 196.00 \\ 58.00 & 9.6805 & 134.72 & 80.97 & 7.663 & 198.00 \\ 60.00 & 10.0688 & 140.01 & 84.12 & 7.753 & 200.00 \\ & & & & & \\ 62.00 & 10.4545 & 145.28 & 87.25 & 7.840 & 202.00 \\ 64.00 & 10.8379 & 150.53 & 90.36 & 7.923 & 204.00 \\ 66.00 & 11.2193 & 155.75 & 93.47 & 8.003 & 206.00 \\ 68.00 & 11.5990 & 160.96 & 96.56 & 8.081 & 208.00 \\ 70.00 & 11.9771 & 166.15 & 99.65 & 8.156 & 210.00 \\ 72.00 & 12.3537 & 171.32 & 102.74 & 8.229 & 212.00 \\ 74.00 & 12.7290 & 176.48 & 105.82 & 8.300 & 214.00 \\ 76.00 & 13.1031 & 181.64 & 108.89 & 8.368 & 216.00 \\ 78.00 & 13.4762 & 186.78 & 111.97 & 8.435 & 218.00 \\ 80.00 & 13.8482 & 191.92 & 115.04 & 8.500 & 220.00 \\ 82.00 & 14.2193 & 197.05 & 118.11 & 8.564 & 222.00 \\ 84.00 & 14.5895 & 202.18 & 121.18 & 8.625 & 224.00 \\ 86.00 & 14.9590 & 207.30 & 124.25 & 8.686 & 226.00 \\ 88.00 & 15.3277 & 212.42 & 127.33 & 8.745 & 228.00 \\ 90.00 & 15.6957 & 217.54 & 130.40 & 8.802 & 230.00 \\ 92.00 & 16.0631 & 222.66 & 133.49 & 8.858 & 232.00 \\ 94.00 & 16.4299 & 227.78 & 136.57 & 8.913 & 234.00 \\ 96.00 & 16.7962 & 232.91 & 139.66 & 8.967 & 236.00 \\ 98.00 & 17.1619 & 238.04 & 142.77 & 9.020 & 238.00 \\ 100.00 & 17.5272 & 243.18 & 145.88 & 9.072 & 240.00 \\ & & & & & \\ 102.00 & 17.8920 & 248.32 & 148.99 & 9.123 & 242.00 \\ 104.00 & 18.2563 & 253.47 & 152.12 & 9.173 & 244.00 \\ 106.00 & 18.6203 & 258.64 & 155.26 & 9.222 & 246.00 \\ 108.00 & 18.9839 & 263.81 & 158.42 & 9.271 & 248.00 \\ 110.00 & 19.3471 & 269.00 & 161.59 & 9.318 & 250.00 \\ 112.00 & 19.7100 & 274.20 & 164.78 & 9.365 & 252.00 \\ 114.00 & 20.0726 & 279.42 & 167.98 & 9.411 & 254.00 \\ 116.00 & 20.4349 & 284.65 & 171.21 & 9.457 & 256.00 \\ 118.00 & 20.7969 & 289.91 & 174.45 & 9.502 & 258.00 \\ 120.00 & 21.1586 & 295.18 & 177.72 & 9.546 & 260.00 \\ & & & & & \end{array}$

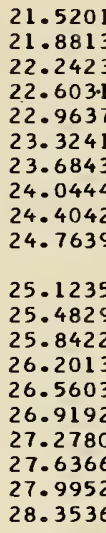

28.7120

29.0702

29.4284

29.7865

30.1445

30.5024

30.8602

31.2180

31.5757

31.9333

32.2908

32.6481

33.0054

33.3626

33.7198

34.0769

34.4339

34.7909

35.1478

35.5047

35.8615

36.2183

36.5750

36.9316

37.2883

37.6448

38.0014

38.3579

38.7144

39.0708

39.4272

39.7836

40.1399

40.4963

40.8526

41.2088

41.5651

41.9213

42.2775

42.6337

42.9899

43.3461

43.7022

44.0584

44.4145

44.7706

45.1266

45.4827

45.8387

46.1947

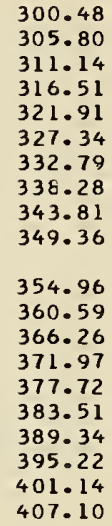

413.11

419.16

425.26

431.41

437.60

443.83

450.12

456.44

462.81

469.22

475.68

482.17

488.72

495.30

501.93

508.60

515.32

522.08

528.88

535.72

542.60

549.52

556.48

563.48

570.51

577.58

584.69

591.84

599.01

606.21

613.45

620.72

628.02

635.36

642.72

650.10

657.52

664.96

672.43

679.92

687.44

694.98

702.54

710.12

717.73

725.35

32.99

740.66

748.33

756.03

$\begin{array}{ll}181.01 & 9.590 \\ 184.32 & 9.633 \\ 187.66 & 9.676 \\ 191.03 & 9.718 \\ 194.42 & 9.760 \\ 197.85 & 9.801 \\ 201.31 & 9.842 \\ 204.80 & 9.883 \\ 208.32 & 9.923 \\ 211.89 & 9.963 \\ 215.48 & 10.003 \\ 219.12 & 10.042 \\ 222.79 & 10.082 \\ 226.51 & 10.120 \\ 230.26 & 10.159 \\ 234.06 & 10.197 \\ 237.90 & 10.235 \\ 241.79 & 10.273 \\ 245.72 & 10.311 \\ 249.69 & 10.349\end{array}$

$253.71 \quad 10.386$ $257.78 \quad 10.423$

$261.89 \quad 10.460$

$\begin{array}{ll}266.05 & 10.497\end{array}$

$270.25 \quad 10.533$

$274.50 \quad 10.570$

$278.79 \quad 10.606$

$283.13 \quad 10.642$

$287.52 \quad 10.678$

$291.94 \quad 10.714$

$296.41 \quad 10.750$

$300.93 \quad 10.785$

$305.48 \quad 10.821$

$310.09 \quad 10.856$

$314.73 \quad 10.891$

$319.42 \quad 10.926$

$324.16 \quad 10.961$

$328.93 \quad 10.995$

$333.75 \quad 11.030$

$338.61 \quad 11.064$

$343.51 \quad 11.098$

$348.45 \quad 11.133$

$353.43 \quad 11.167$

$358.45 \quad 11.200$

$363.50 \quad 11.234$

$368.59 \quad 11.267$

$373.72 \quad 11.301$

$378.89 \quad 11.334$

$384.08 \quad 11.367$

$389.31 \quad 11.400$

394.57

399.86

405.18

410.54

415.92

421.33

426.77

432.23

437.72

443.24

11.433

11.465

11.498

11.530

11.562

11.594

11.626

11.658

11.689

$448.78 \quad 11.752$

$454.34 \quad 11.783$

$459.92 \quad 11.814$

$465.53 \quad 11.844$

$471.16 \quad 11.875$

$476.80 \quad 11.905$

$482.47 \quad 11.936$

$488.15 \quad 11.966$

$493.86 \quad 11.995$

$499.57 \quad 12.025$ 
TEMPER- SPECIFIC ENTHALPY INTERNAL ENTROPY ATURE VOLUME (BTU/LB) ENERGY (BTU/L8-R) (R) (CU FT/LB) (BTU/L8)

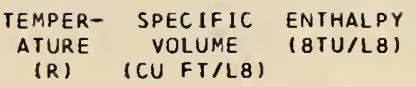

INTERNAL ENTROPY ENERGY (BTU/LB-R) (BTU/L8)

\begin{tabular}{|c|c|c|c|c|c|c|c|c|c|}
\hline $\begin{array}{l}262.00 \\
264.00 \\
266.00 \\
268.00 \\
270.00 \\
272.00 \\
274.00 \\
276.00 \\
278.00 \\
280.00\end{array}$ & $\begin{array}{l}46.5508 \\
46.9067 \\
47.2627 \\
47.6187 \\
47.9746 \\
48.3305 \\
48.6864 \\
49.0423 \\
49.3982 \\
49.7540\end{array}$ & $\begin{array}{l}763.74 \\
771.46 \\
779.20 \\
786.95 \\
794.71 \\
802.46 \\
810.22 \\
817.98 \\
825.76 \\
833.54\end{array}$ & $\begin{array}{l}505.31 \\
511.05 \\
516.81 \\
522.59 \\
528.37 \\
534.15 \\
539.93 \\
545.72 \\
551.52 \\
557.32\end{array}$ & $\begin{array}{l}12.055 \\
12.084 \\
12.113 \\
12.142 \\
12.171 \\
12.200 \\
12.228 \\
12.256 \\
12.284 \\
12.312\end{array}$ & $\begin{array}{l}402.00 \\
404.00 \\
406.00 \\
408.00 \\
410.00 \\
412.00 \\
414.00 \\
416.00 \\
418.00 \\
420.00\end{array}$ & $\begin{array}{l}71.4398 \\
71.7951 \\
72.1505 \\
72.5059 \\
72.8613 \\
73.2167 \\
73.5721 \\
73.9277 \\
74.2832 \\
74.6388\end{array}$ & $\begin{array}{l}1304.64 \\
1312.15 \\
1319.65 \\
1327.13 \\
1334.61 \\
1342.08 \\
1349.55 \\
1357.00 \\
1364.45 \\
1371.89\end{array}$ & $\begin{array}{l}908.04 \\
913.57 \\
919.10 \\
924.61 \\
930.12 \\
935.61 \\
941.10 \\
946.59 \\
952.06 \\
957.53\end{array}$ & $\begin{array}{l}13.711 \\
13.729 \\
13.748 \\
13.766 \\
13.784 \\
13.803 \\
13.821 \\
13.839 \\
13.856 \\
13.874\end{array}$ \\
\hline $\begin{array}{l}282.00 \\
284.00 \\
286.00 \\
288.00 \\
290.00 \\
292.00 \\
294.00 \\
296.00 \\
298.00 \\
300.00\end{array}$ & $\begin{array}{l}50.1099 \\
50.4657 \\
50.8215 \\
51.1773 \\
51.5330 \\
51.8888 \\
52.2445 \\
52.6002 \\
52.9559 \\
53.3116\end{array}$ & $\begin{array}{l}841.32 \\
849.12 \\
856.92 \\
864.72 \\
872.53 \\
880.34 \\
888.16 \\
895.98 \\
903.80 \\
911.62\end{array}$ & $\begin{array}{l}563.13 \\
568.95 \\
574.78 \\
580.61 \\
586.44 \\
592.28 \\
598.12 \\
603.96 \\
609.81 \\
615.65\end{array}$ & $\begin{array}{l}12.340 \\
12.368 \\
12.395 \\
12.422 \\
12.449 \\
12.476 \\
12.503 \\
12.529 \\
12.556 \\
12.582\end{array}$ & $\begin{array}{l}422.00 \\
424.00 \\
426.00 \\
428.00 \\
430.00 \\
432.00 \\
434.00 \\
436.00 \\
438.00 \\
440.00\end{array}$ & $\begin{array}{l}74.9943 \\
75.3498 \\
75.7052 \\
76.0606 \\
76.4160 \\
76.7713 \\
77.1265 \\
77.4817 \\
77.8368 \\
78.1919\end{array}$ & $\begin{array}{l}1379.32 \\
1386.74 \\
1394.15 \\
1401.56 \\
1408.95 \\
1416.34 \\
1423.72 \\
1431.09 \\
1438.45 \\
1445.80\end{array}$ & $\begin{array}{r}962.98 \\
968.43 \\
973.87 \\
979.30 \\
984.72 \\
990.14 \\
995.54 \\
1000.94 \\
1006.33 \\
1011.71\end{array}$ & $\begin{array}{l}13.892 \\
13.909 \\
13.927 \\
13.944 \\
13.961 \\
13.979 \\
13.996 \\
14.013 \\
14.029 \\
14.046\end{array}$ \\
\hline $\begin{array}{l}302.00 \\
304.00 \\
306.00 \\
308.00 \\
310.00 \\
312.00 \\
314.00 \\
316.00 \\
318.00 \\
320.00\end{array}$ & $\begin{array}{l}53.6673 \\
54.0229 \\
54.3786 \\
54.7342 \\
55.0898 \\
55.4454 \\
55.8010 \\
56.1566 \\
56.5122 \\
56.8678\end{array}$ & $\begin{array}{l}919.44 \\
927.26 \\
935.09 \\
942.91 \\
950.73 \\
958.56 \\
966.38 \\
974.19 \\
982.01 \\
989.82\end{array}$ & $\begin{array}{l}621.50 \\
627.35 \\
633.20 \\
639.05 \\
644.90 \\
650.75 \\
656.59 \\
662.44 \\
668.28 \\
674.12\end{array}$ & $\begin{array}{l}12.608 \\
12.634 \\
12.659 \\
12.685 \\
12.710 \\
12.735 \\
12.760 \\
12.785 \\
12.810 \\
12.834\end{array}$ & $\begin{array}{l}442.00 \\
444.00 \\
446.00 \\
448.00 \\
450.00 \\
452.00 \\
454.00 \\
456.00 \\
458.00 \\
460.00\end{array}$ & $\begin{array}{l}78.5469 \\
78.9018 \\
79.2567 \\
79.6114 \\
79.9661 \\
80.3207 \\
80.6753 \\
81.0297 \\
81.3841 \\
81.7384\end{array}$ & $\begin{array}{l}1453.14 \\
1460.47 \\
1467.80 \\
1475.12 \\
1482.43 \\
1489.75 \\
1497.06 \\
1504.36 \\
1511.66 \\
1518.94\end{array}$ & $\begin{array}{l}1017.08 \\
1022.44 \\
1027.80 \\
1033.15 \\
1038.49 \\
1043.84 \\
1049.18 \\
1054.52 \\
1059.84 \\
1065.17\end{array}$ & $\begin{array}{l}14.063 \\
14.079 \\
14.096 \\
14.112 \\
14.128 \\
14.145 \\
14.161 \\
14.177 \\
14.193 \\
14.209\end{array}$ \\
\hline $\begin{array}{l}322.00 \\
324.00 \\
326.00 \\
328.00 \\
330.00 \\
332.00 \\
334.00 \\
336.00 \\
338.00 \\
340.00\end{array}$ & $\begin{array}{l}57.2233 \\
57.5789 \\
57.9344 \\
58.2900 \\
58.6455 \\
59.0010 \\
59.3566 \\
59.7121 \\
60.0676 \\
60.4231\end{array}$ & $\begin{array}{r}997.63 \\
1005.44 \\
1013.24 \\
1021.04 \\
1028.84 \\
1036.63 \\
1044.41 \\
1052.19 \\
1059.96 \\
1067.73\end{array}$ & $\begin{array}{l}679.95 \\
685.79 \\
691.61 \\
697.44 \\
703.26 \\
709.08 \\
714.89 \\
720.69 \\
726.49 \\
732.29\end{array}$ & $\begin{array}{l}12.858 \\
12.883 \\
12.907 \\
12.930 \\
12.954 \\
12.978 \\
13.001 \\
13.024 \\
13.047 \\
13.070\end{array}$ & $\begin{array}{l}462.00 \\
464.00 \\
466.00 \\
468.00 \\
470.00 \\
472.00 \\
474.00 \\
476.00 \\
478.00 \\
480.00\end{array}$ & $\begin{array}{l}82.0926 \\
82.4468 \\
82.8009 \\
83.1550 \\
83.5090 \\
83.8630 \\
84.2170 \\
84.5709 \\
84.9248 \\
85.2788\end{array}$ & $\begin{array}{l}1526.22 \\
1533.50 \\
1540.76 \\
1548.02 \\
1555.27 \\
1562.52 \\
1569.75 \\
1576.99 \\
1584.21 \\
1591.43\end{array}$ & $\begin{array}{l}1070.48 \\
1075.78 \\
1081.08 \\
1086.38 \\
1091.66 \\
1096.94 \\
1102.22 \\
1107.48 \\
1112.74 \\
1118.00\end{array}$ & $\begin{array}{l}14.225 \\
14.240 \\
14.256 \\
14.271 \\
14.287 \\
14.302 \\
14.318 \\
14.333 \\
14.348 \\
14.363\end{array}$ \\
\hline $\begin{array}{l}342.00 \\
344.00 \\
346.00 \\
348.00 \\
350.00 \\
352.00 \\
354.00 \\
356.00 \\
358.00 \\
360.00\end{array}$ & $\begin{array}{l}60.7786 \\
61.1341 \\
61.4896 \\
61.8450 \\
62.2005 \\
62.5560 \\
62.9115 \\
63.2669 \\
63.6224 \\
63.9778\end{array}$ & $\begin{array}{l}1075.49 \\
1083.25 \\
1091.00 \\
1098.74 \\
1106.47 \\
1114.20 \\
1121.92 \\
1129.63 \\
1137.34 \\
1145.04\end{array}$ & $\begin{array}{l}738.07 \\
743.86 \\
749.63 \\
755.40 \\
761.16 \\
766.92 \\
772.66 \\
778.40 \\
784.13 \\
789.86\end{array}$ & $\begin{array}{l}13.093 \\
13.116 \\
13.138 \\
13.160 \\
13.183 \\
13.205 \\
13.226 \\
13.248 \\
13.270 \\
13.291\end{array}$ & $\begin{array}{l}482.00 \\
484.00 \\
486.00 \\
488.00 \\
490.00 \\
492.00 \\
494.00 \\
496.00 \\
498.00 \\
500.00\end{array}$ & $\begin{array}{l}85.6327 \\
85.9867 \\
86.3407 \\
86.6948 \\
87.0489 \\
87.4031 \\
87.7574 \\
88.1118 \\
88.4663 \\
88.8209\end{array}$ & $\begin{array}{l}1598.65 \\
1605.86 \\
1613.06 \\
1620.26 \\
1627.45 \\
1634.64 \\
1641.83 \\
1649.01 \\
1656.19 \\
1663.36\end{array}$ & $\begin{array}{l}1123.25 \\
1128.49 \\
1133.73 \\
1138.97 \\
1144.19 \\
1149.42 \\
1154.64 \\
1159.85 \\
1165.06 \\
1170.26\end{array}$ & $\begin{array}{l}14.378 \\
14.393 \\
14.408 \\
14.423 \\
14.437 \\
14.452 \\
14.466 \\
14.481 \\
14.495 \\
14.510\end{array}$ \\
\hline $\begin{array}{l}362.00 \\
364.000 \\
366.00 \\
368.00 \\
370.00 \\
372.00 \\
374.00 \\
376.00 \\
378.00 \\
380.00\end{array}$ & $\begin{array}{l}64.3332 \\
64.6885 \\
65.0438 \\
65.3991 \\
65.7544 \\
66.1097 \\
66.4651 \\
66.8204 \\
67.1757 \\
67.5310\end{array}$ & $\begin{array}{l}1152.72 \\
1160.40 \\
1168.07 \\
1175.73 \\
1183.38 \\
1191.02 \\
1198.66 \\
1206.29 \\
1213.90 \\
1221.51\end{array}$ & $\begin{array}{l}795.57 \\
801.27 \\
806.97 \\
812.66 \\
818.34 \\
824.01 \\
829.67 \\
835.33 \\
840.97 \\
846.61\end{array}$ & $\begin{array}{l}13.312 \\
13.334 \\
13.355 \\
13.375 \\
13.396 \\
13.417 \\
13.437 \\
13.458 \\
13.478 \\
13.498\end{array}$ & $\begin{array}{l}502.00 \\
504.00 \\
506.00 \\
508.00 \\
510.00 \\
512.00 \\
514.00 \\
516.00 \\
518.00 \\
520.00\end{array}$ & $\begin{array}{l}89.1757 \\
89.5306 \\
89.8857 \\
90.2409 \\
90.5964 \\
90.9520 \\
91.3077 \\
91.6637 \\
92.0199 \\
92.3762\end{array}$ & $\begin{array}{l}1670.53 \\
1677.70 \\
1684.86 \\
1692.02 \\
1699.18 \\
1706.34 \\
1713.49 \\
1720.64 \\
1727.78 \\
1734.93\end{array}$ & $\begin{array}{l}1175.46 \\
1180.66 \\
1185.85 \\
1191.04 \\
1196.23 \\
1201.41 \\
1206.58 \\
1211.76 \\
1216.93 \\
1222.09\end{array}$ & $\begin{array}{l}14.524 \\
14.538 \\
14.553 \\
14.567 \\
14.581 \\
14.595 \\
14.609 \\
14.623 \\
14.636 \\
14.650\end{array}$ \\
\hline $\begin{array}{l}382.00 \\
384.00 \\
386.00 \\
388.00 \\
390.00 \\
392.00 \\
394.00 \\
396.00 \\
398.00 \\
400.00\end{array}$ & $\begin{array}{l}67.8863 \\
68.2416 \\
68.5969 \\
68.9523 \\
69.3076 \\
69.6629 \\
70.0183 \\
70.3736 \\
70.7290 \\
71.0844\end{array}$ & $\begin{array}{l}1229.11 \\
1236.71 \\
1244.29 \\
1251.86 \\
1259.43 \\
1266.99 \\
1274.54 \\
1282.08 \\
1289.61 \\
1297.13\end{array}$ & $\begin{array}{l}852.24 \\
857.86 \\
863.47 \\
869.07 \\
874.66 \\
880.25 \\
885.82 \\
891.39 \\
896.95 \\
902.50\end{array}$ & $\begin{array}{l}13.518 \\
13.538 \\
13.557 \\
13.577 \\
13.596 \\
13.616 \\
13.635 \\
13.654 \\
13.673 \\
13.692\end{array}$ & $\begin{array}{l}522.00 \\
524.00 \\
526.00 \\
528.00 \\
530.00 \\
532.00 \\
534.00 \\
536.00 \\
538.00 \\
540.00\end{array}$ & $\begin{array}{l}92.7327 \\
93.0894 \\
93.4462 \\
93.8032 \\
94.1604 \\
94.5176 \\
94.8749 \\
95.2323 \\
95.5898 \\
95.9472\end{array}$ & $\begin{array}{l}1742.07 \\
1749.21 \\
1756.35 \\
1763.48 \\
1770.61 \\
1777.74 \\
1784.86 \\
1791.98 \\
1799.10 \\
1806.22\end{array}$ & $\begin{array}{l}1227.26 \\
1232.41 \\
1237.57 \\
1242.72 \\
1247.87 \\
1253.02 \\
1258.16 \\
1263.29 \\
1268.43 \\
1273.56\end{array}$ & $\begin{array}{l}14.664 \\
14.678 \\
14.691 \\
14.705 \\
14.718 \\
14.732 \\
14.745 \\
14.758 \\
14.771 \\
14.785\end{array}$ \\
\hline
\end{tabular}



$\begin{array}{ccccc}\text { TEMPER- } & \text { SPECIFIC } & \text { ENTHALPY } & \text { INTERNAL ENTROPY } \\ \text { ATURE } & \text { VOLUME } & \text { (8TU/L8) ENERGY } & \text { (8TU/LB-R) }\end{array}$ (R) (CU FT/L8)

\section{( 8 TU/LE)} ATURE

(R)
SPECIFIC VOLUME
ENTHALPY (BTU/L8) )
INTERNAL ENTROPY ENERGY (BTU/L8-R) (8TU/L8)

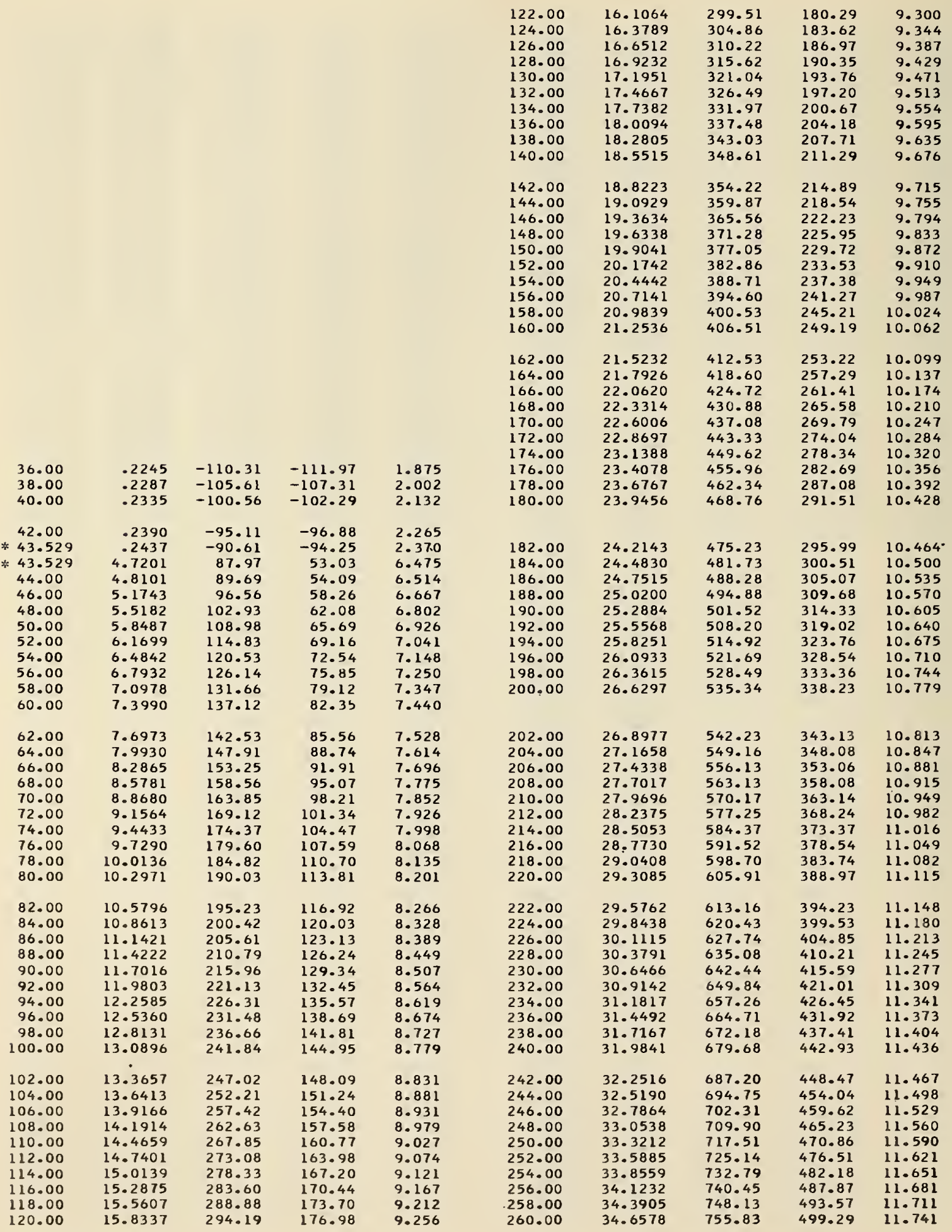


TEMPER- SPECIFIC ENTHALPY INTERNAL ENTROPY

ATURE VOLUME (BTU/L 8 ) ENERGY (BTU/LB-R) (R) (CU FT/LB)
(8TU/L8)
TEMPER- SPECIFIC ENTHALPY

ATURE VDLUME (8TU/L8) (R) (CU FT/LB)
INTERNAL ENTROPY

ENERGY (8TU/L8-R) (8TU/L8)

\begin{tabular}{|c|c|c|c|c|c|c|c|c|c|}
\hline $\begin{array}{l}262.00 \\
264.00 \\
266.00 \\
268.00 \\
270.00 \\
272.00 \\
274.00 \\
276.00 \\
278.00 \\
280.00\end{array}$ & $\begin{array}{l}34.9250 \\
35.1923 \\
35.4595 \\
35.7268 \\
35.9940 \\
36.2612 \\
36.5283 \\
36.7955 \\
37.0626 \\
37.3297\end{array}$ & $\begin{array}{l}763.55 \\
771.27 \\
779.02 \\
786.77 \\
794.54 \\
802.29 \\
810.05 \\
817.82 \\
825.60 \\
833.38\end{array}$ & $\begin{array}{l}505.03 \\
510.78 \\
516.54 \\
522.32 \\
528.11 \\
533.88 \\
539.66 \\
545.46 \\
551.26 \\
557.06\end{array}$ & $\begin{array}{l}11.770 \\
11.799 \\
11.829 \\
11.858 \\
11.887 \\
11.915 \\
11.944 \\
11.972 \\
12.000 \\
12.028\end{array}$ & $\begin{array}{l}402.00 \\
404.00 \\
406.00 \\
408.00 \\
410.00 \\
412.00 \\
414.00 \\
416.00 \\
418.00 \\
420.00\end{array}$ & $\begin{array}{l}53.6031 \\
53.8698 \\
54.1364 \\
54.4031 \\
54.6697 \\
54.9364 \\
55.2030 \\
55.4698 \\
55.7365 \\
56.0033\end{array}$ & $\begin{array}{l}1304.65 \\
1312.16 \\
1319.66 \\
1327.15 \\
1334.63 \\
1342.10 \\
1349.57 \\
1357.02 \\
1364.47 \\
1371.91\end{array}$ & $\begin{array}{l}907.88 \\
913.41 \\
918.93 \\
924.45 \\
929.96 \\
935.46 \\
940.95 \\
946.43 \\
951.90 \\
957.37\end{array}$ & $\begin{array}{l}13.427 \\
13.445 \\
13.464 \\
13.482 \\
13.500 \\
13.519 \\
13.537 \\
13.555 \\
13.573 \\
13.590\end{array}$ \\
\hline $\begin{array}{l}282.00 \\
284.00 \\
286.00 \\
288.00 \\
290.00 \\
292.00 \\
294.00 \\
296.00 \\
298.00 \\
300.00\end{array}$ & $\begin{array}{l}37.5969 \\
37.8639 \\
38.1310 \\
38.3981 \\
38.6651 \\
38.9322 \\
39.1992 \\
39.4662 \\
39.7331 \\
40.0001\end{array}$ & $\begin{array}{l}841.17 \\
848.97 \\
856.77 \\
864.58 \\
872.39 \\
880.21 \\
888.03 \\
895.85 \\
903.67 \\
911.50\end{array}$ & $\begin{array}{l}562.88 \\
568.70 \\
574.52 \\
580.36 \\
586.19 \\
592.03 \\
597.87 \\
603.72 \\
609.56 \\
615.41\end{array}$ & $\begin{array}{l}12.056 \\
12.083 \\
12.111 \\
12.138 \\
12.165 \\
12.192 \\
12.218 \\
12.245 \\
12.271 \\
12.297\end{array}$ & $\begin{array}{l}422.00 \\
424.00 \\
426.00 \\
428.00 \\
430.00 \\
432.00 \\
434.00 \\
436.00 \\
438.00 \\
440.00\end{array}$ & $\begin{array}{l}56.2700 \\
56.5367 \\
56.8033 \\
57.0700 \\
57.3366 \\
57.6032 \\
57.8697 \\
58.1362 \\
58.4026 \\
58.6690\end{array}$ & $\begin{array}{l}1379.34 \\
1386.77 \\
1394.18 \\
1401.59 \\
1408.98 \\
1416.37 \\
1423.75 \\
1431.12 \\
1438.48 \\
1445.83\end{array}$ & $\begin{array}{r}962.83 \\
968.28 \\
973.72 \\
979.15 \\
984.57 \\
989.99 \\
995.39 \\
1000.79 \\
1006.18 \\
1011.56\end{array}$ & $\begin{array}{l}13.608 \\
13.626 \\
13.643 \\
13.660 \\
13.678 \\
13.695 \\
13.712 \\
13.729 \\
13.746 \\
13.762\end{array}$ \\
\hline $\begin{array}{l}302.00 \\
304.00 \\
306.00 \\
308.00 \\
310.00 \\
312.00 \\
314.00 \\
316.00 \\
318.00 \\
320.00\end{array}$ & $\begin{array}{l}40.2671 \\
40.5340 \\
40.8009 \\
41.0678 \\
41.3347 \\
41.6016 \\
41.8685 \\
42.1354 \\
42.4022 \\
42.6691\end{array}$ & $\begin{array}{l}919.32 \\
927.15 \\
934.98 \\
942.80 \\
950.63 \\
958.46 \\
966.28 \\
974.10 \\
981.92 \\
989.73\end{array}$ & $\begin{array}{l}621.26 \\
627.11 \\
632.96 \\
638.82 \\
644.67 \\
650.52 \\
656.36 \\
662.21 \\
668.05 \\
673.89\end{array}$ & $\begin{array}{l}12.323 \\
12.349 \\
12.375 \\
12.400 \\
12.426 \\
12.451 \\
12.476 \\
12.501 \\
12.525 \\
12.550\end{array}$ & $\begin{array}{l}442.00 \\
444.00 \\
446.00 \\
448.00 \\
450.00 \\
452.00 \\
454.00 \\
456.00 \\
458.00 \\
460.00\end{array}$ & $\begin{array}{l}58.9353 \\
59.2016 \\
59.4678 \\
59.7340 \\
60.0001 \\
60.2661 \\
60.5321 \\
60.7980 \\
61.0639 \\
61.3297\end{array}$ & $\begin{array}{l}1453.18 \\
1460.52 \\
1467.84 \\
1475.16 \\
1482.47 \\
1489.79 \\
1497.11 \\
1504.41 \\
1511.71 \\
1519.00\end{array}$ & $\begin{array}{l}1016.93 \\
1022.30 \\
1027.66 \\
1033.00 \\
1038.35 \\
1043.70 \\
1049.04 \\
1054.38 \\
1059.70 \\
1065.03\end{array}$ & $\begin{array}{l}13.779 \\
13.795 \\
13.812 \\
13.828 \\
13.845 \\
13.861 \\
13.877 \\
13.893 \\
13.909 \\
13.925\end{array}$ \\
\hline $\begin{array}{l}322.00 \\
324.00 \\
326.00 \\
328.00 \\
330.00 \\
332.00 \\
334.00 \\
336.00 \\
338.00 \\
340.00\end{array}$ & $\begin{array}{l}42.9359 \\
43.2027 \\
43.4696 \\
43.7364 \\
44.0032 \\
44.2700 \\
44.5367 \\
44.8035 \\
45.0703 \\
45.3371\end{array}$ & $\begin{array}{r}997.55 \\
1005.36 \\
1013.16 \\
1020.96 \\
1028.76 \\
1036.55 \\
1044.34 \\
1052.12 \\
1059.90 \\
1067.67\end{array}$ & $\begin{array}{l}679.73 \\
685.57 \\
691.40 \\
697.22 \\
703.05 \\
708.86 \\
714.68 \\
720.48 \\
726.28 \\
732.08\end{array}$ & $\begin{array}{l}12.574 \\
12.598 \\
12.622 \\
12.646 \\
12.670 \\
12.693 \\
12.717 \\
12.740 \\
12.763 \\
12.786\end{array}$ & $\begin{array}{l}462.00 \\
464.00 \\
466.00 \\
468.00 \\
470.00 \\
472.00 \\
474.00 \\
476.00 \\
478.00 \\
480.00\end{array}$ & $\begin{array}{l}61.5955 \\
61.8612 \\
62.1269 \\
62.3925 \\
62.6581 \\
62.9237 \\
63.1892 \\
63.4548 \\
63.7203 \\
63.9858\end{array}$ & $\begin{array}{l}1526.28 \\
1533.55 \\
1540.82 \\
1548.08 \\
1555.33 \\
1562.58 \\
1569.82 \\
1577.05 \\
1584.28 \\
1591.50\end{array}$ & $\begin{array}{l}1070.34 \\
1075.65 \\
1080.95 \\
1086.24 \\
1091.53 \\
1096.81 \\
1102.08 \\
1107.35 \\
1112.61 \\
1117.87\end{array}$ & $\begin{array}{l}13.941 \\
13.956 \\
13.972 \\
13.988 \\
14.003 \\
14.018 \\
14.034 \\
14.049 \\
14.064 \\
14.079\end{array}$ \\
\hline $\begin{array}{l}342.00 \\
344.00 \\
346.00 \\
348.00 \\
350.00 \\
352.00 \\
354.00 \\
356.00 \\
358.00 \\
360.00\end{array}$ & $\begin{array}{l}45.6038 \\
45.8706 \\
46.1373 \\
46.4040 \\
46.6708 \\
46.9375 \\
47.2042 \\
47.4709 \\
47.7377 \\
48.0044\end{array}$ & $\begin{array}{l}1075.43 \\
1083.19 \\
1090.94 \\
1098.69 \\
1106.43 \\
1114.16 \\
1121.88 \\
1129.60 \\
1137.30 \\
1145.00\end{array}$ & $\begin{array}{l}737.87 \\
743.65 \\
749.43 \\
755.20 \\
760.96 \\
766.72 \\
772.47 \\
778.21 \\
783.94 \\
789.67\end{array}$ & $\begin{array}{l}12.809 \\
12.831 \\
12.854 \\
12.876 \\
12.898 \\
12.920 \\
12.942 \\
12.964 \\
12.986 \\
13.007\end{array}$ & $\begin{array}{l}482.00 \\
484.00 \\
486.00 \\
488.00 \\
490.00 \\
492.00 \\
494.00 \\
496.00 \\
498.00 \\
500.00\end{array}$ & $\begin{array}{l}64.2514 \\
64.5169 \\
64.7825 \\
65.0481 \\
65.3138 \\
65.5795 \\
65.8453 \\
66.1112 \\
66.3771 \\
66.6432\end{array}$ & $\begin{array}{l}1598.72 \\
1605.93 \\
1613.13 \\
1620.33 \\
1627.53 \\
1634.72 \\
1641.91 \\
1649.09 \\
1656.27 \\
1663.45\end{array}$ & $\begin{array}{l}1123.12 \\
1128.37 \\
1133.61 \\
1138.84 \\
1144.07 \\
1149.29 \\
1154.51 \\
1159.73 \\
1164.94 \\
1170.14\end{array}$ & $\begin{array}{l}14.094 \\
14.109 \\
14.124 \\
14.139 \\
14.153 \\
14.168 \\
14.183 \\
14.197 \\
14.212 \\
14.226\end{array}$ \\
\hline $\begin{array}{l}362.00 \\
364.00 \\
366.00 \\
368.00 \\
370.00 \\
372.00 \\
374.00 \\
376.00 \\
379.00 \\
380.00\end{array}$ & $\begin{array}{l}48.2710 \\
48.5376 \\
48.8042 \\
49.0708 \\
49.3374 \\
49.6040 \\
49.8706 \\
50.1372 \\
50.4038 \\
50.6704\end{array}$ & $\begin{array}{l}1152.69 \\
1160.37 \\
1168.04 \\
1175.70 \\
1183.36 \\
1191.00 \\
1198.64 \\
1206.27 \\
1213.89 \\
1221.50\end{array}$ & $\begin{array}{l}795.38 \\
801.09 \\
806.79 \\
812.48 \\
818.16 \\
823.83 \\
829.49 \\
835.15 \\
840.80 \\
846.43\end{array}$ & $\begin{array}{l}13.028 \\
13.050 \\
13.071 \\
13.091 \\
13.112 \\
13.133 \\
13.153 \\
13.174 \\
13.194 \\
13.214\end{array}$ & $\begin{array}{l}502.00 \\
504.00 \\
506.00 \\
508.00 \\
510.00 \\
512.00 \\
514.00 \\
516.00 \\
518.00 \\
520.00\end{array}$ & $\begin{array}{l}66.9093 \\
67.1755 \\
67.4419 \\
67.7084 \\
67.9750 \\
68.2417 \\
68.5086 \\
68.7756 \\
69.0427 \\
69.3100\end{array}$ & $\begin{array}{l}1670.62 \\
1677.78 \\
1684.95 \\
1692.11 \\
1699.27 \\
1706.43 \\
1713.58 \\
1720.73 \\
1727.88 \\
1735.02\end{array}$ & $\begin{array}{l}1175.35 \\
1180.54 \\
1185.74 \\
1190.93 \\
1196.11 \\
1201.29 \\
1206.47 \\
1211.64 \\
1216.81 \\
1221.98\end{array}$ & $\begin{array}{l}14.240 \\
14.255 \\
14.269 \\
14.283 \\
14.297 \\
14.311 \\
14.325 \\
14.339 \\
14.353 \\
14.366\end{array}$ \\
\hline $\begin{array}{l}382.00 \\
384.00 \\
386.00 \\
388.00 \\
390.00 \\
392.00 \\
344.00 \\
336.00 \\
338.00 \\
400.00\end{array}$ & $\begin{array}{l}50.9370 \\
51.2036 \\
51.4702 \\
51.7368 \\
52.0034 \\
52.2700 \\
32.5366 \\
52.8032 \\
53.0619 \\
53.3365\end{array}$ & $\begin{array}{l}1229.10 \\
1236.70 \\
1244.28 \\
1251.86 \\
1259.43 \\
1266.99 \\
1274.54 \\
1282.08 \\
1289.61 \\
1297.14\end{array}$ & $\begin{array}{l}852.06 \\
857.68 \\
863.30 \\
868.90 \\
874.49 \\
880.08 \\
885.66 \\
891.23 \\
896.78 \\
902.34\end{array}$ & $\begin{array}{l}13.234 \\
13.254 \\
13.273 \\
13.293 \\
13.312 \\
13.332 \\
13.351 \\
13.370 \\
13.389 \\
13.408\end{array}$ & $\begin{array}{l}522.00 \\
524.00 \\
526.00 \\
528.00 \\
530.00 \\
532.00 \\
534.00 \\
536.00 \\
538.00 \\
540.00\end{array}$ & $\begin{array}{l}69.5774 \\
69.8449 \\
70.1126 \\
70.3803 \\
70.6482 \\
70.9161 \\
71.1841 \\
71.4522 \\
71.7202 \\
71.9883\end{array}$ & $\begin{array}{l}1742.16 \\
1749.30 \\
1756.44 \\
1763.57 \\
1770.71 \\
1777.83 \\
1784.96 \\
1792.08 \\
1799.20 \\
1806.31\end{array}$ & $\begin{array}{l}1227.14 \\
1232.30 \\
1237.46 \\
1242.61 \\
1247.76 \\
1252.90 \\
1258.05 \\
1263.18 \\
1268.32 \\
1273.45\end{array}$ & $\begin{array}{l}14.380 \\
14.394 \\
14.407 \\
14.421 \\
14.434 \\
14.448 \\
14.461 \\
14.474 \\
14.488 \\
14.501\end{array}$ \\
\hline
\end{tabular}


IEMPER- SPECIFIC ENTHALPY INTERNAL ENTROPY ATURE VOLUME (BTU/L8) ENERGY (BTU/LB-R) (R) (CU FT/L8)
(8TU/LB)
TEMPER- SPECIFIC ENTHALPY ATURE VOLUME (BTU/L8) (R) (CU FT/LB)
INTERNAL ENTROPY ( $8 \mathrm{TU} / \mathrm{L} 8$ )

\begin{tabular}{|c|c|c|c|c|c|c|c|c|c|}
\hline & & & & & $\begin{array}{l}122.00 \\
124.00 \\
126.00 \\
128.00 \\
130.00 \\
132.00 \\
134.00 \\
136.00 \\
138.00 \\
140.00\end{array}$ & $\begin{array}{l}12.8585 \\
13.0777 \\
13.2967 \\
13.5155 \\
13.7341 \\
13.9525 \\
14.1707 \\
14.3887 \\
14.6065 \\
14.8242\end{array}$ & $\begin{array}{l}298.54 \\
303.92 \\
309.31 \\
314.73 \\
320.18 \\
325.65 \\
331.15 \\
336.69 \\
342.25 \\
347.85\end{array}$ & $\begin{array}{l}179.57 \\
182.91 \\
186.28 \\
189.68 \\
193.10 \\
196.55 \\
200.04 \\
203.55 \\
207.10 \\
210.69\end{array}$ & $\begin{array}{l}9.075 \\
9.118 \\
9.162 \\
9.204 \\
9.246 \\
9.288 \\
9.330 \\
9.371 \\
9.411 \\
9.451\end{array}$ \\
\hline & & & & & $\begin{array}{l}142.00 \\
144.00 \\
146.00 \\
148.00 \\
150.00 \\
152.00 \\
154.00 \\
156.00 \\
158.00 \\
160.00\end{array}$ & $\begin{array}{l}15.0418 \\
15.2591 \\
15.4764 \\
15.6935 \\
15.9105 \\
16.1274 \\
16.3441 \\
16.5608 \\
16.7773 \\
16.9937\end{array}$ & $\begin{array}{l}353.48 \\
359.15 \\
364.86 \\
370.60 \\
376.38 \\
382.21 \\
388.07 \\
393.98 \\
399.93 \\
405.92\end{array}$ & $\begin{array}{l}214.31 \\
217.96 \\
221.66 \\
225.39 \\
229.17 \\
232.99 \\
236.85 \\
240.75 \\
244.70 \\
248.69\end{array}$ & $\begin{array}{l}9.491 \\
9.531 \\
9.570 \\
9.609 \\
9.648 \\
9.687 \\
9.725 \\
9.763 \\
9.801 \\
9.839\end{array}$ \\
\hline $\begin{array}{l}36.00 \\
38.00 \\
40.00\end{array}$ & $\begin{array}{l}.2242 \\
.2284 \\
.2331\end{array}$ & $\begin{array}{l}-110.03 \\
-105.35 \\
-100.32\end{array}$ & $\begin{array}{l}-112.10 \\
-107.46 \\
-102.48\end{array}$ & $\begin{array}{l}1.872 \\
1.998 \\
2.127\end{array}$ & $\begin{array}{l}162.00 \\
164.00 \\
166.00 \\
168.00 \\
170.00 \\
172.00 \\
174.00 \\
176.00 \\
178.00 \\
180.00\end{array}$ & $\begin{array}{l}17.2100 \\
17.4263 \\
17.6424 \\
17.8585 \\
18.0744 \\
18.2903 \\
18.5061 \\
18.7219 \\
18.9376 \\
19.1532\end{array}$ & $\begin{array}{l}411.96 \\
418.04 \\
424.17 \\
430.34 \\
436.56 \\
442.82 \\
449.13 \\
455.48 \\
461.87 \\
468.30\end{array}$ & $\begin{array}{l}252.72 \\
256.81 \\
260.93 \\
265.11 \\
269.32 \\
273.59 \\
277.90 \\
282.25 \\
286.65 \\
291.08\end{array}$ & $\begin{array}{l}9.876 \\
9.914 \\
9.951 \\
9.988 \\
10.025 \\
10.061 \\
10.098 \\
10.134 \\
10.170 \\
10.206\end{array}$ \\
\hline $\begin{array}{l}42.00 \\
44.00 \\
* 45.400 \\
* 45.400 \\
46.00 \\
48.00 \\
50.00 \\
52.00 \\
54.00 \\
56.00 \\
58.00 \\
60.00\end{array}$ & $\begin{array}{l}.2385 \\
.2449 \\
.2498 \\
3.7977 \\
3.8957 \\
4.2032 \\
4.4908 \\
4.7655 \\
5.0312 \\
5.2903 \\
5.5442 \\
5.7940\end{array}$ & $\begin{array}{r}-94.89 \\
-88.99 \\
-84.51 \\
88.30 \\
90.65 \\
97.90 \\
104.55 \\
110.83 \\
116.87 \\
122.73 \\
128.48 \\
134.13\end{array}$ & $\begin{array}{r}-97.10 \\
-91.26 \\
-89.17 \\
53.17 \\
54.61 \\
59.01 \\
63.00 \\
66.74 \\
70.31 \\
73.78 \\
77.18 \\
80.52\end{array}$ & $\begin{array}{l}2.259 \\
2.397 \\
2.497 \\
6.306 \\
6.358 \\
6.512 \\
6.648 \\
6.771 \\
6.885 \\
6.991 \\
7.092 \\
7.188\end{array}$ & $\begin{array}{l}182.00 \\
184.00 \\
186.00 \\
188.00 \\
190.00 \\
192.00 \\
194.00 \\
196.00 \\
198.00 \\
200.00\end{array}$ & $\begin{array}{l}19.3686 \\
19.5840 \\
19.7993 \\
20.0145 \\
20.2297 \\
20.4448 \\
20.6599 \\
20.8749 \\
21.0898 \\
21.3047\end{array}$ & $\begin{array}{l}474.78 \\
481.29 \\
487.85 \\
494.46 \\
501.10 \\
507.79 \\
514.53 \\
521.30 \\
528.12 \\
534.97\end{array}$ & $\begin{array}{l}295.57 \\
300.09 \\
304.66 \\
309.27 \\
313.93 \\
318.62 \\
323.37 \\
328.15 \\
332.98 \\
337.85\end{array}$ & $\begin{array}{l}10.242 \\
10.277 \\
10.313 \\
10.348 \\
10.383 \\
10.418 \\
10.453 \\
10.488 \\
10.523 \\
10.557\end{array}$ \\
\hline $\begin{array}{l}62.00 \\
64.00 \\
66.00 \\
68.00 \\
70.00 \\
72.00 \\
74.00 \\
76.00 \\
78.00 \\
80.00\end{array}$ & $\begin{array}{l}6.0404 \\
6.2840 \\
6.5252 \\
6.7643 \\
7.0015 \\
7.2371 \\
7.4712 \\
7.7040 \\
7.9356 \\
8.1661\end{array}$ & $\begin{array}{l}139.70 \\
145.22 \\
150.69 \\
156.12 \\
161.52 \\
166.89 \\
172.23 \\
177.55 \\
182.85 \\
188.13\end{array}$ & $\begin{array}{r}83.81 \\
87.08 \\
90.32 \\
93.53 \\
96.74 \\
99.92 \\
103.10 \\
106.26 \\
109.42 \\
112.57\end{array}$ & $\begin{array}{l}7.279 \\
7.367 \\
7.451 \\
7.532 \\
7.610 \\
7.686 \\
7.759 \\
7.830 \\
7.899 \\
7.966\end{array}$ & $\begin{array}{l}202.00 \\
204.00 \\
206.00 \\
208.00 \\
210.00 \\
212.00 \\
214.00 \\
216.00 \\
218.00 \\
220.00\end{array}$ & $\begin{array}{l}21.5196 \\
21.7344 \\
21.9491 \\
22.1638 \\
22.3785 \\
22.5931 \\
22.8077 \\
23.0223 \\
23.2368 \\
23.4512\end{array}$ & $\begin{array}{l}541.87 \\
548.80 \\
555.78 \\
562.79 \\
569.84 \\
576.93 \\
584.05 \\
591.21 \\
598.39 \\
605.61\end{array}$ & $\begin{array}{l}342.75 \\
347.70 \\
352.69 \\
357.72 \\
362.78 \\
367.88 \\
373.02 \\
378.19 \\
383.39 \\
388.63\end{array}$ & $\begin{array}{l}10.591 \\
10.626 \\
10.660 \\
10.693 \\
10.727 \\
10.761 \\
10.794 \\
10.827 \\
10.861 \\
10.894\end{array}$ \\
\hline $\begin{array}{r}82.00 \\
84.00 \\
86.00 \\
88.00 \\
90.00 \\
92.00 \\
94.00 \\
96.00 \\
98.00 \\
100.00\end{array}$ & $\begin{array}{r}8.3956 \\
8.6242 \\
8.8519 \\
9.0789 \\
9.3051 \\
9.5307 \\
9.7556 \\
9.9800 \\
10.2039 \\
10.4272\end{array}$ & $\begin{array}{l}193.40 \\
198.66 \\
203.90 \\
209.14 \\
214.37 \\
219.60 \\
224.82 \\
230.05 \\
235.27 \\
240.49\end{array}$ & $\begin{array}{l}115.72 \\
118.86 \\
122.00 \\
125.14 \\
128.28 \\
131.42 \\
134.56 \\
137.70 \\
140.86 \\
144.01\end{array}$ & $\begin{array}{l}8.031 \\
8.094 \\
8.156 \\
8.216 \\
8.275 \\
8.332 \\
8.389 \\
8.444 \\
8.497 \\
8.550\end{array}$ & $\begin{array}{l}222.00 \\
224.00 \\
226.00 \\
228.00 \\
230.00 \\
232.00 \\
234.00 \\
236.00 \\
238.00 \\
240.00\end{array}$ & $\begin{array}{l}23.6657 \\
23.8801 \\
24.0945 \\
24.3088 \\
24.5232 \\
24.7375 \\
24.9518 \\
25.1660 \\
25.3803 \\
25.5945\end{array}$ & $\begin{array}{l}612.87 \\
620.15 \\
627.46 \\
634.80 \\
642.18 \\
649.58 \\
657.00 \\
664.46 \\
671.94 \\
679.44\end{array}$ & $\begin{array}{l}393.90 \\
399.19 \\
404.52 \\
409.88 \\
415.27 \\
420.69 \\
426.13 \\
431.61 \\
437.10 \\
442.62\end{array}$ & $\begin{array}{l}10.926 \\
10.959 \\
10.992 \\
11.024 \\
11.056 \\
11.088 \\
11.120 \\
11.152 \\
11.183 \\
11.215\end{array}$ \\
\hline $\begin{array}{l}102.00 \\
104.00 \\
106.00 \\
108.00 \\
110.00 \\
112.00 \\
114.00 \\
116.00 \\
118.00 \\
120.00\end{array}$ & $\begin{array}{l}10.6501 \\
10.8725 \\
11.0945 \\
11.3161 \\
11.5374 \\
11.7583 \\
11.9789 \\
12.1992 \\
12.4192 \\
12.6390\end{array}$ & $\begin{array}{l}245.72 \\
250.95 \\
256.19 \\
261.44 \\
266.70 \\
271.97 \\
277.25 \\
282.55 \\
287.86 \\
293.19\end{array}$ & $\begin{array}{l}147.18 \\
150.35 \\
153.54 \\
156.73 \\
159.95 \\
163.17 \\
166.41 \\
169.67 \\
172.95 \\
176.25\end{array}$ & $\begin{array}{l}8.602 \\
8.653 \\
8.703 \\
8.752 \\
8.800 \\
8.847 \\
8.894 \\
8.940 \\
8.986 \\
9.030\end{array}$ & $\begin{array}{l}242.00 \\
244.00 \\
246.00 \\
248.00 \\
250.00 \\
252.00 \\
254.00 \\
256.00 \\
258.00 \\
260.00\end{array}$ & $\begin{array}{l}25.8087 \\
26.0229 \\
26.2370 \\
26.4512 \\
26.6653 \\
26.8794 \\
27.0935 \\
27.3076 \\
27.5217 \\
27.7357\end{array}$ & $\begin{array}{l}686.97 \\
694.52 \\
702.09 \\
709.68 \\
717.30 \\
724.93 \\
732.58 \\
740.25 \\
747.94 \\
755.64\end{array}$ & $\begin{array}{l}448.17 \\
453.74 \\
459.33 \\
464.94 \\
470.57 \\
476.22 \\
481.90 \\
487.58 \\
493.29 \\
499.01\end{array}$ & $\begin{array}{l}11.246 \\
11.277 \\
11.308 \\
11.339 \\
11.369 \\
11.400 \\
11.430 \\
11.460 \\
11.490 \\
11.520\end{array}$ \\
\hline
\end{tabular}


TEMPER- SPECIFIC ENTHALPY INTERNAL ENTROPY ATURE VOLUME (BTU/L8) ENERGY (8TU/L8-R) (R) (CU FT/LB) (8TU/L8)
TEMPERATURE (R)
SPECIFIC ENTHALPY IN VOLUME (CU FT/LB)
ENTHALPY
(BTU/L8) ENERGY
[8TU/L8)

\begin{tabular}{|c|c|c|c|c|c|c|c|c|c|}
\hline & 27.9497 & $\begin{array}{l}763.36 \\
771.09\end{array}$ & 504.75 & $\begin{array}{l}11.549 \\
11.579\end{array}$ & $\begin{array}{l}402.00 \\
404.00\end{array}$ & $\begin{array}{l}42.9013 \\
43.1147\end{array}$ & $\begin{array}{l}1304.66 \\
1312.17\end{array}$ & $\begin{array}{l}907.71 \\
913.25\end{array}$ & $\begin{array}{l}13.206 \\
13.225\end{array}$ \\
\hline $\begin{array}{l}264.00 \\
266.00\end{array}$ & $\begin{array}{l}28.1037 \\
28.3777\end{array}$ & 778.84 & 516.27 & 11.608 & $\begin{array}{l}404.00 \\
406.00\end{array}$ & $\begin{array}{l}43.1147 \\
43.3281\end{array}$ & $\begin{array}{l}1312.17 \\
1319.67\end{array}$ & $\begin{array}{r}913.25 \\
918.77\end{array}$ & $\begin{array}{l}13.225 \\
13.243\end{array}$ \\
\hline 268.00 & 28.5917 & 786.60 & 522.05 & 11.637 & 408.00 & 43.5415 & 1327.16 & 924.29 & 13.262 \\
\hline 270.00 & 28.8057 & 794.37 & 527.84 & 11.666 & 410.00 & 43.7549 & 1334.65 & 929.80 & 13.280 \\
\hline 272.00 & 29.0196 & 802.12 & 533.61 & 11.694 & 412.00 & 43.9683 & 1342.12 & 935.30 & 13.298 \\
\hline 274.00 & 29.2335 & 809.89 & 539.40 & 11.723 & 414.00 & 44.1817 & 1349.58 & 940.79 & 13.316 \\
\hline 276.00 & 29.4474 & 817.66 & 545.19 & 11.751 & 416.00 & 44.3952 & 1357.04 & 946.27 & 13.334 \\
\hline 278.00 & 29.6613 & 825.44 & 551.00 & 11.779 & 418.00 & 44.6086 & 1364.49 & 951.75 & 13.352 \\
\hline 280.00 & 29.8752 & 833.23 & 556.80 & 11.807 & 420.00 & 44.8221 & 1371.94 & 957.21 & 13.370 \\
\hline 282.00 & 30.0891 & 841.02 & 562.62 & 11.835 & 422.00 & 45.0355 & 1379.37 & 962.67 & 13.388 \\
\hline 284.00 & 30.3029 & 848.83 & 568.44 & 11.862 & 424.00 & 45.2490 & 1386.79 & 968.12 & 13.405 \\
\hline 286.00 & 30.5168 & 856.63 & 574.27 & 11.890 & 426.00 & 45.4624 & 1394.21 & 973.56 & 13.423 \\
\hline 288.00 & 30.7306 & 864.44 & 580.10 & 11.917 & 428.00 & 45.6757 & 1401.62 & 979.00 & 13.440 \\
\hline 290.00 & 30.9444 & 872.26 & 585.94 & 11.944 & 430.00 & 45.8891 & 1409.01 & 984.42 & 13.457 \\
\hline 292.00 & 31.1582 & 880.08 & 591.78 & 11.971 & 432.00 & 46.1024 & 1416.40 & 989.83 & 13.474 \\
\hline 294.00 & 31.3719 & 887.90 & 597.62 & 11.998 & 434.00 & 46.3157 & 1423.78 & 995.24 & 13.491 \\
\hline 296.00 & 31.5857 & 895.72 & 603.47 & 12.024 & 436.00 & 46.5289 & 1431.16 & 1000.64 & 13.508 \\
\hline 298.00 & 31.7995 & 903.55 & 609.32 & 12.050 & 438.00 & 46.7422 & 1438.52 & 1006.03 & 13.525 \\
\hline 300.00 & 32.0132 & 911.38 & 615.17 & 12.077 & 440.00 & 46.9553 & 1445.87 & 1011.41 & 13.542 \\
\hline 302.00 & 32.2269 & 919.21 & 621.02 & 12.103 & 442.00 & 47.1685 & 1453.22 & 1016.79 & 13.559 \\
\hline 304.00 & 32.4406 & 927.04 & 626.87 & 12.128 & 444.00 & 47.3815 & 1460.56 & 1022.15 & 13.575 \\
\hline 306.00 & 32.6543 & 934.87 & 632.73 & 12.154 & 446.00 & 47.5946 & 1467.89 & 1027.51 & 13.592 \\
\hline 308.00 & 32.8680 & 942.70 & 638.58 & 12.180 & 448.00 & 47.8076 & 1475.21 & 1032.86 & 13.608 \\
\hline 310.00 & 33.0816 & 950.52 & 644.43 & 12.205 & 450.00 & 48.0205 & 1482.52 & 1038.20 & 13.624 \\
\hline 312.00 & 33.2953 & 958.35 & 650.28 & 12.230 & 452.00 & 48.2334 & 1489.84 & 1043.55 & 13.641 \\
\hline 314.00 & 33.5089 & 966.18 & 656.13 & 12.255 & 454.00 & 48.4463 & 1497.16 & 1048.90 & 13.657 \\
\hline 316.00 & 33.7226 & 974.00 & 661.98 & 12.280 & 456.00 & 48.6591 & 1504.46 & 1054.24 & 13.673 \\
\hline 318.00 & 33.9362 & 981.82 & 667.82 & 12.305 & 458.00 & 48.8718 & 1511.76 & 1059.57 & 13.689 \\
\hline 320.00 & 34.1498 & 989.64 & 673.67 & 12.329 & 460.00 & 49.0846 & 1519.05 & 1064.89 & 13.705 \\
\hline 322.00 & 34.3634 & 997.46 & 679.51 & 12.354 & 462.00 & 49.2972 & 1526.33 & 1070.20 & 13.720 \\
\hline 324.00 & 34.5770 & 1005.27 & 685.34 & 12.378 & 464.00 & 49.5099 & 1533.61 & 1075.51 & 13.736 \\
\hline 326.00 & 34.7906 & 1013.08 & 691.18 & 12.402 & 466.00 & 49.7225 & 1540.88 & 1080.81 & 13.752 \\
\hline 328.00 & 35.0042 & 1020.88 & 697.00 & 12.426 & 468.00 & 49.9350 & 1548.14 & 1086.11 & 13.767 \\
\hline 330.00 & 35.2177 & 1028.68 & 702.83 & 12.449 & 470.00 & 50.1476 & 1555.39 & 1091.40 & 13.783 \\
\hline 332.00 & 35.4313 & 1036.48 & 708.65 & 12.473 & 472.00 & 50.3601 & 1562.64 & 1096.68 & 13.798 \\
\hline 334.00 & 35.6448 & 1044.27 & 714.46 & 12.496 & 474.00 & 50.5726 & 1569.88 & 1101.95 & 13.813 \\
\hline 336.00 & 35.8584 & 1052.05 & 720.27 & 12.519 & 476.00 & 50.7851 & 1577.12 & 1107.22 & 13.829 \\
\hline 338.00 & 36.0719 & 1059.83 & 726.07 & 12.543 & 478.00 & 50.9976 & 1584.35 & 1112.49 & 13.844 \\
\hline 340.00 & 36.2854 & 1067.61 & 731.87 & 12.565 & 480.00 & 51.2101 & 1591.57 & 1117.74 & 13.859 \\
\hline 342.00 & 36.4990 & 1075.37 & 737.66 & 12.588 & 482.00 & 51.4225 & 1598.79 & 1122.99 & 13.874 \\
\hline 344.00 & 36.7125 & 1083.14 & 743.45 & 12.611 & 484.00 & 51.6350 & 1606.00 & 1128.24 & 13.889 \\
\hline 346.00 & 36.9260 & 1090.89 & 749.23 & 12.633 & 486.00 & 51.8476 & 1613.21 & 1133.48 & 13.904 \\
\hline 348.00 & 37.1395 & 1098.64 & 755.00 & 12.656 & 488.00 & 52.0601 & 1620.41 & 1138.72 & 13.919 \\
\hline 350.00 & 37.3530 & 1106.38 & 760.76 & 12.678 & 490.00 & 52.2727 & 1627.61 & 1143.95 & 13.933 \\
\hline 352.00 & 37.5665 & 1114.11 & 766.52 & 12.700 & 492.00 & 52.4853 & 1634.80 & 1149.17 & 13.948 \\
\hline 354.00 & 37.7800 & 1121.84 & 772.27 & 12.722 & 494.00 & 52.6 .980 & 1641.99 & 1154.39 & 13.962 \\
\hline 356.00 & 37.9935 & 1129.55 & 778.01 & 12.744 & 496.00 & 52.9108 & 1649.17 & 1159.61 & 13.977 \\
\hline 358.00 & 38.2069 & 1137.26 & 783.75 & 12.765 & 498.00 & 53.1236 & 1656.35 & 1164.82 & 13.991 \\
\hline 360.00 & 38.4204 & 1144.97 & 789.48 & 12.787 & 500.00 & 53.3364 & 1663.53 & 1170.03 & 14.006 \\
\hline 362.00 & 38.6338 & 1152.66 & 795.19 & 12.808 & 502.00 & 53.5494 & 1670.70 & 1175.23 & 14.020 \\
\hline 364.00 & 38.8 & 1160.34 & 800.90 & 12.829 & 504.00 & & 7.87 & 1180.43 & 14.034 \\
\hline 366.00 & 39.0606 & 1168.01 & 806.60 & 12.850 & 506.00 & 53.9755 & 1685.04 & 1185.62 & 14.049 \\
\hline 368.00 & 39.2740 & 1175.68 & 812.29 & 12.871 & 508.00 & 54.1888 & 1692.20 & 1190.81 & 14.063 \\
\hline 370.00 & 39.4873 & 1183.33 & 817.97 & 12.892 & 510.00 & 54.4021 & 1699.36 & 1196.00 & 14.077 \\
\hline 372.00 & 39.7007 & 1190.98 & 823.65 & 12.912 & 512.00 & 54.6155 & 1706.52 & 1201.18 & 14.091 \\
\hline 374.00 & 39.9141 & 1198.62 & 829.31 & 12.933 & 514.00 & 54.8291 & 1713.67 & 1206.36 & 14.105 \\
\hline 376.00 & 40.1274 & 1206.25 & 834.97 & 12.953 & 516.00 & 55.0427 & 1720.82 & 1211.53 & 14.119 \\
\hline 378.00 & 40.3408 & 1213.88 & 840.62 & 12.973 & 518.00 & 55.2564 & 1727.97 & 1216.70 & 14.132 \\
\hline 380.00 & 40.5542 & 1221.49 & 846.26 & 12.993 & 520.00 & 55.4703 & 1735.11 & 1221.87 & 14.146 \\
\hline 382.00 & 40.7675 & 1229.09 & 851.89 & 13.013 & 522.00 & 55.6842 & 1742.26 & 1227.03 & 14.160 \\
\hline 384.00 & 40.9809 & 1236.69 & 857.51 & 13.033 & 524.00 & & 1749.40 & 1232.19 & 14.174 \\
\hline 386.00 & 41.1943 & 1244.28 & 863.12 & 13.053 & 526.00 & 56.1124 & 1756.54 & 1237.35 & 14.187 \\
\hline 388.00 & 41.4076 & 1251.86 & 868.73 & 13.073 & 528.00 & 56.3266 & 1763.67 & 1242.50 & 14.201 \\
\hline 390.00 & 41.6210 & 1259.43 & 874.32 & 13.092 & 530.00 & 56.5409 & 1770.80 & 1247.65 & 14.214 \\
\hline 392.00 & 41.8344 & 1266.99 & 879.91 & 13.111 & 532.00 & 56.7553 & 1777.93 & 1252.79 & 14.228 \\
\hline 394.00 & 42.0478 & 1274.54 & 885.49 & 13.131 & 534.00 & 56.9697 & 1785.06 & 1257.94 & 14.241 \\
\hline 396.00 & 42.2612 & 1282.08 & 891.06 & 13.150 & 536.00 & 57.1842 & 1792.18 & 1263.07 & 14.254 \\
\hline 398.00 & 42.4745 & 1289.62 & 896.62 & 13.169 & 538.00 & 57.3986 & 1799.30 & 1268.21 & 14.268 \\
\hline 400.00 & 42.6879 & 1297.15 & 902.17 & 13.188 & 540.00 & 57.6131 & 1806.41 & 1273.34 & 14.281 \\
\hline
\end{tabular}


TEMPER- SPECIFIC ENTHALPY INTERNAL ENTROPY ATURE VOLUME (BTU/LB) ENERGY (BTU/L8-R) (R) (CU FT/LB)
(BTU/LO)
TEMPER- SPECIFIC ENTHALPY (R) (CU FT/LB)
INTERNAL ENTROPY (BTU/L)
(BTU/LB-R)

\begin{tabular}{|c|c|c|c|c|c|c|c|c|c|}
\hline & & & & & $\begin{array}{l}122.00 \\
124.00 \\
126.00 \\
128.00 \\
130.00 \\
132.00 \\
134.00 \\
136.00 \\
138.00 \\
140.00\end{array}$ & $\begin{array}{l}10.6933 \\
10.8771 \\
11.0606 \\
11.2439 \\
11.4269 \\
11.6098 \\
11.7925 \\
11.9750 \\
12.1574 \\
12.3396\end{array}$ & $\begin{array}{l}297.58 \\
302.98 \\
308.40 \\
313.84 \\
319.31 \\
324.81 \\
330.33 \\
335.89 \\
341.48 \\
347.09\end{array}$ & $\begin{array}{l}178.85 \\
182.21 \\
185.59 \\
189.00 \\
192.44 \\
195.90 \\
199.40 \\
202.93 \\
206.49 \\
210.09\end{array}$ & $\begin{array}{l}8.889 \\
8.933 \\
8.976 \\
9.019 \\
9.062 \\
9.104 \\
9.145 \\
9.186 \\
9.227 \\
9.268\end{array}$ \\
\hline & & & & & $\begin{array}{l}142.00 \\
144.00 \\
146.00 \\
148.00 \\
150.00 \\
152.00 \\
154.00 \\
156.00 \\
158.00 \\
160.00\end{array}$ & $\begin{array}{l}12.5216 \\
12.7035 \\
12.8852 \\
13.0668 \\
13.2483 \\
13.4296 \\
13.6109 \\
13.7920 \\
13.9730 \\
14.1540\end{array}$ & $\begin{array}{l}352.75 \\
358.43 \\
364.16 \\
369.92 \\
375.72 \\
381.56 \\
387.44 \\
393.37 \\
399.33 \\
405.34\end{array}$ & $\begin{array}{l}213.72 \\
217.39 \\
221.09 \\
224.84 \\
228.62 \\
232.45 \\
236.32 \\
240.23 \\
244.19 \\
248.19\end{array}$ & $\begin{array}{l}9.308 \\
9.347 \\
9.387 \\
9.426 \\
9.465 \\
9.504 \\
9.542 \\
9.580 \\
9.618 \\
9.656\end{array}$ \\
\hline $\begin{array}{l}36.00 \\
38.00 \\
40.00\end{array}$ & $\begin{array}{r}.2239 \\
.2281 \\
.2327\end{array}$ & $\begin{array}{l}-109.74 \\
-105.08 \\
-100.08\end{array}$ & $\begin{array}{l}-112.23 \\
-107.61 \\
-102.66\end{array}$ & $\begin{array}{l}1.868 \\
1.994 \\
2.122\end{array}$ & $\begin{array}{l}162.00 \\
164.00 \\
166.00 \\
168.00 \\
170.00 \\
172.00 \\
174.00 \\
176.00 \\
178.00 \\
180.00\end{array}$ & $\begin{array}{l}14.3348 \\
14.5155 \\
14.6961 \\
14.8767 \\
15.0571 \\
15.2375 \\
15.4178 \\
15.5981 \\
15.7782 \\
15.9583\end{array}$ & $\begin{array}{l}411.39 \\
417.49 \\
423.63 \\
429.81 \\
436.04 \\
442.32 \\
448.63 \\
454.99 \\
461.40 \\
467.84\end{array}$ & $\begin{array}{l}252.23 \\
256.32 \\
260.46 \\
264.64 \\
268.86 \\
273.13 \\
277.45 \\
281.81 \\
286.21 \\
290.66\end{array}$ & $\begin{array}{r}9.694 \\
9.731 \\
9.768 \\
9.805 \\
9.842 \\
9.879 \\
9.915 \\
9.952 \\
9.988 \\
10.024\end{array}$ \\
\hline $\begin{array}{l}42.00 \\
44.00 \\
46.00 \\
* 47.022 \\
* 47.022 \\
48.00 \\
50.00 \\
52.00 \\
54.00 \\
56.00 \\
58.00 \\
60.00\end{array}$ & $\begin{array}{l}.2381 \\
.2443 \\
.2517 \\
.2559 \\
3.1644 \\
3.3052 \\
3.5718 \\
3.8198 \\
4.0558 \\
4.2833 \\
4.5046 \\
4.7210\end{array}$ & $\begin{array}{r}-94.68 \\
-88.81 \\
-82.37 \\
-78.80 \\
88.04 \\
92.10 \\
99.62 \\
106.49 \\
112.95 \\
119.15 \\
125.15 \\
131.02\end{array}$ & $\begin{array}{r}-97.32 \\
-91.53 \\
-85.17 \\
-84.53 \\
52.91 \\
55.40 \\
59.96 \\
64.08 \\
67.92 \\
71.59 \\
75.14 \\
78.60\end{array}$ & $\begin{array}{l}2.254 \\
2.390 \\
2.533 \\
2.610 \\
6.162 \\
6.247 \\
6.401 \\
6.536 \\
6.658 \\
6.770 \\
6.876 \\
6.975\end{array}$ & $\begin{array}{l}182.00 \\
184.00 \\
186.00 \\
188.00 \\
190.00 \\
192.00 \\
194.00 \\
196.00 \\
198.00 \\
200.00\end{array}$ & $\begin{array}{l}16.1383 \\
16.3182 \\
16.4980 \\
16.6777 \\
16.8574 \\
17.0370 \\
17.2166 \\
17.3961 \\
17.5755 \\
17.7549\end{array}$ & $\begin{array}{l}474.33 \\
480.86 \\
487.43 \\
494.04 \\
500.69 \\
507.39 \\
514.13 \\
520.92 \\
527.74 \\
534.60\end{array}$ & $\begin{array}{l}295.14 \\
299.67 \\
304.25 \\
308.86 \\
313.52 \\
318.23 \\
322.97 \\
327.76 \\
332.59 \\
337.47\end{array}$ & $\begin{array}{l}10.060 \\
10.095 \\
10.131 \\
10.166 \\
10.202 \\
10.237 \\
10.272 \\
10.306 \\
10.341 \\
10.375\end{array}$ \\
\hline $\begin{array}{l}62.00 \\
64.00 \\
66.00 \\
68.00 \\
70.00 \\
72.00 \\
74.00 \\
76.00 \\
78.00 \\
80.00\end{array}$ & $\begin{array}{l}4.9335 \\
5.1429 \\
5.3495 \\
5.5539 \\
5.7563 \\
5.9569 \\
6.1559 \\
6.3535 \\
6.5499 \\
6.7452\end{array}$ & $\begin{array}{l}136.78 \\
142.46 \\
148.08 \\
153.63 \\
159.14 \\
164.62 \\
170.06 \\
175.46 \\
180.85 \\
186.21\end{array}$ & $\begin{array}{l}82.01 \\
85.36 \\
88.68 \\
91.97 \\
95.23 \\
98.48 \\
101.71 \\
104.92 \\
108.12 \\
111.32\end{array}$ & $\begin{array}{l}7.070 \\
7.160 \\
7.246 \\
7.329 \\
7.409 \\
7.486 \\
7.560 \\
7.633 \\
7.703 \\
7.770\end{array}$ & $\begin{array}{l}202.00 \\
204.00 \\
206.00 \\
208.00 \\
210.00 \\
212.00 \\
214.00 \\
216.00 \\
218.00 \\
220.00\end{array}$ & $\begin{array}{l}17.9343 \\
18.1136 \\
18.2928 \\
18.4721 \\
18.6512 \\
18.8304 \\
19.0094 \\
19.1885 \\
19.3675 \\
19.5465\end{array}$ & $\begin{array}{l}541.51 \\
548.45 \\
555.43 \\
562.45 \\
569.51 \\
576.60 \\
583.73 \\
590.90 \\
598.09 \\
605.32\end{array}$ & $\begin{array}{l}342.38 \\
347.33 \\
352.32 \\
357.35 \\
362.42 \\
367.53 \\
372.67 \\
377.84 \\
383.05 \\
388.29\end{array}$ & $\begin{array}{l}10.410 \\
10.444 \\
10.478 \\
10.512 \\
10.546 \\
10.579 \\
10.613 \\
10.646 \\
10.679 \\
10.712\end{array}$ \\
\hline $\begin{array}{l}82.00 \\
84.00 \\
86.00 \\
88.00 \\
90.00 \\
92.00 \\
94.00 \\
96.00 \\
98.00 \\
100.00\end{array}$ & $\begin{array}{l}6.9394 \\
7.1326 \\
7.3250 \\
7.5166 \\
7.7074 \\
7.8976 \\
8.0871 \\
8.2761 \\
8.4645 \\
8.6524\end{array}$ & $\begin{array}{l}191.55 \\
196.88 \\
202.19 \\
207.49 \\
212.78 \\
218.06 \\
223.33 \\
228.61 \\
233.88 \\
239.14\end{array}$ & $\begin{array}{l}114.50 \\
117.68 \\
120.86 \\
124.03 \\
127.20 \\
130.37 \\
133.54 \\
136.71 \\
139.89 \\
143.07\end{array}$ & $\begin{array}{l}7.836 \\
7.901 \\
7.963 \\
8.024 \\
8.083 \\
8.141 \\
8.198 \\
8.254 \\
8.308 \\
8.361\end{array}$ & $\begin{array}{l}222.00 \\
224.00 \\
226.00 \\
228.00 \\
230.00 \\
232.00 \\
234.00 \\
236.00 \\
238.00 \\
240.00\end{array}$ & $\begin{array}{l}19.7255 \\
19.9044 \\
20.0833 \\
20.2622 \\
20.4410 \\
20.6198 \\
20.7986 \\
20.9774 \\
21.1561 \\
21.3349\end{array}$ & $\begin{array}{l}612.57 \\
619.86 \\
627.18 \\
634.53 \\
641.91 \\
649.32 \\
656.75 \\
664.21 \\
671.69 \\
679.20\end{array}$ & $\begin{array}{l}393.56 \\
398.86 \\
404.19 \\
409.56 \\
414.95 \\
420.37 \\
425.82 \\
431.29 \\
436.79 \\
442.32\end{array}$ & $\begin{array}{l}10.745 \\
10.778 \\
10.810 \\
10.843 \\
10.875 \\
10.907 \\
10.939 \\
10.971 \\
11.002 \\
11.034\end{array}$ \\
\hline $\begin{array}{l}102.00 \\
104.00 \\
106.00 \\
108.00 \\
110.00 \\
112.00 \\
114.00 \\
116.00 \\
118.00 \\
120.00\end{array}$ & $\begin{array}{r}8.8398 \\
9.0267 \\
9.2133 \\
9.3994 \\
9.5852 \\
9.7707 \\
9.9558 \\
10.1406 \\
10.3251 \\
10.5093\end{array}$ & $\begin{array}{l}244.41 \\
249.69 \\
254.97 \\
260.25 \\
265.54 \\
270.85 \\
276.16 \\
281.49 \\
286.84 \\
292.20\end{array}$ & $\begin{array}{l}146.26 \\
149.46 \\
152.67 \\
155.89 \\
159.12 \\
162.36 \\
165.62 \\
168.90 \\
172.19 \\
175.51\end{array}$ & $\begin{array}{l}8.413 \\
8.465 \\
8.515 \\
8.564 \\
8.613 \\
8.661 \\
8.708 \\
8.754 \\
8.800 \\
8.845\end{array}$ & $\begin{array}{l}242.00 \\
244.00 \\
246.00 \\
248.00 \\
250.00 \\
252.00 \\
254.00 \\
256.00 \\
258.00 \\
260.00\end{array}$ & $\begin{array}{l}21.5136 \\
21.6923 \\
21.8709 \\
22.0496 \\
22.2282 \\
22.4068 \\
22.5854 \\
22.7640 \\
22.9426 \\
23.1211\end{array}$ & $\begin{array}{l}686.73 \\
694.29 \\
701.87 \\
709.47 \\
717.08 \\
724.72 \\
732.38 \\
740.05 \\
747.74 \\
755.45\end{array}$ & $\begin{array}{l}447.87 \\
453.44 \\
459.03 \\
464.64 \\
470.28 \\
475.94 \\
481.61 \\
487.30 \\
493.01 \\
498.73\end{array}$ & $\begin{array}{l}11.065 \\
11.096 \\
11.127 \\
11.158 \\
11.188 \\
11.219 \\
11.249 \\
11.279 \\
11.309 \\
11.339\end{array}$ \\
\hline
\end{tabular}


TEMPER- SPECIFIC ENTHALPY INTERNAL ENTROPY ATURE VOLUME (BTU/LB) ENERGY (BTU/L8-R) (R) (CUFT/LB)
(8TU/L8) ATURE (R)
SPECIFIC (CU FT/L8)
ENTHALPY

(8TU/LB)

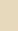

INTERNAL ENTROPY

ENERGY (8TU/L8-R) (BTU/L8)

\begin{tabular}{|c|c|c|c|c|c|c|c|c|c|}
\hline $\begin{array}{l}262.00 \\
264.00 \\
266.00 \\
268.00 \\
270.00 \\
272.00 \\
274.00 \\
276.00 \\
278.00 \\
280.00\end{array}$ & $\begin{array}{l}23.2996 \\
23.4782 \\
23.6567 \\
23.8351 \\
24.0136 \\
24.1921 \\
24.3705 \\
24.5489 \\
24.7273 \\
24.9057\end{array}$ & $\begin{array}{l}763.17 \\
770.91 \\
778.66 \\
786.42 \\
794.20 \\
801.96 \\
809.72 \\
817.50 \\
825.29 \\
833.08\end{array}$ & $\begin{array}{l}504.47 \\
510.23 \\
515.99 \\
521.78 \\
527.57 \\
533.35 \\
539.13 \\
544.93 \\
550.73 \\
556.55\end{array}$ & $\begin{array}{l}11.368 \\
11.398 \\
11.427 \\
11.456 \\
11.485 \\
11.514 \\
11.542 \\
11.570 \\
11.598 \\
11.626\end{array}$ & $\begin{array}{l}402.00 \\
404.00 \\
406.00 \\
408.00 \\
410.00 \\
412.00 \\
414.00 \\
416.00 \\
418.00 \\
420.00\end{array}$ & $\begin{array}{l}35.7668 \\
35.9447 \\
36.1226 \\
36.3005 \\
36.4784 \\
36.6562 \\
36.8341 \\
37.0121 \\
37.1900 \\
37.3679\end{array}$ & $\begin{array}{l}1304.67 \\
1312.18 \\
1319.69 \\
1327.18 \\
1334.66 \\
1342.14 \\
1349.60 \\
1357.07 \\
1364.52 \\
1371.96\end{array}$ & $\begin{array}{l}907.55 \\
913.08 \\
918.61 \\
924.13 \\
929.64 \\
935.14 \\
940.63 \\
946.11 \\
951.59 \\
957.06\end{array}$ & $\begin{array}{l}13.026 \\
13.045 \\
13.063 \\
13.082 \\
13.100 \\
13.118 \\
13.136 \\
13.154 \\
13.172 \\
13.190\end{array}$ \\
\hline $\begin{array}{l}282.00 \\
284.00 \\
286.00 \\
288.00 \\
290.00 \\
292.00 \\
294.00 \\
296.00 \\
298.00 \\
300.00\end{array}$ & $\begin{array}{l}25.0841 \\
25.2624 \\
25.4408 \\
25.6191 \\
25.7974 \\
25.9757 \\
26.1540 \\
26.3322 \\
26.5105 \\
26.6887\end{array}$ & $\begin{array}{l}840.88 \\
848.68 \\
856.49 \\
864.30 \\
872.12 \\
879.94 \\
887.77 \\
895.60 \\
903.43 \\
911.26\end{array}$ & $\begin{array}{l}562.36 \\
568.19 \\
574.02 \\
579.85 \\
585.69 \\
591.53 \\
597.38 \\
603.23 \\
609.08 \\
614.93\end{array}$ & $\begin{array}{l}11.654 \\
11.682 \\
11.709 \\
11.736 \\
11.763 \\
11.790 \\
11.817 \\
11.844 \\
11.870 \\
11.896\end{array}$ & $\begin{array}{l}422.00 \\
424.00 \\
426.00 \\
428.00 \\
430.00 \\
432.00 \\
434.00 \\
436.00 \\
438.00 \\
440.00\end{array}$ & $\begin{array}{l}37.5459 \\
37.7238 \\
37.9016 \\
38.0795 \\
38.2573 \\
36.4352 \\
38.6130 \\
38.7907 \\
38.9684 \\
39.1461\end{array}$ & $\begin{array}{l}1379.40 \\
1386.82 \\
1394.24 \\
1401.65 \\
1409.05 \\
1416.44 \\
1423.82 \\
1431.19 \\
1438.56 \\
1445.91\end{array}$ & $\begin{array}{r}962.52 \\
967.97 \\
973.41 \\
978.84 \\
984.27 \\
989.69 \\
995.09 \\
1000.49 \\
1005.89 \\
1011.27\end{array}$ & $\begin{array}{l}13.208 \\
13.225 \\
13.243 \\
13.260 \\
13.277 \\
13.294 \\
13.311 \\
13.328 \\
13.345 \\
13.362\end{array}$ \\
\hline $\begin{array}{l}302.00 \\
304.00 \\
306.00 \\
308.00 \\
310.00 \\
312.00 \\
314.00 \\
316.00 \\
318.00 \\
320.00\end{array}$ & $\begin{array}{l}26.8670 \\
27.0452 \\
27.2234 \\
27.4016 \\
27.5797 \\
27.7579 \\
27.9361 \\
28.1142 \\
28.2923 \\
28.4704\end{array}$ & $\begin{array}{l}919.09 \\
926.92 \\
934.76 \\
942.59 \\
950.42 \\
958.25 \\
966.08 \\
973.91 \\
981.73 \\
989.55\end{array}$ & $\begin{array}{l}620.78 \\
626.64 \\
632.49 \\
638.35 \\
644.20 \\
650.05 \\
655.90 \\
661.75 \\
667.60 \\
673.44\end{array}$ & $\begin{array}{l}11.922 \\
11.948 \\
11.974 \\
11.999 \\
12.025 \\
12.050 \\
12.075 \\
12.100 \\
12.124 \\
12.149\end{array}$ & $\begin{array}{l}442.00 \\
444.00 \\
446.00 \\
448.00 \\
450.00 \\
452.00 \\
454.00 \\
456.00 \\
458.00 \\
460.00\end{array}$ & $\begin{array}{l}39.3238 \\
39.5014 \\
39.6790 \\
39.8565 \\
40.0340 \\
40.2115 \\
40.3889 \\
40.5663 \\
40.7437 \\
40.9210\end{array}$ & $\begin{array}{l}1453.26 \\
1460.60 \\
1467.93 \\
1475.25 \\
1482.57 \\
1489.89 \\
1497.21 \\
1504.51 \\
1511.81 \\
1519.11\end{array}$ & $\begin{array}{l}1016.64 \\
1022.01 \\
1027.37 \\
1032.72 \\
1038.06 \\
1043.42 \\
1048.76 \\
1054.10 \\
1059.43 \\
1064.75\end{array}$ & $\begin{array}{l}13.379 \\
13.395 \\
13.412 \\
13.428 \\
13.444 \\
13.461 \\
13.477 \\
13.493 \\
13.509 \\
13.525\end{array}$ \\
\hline $\begin{array}{l}322.00 \\
324.00 \\
326.00 \\
328.00 \\
330.00 \\
332.00 \\
334.00 \\
336.00 \\
338.00 \\
340.00\end{array}$ & $\begin{array}{l}28.6486 \\
28.8267 \\
29.0048 \\
29.1828 \\
29.3609 \\
29.5390 \\
29.7170 \\
29.8951 \\
30.0731 \\
30.2512\end{array}$ & $\begin{array}{r}997.37 \\
1005.19 \\
1013.00 \\
1020.81 \\
1028.61 \\
1036.41 \\
1044.20 \\
1051.99 \\
1059.77 \\
1067.55\end{array}$ & $\begin{array}{l}679.28 \\
685.12 \\
690.96 \\
696.79 \\
702.61 \\
708.43 \\
714.25 \\
720.06 \\
725.86 \\
731.66\end{array}$ & $\begin{array}{l}12.173 \\
12.197 \\
12.221 \\
12.245 \\
12.269 \\
12.292 \\
12.316 \\
12.339 \\
12.362 \\
12.385\end{array}$ & $\begin{array}{l}462.00 \\
464.00 \\
466.00 \\
468.00 \\
470.00 \\
472.00 \\
474.00 \\
476.00 \\
478.00 \\
480.00\end{array}$ & $\begin{array}{l}41.0983 \\
41.2756 \\
41.4528 \\
41.6300 \\
41.8072 \\
41.9843 \\
42.1615 \\
42.3386 \\
42.5157 \\
42.6929\end{array}$ & $\begin{array}{l}1526.39 \\
1533.67 \\
1540.94 \\
1548.20 \\
1555.46 \\
1562.71 \\
1569.95 \\
1577.19 \\
1584.42 \\
1591.64\end{array}$ & $\begin{array}{l}1070.07 \\
1075.38 \\
1080.68 \\
1085.98 \\
1091.27 \\
1096.55 \\
1101.83 \\
1107.10 \\
1112.36 \\
1117.62\end{array}$ & $\begin{array}{l}13.540 \\
13.556 \\
13.572 \\
13.587 \\
13.603 \\
13.618 \\
13.633 \\
13.649 \\
13.664 \\
13.679\end{array}$ \\
\hline $\begin{array}{l}342.00 \\
344.00 \\
346.00 \\
348.00 \\
350.00 \\
352.00 \\
354.00 \\
356.00 \\
358.00 \\
360.00\end{array}$ & $\begin{array}{l}30.4292 \\
30.6072 \\
30.7852 \\
30.9633 \\
31.1413 \\
31.3193 \\
31.4972 \\
31.6752 \\
31.8532 \\
32.0312\end{array}$ & $\begin{array}{l}1075.32 \\
1083.08 \\
1090.84 \\
1098.59 \\
1106.33 \\
1114.07 \\
1121.79 \\
1129.51 \\
1137.23 \\
1144.93\end{array}$ & $\begin{array}{l}737.46 \\
743.24 \\
749.02 \\
754.80 \\
760.56 \\
766.32 \\
772.07 \\
777.82 \\
783.56 \\
789.28\end{array}$ & $\begin{array}{l}12.408 \\
12.431 \\
12.453 \\
12.475 \\
12.498 \\
12.520 \\
12.542 \\
12.563 \\
12.585 \\
12.606\end{array}$ & $\begin{array}{l}482.00 \\
484.00 \\
486.00 \\
488.00 \\
490.00 \\
492.00 \\
494.00 \\
496.00 \\
498.00 \\
500.00\end{array}$ & $\begin{array}{l}42.8700 \\
43.0472 \\
43.2243 \\
43.4015 \\
43.5787 \\
43.7560 \\
43.9332 \\
44.1106 \\
44.2880 \\
44.4654\end{array}$ & $\begin{array}{l}1598.86 \\
1606.08 \\
1613.28 \\
1620.49 \\
1627.69 \\
1634.88 \\
1642.07 \\
1649.26 \\
1656.44 \\
1663.61\end{array}$ & $\begin{array}{l}1122.87 \\
1128.12 \\
1133.36 \\
1138.59 \\
1143.82 \\
1149.05 \\
1154.27 \\
1159.49 \\
1164.70 \\
1169.91\end{array}$ & $\begin{array}{l}13.694 \\
13.709 \\
13.724 \\
13.739 \\
13.753 \\
13.768 \\
13.782 \\
13.797 \\
13.811 \\
13.826\end{array}$ \\
\hline $\begin{array}{l}362.00 \\
364.00 \\
366.00 \\
368.00 \\
370.00 \\
372.00 \\
374.00 \\
376.00 \\
378.00 \\
380.00\end{array}$ & $\begin{array}{l}32.2091 \\
32.3870 \\
32.5649 \\
32.7428 \\
32.9207 \\
33.0986 \\
33.2765 \\
33.4544 \\
33.6322 \\
33.8101\end{array}$ & $\begin{array}{l}1152.62 \\
1160.31 \\
1167.98 \\
1175.65 \\
1183.31 \\
1190.96 \\
1198.60 \\
1206.24 \\
1213.86 \\
1221.48\end{array}$ & $\begin{array}{l}795.00 \\
800.71 \\
806.41 \\
812.10 \\
817.79 \\
823.46 \\
829.13 \\
834.79 \\
840.44 \\
846.08\end{array}$ & $\begin{array}{l}12.628 \\
12.649 . \\
12.670 \\
12.691 \\
12.711 \\
12.732 \\
12.753 \\
12.773 \\
12.793 \\
12.813\end{array}$ & $\begin{array}{l}502.00 \\
504.00 \\
506.00 \\
508.00 \\
510.00 \\
512.00 \\
514.00 \\
516.00 \\
518.00 \\
520.00\end{array}$ & $\begin{array}{l}44.6429 \\
44.8205 \\
44.9981 \\
45.1759 \\
45.3537 \\
45.5315 \\
45.7095 \\
45.8876 \\
46.0657 \\
46.2439\end{array}$ & $\begin{array}{l}1670.79 \\
1677.96 \\
1685.12 \\
1692.29 \\
1699.45 \\
1706.61 \\
1713.76 \\
1720.91 \\
1728.06 \\
1735.21\end{array}$ & $\begin{array}{l}1175.11 \\
1180.31 \\
1185.50 \\
1190.69 \\
1195.88 \\
1201.06 \\
1206.24 \\
1211.41 \\
1216.59 \\
1221.75\end{array}$ & $\begin{array}{l}13.840 \\
13.854 \\
13.869 \\
13.883 \\
13.897 \\
13.911 \\
13.925 \\
13.939 \\
13.952 \\
13.966\end{array}$ \\
\hline $\begin{array}{l}382.00 \\
384.00 \\
386.00 \\
388.00 \\
390.00 \\
392.00 \\
394.00 \\
396.00 \\
398.00 \\
400.00\end{array}$ & $\begin{array}{l}33.9880 \\
34.1659 \\
34.3438 \\
34.5216 \\
34.6995 \\
34.8774 \\
35.0553 \\
35.2332 \\
35.4110 \\
35.5889\end{array}$ & $\begin{array}{l}1229.09 \\
1236.68 \\
1244.27 \\
1251.85 \\
1259.43 \\
1266.99 \\
1274.54 \\
1282.09 \\
1289.63 \\
1297.15\end{array}$ & $\begin{array}{l}851.71 \\
857.33 \\
862.95 \\
868.55 \\
874.15 \\
879.74 \\
885.32 \\
890.89 \\
896.45 \\
902.00\end{array}$ & $\begin{array}{l}12.833 \\
12.853 \\
12.873 \\
12.892 \\
12.912 \\
12.931 \\
12.950 \\
12.970 \\
12.989 \\
13.007\end{array}$ & $\begin{array}{l}522.00 \\
524.00 \\
526.00 \\
528.00 \\
530.00 \\
532.00 \\
534.00 \\
536.00 \\
538.00 \\
540.00\end{array}$ & $\begin{array}{l}46.4222 \\
46.6006 \\
46.7791 \\
46.9576 \\
47.1362 \\
47.3148 \\
47.4935 \\
47.6722 \\
47.8509 \\
48.0296\end{array}$ & $\begin{array}{l}1742.35 \\
1749.49 \\
1756.63 \\
1763.76 \\
1770.90 \\
1778.03 \\
1785.15 \\
1792.28 \\
1799.40 \\
1806.51\end{array}$ & $\begin{array}{l}1226.92 \\
1232.08 \\
1237.23 \\
1242.39 \\
1247.54 \\
1252.68 \\
1257.82 \\
1262.96 \\
1268.10 \\
1273.23\end{array}$ & $\begin{array}{l}13.980 \\
13.994 \\
14.007 \\
14.021 \\
14.034 \\
14.048 \\
14.061 \\
14.074 \\
14.088 \\
14.101\end{array}$ \\
\hline
\end{tabular}


TEMPER- SPECIFIC ENTHALPY INTERNAL ENTROPY ATURE VOLUME (BTU/LB) ENERGY (BTU/LB-R) (R) (CUFT/LG) (BTU/LB)
TEMPER- SPECIFIC ENTHALPY ATURE VOLUME (8TU/LB) $(R) \quad(C U F T / L B)$
INTERNAL ENTROPY ENERGY (BTU/L8-R) (8TU/LB)

\begin{tabular}{|c|c|c|c|}
\hline $\begin{array}{l}36.00 \\
38.00 \\
40.00\end{array}$ & $\begin{array}{l}.2237 \\
.2277 \\
.2324\end{array}$ & $\begin{array}{r}-109.46 \\
-104.81 \\
-99.83\end{array}$ & $\begin{array}{l}-112.35 \\
-107.76 \\
-102.84\end{array}$ \\
\hline $\begin{array}{l}42.00 \\
44.00 \\
46.00 \\
48.00 \\
* 48.464 \\
* 48.464 \\
50.00 \\
52.00 \\
54.00 \\
56.00 \\
58.00 \\
60.00\end{array}$ & $\begin{array}{l}.2376 \\
.2437 \\
.2509 \\
.2598 \\
.2620 \\
2.7007 \\
2.8995 \\
3.1342 \\
3.3521 \\
3.5591 \\
3.7583 \\
3.9518\end{array}$ & $\begin{array}{r}-94.46 \\
-88.63 \\
-82.25 \\
-75.13 \\
-73.35 \\
87.34 \\
94.02 \\
101.72 \\
108.74 \\
115.35 \\
121.67 \\
127.79\end{array}$ & $\begin{array}{r}-97.54 \\
-91.79 \\
-85.50 \\
-78.50 \\
-80.19 \\
52.35 \\
56.46 \\
61.12 \\
65.32 \\
69.25 \\
72.99 \\
76.60\end{array}$ \\
\hline $\begin{array}{l}62.00 \\
64.00 \\
66.00 \\
68.00 \\
70.00 \\
72.00 \\
74.00 \\
76.00 \\
78.00 \\
80.00\end{array}$ & $\begin{array}{l}4.1408 \\
4.3261 \\
4.5085 \\
4.6883 \\
4.8660 \\
5.0418 \\
5.2159 \\
5.3885 \\
5.5599 \\
5.7300\end{array}$ & $\begin{array}{l}133.77 \\
139.63 \\
145.40 \\
151.09 \\
156.73 \\
162.31 \\
167.85 \\
173.36 \\
178.83 \\
184.27\end{array}$ & $\begin{array}{r}80.13 \\
83.59 \\
87.00 \\
90.36 \\
93.70 \\
97.00 \\
100.29 \\
103.56 \\
106.81 \\
110.05\end{array}$ \\
\hline $\begin{array}{r}82.00 \\
84.00 \\
86.00 \\
88.00 \\
90.00 \\
92.00 \\
94.00 \\
96.00 \\
98.00 \\
100.00\end{array}$ & $\begin{array}{l}5.8991 \\
6.0671 \\
6.2343 \\
6.4007 \\
6.5663 \\
6.7312 \\
6.8954 \\
7.0591 \\
7.2222 \\
7.3847\end{array}$ & $\begin{array}{l}189.69 \\
195.08 \\
200.46 \\
205.82 \\
211.17 \\
216.51 \\
221.84 \\
227.16 \\
232.48 \\
237.79\end{array}$ & $\begin{array}{l}113.27 \\
116.49 \\
119.70 \\
122.91 \\
126.11 \\
129.32 \\
132.52 \\
135.72 \\
138.92 \\
142.13\end{array}$ \\
\hline $\begin{array}{l}102.00 \\
104.00 \\
106.00 \\
108.00 \\
110.00 \\
112.00 \\
114.00 \\
116.00 \\
118.00 \\
120.00\end{array}$ & $\begin{array}{l}7.5468 \\
7.7085 \\
7.8697 \\
8.0305 \\
8.1909 \\
8.3510 \\
8.5108 \\
8.6703 \\
8.8294 \\
8.9883\end{array}$ & $\begin{array}{l}243.10 \\
248.42 \\
253.74 \\
259.06 \\
264.39 \\
269.73 \\
275.08 \\
280.44 \\
285.81 \\
291.20\end{array}$ & $\begin{array}{l}145.35 \\
148.57 \\
151.80 \\
155.04 \\
158.29 \\
161.55 \\
164.83 \\
168.13 \\
171.44 \\
174.77\end{array}$ \\
\hline
\end{tabular}

* PHASE ChanGE

\begin{tabular}{|c|c|c|c|c|}
\hline $\begin{array}{l}122.00 \\
124.00 \\
126.00 \\
128.00 \\
130.00 \\
132.00 \\
134.00 \\
136.00 \\
138.00 \\
140.00\end{array}$ & $\begin{array}{r}9.1469 \\
9.3053 \\
9.4635 \\
9.6214 \\
9.7791 \\
9.9366 \\
10.0940 \\
10.2511 \\
10.4081 \\
10.5649\end{array}$ & $\begin{array}{l}296.61 \\
302.04 \\
307.48 \\
312.96 \\
318.45 \\
323.97 \\
329.52 \\
335.09 \\
340.70 \\
346.34\end{array}$ & $\begin{array}{l}178.12 \\
181.50 \\
184.90 \\
188.32 \\
191.77 \\
195.25 \\
198.76 \\
202.30 \\
205.88 \\
209.49\end{array}$ & $\begin{array}{l}8.731 \\
8.775 \\
8.819 \\
8.862 \\
8.905 \\
8.947 \\
8.989 \\
9.030 \\
9.071 \\
9.111\end{array}$ \\
\hline $\begin{array}{l}142.00 \\
144.00 \\
146.00 \\
148.00 \\
150.00 \\
152.00 \\
154.00 \\
156.00 \\
158.00 \\
160.00\end{array}$ & $\begin{array}{l}10.7216 \\
10.8781 \\
11.0345 \\
11.1907 \\
11.3469 \\
11.5028 \\
11.6587 \\
11.8145 \\
11.9701 \\
12.1257\end{array}$ & $\begin{array}{l}352.01 \\
357.72 \\
363.46 \\
369.24 \\
375.06 \\
380.92 \\
386.81 \\
392.75 \\
398.73 \\
404.76\end{array}$ & $\begin{array}{l}213.13 \\
216.81 \\
220.52 \\
224.28 \\
228.08 \\
231.91 \\
235.79 \\
239.71 \\
243.68 \\
247.69\end{array}$ & $\begin{array}{l}9.152 \\
9.192 \\
9.231 \\
9.270 \\
9.309 \\
9.348 \\
9.387 \\
9.425 \\
9.463 \\
9.501\end{array}$ \\
\hline $\begin{array}{l}162.00 \\
164.00 \\
166.00 \\
168.00 \\
170.00 \\
172.00 \\
174.00 \\
176.00 \\
178.00 \\
180.00\end{array}$ & $\begin{array}{l}12.2811 \\
12.4365 \\
12.5918 \\
12.7470 \\
12.9021 \\
13.0571 \\
13.2120 \\
13.3669 \\
13.5217 \\
13.6764\end{array}$ & $\begin{array}{l}410.82 \\
416.93 \\
423.09 \\
429.29 \\
435.53 \\
441.81 \\
448.14 \\
454.52 \\
460.93 \\
467.39\end{array}$ & $\begin{array}{l}251.74 \\
255.84 \\
259.98 \\
264.17 \\
268.40 \\
272.68 \\
277.00 \\
281.37 \\
285.78 \\
290.23\end{array}$ & $\begin{array}{l}9.539 \\
9.576 \\
9.614 \\
9.651 \\
9.688 \\
9.724 \\
9.761 \\
9.797 \\
9.834 \\
9.870\end{array}$ \\
\hline $\begin{array}{l}182.00 \\
184.00 \\
186.00 \\
188.00 \\
190.00 \\
192.00 \\
194.00 \\
196.00 \\
198.00 \\
200.00\end{array}$ & $\begin{array}{l}13.8310 \\
13.9855 \\
14.1399 \\
14.2943 \\
14.4486 \\
14.6029 \\
14.7571 \\
14.9112 \\
15.0653 \\
15.2194\end{array}$ & $\begin{array}{l}473.88 \\
480.42 \\
487.00 \\
493.62 \\
500.29 \\
507.00 \\
513.74 \\
520.54 \\
527.37 \\
534.24\end{array}$ & $\begin{array}{l}294.72 \\
299.26 \\
303.84 \\
308.46 \\
313.12 \\
317.83 \\
322.59 \\
327.38 \\
332.22 \\
337.09\end{array}$ & $\begin{array}{r}9.906 \\
9.941 \\
9.977 \\
10.012 \\
10.048 \\
10.083 \\
10.118 \\
10.152 \\
10.187 \\
10.222\end{array}$ \\
\hline $\begin{array}{l}202.00 \\
204.00 \\
206.00 \\
208.00 \\
210.00 \\
212.00 \\
214.00 \\
216.00 \\
218.00 \\
220.00\end{array}$ & $\begin{array}{l}15.3734 \\
15.5273 \\
15.6813 \\
15.8351 \\
15.9889 \\
16.1427 \\
16.2965 \\
16.4502 \\
16.6038 \\
16.7575\end{array}$ & $\begin{array}{l}541.15 \\
548.10 \\
555.09 \\
562.12 \\
569.18 \\
576.28 \\
583.42 \\
590.59 \\
597.79 \\
605.02\end{array}$ & $\begin{array}{l}342.01 \\
346.97 \\
351.96 \\
357.00 \\
362.07 \\
367.18 \\
372.32 \\
377.50 \\
382.71 \\
387.95\end{array}$ & $\begin{array}{l}10.256 \\
10.290 \\
10.324 \\
10.358 \\
10.392 \\
10.426 \\
10.459 \\
10.493 \\
10.526 \\
10.559\end{array}$ \\
\hline $\begin{array}{l}222.00 \\
224.00 \\
226.00 \\
228.00 \\
230.00 \\
232.00 \\
234.00 \\
236.00 \\
238.00 \\
240.00\end{array}$ & $\begin{array}{l}16.9111 \\
17.0647 \\
17.2182 \\
17.3717 \\
17.5252 \\
17.6787 \\
17.8321 \\
17.9856 \\
18.1390 \\
18.2923\end{array}$ & $\begin{array}{l}612.29 \\
619.58 \\
626.91 \\
634.26 \\
641.65 \\
649.06 \\
656.50 \\
663.96 \\
671.45 \\
678.96\end{array}$ & $\begin{array}{l}393.23 \\
398.53 \\
403.87 \\
409.23 \\
414.63 \\
420.05 \\
425.50 \\
430.98 \\
436.48 \\
442.01\end{array}$ & $\begin{array}{l}10.592 \\
10.624 \\
10.657 \\
10.689 \\
10.722 \\
10.754 \\
10.786 \\
10.817 \\
10.849 \\
10.880\end{array}$ \\
\hline $\begin{array}{l}242.00 \\
244.00 \\
246.00 \\
248.00 \\
250.00 \\
252.00 \\
254.00 \\
256.00 \\
258.00 \\
260.00\end{array}$ & $\begin{array}{l}18.4457 \\
18.5990 \\
18.7524 \\
18.9057 \\
19.0589 \\
19.2122 \\
19.3654 \\
19.5187 \\
19.6719 \\
19.8251\end{array}$ & $\begin{array}{l}686.50 \\
694.06 \\
701.64 \\
709.25 \\
716.87 \\
724.51 \\
732.17 \\
739.85 \\
747.55 \\
755.26\end{array}$ & $\begin{array}{l}447.56 \\
453.14 \\
458.73 \\
464.35 \\
469.99 \\
475.64 \\
481.32 \\
487.01 \\
492.73 \\
498.45\end{array}$ & $\begin{array}{l}10.912 \\
10.943 \\
10.974 \\
11.005 \\
11.035 \\
11.066 \\
11.096 \\
11.126 \\
11.156 \\
11.186\end{array}$ \\
\hline
\end{tabular}


TEMPER- SPECIFIC ENTHALPY INTERNAL ENTROPY

ATURE VOLUME (8TU/L8) ENERGY (8TU/L8-R) (R) (CU FT/LE)
TEMPER-

ATURE (8TU/L8)
SPECIFIC ENTHALPY VOLUME (BRU/LB) (R) (CU FT/L8)
INTERNAL ENTROPY

ENERGY (8TU/L8-R) $(8 \mathrm{TU} / \mathrm{L} 8)$

\begin{tabular}{|c|c|c|c|c|c|c|c|c|c|}
\hline $\begin{array}{l}262.00 \\
264.00 \\
266.00 \\
268.00 \\
270.00 \\
272.00 \\
274.00 \\
276.00 \\
278.00 \\
280.00\end{array}$ & $\begin{array}{l}19.9782 \\
20.1314 \\
20.2845 \\
20.4377 \\
20.5908 \\
20.7439 \\
20.8970 \\
21.0500 \\
21.2031 \\
21.3561\end{array}$ & $\begin{array}{l}762.99 \\
770.73 \\
778.48 \\
786.25 \\
794.03 \\
801.79 \\
809.56 \\
817.34 \\
825.13 \\
832.93\end{array}$ & $\begin{array}{l}504.19 \\
509.95 \\
515.72 \\
521.50 \\
527.30 \\
533.08 \\
538.87 \\
544.67 \\
550.47 \\
556.29\end{array}$ & $\begin{array}{l}11.215 \\
11.245 \\
11.274 \\
11.303 \\
11.332 \\
11.361 \\
11.389 \\
11.417 \\
11.446 \\
11.474\end{array}$ & $\begin{array}{l}402.00 \\
404.00 \\
406.00 \\
408.00 \\
410.00 \\
412.00 \\
414.00 \\
416.00 \\
418.00 \\
420.00\end{array}$ & $\begin{array}{l}30.6707 \\
30.8232 \\
30.9758 \\
31.1283 \\
31.2808 \\
31.4334 \\
31.5859 \\
31.7385 \\
31.8910 \\
32.0436\end{array}$ & $\begin{array}{l}1304.68 \\
1312.20 \\
1319.70 \\
1327.19 \\
1334.68 \\
1342.16 \\
1349.63 \\
1357.09 \\
1364.54 \\
1371.99\end{array}$ & $\begin{array}{l}907.38 \\
912.92 \\
918.45 \\
923.97 \\
929.48 \\
934.98 \\
940.47 \\
945.96 \\
951.44 \\
956.91\end{array}$ & $\begin{array}{l}12.874 \\
12.892 \\
12.911 \\
12.929 \\
12.948 \\
12.966 \\
12.984 \\
13.002 \\
13.020 \\
13.038\end{array}$ \\
\hline $\begin{array}{l}282.00 \\
284.00 \\
286.00 \\
288.00 \\
290.00 \\
292.00 \\
294.00 \\
296.00 \\
298.00 \\
300.00\end{array}$ & $\begin{array}{l}21.5091 \\
21.6621 \\
21.8151 \\
21.9681 \\
22.1210 \\
22.2740 \\
22.4269 \\
22.5798 \\
22.7327 \\
22.8856\end{array}$ & $\begin{array}{l}840.73 \\
848.54 \\
856.35 \\
864.17 \\
871.99 \\
879.82 \\
887.65 \\
895.48 \\
903.31 \\
911.14\end{array}$ & $\begin{array}{l}562.11 \\
567.93 \\
573.76 \\
579.60 \\
585.44 \\
591.29 \\
597.13 \\
602.38 \\
608.84 \\
614.69\end{array}$ & $\begin{array}{l}11.501 \\
11.529 \\
11.556 \\
11.584 \\
11.611 \\
11.638 \\
11.664 \\
11.691 \\
11.717 \\
11.743\end{array}$ & $\begin{array}{l}422.00 \\
424.00 \\
426.00 \\
428.00 \\
430.00 \\
432.00 \\
434.00 \\
436.00 \\
438.00 \\
440.00\end{array}$ & $\begin{array}{l}32.1961 \\
32.3487 \\
32.5012 \\
32.6537 \\
32.8062 \\
32.9586 \\
33.1111 \\
33.2635 \\
33.4159 \\
33.5682\end{array}$ & $\begin{array}{l}1379.42 \\
1386.85 \\
1394.27 \\
1401.68 \\
1409.08 \\
1416.48 \\
1423.86 \\
1431.23 \\
1438.60 \\
1445.96\end{array}$ & $\begin{array}{r}962.37 \\
967.82 \\
973.26 \\
978.70 \\
984.12 \\
989.54 \\
994.95 \\
1000.35 \\
1005.74 \\
1011.13\end{array}$ & $\begin{array}{l}13.055 \\
13.073 \\
13.090 \\
13.108 \\
13.125 \\
13.142 \\
13.159 \\
13.176 \\
13.193 \\
13.210\end{array}$ \\
\hline $\begin{array}{l}302.00 \\
304.00 \\
306.00 \\
308.00 \\
310.00 \\
312.00 \\
314.00 \\
316.00 \\
318.00 \\
320.00\end{array}$ & $\begin{array}{l}23.0385 \\
23.1913 \\
23.3442 \\
23.4970 \\
23.6498 \\
23.8026 \\
23.9554 \\
24.1082 \\
24.2610 \\
24.4138\end{array}$ & $\begin{array}{l}918.98 \\
926.82 \\
934.65 \\
942.49 \\
950.32 \\
958.16 \\
965.99 \\
973.82 \\
981.65 \\
989.47\end{array}$ & $\begin{array}{l}620.55 \\
626.40 \\
632.26 \\
638.11 \\
643.97 \\
649.82 \\
655.68 \\
661.53 \\
667.38 \\
673.22\end{array}$ & $\begin{array}{l}11.769 \\
11.795 \\
11.821 \\
11.846 \\
11.872 \\
11.897 \\
11.922 \\
11.947 \\
11.972 \\
11.996\end{array}$ & $\begin{array}{l}442.00 \\
444.00 \\
446.00 \\
448.00 \\
450.00 \\
452.00 \\
454.00 \\
456.00 \\
458.00 \\
460.00\end{array}$ & $\begin{array}{l}33.7206 \\
33.8729 \\
34.0251 \\
34.1774 \\
34.3296 \\
34.4817 \\
34.6338 \\
34.7859 \\
34.9380 \\
35.0900\end{array}$ & $\begin{array}{l}1453.31 \\
1460.65 \\
1467.98 \\
1475.30 \\
1482.62 \\
1489.95 \\
1497.26 \\
1504.57 \\
1511.87 \\
1519.17\end{array}$ & $\begin{array}{l}1016.50 \\
1021.87 \\
1027.23 \\
1032.58 \\
1037.93 \\
1043.28 \\
1048.63 \\
1053.96 \\
1059.30 \\
1064.62\end{array}$ & $\begin{array}{l}13.226 \\
13.243 \\
13.259 \\
13.276 \\
13.292 \\
13.308 \\
13.324 \\
13.340 \\
13.356 \\
13.372\end{array}$ \\
\hline $\begin{array}{l}322.00 \\
324.00 \\
326.00 \\
328.00 \\
330.00 \\
332.00 \\
334.00 \\
336.00 \\
338.00 \\
340.00\end{array}$ & $\begin{array}{l}24.5665 \\
24.7193 \\
24.8720 \\
25.0248 \\
25.1775 \\
25.3302 \\
25.4829 \\
25.6356 \\
25.7883 \\
25.9410\end{array}$ & $\begin{array}{r}997.29 \\
1005.11 \\
1012.93 \\
1020.74 \\
1028.54 \\
1036.34 \\
1044.14 \\
1051.93 \\
1059.71 \\
1067.49\end{array}$ & $\begin{array}{l}679.07 \\
684.90 \\
690.74 \\
696.57 \\
702.40 \\
708.22 \\
714.04 \\
719.85 \\
725.66 \\
731.46\end{array}$ & $\begin{array}{l}12.020 \\
12.045 \\
12.069 \\
12.093 \\
12.116 \\
12.140 \\
12.163 \\
12.187 \\
12.210 \\
12.233\end{array}$ & $\begin{array}{l}462.00 \\
464.00 \\
466.00 \\
468.00 \\
470.00 \\
472.00 \\
474.00 \\
476.00 \\
478.00 \\
480.00\end{array}$ & $\begin{array}{l}35.2421 \\
35.3940 \\
35.5460 \\
35.6979 \\
35.8498 \\
36.0017 \\
36.1536 \\
36.3055 \\
36.4574 \\
36.6092\end{array}$ & $\begin{array}{l}1526.45 \\
1533.73 \\
1541.00 \\
1548.27 \\
1555.53 \\
1562.78 \\
1570.02 \\
1577.26 \\
1584.49 \\
1591.72\end{array}$ & $\begin{array}{l}1069.94 \\
1075.25 \\
1080.55 \\
1085.85 \\
1091.14 \\
1096.42 \\
1101.70 \\
1106.97 \\
1112.23 \\
1117.49\end{array}$ & $\begin{array}{l}13.388 \\
13.404 \\
13.419 \\
13.435 \\
13.451 \\
13.466 \\
13.481 \\
13.496 \\
13.512 \\
13.527\end{array}$ \\
\hline $\begin{array}{l}342.00 \\
344.00 \\
346.00 \\
348.00 \\
350.00 \\
352.00 \\
354.00 \\
356.00 \\
358.00 \\
360.00\end{array}$ & $\begin{array}{l}26.0937 \\
26.2463 \\
26.3990 \\
26.5517 \\
26.7043 \\
26.8570 \\
27.0096 \\
27.1622 \\
27.3149 \\
27.4675\end{array}$ & $\begin{array}{l}1075.26 \\
1083.03 \\
1090.79 \\
1098.54 \\
1106.29 \\
1114.02 \\
1121.75 \\
1129.48 \\
1137.19 \\
1144.90\end{array}$ & $\begin{array}{l}737.25 \\
743.04 \\
748.82 \\
754.60 \\
760.37 \\
766.13 \\
771.88 \\
777.63 \\
783.36 \\
789.09\end{array}$ & $\begin{array}{l}12.255 \\
12.278 \\
12.301 \\
12.323 \\
12.345 \\
12.367 \\
12.389 \\
12.411 \\
12.432 \\
12.454\end{array}$ & $\begin{array}{l}482.00 \\
484.00 \\
486.00 \\
488.00 \\
490.00 \\
492.00 \\
494.00 \\
496.00 \\
498.00 \\
500.00\end{array}$ & $\begin{array}{l}36.7611 \\
36.9130 \\
37.0649 \\
37.2168 \\
37.3687 \\
37.5207 \\
37.6726 \\
37.8247 \\
37.9768 \\
38.1289\end{array}$ & $\begin{array}{l}1598.94 \\
1606.15 \\
1613.36 \\
1620.57 \\
1627.77 \\
1634.96 \\
1642.15 \\
1649.34 \\
1656.52 \\
1663.70\end{array}$ & $\begin{array}{l}1122.75 \\
1127.99 \\
1133.24 \\
1138.47 \\
1143.70 \\
1148.93 \\
1154.15 \\
1159.37 \\
1164.58 \\
1169.79\end{array}$ & $\begin{array}{l}13.542 \\
13.557 \\
13.572 \\
13.586 \\
13.601 \\
13.616 \\
13.630 \\
13.645 \\
13.659 \\
13.674\end{array}$ \\
\hline $\begin{array}{l}362.00 \\
364.00 \\
366.00 \\
368.00 \\
370.00 \\
372.00 \\
374.00 \\
376.00 \\
378.00 \\
380.00\end{array}$ & $\begin{array}{l}27.6201 \\
27.7726 \\
27.9252 \\
28.0777 \\
28.2303 \\
28.3828 \\
28.5353 \\
28.6879 \\
28.8404 \\
28.9929\end{array}$ & $\begin{array}{l}1152.59 \\
1160.28 \\
1167.96 \\
1175.63 \\
1183.29 \\
1190.94 \\
1198.59 \\
1206.22 \\
1213.85 \\
1221.47\end{array}$ & $\begin{array}{l}794.81 \\
800.52 \\
806.22 \\
811.92 \\
817.60 \\
823.28 \\
828.95 \\
834.61 \\
840.26 \\
845.90\end{array}$ & $\begin{array}{l}12.475 \\
12.496 \\
12.517 \\
12.538 \\
12.559 \\
12.580 \\
12.600 \\
12.621 \\
12.641 \\
12.661\end{array}$ & $\begin{array}{l}502.00 \\
504.00 \\
506.00 \\
508.00 \\
510.00 \\
512.00 \\
514.00 \\
516.00 \\
518.00 \\
520.00\end{array}$ & $\begin{array}{l}38.2810 \\
38.4333 \\
38.5856 \\
38.7379 \\
38.8903 \\
39.0428 \\
39.1954 \\
39.3480 \\
39.5007 \\
39.6535\end{array}$ & $\begin{array}{l}1670.87 \\
1678.04 \\
1685.21 \\
1692.38 \\
1699.54 \\
1706.70 \\
1713.85 \\
1721.00 \\
1728.15 \\
1735.30\end{array}$ & $\begin{array}{l}1174.99 \\
1180.19 \\
1185.99 \\
1190.58 \\
1195.76 \\
1200.95 \\
1206.13 \\
1211.30 \\
1216.47 \\
1221.64\end{array}$ & $\begin{array}{l}13.688 \\
13.702 \\
13.716 \\
13.731 \\
13.745 \\
13.759 \\
13.773 \\
13.786 \\
13.800 \\
13.814\end{array}$ \\
\hline $\begin{array}{l}382.00 \\
384.00 \\
386.00 \\
388.00 \\
390.00 \\
392.00 \\
394.00 \\
396.00 \\
398.00 \\
400.00\end{array}$ & $\begin{array}{l}29.1455 \\
29.2980 \\
29.4505 \\
29.6030 \\
29.7556 \\
29.9081 \\
30.0606 \\
30.2131 \\
30.3657 \\
30.5182\end{array}$ & $\begin{array}{l}1229.08 \\
1236.68 \\
1244.27 \\
1251.85 \\
1259.42 \\
1266.99 \\
1274.55 \\
1282.09 \\
1289.63 \\
1297.16\end{array}$ & $\begin{array}{l}851.53 \\
857.16 \\
862.77 \\
868.38 \\
873.98 \\
879.57 \\
885.15 \\
890.72 \\
896.28 \\
901.84\end{array}$ & $\begin{array}{l}12.681 \\
12.701 \\
12.720 \\
12.740 \\
12.759 \\
12.779 \\
12.798 \\
12.817 \\
12.836 \\
12.855\end{array}$ & $\begin{array}{l}522.00 \\
524.00 \\
526.00 \\
528.00 \\
530.00 \\
532.00 \\
534.00 \\
536.00 \\
538.00 \\
540.00\end{array}$ & $\begin{array}{l}39.8064 \\
39.9593 \\
40.1122 \\
40.2653 \\
40.4183 \\
40.5715 \\
40.7246 \\
40.8778 \\
41.0310 \\
41.1842\end{array}$ & $\begin{array}{l}1742.44 \\
1749.59 \\
1756.72 \\
1763.86 \\
1770.99 \\
1778.12 \\
1785.25 \\
1792.37 \\
1799.49 \\
1806.61\end{array}$ & $\begin{array}{l}1226.81 \\
1231.97 \\
1237.12 \\
1242.28 \\
1247.43 \\
1252.57 \\
1257.72 \\
1262.85 \\
1267.99 \\
1273.12\end{array}$ & $\begin{array}{l}13.828 \\
13.841 \\
13.855 \\
13.869 \\
13.882 \\
13.895 \\
13.909 \\
13.922 \\
13.935 \\
13.949\end{array}$ \\
\hline
\end{tabular}


IEMPER- SPECIFIC ENTHALPY INTERNAL ENTROPY ATURE VOLUME (BTU/LB) ENERGY (BTU/L8 B (R) (CU FT/LB)

\section{(BTU/LB)}

$\begin{array}{ccc}\text { TEMPER- } & \text { SPECIFIC } & \text { ENTHALPY } \\ \text { ATURE } & \text { VOLUME } & \text { (8TU/L } 8)\end{array}$ (R) (CU FT/LB)
INTERNAL ENTROPY ENERGY (BTU/L8-R) $(8 \mathrm{TU} / \mathrm{LB})$

$\begin{array}{lrrr}36.00 & .2234 & -109.17 & -112.48 \\ 38.00 & .2274 & -104.54 & -107.91 \\ 40.00 & .2320 & -99.58 & -103.01 \\ & & & \\ 42.00 & .2372 & -94.24 & -97.75 \\ 44.00 & .2432 & -88.45 & -92.05 \\ 46.00 & .2502 & -82.12 & -85.82 \\ 48.00 & .2588 & -75.09 & -78.92 \\ * 49.768 & .2683 & -68.06 & -76.06 \\ * 49.768 & 2.3452 & 86.26 & 51.54 \\ 50.00 & 2.3747 & 87.42 & 52.26 \\ 52.00 & 2.6082 & 96.38 & 57.76 \\ 54.00 & 2.8169 & 104.17 & 62.47 \\ 56.00 & 3.0108 & 111.30 & 66.73 \\ 58.00 & 3.1950 & 118.01 & 70.71 \\ 60.00 & 3.3722 & 124.43 & 74.51 \\ & & & \\ 62.00 & 3.5442 & 130.65 & 78.18 \\ 64.00 & 3.7120 & 136.71 & 81.76 \\ 66.00 & 3.8765 & 142.65 & 85.26 \\ 68.00 & 4.0382 & 148.50 & 88.72 \\ 70.00 & 4.1975 & 154.27 & 92.13 \\ 72.00 & 4.3549 & 159.97 & 95.50 \\ 74.00 & 4.5104 & 165.62 & 98.85 \\ 76.00 & 4.6644 & 171.22 & 102.17 \\ 78.00 & 4.8170 & 176.78 & 105.47 \\ 80.00 & 4.9684 & 182.31 & 108.76 \\ 82.00 & 5.1187 & 187.81 & 112.03 \\ 84.00 & 5.2679 & 193.28 & 115.29 \\ 86.00 & 5.4162 & 198.72 & 118.54 \\ 88.00 & 5.5637 & 204.15 & 121.78 \\ 90.00 & 5.7104 & 209.56 & 125.02 \\ 92.00 & 5.8564 & 214.95 & 128.25 \\ 94.00 & 6.0017 & 220.34 & 131.49 \\ 96.00 & 6.1464 & 225.71 & 134.72 \\ 98.00 & 6.2905 & 231.08 & 137.95 \\ 100.00 & 6.4341 & 236.44 & 141.18 \\ 102.00 & 6.5772 & 241.79 & 144.42 \\ 104.00 & 6.7199 & 247.15 & 147.67 \\ 106.00 & 6.8621 & 252.51 & 150.92 \\ 108.00 & 7.0039 & 257.87 & 154.18 \\ 110.00 & 7.1453 & 263.24 & 157.45 \\ 112.00 & 7.2864 & 268.61 & 160.74 \\ 114.00 & 7.4272 & 273.99 & 164.04 \\ 116.00 & 7.5677 & 279.38 & 167.35 \\ 118.00 & 7.7078 & 284.79 & 170.68 \\ 120.00 & 7.8477 & 290.21 & 174.03\end{array}$

1.861

1.986

2.113

2.243

2.378

2.519

2.668

2.812

5.917

5.940

6.116

6.263

6.393

6.511

6.620

6.721

6.818

6.909

6.996

7.080

7.160

7.238

7.312

7.385

7.455

7.523

7.588

7.653

7.715

7.776

7.835

7.893

7.949

8.005

8.059

8.112

8.164

8.215

8.265

8.314

8.363

8.410

8.457

8.503

8.549

122.00
124.00
126.00
128.00
130.00
132.00
134.00
136.00
138.00
140.00

142.00
144.00
146.00
148.00
150.00
152.00
154.00
156.00
158.00
160.00
162.00
164.00
166.00
168.00
170.00
172.00
174.00
176.00
178.00
180.00

8.1267

8.2658

8.4047

8. 5434

8.6819

8.8202

8.9583

9.0963

9.2341

9.3717

9.5092

9.6466

9.7838

9.9209

10.0579

10.1947

10.3315

10.4681

10.6046

10.7410

10.8774

11.0136

11.1498

11.2859

11.4218

11.5578

11.6936

11.8294

11.9651

12.1007

12.2361

12.3715

12.5069

12.6422

12.7774

12.9126

13.0477

13. 1828

13.3179

13.5878

13.7227

13.8575

13.9923

14.1271

14.2618

14.3965

14.5312

14.6658

14.8004

14.9350

15.0695

15.2040

15.3385

15.4729

15.6074

15.7418

15.8762

16.0105

16.1449

16.2792

16.4135

16.5478

16.6821

16.8163

16. 9505

17.0847

17.2189

17.3531
295.64

301.10

306.57

312.07

317.59

323.13

328.70

334.30

339.93

345.59

351.28

357.01

362.77

368.56

374.40

380.27

386.19

392.14

398.14

404.18

410.26

416.38

422.55

428.76

435.01

441.31

447.65

454.04

460.47

466.93

473.44

479.99

486.58

493.21

499.88

506.60

513.36

520.16

527.00

533.88

540.80

547.75

554.75

561.79

568.86

575.97

583.11

590.29

597.49

604.73

612.00

619.30

626.63

633.99

641.38

648.80

656.24

663.71

671.21

678.73

686.27

693.84

701.42

709.03

716.66

724.31

731.97

739.66

747.36

755.07
177.40

180.79

184.20

187.64

191.11

194.60

198.13

201.68

205.26

208.88

212.54

216.23

219.96

223.72

227.53

231.38

235.26

239.19

243.17

247.18

251.24

255.35

259.50

263.70

267.94

272.22

276.55

280.92

285.34

289.80

294.30

298.84

303.42

308.05

312.72

317.44

322.20

326.99

331.83

336.72

341.64

346.60

351.60

356.64

361.71

366.82

371.97

377.16

382.37

387.61

392.89

398.20

403.54

408.91

414.31

419.74

425.19

430.67

436.17

441.70

447.26

452.83

458.43

464.05

469.69

475.35

481.03

486.73

492.44

498.17

8.594

.638

8.682

8.725

8.768

8.810

8.852

8.894

8.935

8.976

9.016

9.056 
TEMPER- SPECIFIC ENTHALPY INTERNAL ENTROPY ATURE VOLUME (8TU/L8) ENERGY (8TU/L8-R) (R) (CU FT/L8)
ENERGY
$(8 \mathrm{TU} / \mathrm{L} 8)$
TEMPER- SPECIFIC ENTHALPY ATURE (R)
VOLUME (CU FT/L8)
(BTU/L8)

(8)

INTERNAL ENTROPY

ENERGY (8TU/L8-R) $(8 \mathrm{TU} / \mathrm{LB})$

\begin{tabular}{|c|c|c|c|c|c|c|c|c|c|}
\hline $\begin{array}{l}262.00 \\
264.00 \\
266.00 \\
268.00 \\
270.00 \\
272.00 \\
274.00 \\
276.00 \\
278.00 \\
280.00\end{array}$ & $\begin{array}{l}17.4873 \\
17.6214 \\
17.7555 \\
17.8897 \\
18.0237 \\
18.1578 \\
18.2919 \\
18.4259 \\
18.5600 \\
18.6940\end{array}$ & $\begin{array}{l}762.80 \\
770.55 \\
778.30 \\
786.08 \\
793.86 \\
801.63 \\
809.40 \\
817.19 \\
824.98 \\
832.78\end{array}$ & $\begin{array}{l}503.92 \\
509.67 \\
515.45 \\
521.23 \\
527.03 \\
532.81 \\
538.60 \\
544.41 \\
550.21 \\
556.03\end{array}$ & $\begin{array}{l}11.083 \\
11.112 \\
11.141 \\
11.171 \\
11.197 \\
11.228 \\
11.257 \\
11.285 \\
11.313 \\
11.341\end{array}$ & $\begin{array}{l}402.00 \\
404.00 \\
406.00 \\
408.00 \\
410.00 \\
412.00 \\
414.00 \\
416.00 \\
418.00 \\
420.00\end{array}$ & $\begin{array}{l}26.8487 \\
26.9822 \\
27.1157 \\
27.2492 \\
27.3828 \\
27.5163 \\
27.6498 \\
27.7833 \\
27.9168 \\
28.0504\end{array}$ & $\begin{array}{l}1304.69 \\
1312.21 \\
1319.71 \\
1327.21 \\
1334.70 \\
1342.18 \\
1349.65 \\
1357.11 \\
1364.57 \\
1372.02\end{array}$ & $\begin{array}{l}907.22 \\
912.76 \\
918.29 \\
923.81 \\
929.32 \\
934.82 \\
940.31 \\
945.80 \\
951.28 \\
956.75\end{array}$ & $\begin{array}{l}12.742 \\
12.760 \\
12.779 \\
12.797 \\
12.816 \\
12.834 \\
12.852 \\
12.870 \\
12.888 \\
12.906\end{array}$ \\
\hline $\begin{array}{l}282.00 \\
284.00 \\
286.00 \\
288.00 \\
290.00 \\
292.00 \\
294.00 \\
296.00 \\
298.00 \\
300.00\end{array}$ & $\begin{array}{l}18.8280 \\
18.9619 \\
19.0959 \\
19.2299 \\
19.3638 \\
19.4977 \\
19.6316 \\
19.7655 \\
19.8994 \\
20.0333\end{array}$ & $\begin{array}{l}840.58 \\
848.40 \\
856.21 \\
864.04 \\
871.86 \\
879.69 \\
887.52 \\
895.36 \\
903.19 \\
911.03\end{array}$ & $\begin{array}{l}561.85 \\
567.68 \\
573.51 \\
579.35 \\
585.19 \\
591.04 \\
596.84 \\
602.74 \\
608.60 \\
614.45\end{array}$ & $\begin{array}{l}11.369 \\
11.396 \\
11.424 \\
11.451 \\
11.478 \\
11.505 \\
11.532 \\
11.558 \\
11.585 \\
11.611\end{array}$ & $\begin{array}{l}422.00 \\
424.00 \\
426.00 \\
428.00 \\
430.00 \\
432.00 \\
434.00 \\
436.00 \\
438.00 \\
440.00\end{array}$ & $\begin{array}{l}28.1839 \\
28.3174 \\
28.4509 \\
28.5844 \\
28.7178 \\
28.8513 \\
28.9847 \\
29.1181 \\
29.2515 \\
29.3848\end{array}$ & $\begin{array}{l}1379.45 \\
1386.88 \\
1394.30 \\
1401.72 \\
1409.12 \\
1416.51 \\
1423.90 \\
1431.28 \\
1438.64 \\
1446.00\end{array}$ & $\begin{array}{r}962.21 \\
967.67 \\
973.11 \\
978.55 \\
983.97 \\
989.39 \\
994.80 \\
1000.20 \\
1005.60 \\
1010.98\end{array}$ & $\begin{array}{l}12.923 \\
12.941 \\
12.958 \\
12.976 \\
12.993 \\
13.010 \\
13.027 \\
13.044 \\
13.061 \\
13.078\end{array}$ \\
\hline $\begin{array}{l}302.00 \\
304.00 \\
306.00 \\
308.00 \\
310.00 \\
312.00 \\
314.00 \\
316.00 \\
318.00 \\
320.00\end{array}$ & $\begin{array}{l}20.1671 \\
20.3010 \\
20.4348 \\
20.5686 \\
20.7024 \\
20.8362 \\
20.9700 \\
21.1038 \\
21.2376 \\
21.3713\end{array}$ & $\begin{array}{l}918.87 \\
926.71 \\
934.55 \\
942.39 \\
950.23 \\
958.06 \\
965.90 \\
973.73 \\
981.56 \\
989.39\end{array}$ & $\begin{array}{l}620.31 \\
626.17 \\
632.03 \\
637.88 \\
643.74 \\
649.60 \\
655.45 \\
661.31 \\
667.16 \\
673.00\end{array}$ & $\begin{array}{l}11.637 \\
11.663 \\
11.689 \\
11.714 \\
11.739 \\
11.765 \\
11.790 \\
11.815 \\
11.839 \\
11.864\end{array}$ & $\begin{array}{l}442.00 \\
444.00 \\
446.00 \\
448.00 \\
450.00 \\
452.00 \\
454.00 \\
456.00 \\
458.00 \\
460.00\end{array}$ & $\begin{array}{l}29.5182 \\
29.6515 \\
29.7847 \\
29.9180 \\
30.0512 \\
30.1844 \\
30.3175 \\
30.4506 \\
30.5837 \\
30.7168\end{array}$ & $\begin{array}{l}1453.35 \\
1460.70 \\
1468.03 \\
1475.36 \\
1482.67 \\
1490.00 \\
1497.32 \\
1504.63 \\
1511.93 \\
1519.23\end{array}$ & $\begin{array}{l}1016.36 \\
1021.73 \\
1027.09 \\
1032.44 \\
1037.79 \\
1043.14 \\
1048.49 \\
1053.83 \\
1059.16 \\
1064.49\end{array}$ & $\begin{array}{l}13.094 \\
13.111 \\
13.127 \\
13.144 \\
13.160 \\
13.176 \\
13.192 \\
13.208 \\
13.224 \\
13.240\end{array}$ \\
\hline $\begin{array}{l}322.00 \\
324.00 \\
326.00 \\
328.00 \\
330.00 \\
332.00 \\
334.00 \\
336.00 \\
338.00 \\
340.00\end{array}$ & $\begin{array}{l}21.5051 \\
21.6388 \\
21.7725 \\
21.9062 \\
22.0399 \\
22.1737 \\
22.3073 \\
22.4410 \\
22.5747 \\
22.7084\end{array}$ & $\begin{array}{r}997.21 \\
1005.03 \\
1012.85 \\
1020.66 \\
1028.47 \\
1036.28 \\
1044.07 \\
1051.87 \\
1059.65 \\
1067.44\end{array}$ & $\begin{array}{l}678.85 \\
684.69 \\
690.53 \\
696.36 \\
702.19 \\
708.01 \\
713.83 \\
719.65 \\
725.45 \\
731.26\end{array}$ & $\begin{array}{l}11.888 \\
11.912 \\
11.936 \\
11.960 \\
11.984 \\
12.008 \\
12.031 \\
12.054 \\
12.077 \\
12.100\end{array}$ & $\begin{array}{l}462.00 \\
464.00 \\
466.00 \\
468.00 \\
470.00 \\
472.00 \\
474.00 \\
476.00 \\
478.00 \\
480.00\end{array}$ & $\begin{array}{l}30.8499 \\
30.9829 \\
31.1159 \\
31.2489 \\
31.3818 \\
31.5148 \\
31.6477 \\
31.7806 \\
31.9136 \\
32.0465\end{array}$ & $\begin{array}{l}1526.51 \\
1533.80 \\
1541.07 \\
1548.33 \\
1555.59 \\
1562.85 \\
1570.09 \\
1577.33 \\
1584.56 \\
1591.79\end{array}$ & $\begin{array}{l}1069.81 \\
1075.12 \\
1080.42 \\
1085.72 \\
1091.01 \\
1096.29 \\
1101.57 \\
1106.84 \\
1112.11 \\
1117.37\end{array}$ & $\begin{array}{l}13.256 \\
13.272 \\
13.288 \\
13.303 \\
13.319 \\
13.334 \\
13.349 \\
13.365 \\
13.380 \\
13.395\end{array}$ \\
\hline $\begin{array}{l}342.00 \\
344.00 \\
346.00 \\
348.00 \\
350.00 \\
352.00 \\
354.00 \\
356.00 \\
358.00 \\
360.00\end{array}$ & $\begin{array}{l}22.8420 \\
22.9757 \\
23.1094 \\
23.2430 \\
23.3766 \\
23.5103 \\
23.6439 \\
23.7775 \\
23.9111 \\
24.0447\end{array}$ & $\begin{array}{l}1075.21 \\
1082.98 \\
1090.74 \\
1098.50 \\
1106.24 \\
1113.98 \\
1121.72 \\
1129.44 \\
1137.16 \\
1144.87\end{array}$ & $\begin{array}{l}737.05 \\
742.84 \\
748.62 \\
754.40 \\
760.17 \\
765.93 \\
771.69 \\
777.43 \\
783.17 \\
788.90\end{array}$ & $\begin{array}{l}12.123 \\
12.146 \\
12.168 \\
12.191 \\
12.213 \\
12.235 \\
12.257 \\
12.279 \\
12.300 \\
12.322\end{array}$ & $\begin{array}{l}482.00 \\
484.00 \\
486.00 \\
488.00 \\
490.00 \\
492.00 \\
494.00 \\
496.00 \\
498.00 \\
500.00\end{array}$ & $\begin{array}{l}32.1794 \\
32.3124 \\
32.4453 \\
32.5783 \\
32.7112 \\
32.8442 \\
32.9773 \\
33.1103 \\
33.2434 \\
33.3766\end{array}$ & $\begin{array}{l}1599.01 \\
1606.23 \\
1613.44 \\
1620.65 \\
1627.85 \\
1635.04 \\
1642.24 \\
1649.42 \\
1656.61 \\
1663.78\end{array}$ & $\begin{array}{l}1122.62 \\
1127.87 \\
1133.11 \\
1138.35 \\
1143.58 \\
1148.81 \\
1154.03 \\
1159.25 \\
1164.46 \\
1169.67\end{array}$ & $\begin{array}{l}13.410 \\
13.425 \\
13.440 \\
13.454 \\
13.469 \\
13.484 \\
13.498 \\
13.513 \\
13.527 \\
13.542\end{array}$ \\
\hline $\begin{array}{l}362.00 \\
364.00 \\
366.00 \\
368.00 \\
370.00 \\
372.00 \\
374.00 \\
376.00 \\
378.00 \\
380.00\end{array}$ & $\begin{array}{l}24.1783 \\
24.3118 \\
24.4454 \\
24.5789 \\
24.7125 \\
24.8460 \\
24.9795 \\
25.1130 \\
25.2466 \\
25.3801\end{array}$ & $\begin{array}{l}1152.56 \\
1160.25 \\
1167.93 \\
1175.61 \\
1183.27 \\
1190.92 \\
1198.57 \\
1206.21 \\
1213.84 \\
1221.46\end{array}$ & $\begin{array}{l}794.62 \\
800.34 \\
806.04 \\
811.73 \\
817.42 \\
823.10 \\
828.77 \\
834.43 \\
840.08 \\
845.72\end{array}$ & $\begin{array}{l}12.343 \\
12.364 \\
12.385 \\
12.406 \\
12.427 \\
12.448 \\
12.468 \\
12.488 \\
12.509 \\
12.529\end{array}$ & $\begin{array}{l}502.00 \\
504.00 \\
506.00 \\
508.00 \\
510.00 \\
512.00 \\
514.00 \\
516.00 \\
518.00 \\
520.00\end{array}$ & $\begin{array}{l}33.5097 \\
33.6430 \\
33.7762 \\
33.9096 \\
34.0430 \\
34.1764 \\
34.3099 \\
34.4435 \\
34.5771 \\
34.7108\end{array}$ & $\begin{array}{l}1670.96 \\
1678.13 \\
1685.30 \\
1692.47 \\
1699.63 \\
1706.79 \\
1713.94 \\
1721.10 \\
1728.25 \\
1735.39\end{array}$ & $\begin{array}{l}1174.87 \\
1180.07 \\
1185.27 \\
1190.46 \\
1195.65 \\
1200.83 \\
1206.01 \\
1211.19 \\
1216.36 \\
1221.53\end{array}$ & $\begin{array}{l}13.556 \\
13.570 \\
13.585 \\
13.599 \\
13.613 \\
13.627 \\
13.641 \\
13.655 \\
13.668 \\
13.682\end{array}$ \\
\hline $\begin{array}{l}382.00 \\
384.00 \\
386.00 \\
388.00 \\
390.00 \\
392.00 \\
394.00 \\
396.00 \\
398.00 \\
400.00\end{array}$ & $\begin{array}{l}25.5136 \\
25.6471 \\
25.7806 \\
25.9141 \\
26.0477 \\
26.1812 \\
26.3147 \\
26.4482 \\
26.5817 \\
26.7152\end{array}$ & $\begin{array}{l}1229.07 \\
1236.67 \\
1244.26 \\
1251.85 \\
1259.42 \\
1266.99 \\
1274.55 \\
1282.10 \\
1289.64 \\
1297.17\end{array}$ & $\begin{array}{l}851.36 \\
856.98 \\
862.60 \\
868.21 \\
873.81 \\
879.40 \\
884.98 \\
890.55 \\
896.12 \\
901.67\end{array}$ & $\begin{array}{l}12.549 \\
12.569 \\
12.588 \\
12.608 \\
12.627 \\
12.647 \\
12.666 \\
12.685 \\
12.704 \\
12.723\end{array}$ & $\begin{array}{l}522.00 \\
524.00 \\
526.00 \\
528.00 \\
530.00 \\
532.00 \\
534.00 \\
536.00 \\
538.00 \\
540.00\end{array}$ & $\begin{array}{l}34.8446 \\
34.9784 \\
35.1122 \\
35.2462 \\
35.3801 \\
35.5141 \\
35.6481 \\
35.7821 \\
35.9162 \\
36.0503\end{array}$ & $\begin{array}{l}1742.54 \\
1749.68 \\
1756.82 \\
1763.96 \\
1771.09 \\
1778.22 \\
1785.35 \\
1792.47 \\
1799.59 \\
1806.71\end{array}$ & $\begin{array}{l}1226.69 \\
1231.85 \\
1237.01 \\
1242.16 \\
1247.31 \\
1252.46 \\
1257.60 \\
1262.74 \\
1267.88 \\
1273.01\end{array}$ & $\begin{array}{l}13.696 \\
13.710 \\
13.723 \\
13.737 \\
13.750 \\
13.764 \\
13.777 \\
13.790 \\
13.804 \\
13.817\end{array}$ \\
\hline
\end{tabular}


TEMPER- SPECIFIC ENTHALPY INTERNAL ENTROPY ATURE VOLUME (BTU/L8) ENERGY (8TU/L8-R) (R) (CU FT/LB)
( $8 \mathrm{TU} / \mathrm{LB}$ )
TEMPER-

A TURE

(R)
SPECIFIC

VOLUME ( CU FT/LB)
ENTHALPY

(8TU/LB)

INTERNAL ENTROPY

ENERGY (8TU/L8)
(BTU/L8-R

\begin{tabular}{|c|c|c|c|c|c|c|c|c|c|}
\hline & & & & & $\begin{array}{l}122.00 \\
124.00 \\
126.00 \\
128.00 \\
130.00 \\
132.00 \\
134.00 \\
136.00 \\
138.00 \\
140.00\end{array}$ & $\begin{array}{l}7.0855 \\
7.2101 \\
7.3344 \\
7.4585 \\
7.5824 \\
7.7061 \\
7.8296 \\
7.9530 \\
8.0761 \\
8.1991\end{array}$ & $\begin{array}{l}294.68 \\
300.16 \\
305.66 \\
311.18 \\
316.73 \\
322.29 \\
327.89 \\
333.51 \\
339.16 \\
344.84\end{array}$ & $\begin{array}{l}176.67 \\
180.08 \\
183.51 \\
186.96 \\
190.44 \\
193.95 \\
197.49 \\
201.05 \\
204.65 \\
208.28\end{array}$ & $\begin{array}{l}8.472 \\
8.517 \\
8.561 \\
8.604 \\
8.647 \\
8.689 \\
8.732 \\
8.773 \\
8.814 \\
8.855\end{array}$ \\
\hline & & & & & $\begin{array}{l}142.00 \\
144.00 \\
146.00 \\
148.00 \\
150.00 \\
152.00 \\
154.00 \\
156.00 \\
158.00 \\
160.00\end{array}$ & $\begin{array}{l}8.3220 \\
8.4447 \\
8.5672 \\
8.6896 \\
8.8119 \\
8.9341 \\
9.0562 \\
9.1781 \\
9.2999 \\
9.4217\end{array}$ & $\begin{array}{l}350.55 \\
356.29 \\
362.07 \\
367.89 \\
373.74 \\
379.63 \\
385.56 \\
391.53 \\
397.54 \\
403.60\end{array}$ & $\begin{array}{l}211.95 \\
215.65 \\
219.39 \\
223.16 \\
226.98 \\
230.84 \\
234.73 \\
238.67 \\
242.66 \\
246.68\end{array}$ & $\begin{array}{l}8.896 \\
8.936 \\
8.976 \\
9.015 \\
9.055 \\
9.094 \\
9.132 \\
9.171 \\
9.209 \\
9.247\end{array}$ \\
\hline $\begin{array}{l}36.00 \\
38.00 \\
40.00\end{array}$ & $\begin{array}{l}.2231 \\
.2271 \\
.2316\end{array}$ & $\begin{array}{r}-108.89 \\
-104.27 \\
-99.33\end{array}$ & $\begin{array}{l}-112.60 \\
-108.05 \\
-103.19\end{array}$ & $\begin{array}{l}1.857 \\
1.982 \\
2.109\end{array}$ & $\begin{array}{l}162.00 \\
164.00 \\
166.00 \\
168.00 \\
170.00 \\
172.00 \\
174.00 \\
176.00 \\
178.00 \\
180.00\end{array}$ & $\begin{array}{r}9.5433 \\
9.6648 \\
9.7863 \\
9.9076 \\
10.0289 \\
10.1501 \\
10.2712 \\
10.3922 \\
10.5132 \\
10.6341\end{array}$ & $\begin{array}{l}409.69 \\
415.83 \\
422.01 \\
428.24 \\
434.50 \\
440.81 \\
447.17 \\
453.56 \\
460.00 \\
466.48\end{array}$ & $\begin{array}{l}250.75 \\
254.87 \\
259.02 \\
263.23 \\
267.48 \\
271.77 \\
276.10 \\
280.48 \\
284.91 \\
289.37\end{array}$ & $\begin{array}{l}9.285 \\
9.323 \\
9.360 \\
9.398 \\
9.435 \\
9.472 \\
9.508 \\
9.545 \\
9.581 \\
9.617\end{array}$ \\
\hline $\begin{array}{l}42.00 \\
44.00 \\
46.00 \\
48.00 \\
50.00 \\
* 50.962 \\
* 50.962 \\
52.00 \\
54.00 \\
56.00 \\
58.00 \\
60.00\end{array}$ & $\begin{array}{r}.2367 \\
.2426 \\
.2495 \\
.2579 \\
.2685 \\
.2749 \\
2.0629 \\
2.1845 \\
2.3921 \\
2.5789 \\
2.7531 \\
2.9187\end{array}$ & $\begin{array}{r}-94.01 \\
-88.26 \\
-81.98 \\
-75.03 \\
-67.13 \\
-62.86 \\
84.88 \\
90.23 \\
99.12 \\
106.94 \\
114.13 \\
120.91\end{array}$ & $\begin{array}{r}-97.96 \\
-92.30 \\
-86.14 \\
-79.32 \\
-71.61 \\
-72.09 \\
50.52 \\
53.85 \\
59.28 \\
63.99 \\
68.28 \\
72.30\end{array}$ & $\begin{array}{l}2.238 \\
2.372 \\
2.512 \\
2.660 \\
2.821 \\
2.905 \\
5.809 \\
5.913 \\
6.081 \\
6.223 \\
6.349 \\
6.464\end{array}$ & $\begin{array}{l}182.00 \\
184.00 \\
186.00 \\
188.00 \\
190.00 \\
192.00 \\
194.00 \\
196.00 \\
198.00 \\
200.00\end{array}$ & $\begin{array}{l}10.7549 \\
10.8756 \\
10.9962 \\
11.1167 \\
11.2372 \\
11.3577 \\
11.4781 \\
11.5984 \\
11.7187 \\
11.8389\end{array}$ & $\begin{array}{l}473.00 \\
479.55 \\
486.15 \\
492.79 \\
499.48 \\
506.20 \\
512.97 \\
519.78 \\
526.63 \\
533.51\end{array}$ & $\begin{array}{l}293.88 \\
298.42 \\
303.01 \\
307.65 \\
312.32 \\
317.04 \\
321.81 \\
326.61 \\
331.45 \\
336.34\end{array}$ & $\begin{array}{l}9.653 \\
9.689 \\
9.725 \\
9.760 \\
9.796 \\
9.831 \\
9.866 \\
9.901 \\
9.936 \\
9.970\end{array}$ \\
\hline $\begin{array}{l}62.00 \\
64.00 \\
66.00 \\
68.00 \\
70.00 \\
72.00 \\
74.00 \\
76.00 \\
78.00 \\
80.00\end{array}$ & $\begin{array}{l}3.0781 \\
3.2328 \\
3.3838 \\
3.5316 \\
3.6770 \\
3.8201 \\
3.9613 \\
4.1009 \\
4.2391 \\
4.3759\end{array}$ & $\begin{array}{l}127.41 \\
133.70 \\
139.83 \\
145.84 \\
151.76 \\
157.59 \\
163.35 \\
169.06 \\
174.72 \\
180.33\end{array}$ & $\begin{array}{r}76.14 \\
79.86 \\
83.48 \\
87.03 \\
90.52 \\
93.97 \\
97.38 \\
100.76 \\
104.12 \\
107.45\end{array}$ & $\begin{array}{l}6.571 \\
6.671 \\
6.765 \\
6.855 \\
6.940 \\
7.023 \\
7.101 \\
7.178 \\
7.251 \\
7.322\end{array}$ & $\begin{array}{l}202.00 \\
204.00 \\
206.00 \\
208.00 \\
210.00 \\
212.00 \\
214.00 \\
216.00 \\
218.00 \\
220.00\end{array}$ & $\begin{array}{l}11.9591 \\
12.0793 \\
12.1994 \\
12.3194 \\
12.4394 \\
12.5594 \\
12.6793 \\
12.7993 \\
12.9191 \\
13.0390\end{array}$ & $\begin{array}{l}540.44 \\
547.41 \\
554.41 \\
561.46 \\
568.53 \\
575.65 \\
582.80 \\
589.98 \\
597.19 \\
604.44\end{array}$ & $\begin{array}{l}341.27 \\
346.23 \\
351.24 \\
356.28 \\
361.36 \\
366.47 \\
371.63 \\
376.81 \\
382.03 \\
387.28\end{array}$ & $\begin{array}{l}10.005 \\
10.039 \\
10.073 \\
10.107 \\
10.141 \\
10.175 \\
10.208 \\
10.242 \\
10.275 \\
10.308\end{array}$ \\
\hline $\begin{array}{r}82.00 \\
84.00 \\
86.00 \\
88.00 \\
90.00 \\
92.00 \\
94.00 \\
96.00 \\
98.00 \\
100.00\end{array}$ & $\begin{array}{l}4.5116 \\
4.6462 \\
4.7799 \\
4.9127 \\
5.0447 \\
5.1760 \\
5.3066 \\
5.4366 \\
5.5659 \\
5.6948\end{array}$ & $\begin{array}{l}185.91 \\
191.46 \\
196.97 \\
202.47 \\
207.94 \\
213.39 \\
218.83 \\
224.25 \\
229.67 \\
235.08\end{array}$ & $\begin{array}{l}110.77 \\
114.07 \\
117.37 \\
120.65 \\
123.92 \\
127.19 \\
130.45 \\
133.71 \\
136.97 \\
140.23\end{array}$ & $\begin{array}{l}7.391 \\
7.458 \\
7.523 \\
7.586 \\
7.647 \\
7.707 \\
7.766 \\
7.823 \\
7.879 \\
7.933\end{array}$ & $\begin{array}{l}222.00 \\
224.00 \\
226.00 \\
228.00 \\
230.00 \\
232.00 \\
234.00 \\
236.00 \\
238.00 \\
240.00\end{array}$ & $\begin{array}{l}13.1588 \\
13.2785 \\
13.3983 \\
13.5180 \\
13.6377 \\
13.7574 \\
13.8770 \\
13.9966 \\
14.1162 \\
14.2358\end{array}$ & $\begin{array}{l}611.72 \\
619.02 \\
626.36 \\
633.73 \\
641.12 \\
648.54 \\
655.99 \\
663.47 \\
670.97 \\
678.49\end{array}$ & $\begin{array}{l}392.56 \\
397.87 \\
403.21 \\
408.59 \\
413.99 \\
419.42 \\
424.87 \\
430.36 \\
435.87 \\
441.40\end{array}$ & $\begin{array}{l}10.341 \\
10.374 \\
10.406 \\
10.439 \\
10.471 \\
10.503 \\
10.535 \\
10.567 \\
10.599 \\
10.630\end{array}$ \\
\hline $\begin{array}{l}102.00 \\
104.00 \\
106.00 \\
108.00 \\
110.00 \\
112.00 \\
114.00 \\
116.00 \\
118.00 \\
120.00\end{array}$ & $\begin{array}{l}5.8232 \\
5.9511 \\
6.0785 \\
6.2056 \\
6.3322 \\
6.4585 \\
6.5845 \\
6.7102 \\
6.8356 \\
6.9607\end{array}$ & $\begin{array}{l}240.48 \\
245.88 \\
251.28 \\
256.68 \\
262.08 \\
267.49 \\
272.90 \\
278.33 \\
283.77 \\
289.22\end{array}$ & $\begin{array}{l}143.50 \\
146.77 \\
150.04 \\
153.32 \\
156.62 \\
159.92 \\
163.24 \\
166.57 \\
169.92 \\
173.29\end{array}$ & $\begin{array}{l}7.987 \\
8.039 \\
8.091 \\
8.141 \\
8.191 \\
8.239 \\
8.287 \\
8.335 \\
8.381 \\
8.427\end{array}$ & $\begin{array}{l}242.00 \\
244.00 \\
246.00 \\
248.00 \\
250.00 \\
252.00 \\
254.00 \\
256.00 \\
258.00 \\
260.00\end{array}$ & $\begin{array}{l}14.3554 \\
14.4749 \\
14.5944 \\
14.7139 \\
14.8334 \\
14.9528 \\
15.0723 \\
15.1917 \\
15.3111 \\
15.4305\end{array}$ & $\begin{array}{l}686.04 \\
693.61 \\
701.20 \\
708.82 \\
716.45 \\
724.10 \\
731.77 \\
739.46 \\
747.16 \\
754.88\end{array}$ & $\begin{array}{l}446.96 \\
452.53 \\
458.14 \\
463.76 \\
469.40 \\
475.06 \\
480.75 \\
486.44 \\
492.16 \\
497.89\end{array}$ & $\begin{array}{l}10.662 \\
10.693 \\
10.724 \\
10.755 \\
10.785 \\
10.816 \\
10.846 \\
10.876 \\
10.906 \\
10.936\end{array}$ \\
\hline
\end{tabular}


TEMPER- SPECIFIC ENTHALPY INTERNAL ENTROPY ATURE VOLUME (BTU/L8) ENERGY (BTU/L8-R) (R) (CU FT/L8)
TEMPER(8RU/L8) $\begin{array}{lll}\text { TEMPER- } & \text { SPECIFIC } & \text { ENTHALPY } \\ \text { ATURE } & \text { VOLUME } & \text { (8TU/LB) }\end{array}$

(R) (CU FT/L8)
INTERNAL ENTROPY

ENERGY (BTU/LB-R)

(BrU/L8)

\begin{tabular}{|c|c|c|c|c|c|c|c|c|c|}
\hline $\begin{array}{l}262.00 \\
264.00 \\
266.00 \\
268.00 \\
270.00 \\
272.00 \\
274.00 \\
276.00 \\
278.00 \\
280.00\end{array}$ & $\begin{array}{l}15.5498 \\
15.6692 \\
15.7885 \\
15.9078 \\
16.0271 \\
16.1464 \\
16.2657 \\
16.3850 \\
16.5042 \\
16.6234\end{array}$ & $\begin{array}{l}762.62 \\
770.37 \\
778.13 \\
785.90 \\
793.69 \\
801.46 \\
809.24 \\
817.03 \\
824.83 \\
832.63\end{array}$ & $\begin{array}{l}503.64 \\
509.40 \\
515.17 \\
520.96 \\
526.76 \\
532.55 \\
538.34 \\
544.14 \\
549.95 \\
555.77\end{array}$ & $\begin{array}{l}10.966 \\
10.995 \\
11.024 \\
11.053 \\
11.082 \\
11.111 \\
11.140 \\
11.168 \\
11.196 \\
11.224\end{array}$ & $\begin{array}{l}402.00 \\
404.00 \\
406.00 \\
408.00 \\
410.00 \\
412.00 \\
414.00 \\
416.00 \\
418.00 \\
420.00\end{array}$ & $\begin{array}{l}23.8761 \\
23.9948 \\
24.1135 \\
24.2322 \\
24.3509 \\
24.4696 \\
24.5883 \\
24.7071 \\
24.8258 \\
24.9446\end{array}$ & $\begin{array}{l}1304.70 \\
1312.22 \\
1319.73 \\
1327.23 \\
1334.72 \\
1342.20 \\
1349.67 \\
1357.14 \\
1364.59 \\
1372.04\end{array}$ & $\begin{array}{l}907.05 \\
912.59 \\
918.12 \\
923.64 \\
929.16 \\
934.66 \\
940.16 \\
945.65 \\
951.13 \\
956.60\end{array}$ & $\begin{array}{l}12.625 \\
12.644 \\
12.662 \\
12.681 \\
12.699 \\
12.717 \\
12.735 \\
12.753 \\
12.771 \\
12.789\end{array}$ \\
\hline $\begin{array}{l}282.00 \\
284.00 \\
286.00 \\
288.00 \\
290.00 \\
292.00 \\
294.00 \\
296.00 \\
298.00 \\
300.00\end{array}$ & $\begin{array}{l}16.7426 \\
16.8618 \\
16.9810 \\
17.1001 \\
17.2193 \\
17.3384 \\
17.4575 \\
17.5766 \\
17.6957 \\
17.8148\end{array}$ & $\begin{array}{l}840.44 \\
848.26 \\
856.08 \\
863.90 \\
871.73 \\
879.56 \\
887.40 \\
895.24 \\
903.08 \\
910.92\end{array}$ & $\begin{array}{l}561.60 \\
567.43 \\
573.26 \\
579.10 \\
584.95 \\
590.80 \\
596.65 \\
602.50 \\
608.36 \\
614.22\end{array}$ & $\begin{array}{l}11.252 \\
11.279 \\
11.307 \\
11.334 \\
11.361 \\
11.388 \\
11.415 \\
11.441 \\
11.468 \\
11.494\end{array}$ & $\begin{array}{l}422.00 \\
424.00 \\
426.00 \\
428.00 \\
430.00 \\
432.00 \\
434.00 \\
436.00 \\
438.00 \\
440.00\end{array}$ & $\begin{array}{l}25.0633 \\
25.1820 \\
25.3007 \\
25.4194 \\
25.5380 \\
25.6567 \\
25.7753 \\
25.8939 \\
26.0125 \\
26.1311\end{array}$ & $\begin{array}{l}1379.48 \\
1386.91 \\
1394.34 \\
1401.75 \\
1409.15 \\
1416.55 \\
1423.94 \\
1431.32 \\
1438.69 \\
1446.05\end{array}$ & $\begin{array}{r}962.06 \\
967.51 \\
972.96 \\
978.40 \\
983.82 \\
989.24 \\
994.66 \\
1000.06 \\
1005.45 \\
1010.84\end{array}$ & $\begin{array}{l}12.807 \\
12.824 \\
12.842 \\
12.859 \\
12.876 \\
12.894 \\
12.911 \\
12.928 \\
12.944 \\
12.961\end{array}$ \\
\hline $\begin{array}{l}302.00 \\
304.00 \\
306.00 \\
308.00 \\
310.00 \\
312.00 \\
314.00 \\
316.00 \\
318.00 \\
320.00\end{array}$ & $\begin{array}{l}17.9339 \\
18.0529 \\
18.1720 \\
18.2910 \\
18.4100 \\
18.5290 \\
18.6480 \\
18.7670 \\
18.8860 \\
19.0049\end{array}$ & $\begin{array}{l}918.76 \\
926.60 \\
934.45 \\
942.29 \\
950.13 \\
957.97 \\
965.81 \\
973.64 \\
981.48 \\
989.31\end{array}$ & $\begin{array}{l}620.08 \\
625.94 \\
631.80 \\
637.66 \\
643.51 \\
649.37 \\
655.23 \\
661.08 \\
666.93 \\
672.78\end{array}$ & $\begin{array}{l}11.520 \\
11.546 \\
11.572 \\
11.597 \\
11.623 \\
11.648 \\
11.673 \\
11.698 \\
11.722 \\
11.747\end{array}$ & $\begin{array}{l}442.00 \\
444.00 \\
446.00 \\
448.00 \\
450.00 \\
452.00 \\
454.00 \\
456.00 \\
458.00 \\
460.00\end{array}$ & $\begin{array}{l}26.2496 \\
26.3682 \\
26.4867 \\
26.6051 \\
26.7236 \\
26.8420 \\
26.9604 \\
27.0788 \\
27.1971 \\
27.3154\end{array}$ & $\begin{array}{l}1453.40 \\
1460.74 \\
1468.08 \\
1475.41 \\
1482.73 \\
1490.05 \\
1497.37 \\
1504.69 \\
1511.99 \\
1519.29\end{array}$ & $\begin{array}{l}1016.22 \\
1021.59 \\
1026.95 \\
1032.30 \\
1037.65 \\
1043.01 \\
1048.35 \\
1053.70 \\
1059.03 \\
1064.35\end{array}$ & $\begin{array}{l}12.978 \\
12.994 \\
13.011 \\
13.027 \\
13.044 \\
13.060 \\
13.076 \\
13.092 \\
13.108 \\
13.124\end{array}$ \\
\hline $\begin{array}{l}322.00 \\
324.00 \\
326.00 \\
328.00 \\
330.00 \\
332.00 \\
334.00 \\
336.00 \\
338.00 \\
340.00\end{array}$ & $\begin{array}{l}19.1239 \\
19.2429 \\
19.3618 \\
19.4807 \\
19.5996 \\
19.7186 \\
19.8375 \\
19.9564 \\
20.0752 \\
20.1941\end{array}$ & $\begin{array}{r}997.13 \\
1004.96 \\
1012.78 \\
1020.59 \\
1028.40 \\
1036.21 \\
1044.01 \\
1051.81 \\
1059.60 \\
1067.38\end{array}$ & $\begin{array}{l}678.63 \\
684.47 \\
690.31 \\
696.15 \\
701.98 \\
707.80 \\
713.62 \\
719.44 \\
725.25 \\
731.05\end{array}$ & $\begin{array}{l}11.771 \\
11.796 \\
11.820 \\
11.844 \\
11.867 \\
11.891 \\
11.914 \\
11.938 \\
11.961 \\
11.984\end{array}$ & $\begin{array}{l}462.00 \\
464.00 \\
466.00 \\
468.00 \\
470.00 \\
472.00 \\
474.00 \\
476.00 \\
478.00 \\
480.00\end{array}$ & $\begin{array}{l}27.4337 \\
27.5520 \\
27.6703 \\
27.7885 \\
27.9067 \\
28.0249 \\
28.1431 \\
28.2613 \\
28.3795 \\
28.4977\end{array}$ & $\begin{array}{l}1526.58 \\
1533.86 \\
1541.13 \\
1548.40 \\
1555.66 \\
1562.91 \\
1570.16 \\
1577.40 \\
1584.64 \\
1591.87\end{array}$ & $\begin{array}{l}1069.67 \\
1074.99 \\
1080.29 \\
1085.59 \\
1090.88 \\
1096.17 \\
1101.44 \\
1106.72 \\
1111.98 \\
1117.24\end{array}$ & $\begin{array}{l}13.140 \\
13.155 \\
13.171 \\
13.187 \\
13.202 \\
13.218 \\
13.233 \\
13.248 \\
13.263 \\
13.278\end{array}$ \\
\hline $\begin{array}{l}342.00 \\
344.00 \\
346.00 \\
348.00 \\
350.00 \\
352.00 \\
354.00 \\
356.00 \\
358.00 \\
360.00\end{array}$ & $\begin{array}{l}20.3130 \\
20.4319 \\
20.5507 \\
20.6696 \\
20.7885 \\
20.9073 \\
21.0261 \\
21.1450 \\
21.2638 \\
21.3826\end{array}$ & $\begin{array}{l}1075.16 \\
1082.93 \\
1090.69 \\
1098.45 \\
1106.20 \\
1113.94 \\
1121.68 \\
1129.41 \\
1137.13 \\
1144.84\end{array}$ & $\begin{array}{l}736.85 \\
742.64 \\
748.43 \\
754.20 \\
759.97 \\
765.74 \\
771.49 \\
777.24 \\
782.98 \\
788.71\end{array}$ & $\begin{array}{l}12.006 \\
12.029 \\
12.052 \\
12.074 \\
12.096 \\
12.118 \\
12.140 \\
12.162 \\
12.184 \\
12.205\end{array}$ & $\begin{array}{l}482.00 \\
484.00 \\
486.00 \\
488.00 \\
490.00 \\
492.00 \\
494.00 \\
496.00 \\
498.00 \\
500.00\end{array}$ & $\begin{array}{l}28.6159 \\
28.7341 \\
28.8523 \\
28.9705 \\
29.0887 \\
29.2070 \\
29.3253 \\
29.4436 \\
29.5619 \\
29.6803\end{array}$ & $\begin{array}{l}1599.09 \\
1606.31 \\
1613.52 \\
1620.73 \\
1627.93 \\
1635.13 \\
1642.32 \\
1649.51 \\
1656.69 \\
1663.87\end{array}$ & $\begin{array}{l}1122.50 \\
1127.75 \\
1132.99 \\
1138.23 \\
1143.46 \\
1148.69 \\
1153.91 \\
1159.13 \\
1164.34 \\
1169.55\end{array}$ & $\begin{array}{l}13.293 \\
13.308 \\
13.323 \\
13.338 \\
13.353 \\
13.367 \\
13.382 \\
13.397 \\
13.411 \\
13.425\end{array}$ \\
\hline $\begin{array}{l}362.00 \\
364.00 \\
366.00 \\
368.00 \\
370.00 \\
372.00 \\
374.00 \\
376.00 \\
378.00 \\
380.00\end{array}$ & $\begin{array}{l}21.5014 \\
21.6202 \\
21.7389 \\
21.8577 \\
21.9764 \\
22.0952 \\
22.2139 \\
22.3327 \\
22.4514 \\
22.5701\end{array}$ & $\begin{array}{l}1152.54 \\
1160.23 \\
1167.91 \\
1175.58 \\
1183.25 \\
1190.91 \\
1198.55 \\
1206.19 \\
1213.82 \\
1221.45\end{array}$ & $\begin{array}{l}794.44 \\
800.15 \\
805.85 \\
811.55 \\
817.24 \\
822.92 \\
828.59 \\
834.25 \\
839.90 \\
845.55\end{array}$ & $\begin{array}{l}12.226 \\
12.248 \\
12.269 \\
12.290 \\
12.310 \\
12.331 \\
12.351 \\
12.372 \\
12.392 \\
12.412\end{array}$ & $\begin{array}{l}502.00 \\
504.00 \\
506.00 \\
508.00 \\
510.00 \\
512.00 \\
514.00 \\
516.00 \\
518.00 \\
520.00\end{array}$ & $\begin{array}{l}29.7987 \\
29.9171 \\
30.0356 \\
30.1542 \\
30.2728 \\
30.3914 \\
30.5101 \\
30.6288 \\
30.7476 \\
30.8665\end{array}$ & $\begin{array}{l}1671.05 \\
1678.22 \\
1685.39 \\
1692.55 \\
1699.72 \\
1706.88 \\
1714.03 \\
1721.19 \\
1728.34 \\
1735.49\end{array}$ & $\begin{array}{l}1174.76 \\
1179.96 \\
1185.15 \\
1190.34 \\
1195.53 \\
1200.72 \\
1205.90 \\
1211.07 \\
1216.24 \\
1221.41\end{array}$ & $\begin{array}{l}13.440 \\
13.454 \\
13.468 \\
13.482 \\
13.496 \\
13.510 \\
13.524 \\
13.538 \\
13.552 \\
13.566\end{array}$ \\
\hline $\begin{array}{l}382.00 \\
384.00 \\
386.00 \\
388.00 \\
390.00 \\
392.00 \\
394.00 \\
396.00 \\
398.00 \\
400.00\end{array}$ & $\begin{array}{l}22.6889 \\
22.8076 \\
22.9263 \\
23.0450 \\
23.1638 \\
23.2825 \\
23.4012 \\
23.5199 \\
23.6386 \\
23.7574\end{array}$ & $\begin{array}{l}1229.06 \\
1236.66 \\
1244.26 \\
1251.85 \\
1259.42 \\
1266.99 \\
1274.55 \\
1282.10 \\
1289.65 \\
1297.18\end{array}$ & $\begin{array}{l}851.18 \\
856.81 \\
862.43 \\
868.04 \\
873.64 \\
879.23 \\
884.81 \\
890.39 \\
895.95 \\
901.51\end{array}$ & $\begin{array}{l}12.432 \\
12.452 \\
12.472 \\
12.491 \\
12.511 \\
12.530 \\
12.549 \\
12.569 \\
12.588 \\
12.606\end{array}$ & $\begin{array}{l}522.00 \\
524.00 \\
526.00 \\
528.00 \\
530.00 \\
532.00 \\
534.00 \\
536.00 \\
538.00 \\
540.00\end{array}$ & $\begin{array}{l}30.9854 \\
31.1043 \\
31.2233 \\
31.3424 \\
31.4614 \\
31.5805 \\
31.6997 \\
31.8188 \\
31.9380 \\
32.0571\end{array}$ & $\begin{array}{l}1742.63 \\
1749.78 \\
1756.92 \\
1764.05 \\
1771.19 \\
1778.32 \\
1785.44 \\
1792.57 \\
1799.69 \\
1806.81\end{array}$ & $\begin{array}{l}1226.58 \\
1231.74 \\
1236.90 \\
1242.05 \\
1247.20 \\
1252.35 \\
1257.49 \\
1262.63 \\
1267.77 \\
1272.90\end{array}$ & $\begin{array}{l}13.580 \\
13.593 \\
13.607 \\
13.620 \\
13.634 \\
13.647 \\
13.661 \\
13.674 \\
13.687 \\
13.700\end{array}$ \\
\hline
\end{tabular}



$\begin{array}{ccccc}\text { TEMPER- } & \text { SPECIFIC } & \text { ENTHALPY } & \text { INTERNAL ENTROPY } \\ \text { ATURE } & \text { VOLUME } & \text { (8TU/L8) ENERGY } & \text { (8TU/L8-R) }\end{array}$ (R) (CU FT/L8) ( $8 T U / L 8$ )
TEMPER-

VPECIFIC ENTHALPY (R) (CU FT/L8)
INTERNAL ENTROPY (BTU/L8)

( $8 T U / L B-R$ )

\begin{tabular}{|c|c|c|c|c|c|c|c|c|c|}
\hline & & & & & $\begin{array}{l}122.00 \\
124.00 \\
126.00 \\
128.00 \\
130.00 \\
132.00 \\
134.00 \\
136.00 \\
138.00 \\
140.00\end{array}$ & $\begin{array}{l}6.3641 \\
6.4769 \\
6.5894 \\
6.7017 \\
6.8137 \\
6.9256 \\
7.0373 \\
7.1488 \\
7.2601 \\
7.3713\end{array}$ & $\begin{array}{l}293.72 \\
299.23 \\
304.75 \\
310.30 \\
315.87 \\
321.46 \\
327.07 \\
332.72 \\
338.39 \\
344.09\end{array}$ & $\begin{array}{l}175.95 \\
179.37 \\
182.81 \\
186.28 \\
189.78 \\
193.30 \\
196.85 \\
200.43 \\
204.04 \\
207.68\end{array}$ & $\begin{array}{l}8.362 \\
8.407 \\
8.451 \\
8.495 \\
8.538 \\
8.581 \\
8.623 \\
8.665 \\
8.706 \\
8.747\end{array}$ \\
\hline & & & & & $\begin{array}{l}142.00 \\
144.00 \\
146.00 \\
148.00 \\
150.00 \\
152.00 \\
154.00 \\
156.00 \\
158.00 \\
160.00\end{array}$ & $\begin{array}{l}7.4823 \\
7.5931 \\
7.7038 \\
7.8144 \\
7.9249 \\
8.0352 \\
8.1454 \\
8.2555 \\
8.3655 \\
8.4754\end{array}$ & $\begin{array}{l}349.82 \\
355.58 \\
361.38 \\
367.21 \\
373.08 \\
378.99 \\
384.94 \\
390.92 \\
396.95 \\
403.02\end{array}$ & $\begin{array}{l}211.36 \\
215.07 \\
218.82 \\
222.61 \\
226.43 \\
230.30 \\
234.21 \\
238.15 \\
242.15 \\
246.18\end{array}$ & $\begin{array}{l}8.788 \\
8.828 \\
8.868 \\
8.908 \\
8.947 \\
8.986 \\
9.025 \\
9.064 \\
9.102 \\
9.140\end{array}$ \\
\hline $\begin{array}{l}36.00 \\
38.00 \\
40.00\end{array}$ & $\begin{array}{r}.2228 \\
.2268 \\
.2313\end{array}$ & $\begin{array}{r}-108.60 \\
-104.00 \\
-99.08\end{array}$ & $\begin{array}{l}-112.72 \\
-108.20 \\
-103.36\end{array}$ & $\begin{array}{l}1.854 \\
1.978 \\
2.104\end{array}$ & $\begin{array}{l}162.00 \\
164.00 \\
166.00 \\
168.00 \\
170.00 \\
172.00 \\
174.00 \\
176.00 \\
178.00 \\
180.00\end{array}$ & $\begin{array}{l}8.5852 \\
8.6949 \\
8.8045 \\
8.9140 \\
9.0234 \\
9.1328 \\
9.2421 \\
9.3513 \\
9.4604 \\
9.5694\end{array}$ & $\begin{array}{l}409.13 \\
415.28 \\
421.48 \\
427.71 \\
433.99 \\
440.32 \\
446.68 \\
453.09 \\
459.54 \\
466.03\end{array}$ & $\begin{array}{l}250.26 \\
254.38 \\
258.55 \\
262.76 \\
267.01 \\
271.31 \\
275.66 \\
280.04 \\
284.47 \\
288.95\end{array}$ & $\begin{array}{l}9.178 \\
9.216 \\
9.254 \\
9.291 \\
9.328 \\
9.365 \\
9.402 \\
9.438 \\
9.475 \\
9.511\end{array}$ \\
\hline $\begin{array}{l}42.00 \\
44.00 \\
46.00 \\
48.00 \\
50.00 \\
52.00 \\
* 52.067 \\
* 52.067 \\
54.00 \\
56.00 \\
58.00 \\
60.00\end{array}$ & $\begin{array}{r}.2363 \\
.2421 \\
.2489 \\
.2570 \\
.2673 \\
.2811 \\
.2818 \\
1.8328 \\
2.0419 \\
2.2272 \\
2.3956 \\
2.5532\end{array}$ & $\begin{array}{r}-93.79 \\
-88.07 \\
-81.84 \\
-74.96 \\
-67.19 \\
-58.05 \\
-57.71 \\
83.21 \\
93.44 \\
102.20 \\
110.00 \\
117.21\end{array}$ & $\begin{array}{l}-98.16 \\
-92.55 \\
-86.44 \\
-79.71 \\
-72.13 \\
-63.25 \\
-68.22 \\
49.29 \\
55.65 \\
60.99 \\
65.67 \\
69.96\end{array}$ & $\begin{array}{l}2.233 \\
2.366 \\
2.505 \\
2.651 \\
2.810 \\
2.989 \\
2.995 \\
5.707 \\
5.900 \\
6.059 \\
6.196 \\
6.318\end{array}$ & $\begin{array}{l}182.00 \\
184.00 \\
186.00 \\
188.00 \\
190.00 \\
192.00 \\
194.00 \\
196.00 \\
198.00 \\
200.00\end{array}$ & $\begin{array}{r}9.6784 \\
9.7872 \\
9.8960 \\
10.0047 \\
10.1134 \\
10.2220 \\
10.3305 \\
10.4390 \\
10.5475 \\
10.6559\end{array}$ & $\begin{array}{l}472.56 \\
479.12 \\
485.73 \\
492.38 \\
499.08 \\
505.81 \\
512.59 \\
519.40 \\
526.26 \\
533.16\end{array}$ & $\begin{array}{l}293.46 \\
298.01 \\
302.60 \\
307.24 \\
311.93 \\
316.65 \\
321.42 \\
326.23 \\
331.08 \\
335.97\end{array}$ & $\begin{array}{l}9.547 \\
9.583^{\circ} \\
9.619 \\
9.654 \\
9.690 \\
9.725 \\
9.760 \\
9.795 \\
9.830 \\
9.865\end{array}$ \\
\hline $\begin{array}{l}62.00 \\
64.00 \\
66.00 \\
68.00 \\
70.00 \\
72.00 \\
74.00 \\
76.00 \\
78.00 \\
80.00\end{array}$ & $\begin{array}{l}2.7034 \\
2.8481 \\
2.9885 \\
3.1256 \\
3.2599 \\
3.3918 \\
3.5217 \\
3.6499 \\
3.7765 \\
3.9018\end{array}$ & $\begin{array}{l}124.04 \\
130.59 \\
136.94 \\
143.13 \\
149.20 \\
155.17 \\
161.06 \\
166.87 \\
172.63 \\
178.34\end{array}$ & $\begin{array}{r}74.01 \\
77.88 \\
81.63 \\
85.29 \\
88.87 \\
92.40 \\
95.89 \\
99.33 \\
102.74 \\
106.13\end{array}$ & $\begin{array}{l}6.430 \\
6.534 \\
6.632 \\
6.724 \\
6.812 \\
6.896 \\
6.977 \\
7.055 \\
7.129 \\
7.202\end{array}$ & $\begin{array}{l}202.00 \\
204.00 \\
206.00 \\
208.00 \\
210.00 \\
212.00 \\
214.00 \\
216.00 \\
218.00 \\
220.00\end{array}$ & $\begin{array}{l}10.7642 \\
10.8725 \\
10.9808 \\
11.0890 \\
11.1972 \\
11.3053 \\
11.4134 \\
11.5215 \\
11.6295 \\
11.7375\end{array}$ & $\begin{array}{l}540.09 \\
547.06 \\
554.08 \\
561.13 \\
568.21 \\
575.33 \\
582.49 \\
589.68 \\
596.90 \\
604.15\end{array}$ & $\begin{array}{l}340.90 \\
345.87 \\
350.87 \\
355.92 \\
361.00 \\
366.12 \\
371.28 \\
376.47 \\
381.69 \\
386.94\end{array}$ & $\begin{array}{l}9.899 \\
9.933 \\
9.968 \\
10.002 \\
10.036 \\
10.069 \\
10.103 \\
10.136 \\
10.170 \\
10.203\end{array}$ \\
\hline $\begin{array}{r}82.00 \\
84.00 \\
86.00 \\
88.00 \\
90.00 \\
92.00 \\
94.00 \\
96.00 \\
98.00 \\
100.00\end{array}$ & $\begin{array}{l}4.0259 \\
4.1488 \\
4.2708 \\
4.3919 \\
4.5122 \\
4.6317 \\
4.7506 \\
4.8688 \\
4.9864 \\
5.1035\end{array}$ & $\begin{array}{l}184.00 \\
189.62 \\
195.21 \\
200.77 \\
206.31 \\
211.82 \\
217.31 \\
222.79 \\
228.26 \\
233.71\end{array}$ & $\begin{array}{l}109.50 \\
112.85 \\
116.18 \\
119.50 \\
122.81 \\
126.11 \\
129.40 \\
132.70 \\
135.98 \\
139.27\end{array}$ & $\begin{array}{l}7.272 \\
7.339 \\
7.405 \\
7.469 \\
7.531 \\
7.592 \\
7.651 \\
7.709 \\
7.765 \\
7.820\end{array}$ & $\begin{array}{l}222.00 \\
224.00 \\
226.00 \\
228.00 \\
230.00 \\
232.00 \\
234.00 \\
236.00 \\
238.00 \\
240.00\end{array}$ & $\begin{array}{l}11.8455 \\
11.9534 \\
12.0613 \\
12.1692 \\
12.2771 \\
12.3849 \\
12.4927 \\
12.6005 \\
12.7083 \\
12.8160\end{array}$ & $\begin{array}{l}611.43 \\
618.75 \\
626.09 \\
633.46 \\
640.86 \\
648.29 \\
655.74 \\
663.22 \\
670.73 \\
678.26\end{array}$ & $\begin{array}{l}392.23 \\
397.54 \\
402.89 \\
408.27 \\
413.67 \\
419.10 \\
424.56 \\
430.05 \\
435.56 \\
441.09\end{array}$ & $\begin{array}{l}10.236 \\
10.269 \\
10.301 \\
10.334 \\
10.366 \\
10.398 \\
10.430 \\
10.462 \\
10.494 \\
10.525\end{array}$ \\
\hline $\begin{array}{l}102.00 \\
104.00 \\
106.00 \\
108.00 \\
110.00 \\
112.00 \\
114.00 \\
116.00 \\
118.00 \\
120.00\end{array}$ & $\begin{array}{l}5: 2200 \\
5.3361 \\
5.4518 \\
5.5670 \\
5.6819 \\
5.7963 \\
5.9105 \\
6.0243 \\
6.1379 \\
6.2511\end{array}$ & $\begin{array}{l}239.16 \\
244.60 \\
250.04 \\
255.48 \\
260.92 \\
266.37 \\
271.82 \\
277.28 \\
282.74 \\
288.22\end{array}$ & $\begin{array}{l}142.56 \\
145.86 \\
149.16 \\
152.46 \\
155.78 \\
159.10 \\
162.44 \\
165.79 \\
169.16 \\
172.54\end{array}$ & $\begin{array}{l}7.874 \\
7.927 \\
7.979 \\
8.029 \\
8.079 \\
8.128 \\
8.177 \\
8.224 \\
8.271 \\
8.317\end{array}$ & $\begin{array}{l}242.00 \\
244.00 \\
246.00 \\
248.00 \\
250.00 \\
252.00 \\
254.00 \\
256.00 \\
258.00 \\
260.00\end{array}$ & $\begin{array}{l}12.9237 \\
13.0314 \\
13.1391 \\
13.2468 \\
13.3544 \\
13.4621 \\
13.5697 \\
13.6772 \\
13.7848 \\
13.8924\end{array}$ & $\begin{array}{l}685.81 \\
693.39 \\
700.98 \\
708.60 \\
716.24 \\
723.90 \\
731.57 \\
739.26 \\
746.97 \\
754.69\end{array}$ & $\begin{array}{l}446.65 \\
452.24 \\
457.84 \\
463.47 \\
469.11 \\
474.78 \\
480.46 \\
486.16 \\
491.88 \\
497.61\end{array}$ & $\begin{array}{l}10.556 \\
10.588 \\
10.619 \\
10.650 \\
10.680 \\
10.711 \\
10.741 \\
10.771 \\
10.801 \\
10.831\end{array}$ \\
\hline
\end{tabular}


TEMPER- SPECIFIC ENTHALPY INTERNAL ENTROPY

ATURE VOLUME (BTU/LB) ENERGY (BTU/LB-R) (R) (CU FT/LE)
TEMPER - SPECIFIC ENTHALPY (R)

VOLUME (BTU/LB)
INTERNAL ENTROPY (BTU/LB)

\begin{tabular}{|c|c|c|c|c|c|c|c|c|c|}
\hline 262.00 & 13.9999 & 762.43 & 503.36 & 10.861 & 402.00 & 21.4979 & 1304.72 & 906.89 & 12.521 \\
\hline 264.00 & 14.1074 & 770.19 & 509.13 & 10.890 & 404.00 & 21.6048 & 1312.24 & 912.43 & 12.540 \\
\hline 266.00 & 14.2149 & 777.95 & 514.90 & 10.919 & 406.00 & 21.7117 & 1319.75 & 917.96 & 12.558 \\
\hline 268.00 & 14.3224 & 785.73 & 520.69 & 10.949 & 408.00 & 21.8186 & 1327.25 & 923.49 & 12.577 \\
\hline 270.00 & 14.4299 & 793.52 & 526.49 & 10.978 & 410.00 & 21.9255 & 1334.74 & 929.00 & 12.595 \\
\hline 272.00 & 14.5373 & 801.30 & 532.28 & 11.006 & 412.00 & 22.0323 & 1342.22 & 934.50 & 12.613 \\
\hline 274.00 & 14.6448 & 809.08 & 538.08 & 11.035 & 414.00 & 22.1392 & 1349.69 & 940.00 & 12.631 \\
\hline 276.00 & 14.7522 & 816.88 & 543.88 & 11.063 & 416.00 & 22.2461 & 1357.16 & 945.49 & 12.649 \\
\hline 278.00 & 14.8596 & 824.68 & 549.70 & 11.091 & 418.00 & 22.3530 & 1364.62 & 950.97 & 12.667 \\
\hline 280.00 & $14.967 \mathrm{C}$ & 832.48 & 555.52 & 11.119 & 420.00 & 22.4599 & 1372.07 & 956.44 & 12.685 \\
\hline 282.00 & 15.0744 & 840.30 & 561.34 & 11.147 & 422.00 & 22.5668 & 1379.51 & 961.91 & 12.702 \\
\hline 284.00 & 15.1817 & 848.12 & 567.17 & 11.175 & 424.00 & 22.6736 & 1386.95 & 967.36 & 12.720 \\
\hline 286.00 & 15.2891 & 855.94 & 573.01 & 11.202 & 426.00 & 22.7805 & 1394.37 & 972.81 & 12.738 \\
\hline 288.00 & 15.3964 & 863.77 & 578.85 & 11.229 & 428.00 & 22.8873 & 1401.78 & 978.25 & 12.755 \\
\hline 290.00 & 15.5037 & 871.60 & 584.70 & 11.256 & 430.00 & 22.9942 & 1409.19 & 983.68 & 12.772 \\
\hline 292.00 & 15.6110 & 879.44 & 590.55 & 11.283 & 432.00 & 23.1010 & 1416.59 & 989.10 & 12.789 \\
\hline 294.00 & 15.7183 & 887.28 & 596.41 & 11.310 & 434.00 & 23.2078 & 1423.98 & 994.51 & 12.806 \\
\hline 296.00 & 15.8256 & 895.12 & 602.26 & 11.337 & 436.00 & 23.3146 & 1431.36 & 999.91 & 12.823 \\
\hline$\angle 98.00$ & 15.9328 & 902.96 & 608.12 & 11.363 & 438.00 & 23.4213 & 1438.73 & 1005.31 & 12.840 \\
\hline 300.00 & 16.0401 & 910.81 & 613.98 & 11.389 & 440.00 & 23.5281 & 1446.09 & 1010.70 & 12.857 \\
\hline 302.00 & 16.1473 & 918.65 & 619.84 & 11.415 & 442.00 & 23.6348 & 1453.45 & 1016.08 & 12.874 \\
\hline 304.00 & 16.2545 & 726.50 & 625.70 & 11.441 & 444.00 & 23.7415 & 1460.79 & 1021.45 & 12.890 \\
\hline 306.00 & 16.3617 & 934.34 & 631.57 & 11.467 & 446.00 & 23.8482 & 1468.13 & 1026.81 & 12.907 \\
\hline 308.00 & 16.4689 & 942.19 & 637.43 & 11.493 & 448.00 & 23.9548 & 1475.46 & 1032.17 & 12.923 \\
\hline 310.00 & 16.5761 & 950.03 & 643.29 & 11.518 & 450.00 & 24.0614 & 1482.78 & 1037.51 & 12.939 \\
\hline 312.00 & 16.6833 & 957.88 & 649.15 & 11.543 & 452.00 & 24.1680 & 1490.11 & 1042.87 & 12.956 \\
\hline 314.00 & 16.7904 & 965.72 & 655.01 & 11.568 & 454.00 & 24.2746 & 1497.43 & 1048.22 & 12.972 \\
\hline 316.00 & 16.8976 & 973.56 & 660.86 & 11.593 & 456.00 & 24.3812 & 1504.74 & 1053.56 & 12.988 \\
\hline 318.00 & 17.0047 & 981.39 & 666.72 & 11.618 & 458.00 & 24.4877 & 1512.05 & 1058.90 & 13.004 \\
\hline 320.00 & 17.1119 & 989.23 & 672.57 & 11.642 & 460.00 & 24.5943 & 1519.35 & 1064.22 & 13.020 \\
\hline 322.00 & 17.2190 & 997.06 & 678.42 & 11.667 & 462.00 & 24.7008 & 1526.64 & 1069.54 & 13.036 \\
\hline 324.00 & 17.3261 & 1004.88 & 684.26 & 11.691 & 464.00 & 24.8072 & 1533.92 & 1074.86 & 13.051 \\
\hline 326.00 & 17.4332 & 1012.71 & 690.10 & 11.715 & 466.00 & 24.9137 & 1541.20 & 1080.16 & 13.067 \\
\hline 328.00 & 17.5403 & 1020.53 & 695.94 & 11.739 & 468.00 & 25.0201 & 1548.47 & 1085.46 & 13.083 \\
\hline 330.00 & 17.6474 & 1028.34 & 701.77 & 11.763 & 470.00 & 25.1266 & 1555.73 & 1090.75 & 13.098 \\
\hline 332.00 & 17.7545 & 1036.15 & 707.60 & 11.786 & 472.00 & 25.2330 & 1562.99 & 1096.04 & 13.113 \\
\hline 334.00 & 17.8616 & 1043.95 & 713.42 & 11.810 & 474.00 & 25.3394 & 1570.23 & 1101.32 & 13.129 \\
\hline 336.00 & 17.9686 & 1051.75 & 719.24 & 11.833 & 476.00 & 25.4458 & 1577.48 & 1106.59 & 13.144 \\
\hline 338.00 & 18.0757 & 1059.54 & 725.05 & 11.856 & 478.00 & 25.5522 & 1584.71 & 1111.86 & 13.159 \\
\hline 340.00 & 18.1827 & 1067.33 & 730.85 & 11.879 & 480.00 & 25.6586 & 1591.94 & 1117.12 & 13.174 \\
\hline 342.00 & 18.2898 & 1075.11 & 736.65 & 11.902 & 482.00 & 25.7650 & 1599.17 & 1122.38 & 13.189 \\
\hline 344.00 & 18.3968 & 1082.88 & 742.44 & 11.925 & 484.00 & 25.8714 & 1606.39 & 1127.63 & 13.204 \\
\hline 346.00 & 18.5038 & 1090.65 & 748.23 & 11.947 & 486.00 & 25.9778 & 1613.60 & 1132.87 & 13.219 \\
\hline 348.00 & 18.6109 & 1098.41 & 754.01 & 11.970 & 488.00 & 26.0843 & 1620.81 & 1138.11 & 13.234 \\
\hline 350.00 & 18.7179 & 1106.16 & 759.78 & 11.992 & 490.00 & 26.1907 & 1628.01 & 1143.34 & 13.249 \\
\hline 352.00 & 18.8249 & 1113.91 & 765.55 & 12.014 & 492.00 & 26.2972 & 1635.21 & 1148.57 & 13.263 \\
\hline 354.00 & 18.9319 & 1121.64 & 771.30 & 12.036 & 494.00 & 26.4036 & 1642.40 & 1153.80 & 13.278 \\
\hline 356.00 & 19.0389 & 1129.37 & 777.05 & 12.058 & 496.00 & 26.5101 & 1649.59 & 1159.02 & 13.292 \\
\hline 358.00 & 19.1459 & 1137.09 & 782.79 & 12.079 & 498.00 & 26.6167 & 1656.78 & 1164.23 & 13.307 \\
\hline 360.00 & 19.2529 & 1144.81 & 788.53 & 12.101 & 500.00 & 26.7232 & 1663.96 & 1169.44 & 13.321 \\
\hline 362.00 & 19.3598 & 1152.51 & 794.25 & 12.122 & 502.00 & 26.8298 & 1671.14 & 1174.64 & 13.336 \\
\hline 364.00 & 19.4668 & 1160.20 & 799.96 & 12.1 .43 & 504.00 & 26.9364 & 1678.31 & 1179.84 & 13.350 \\
\hline 366.00 & 19.5737 & 1167.89 & 805.67 & 12.164 & 506.00 & 27.0431 & 1685.48 & 1185.04 & 13.364 \\
\hline 368.00 & 19.6807 & 1175.56 & 811.37 & 12.185 & 508.00 & 27.1498 & 1692.65 & 1190.23 & 13.378 \\
\hline 370.00 & 19.7876 & 1183.23 & 817.06 & 12.206 & 510.00 & 27.2566 & 1699.81 & 1195.42 & 13.392 \\
\hline 372.00 & 19.8945 & 1190.89 & 822.74 & 12.227 & 512.00 & 27.3634 & 1706.97 & 1200.60 & 13.406 \\
\hline 374.00 & 20.0014 & 1198.54 & 828.41 & 12.247 & 514.00 & 27.4702 & 1714.13 & 1205.78 & 13.420 \\
\hline 376.00 & 20.1083 & 1206.18 & 834.07 & 12.267 & 516.00 & 27.5771 & 1721.28 & 1210.96 & 13.434 \\
\hline 378.00 & 20.2152 & 1213.82 & 839.73 & 12.288 & 518.00 & 27.6840 & 1728.43 & 1216.13 & 13.448 \\
\hline 380.00 & 20.3221 & 1221.44 & 845.37 & $12 \cdot 308$ & 520.00 & 27.7910 & 1735.58 & 1221.30 & 13.462 \\
\hline 382.00 & 20.4290 & 1229.05 & 851.01 & 12.328 & 522.00 & 27.8980 & 1742.73 & 1226.47 & 13.475 \\
\hline 384.00 & 20.5359 & 1236.66 & 856.64 & 12.348 & 524.00 & 28.0051 & 1749.87 & 1231.63 & 13.489 \\
\hline 386.00 & 20.6428 & 1244.26 & 862.26 & 12.367 & 526.00 & 28.1122 & 1757.01 & 1236.79 & 13.503 \\
\hline 388.00 & 20.7497 & 1251.85 & 867.87 & 12.387 & 528.00 & 28.2193 & 1764.15 & 1241.94 & 13.516 \\
\hline 390.00 & 20.8566 & 1259.43 & 873.47 & 12.406 & 530.00 & 28.3265 & 1771.28 & 1247.09 & 13.530 \\
\hline 392.00 & 20.9635 & 1267.00 & 879.06 & 12.426 & 532.00 & 28.4337 & 1778.42 & 1252.24 & 13.543 \\
\hline 394.00 & 21.0704 & 1274.56 & 884.65 & 12.445 & 534.00 & 28.5409 & 1785.54 & 1257.39 & 13.557 \\
\hline 396.00 & 21.1773 & 1282.11 & 890.22 & 12.464 & 536.00 & 28.6481 & 1792.67 & 1262.53 & 13.570 \\
\hline 398.00 & 21.2842 & 1289.66 & 895.79 & 12.483 & 538.00 & 28.7554 & 1799.79 & 1267.66 & 13.583 \\
\hline 400.00 & 21.3911 & 1297.19 & 901.34 & 12.502 & 540.00 & 28.8626 & 1806.91 & 1272.80 & 13.596 \\
\hline
\end{tabular}


TEMPER- SPECIFIC ENTHALPY INTERNAL ENTROPY ATURE VOLUME (8TU/L8) ENERGY (BTU/L8-R) (R) (CU FT/L8) ENERGY (BTU/L8-R)
$(8 T U / L 8)$
TEMPER-

ATURE

(R)
SPECIFIC ( CU FT/L 8 )
ENTHALPY ( $8 \mathrm{TU} / L 8$ ) )
INTERNAL ENTROPY

ENERGY ( $8 T U / L 8-R)$ ( $8 \mathrm{TU} / \mathrm{L} 8$ )

\begin{tabular}{|c|c|c|c|c|c|c|c|c|c|}
\hline & & & & & $\begin{array}{l}122.00 \\
124.00 \\
126.00 \\
128.00 \\
130.00 \\
132.00 \\
134.00 \\
136.00 \\
138.00 \\
140.00\end{array}$ & $\begin{array}{l}5.2824 \\
5.3774 \\
5.4722 \\
5.5667 \\
5.6610 \\
5.7551 \\
5.8490 \\
5.9428 \\
6.0363 \\
6.1297\end{array}$ & $\begin{array}{l}291.79 \\
297.36 \\
302.94 \\
308.53 \\
314.15 \\
319.79 \\
325.45 \\
331.14 \\
336.85 \\
342.59\end{array}$ & $\begin{array}{l}174.49 \\
177.94 \\
181.42 \\
184.92 \\
188.44 \\
191.99 \\
195.57 \\
199.17 \\
202.81 \\
206.48\end{array}$ & $\begin{array}{l}8.171 \\
8.216 \\
8.261 \\
8.305 \\
8.348 \\
8.391 \\
8.434 \\
8.476 \\
8.518 \\
8.559\end{array}$ \\
\hline & & & & & $\begin{array}{l}142.00 \\
144.00 \\
146.00 \\
148.00 \\
150.00 \\
152.00 \\
154.00 \\
156.00 \\
158.00 \\
160.00\end{array}$ & $\begin{array}{l}6.2230 \\
6.3161 \\
6.4090 \\
6.5018 \\
6.5945 \\
6.6871 \\
6.7795 \\
6.8719 \\
6.9641 \\
7.0562\end{array}$ & $\begin{array}{l}348.37 \\
354.17 \\
360.00 \\
365.87 \\
371.78 \\
377.72 \\
383.70 \\
389.71 \\
395.77 \\
401.87\end{array}$ & $\begin{array}{l}210.18 \\
213.91 \\
217.68 \\
221.49 \\
225.34 \\
229.22 \\
233.15 \\
237.12 \\
241.13 \\
245.18\end{array}$ & $\begin{array}{l}8.600 \\
8.641 \\
8.681 \\
8.721 \\
8.760 \\
8.800 \\
8.839 \\
8.878 \\
8.916 \\
8.955\end{array}$ \\
\hline $\begin{array}{l}36.00 \\
38.00 \\
40.00\end{array}$ & $\begin{array}{l}.2223 \\
.2262 \\
.2305\end{array}$ & $\begin{array}{r}-108.02 \\
-103.45 \\
-98.57\end{array}$ & $\begin{array}{l}-112.96 \\
-108.48 \\
-103.69\end{array}$ & $\begin{array}{l}1.847 \\
1.970 \\
2.096\end{array}$ & $\begin{array}{l}162.00 \\
164.00 \\
166.00 \\
168.00 \\
170.00 \\
172.00 \\
174.00 \\
176.00 \\
178.00 \\
180.00\end{array}$ & $\begin{array}{l}7.1482 \\
7.2402 \\
7.3320 \\
7.4238 \\
7.5154 \\
7.6070 \\
7.6985 \\
7.7900 \\
7.8813 \\
7.9726\end{array}$ & $\begin{array}{l}408.01 \\
414.19 \\
420.41 \\
426.67 \\
432.98 \\
439.33 \\
445.72 \\
452.15 \\
458.62 \\
465.13\end{array}$ & $\begin{array}{l}249.27 \\
253.41 \\
257.59 \\
261.82 \\
266.09 \\
270.40 \\
274.76 \\
279.16 \\
283.61 \\
288.09\end{array}$ & $\begin{array}{l}8.993 \\
9.031 \\
9.068 \\
9.106 \\
9.143 \\
9.180 \\
9.217 \\
9.254 \\
9.290 \\
9.327\end{array}$ \\
\hline $\begin{array}{l}42.00 \\
44.00 \\
46.00 \\
48.00 \\
50.00 \\
52.00 \\
54.00 \\
+54.062 \\
+54.062 \\
56.00 \\
58.00 \\
60.00\end{array}$ & $\begin{array}{l}.2355 \\
.2411 \\
.2476 \\
.2554 \\
.2650 \\
.2775 \\
.2961 \\
.2975 \\
1.4780 \\
1.6777 \\
1.8464 \\
1.9965\end{array}$ & $\begin{array}{r}-93.33 \\
-87.67 \\
-81.53 \\
-74.79 \\
-67.24 \\
-58.52 \\
-47.71 \\
-47.31 \\
79.07 \\
91.04 \\
100.74 \\
109.16\end{array}$ & $\begin{array}{l}-98.55 \\
-93.03 \\
-87.03 \\
-80.46 \\
-73.13 \\
-64.68 \\
-54.28 \\
-60.63 \\
46.25 \\
53.79 \\
59.74 \\
64.82\end{array}$ & $\begin{array}{l}2.223 \\
2.355 \\
2.491 \\
2.635 \\
2.789 \\
2.960 \\
3.164 \\
3.171 \\
5.514 \\
5.7 .32 \\
5.902 \\
6.045\end{array}$ & $\begin{array}{l}182.00 \\
184.00 \\
186.00 \\
188.00 \\
190.00 \\
192.00 \\
194.00 \\
196.00 \\
198.00 \\
200.00\end{array}$ & $\begin{array}{l}8.0638 \\
8.1549 \\
8.2459 \\
8.3368 \\
8.4277 \\
8.5185 \\
8.6093 \\
8.7000 \\
8.7906 \\
8.8812\end{array}$ & $\begin{array}{l}471.68 \\
478.27 \\
484.90 \\
491.57 \\
498.28 \\
505.03 \\
511.82 \\
518.66 \\
525.53 \\
532.44\end{array}$ & $\begin{array}{l}292.62 \\
297.18 \\
301.79 \\
306.44 \\
311.13 \\
315.87 \\
320.64 \\
325.46 \\
330.32 \\
335.22\end{array}$ & $\begin{array}{l}9.363 \\
9.399 \\
9.435 \\
9.471 \\
9.506 \\
9.541 \\
9.577 \\
9.612 \\
9.647 \\
9.681\end{array}$ \\
\hline $\begin{array}{l}62.00 \\
64.00 \\
66.00 \\
68.00 \\
70.00 \\
72.00 \\
74.00 \\
76.00 \\
78.00 \\
80.00\end{array}$ & $\begin{array}{l}2.1355 \\
2.2669 \\
2.3927 \\
2.5143 \\
2.6326 \\
2.7481 \\
2.8614 \\
2.9726 \\
3.0822 \\
3.1903\end{array}$ & $\begin{array}{l}116.83 \\
124.03 \\
130.89 \\
137.50 \\
143.92 \\
150.20 \\
156.35 \\
162.41 \\
168.38 \\
174.28\end{array}$ & $\begin{array}{l}69.41 \\
73.69 \\
77.75 \\
81.66 \\
85.46 \\
89.17 \\
92.81 \\
96.40 \\
99.94 \\
103.44\end{array}$ & $\begin{array}{l}6.171 \\
6.285 \\
6.390 \\
6.489 \\
6.582 \\
6.671 \\
6.755 \\
6.836 \\
6.913 \\
6.988\end{array}$ & $\begin{array}{l}202.00 \\
204.00 \\
206.00 \\
208.00 \\
210.00 \\
212.00 \\
214.00 \\
216.00 \\
218.00 \\
220.00\end{array}$ & $\begin{array}{l}8.9718 \\
9.0623 \\
9.1528 \\
9.2432 \\
9.3336 \\
9.4240 \\
9.5143 \\
9.6046 \\
9.6949 \\
9.7851\end{array}$ & $\begin{array}{l}539.39 \\
546.38 \\
553.41 \\
560.47 \\
567.57 \\
574.71 \\
581.87 \\
589.08 \\
596.31 \\
603.57\end{array}$ & $\begin{array}{l}340.16 \\
345.14 \\
350.16 \\
355.21 \\
360.30 \\
365.43 \\
370.60 \\
375.80 \\
381.02 \\
386.28\end{array}$ & $\begin{array}{r}9.716 \\
9.750 \\
9.785 \\
9.819 \\
9.853 \\
9.886 \\
9.920 \\
9.954 \\
9.987 \\
10.020\end{array}$ \\
\hline $\begin{array}{l}82.00 \\
84.00 \\
86.00 \\
88.00 \\
90.00 \\
92.00 \\
94.00 \\
96.00 \\
98.00 \\
100.00\end{array}$ & $\begin{array}{l}3.2971 \\
3.4027 \\
3.5072 \\
3.6108 \\
3.7135 \\
3.8155 \\
3.9167 \\
4.0173 \\
4.1173 \\
4.2167\end{array}$ & $\begin{array}{l}180.13 \\
185.91 \\
191.65 \\
197.36 \\
203.02 \\
208.66 \\
214.27 \\
219.86 \\
225.43 \\
230.98\end{array}$ & $\begin{array}{l}106.91 \\
110.35 \\
113.77 \\
117.17 \\
120.56 \\
123.93 \\
127.30 \\
130.65 \\
134.00 \\
137.34\end{array}$ & $\begin{array}{l}7.060 \\
7.130 \\
7.197 \\
7.263 \\
7.327 \\
7.388 \\
7.449 \\
7.508 \\
7.565 \\
7.621\end{array}$ & $\begin{array}{l}222.00 \\
224.00 \\
226.00 \\
228.00 \\
230.00 \\
232.00 \\
234.00 \\
236.00 \\
238.00 \\
240.00\end{array}$ & $\begin{array}{r}9.8753 \\
9.9655 \\
10.0556 \\
10.1457 \\
10.2358 \\
10.3259 \\
10.4160 \\
10.5060 \\
10.5960 \\
10.6860\end{array}$ & $\begin{array}{l}610.87 \\
618.19 \\
625.55 \\
632.93 \\
640.34 \\
647.78 \\
655.25 \\
662.74 \\
670.25 \\
677.79\end{array}$ & $\begin{array}{l}391.57 \\
396.90 \\
402.25 \\
407.63 \\
413.04 \\
418.48 \\
423.95 \\
429.44 \\
434.95 \\
440.50\end{array}$ & $\begin{array}{l}10.053 \\
10.086 \\
10.119 \\
10.151 \\
10.184 \\
10.216 \\
10.248 \\
10.280 \\
10.311 \\
10.343\end{array}$ \\
\hline $\begin{array}{l}102.00 \\
104.00 \\
106.00 \\
108.00 \\
110.00 \\
112.00 \\
114.00 \\
116.00 \\
118.00 \\
120.00\end{array}$ & $\begin{array}{l}4.3156 \\
4.4140 \\
4.5119 \\
4.6094 \\
4.7066 \\
4.8033 \\
4.8998 \\
4.9959 \\
5.0917 \\
5.1872\end{array}$ & $\begin{array}{l}236.52 \\
242.05 \\
247.57 \\
253.09 \\
258.61 \\
264.13 \\
269.64 \\
275.17 \\
280.70 \\
286.24\end{array}$ & $\begin{array}{l}140.69 \\
144.03 \\
147.38 \\
150.73 \\
154.09 \\
157.46 \\
160.84 \\
164.23 \\
167.63 \\
171.05\end{array}$ & $\begin{array}{l}7.676 \\
7.730 \\
7.782 \\
7.834 \\
7.884 \\
7.934 \\
7.983 \\
8.031 \\
8.078 \\
8.125\end{array}$ & $\begin{array}{l}242.00 \\
244.00 \\
246.00 \\
248.00 \\
250.00 \\
252.00 \\
254.00 \\
256.00 \\
258.00 \\
260.00\end{array}$ & $\begin{array}{l}10.7760 \\
10.8659 \\
10.9558 \\
11.0457 \\
11.1356 \\
11.2255 \\
11.3154 \\
11.4052 \\
11.4950 \\
11.5849\end{array}$ & $\begin{array}{l}685.36 \\
692.94 \\
700.55 \\
708.17 \\
715.82 \\
723.49 \\
731.17 \\
738.87 \\
746.59 \\
754.32\end{array}$ & $\begin{array}{l}446.06 \\
451.65 \\
457.26 \\
462.89 \\
468.54 \\
474.21 \\
479.90 \\
485.60 \\
491.33 \\
497.06\end{array}$ & $\begin{array}{l}10.374 \\
10.406 \\
10.437 \\
10.467 \\
10.498 \\
10.529 \\
10.559 \\
10.589 \\
10.619 \\
10.649\end{array}$ \\
\hline
\end{tabular}


TEMPER- SPECIFIC ENTHALPY INTERNAL ENTROPY ATURE VOLUME (BTU/LB) ENERGY (BTU/LB-K) (R) (CUFT/LB)
(BTU/LB) $\begin{array}{ccccc}\text { TEMPER- } & \text { SPECIFIC } & \text { ENTHALPY } & \text { INTERNAL } & \text { ENTROPY } \\ \text { ATURE } & \text { VOLUME } & \text { (8TU/L8) } & \text { ENERGY } & \text { (8TU/L8-R) } \\ (R) & (\text { CU FT/L8) } & & \text { (BTU/L8) } & \end{array}$

\begin{tabular}{|c|c|c|c|c|c|c|c|c|c|}
\hline $\begin{array}{l}262.00 \\
264.00 \\
266.00 \\
268.00 \\
270.00 \\
272.00 \\
274.00 \\
276.00 \\
278.00 \\
280.00\end{array}$ & $\begin{array}{l}11.6746 \\
11.7644 \\
11.8542 \\
11.9439 \\
12.0336 \\
12.1233 \\
12.2130 \\
12.3027 \\
12.3924 \\
12.4820\end{array}$ & $\begin{array}{l}762.07 \\
769.83 \\
777.61 \\
785.39 \\
793.19 \\
800.98 \\
808.77 \\
816.57 \\
824.38 \\
832.19\end{array}$ & $\begin{array}{l}502.82 \\
508.59 \\
514.37 \\
520.16 \\
525.97 \\
531.76 \\
537.56 \\
543.31 \\
549.19 \\
555.01\end{array}$ & $\begin{array}{l}10.679 \\
10.708 \\
10.738 \\
10.767 \\
10.796 \\
10.825 \\
10.853 \\
10.881 \\
10.910 \\
10.938\end{array}$ & $\begin{array}{l}402.00 \\
404.00 \\
406.00 \\
408.00 \\
410.00 \\
412.00 \\
414.00 \\
416.00 \\
418.00 \\
420.00\end{array}$ & $\begin{array}{l}17.9303 \\
18.0194 \\
18.1086 \\
18.1977 \\
18.2868 \\
18.3759 \\
18.4651 \\
18.5542 \\
18.6433 \\
18.7325\end{array}$ & $\begin{array}{l}1304.74 \\
1312.27 \\
1319.78 \\
1327.28 \\
1334.78 \\
1342.26 \\
1349.74 \\
1357.21 \\
1364.67 \\
1372.13\end{array}$ & $\begin{array}{l}906.58 \\
912.12 \\
917.65 \\
923.18 \\
928.69 \\
934.20 \\
939.70 \\
945.19 \\
950.67 \\
956.15\end{array}$ & $\begin{array}{l}12.340 \\
12.359 \\
12.377 \\
12.396 \\
12.414 \\
12.432 \\
12.451 \\
12.469 \\
12.486 \\
12.504\end{array}$ \\
\hline $\begin{array}{l}282.00 \\
284.00 \\
286.00 \\
288.00 \\
290.00 \\
292.00 \\
294.00 \\
296.00 \\
298.00 \\
300.00\end{array}$ & $\begin{array}{l}12.5716 \\
12.6612 \\
12.7508 \\
12.8404 \\
12.9300 \\
13.0195 \\
13.1090 \\
13.1986 \\
13.2881 \\
13.3776\end{array}$ & $\begin{array}{l}840.01 \\
847.84 \\
855.67 \\
863.51 \\
871.35 \\
879.19 \\
887.04 \\
894.89 \\
902.74 \\
910.59\end{array}$ & $\begin{array}{l}560.84 \\
566.68 \\
572.52 \\
578.37 \\
584.22 \\
590.08 \\
595.93 \\
601.79 \\
607.66 \\
613.52\end{array}$ & $\begin{array}{l}10.965 \\
10.993 \\
11.021 \\
11.048 \\
11.075 \\
11.102 \\
11.129 \\
11.155 \\
11.182 \\
11.208\end{array}$ & $\begin{array}{l}422.00 \\
424.00 \\
426.00 \\
428.00 \\
430.00 \\
432.00 \\
434.00 \\
436.00 \\
438.00 \\
440.00\end{array}$ & $\begin{array}{l}18.8216 \\
18.9107 \\
18.9998 \\
19.0889 \\
19.1780 \\
19.2670 \\
19.3561 \\
19.4451 \\
19.5341 \\
19.6232\end{array}$ & $\begin{array}{l}1379.57 \\
1387.01 \\
1394.44 \\
1401.85 \\
1409.26 \\
1416.66 \\
1424.06 \\
1431.44 \\
1438.81 \\
1446.18\end{array}$ & $\begin{array}{r}961.61 \\
967.07 \\
972.52 \\
977.96 \\
983.39 \\
988.81 \\
994.23 \\
999.63 \\
1005.03 \\
1010.42\end{array}$ & $\begin{array}{l}12.522 \\
12.540 \\
12.557 \\
12.574 \\
12.592 \\
12.609 \\
12.626 \\
12.643 \\
12.660 \\
12.677\end{array}$ \\
\hline $\begin{array}{l}302.00 \\
304.00 \\
306.00 \\
308.00 \\
310.00 \\
312.00 \\
314.00 \\
316.00 \\
318.00 \\
320.00\end{array}$ & $\begin{array}{l}13.4670 \\
13.5565 \\
13.6460 \\
13.7354 \\
13.8248 \\
13.9143 \\
14.0037 \\
14.0931 \\
14.1825 \\
14.2718\end{array}$ & $\begin{array}{l}918.44 \\
926.29 \\
934.14 \\
942.00 \\
949.85 \\
957.70 \\
965.54 \\
973.39 \\
981.23 \\
989.07\end{array}$ & $\begin{array}{l}619.39 \\
625.25 \\
631.12 \\
636.98 \\
642.85 \\
648.71 \\
654.57 \\
660.43 \\
666.29 \\
672.14\end{array}$ & $\begin{array}{l}11.234 \\
11.260 \\
11.286 \\
11.311 \\
11.337 \\
11.362 \\
11.387 \\
11.412 \\
11.437 \\
11.461\end{array}$ & $\begin{array}{l}442.00 \\
444.00 \\
446.00 \\
448.00 \\
450.00 \\
452.00 \\
454.00 \\
456.00 \\
458.00 \\
460.00\end{array}$ & $\begin{array}{l}19.7121 \\
19.8011 \\
19.8901 \\
19.9790 \\
20.0679 \\
20.1568 \\
20.2457 \\
20.3345 \\
20.4233 \\
20.5122\end{array}$ & $\begin{array}{l}1453.54 \\
1460.89 \\
1468.23 \\
1475.56 \\
1482.88 \\
1490.22 \\
1497.54 \\
1504.86 \\
1512.17 \\
1519.47\end{array}$ & $\begin{array}{l}1015.80 \\
1021.18 \\
1026.54 \\
1031.90 \\
1037.25 \\
1042.61 \\
1047.96 \\
1053.30 \\
1058.64 \\
1063.97\end{array}$ & $\begin{array}{l}12.693 \\
12.710 \\
12.726 \\
12.743 \\
12.759 \\
12.775 \\
12.791 \\
12.808 \\
12.824 \\
12.839\end{array}$ \\
\hline $\begin{array}{l}322.00 \\
324.00 \\
326.00 \\
328.00 \\
330.00 \\
332.00 \\
334.00 \\
336.00 \\
338.00 \\
340.00\end{array}$ & $\begin{array}{l}14.3612 \\
14.4506 \\
14.5399 \\
14.6293 \\
14.7186 \\
14.8080 \\
14.8973 \\
14.9866 \\
15.0759 \\
15.1652\end{array}$ & $\begin{array}{r}996.91 \\
1004.74 \\
1012.57 \\
1020.39 \\
1028.21 \\
1036.02 \\
1043.83 \\
1051.64 \\
1059.43 \\
1067.22\end{array}$ & $\begin{array}{l}678.00 \\
683.84 \\
689.69 \\
695.53 \\
701.36 \\
707.19 \\
713.02 \\
718.84 \\
724.65 \\
730.46\end{array}$ & $\begin{array}{l}11.486 \\
11.510 \\
11.534 \\
11.558 \\
11.582 \\
11.605 \\
11.629 \\
11.652 \\
11.675 \\
11.698\end{array}$ & $\begin{array}{l}462.00 \\
464.00 \\
466.00 \\
468.00 \\
470.00 \\
472.00 \\
474.00 \\
476.00 \\
478.00 \\
480.00\end{array}$ & $\begin{array}{l}20.6010 \\
20.6898 \\
20.7785 \\
20.8673 \\
20.9560 \\
21.0448 \\
21.1335 \\
21.2222 \\
21.3109 \\
21.3996\end{array}$ & $\begin{array}{l}1526.76 \\
1534.05 \\
1541.33 \\
1548.60 \\
1555.86 \\
1563.12 \\
1570.37 \\
1577.62 \\
1584.86 \\
1592.09\end{array}$ & $\begin{array}{l}1069.29 \\
1074.60 \\
1079.91 \\
1085.21 \\
1090.51 \\
1095.80 \\
1101.08 \\
1106.35 \\
1111.62 \\
1116.88\end{array}$ & $\begin{array}{l}12.855 \\
12.871 \\
12.887 \\
12.902 \\
12.918 \\
12.933 \\
12.948 \\
12.964 \\
12.979 \\
12.994\end{array}$ \\
\hline $\begin{array}{l}342.00 \\
344.00 \\
346.00 \\
348.00 \\
350.00 \\
352.00 \\
354.00 \\
356.00 \\
358.00 \\
360.00\end{array}$ & $\begin{array}{l}15.2545 \\
15.3438 \\
15.4331 \\
15.5224 \\
15.6116 \\
15.7009 \\
15.7902 \\
15.8794 \\
15.9687 \\
16.0579\end{array}$ & $\begin{array}{l}1075.01 \\
1082.79 \\
1090.56 \\
1098.32 \\
1106.08 \\
1113.83 \\
1121.57 \\
1129.31 \\
1137.03 \\
1144.75\end{array}$ & $\begin{array}{l}736.26 \\
742.06 \\
747.85 \\
753.63 \\
759.40 \\
765.17 \\
770.93 \\
776.68 \\
782.43 \\
788.16\end{array}$ & $\begin{array}{l}11.721 \\
11.744 \\
11.766 \\
11.789 \\
11.811 \\
11.833 \\
11.855 \\
11.877 \\
11.898 \\
11.920\end{array}$ & $\begin{array}{l}482.00 \\
484.00 \\
486.00 \\
488.00 \\
490.00 \\
492.00 \\
494.00 \\
496.00 \\
498.00 \\
500.00\end{array}$ & $\begin{array}{l}21.4884 \\
21.5771 \\
21.6658 \\
21.7545 \\
21.8433 \\
21.9320 \\
22.0208 \\
22.1096 \\
22.1984 \\
22.2872\end{array}$ & $\begin{array}{l}1599.32 \\
1606.54 \\
1613.76 \\
1620.97 \\
1628.17 \\
1635.37 \\
1642.57 \\
1649.76 \\
1656.95 \\
1664.13\end{array}$ & $\begin{array}{l}1122.14 \\
1127.39 \\
1132.64 \\
1137.88 \\
1143.11 \\
1148.34 \\
1153.57 \\
1158.79 \\
1164.00 \\
1169.22\end{array}$ & $\begin{array}{l}13.009 \\
13.024 \\
13.039 \\
13.054 \\
13.068 \\
13.083 \\
13.098 \\
13.112 \\
13.127 \\
13.141\end{array}$ \\
\hline $\begin{array}{l}362.00 \\
364.00 \\
366.00 \\
368.00 \\
370.00 \\
372.00 \\
374.00 \\
376.00 \\
378.00 \\
380.00\end{array}$ & $\begin{array}{l}16.1471 \\
16.2363 \\
16.3255 \\
16.4147 \\
16.5039 \\
16.5931 \\
16.6822 \\
16.7714 \\
16.8606 \\
16.9497\end{array}$ & $\begin{array}{l}1152.46 \\
1160.15 \\
1167.84 \\
1175.52 \\
1183.20 \\
1190.86 \\
1198.51 \\
1206.16 \\
1213.80 \\
1221.42\end{array}$ & $\begin{array}{l}793.89 \\
799.60 \\
805.31 \\
811.01 \\
816.70 \\
822.39 \\
828.06 \\
833.73 \\
839.38 \\
845.03\end{array}$ & $\begin{array}{l}11.941 \\
11.962 \\
11.983 \\
12.004 \\
12.025 \\
12.046 \\
12.066 \\
12.087 \\
12.107 \\
12.127\end{array}$ & $\begin{array}{l}502.00 \\
504.00 \\
506.00 \\
508.00 \\
510.00 \\
512.00 \\
514.00 \\
516.00 \\
518.00 \\
520.00\end{array}$ & $\begin{array}{l}22.3761 \\
22.4650 \\
22.5539 \\
22.6428 \\
22.7318 \\
22.8209 \\
22.9099 \\
22.9990 \\
23.0881 \\
23.1773\end{array}$ & $\begin{array}{l}1671.31 \\
1678.49 \\
1685.66 \\
1692.83 \\
1699.99 \\
1707.16 \\
1714.32 \\
1721.47 \\
1728.62 \\
1735.78\end{array}$ & $\begin{array}{l}1174.42 \\
1179.62 \\
1184.82 \\
1190.01 \\
1195.20 \\
1200.39 \\
1205.57 \\
1210.75 \\
1215.92 \\
1221.09\end{array}$ & $\begin{array}{l}13.155 \\
13.170 \\
13.184 \\
13.198 \\
13.212 \\
13.226 \\
13.240 \\
13.254 \\
13.268 \\
13.282\end{array}$ \\
\hline $\begin{array}{l}382.00 \\
384.00 \\
386.00 \\
388.00 \\
390.00 \\
392.00 \\
394.00 \\
396.00 \\
398.00 \\
400.00\end{array}$ & $\begin{array}{l}17.0389 \\
17.1280 \\
17.2172 \\
17.3063 \\
17.3955 \\
17.4846 \\
17.5738 \\
17.6629 \\
17.7521 \\
17.8412\end{array}$ & $\begin{array}{l}1229.04 \\
1236.65 \\
1244.25 \\
1251.85 \\
1259.43 \\
1267.00 \\
1274.57 \\
1282.13 \\
1289.68 \\
1297.21\end{array}$ & $\begin{array}{l}850.67 \\
856.30 \\
861.92 \\
867.54 \\
873.14 \\
878.73 \\
884.32 \\
889.90 \\
895.47 \\
901.03\end{array}$ & $\begin{array}{l}12.147 \\
12.167 \\
12.187 \\
12.206 \\
12.226 \\
12.245 \\
12.264 \\
12.284 \\
12.303 \\
12.322\end{array}$ & $\begin{array}{l}522.00 \\
524.00 \\
526.00 \\
528.00 \\
530.00 \\
532.00 \\
534.00 \\
536.00 \\
538.00 \\
540.00\end{array}$ & $\begin{array}{l}23.2665 \\
23.3557 \\
23.4450 \\
23.5343 \\
23.6236 \\
23.7130 \\
23.8023 \\
23.8917 \\
23.9811 \\
24.0704\end{array}$ & $\begin{array}{l}1742.92 \\
1750.07 \\
1757.21 \\
1764.35 \\
1771.48 \\
1778.61 \\
1785.74 \\
1792.87 \\
1799.99 \\
1807.11\end{array}$ & $\begin{array}{l}1226.26 \\
1231.42 \\
1236.58 \\
1241.74 \\
1246.89 \\
1252.04 \\
1257.18 \\
1262.32 \\
1267.46 \\
1272.59\end{array}$ & $\begin{array}{l}13.295 \\
13.309 \\
13.322 \\
13.336 \\
13.350 \\
13.363 \\
13.376 \\
13.390 \\
13.403 \\
13.416\end{array}$ \\
\hline
\end{tabular}


TEMPER- SPECIFIC ENTHALPY INTERNAL ENTROPY ATURE VOLUME (BTU/LB) ENERGY (BTU/L8-R)

(R) (CU FT/LB)
(BTU/LB)
TEMPER- SPECIFIC ENTHALPY VOLUME (R) (CU FT/L8)
INTERNAL ENTROPY

(BIU/LB)




IEMPER- SPECIFIC ENTHALPY INTERNAL ENTROPY ATURE VOLUME (BTU/LB) ENERGY (BTU/LB-R) (R) (CU FT/LB)
(BTU/LB) $\begin{array}{ccccc}\text { TEMPER- } & \text { SPECIFIC } & \text { ENTHALPY } & \text { INTERNAL } & \text { ENTROPY } \\ \text { ATURE } & \text { VOLUME } & \text { (8TU/L8) } & \text { ENERGY } & \text { (8TU/L8-R) } \\ \text { (R) } & \text { (CUFT/LB) } & & \text { (8TU/LB) } & \end{array}$

\begin{tabular}{|c|c|c|c|c|c|c|c|c|c|}
\hline $\begin{array}{l}262.00 \\
264.00 \\
266.00 \\
268.00 \\
270.00 \\
272.00 \\
274.00 \\
276.00 \\
278.00 \\
280.00\end{array}$ & $\begin{array}{l}10.0135 \\
10.0906 \\
10.1677 \\
10.2447 \\
10.3218 \\
10.3988 \\
10.4758 \\
10.5528 \\
10.6298 \\
10.7068\end{array}$ & $\begin{array}{l}761.71 \\
769.48 \\
777.26 \\
785.06 \\
792.87 \\
800.66 \\
808.46 \\
816.27 \\
824.08 \\
831.90\end{array}$ & $\begin{array}{l}502.29 \\
508.06 \\
513.85 \\
519.64 \\
525.46 \\
531.25 \\
537.06 \\
542.87 \\
548.69 \\
554.52\end{array}$ & $\begin{array}{l}10.525 \\
10.554 \\
10.584 \\
10.613 \\
10.642 \\
10.671 \\
10.699 \\
10.728 \\
10.756 \\
10.784\end{array}$ & $\begin{array}{l}402.00 \\
404.00 \\
406.00 \\
408.00 \\
410.00 \\
412.00 \\
414.00 \\
416.00 \\
418.00 \\
420.00\end{array}$ & $\begin{array}{l}15.3817 \\
15.4582 \\
15.5346 \\
15.6111 \\
15.6875 \\
15.7639 \\
15.8404 \\
15.9168 \\
15.9933 \\
16.0697\end{array}$ & $\begin{array}{l}1304.77 \\
1312.30 \\
1319.81 \\
1327.32 \\
1334.82 \\
1342.31 \\
1349.79 \\
1357.26 \\
1364.73 \\
1372.19\end{array}$ & $\begin{array}{l}906.27 \\
911.81 \\
917.35 \\
922.88 \\
928.39 \\
933.90 \\
939.40 \\
944.90 \\
950.38 \\
955.86\end{array}$ & $\begin{array}{l}12.187 \\
12.206 \\
12.225 \\
12.243 \\
12.261 \\
12.280 \\
12.298 \\
12.316 \\
12.334 \\
12.352\end{array}$ \\
\hline $\begin{array}{l}282.00 \\
284.00 \\
286.00 \\
288.00 \\
290.00 \\
292.00 \\
294.00 \\
296.00 \\
298.00 \\
300.00\end{array}$ & $\begin{array}{l}10.7837 \\
10.8606 \\
10.9375 \\
11.0144 \\
11.0913 \\
11.1682 \\
11.2450 \\
11.3219 \\
11.3987 \\
11.4755\end{array}$ & $\begin{array}{l}839.73 \\
847.57 \\
855.41 \\
863.25 \\
871.10 \\
878.95 \\
886.80 \\
894.66 \\
902.51 \\
910.37\end{array}$ & $\begin{array}{l}560.36 \\
566.20 \\
572.04 \\
577.89 \\
583.75 \\
589.61 \\
595.47 \\
601.34 \\
607.20 \\
613.07\end{array}$ & $\begin{array}{l}10.812 \\
10.839 \\
10.867 \\
10.894 \\
10.921 \\
10.948 \\
10.975 \\
11.002 \\
11.028 \\
11.055\end{array}$ & $\begin{array}{l}422.00 \\
424.00 \\
426.00 \\
428.00 \\
430.00 \\
432.00 \\
434.00 \\
436.00 \\
438.00 \\
440.00\end{array}$ & $\begin{array}{l}16.1462 \\
16.2226 \\
16.2990 \\
16.3754 \\
16.4518 \\
16.5282 \\
16.6046 \\
16.6809 \\
16.7573 \\
16.8336\end{array}$ & $\begin{array}{l}1379.63 \\
1387.07 \\
1394.50 \\
1401.93 \\
1409.34 \\
1416.74 \\
1424.14 \\
1431.52 \\
1438.90 \\
1446.27\end{array}$ & $\begin{array}{r}961.33 \\
966.79 \\
972.24 \\
977.68 \\
983.11 \\
988.54 \\
993.96 \\
999.36 \\
1004.76 \\
1010.15\end{array}$ & $\begin{array}{l}12.369 \\
12.387 \\
12.404 \\
12.422 \\
12.439 \\
12.456 \\
12.473 \\
12.490 \\
12.507 \\
12.524\end{array}$ \\
\hline $\begin{array}{l}302.00 \\
304.00 \\
306.00 \\
308.00 \\
310.00 \\
312.00 \\
314.00 \\
316.00 \\
318.00 \\
320.00\end{array}$ & $\begin{array}{l}11.5523 \\
11.6291 \\
11.7059 \\
11.7826 \\
11.8594 \\
11.9361 \\
12.0128 \\
12.0896 \\
12.1663 \\
12.2430\end{array}$ & $\begin{array}{l}918.23 \\
926.09 \\
933.95 \\
941.81 \\
949.66 \\
957.52 \\
965.37 \\
973.22 \\
981.07 \\
988.92\end{array}$ & $\begin{array}{l}618.94 \\
624.81 \\
630.68 \\
636.55 \\
642.42 \\
648.29 \\
654.15 \\
660.01 \\
665.88 \\
671.73\end{array}$ & $\begin{array}{l}11.081 \\
11.107 \\
11.132 \\
11.158 \\
11.183 \\
11.209 \\
11.234 \\
11.259 \\
11.283 \\
11.308\end{array}$ & $\begin{array}{l}442.00 \\
444.00 \\
446.00 \\
448.00 \\
450.00 \\
452.00 \\
454.00 \\
456.00 \\
458.00 \\
460.00\end{array}$ & $\begin{array}{l}16.9099 \\
16.9862 \\
17.0625 \\
17.1388 \\
17.2151 \\
17.2913 \\
17.3675 \\
17.4437 \\
17.5199 \\
17.5961\end{array}$ & $\begin{array}{l}1453.63 \\
1460.98 \\
1468.33 \\
1475.66 \\
1482.99 \\
1490.33 \\
1497.65 \\
1504.97 \\
1512.29 \\
1519.59\end{array}$ & $\begin{array}{l}1015.54 \\
1020.91 \\
1026.28 \\
1031.64 \\
1036.99 \\
1042.35 \\
1047.71 \\
1053.05 \\
1058.39 \\
1063.72\end{array}$ & $\begin{array}{l}12.541 \\
12.557 \\
12.574 \\
12.590 \\
12.606 \\
12.623 \\
12.639 \\
12.655 \\
12.671 \\
12.687\end{array}$ \\
\hline $\begin{array}{l}322.00 \\
324.00 \\
326.00 \\
328.00 \\
330.00 \\
332.00 \\
334.00 \\
336.00 \\
338.00 \\
340.00\end{array}$ & $\begin{array}{l}12.3197 \\
12.3963 \\
12.4730 \\
12.5497 \\
12.6263 \\
12.7030 \\
12.7796 \\
12.8563 \\
12.9329 \\
13.0095\end{array}$ & $\begin{array}{r}996.76 \\
1004.60 \\
1012.43 \\
1020.26 \\
1028.09 \\
1035.90 \\
1043.72 \\
1051.53 \\
1059.33 \\
1067.13\end{array}$ & $\begin{array}{l}677.59 \\
683.44 \\
689.29 \\
695.13 \\
700.97 \\
706.80 \\
712.63 \\
718.45 \\
724.27 \\
730.08\end{array}$ & $\begin{array}{l}11.332 \\
11.357 \\
11.381 \\
11.405 \\
11.429 \\
11.452 \\
11.476 \\
11.499 \\
11.522 \\
11.545\end{array}$ & $\begin{array}{l}462.00 \\
464.00 \\
466.00 \\
468.00 \\
470.00 \\
472.00 \\
474.00 \\
476.00 \\
478.00 \\
480.00\end{array}$ & $\begin{array}{l}17.6722 \\
17.7484 \\
17.8245 \\
17.9006 \\
17.9768 \\
18.0529 \\
18.1289 \\
18.2050 \\
18.2811 \\
18.3572\end{array}$ & $\begin{array}{l}1526.89 \\
1534.18 \\
1541.46 \\
1548.73 \\
1556.00 \\
1563.26 \\
1570.52 \\
1577.77 \\
1585.01 \\
1592.24\end{array}$ & $\begin{array}{l}1069.04 \\
1074.36 \\
1079.67 \\
1084.97 \\
1090.27 \\
1095.56 \\
1100.84 \\
1106.12 \\
1111.39 \\
1116.66\end{array}$ & $\begin{array}{l}12.703 \\
12.718 \\
12.734 \\
12.750 \\
12.765 \\
12.781 \\
12.796 \\
12.811 \\
12.826 \\
12.841\end{array}$ \\
\hline $\begin{array}{l}342.00 \\
344.00 \\
346.00 \\
348.00 \\
350.00 \\
352.00 \\
354.00 \\
356.00 \\
358.00 \\
360.00\end{array}$ & $\begin{array}{l}13.0862 \\
13.1628 \\
13.2394 \\
13.3160 \\
13.3926 \\
13.4692 \\
13.5458 \\
13.6224 \\
13.6990 \\
13.7755\end{array}$ & $\begin{array}{l}1074.92 \\
1082.70 \\
1090.47 \\
1098.24 \\
1106.01 \\
1113.76 \\
1121.51 \\
1129.24 \\
1136.98 \\
1144.70\end{array}$ & $\begin{array}{l}735.89 \\
741.69 \\
747.48 \\
753.26 \\
759.04 \\
764.81 \\
770.57 \\
776.32 \\
782.07 \\
787.81\end{array}$ & $\begin{array}{l}11.568 \\
11.591 \\
11.613 \\
11.636 \\
11.658 \\
11.680 \\
11.702 \\
11.724 \\
11.745 \\
11.767\end{array}$ & $\begin{array}{l}482.00 \\
484.00 \\
486.00 \\
488.00 \\
490.00 \\
492.00 \\
494.00 \\
496.00 \\
498.00 \\
500.00\end{array}$ & $\begin{array}{l}18.4333 \\
18.5094 \\
18.5855 \\
18.6615 \\
18.7376 \\
18.8138 \\
18.8899 \\
18.9660 \\
19.0422 \\
19.1184\end{array}$ & $\begin{array}{l}1599.47 \\
1606.70 \\
1613.92 \\
1621.13 \\
1628.34 \\
1635.54 \\
1642.74 \\
1649.93 \\
1657.12 \\
1664.31\end{array}$ & $\begin{array}{l}1121.91 \\
1127.17 \\
1132.41 \\
1137.66 \\
1142.89 \\
1148.12 \\
1153.35 \\
1158.57 \\
1163.79 \\
1169.00\end{array}$ & $\begin{array}{l}12.856 \\
12.871 \\
12.886 \\
12.901 \\
12.916 \\
12.931 \\
12.945 \\
12.960 \\
12.974 \\
12.989\end{array}$ \\
\hline $\begin{array}{l}362.00 \\
364.00 \\
366.00 \\
368.00 \\
370.00 \\
372.00 \\
374.00 \\
376.00 \\
378.00 \\
380.00\end{array}$ & $\begin{array}{l}13.8521 \\
13.9286 \\
14.0051 \\
14.0816 \\
14.1581 \\
14.2346 \\
14.3111 \\
14.3876 \\
14.4641 \\
14.5406\end{array}$ & $\begin{array}{l}1152.41 \\
1160.11 \\
1167.80 \\
1175.49 \\
1183.16 \\
1190.83 \\
1198.49 \\
1206.14 \\
1213.78 \\
1221.41\end{array}$ & $\begin{array}{l}793.54 \\
799.26 \\
804.97 \\
810.67 \\
816.36 \\
822.05 \\
827.72 \\
833.39 \\
839.05 \\
844.70\end{array}$ & $\begin{array}{l}11.788 \\
11.809 \\
11.830 \\
11.851 \\
11.872 \\
11.893 \\
11.913 \\
11.934 \\
11.954 \\
11.974\end{array}$ & $\begin{array}{l}502.00 \\
504.00 \\
506.00 \\
508.00 \\
510.00 \\
512.00 \\
514.00 \\
516.00 \\
518.00 \\
520.00\end{array}$ & $\begin{array}{l}19.1946 \\
19.2708 \\
19.3470 \\
19.4233 \\
19.4996 \\
19.5759 \\
19.6523 \\
19.7286 \\
19.8051 \\
19.8815\end{array}$ & $\begin{array}{l}1671.49 \\
1678.67 \\
1685.84 \\
1693.01 \\
1700.18 \\
1707.34 \\
1714.50 \\
1721.66 \\
1728.82 \\
1735.97\end{array}$ & $\begin{array}{l}1174.21 \\
1179.41 \\
1184.61 \\
1189.80 \\
1195.00 \\
1200.18 \\
1205.36 \\
1210.54 \\
1215.72 \\
1220.89\end{array}$ & $\begin{array}{l}13.003 \\
13.017 \\
13.031 \\
13.045 \\
13.060 \\
13.074 \\
13.088 \\
13.101 \\
13.115 \\
13.129\end{array}$ \\
\hline $\begin{array}{l}382.00 \\
384.00 \\
386.00 \\
388.00 \\
390.00 \\
392.00 \\
394.00 \\
396.00 \\
398.00 \\
400.00\end{array}$ & $\begin{array}{l}14.6171 \\
14.6935 \\
14.7700 \\
14.8465 \\
14.9230 \\
14.9994 \\
15.0759 \\
15.1524 \\
15.2288 \\
15.3053\end{array}$ & $\begin{array}{l}1229.03 \\
1236.65 \\
1244.25 \\
1251.85 \\
1259.44 \\
1267.01 \\
1274.58 \\
1282.14 \\
1289.69 \\
1297.24\end{array}$ & $\begin{array}{l}850.34 \\
855.98 \\
861.60 \\
867.21 \\
872.82 \\
878.42 \\
884.01 \\
889.59 \\
895.16 \\
900.72\end{array}$ & $\begin{array}{l}11.994 \\
12.014 \\
12.034 \\
12.053 \\
12.073 \\
12.092 \\
12.112 \\
12.131 \\
12.150 \\
12.169\end{array}$ & $\begin{array}{l}522.00 \\
524.00 \\
526.00 \\
528.00 \\
530.00 \\
532.00 \\
534.00 \\
536.00 \\
538.00 \\
540.00\end{array}$ & $\begin{array}{l}19.9580 \\
20.0345 \\
20.1110 \\
20.1875 \\
20.2641 \\
20.3407 \\
20.4173 \\
20.4939 \\
20.5705 \\
20.6472\end{array}$ & $\begin{array}{l}1743.12 \\
1750.26 \\
1757.40 \\
1764.54 \\
1771.68 \\
1778.82 \\
1785.95 \\
1793.07 \\
1800.20 \\
1807.32\end{array}$ & $\begin{array}{l}1226.06 \\
1231.22 \\
1236.38 \\
1241.54 \\
1246.69 \\
1251.84 \\
1256.99 \\
1262.13 \\
1267.27 \\
1272.40\end{array}$ & $\begin{array}{l}13.143 \\
13.156 \\
13.170 \\
13.184 \\
13.197 \\
13.211 \\
13.224 \\
13.237 \\
13.250 \\
13.264\end{array}$ \\
\hline
\end{tabular}


TEMPER- SPECIFIC ENTHALPY INTERNAL ENTROPY ATURE VDLUME (BTU/L8) ENERGY (BTU/L8-R) (R) (CU FT/L8)
( $8 \mathrm{TU} / \mathrm{L} 8$ )
SPECIFIC ENTHALPY VDLUME (CU FT/LB)
INTERNAL ENTROPY

ENERGY
( 8 TU/LB) ATURE

(R)
(BTU/LB-R)

\begin{tabular}{|c|c|c|c|c|c|c|c|c|c|}
\hline & & : & & & $\begin{array}{l}122.00 \\
124.00 \\
126.00 \\
128.00 \\
130.00 \\
132.00 \\
134.00 \\
136.00 \\
138.00 \\
140.00\end{array}$ & $\begin{array}{l}3.9312 \\
4.0040 \\
4.0766 \\
4.1489 \\
4.2210 \\
4.2929 \\
4.3646 \\
4.4362 \\
4.5075 \\
4.5787\end{array}$ & $\begin{array}{l}287.95 \\
293.63 \\
299.32 \\
305.02 \\
310.74 \\
316.48 \\
322.23 \\
328.01 \\
333.80 \\
339.63\end{array}$ & $\begin{array}{l}171.56 \\
175.08 \\
178.62 \\
182.18 \\
185.76 \\
189.37 \\
193.00 \\
196.66 \\
200.34 \\
204.06\end{array}$ & $\begin{array}{l}7.864 \\
7.910 \\
7.955 \\
8.000 \\
8.045 \\
8.088 \\
8.132 \\
8.174 \\
8.217 \\
8.259\end{array}$ \\
\hline & & & & & $\begin{array}{l}142.00 \\
144.00 \\
146.00 \\
148.00 \\
150.00 \\
152.00 \\
154.00 \\
156.00 \\
158.00 \\
160.00\end{array}$ & $\begin{array}{l}4.6497 \\
4.7206 \\
4.7913 \\
4.8619 \\
4.9324 \\
5.0027 \\
5.0730 \\
5.1431 \\
5.2131 \\
5.2830\end{array}$ & $\begin{array}{l}345.48 \\
351.36 \\
357.27 \\
363.21 \\
369.19 \\
375.19 \\
381.24 \\
387.32 \\
393.44 \\
399.59\end{array}$ & $\begin{array}{l}207.81 \\
211.59 \\
215.41 \\
219.26 \\
223.15 \\
227.07 \\
231.04 \\
235.04 \\
239.09 \\
243.17\end{array}$ & $\begin{array}{l}8.300 \\
8.341 \\
8.382 \\
8.422 \\
8.463 \\
8.502 \\
8.542 \\
8.581 \\
8.620 \\
8.659\end{array}$ \\
\hline $\begin{array}{l}36.00 \\
38.00 \\
40.00\end{array}$ & $\begin{array}{l}.2213 \\
.2250 \\
.2292\end{array}$ & $\begin{array}{r}-106.86 \\
-102.35 \\
-97.53\end{array}$ & $\begin{array}{l}-113.41 \\
-109.01 \\
-104.32\end{array}$ & $\begin{array}{l}1.834 \\
1.955 \\
2.079\end{array}$ & $\begin{array}{l}162.00 \\
164.00 \\
166.00 \\
168.00 \\
170.00 \\
172.00 \\
174.00 \\
176.00 \\
178.00 \\
180.00\end{array}$ & $\begin{array}{l}5.3528 \\
5.4225 \\
5.4921 \\
5.5616 \\
5.6311 \\
5.7005 \\
5.7698 \\
5.8390 \\
5.9081 \\
5.9772\end{array}$ & $\begin{array}{l}405.79 \\
412.03 \\
418.30 \\
424.62 \\
430.98 \\
437.37 \\
443.81 \\
450.29 \\
456.81 \\
463.36\end{array}$ & $\begin{array}{l}247.30 \\
251.47 \\
255.69 \\
259.95 \\
264.25 \\
268.59 \\
272.98 \\
277.41 \\
281.88 \\
286.39\end{array}$ & $\begin{array}{l}8.697 \\
8.736 \\
8.774 \\
8.811 \\
8.849 \\
8.880 \\
8.924 \\
8.961 \\
8.998 \\
9.034\end{array}$ \\
\hline $\begin{array}{l}42.00 \\
44.00 \\
46.00 \\
48.00 \\
50.00 \\
52.00 \\
54.00 \\
56.00 \\
57.424 \\
57.424 \\
58.00 \\
60.00\end{array}$ & $\begin{array}{l}.2339 \\
.2392 \\
.2453 \\
.2524 \\
.2609 \\
.2717 \\
.2861 \\
.3086 \\
.3446 \\
1.0064 \\
1.0790 \\
1.2612\end{array}$ & $\begin{array}{r}-92.38 \\
-86.85 \\
-80.87 \\
-74.36 \\
-67.16 \\
-59.06 \\
-49.60 \\
-37.46 \\
-24.27 \\
67.33 \\
73.70 \\
88.77\end{array}$ & $\begin{array}{r}-99.31 \\
-93.93 \\
-88.13 \\
-81.83 \\
-74.89 \\
-67.10 \\
-58.07 \\
-46.60 \\
-44.84 \\
37.53 \\
41.75 \\
51.43\end{array}$ & $\begin{array}{l}2.205 \\
2.333 \\
2.466 \\
2.605 \\
2.751 \\
2.910 \\
3.089 \\
3.309 \\
3.541 \\
5.141 \\
5.252 \\
5.507\end{array}$ & $\begin{array}{l}182.00 \\
184.00 \\
186.00 \\
188.00 \\
190.00 \\
192.00 \\
194.00 \\
196.00 \\
198.00 \\
200.00\end{array}$ & $\begin{array}{l}6.0462 \\
6.1150 \\
6.1838 \\
6.2525 \\
6.3211 \\
6.3896 \\
6.4581 \\
6.5265 \\
6.5949 \\
6.6632\end{array}$ & $\begin{array}{l}469.95 \\
476.58 \\
483.25 \\
489.95 \\
496.70 \\
503.49 \\
510.32 \\
517.18 \\
524.09 \\
531.03\end{array}$ & $\begin{array}{l}290.94 \\
295.52 \\
300.15 \\
304.83 \\
309.54 \\
314.30 \\
319.10 \\
323.94 \\
328.82 \\
333.74\end{array}$ & $\begin{array}{l}9.071 \\
9.107 \\
9.143 \\
9.179 \\
9.214 \\
9.250 \\
9.285 \\
9.320 \\
9.356 \\
9.390\end{array}$ \\
\hline $\begin{array}{l}62.00 \\
64.00 \\
66.00 \\
68.00 \\
70.00 \\
72.00 \\
74.00 \\
76.00 \\
78.00 \\
80.00\end{array}$ & $\begin{array}{l}1.4021 \\
1.5251 \\
1.6376 \\
1.7430 \\
1.8434 \\
1.9399 \\
2.0333 \\
2.1242 \\
2.2131 \\
2.3001\end{array}$ & $\begin{array}{r}99.80 \\
109.13 \\
117.52 \\
125.29 \\
132.65 \\
139.69 \\
146.50 \\
153.12 \\
159.59 \\
165.94\end{array}$ & $\begin{array}{l}58.28 \\
63.98 \\
69.03 \\
73.69 \\
78.07 \\
82.25 \\
86.30 \\
90.23 \\
94.07 \\
97.83\end{array}$ & $\begin{array}{l}5.688 \\
5.837 \\
5.966 \\
6.082 \\
6.188 \\
6.288 \\
6.381 \\
6.469 \\
6.553 \\
6.634\end{array}$ & $\begin{array}{l}202.00 \\
204.00 \\
206.00 \\
208.00 \\
210.00 \\
212.00 \\
214.00 \\
216.00 \\
218.00 \\
220.00\end{array}$ & $\begin{array}{l}6.7315 \\
6.7997 \\
6.8679 \\
6.9361 \\
7.0042 \\
7.0723 \\
7.1403 \\
7.2083 \\
7.2763 \\
7.3443\end{array}$ & $\begin{array}{l}536.01 \\
545.03 \\
552.09 \\
559.18 \\
566.30 \\
573.47 \\
580.66 \\
587.89 \\
595.15 \\
602.43\end{array}$ & $\begin{array}{l}338.70 \\
343.70 \\
348.74 \\
353.81 \\
358.92 \\
364.07 \\
369.25 \\
374.46 \\
379.71 \\
384.98\end{array}$ & $\begin{array}{l}9.425 \\
9.460 \\
9.494 \\
9.528 \\
9.563 \\
9.596 \\
9.630 \\
9.664 \\
9.697 \\
9.731\end{array}$ \\
\hline $\begin{array}{r}82.00 \\
84.00 \\
86.00 \\
88.00 \\
90.00 \\
92.00 \\
94.00 \\
96.00 \\
98.00 \\
100.00\end{array}$ & $\begin{array}{l}2.3856 \\
2.4698 \\
2.5528 \\
2.6347 \\
2.7157 \\
2.7958 \\
2.8751 \\
2.9537 \\
3.0317 \\
3.1090\end{array}$ & $\begin{array}{l}172.18 \\
178.33 \\
184.40 \\
190.41 \\
196.36 \\
202.26 \\
208.12 \\
213.94 \\
219.73 \\
225.48\end{array}$ & $\begin{array}{l}101.54 \\
105.20 \\
108.82 \\
112.40 \\
115.96 \\
119.49 \\
122.99 \\
126.49 \\
129.96 \\
133.43\end{array}$ & $\begin{array}{l}6.711 \\
6.785 \\
6.856 \\
6.925 \\
6.992 \\
7.057 \\
7.120 \\
7.181 \\
7.241 \\
7.299\end{array}$ & $\begin{array}{l}222.00 \\
224.00 \\
226.00 \\
228.00 \\
230.00 \\
232.00 \\
234.00 \\
236.00 \\
238.00 \\
240.00\end{array}$ & $\begin{array}{l}7.4123 \\
7.4802 \\
7.5481 \\
7.6160 \\
7.6839 \\
7.7517 \\
7.8196 \\
7.8874 \\
7.9552 \\
8.0230\end{array}$ & $\begin{array}{l}609.75 \\
617.10 \\
624.48 \\
631.88 \\
639.32 \\
646.78 \\
654.26 \\
661.78 \\
669.31 \\
676.87\end{array}$ & $\begin{array}{l}390.29 \\
395.62 \\
400.99 \\
406.39 \\
411.81 \\
417.26 \\
422.74 \\
428.24 \\
433.77 \\
439.32\end{array}$ & $\begin{array}{r}9.764 \\
9.797 \\
9.829 \\
9.862 \\
9.895 \\
9.927 \\
9.959 \\
9.991 \\
10.023 \\
10.054\end{array}$ \\
\hline $\begin{array}{l}102.00 \\
104.00 \\
106.00 \\
108.00 \\
110.00 \\
112.00 \\
114.00 \\
116.00 \\
118.00 \\
120.00\end{array}$ & $\begin{array}{l}3.1859 \\
3.2622 \\
3.3380 \\
3.4134 \\
3.4884 \\
3.5630 \\
3.6373 \\
3.7112 \\
3.7849 \\
3.8582\end{array}$ & $\begin{array}{l}231.22 \\
236.93 \\
242.62 \\
248.30 \\
253.98 \\
259.64 \\
265.30 \\
270.96 \\
276.62 \\
282.28\end{array}$ & $\begin{array}{l}136.89 \\
140.34 \\
143.79 \\
147.24 \\
150.69 \\
154.15 \\
157.61 \\
161.08 \\
164.56 \\
168.05\end{array}$ & $\begin{array}{l}7.356 \\
7.411 \\
7.465 \\
7.519 \\
7.571 \\
7.622 \\
7.672 \\
7.721 \\
7.769 \\
7.817\end{array}$ & $\begin{array}{l}242.00 \\
244.00 \\
246.00 \\
248.00 \\
250.00 \\
252.00 \\
254.00 \\
256.00 \\
258.00 \\
260.00\end{array}$ & $\begin{array}{l}8.0907 \\
8.1585 \\
8.2262 \\
8.2940 \\
8.3617 \\
8.4294 \\
8.4970 \\
8.5647 \\
8.6323 \\
8.7000\end{array}$ & $\begin{array}{l}684.45 \\
692.06 \\
699.68 \\
707.33 \\
715.00 \\
722.68 \\
730.38 \\
738.10 \\
745.84 \\
753.59\end{array}$ & $\begin{array}{l}444.90 \\
450.50 \\
456.12 \\
461.76 \\
467.42 \\
473.10 \\
478.80 \\
484.51 \\
490.24 \\
495.99\end{array}$ & $\begin{array}{l}10.086 \\
10.117 \\
10.148 \\
10.179 \\
10.210 \\
10.241 \\
10.271 \\
10.301 \\
10.331 \\
10.361\end{array}$ \\
\hline
\end{tabular}


TEMPER- SPECIFIC ENTHALPY INTERNAL ENTROPY ATURE VDLUME (BTU/L8) ENERGY (BTU/L8-R) (R) (CU FT/L8)
TEMPERSPECIFIC ENTHALPY (R) (CU FT/L )
INTERNAL ENTROPY ENERGY (BTU/L8-R) (8 $\mathrm{TU} / \mathrm{L} 8$ )

\begin{tabular}{|c|c|c|c|c|c|c|c|c|c|}
\hline $\begin{array}{l}262.00 \\
264.00 \\
266.00 \\
268.00 \\
270.00 \\
272.00 \\
274.00 \\
276.00 \\
278.00 \\
280.00\end{array}$ & $\begin{array}{l}8.7676 \\
8.8352 \\
8.9028 \\
8.9703 \\
9.0379 \\
9.1054 \\
9.1729 \\
9.2404 \\
9.3079 \\
9.3753\end{array}$ & $\begin{array}{l}761.35 \\
769.13 \\
776.92 \\
784.72 \\
792.54 \\
800.34 \\
808.15 \\
815.96 \\
823.79 \\
831.62\end{array}$ & $\begin{array}{l}501.76 \\
507.53 \\
513.32 \\
519.13 \\
524.94 \\
530.74 \\
536.55 \\
542.37 \\
548.20 \\
554.03\end{array}$ & $\begin{array}{l}10.391 \\
10.421 \\
10.450 \\
10.479 \\
10.508 \\
10.537 \\
10.566 \\
10.594 \\
10.622 \\
10.650\end{array}$ & $\begin{array}{l}402.00 \\
404.00 \\
406.00 \\
408.00 \\
410.00 \\
412.00 \\
414.00 \\
416.00 \\
418.00 \\
420.00\end{array}$ & $\begin{array}{l}13.4702 \\
13.5371 \\
13.6041 \\
13.6710 \\
13.7379 \\
13.8049 \\
13.8718 \\
13.9387 \\
14.0057 \\
14.0726\end{array}$ & $\begin{array}{l}1304.80 \\
1312.33 \\
1319.85 \\
1327.36 \\
1334.86 \\
1342.35 \\
1349.83 \\
1357.31 \\
1364.78 \\
1372.24\end{array}$ & $\begin{array}{l}905.97 \\
911.51 \\
917.05 \\
922.58 \\
928.10 \\
933.61 \\
939.11 \\
944.61 \\
950.10 \\
955.57\end{array}$ & $\begin{array}{l}12.055 \\
12.074 \\
12.092 \\
12.111 \\
12.129 \\
12.147 \\
12.165 \\
12.183 \\
12.201 \\
12.219\end{array}$ \\
\hline $\begin{array}{l}282.00 \\
284.00 \\
286.00 \\
288.00 \\
290.00 \\
292.00 \\
294.00 \\
296.00 \\
298.00 \\
300.00\end{array}$ & $\begin{array}{r}9.4427 \\
9.5102 \\
9.5776 \\
9.6450 \\
9.7123 \\
9.7797 \\
9.8470 \\
9.9144 \\
9.9817 \\
10.0490\end{array}$ & $\begin{array}{l}839.45 \\
847.30 \\
855.14 \\
862.99 \\
870.85 \\
878.71 \\
886.57 \\
894.43 \\
902.29 \\
910.16\end{array}$ & $\begin{array}{l}559.87 \\
565.71 \\
571.57 \\
577.42 \\
583.28 \\
589.14 \\
595.01 \\
600.88 \\
606.75 \\
612.62\end{array}$ & $\begin{array}{l}10.678 \\
10.706 \\
10.734 \\
10.761 \\
10.788 \\
10.815 \\
10.842 \\
10.869 \\
10.895 \\
10.921\end{array}$ & $\begin{array}{l}422.00 \\
424.00 \\
426.00 \\
428.00 \\
430.00 \\
432.00 \\
434.00 \\
436.00 \\
438.00 \\
440.00\end{array}$ & $\begin{array}{l}14.1395 \\
14.2064 \\
14.2733 \\
14.3402 \\
14.4071 \\
14.4740 \\
14.5408 \\
14.6077 \\
14.6745 \\
14.7414\end{array}$ & $\begin{array}{l}1379.69 \\
1387.14 \\
1394.57 \\
1401.99 \\
1409.41 \\
1416.82 \\
1424.22 \\
1431.60 \\
1438.99 \\
1446.36\end{array}$ & $\begin{array}{r}961.04 \\
966.51 \\
971.96 \\
977.40 \\
982.84 \\
988.26 \\
993.68 \\
999.09 \\
1004.49 \\
1009.89\end{array}$ & $\begin{array}{l}12.237 \\
12.254 \\
12.272 \\
12.289 \\
12.307 \\
12.324 \\
12.341 \\
12.358 \\
12.375 \\
12.391\end{array}$ \\
\hline $\begin{array}{l}302.00 \\
304.00 \\
306.00 \\
308.00 \\
310.00 \\
312.00 \\
314.00 \\
316.00 \\
318.00 \\
320.00\end{array}$ & $\begin{array}{l}10.1162 \\
10.1835 \\
10.2508 \\
10.3180 \\
10.3852 \\
10.4525 \\
10.5197 \\
10.5869 \\
10.6541 \\
10.7212\end{array}$ & $\begin{array}{l}918.02 \\
925.89 \\
933.75 \\
941.62 \\
949.48 \\
957.34 \\
965.20 \\
973.06 \\
980.91 \\
988.76\end{array}$ & $\begin{array}{l}618.50 \\
624.37 \\
630.24 \\
636.12 \\
641.99 \\
647.86 \\
653.73 \\
659.60 \\
665.46 \\
671.32\end{array}$ & $\begin{array}{l}10.947 \\
10.973 \\
10.999 \\
11.025 \\
11.050 \\
11.076 \\
11.101 \\
11.126 \\
11.150 \\
11.175\end{array}$ & $\begin{array}{l}442.00 \\
444.00 \\
446.00 \\
448.00 \\
450.00 \\
452.00 \\
454.00 \\
456.00 \\
458.00 \\
460.00\end{array}$ & $\begin{array}{l}14.8082 \\
14.8750 \\
14.9418 \\
15.0085 \\
15.0753 \\
15.1420 \\
15.2088 \\
15.2755 \\
15.3422 \\
15.4089\end{array}$ & $\begin{array}{l}1453.72 \\
1461.08 \\
1468.42 \\
1475.76 \\
1483.09 \\
1490.43 \\
1497.76 \\
1505.08 \\
1512.40 \\
1519.71\end{array}$ & $\begin{array}{l}1015.27 \\
1020.65 \\
1026.02 \\
1031.38 \\
1036.74 \\
1042.10 \\
1047.45 \\
1052.80 \\
1058.14 \\
1063.47\end{array}$ & $\begin{array}{l}12.408 \\
12.425 \\
12.441 \\
12.458 \\
12.474 \\
12.490 \\
12.506 \\
12.523 \\
12.539 \\
12.555\end{array}$ \\
\hline $\begin{array}{l}322.00 \\
324.00 \\
326.00 \\
328.00 \\
330.00 \\
332.00 \\
334.00 \\
336.00 \\
338.00 \\
340.00\end{array}$ & $\begin{array}{l}10.7884 \\
10.8556 \\
10.9228 \\
10.9899 \\
11.0571 \\
11.1242 \\
11.1913 \\
11.2585 \\
11.3256 \\
11.3927\end{array}$ & $\begin{array}{r}996.61 \\
1004.46 \\
1012.29 \\
1020.13 \\
1027.96 \\
1035.78 \\
1043.60 \\
1051.42 \\
1059.22 \\
1067.03\end{array}$ & $\begin{array}{l}677.18 \\
683.04 \\
688.89 \\
694.74 \\
700.58 \\
706.41 \\
712.25 \\
718.07 \\
723.89 \\
729.70\end{array}$ & $\begin{array}{l}11.199 \\
11.224 \\
11.248 \\
11.272 \\
11.296 \\
11.319 \\
11.343 \\
11.366 \\
11.389 \\
11.412\end{array}$ & $\begin{array}{l}462.00 \\
464.00 \\
466.00 \\
468.00 \\
470.00 \\
472.00 \\
474.00 \\
476.00 \\
478.00 \\
480.00\end{array}$ & $\begin{array}{l}15.4756 \\
15.5422 \\
15.6089 \\
15.6755 \\
15.7422 \\
15.8088 \\
15.8754 \\
15.9420 \\
16.0086 \\
16.0752\end{array}$ & $\begin{array}{l}1527.01 \\
1534.30 \\
1541.59 \\
1548.86 \\
1556.13 \\
1563.40 \\
1570.66 \\
1577.91 \\
1585.15 \\
1592.39\end{array}$ & $\begin{array}{l}1068.80 \\
1074.12 \\
1079.43 \\
1084.74 \\
1090.03 \\
1095.32 \\
1100.61 \\
1105.89 \\
1111.16 \\
1116.43\end{array}$ & $\begin{array}{l}12.570 \\
12.586 \\
12.602 \\
12.617 \\
12.633 \\
12.648 \\
12.664 \\
12.679 \\
12.694 \\
12.709\end{array}$ \\
\hline $\begin{array}{l}342.00 \\
344.00 \\
346.00 \\
348.00 \\
350.00 \\
352.00 \\
354.00 \\
356.00 \\
358.00 \\
360.00\end{array}$ & $\begin{array}{l}11.4598 \\
11.5269 \\
11.5940 \\
11.6612 \\
11.7282 \\
11.7953 \\
11.8624 \\
11.9295 \\
11.9966 \\
12.0637\end{array}$ & $\begin{array}{l}1074.82 \\
1082.61 \\
1090.39 \\
1098.16 \\
1105.93 \\
1113.69 \\
1121.44 \\
1129.18 \\
1136.92 \\
1144.64\end{array}$ & $\begin{array}{l}735.51 \\
741.31 \\
747.11 \\
752.89 \\
758.67 \\
764.45 \\
770.21 \\
775.97 \\
781.72 \\
787.46\end{array}$ & $\begin{array}{l}11.435 \\
11.458 \\
11.480 \\
11.503 \\
11.525 \\
11.547 \\
11.569 \\
11.591 \\
11.613 \\
11.634\end{array}$ & $\begin{array}{l}482.00 \\
484.00 \\
486.00 \\
488.00 \\
490.00 \\
492.00 \\
494.00 \\
496.00 \\
498.00 \\
500.00\end{array}$ & $\begin{array}{l}16.1418 \\
16.2085 \\
16.2751 \\
16.3417 \\
16.4083 \\
16.4749 \\
16.5416 \\
16.6082 \\
16.6749 \\
16.7416\end{array}$ & $\begin{array}{l}1599.62 \\
1606.85 \\
1614.07 \\
1621.29 \\
1628.50 \\
1635.70 \\
1642.90 \\
1650.10 \\
1657.29 \\
1664.48\end{array}$ & $\begin{array}{l}1121.69 \\
1126.94 \\
1132.19 \\
1137.43 \\
1142.67 \\
1147.91 \\
1153.13 \\
1158.36 \\
1163.57 \\
1168.79\end{array}$ & $\begin{array}{l}12.724 \\
12.739 \\
12.754 \\
12.769 \\
12.784 \\
12.798 \\
12.813 \\
12.827 \\
12.842 \\
12.856\end{array}$ \\
\hline $\begin{array}{l}362.00 \\
364.00 \\
366.00 \\
368.00 \\
370.00 \\
372.00 \\
374.00 \\
376.00 \\
378.00 \\
380.00\end{array}$ & $\begin{array}{l}12.1307 \\
12.1977 \\
12.2647 \\
12.3317 \\
12.3987 \\
12.4657 \\
12.5327 \\
12.5997 \\
12.6667 \\
12.7337\end{array}$ & $\begin{array}{l}1152.36 \\
1160.06 \\
1167.76 \\
1175.45 \\
1183.13 \\
1190.80 \\
1198.46 \\
1206.12 \\
1213.76 \\
1221.40\end{array}$ & $\begin{array}{l}793.19 \\
798.91 \\
804.62 \\
810.33 \\
816.02 \\
821.71 \\
827.39 \\
833.06 \\
838.72 \\
844.37\end{array}$ & $\begin{array}{l}11.655 \\
11.677 \\
11.698 \\
11.719 \\
11.740 \\
11.760 \\
11.781 \\
11.801 \\
11.821 \\
11.842\end{array}$ & $\begin{array}{l}502.00 \\
504.00 \\
506.00 \\
508.00 \\
510.00 \\
512.00 \\
514.00 \\
516.00 \\
518.00 \\
520.00\end{array}$ & $\begin{array}{l}16.8083 \\
16.8750 \\
16.9417 \\
17.0085 \\
17.0753 \\
17.1421 \\
17.2089 \\
17.2758 \\
17.3427 \\
17.4096\end{array}$ & $\begin{array}{l}1671.66 \\
1678.84 \\
1686.02 \\
1693.19 \\
1700.36 \\
1707.53 \\
1714.69 \\
1721.85 \\
1729.00 \\
1736.16\end{array}$ & $\begin{array}{l}1174.00 \\
1179.20 \\
1184.40 \\
1189.60 \\
1194.79 \\
1199.98 \\
1205.16 \\
1210.34 \\
1215.52 \\
1220.69\end{array}$ & $\begin{array}{l}12.871 \\
12.885 \\
12.899 \\
12.913 \\
12.927 \\
12.941 \\
12.955 \\
12.969 \\
12.983 \\
12.997\end{array}$ \\
\hline $\begin{array}{l}382.00 \\
384.00 \\
386.00 \\
388.00 \\
390.00 \\
392.00 \\
394.00 \\
396.00 \\
398.00 \\
400.00\end{array}$ & $\begin{array}{l}12.8006 \\
12.8676 \\
12.9346 \\
13.0015 \\
13.0685 \\
13.1355 \\
13.2024 \\
13.2694 \\
13.3363 \\
13.4033\end{array}$ & $\begin{array}{l}1229.02 \\
1236.64 \\
1244.25 \\
1251.85 \\
1259.44 \\
1267.02 \\
1274.60 \\
1282.16 \\
1289.71 \\
1297.26\end{array}$ & $\begin{array}{l}850.02 \\
855.65 \\
861.28 \\
866.89 \\
872.50 \\
878.10 \\
883.69 \\
889.27 \\
894.85 \\
900.41\end{array}$ & $\begin{array}{l}11.862 \\
11.881 \\
11.901 \\
11.921 \\
11.940 \\
11.960 \\
11.979 \\
11.998 \\
12.017 \\
12.036\end{array}$ & $\begin{array}{l}522.00 \\
524.00 \\
526.00 \\
528.00 \\
530.00 \\
532.00 \\
534.00 \\
536.00 \\
538.00 \\
540.00\end{array}$ & $\begin{array}{l}17.4765 \\
17.5434 \\
17.6104 \\
17.6774 \\
17.7444 \\
17.8114 \\
17.8785 \\
17.9455 \\
18.0125 \\
18.0796\end{array}$ & $\begin{array}{l}1743.31 \\
1750.45 \\
1757.60 \\
1764.74 \\
1771.88 \\
1779.01 \\
1786.14 \\
1793.27 \\
1800.40 \\
1807.52\end{array}$ & $\begin{array}{l}1225.86 \\
1231.02 \\
1236.18 \\
1241.34 \\
1246.49 \\
1251.64 \\
1256.79 \\
1261.93 \\
1267.07 \\
1272.21\end{array}$ & $\begin{array}{l}13.011 \\
13.024 \\
13.038 \\
13.051 \\
13.065 \\
13.078 \\
13.092 \\
13.105 \\
13.118 \\
13.132\end{array}$ \\
\hline
\end{tabular}


TEMPER- SPECIFIC ENTHALPY INTERNAL ENTROPY ATURE VOLUME (8TU/L8) ENERGY (8TU/L8-R

(R) (CU FT/LB) (8TU/L8)
TEMPER- SPECIFIC ENTHALPY INTERNAL ENTROPY ATURE VOLUME (8TU/L8) ENERGY (R) (CU FT/L8) (8TU/L8)
$(8 T U / L B \rightarrow R)$

(8TU/L8)

\begin{tabular}{|c|c|c|c|c|c|c|c|c|c|}
\hline & & & & & $\begin{array}{l}122.00 \\
124.00 \\
126.00 \\
128.00 \\
130.00 \\
132.00 \\
134.00 \\
136.00 \\
138.00 \\
140.00\end{array}$ & $\begin{array}{l}3.4813 \\
3.5467 \\
3.6118 \\
3.6767 \\
3.7414 \\
3.8059 \\
3.8702 \\
3.9343 \\
3.9983 \\
4.0621\end{array}$ & $\begin{array}{l}286.04 \\
291.78 \\
297.52 \\
303.28 \\
309.04 \\
314.83 \\
320.63 \\
326.45 \\
332.29 \\
338.16\end{array}$ & $\begin{array}{l}170.08 \\
173.64 \\
177.21 \\
180.81 \\
184.42 \\
188.05 \\
191.71 \\
195.40 \\
199.11 \\
202.85\end{array}$ & $\begin{array}{l}7.736 \\
7.783 \\
7.828 \\
7.874 \\
7.919 \\
7.963 \\
8.006 \\
8.049 \\
8.092 \\
8.134\end{array}$ \\
\hline & & & & & $\begin{array}{l}142.00 \\
144.00 \\
146.00 \\
148.00 \\
150.00 \\
152.00 \\
154.00 \\
156.00 \\
158.00 \\
160.00\end{array}$ & $\begin{array}{l}4.1257 \\
4.1891 \\
4.2524 \\
4.3156 \\
4.3787 \\
4.4416 \\
4.5044 \\
4.5671 \\
4.6297 \\
4.6922\end{array}$ & $\begin{array}{l}344.05 \\
349.97 \\
355.91 \\
361.89 \\
367.90 \\
373.94 \\
380.02 \\
386.13 \\
392.28 \\
398.47\end{array}$ & $\begin{array}{l}206.63 \\
210.43 \\
214.27 \\
218.14 \\
222.05 \\
226.00 \\
229.98 \\
234.00 \\
238.07 \\
242.17\end{array}$ & $\begin{array}{l}8.176 \\
8.217 \\
8.258 \\
8.299 \\
8.339 \\
8.379 \\
8.419 \\
8.459 \\
8.498 \\
8.537\end{array}$ \\
\hline $\begin{array}{l}36.00 \\
38.00 \\
40.00\end{array}$ & $\begin{array}{r}.2208 \\
.2244 \\
.2285\end{array}$ & $\begin{array}{r}-106.28 \\
-101.79 \\
-97.01\end{array}$ & $\begin{array}{r}-113.63 \\
-109.27 \\
-104.62\end{array}$ & $\begin{array}{l}1.827 \\
1.948 \\
2.071\end{array}$ & $\begin{array}{l}162.00 \\
164.00 \\
166.00 \\
168.00 \\
170.00 \\
172.00 \\
174.00 \\
176.00 \\
178.00 \\
180.00\end{array}$ & $\begin{array}{l}4.7546 \\
4.8169 \\
4.8791 \\
4.9412 \\
5.0033 \\
5.0652 \\
5.1271 \\
5.1889 \\
5.2507 \\
5.3123\end{array}$ & $\begin{array}{l}404.69 \\
410.96 \\
417.26 \\
423.60 \\
429.98 \\
436.41 \\
442.87 \\
449.37 \\
455.91 \\
462.49\end{array}$ & $\begin{array}{l}246.32 \\
250.51 \\
254.74 \\
259.01 \\
263.33 \\
267.69 \\
272.09 \\
276.53 \\
281.01 \\
285.54\end{array}$ & $\begin{array}{l}8.575 \\
8.614 \\
8.652 \\
8.690 \\
8.728 \\
8.765 \\
8.803 \\
8.840 \\
8.877 \\
8.913\end{array}$ \\
\hline $\begin{array}{l}42.00 \\
44.00 \\
46.00 \\
48.00 \\
50.00 \\
52.00 \\
54.00 \\
56.00 \\
58.00 \\
* 58.860 \\
* 58.860 \\
60.00\end{array}$ & $\begin{array}{l}.2331 \\
.2383 \\
.2442 \\
.2510 \\
.2591 \\
.2692 \\
.2822 \\
.3012 \\
.3388 \\
.3978\end{array}$ & $\begin{array}{r}-91.90 \\
-86.42 \\
-80.51 \\
-74.10 \\
-67.05 \\
-59.19 \\
-50.16 \\
-39.13 \\
-22.53 \\
-8.28\end{array}$ & $\begin{array}{l}-99.66 \\
-94.36 \\
-88.65 \\
-82.46 \\
-75.69 \\
-68.15 \\
-59.56 \\
-47.17 \\
-33.81 \\
-34.99\end{array}$ & $\begin{array}{l}2.196 \\
2.323 \\
2.454 \\
2.591 \\
2.734 \\
2.889 \\
3.059 \\
3.259 \\
3.550 \\
3.793\end{array}$ & $\begin{array}{l}182.00 \\
184.00 \\
186.00 \\
188.00 \\
190.00 \\
192.00 \\
194.00 \\
196.00 \\
198.00 \\
200.00\end{array}$ & $\begin{array}{l}5.3739 \\
5.4353 \\
5.4967 \\
5.5579 \\
5.6191 \\
5.6803 \\
5.7413 \\
5.8023 \\
5.8632 \\
5.9241\end{array}$ & $\begin{array}{l}469.10 \\
475.75 \\
482.43 \\
489.16 \\
495.92 \\
502.73 \\
509.57 \\
516.45 \\
523.37 \\
530.33\end{array}$ & $\begin{array}{l}290.10 \\
294.70 \\
299.34 \\
304.02 \\
308.75 \\
313.52 \\
318.33 \\
323.18 \\
328.07 \\
333.00\end{array}$ & $\begin{array}{l}8.950 \\
8.986 \\
9.022 \\
9.058 \\
9.094 \\
9.130 \\
9.165 \\
9.201 \\
9.236 \\
9.271\end{array}$ \\
\hline $\begin{array}{l}62.00 \\
64.00 \\
66.00 \\
68.00 \\
70.00 \\
72.00 \\
74.00 \\
76.00 \\
78.00 \\
80.00\end{array}$ & $\begin{array}{l}1.5778 \\
1.6688 \\
1.7562 \\
1.8407 \\
1.9229 \\
2.0032\end{array}$ & $\begin{array}{l}126.59 \\
134.12 \\
141.33 \\
148.28 \\
155.04 \\
161.64\end{array}$ & $\begin{array}{l}74.03 \\
78.53 \\
82.83 \\
86.97 \\
90.99 \\
94.92\end{array}$ & $\begin{array}{l}6.012 \\
6.118 \\
6.216 \\
6.309 \\
6.397 \\
6.481\end{array}$ & $\begin{array}{l}202.00 \\
204.00 \\
206.00 \\
208.00 \\
210.00 \\
212.00 \\
214.00 \\
216.00 \\
218.00 \\
220.00\end{array}$ & $\begin{array}{l}5.9850 \\
6.0458 \\
6.1065 \\
6.1672 \\
6.2279 \\
6.2886 \\
6.3492 \\
6.4098 \\
6.4703 \\
6.5309\end{array}$ & $\begin{array}{l}537.33 \\
544.36 \\
551.43 \\
558.54 \\
565.68 \\
572.85 \\
580.06 \\
587.30 \\
594.57 \\
601.87\end{array}$ & $\begin{array}{l}337.97 \\
342.98 \\
348.03 \\
353.11 \\
358.23 \\
363.39 \\
368.58 \\
373.80 \\
379.05 \\
384.33\end{array}$ & $\begin{array}{l}9.306 \\
9.340 \\
9.375 \\
9.409 \\
9.443 \\
9.477 \\
9.511 \\
9.545 \\
9.578 \\
9.611\end{array}$ \\
\hline $\begin{array}{r}82.00 \\
84.00 \\
86.00 \\
88.00 \\
90.00 \\
92.00 \\
94.00 \\
96.00 \\
98.00 \\
100.00\end{array}$ & $\begin{array}{l}2.0818 \\
2.1589 \\
2.2348 \\
2.3096 \\
2.3833 \\
2.4562 \\
2.5282 \\
2.5996 \\
2.6702 \\
2.7403\end{array}$ & $\begin{array}{l}168.10 \\
174.46 \\
180.71 \\
186.89 \\
192.99 \\
199.03 \\
205.02 \\
210.96 \\
216.86 \\
222.72\end{array}$ & $\begin{array}{r}98.76 \\
102.54 \\
106.27 \\
109.96 \\
113.60 \\
117.22 \\
120.80 \\
124.37 \\
127.91 \\
131.44\end{array}$ & $\begin{array}{l}6.560 \\
6.637 \\
6.710 \\
6.781 \\
6.850 \\
6.916 \\
6.981 \\
7.043 \\
7.104 \\
7.163\end{array}$ & $\begin{array}{l}222.00 \\
224.00 \\
226.00 \\
228.00 \\
230.00 \\
232.00 \\
234.00 \\
236.00 \\
238.00 \\
240.00\end{array}$ & $\begin{array}{l}6.5914 \\
6.6519 \\
6.7124 \\
6.7729 \\
6.8333 \\
6.8938 \\
6.9542 \\
7.0146 \\
7.0750 \\
7.1354\end{array}$ & $\begin{array}{l}609.20 \\
616.56 \\
623.95 \\
631.37 \\
638.81 \\
646.28 \\
653.78 \\
661.30 \\
668.85 \\
676.42\end{array}$ & $\begin{array}{l}389.65 \\
394.99 \\
400.36 \\
405.77 \\
411.20 \\
416.66 \\
422.14 \\
427.65 \\
433.18 \\
438.74\end{array}$ & $\begin{array}{l}9.645 \\
9.678 \\
9.711 \\
9.743 \\
9.776 \\
9.808 \\
9.840 \\
9.872 \\
9.904 \\
9.936\end{array}$ \\
\hline $\begin{array}{l}102.00 \\
104.00 \\
106.00 \\
108.00 \\
110.00 \\
112.00 \\
114.00 \\
116.00 \\
118.00 \\
120.00\end{array}$ & $\begin{array}{l}2.8097 \\
2.8787 \\
2.9472 \\
3.0152 \\
3.0828 \\
3.1501 \\
3.2169 \\
3.2835 \\
3.3497 \\
3.4156\end{array}$ & $\begin{array}{l}228.55 \\
234.36 \\
240.14 \\
245.91 \\
251.66 \\
257.40 \\
263.13 \\
268.86 \\
274.59 \\
280.31\end{array}$ & $\begin{array}{l}134.96 \\
138.47 \\
141.97 \\
145.47 \\
148.97 \\
152.48 \\
155.98 \\
159.49 \\
163.01 \\
166.54\end{array}$ & $\begin{array}{l}7.221 \\
7.278 \\
7.333 \\
7.386 \\
7.439 \\
7.491 \\
7.542 \\
7.592 \\
7.640 \\
7.689\end{array}$ & $\begin{array}{l}242.00 \\
244.00 \\
246.00 \\
248.00 \\
250.00 \\
252.00 \\
254.00 \\
256.00 \\
258.00 \\
260.00\end{array}$ & $\begin{array}{l}7.1958 \\
7.2561 \\
7.3165 \\
7.3768 \\
7.4371 \\
7.4974 \\
7.5577 \\
7.6180 \\
7.6782 \\
7.7385\end{array}$ & $\begin{array}{l}684.01 \\
691.63 \\
699.26 \\
706.92 \\
714.59 \\
722.28 \\
730.00 \\
737.72 \\
745.47 \\
753.23\end{array}$ & $\begin{array}{l}444.32 \\
449.93 \\
455.55 \\
461.20 \\
466.86 \\
472.55 \\
478.25 \\
483.97 \\
489.71 \\
495.46\end{array}$ & $\begin{array}{r}9.967 \\
9.999 \\
10.030 \\
10.061 \\
10.092 \\
10.122 \\
10.153 \\
10.183 \\
10.213 \\
10.243\end{array}$ \\
\hline
\end{tabular}

* PHASE CHANGE 
TEMPER- SPECIFIC ENTHALPY INTERNAL ENTROPY ATURE VOLUME (8TU/L8) ENERGY (8TU/L8-R) (R) (CU FT/L8)
( $8 T$ TU/L8)
TEMPER- SPECIFIC ENTHALPY INTERNAL ENTROPY
ATURE VOLUME (8TU/L8) ENERGY (8TU/L8-R)
(R) (CU FT/L8) ( $)$ TU/L8)
( $) ~$

\begin{tabular}{|c|c|c|c|c|c|c|c|c|c|}
\hline $\begin{array}{l}262.00 \\
264.00 \\
266.00 \\
268.00 \\
270.00 \\
272.00 \\
274.00 \\
276.00 \\
278.00 \\
280.00\end{array}$ & $\begin{array}{l}7.7987 \\
7.8589 \\
7.9191 \\
7.9792 \\
8.0394 \\
8.0995 \\
8.1596 \\
8.2197 \\
8.2798 \\
8.3398\end{array}$ & $\begin{array}{l}761.00 \\
768.79 \\
776.59 \\
784.40 \\
792.22 \\
800.03 \\
807.85 \\
815.67 \\
823.50 \\
831.34\end{array}$ & $\begin{array}{l}501.23 \\
507.01 \\
512.81 \\
518.61 \\
524.43 \\
530.24 \\
536.05 \\
541.88 \\
547.71 \\
553.54\end{array}$ & $\begin{array}{l}10.273 \\
10.302 \\
10.332 \\
10.361 \\
10.390 \\
10.419 \\
10.448 \\
10.476 \\
10.504 \\
10.532\end{array}$ & $\begin{array}{l}402.00 \\
404.00 \\
406.00 \\
408.00 \\
410.00 \\
412.00 \\
414.00 \\
416.00 \\
418.00 \\
420.00\end{array}$ & $\begin{array}{l}11.9835 \\
12.0431 \\
12.1026 \\
12.1621 \\
12.2217 \\
12.2812 \\
12.3407 \\
12.4003 \\
12.4598 \\
12.5193\end{array}$ & $\begin{array}{l}1304.83 \\
1312.36 \\
1319.88 \\
1327.39 \\
1334.90 \\
1342.40 \\
1349.88 \\
1357.36 \\
1364.84 \\
1372.30\end{array}$ & $\begin{array}{l}905.66 \\
911.21 \\
916.75 \\
922.28 \\
927.80 \\
933.32 \\
938.82 \\
944.32 \\
949.81 \\
955.29\end{array}$ & $\begin{array}{l}11.938 \\
11.957 \\
11.975 \\
11.994 \\
12.012 \\
12.030 \\
12.048 \\
12.066 \\
12.084 \\
12.102\end{array}$ \\
\hline $\begin{array}{l}282.00 \\
284.00 \\
286.00 \\
288.00 \\
290.00 \\
292.00 \\
294.00 \\
296.00 \\
298.00 \\
300.00\end{array}$ & $\begin{array}{l}8.3999 \\
8.4599 \\
8.5199 \\
8.5799 \\
8.6399 \\
8.6998 \\
8.7598 \\
8.8197 \\
8.8796 \\
8.9395\end{array}$ & $\begin{array}{l}839.18 \\
847.03 \\
854.89 \\
862.74 \\
870.61 \\
878.47 \\
886.34 \\
894.21 \\
902.08 \\
909.95\end{array}$ & $\begin{array}{l}559.39 \\
565.24 \\
571.09 \\
576.95 \\
582.82 \\
588.68 \\
594.55 \\
600.43 \\
606.30 \\
612.18\end{array}$ & $\begin{array}{l}10.560 \\
10.588 \\
10.616 \\
10.643 \\
10.670 \\
10.697 \\
10.724 \\
10.751 \\
10.777 \\
10.804\end{array}$ & $\begin{array}{l}422.00 \\
424.00 \\
426.00 \\
428.00 \\
430.00 \\
432.00 \\
434.00 \\
436.00 \\
438.00 \\
440.00\end{array}$ & $\begin{array}{l}12.5788 \\
12.6383 \\
12.6978 \\
12.7573 \\
12.8168 \\
12.8763 \\
12.9358 \\
12.9952 \\
13.0547 \\
13.1141\end{array}$ & $\begin{array}{l}1379.76 \\
1387.20 \\
1394.64 \\
1402.07 \\
1409.49 \\
1416.90 \\
1424.30 \\
1431.69 \\
1439.07 \\
1446.45\end{array}$ & $\begin{array}{r}960.76 \\
966.22 \\
971.68 \\
977.13 \\
982.56 \\
987.99 \\
993.41 \\
998.83 \\
1004.23 \\
1009.62\end{array}$ & $\begin{array}{l}12.120 \\
12.137 \\
12.155 \\
12.172 \\
12.190 \\
12.207 \\
12.224 \\
12.241 \\
12.258 \\
12.275\end{array}$ \\
\hline $\begin{array}{l}302.00 \\
304.00 \\
306.00 \\
308.00 \\
310.00 \\
312.00 \\
314.00 \\
316.00 \\
318.00 \\
32.0 .00\end{array}$ & $\begin{array}{l}8.9994 \\
9.0592 \\
9.1191 \\
9.1789 \\
9.2388 \\
9.2986 \\
9.3584 \\
9.4182 \\
9.4780 \\
9.5378\end{array}$ & $\begin{array}{l}917.82 \\
925.69 \\
933.56 \\
941.43 \\
949.30 \\
957.17 \\
965.04 \\
972.90 \\
980.76 \\
988.62\end{array}$ & $\begin{array}{l}618.06 \\
623.93 \\
629.81 \\
635.69 \\
641.56 \\
647.44 \\
653.31 \\
659.18 \\
665.05 \\
670.92\end{array}$ & $\begin{array}{l}10.830 \\
10.856 \\
10.882 \\
10.907 \\
10.933 \\
10.958 \\
10.983 \\
11.008 \\
11.033 \\
11.058\end{array}$ & $\begin{array}{l}442.00 \\
444.00 \\
446.00 \\
448.00 \\
450.00 \\
452.00 \\
454.00 \\
456.00 \\
458.00 \\
460.00\end{array}$ & $\begin{array}{l}13.1735 \\
13.2329 \\
13.2923 \\
13.3517 \\
13.4111 \\
13.4705 \\
13.5298 \\
13.5891 \\
13.6485 \\
13.7078\end{array}$ & $\begin{array}{l}1453.82 \\
1461.17 \\
1468.52 \\
1475.87 \\
1483.20 \\
1490.54 \\
1497.88 \\
1505.20 \\
1512.52 \\
1519.83\end{array}$ & $\begin{array}{l}1015.01 \\
1020.39 \\
1025.76 \\
1031.13 \\
1036.48 \\
1041.85 \\
1047.21 \\
1052.56 \\
1057.90 \\
1063.23\end{array}$ & $\begin{array}{l}12.291 \\
12.308 \\
12.324 \\
12.341 \\
12.357 \\
12.374 \\
12.390 \\
12.406 \\
12.422 \\
12.438\end{array}$ \\
\hline $\begin{array}{l}322.00 \\
324.00 \\
326.00 \\
328.00 \\
330.00 \\
332.00 \\
334.00 \\
336.00 \\
338.00 \\
340.00\end{array}$ & $\begin{array}{r}9.5976 \\
9.6573 \\
9.7171 \\
9.7768 \\
9.8366 \\
9.8963 \\
9.9561 \\
10.0158 \\
10.0755 \\
10.1353\end{array}$ & $\begin{array}{r}996.47 \\
1004.32 \\
1012.17 \\
1020.01 \\
1027.84 \\
1035.67 \\
1043.50 \\
1051.31 \\
1059.13 \\
1066.93\end{array}$ & $\begin{array}{l}676.78 \\
682.64 \\
688.49 \\
694.34 \\
700.19 \\
706.03 \\
711.86 \\
717.69 \\
723.52 \\
729.33\end{array}$ & $\begin{array}{l}11.082 \\
11.106 \\
11.130 \\
11.154 \\
11.178 \\
11.202 \\
11.225 \\
11.249 \\
11.272 \\
11.295\end{array}$ & $\begin{array}{l}462.00 \\
464.00 \\
466.00 \\
468.00 \\
470.00 \\
472.00 \\
474.00 \\
476.00 \\
478.00 \\
480.00\end{array}$ & $\begin{array}{l}13.7671 \\
13.8264 \\
13.8857 \\
13.9449 \\
14.0042 \\
14.0635 \\
14.1227 \\
14.1819 \\
14.2412 \\
14.3004\end{array}$ & $\begin{array}{l}1527.14 \\
1534.43 \\
1541.72 \\
1549.00 \\
1556.27 \\
1563.54 \\
1570.80 \\
1578.06 \\
1585.30 \\
1592.54\end{array}$ & $\begin{array}{l}1068.56 \\
1073.88 \\
1079.19 \\
1084.50 \\
1089.80 \\
1095.09 \\
1100.38 \\
1105.66 \\
1110.94 \\
1116.20\end{array}$ & $\begin{array}{l}12.454 \\
12.469 \\
12.485 \\
12.501 \\
12.516 \\
12.532 \\
12.547 \\
12.562 \\
12.577 \\
12.592\end{array}$ \\
\hline $\begin{array}{l}342.00 \\
344.00 \\
346.00 \\
348.00 \\
350.00 \\
352.00 \\
354.00 \\
356.00 \\
358.00 \\
360.00\end{array}$ & $\begin{array}{l}10.1950 \\
10.2547 \\
10.3144 \\
10.3741 \\
10.4338 \\
10.4935 \\
10.5532 \\
10.6129 \\
10.6726 \\
10.7323\end{array}$ & $\begin{array}{l}1074.73 \\
1082.52 \\
1090.31 \\
1098.09 \\
1105.86 \\
1113.62 \\
1121.38 \\
1129.13 \\
1136.86 \\
1144.60\end{array}$ & $\begin{array}{l}735.14 \\
740.95 \\
746.74 \\
752.53 \\
758.31 \\
764.09 \\
769.86 \\
775.61 \\
781.37 \\
787.11\end{array}$ & $\begin{array}{l}11.318 \\
11.341 \\
11.363 \\
11.385 \\
11.408 \\
11.430 \\
11.452 \\
11.474 \\
11.495 \\
11.517\end{array}$ & $\begin{array}{l}482.00 \\
484.00 \\
486.00 \\
488.00 \\
490.00 \\
492.00 \\
494.00 \\
496.00 \\
498.00 \\
500.00\end{array}$ & $\begin{array}{l}14.3597 \\
14.4189 \\
14.4782 \\
14.5374 \\
14.5966 \\
14.6559 \\
14.7152 \\
14.7744 \\
14.8337 \\
14.8930\end{array}$ & $\begin{array}{l}1599.78 \\
1607.01 \\
1614.23 \\
1621.45 \\
1628.66 \\
1635.87 \\
1643.08 \\
1650.27 \\
1657.47 \\
1664.66\end{array}$ & $\begin{array}{l}1121.47 \\
1126.72 \\
1131.97 \\
1137.22 \\
1142.46 \\
1147.69 \\
1152.92 \\
1158.14 \\
1163.36 \\
1168.58\end{array}$ & $\begin{array}{l}12.608 \\
12.622 \\
12.637 \\
12.652 \\
12.667 \\
12.682 \\
12.696 \\
12.711 \\
12.725 \\
12.740\end{array}$ \\
\hline $\begin{array}{l}362.00 \\
364.00 \\
366.00 \\
368.00 \\
370.00 \\
372.00 \\
374.00 \\
376.00 \\
378.00 \\
380.00\end{array}$ & $\begin{array}{l}10.7919 \\
10.8515 \\
10.9112 \\
10.9708 \\
11.0304 \\
11.0900 \\
11.1496 \\
11.2092 \\
11.2688 \\
11.3283\end{array}$ & $\begin{array}{l}1152.31 \\
1160.02 \\
1167.73 \\
11175.42 \\
1183.10 \\
11190.78 \\
1198.44 \\
1206.10 \\
1213.75 \\
1221.39\end{array}$ & $\begin{array}{l}792.84 \\
798.56 \\
804.28 \\
809.99 \\
815.69 \\
821.37 \\
827.06 \\
832.73 \\
838.39 \\
844.05\end{array}$ & $\begin{array}{l}11.538 \\
11.560 \\
11.581 \\
11.602 \\
11.622 \\
11.643 \\
11.664 \\
11.684 \\
11.704 \\
11.724\end{array}$ & $\begin{array}{l}502.00 \\
504.00 \\
506.00 \\
508.00 \\
510.00 \\
512.00 \\
514.00 \\
516.00 \\
518.00 \\
520.00\end{array}$ & $\begin{array}{l}14.9524 \\
15.0117 \\
15.0710 \\
15.1304 \\
15.1898 \\
15.2492 \\
15.3086 \\
15.3680 \\
15.4275 \\
15.4870\end{array}$ & $\begin{array}{l}1671.84 \\
1679.03 \\
1686.20 \\
1693.38 \\
1700.55 \\
1707.72 \\
1714.88 \\
1722.04 \\
1729.20 \\
1736.35\end{array}$ & $\begin{array}{l}1173.79 \\
1178.99 \\
1184.19 \\
1189.39 \\
1194.58 \\
1199.77 \\
1204.96 \\
1210.14 \\
1215.31 \\
1220.49\end{array}$ & $\begin{array}{l}12.754 \\
12.768 \\
12.783 \\
12.797 \\
12.811 \\
12.825 \\
12.839 \\
12.853 \\
12.866 \\
12.880\end{array}$ \\
\hline $\begin{array}{l}382.00 \\
384.00 \\
386.00 \\
388.00 \\
390.00 \\
392.00 \\
394.00 \\
396.00 \\
398.00 \\
400.00\end{array}$ & $\begin{array}{l}11.3879 \\
11.4475 \\
11.5071 \\
11.5666 \\
11.6262 \\
11.6858 \\
11.7453 \\
11.8049 \\
11.8644 \\
11.9240\end{array}$ & $\begin{array}{l}1229.02 \\
1236.64 \\
1244.25 \\
1251.86 \\
1259.45 \\
1267.04 \\
1274.61 \\
1282.18 \\
1289.74 \\
1297.29\end{array}$ & $\begin{array}{l}849.69 \\
855.33 \\
860.96 \\
866.58 \\
872.19 \\
877.79 \\
883.38 \\
888.96 \\
894.54 \\
900.10\end{array}$ & $\begin{array}{l}11.745 \\
11.764 \\
11.784 \\
11.804 \\
11.823 \\
11.843 \\
11.862 \\
11.881 \\
11.900 \\
11.919\end{array}$ & $\begin{array}{l}522.00 \\
524.00 \\
526.00 \\
528.00 \\
530.00 \\
532.00 \\
534.00 \\
536.00 \\
538.00 \\
540.00\end{array}$ & $\begin{array}{l}15.5465 \\
15.6060 \\
15.6655 \\
15.7251 \\
15.7847 \\
15.8442 \\
15.9038 \\
15.9634 \\
16.0230 \\
16.0826\end{array}$ & $\begin{array}{l}1743.50 \\
1750.65 \\
1757.80 \\
1764.94 \\
1772.08 \\
1779.21 \\
1786.35 \\
1793.48 \\
1800.60 \\
1807.72\end{array}$ & $\begin{array}{l}1225.66 \\
1230.82 \\
1235.99 \\
1241.14 \\
1246.30 \\
1251.45 \\
1256.60 \\
1261.74 \\
1266.88 \\
1272.02\end{array}$ & $\begin{array}{l}12.894 \\
12.908 \\
12.921 \\
12.935 \\
12.948 \\
12.962 \\
12.975 \\
12.988 \\
13.002 \\
13.015\end{array}$ \\
\hline
\end{tabular}


$\begin{array}{ccccc}\text { TEMPER- } & \text { SPECIFIC } & \text { ENTHALPY } & \text { INTERNAL } & \text { ENTROPY } \\ \text { ATURE } & \text { VOLUME } & \text { (BTU/L8) } & \text { ENERGY } & (8 T U / L-R)\end{array}$ (R) (CU FT/LB) (BTU/L. B)
TEMPER-

ATURE VOLUME (BTU/L B) (R) (CU FT/L8)
INTERNAL ENTROPY

ENERGY (8TU/L.8-R) (BTU/L8)

\begin{tabular}{|c|c|c|c|c|c|c|c|c|c|}
\hline & & & & & $\begin{array}{l}122.00 \\
124.00 \\
126.00 \\
128.00 \\
130.00 \\
132.00 \\
134.00 \\
136.00 \\
138.00 \\
140.00\end{array}$ & $\begin{array}{l}3.1216 \\
3.1811 \\
3.2403 \\
3.2993 \\
3.3580 \\
3.4166 \\
3.4750 \\
3.5331 \\
3.5911 \\
3.6490\end{array}$ & $\begin{array}{l}284.14 \\
289.93 \\
295.73 \\
301.54 \\
307.36 \\
313.19 \\
319.04 \\
324.90 \\
330.79 \\
336.70\end{array}$ & $\begin{array}{l}168.61 \\
172.20 \\
175.81 \\
179.43 \\
183.07 \\
186.74 \\
190.43 \\
194.14 \\
197.88 \\
201.64\end{array}$ & $\begin{array}{l}7.620 \\
7.667 \\
7.714 \\
7.760 \\
7.805 \\
7.849 \\
7.893 \\
7.937 \\
7.980 \\
8.022\end{array}$ \\
\hline & & & & & $\begin{array}{l}142.00 \\
144.00 \\
146.00 \\
148.00 \\
150.00 \\
152.00 \\
154.00 \\
156.00 \\
158.00 \\
160.00\end{array}$ & $\begin{array}{l}3.7067 \\
3.7642 \\
3.8216 \\
3.8788 \\
3.9360 \\
3.9929 \\
4.0498 \\
4.1066 \\
4.1633 \\
4.2198\end{array}$ & $\begin{array}{l}342.63 \\
348.58 \\
354.57 \\
360.58 \\
366.63 \\
372.70 \\
378.81 \\
384.96 \\
391.13 \\
397.35\end{array}$ & $\begin{array}{l}205.44 \\
209.27 \\
213.13 \\
217.02 \\
220.95 \\
224.92 \\
228.93 \\
232.97 \\
237.05 \\
241.17\end{array}$ & $\begin{array}{l}8.064 \\
8.106 \\
8.147 \\
8.188 \\
8.229 \\
8.269 \\
8.309 \\
8.348 \\
8.388 \\
8.427\end{array}$ \\
\hline 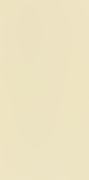 & 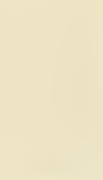 & 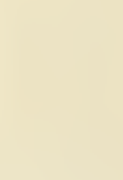 & & 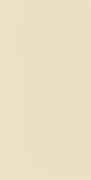 & $\begin{array}{l}162.00 \\
164.00 \\
166.00 \\
168.00 \\
170.00 \\
172.00 \\
174.00\end{array}$ & $\begin{array}{l}4.2763 \\
4.3326 \\
4.3889 \\
4.4451 \\
4.5012 \\
4.5572 \\
4.6132\end{array}$ & $\begin{array}{l}403.60 \\
409.90 \\
416.22 \\
422.59 \\
429.00 \\
435.45 \\
441.93\end{array}$ & $\begin{array}{l}245.34 \\
249.54 \\
253.79 \\
258.08 \\
262.41 \\
266.78 \\
271.20\end{array}$ & $\begin{array}{l}8.466 \\
8.504 \\
8.543 \\
8.581 \\
8.619 \\
8.656 \\
8.694\end{array}$ \\
\hline $\begin{array}{l}36.00 \\
38.00 \\
40.00\end{array}$ & $\begin{array}{l}.2203 \\
.2239 \\
.2279\end{array}$ & $\begin{array}{r}-105.69 \\
-101.23 \\
-96.48\end{array}$ & $\begin{array}{l}-113.84 \\
-109.52 \\
-104.92\end{array}$ & $\begin{array}{l}1.821 \\
1.941 \\
2.063\end{array}$ & $\begin{array}{l}176.00 \\
178.00 \\
180.00\end{array}$ & $\begin{array}{l}4.6691 \\
4.7249 \\
4.7806\end{array}$ & $\begin{array}{l}448.46 \\
455.02 \\
461.62\end{array}$ & $\begin{array}{l}275.66 \\
280.15 \\
284.69\end{array}$ & $\begin{array}{l}8.731 \\
8.768 \\
8.805\end{array}$ \\
\hline $\begin{array}{l}42.00 \\
44.00 \\
46.00 \\
48.00 \\
50.00 \\
52.00 \\
54.00 \\
56.00 \\
58.00 \\
60.00\end{array}$ & $\begin{array}{l}.2324 \\
.2374 \\
.2431 \\
.2497 \\
.2575 \\
.2669 \\
.2789 \\
.2955\end{array}$ & $\begin{array}{l}-91.41 \\
-85.98 \\
-80.14 \\
-73.82 \\
-66.90 \\
-59.24 \\
-50.57 \\
-40.30\end{array}$ & $\begin{array}{r}-100.01 \\
-94.77 \\
-89.14 \\
-83.06 \\
-76.43 \\
-69.12 \\
-60.90 \\
-51.24\end{array}$ & $\begin{array}{l}2.187 \\
2.313 \\
2.443 \\
2.577 \\
2.718 \\
2.868 \\
3.032 \\
3.219\end{array}$ & $\begin{array}{l}182.00 \\
184.00 \\
186.00 \\
188.00 \\
190.00 \\
192.00 \\
194.00 \\
196.00 \\
198.00 \\
200.00\end{array}$ & $\begin{array}{l}4.8362 \\
4.8917 \\
4.9472 \\
5.0025 \\
5.0577 \\
5.1129 \\
5.1680 \\
5.2231 \\
5.2781 \\
5.3330\end{array}$ & $\begin{array}{l}468.26 \\
474.92 \\
481.62 \\
488.37 \\
495.15 \\
501.97 \\
508.83 \\
515.73 \\
522.67 \\
529.64\end{array}$ & $\begin{array}{l}289.26 \\
293.87 \\
298.53 \\
303.22 \\
307.96 \\
312.74 \\
317.56 \\
322.42 \\
327.32 \\
332.27\end{array}$ & $\begin{array}{l}8.842 \\
8.878 \\
8.914 \\
8.950 \\
8.986 \\
9.022 \\
9.058 \\
9.093 \\
9.128 \\
9.163\end{array}$ \\
\hline $\begin{array}{l}62.00 \\
64.00 \\
66.00 \\
68.00 \\
70.00 \\
72.00 \\
74.00 \\
76.00 \\
78.00 \\
80.00\end{array}$ & $\begin{array}{l}1.2710 \\
1.3638 \\
1.4509 \\
1.5338 \\
1.6135 \\
1.6906 \\
1.7656\end{array}$ & $\begin{array}{l}111.46 \\
120.20 \\
128.31 \\
135.98 \\
143.31 \\
150.39 \\
157.26\end{array}$ & $\begin{array}{l}64.42 \\
69.73 \\
74.61 \\
79.21 \\
83.59 \\
87.82 \\
91.92\end{array}$ & $\begin{array}{l}5.716 \\
5.843 \\
5.957 \\
6.062 \\
6.160 \\
6.252 \\
6.339\end{array}$ & $\begin{array}{l}202.00 \\
204.00 \\
206.00 \\
208.00 \\
210.00 \\
212.00 \\
214.00 \\
216.00 \\
218.00 \\
220.00\end{array}$ & $\begin{array}{l}5.3879 \\
5.4427 \\
5.4975 \\
5.5523 \\
5.6071 \\
5.6618 \\
5.7164 \\
5.7711 \\
5.8257 \\
5.8803\end{array}$ & $\begin{array}{l}536.65 \\
543.70 \\
550.79 \\
557.91 \\
565.06 \\
572.25 \\
579.47 \\
586.73 \\
594.01 \\
601.32\end{array}$ & $\begin{array}{l}337.25 \\
342.26 \\
347.32 \\
352.41 \\
357.54 \\
362.70 \\
367.90 \\
373.13 \\
378.39 \\
383.68\end{array}$ & $\begin{array}{l}9.198 \\
9.233 \\
9.267 \\
9.302 \\
9.336 \\
9.370 \\
9.404 \\
9.438 \\
9.471 \\
9.505\end{array}$ \\
\hline $\begin{array}{l}82.00 \\
84.00 \\
86.00 \\
88.00 \\
90.00 \\
92.00 \\
94.00 \\
96.00 \\
98.00 \\
00.00\end{array}$ & $\begin{array}{l}1.8387 \\
1.9103 \\
1.9806 \\
2.0496 \\
2.1177 \\
2.1848 \\
2.2510 \\
2.3165 \\
2.3814 \\
2.4455\end{array}$ & $\begin{array}{l}163.97 \\
170.53 \\
176.98 \\
183.33 \\
189.59 \\
195.78 \\
201.90 \\
207.96 \\
213.98 \\
219.95\end{array}$ & $\begin{array}{r}95.91 \\
99.83 \\
103.68 \\
107.47 \\
111.21 \\
114.92 \\
118.59 \\
122.23 \\
125.84 \\
129.44\end{array}$ & $\begin{array}{l}6.422 \\
6.501 \\
6.577 \\
6.650 \\
6.720 \\
6.788 \\
6.854 \\
6.918 \\
6.980 \\
7.040\end{array}$ & $\begin{array}{l}222.00 \\
224.00 \\
226.00 \\
228.00 \\
230.00 \\
232.00 \\
234.00 \\
236.00 \\
238.00 \\
240.00\end{array}$ & $\begin{array}{l}5.9349 \\
5.9895 \\
6.0441 \\
6.0986 \\
6.1531 \\
6.2076 \\
6.2621 \\
6.3166 \\
6.3711 \\
6.4256\end{array}$ & $\begin{array}{l}608.66 \\
616.03 \\
623.43 \\
630.86 \\
638.32 \\
645.80 \\
653.31 \\
660.84 \\
668.40 \\
675.98\end{array}$ & $\begin{array}{l}389.01 \\
394.36 \\
399.74 \\
405.15 \\
410.59 \\
416.05 \\
421.54 \\
427.06 \\
432.60 \\
438.16\end{array}$ & $\begin{array}{l}9.538 \\
9.571 \\
9.604 \\
9.637 \\
9.669 \\
9.702 \\
9.734 \\
9.766 \\
9.798 \\
9.829\end{array}$ \\
\hline $\begin{array}{l}02.00 \\
04.00 \\
06.00 \\
08.00 \\
10.00 \\
12.00 \\
14.00 \\
16.00 \\
18.00 \\
20.00\end{array}$ & $\begin{array}{l}2.5091 \\
2.5722 \\
2.6348 \\
2.6969 \\
2.7587 \\
2.8200 \\
2.8809 \\
2.9416 \\
3.0019 \\
3.0619\end{array}$ & $\begin{array}{l}225.89 \\
231.79 \\
237.66 \\
243.52 \\
249.35 \\
255.17 \\
260.97 \\
266.77 \\
272.56 \\
278.35\end{array}$ & $\begin{array}{l}133.02 \\
136.59 \\
140.15 \\
143.70 \\
147.25 \\
150.80 \\
154.35 \\
157.90 \\
161.46 \\
165.03\end{array}$ & $\begin{array}{l}7.099 \\
7.156 \\
7.212 \\
7.267 \\
7.320 \\
7.373 \\
7.424 \\
7.474 \\
7.524 \\
7.572\end{array}$ & $\begin{array}{l}242.00 \\
244.00 \\
246.00 \\
248.00 \\
250.00 \\
252.00 \\
254.00 \\
256.00 \\
258.00 \\
260.00\end{array}$ & $\begin{array}{l}6.4800 \\
6.5345 \\
6.5889 \\
6.6433 \\
6.6977 \\
6.7521 \\
6.8064 \\
6.8608 \\
6.9151 \\
6.9694\end{array}$ & $\begin{array}{l}683.58 \\
691.20 \\
698.85 \\
706.51 \\
714.20 \\
721.90 \\
729.62 \\
737.36 \\
745.11 \\
752.88\end{array}$ & $\begin{array}{l}443.75 \\
449.36 \\
454.99 \\
460.64 \\
466.31 \\
472.00 \\
477.71 \\
483.44 \\
489.18 \\
494.93\end{array}$ & $\begin{array}{r}9.861 \\
9.892 \\
9.924 \\
9.955 \\
9.985 \\
10.016 \\
10.047 \\
10.077 \\
10.107 \\
10.137\end{array}$ \\
\hline
\end{tabular}


TEMPER- SPECIFIC ENTHALPY INTERNAL ENTRCPY ATURE VOLUME (8TU/LB) ENERGY (8TU/L8-R) (R) (CU FI/LB) ATURE VOLUME (BTU/LB) (R) (CU FT/L8)
INTERNAL ENTRGPY (BTU/LB)

\begin{tabular}{|c|c|c|c|c|c|c|c|c|c|}
\hline $\begin{array}{l}262.00 \\
264.00 \\
266.00 \\
268.00 \\
270.00 \\
272.00 \\
274.00 \\
276.00 \\
278.00 \\
280.00\end{array}$ & $\begin{array}{l}7.0237 \\
7.0780 \\
7.1323 \\
7.1865 \\
7.2408 \\
7.2950 \\
7.3492 \\
7.4033 \\
7.4575 \\
7.5116\end{array}$ & $\begin{array}{l}760.66 \\
768.45 \\
776.26 \\
784.08 \\
791.91 \\
799.73 \\
807.55 \\
815.38 \\
823.22 \\
831.07\end{array}$ & $\begin{array}{l}500.71 \\
506.49 \\
512.29 \\
518.10 \\
523.93 \\
529.74 \\
535.55 \\
541.38 \\
547.22 \\
553.06\end{array}$ & $\begin{array}{l}10.167 \\
10.197 \\
10.226 \\
10.255 \\
10.284 \\
10.313 \\
10.342 \\
10.370 \\
10.399 \\
10.427\end{array}$ & $\begin{array}{l}402.00 \\
404.00 \\
406.00 \\
408.00 \\
410.00 \\
412.00 \\
414.00 \\
416.00 \\
418.00 \\
420.00\end{array}$ & $\begin{array}{l}10.7943 \\
10.8479 \\
10.9015 \\
10.9552 \\
11.0088 \\
11.0624 \\
11.1160 \\
11.1696 \\
11.2232 \\
11.2768\end{array}$ & $\begin{array}{l}1304.86 \\
1312.40 \\
1319.92 \\
1327.44 \\
1334.95 \\
1342.45 \\
1349.94 \\
1357.42 \\
1364.90 \\
1372.37\end{array}$ & $\begin{array}{l}905.36 \\
910.91 \\
916.45 \\
921.98 \\
927.51 \\
933.02 \\
938.53 \\
944.03 \\
949.52 \\
955.00\end{array}$ & $\begin{array}{l}11.833 \\
11.852 \\
11.871 \\
11.889 \\
11.907 \\
11.926 \\
11.944 \\
11.962 \\
11.980 \\
11.998\end{array}$ \\
\hline $\begin{array}{l}282.00 \\
284.00 \\
286.00 \\
288.00 \\
290.00 \\
292.00 \\
294.00 \\
296.00 \\
298.00 \\
300.00\end{array}$ & $\begin{array}{l}7.5658 \\
7.6199 \\
7.6739 \\
7.7280 \\
7.7821 \\
7.8361 \\
7.8901 \\
7.9441 \\
7.9981 \\
8.0521\end{array}$ & $\begin{array}{l}838.92 \\
846.77 \\
854.63 \\
862.50 \\
870.37 \\
878.24 \\
886.11 \\
893.99 \\
901.86 \\
909.74\end{array}$ & $\begin{array}{l}558.90 \\
564.76 \\
570.62 \\
576.48 \\
582.35 \\
588.22 \\
594.09 \\
599.97 \\
605.85 \\
611.73\end{array}$ & $\begin{array}{l}10.455 \\
10.483 \\
10.510 \\
10.538 \\
10.565 \\
10.592 \\
10.619 \\
10.645 \\
10.672 \\
10.698\end{array}$ & $\begin{array}{l}422.00 \\
424.00 \\
426.00 \\
428.00 \\
430.00 \\
432.00 \\
434.00 \\
436.00 \\
438.00 \\
440.00\end{array}$ & $\begin{array}{l}11.3304 \\
11.3840 \\
11.4376 \\
11.4911 \\
11.5447 \\
11.5983 \\
11.6518 \\
11.7054 \\
11.7589 \\
11.8124\end{array}$ & $\begin{array}{l}1379.82 \\
1387.27 \\
1394.71 \\
1402.14 \\
1409.57 \\
1416.98 \\
1424.38 \\
1431.78 \\
1439.17 \\
1446.55\end{array}$ & $\begin{array}{r}960.48 \\
965.94 \\
971.40 \\
976.85 \\
982.29 \\
987.72 \\
993.14 \\
998.56 \\
1003.96 \\
1009.36\end{array}$ & $\begin{array}{l}12.015 \\
12.033 \\
12.050 \\
12.068 \\
12.085 \\
12.102 \\
12.119 \\
12.136 \\
12.153 \\
12.170\end{array}$ \\
\hline $\begin{array}{l}302.00 \\
304.00 \\
306.00 \\
308.00 \\
310.00 \\
312.00 \\
314.00 \\
316.00 \\
318.00 \\
320.00\end{array}$ & $\begin{array}{l}8.1060 \\
8.1600 \\
8.2139 \\
8.2678 \\
8.3217 \\
8.3756 \\
8.4295 \\
8.4834 \\
8.5373 \\
8.5911\end{array}$ & $\begin{array}{l}917.62 \\
925.50 \\
933.38 \\
941.25 \\
949.13 \\
957.00 \\
964.87 \\
972.74 \\
980.61 \\
988.47\end{array}$ & $\begin{array}{l}617.61 \\
623.49 \\
629.37 \\
635.26 \\
641.14 \\
647.01 \\
652.89 \\
658.77 \\
664.64 \\
670.51\end{array}$ & $\begin{array}{l}10.724 \\
10.750 \\
10.776 \\
10.802 \\
10.827 \\
10.853 \\
10.878 \\
10.903 \\
10.928 \\
10.952\end{array}$ & $\begin{array}{l}442.00 \\
444.00 \\
446.00 \\
448.00 \\
450.00 \\
452.00 \\
454.00 \\
456.00 \\
458.00 \\
460.00\end{array}$ & $\begin{array}{l}11.8659 \\
11.9194 \\
11.9729 \\
12.0264 \\
12.0798 \\
12.1333 \\
12.1867 \\
12.2402 \\
12.2936 \\
12.3470\end{array}$ & $\begin{array}{l}1453.92 \\
1461.28 \\
1468.63 \\
1475.97 \\
1483.31 \\
1490.66 \\
1497.99 \\
1505.32 \\
1512.64 \\
1519.96\end{array}$ & $\begin{array}{l}1014.75 \\
1020.13 \\
1025.51 \\
1030.87 \\
1036.23 \\
1041.60 \\
1046.96 \\
1052.31 \\
1057.65 \\
1062.99\end{array}$ & $\begin{array}{l}12.187 \\
12.203 \\
12.220 \\
12.236 \\
12.253 \\
12.269 \\
12.285 \\
12.301 \\
12.317 \\
12.333\end{array}$ \\
\hline $\begin{array}{l}322.00 \\
324.00 \\
326.00 \\
328.00 \\
330.00 \\
332.00 \\
334.00 \\
336.00 \\
338.00 \\
340.00\end{array}$ & $\begin{array}{l}8.6450 \\
8.6988 \\
8.7527 \\
8.8065 \\
8.8603 \\
8.9142 \\
8.9680 \\
9.0218 \\
9.0756 \\
9.1294\end{array}$ & $\begin{array}{r}996.33 \\
1004.19 \\
1012.04 \\
1019.88 \\
1027.72 \\
1035.56 \\
1043.39 \\
1051.21 \\
1059.03 \\
1066.84\end{array}$ & $\begin{array}{l}676.37 \\
682.24 \\
688.09 \\
693.95 \\
699.80 \\
705.64 \\
711.48 \\
717.31 \\
723.14 \\
728.95\end{array}$ & $\begin{array}{l}10.977 \\
11.001 \\
11.025 \\
11.049 \\
11.073 \\
11.097 \\
11.120 \\
11.144 \\
11.167 \\
11.190\end{array}$ & $\begin{array}{l}462.00 \\
464.00 \\
466.00 \\
468.00 \\
470.00 \\
472.00 \\
474.00 \\
476.00 \\
478.00 \\
480.00\end{array}$ & $\begin{array}{l}12.4004 \\
12.4538 \\
12.5072 \\
12.5605 \\
12.6139 \\
12.6673 \\
12.7206 \\
12.7740 \\
12.8273 \\
12.8807\end{array}$ & $\begin{array}{l}1527.26 \\
1534.56 \\
1541.85 \\
1549.14 \\
1556.42 \\
1563.69 \\
1570.95 \\
1578.21 \\
1585.46 \\
1592.70\end{array}$ & $\begin{array}{l}1068.32 \\
1073.64 \\
1078.96 \\
1084.27 \\
1089.57 \\
1094.86 \\
1100.15 \\
1105.43 \\
1110.71 \\
1115.98\end{array}$ & $\begin{array}{l}12.349 \\
12.365 \\
12.381 \\
12.396 \\
12.412 \\
12.427 \\
12.442 \\
12.458 \\
12.473 \\
12.488\end{array}$ \\
\hline $\begin{array}{l}342.00 \\
344.00 \\
346.00 \\
348.00 \\
350.00 \\
352.00 \\
354.00 \\
356.00 \\
358.00 \\
360.00\end{array}$ & $\begin{array}{l}9.1832 \\
9.2370 \\
9.2908 \\
9.3446 \\
9.3984 \\
9.4522 \\
9.5060 \\
9.5598 \\
9.6135 \\
9.6673\end{array}$ & $\begin{array}{l}1074.65 \\
1082.44 \\
1090.23 \\
1098.02 \\
1105.79 \\
1113.56 \\
1121.32 \\
1129.07 \\
1136.82 \\
1144.55\end{array}$ & $\begin{array}{l}734.77 \\
740.57 \\
746.37 \\
752.17 \\
757.95 \\
763.73 \\
769.50 \\
775.26 \\
781.01 \\
786.76\end{array}$ & $\begin{array}{l}11.213 \\
11.235 \\
11.258 \\
11.280 \\
11.303 \\
11.325 \\
11.347 \\
11.369 \\
11.390 \\
11.412\end{array}$ & $\begin{array}{l}482.00 \\
484.00 \\
486.00 \\
488.00 \\
490.00 \\
492.00 \\
494.00 \\
496.00 \\
498.00 \\
500.00\end{array}$ & $\begin{array}{l}12.9340 \\
12.9874 \\
13.0407 \\
13.0941 \\
13.1474 \\
13.2008 \\
13.2541 \\
13.3075 \\
13.3609 \\
13.4143\end{array}$ & $\begin{array}{l}1599.94 \\
1607.17 \\
1614.40 \\
1621.62 \\
1628.83 \\
1636.04 \\
1643.25 \\
1650.45 \\
1657.65 \\
1664.84\end{array}$ & $\begin{array}{l}1121.24 \\
1126.50 \\
1131.75 \\
1137.00 \\
1142.24 \\
1147.47 \\
1152.70 \\
1157.93 \\
1163.15 \\
1168.37\end{array}$ & $\begin{array}{l}12.503 \\
12.518 \\
12.533 \\
12.548 \\
12.563 \\
12.577 \\
12.592 \\
12.606 \\
12.621 \\
12.635\end{array}$ \\
\hline $\begin{array}{l}362.00 \\
364.00 \\
366.00 \\
368.00 \\
370.00 \\
372.00 \\
374.00 \\
376.00 \\
378.00 \\
380.00\end{array}$ & $\begin{array}{r}9.7210 \\
9.7747 \\
9.8284 \\
9.8821 \\
9.9358 \\
9.9895 \\
10.0432 \\
10.0969 \\
10.1505 \\
10.2042\end{array}$ & $\begin{array}{l}1152.27 \\
1159.99 \\
1167.69 \\
1175.39 \\
1183.08 \\
1190.76 \\
1198.43 \\
1206.09 \\
1213.74 \\
1221.39\end{array}$ & $\begin{array}{l}792.49 \\
798.22 \\
803.94 \\
809.65 \\
815.35 \\
821.04 \\
826.72 \\
832.40 \\
838.06 \\
843.72\end{array}$ & $\begin{array}{l}11.433 \\
11.455 \\
11.476 \\
11.497 \\
11.518 \\
11.538 \\
11.559 \\
11.579 \\
11.600 \\
11.620\end{array}$ & $\begin{array}{l}502.00 \\
504.00 \\
506.00 \\
508.00 \\
510.00 \\
512.00 \\
514.00 \\
516.00 \\
518.00 \\
520.00\end{array}$ & $\begin{array}{l}13.4677 \\
13.5211 \\
13.5746 \\
13.6280 \\
13.6815 \\
13.7349 \\
13.7884 \\
13.8420 \\
13.8955 \\
13.9490\end{array}$ & $\begin{array}{l}1672.03 \\
1679.21 \\
1686.39 \\
1693.56 \\
1700.74 \\
1707.91 \\
1715.07 \\
1722.23 \\
1729.39 \\
1736.55\end{array}$ & $\begin{array}{l}1173.58 \\
1178.78 \\
1183.99 \\
1189.18 \\
1194.38 \\
1199.57 \\
1204.75 \\
1209.94 \\
1215.11 \\
1220.29\end{array}$ & $\begin{array}{l}12.650 \\
12.664 \\
12.678 \\
12.692 \\
12.706 \\
12.720 \\
12.734 \\
12.748 \\
12.762 \\
12.776\end{array}$ \\
\hline $\begin{array}{l}382.00 \\
384.00 \\
386.00 \\
388.00 \\
390.00 \\
392.00 \\
394.00 \\
396.00 \\
398.00 \\
400.00\end{array}$ & $\begin{array}{l}10.2579 \\
10.3115 \\
10.3652 \\
10.4188 \\
10.4725 \\
10.5261 \\
10.5798 \\
10.6334 \\
10.6870 \\
10.7407\end{array}$ & $\begin{array}{l}1229.02 \\
1236.64 \\
1244.26 \\
1251.87 \\
1259.47 \\
1267.05 \\
1274.63 \\
1282.20 \\
1289.77 \\
1297.32\end{array}$ & $\begin{array}{l}849.37 \\
855.01 \\
860.64 \\
866.26 \\
871.87 \\
877.48 \\
883.07 \\
888.66 \\
894.23 \\
899.80\end{array}$ & $\begin{array}{l}11.640 \\
11.660 \\
11.679 \\
11.699 \\
11.719 \\
11.738 \\
11.757 \\
11.776 \\
11.795 \\
11.814\end{array}$ & $\begin{array}{l}522.00 \\
524.00 \\
526.00 \\
528.00 \\
530.00 \\
532.00 \\
534.00 \\
536.00 \\
538.00 \\
540.00\end{array}$ & $\begin{array}{l}14.0026 \\
14.0562 \\
14.1098 \\
14.1634 \\
14.2170 \\
14.2706 \\
14.3243 \\
14.3779 \\
14.4315 \\
14.4852\end{array}$ & $\begin{array}{l}1743.70 \\
1750.85 \\
1758.00 \\
1765.14 \\
1772.28 \\
1779.42 \\
1786.55 \\
1793.68 \\
1800.81 \\
1807.93\end{array}$ & $\begin{array}{l}1225.46 \\
1230.62 \\
1235.79 \\
1240.95 \\
1246.10 \\
1251.26 \\
1256.40 \\
1261.55 \\
1266.69 \\
1271.83\end{array}$ & $\begin{array}{l}12.790 \\
12.803 \\
12.817 \\
12.830 \\
12.844 \\
12.857 \\
12.871 \\
12.884 \\
12.897 \\
12.911\end{array}$ \\
\hline
\end{tabular}


TEMPER- SPECIFIC ENTHALPY INTERNAL ENTROPY

ATURE VOLUME (8TU/L8) ENERGY (8TU/L8-R)

(R) (CUFT/L8)
(8TU/L8)
TEMPER-

ATURE

(R)
SPECIFIC ENTHALPY

VOLUME (CU FT/L 8 )
INTERNAL ENTROPY

ENERGY (8TU/L8-R) ( $8 T U / L 8$ )

\begin{tabular}{|c|c|c|c|c|}
\hline $\begin{array}{l}122.00 \\
124.00 \\
126.00 \\
128.00 \\
130.00 \\
132.00 \\
134.00 \\
136.00 \\
138.00 \\
140.00\end{array}$ & $\begin{array}{l}2.4752 \\
2.5240 \\
2.5725 \\
2.6208 \\
2.6689 \\
2.7167 \\
2.7644 \\
2.8119 \\
2.8592 \\
2.9063\end{array}$ & $\begin{array}{l}279.41 \\
285.35 \\
291.29 \\
297.23 \\
303.17 \\
309.13 \\
315.09 \\
321.07 \\
327.07 \\
333.08\end{array}$ & $\begin{array}{l}164.90 \\
168.58 \\
172.28 \\
175.98 \\
179.70 \\
183.44 \\
187.20 \\
190.99 \\
194.79 \\
198.62\end{array}$ & $\begin{array}{l}7.371 \\
7.419 \\
7.467 \\
7.514 \\
7.560 \\
7.605 \\
7.650 \\
7.694 \\
7.738 \\
7.781\end{array}$ \\
\hline $\begin{array}{l}142.00 \\
144.00 \\
146.00 \\
148.00 \\
150.00 \\
152.00 \\
154.00 \\
156.00 \\
158.00 \\
160.00\end{array}$ & $\begin{array}{l}2.9533 \\
3.0001 \\
3.0468 \\
3.0934 \\
3.1398 \\
3.1861 \\
3.2323 \\
3.2784 \\
3.3243 \\
3.3702\end{array}$ & $\begin{array}{l}339.11 \\
345.16 \\
351.24 \\
357.35 \\
363.48 \\
369.64 \\
375.83 \\
382.05 \\
388.30 \\
394.59\end{array}$ & $\begin{array}{l}202.48 \\
206.37 \\
210.29 \\
214.24 \\
218.22 \\
222.24 \\
226.29 \\
230.38 \\
234.51 \\
238.68\end{array}$ & $\begin{array}{l}7.824 \\
7.867 \\
7.908 \\
7.950 \\
7.991 \\
8.032 \\
8.072 \\
8.113 \\
8.152 \\
8.192\end{array}$ \\
\hline $\begin{array}{l}162.00 \\
164.00 \\
166.00 \\
168.00 \\
170.00 \\
172.00 \\
174.00 \\
176.00 \\
178.00 \\
180.00\end{array}$ & $\begin{array}{l}3.4159 \\
3.4616 \\
3.5072 \\
3.5527 \\
3.5981 \\
3.6435 \\
3.6887 \\
3.7339 \\
3.7790 \\
3.8241\end{array}$ & $\begin{array}{l}400.92 \\
407.28 \\
413.68 \\
420.11 \\
426.58 \\
433.09 \\
439.63 \\
446.22 \\
452.84 \\
459.49\end{array}$ & $\begin{array}{l}242.89 \\
247.13 \\
251.42 \\
255.75 \\
260.12 \\
264.53 \\
268.98 \\
273.48 \\
278.01 \\
282.58\end{array}$ & $\begin{array}{l}8.231 \\
8.270 \\
8.309 \\
8.348 \\
8.386 \\
8.424 \\
8.462 \\
8.499 \\
8.537 \\
8.574\end{array}$ \\
\hline $\begin{array}{l}182.00 \\
184.00 \\
186.00 \\
188.00 \\
190.00 \\
192.00 \\
194.00 \\
196.00 \\
198.00 \\
200.00\end{array}$ & $\begin{array}{l}3.8690 \\
3.9139 \\
3.9586 \\
4.0033 \\
4.0479 \\
4.0924 \\
4.1369 \\
4.1813 \\
4.2257 \\
4.2700\end{array}$ & $\begin{array}{l}466.18 \\
472.89 \\
479.64 \\
486.43 \\
493.25 \\
500.12 \\
507.02 \\
513.96 \\
520.93 \\
527.95\end{array}$ & $\begin{array}{l}287.18 \\
291.82 \\
296.50 \\
301.22 \\
305.98 \\
310.79 \\
315.63 \\
320.52 \\
325.44 \\
330.40\end{array}$ & $\begin{array}{l}8.611 \\
8.648 \\
8.684 \\
8.720 \\
8.756 \\
8.792 \\
8.828 \\
8.864 \\
8.899 \\
8.934\end{array}$ \\
\hline $\begin{array}{l}202.00 \\
204.00 \\
206.00 \\
208.00 \\
210.00 \\
212.00 \\
214.00 \\
216.00 \\
218.00 \\
220.00\end{array}$ & $\begin{array}{l}4.3143 \\
4.3585 \\
4.4027 \\
4.4469 \\
4.4910 \\
4.5351 \\
4.5791 \\
4.6231 \\
4.6671 \\
.4 .7111\end{array}$ & $\begin{array}{l}535.00 \\
542.08 \\
549.20 \\
556.36 \\
563.55 \\
570.77 \\
578.02 \\
585.31 \\
592.62 \\
599.97\end{array}$ & $\begin{array}{l}335.40 \\
340.44 \\
345.52 \\
350.63 \\
355.78 \\
360.96 \\
366.18 \\
371.43 \\
376.71 \\
382.01\end{array}$ & $\begin{array}{l}8.969 \\
9.004 \\
9.039 \\
9.074 \\
9.108 \\
9.142 \\
9.176 \\
9.210 \\
9.244 \\
9.277\end{array}$ \\
\hline $\begin{array}{l}222.00 \\
224.00 \\
226.00 \\
228.00 \\
230.00 \\
232.00 \\
234.00 \\
236.00 \\
238.00 \\
240.00\end{array}$ & $\begin{array}{l}4.7551 \\
4.7990 \\
4.8429 \\
4.8868 \\
4.9307 \\
4.9746 \\
5.0184 \\
5.0623 \\
5.1061 \\
5.1499\end{array}$ & $\begin{array}{l}607.34 \\
614.74 \\
622.17 \\
629.63 \\
637.11 \\
644.62 \\
652.16 \\
659.72 \\
667.30 \\
674.90\end{array}$ & $\begin{array}{l}387.35 \\
392.72 \\
398.12 \\
403.55 \\
409.00 \\
414.48 \\
419.99 \\
425.52 \\
431.07 \\
436.65\end{array}$ & $\begin{array}{l}9.311 \\
9.344 \\
9.377 \\
9.410 \\
9.443 \\
9.475 \\
9.507 \\
9.540 \\
9.572 \\
9.603\end{array}$ \\
\hline $\begin{array}{l}242.00 \\
244.00 \\
246.00 \\
248.00 \\
250.00 \\
252.00 \\
254.00 \\
256.00 \\
258.00 \\
260.00\end{array}$ & $\begin{array}{l}5.1937 \\
5.2374 \\
5.2812 \\
5.3249 \\
5.3687 \\
5.4124 \\
5.4561 \\
5.4998 \\
5.5434 \\
5.5871\end{array}$ & $\begin{array}{l}682.53 \\
690.18 \\
697.85 \\
705.53 \\
713.24 \\
720.97 \\
728.71 \\
736.47 \\
744.24 \\
752.03\end{array}$ & $\begin{array}{l}442.25 \\
447.88 \\
453.52 \\
459.19 \\
464.87 \\
470.57 \\
476.29 \\
482.03 \\
487.78 \\
493.55\end{array}$ & $\begin{array}{l}9.635 \\
9.667 \\
9.698 \\
9.729 \\
9.760 \\
9.791 \\
9.821 \\
9.852 \\
9.882 \\
9.912\end{array}$ \\
\hline
\end{tabular}


TEMPER - SPECIFIC ENTHALPY INTERNAL ENTROPY ATURE VOLUME (BTU/L8) ENERGY (8TU/L8-R) (R) (CU FT/L8) (8TU/L8)
( $) ~$
TEMPER- SPECIFIC ENTHALPY INTERNAL ENTROPY ATURE VOLUME (8TU/L8) ENERGY (8TU/L8-R) $\begin{array}{ll}\text { (R) (CU FT/L8) } & \text { (8TU/L8) }\end{array}$

\begin{tabular}{|c|c|c|c|c|c|c|c|c|c|}
\hline $\begin{array}{l}262.00 \\
264.00 \\
266.00 \\
268.00 \\
270.00 \\
272.00 \\
274.00 \\
276.00 \\
278.00 \\
280.00\end{array}$ & $\begin{array}{l}5.6307 \\
5.6743 \\
5.7179 \\
5.7615 \\
5.8050 \\
5.8486 \\
5.8921 \\
5.9356 \\
5.9791 \\
6.0226\end{array}$ & $\begin{array}{l}759.83 \\
767.65 \\
775.47 \\
783.31 \\
791.16 \\
799.00 \\
806.84 \\
814.69 \\
822.54 \\
830.41\end{array}$ & $\begin{array}{l}499.34 \\
505.13 \\
510.94 \\
516.77 \\
522.60 \\
528.42 \\
534.25 \\
540.09 \\
545.93 \\
551.78\end{array}$ & $\begin{array}{r}9.942 \\
9.972 \\
10.001 \\
10.031 \\
10.060 \\
10.089 \\
10.117 \\
10.146 \\
10.174 \\
10.202\end{array}$ & $\begin{array}{l}402.00 \\
404.00 \\
406.00 \\
408.00 \\
410.00 \\
412.00 \\
414.00 \\
416.00 \\
418.00 \\
420.00\end{array}$ & $\begin{array}{l}8.6553 \\
8.6983 \\
8.7413 \\
8.7842 \\
8.8272 \\
8.8701 \\
8.9131 \\
8.9560 \\
8.9990 \\
9.0419\end{array}$ & $\begin{array}{l}1304.96 \\
1312.51 \\
1320.04 \\
1327.57 \\
1335.08 \\
1342.59 \\
1350.09 \\
1357.58 \\
1365.07 \\
1372.54\end{array}$ & $\begin{array}{l}904.54 \\
910.10 \\
915.64 \\
921.18 \\
926.71 \\
932.23 \\
937.74 \\
943.25 \\
948.74 \\
954.23\end{array}$ & $\begin{array}{l}11.611 \\
11.630 \\
11.648 \\
11.667 \\
11.685 \\
11.704 \\
11.722 \\
11.740 \\
11.758 \\
11.776\end{array}$ \\
\hline $\begin{array}{l}282.00 \\
284.00 \\
286.00 \\
288.00 \\
290.00 \\
292.00 \\
294.00 \\
296.00 \\
298.00 \\
300.00\end{array}$ & $\begin{array}{l}6.0600 \\
6.1095 \\
6.1529 \\
6.1963 \\
6.2397 \\
6.2831 \\
6.3265 \\
6.3698 \\
6.4131 \\
6.4565\end{array}$ & $\begin{array}{l}838.27 \\
846.15 \\
854.03 \\
861.91 \\
869.79 \\
877.68 \\
885.57 \\
893.46 \\
901.35 \\
909.25\end{array}$ & $\begin{array}{l}557.64 \\
563.50 \\
569.37 \\
575.24 \\
581.12 \\
587.00 \\
592.89 \\
598.77 \\
604.66 \\
610.55\end{array}$ & $\begin{array}{l}10.230 \\
10.258 \\
10.286 \\
10.313 \\
10.341 \\
10.368 \\
10.395 \\
10.421 \\
10.448 \\
10.474\end{array}$ & $\begin{array}{l}422.00 \\
424.00 \\
426.00 \\
428.00 \\
430.00 \\
432.00 \\
434.00 \\
436.00 \\
438.00 \\
440.00\end{array}$ & $\begin{array}{l}9.0848 \\
9.1278 \\
9.1707 \\
9.2136 \\
9.2565 \\
9.2994 \\
9.3423 \\
9.3852 \\
9.4281 \\
9.4710\end{array}$ & $\begin{array}{l}1380.01 \\
1387.46 \\
1394.91 \\
1402.35 \\
1409.78 \\
1417.20 \\
1424.61 \\
1432.02 \\
1439.41 \\
1446.80\end{array}$ & $\begin{array}{r}959.71 \\
965.18 \\
970.64 \\
976.10 \\
981.54 \\
986.98 \\
992.41 \\
997.83 \\
1003.24 \\
1008.64\end{array}$ & $\begin{array}{l}11.793 \\
11.811 \\
11.828 \\
11.846 \\
11.863 \\
11.880 \\
11.898 \\
11.915 \\
11.931 \\
11.948\end{array}$ \\
\hline $\begin{array}{l}302.00 \\
304.00 \\
306.00 \\
308.00 \\
310.00 \\
312.00 \\
314.00 \\
316.00 \\
318.00 \\
320.00\end{array}$ & $\begin{array}{l}6.4998 \\
6.5431 \\
6.5863 \\
6.6296 \\
6.6729 \\
6.7161 \\
6.7593 \\
6.8026 \\
6.8458 \\
6.8890\end{array}$ & $\begin{array}{l}917.14 \\
925.03 \\
932.93 \\
940.82 \\
948.71 \\
956.60 \\
964.48 \\
972.37 \\
980.25 \\
988.12\end{array}$ & $\begin{array}{l}616.44 \\
622.33 \\
628.22 \\
634.11 \\
640.00 \\
645.89 \\
651.77 \\
657.66 \\
663.54 \\
669.42\end{array}$ & $\begin{array}{l}10.501 \\
10.527 \\
10.553 \\
10.578 \\
10.604 \\
10.629 \\
10.654 \\
10.679 \\
10.704 \\
10.729\end{array}$ & $\begin{array}{l}442.00 \\
444.00 \\
446.00 \\
448.00 \\
450.00 \\
452.00 \\
454.00 \\
456.00 \\
458.00 \\
460.00\end{array}$ & $\begin{array}{l}9.5138 \\
9.5567 \\
9.5995 \\
9.6423 \\
9.6852 \\
9.7280 \\
9.7708 \\
9.8136 \\
9.8564 \\
9.8992\end{array}$ & $\begin{array}{l}1454.18 \\
1461.55 \\
1468.91 \\
1476.26 \\
1483.60 \\
1490.96 \\
1498.30 \\
1505.64 \\
1512.97 \\
1520.29\end{array}$ & $\begin{array}{l}1014.04 \\
1019.42 \\
1024.80 \\
1030.17 \\
1035.54 \\
1040.91 \\
1046.27 \\
1051.63 \\
1056.98 \\
1062.32\end{array}$ & $\begin{array}{l}11.965 \\
11.982 \\
11.998 \\
12.015 \\
12.031 \\
12.047 \\
12.064 \\
12.080 \\
12.096 \\
12.112\end{array}$ \\
\hline $\begin{array}{l}322.00 \\
324.00 \\
326.00 \\
328.00 \\
330.00 \\
332.00 \\
334.00 \\
336.00 \\
338.00 \\
340.00\end{array}$ & $\begin{array}{l}6.9322 \\
6.9754 \\
7.0186 \\
7.0618 \\
7.1050 \\
7.1481 \\
7.1913 \\
7.2345 \\
7.2776 \\
7.3208\end{array}$ & $\begin{array}{r}996.00 \\
1003.87 \\
1011.73 \\
1019.59 \\
1027.44 \\
1035.29 \\
1043.14 \\
1050.97 \\
1058.80 \\
1066.63\end{array}$ & $\begin{array}{l}675.29 \\
681.16 \\
687.03 \\
692.89 \\
698.75 \\
704.60 \\
710.44 \\
716.28 \\
722.12 \\
727.95\end{array}$ & $\begin{array}{l}10.753 \\
10.778 \\
10.802 \\
10.826 \\
10.850 \\
10.874 \\
10.897 \\
10.921 \\
10.944 \\
10.967\end{array}$ & $\begin{array}{l}462.00 \\
464.00 \\
466.00 \\
468.00 \\
470.00 \\
472.00 \\
474.00 \\
476.00 \\
478.00 \\
480.00\end{array}$ & $\begin{array}{r}9.9420 \\
9.9847 \\
10.0275 \\
10.0703 \\
10.1130 \\
10.1558 \\
10.1985 \\
10.2413 \\
10.2840 \\
10.3267\end{array}$ & $\begin{array}{l}1527.60 \\
1534.91 \\
1542.21 \\
1549.50 \\
1556.78 \\
1564.06 \\
1571.33 \\
1578.60 \\
1585.85 \\
1593.10\end{array}$ & $\begin{array}{l}1067.66 \\
1072.98 \\
1078.30 \\
1083.62 \\
1088.92 \\
1094.22 \\
1099.52 \\
1104.80 \\
1110.08 \\
1115.36\end{array}$ & $\begin{array}{l}12.128 \\
12.143 \\
12.159 \\
12.175 \\
12.190 \\
12.206 \\
12.221 \\
12.236 \\
12.251 \\
12.267\end{array}$ \\
\hline $\begin{array}{l}342.00 \\
344.00 \\
346.00 \\
348.00 \\
350.00 \\
352.00 \\
354.00 \\
356.00 \\
358.00 \\
360.00\end{array}$ & $\begin{array}{l}7.3639 \\
7.4070 \\
7.4502 \\
7.4933 \\
7.5364 \\
7.5796 \\
7.6227 \\
7.6658 \\
7.7089 \\
7.7520\end{array}$ & $\begin{array}{l}1074.44 \\
1082.25 \\
1090.06 \\
1097.85 \\
1105.64 \\
1113.42 \\
1121.19 \\
1128.96 \\
1136.71 \\
1144.46\end{array}$ & $\begin{array}{l}733.77 \\
739.58 \\
745.39 \\
751.19 \\
756.98 \\
762.77 \\
768.54 \\
774.31 \\
780.07 \\
785.82\end{array}$ & $\begin{array}{l}10.990 \\
11.013 \\
11.035 \\
11.058 \\
11.080 \\
11.102 \\
11.124 \\
11.146 \\
11.168 \\
11.189\end{array}$ & $\begin{array}{l}482.00 \\
484.00 \\
486.00 \\
488.00 \\
490.00 \\
492.00 \\
494.00 \\
496.00 \\
498.00 \\
500.00\end{array}$ & $\begin{array}{l}10.3695 \\
10.4122 \\
10.4549 \\
10.4977 \\
10.5404 \\
10.5832 \\
10.6259 \\
10.6686 \\
10.7114 \\
10.7542\end{array}$ & $\begin{array}{l}1600.35 \\
1607.59 \\
1614.82 \\
1622.05 \\
1629.27 \\
1636.48 \\
1643.70 \\
1650.90 \\
1658.10 \\
1665.30\end{array}$ & $\begin{array}{l}1120.62 \\
1125.88 \\
1131.14 \\
1136.39 \\
1141.63 \\
1146.87 \\
1152.11 \\
1157.34 \\
1162.56 \\
1167.78\end{array}$ & $\begin{array}{l}12.282 \\
12.297 \\
12.312 \\
12.326 \\
12.341 \\
12.356 \\
12.371 \\
12.385 \\
12.400 \\
12.414\end{array}$ \\
\hline $\begin{array}{l}362.00 \\
364.00 \\
366.00 \\
368.00 \\
370.00 \\
372.00 \\
374.00 \\
376.00 \\
378.00 \\
380.00\end{array}$ & $\begin{array}{l}7.7951 \\
7.8382 \\
7.8812 \\
7.9243 \\
7.9673 \\
8.0103 \\
8.0534 \\
8.0964 \\
8.1394 \\
8.1824\end{array}$ & $\begin{array}{l}1152.19 \\
1159.92 \\
1167.63 \\
1175.34 \\
1183.04 \\
1190.73 \\
1198.41 \\
1206.08 \\
1213.74 \\
1221.39\end{array}$ & $\begin{array}{l}791.57 \\
797.30 \\
803.02 \\
808.74 \\
814.44 \\
820.14 \\
825.83 \\
831.51 \\
837.18 \\
842.85\end{array}$ & $\begin{array}{l}11.211 \\
11.232 \\
11.253 \\
11.274 \\
11.295 \\
11.316 \\
11.336 \\
11.357 \\
11.377 \\
11.397\end{array}$ & $\begin{array}{l}502.00 \\
504.00 \\
506.00 \\
508.00 \\
510.00 \\
512.00 \\
514.00 \\
516.00 \\
518.00 \\
520.00\end{array}$ & $\begin{array}{l}10.7969 \\
10.8397 \\
10.8825 \\
10.9253 \\
10.9681 \\
11.0109 \\
11.0538 \\
11.0966 \\
11.1395 \\
11.1823\end{array}$ & $\begin{array}{l}1672.49 \\
1679.68 \\
1686.87 \\
1694.05 \\
1701.22 \\
1708.40 \\
1715.57 \\
1722.73 \\
1729.89 \\
1737.05\end{array}$ & $\begin{array}{l}1172.99 \\
1178.20 \\
1183.41 \\
1188.61 \\
1193.80 \\
1198.99 \\
1204.18 \\
1209.37 \\
1214.55 \\
1219.72\end{array}$ & $\begin{array}{l}12.428 \\
12.443 \\
12.457 \\
12.471 \\
12.485 \\
12.499 \\
12.513 \\
12.527 \\
12.541 \\
12.555\end{array}$ \\
\hline $\begin{array}{l}382.00 \\
384.00 \\
386.00 \\
388.00 \\
390.00 \\
392.00 \\
394.00 \\
396.00 \\
398.00 \\
400.00\end{array}$ & $\begin{array}{l}8.2254 \\
8.2684 \\
8.3115 \\
8.3545 \\
8.3974 \\
8.4404 \\
8.4834 \\
8.5264 \\
8.5694 \\
8.6124\end{array}$ & $\begin{array}{l}1229.03 \\
1236.67 \\
1244.29 \\
1251.91 \\
1259.52 \\
1267.11 \\
1274.70 \\
1282.28 \\
1289.85 \\
1297.41\end{array}$ & $\begin{array}{l}848.50 \\
854.14 \\
859.78 \\
865.41 \\
871.02 \\
876.63 \\
882.23 \\
887.82 \\
893.40 \\
898.98\end{array}$ & $\begin{array}{l}11.417 \\
11.437 \\
11.457 \\
11.477 \\
11.496 \\
11.516 \\
11.535 \\
11.554 \\
11.573 \\
11.592\end{array}$ & $\begin{array}{l}522.00 \\
524.00 \\
526.00 \\
528.00 \\
530.00 \\
532.00 \\
534.00 \\
536.00 \\
538.00 \\
540.00\end{array}$ & $\begin{array}{l}11.2252 \\
11.2681 \\
11.3110 \\
11.3539 \\
11.3968 \\
11.4397 \\
11.4826 \\
11.5255 \\
11.5684 \\
11.6114\end{array}$ & $\begin{array}{l}1744.21 \\
1751.36 \\
1758.51 \\
1765.66 \\
1772.80 \\
1779.94 \\
1787.08 \\
1794.21 \\
1801.34 \\
1808.47\end{array}$ & $\begin{array}{l}1224.90 \\
1230.06 \\
1235.23 \\
1240.39 \\
1245.55 \\
1250.70 \\
1255.86 \\
1261.00 \\
1266.15 \\
1271.29\end{array}$ & $\begin{array}{l}12.568 \\
12.582 \\
12.596 \\
12.609 \\
12.623 \\
12.636 \\
12.650 \\
12.663 \\
12.676 \\
12.689\end{array}$ \\
\hline
\end{tabular}




\begin{tabular}{|c|c|c|c|c|c|c|c|c|c|}
\hline $\begin{array}{l}\text { EMPER- } \\
\text { ATURE } \\
\text { (R) }\end{array}$ & $\begin{array}{l}\text { SPECIFIC } \\
\text { VOLUME } \\
\text { (CU FT/L8) }\end{array}$ & $\begin{array}{l}\text { ENTHALPY } \\
\text { (BTU/L8) }\end{array}$ & $\begin{array}{l}\text { INTERNAL } \\
\text { ENERGY } \\
(8 T U / L 8)\end{array}$ & $\begin{array}{l}\text { ENTROPY } \\
(8 T U / L 8-R)\end{array}$ & $\begin{array}{l}\text { TEMPER- } \\
\text { ATURE } \\
\text { (R) }\end{array}$ & $\begin{array}{c}\text { SPEC IF IC } \\
\text { VOLUME } \\
\{C U F T / L 8 \mid\end{array}$ & $\begin{array}{l}\text { ENTHALPY } \\
(8 T U / L 8)\end{array}$ & $\begin{array}{l}\text { INTERNAL } \\
\text { ENERGY } \\
\text { ( } 8 T U / L 8)\end{array}$ & $\begin{array}{l}E N \\
181\end{array}$ \\
\hline
\end{tabular}

\begin{tabular}{|c|c|c|c|c|c|c|c|c|c|}
\hline & & & & & $\begin{array}{l}122.00 \\
124.00 \\
126.00 \\
128.00 \\
130.00 \\
132.00 \\
134.00 \\
136.00 \\
138.00 \\
140.00\end{array}$ & $\begin{array}{l}2.0456 \\
2.0872 \\
2.1286 \\
2.1697 \\
2.2106 \\
2.2513 \\
2.2918 \\
2.3321 \\
2.3723 \\
2.4123\end{array}$ & $\begin{array}{l}274.74 \\
280.83 \\
286.90 \\
292.98 \\
299.05 \\
305.13 \\
311.21 \\
317.30 \\
323.41 \\
329.52\end{array}$ & $\begin{array}{l}161.18 \\
164.95 \\
168.74 \\
172.53 \\
176.33 \\
180.15 \\
183.98 \\
187.83 \\
191.71 \\
195.60\end{array}$ & $\begin{array}{l}7.162 \\
7.212 \\
7.261 \\
7.308 \\
7.356 \\
7.402 \\
7.448 \\
7.493 \\
7.537 \\
7.581\end{array}$ \\
\hline & & & & & $\begin{array}{l}142.00 \\
144.00 \\
146.00 \\
148.00 \\
150.00 \\
152.00 \\
154.00 \\
156.00 \\
158.00 \\
160.00\end{array}$ & $\begin{array}{l}2.4521 \\
2.4918 \\
2.5313 \\
2.5707 \\
2.6100 \\
2.6491 \\
2.6882 \\
2.7271 \\
2.7659 \\
2.8046\end{array}$ & $\begin{array}{l}335.65 \\
341.80 \\
347.98 \\
354.17 \\
360.39 \\
366.63 \\
372.90 \\
379.20 \\
385.53 \\
391.89\end{array}$ & $\begin{array}{l}199.52 \\
203.47 \\
207.45 \\
211.45 \\
215.49 \\
219.56 \\
223.67 \\
227.81 \\
231.98 \\
236.19\end{array}$ & $\begin{array}{l}7.625 \\
7.668 \\
7.710 \\
7.752 \\
7.794 \\
7.836 \\
7.877 \\
7.917 \\
7.958 \\
7.998\end{array}$ \\
\hline $\begin{array}{l}36.00 \\
38.00 \\
40.00\end{array}$ & $\begin{array}{l}.2180 \\
.2213 \\
.2249\end{array}$ & $\begin{array}{r}-102.73 \\
-98.39 \\
-93.78\end{array}$ & $\begin{array}{l}-114.84 \\
-110.67 \\
-106.26\end{array}$ & $\begin{array}{l}1.790 \\
1.908 \\
2.026\end{array}$ & $\begin{array}{l}162.00 \\
164.00 \\
166.00 \\
168.00 \\
170.00 \\
172.00 \\
174.00 \\
176.00 \\
178.00 \\
180.00\end{array}$ & $\begin{array}{l}2.8432 \\
2.8817 \\
2.9202 \\
2.9585 \\
2.9968 \\
3.0350 \\
3.0731 \\
3.1112 \\
3.1492 \\
3.1871\end{array}$ & $\begin{array}{l}398.29 \\
404.72 \\
411.18 \\
417.68 \\
424.21 \\
430.78 \\
437.39 \\
444.03 \\
450.70 \\
457.41\end{array}$ & $\begin{array}{l}240.45 \\
244.74 \\
249.07 \\
253.43 \\
257.84 \\
262.29 \\
266.78 \\
271.31 \\
275.87 \\
280.48\end{array}$ & $\begin{array}{l}8.037 \\
8.077 \\
8.116 \\
8.155 \\
8.193 \\
8.232 \\
8.270 \\
8.308 \\
8.346 \\
8.383\end{array}$ \\
\hline $\begin{array}{l}42.00 \\
44.00 \\
46.00 \\
48.00 \\
50.00 \\
52.00 \\
54.00 \\
56.00 \\
58.00 \\
60.00\end{array}$ & $\begin{array}{l}.2290 \\
.2334 \\
.2384 \\
.2440 \\
.2504 \\
.2579 \\
.2666 \\
.2773 \\
.2910 \\
.3091\end{array}$ & $\begin{array}{l}-88.88 \\
-83.67 \\
-78.10 \\
-72.14 \\
-65.73 \\
-58.79 \\
-51.23 \\
-42.88 \\
-33.37 \\
-22.70\end{array}$ & $\begin{array}{r}-101.59 \\
-96.62 \\
-91.34 \\
-85.69 \\
-79.63 \\
-73.10 \\
-66.03 \\
-58.28 \\
-49.52 \\
-39.86\end{array}$ & $\begin{array}{l}2.145 \\
2.267 \\
2.390 \\
2.517 \\
2.648 \\
2.784 \\
2.927 \\
3.078 \\
3.246 \\
3.428\end{array}$ & $\begin{array}{l}182.00 \\
184.00 \\
186.00 \\
188.00 \\
190.00 \\
192.00 \\
194.00 \\
196.00 \\
198.00 \\
200.00\end{array}$ & $\begin{array}{l}3.2249 \\
3.2626 \\
3.3002 \\
3.3378 \\
3.3754 \\
3.4128 \\
3.4503 \\
3.4876 \\
3.5250 \\
3.5622\end{array}$ & $\begin{array}{l}464.14 \\
470.90 \\
477.70 \\
484.53 . \\
491.40 \\
498.31 \\
505.25 \\
512.23 \\
519.25 \\
526.30\end{array}$ & $\begin{array}{l}285.11 \\
289.78 \\
294.48 \\
299.23 \\
304.02 \\
308.84 \\
313.71 \\
318.61 \\
323.56 \\
328.54\end{array}$ & $\begin{array}{l}8.420 \\
8.457 \\
8.494 \\
8.531 \\
8.567 \\
8.603 \\
8.639 \\
8.675 \\
8.710 \\
8.746\end{array}$ \\
\hline $\begin{array}{l}62.00 \\
64.00 \\
66.00 \\
68.00 \\
70.00 \\
72.00 \\
74.00 \\
76.00 \\
78.00 \\
80.00\end{array}$ & $\begin{array}{r}.3352 \\
.3814 \\
.4830 \\
.6089 \\
.7080 \\
.7909 \\
.8644 \\
.9319 \\
.9951 \\
1.0550\end{array}$ & $\begin{array}{r}-9.13 \\
9.59 \\
38.43 \\
64.37 \\
81.71 \\
95.05 \\
106.33 \\
116.38 \\
125.59 \\
134.22\end{array}$ & $\begin{array}{r}-27.73 \\
-11.58 \\
11.62 \\
30.56 \\
42.41 \\
51.15 \\
58.35 \\
64.64 \\
70.35 \\
75.65\end{array}$ & $\begin{array}{l}3.651 \\
3.949 \\
4.392 \\
4.779 \\
5.030 \\
5.218 \\
5.373 \\
5.507 \\
5.627 \\
5.736\end{array}$ & $\begin{array}{l}202.00 \\
204.00 \\
206.00 \\
208.00 \\
210.00 \\
212.00 \\
214.00 \\
216.00 \\
218.00 \\
220.00\end{array}$ & $\begin{array}{l}3.5995 \\
3.6366 \\
3.6738 \\
3.7109 \\
3.7480 \\
3.7850 \\
3.8220 \\
3.8590 \\
3.8959 \\
3.9329\end{array}$ & $\begin{array}{l}533.39 \\
540.51 \\
547.67 \\
554.86 \\
562.08 \\
569.34 \\
576.62 \\
583.94 \\
591.29 \\
598.66\end{array}$ & $\begin{array}{l}333.56 \\
338.62 \\
343.71 \\
348.84 \\
354.01 \\
359.21 \\
364.44 \\
369.71 \\
375.00 \\
380.33\end{array}$ & $\begin{array}{l}8.781 \\
8.816 \\
8.851 \\
8.886 \\
8.920 \\
8.955 \\
8.989 \\
9.023 \\
9.057 \\
9.091\end{array}$ \\
\hline $\begin{array}{r}82.00 \\
84.00 \\
86.00 \\
88.00 \\
90.00 \\
92.00 \\
94.00 \\
96.00 \\
98.00 \\
100.00\end{array}$ & $\begin{array}{l}1.1124 \\
1.1678 \\
1.2214 \\
1.2736 \\
1.3245 \\
1.3744 \\
1.4233 \\
1.4713 \\
1.5186 \\
1.5651\end{array}$ & $\begin{array}{l}142.40 \\
150.24 \\
157.81 \\
165.15 \\
172.30 \\
179.29 \\
186.15 \\
192.89 \\
199.53 \\
206.08\end{array}$ & $\begin{array}{r}80.65 \\
85.41 \\
90.00 \\
94.44 \\
98.77 \\
102.99 \\
107.14 \\
111.21 \\
115.23 \\
119.19\end{array}$ & $\begin{array}{l}5.837 \\
5.931 \\
6.020 \\
6.105 \\
6.185 \\
6.262 \\
6.336 \\
6.407 \\
6.475 \\
6.541\end{array}$ & $\begin{array}{l}222.00 \\
224.00 \\
226.00 \\
228.00 \\
230.00 \\
232.00 \\
234.00 \\
236.00 \\
238.00 \\
240.00\end{array}$ & $\begin{array}{l}3.9698 \\
4.0066 \\
4.0435 \\
4.0803 \\
4.1171 \\
4.1538 \\
4.1906 \\
4.2273 \\
4.2640 \\
4.3007\end{array}$ & $\begin{array}{l}606.07 \\
613.50 \\
620.96 \\
628.44 \\
635.95 \\
643.49 \\
651.05 \\
658.64 \\
666.24 \\
673.87\end{array}$ & $\begin{array}{l}385.68 \\
391.07 \\
396.48 \\
401.92 \\
407.39 \\
412.89 \\
418.41 \\
423.95 \\
429.52 \\
435.12\end{array}$ & $\begin{array}{l}9.124 \\
9.157 \\
9.191 \\
9.224 \\
9.256 \\
9.289 \\
9.321 \\
9.354 \\
9.386 \\
9.418\end{array}$ \\
\hline $\begin{array}{l}102.00 \\
104.00 \\
106.00 \\
108.00 \\
110.00 \\
112.00 \\
114.00 \\
116.00 \\
118.00 \\
120.00\end{array}$ & $\begin{array}{l}1.6111 \\
1.6564 \\
1.7013 \\
1.7457 \\
1.7896 \\
1.8331 \\
1.8763 \\
1.9191 \\
1.9615 \\
2.0037\end{array}$ & $\begin{array}{l}212.56 \\
218.96 \\
225.31 \\
231.61 \\
277.86 \\
244.07 \\
250.25 \\
256.41 \\
262.53 \\
268.65\end{array}$ & $\begin{array}{l}123.12 \\
127.01 \\
130.86 \\
134.70 \\
138.51 \\
142.31 \\
146.09 \\
149.87 \\
153.64 \\
157.41\end{array}$ & $\begin{array}{l}6.605 \\
6.668 \\
6.728 \\
6.787 \\
6.844 \\
6.900 \\
6.955 \\
7.008 \\
7.061 \\
7.112\end{array}$ & $\begin{array}{l}242.00 \\
244.00 \\
246.00 \\
248.00 \\
250.00 \\
252.00 \\
254.00 \\
256.00 \\
258.00 \\
260.00\end{array}$ & $\begin{array}{l}4.3374 \\
4.3741 \\
4.4107 \\
4.4473 \\
4.4839 \\
4.5205 \\
4.5571 \\
4.5936 \\
4.6302 \\
4.6667\end{array}$ & $\begin{array}{l}681.53 \\
689.20 \\
696.89 \\
704.60 \\
712.33 \\
720.08 \\
727.84 \\
735.62 \\
743.41 \\
751.22\end{array}$ & $\begin{array}{l}440.73 \\
446.37 \\
452.03 \\
457.70 \\
463.40 \\
469.12 \\
474.85 \\
480.60 \\
486.37 \\
492.15\end{array}$ & $\begin{array}{l}9.450 \\
9.481 \\
9.512 \\
9.544 \\
9.575 \\
9.606 \\
9.636 \\
9.667 \\
9.697 \\
9.727\end{array}$ \\
\hline
\end{tabular}


TEMPER- SPECIFIC ENTHALPY INTERNAL ENTROPY ATURE VOLUME (BTU/L8) ENERGY (BTU/L8-R) (R) (CU FT/LB) (BTU/L8)
TEMPER -

ATURE

(R)
SPECIFIC ENTHALPY VOLUME
INTERNAL ENTROPY

ENERGY (BTU/L8-R) (8TU/L8)

\begin{tabular}{|c|c|c|c|c|c|c|c|c|c|}
\hline $\begin{array}{l}262.00 \\
264.00 \\
266.00 \\
268.00 \\
270.00 \\
272.00 \\
274.00 \\
276.00 \\
278.00 \\
280.00\end{array}$ & $\begin{array}{l}4.7032 \\
4.7397 \\
4.7761 \\
4.8126 \\
4.8490 \\
4.8854 \\
4.9218 \\
4.9582 \\
4.9946 \\
5.0310\end{array}$ & $\begin{array}{l}759.04 \\
766.88 \\
774.73 \\
782.58 \\
790.45 \\
798.30 \\
806.16 \\
814.03 \\
821.90 \\
829.78\end{array}$ & $\begin{array}{l}497.94 \\
503.75 \\
509.57 \\
515.41 \\
521.25 \\
527.08 \\
532.92 \\
538.77 \\
544.62 \\
550.49\end{array}$ & $\begin{array}{r}9.757 \\
9.787 \\
9.817 \\
9.846 \\
9.875 \\
9.904 \\
9.933 \\
9.962 \\
9.990 \\
10.018\end{array}$ & $\begin{array}{l}402.00 \\
404.00 \\
406.00 \\
408.00 \\
410.00 \\
412.00 \\
414.00 \\
416.00 \\
418.00 \\
420.00\end{array}$ & $\begin{array}{l}7.2303 \\
7.2662 \\
7.3020 \\
7.3379 \\
7.3738 \\
7.4096 \\
7.4454 \\
7.4813 \\
7.5171 \\
7.5529\end{array}$ & $\begin{array}{l}1305.10 \\
1312.65 \\
1320.19 \\
1327.72 \\
1335.25 \\
1342.76 \\
1350.27 \\
1357.77 \\
1365.26 \\
1372.75\end{array}$ & $\begin{array}{l}903.70 \\
909.26 \\
914.81 \\
920.35 \\
925.89 \\
931.41 \\
936.93 \\
942.44 \\
947.94 \\
953.44\end{array}$ & $\begin{array}{l}11.429 \\
11.448 \\
11.467 \\
11.485 \\
11.504 \\
11.522 \\
11.540 \\
11.558 \\
11.576 \\
11.594\end{array}$ \\
\hline $\begin{array}{l}282.00 \\
284.00 \\
286.00 \\
288.00 \\
290.00 \\
292.00 \\
294.00 \\
296.00 \\
298.00 \\
300.00\end{array}$ & $\begin{array}{l}5.0673 \\
5.1036 \\
5.1400 \\
5.1762 \\
5.2125 \\
5.2488 \\
5.2851 \\
5.3213 \\
5.3575 \\
5.3938\end{array}$ & $\begin{array}{l}837.67 \\
845.56 \\
853.45 \\
861.35 \\
869.25 \\
877.15 \\
885.06 \\
892.96 \\
900.87 \\
908.78\end{array}$ & $\begin{array}{l}556.35 \\
562.23 \\
568.10 \\
573.99 \\
579.87 \\
585.76 \\
591.65 \\
597.55 \\
603.44 \\
609.34\end{array}$ & $\begin{array}{l}10.046 \\
10.074 \\
10.102 \\
10.130 \\
10.157 \\
10.184 \\
10.211 \\
10.238 \\
10.264 \\
10.291\end{array}$ & $\begin{array}{l}422.00 \\
424.00 \\
426.00 \\
428.00 \\
430.00 \\
432.00 \\
434.00 \\
436.00 \\
438.00 \\
440.00\end{array}$ & $\begin{array}{l}7.5888 \\
7.6246 \\
7.6604 \\
7.6962 \\
7.7320 \\
7.7678 \\
7.8036 \\
7.8394 \\
7.8752 \\
7.9109\end{array}$ & $\begin{array}{l}1380.22 \\
1387.68 \\
1395.14 \\
1402.59 \\
1410.02 \\
1417.45 \\
1424.87 \\
1432.28 \\
1439.69 \\
1447.08\end{array}$ & $\begin{array}{r}958.92 \\
964.40 \\
969.86 \\
975.32 \\
980.77 \\
986.22 \\
991.65 \\
997.07 \\
1002.49 \\
1007.90\end{array}$ & $\begin{array}{l}11.612 \\
11.629 \\
11.647 \\
11.664 \\
11.682 \\
11.699 \\
11.716 \\
11.733 \\
11.750 \\
11.767\end{array}$ \\
\hline $\begin{array}{l}302.00 \\
304.00 \\
30 \% .00 \\
308.00 \\
310.00 \\
312.00 \\
314.00 \\
316.00 \\
318.00 \\
320.00\end{array}$ & $\begin{array}{l}5.4300 \\
5.4662 \\
5.5023 \\
5.5385 \\
5.5747 \\
5.6108 \\
5.6470 \\
5.6831 \\
5.7192 \\
5.7553\end{array}$ & $\begin{array}{l}916.69 \\
924.60 \\
932.51 \\
940.41 \\
948.32 \\
956.22 \\
964.12 \\
972.02 \\
979.91 \\
987.80\end{array}$ & $\begin{array}{l}615.24 \\
621.14 \\
627.04 \\
632.94 \\
638.83 \\
644.73 \\
650.62 \\
656.51 \\
662.40 \\
668.29\end{array}$ & $\begin{array}{l}10.317 \\
10.343 \\
10.369 \\
10.395 \\
10.421 \\
10.446 \\
10.471 \\
10.496 \\
10.521 \\
10.546\end{array}$ & $\begin{array}{l}442.00 \\
444.00 \\
446.00 \\
448.00 \\
450.00 \\
452.00 \\
454.00 \\
456.00 \\
458.00 \\
460.00\end{array}$ & $\begin{array}{l}7.9467 \\
7.9824 \\
8.0182 \\
8.0539 \\
8.0897 \\
8.1254 \\
8.1611 \\
8.1968 \\
8.2325 \\
8.2682\end{array}$ & $\begin{array}{l}1454.47 \\
1461.84 \\
1469.21 \\
1476.57 \\
1483.92 \\
1491.28 \\
1498.64 \\
1505.98 \\
1513.32 \\
1520.64\end{array}$ & $\begin{array}{l}1013.30 \\
1018.69 \\
1024.07 \\
1029.45 \\
1034.82 \\
1040.19 \\
1045.56 \\
1050.93 \\
1056.28 \\
1061.63\end{array}$ & $\begin{array}{l}11.784 \\
11.800 \\
11.817 \\
11.833 \\
11.850 \\
11.866 \\
11.882 \\
11.898 \\
11.914 \\
11.930\end{array}$ \\
\hline $\begin{array}{l}322.00 \\
324.00 \\
326.00 \\
328.00 \\
330.00 \\
332.00 \\
334.00 \\
336.00 \\
338.00 \\
340.00\end{array}$ & $\begin{array}{l}5.7914 \\
5.8275 \\
5.8636 \\
5.8997 \\
5.9358 \\
5.9718 \\
6.0079 \\
6.0440 \\
6.0800 \\
6.1160\end{array}$ & $\begin{array}{r}995.69 \\
1003.57 \\
1011.45 \\
1019.32 \\
1027.19 \\
1035.05 \\
1042.91 \\
1050.76 \\
1058.60 \\
1066.44\end{array}$ & $\begin{array}{l}674.17 \\
680.05 \\
685.92 \\
691.79 \\
697.66 \\
703.52 \\
709.37 \\
715.22 \\
721.06 \\
726.90\end{array}$ & $\begin{array}{l}10.570 \\
10.595 \\
10.619 \\
10.643 \\
10.667 \\
10.691 \\
10.714 \\
10.738 \\
10.761 \\
10.784\end{array}$ & $\begin{array}{l}462.00 \\
464.00 \\
466.00 \\
468.00 \\
470.00 \\
472.00 \\
474.00 \\
476.00 \\
478.00 \\
480.00\end{array}$ & $\begin{array}{l}8.3039 \\
8.3396 \\
8.3753 \\
8.4110 \\
8.4467 \\
8.4823 \\
8.5180 \\
8.5537 \\
8.5893 \\
8.6250\end{array}$ & $\begin{array}{l}1527.97 \\
1535.28 \\
1542.59 \\
1549.88 \\
1557.17 \\
1564.46 \\
1571.74 \\
1579.01 \\
1586.27 \\
1593.53\end{array}$ & $\begin{array}{l}1066.97 \\
1072.30 \\
1077.62 \\
1082.94 \\
1088.25 \\
1093.55 \\
1098.85 \\
1104.14 \\
1109.43 \\
1114.70\end{array}$ & $\begin{array}{l}11.946 \\
11.962 \\
11.978 \\
11.993 \\
12.009 \\
12.024 \\
12.040 \\
12.055 \\
12.070 \\
12.086\end{array}$ \\
\hline $\begin{array}{l}342.00 \\
344.00 \\
346.00 \\
348.00 \\
350.00 \\
352.00 \\
354.00 \\
356.00 \\
358.00 \\
360.00\end{array}$ & $\begin{array}{l}6.1521 \\
6.1881 \\
6.2241 \\
6.2602 \\
6.2962 \\
6.3322 \\
6.3682 \\
6.4042 \\
6.4402 \\
6.4762\end{array}$ & $\begin{array}{l}1074.27 \\
1082.09 \\
1089.90 \\
1097.71 \\
1105.51 \\
1113.30 \\
1121.09 \\
1128.86 \\
1136.63 \\
1144.39\end{array}$ & $\begin{array}{l}732.73 \\
738.55 \\
744.36 \\
750.17 \\
755.97 \\
761.77 \\
767.55 \\
773.33 \\
779.10 \\
784.86\end{array}$ & $\begin{array}{l}10.807 \\
10.830 \\
10.853 \\
10.875 \\
10.898 \\
10.920 \\
10.942 \\
10.964 \\
10.985 \\
11.007\end{array}$ & $\begin{array}{l}482.00 \\
484.00 \\
486.00 \\
488.00 \\
490.00 \\
492.00 \\
494.00 \\
496.00 \\
498.00 \\
500.00\end{array}$ & $\begin{array}{l}8.6607 \\
8.6963 \\
8.7320 \\
8.7676 \\
8.8033 \\
8.8390 \\
8.8746 \\
8.9103 \\
8.9460 \\
8.9816\end{array}$ & $\begin{array}{l}1600.78 \\
1608.02 \\
1615.26 \\
1622.50 \\
1629.72 \\
1636.94 \\
1644.16 \\
1651.37 \\
1658.58 \\
1665.78\end{array}$ & $\begin{array}{l}1119.97 \\
1125.24 \\
1130.50 \\
1135.75 \\
1141.00 \\
1146.24 \\
1151.48 \\
1156.71 \\
1161.94 \\
1167.16\end{array}$ & $\begin{array}{l}12.101 \\
12.116 \\
12.131 \\
12.145 \\
12.160 \\
12.175 \\
12.189 \\
12.204 \\
12.219 \\
12.233\end{array}$ \\
\hline $\begin{array}{l}362.00 \\
364.00 \\
366.00 \\
368.00 \\
370.00 \\
372.00 \\
374.00 \\
376.00 \\
378.00 \\
380.00\end{array}$ & $\begin{array}{l}6.5121 \\
6.5481 \\
6.5840 \\
6.6200 \\
6.6559 \\
6.6919 \\
6.7278 \\
6.7637 \\
6.7996 \\
6.8355\end{array}$ & $\begin{array}{l}1152.13 \\
1159.87 \\
1167.59 \\
1175.31 \\
1183.02 \\
1190.72 \\
1198.41 \\
1206.09 \\
1213.76 \\
1221.42\end{array}$ & $\begin{array}{l}790.60 \\
796.34 \\
802.07 \\
807.80 \\
813.51 \\
819.21 \\
824.91 \\
830.60 \\
836.27 \\
841.94\end{array}$ & $\begin{array}{l}11.028 \\
11.050 \\
11.071 \\
11.092 \\
11.113 \\
11.134 \\
11.154 \\
11.175 \\
11.195 \\
11.215\end{array}$ & $\begin{array}{l}502.00 \\
504.00 \\
506.00 \\
508.00 \\
510.00 \\
512.00 \\
514.00 \\
516.00 \\
518.00 \\
520.00\end{array}$ & $\begin{array}{l}9.0173 \\
9.0530 \\
9.0887 \\
9.1244 \\
9.1601 \\
9.1958 \\
9.2315 \\
9.2672 \\
9.3030 \\
9.3387\end{array}$ & $\begin{array}{l}1672.98 \\
1680.17 \\
1687.36 \\
1694.55 \\
1701.73 \\
1708.90 \\
1716.08 \\
1723.25 \\
1730.41 \\
1737.58\end{array}$ & $\begin{array}{l}1172.38 \\
1177.59 \\
1182.79 \\
1188.00 \\
1193.20 \\
1198.39 \\
1203.58 \\
1208.77 \\
1213.95 \\
1219.13\end{array}$ & $\begin{array}{l}12.247 \\
12.262 \\
12.276 \\
12.290 \\
12.304 \\
12.318 \\
12.332 \\
12.346 \\
12.360 \\
12.374\end{array}$ \\
\hline $\begin{array}{l}382.00 \\
384.00 \\
386.00 \\
388.00 \\
390.00 \\
392.00 \\
394.00 \\
396.00 \\
398.00 \\
400.00\end{array}$ & $\begin{array}{l}6.8715 \\
6.9074 \\
6.9433 \\
6.9792 \\
7.0151 \\
7.0509 \\
7.0868 \\
7.1227 \\
7.1586 \\
7.1945\end{array}$ & $\begin{array}{l}1229.08 \\
1236.72 \\
1244.35 \\
1251.98 \\
1259.60 \\
1267.20 \\
1274.80 \\
1282.39 \\
1289.97 \\
1297.54\end{array}$ & $\begin{array}{l}847.60 \\
853.25 \\
858.89 \\
864.53 \\
870.15 \\
875.76 \\
881.37 \\
886.96 \\
892.55 \\
898.13\end{array}$ & $\begin{array}{l}11.235 \\
11.255 \\
11.275 \\
11.295 \\
11.314 \\
11.334 \\
11.353 \\
11.372 \\
11.392 \\
11.411\end{array}$ & $\begin{array}{l}522.00 \\
524.00 \\
526.00 \\
528.00 \\
530.00 \\
532.00 \\
534.00 \\
536.00 \\
538.00 \\
540.00\end{array}$ & $\begin{array}{l}9.3745 \\
9.4102 \\
9.4460 \\
9.4817 \\
9.5175 \\
9.5533 \\
9.5890 \\
9.6248 \\
9.6606 \\
9.6964\end{array}$ & $\begin{array}{l}1744.73 \\
1751.89 \\
1759.04 \\
1766.19 \\
1773.34 \\
1780.48 \\
1787.62 \\
1794.76 \\
1801.89 \\
1809.02\end{array}$ & $\begin{array}{l}1224.30 \\
1229.47 \\
1234.64 \\
1239.81 \\
1244.97 \\
1250.12 \\
1255.28 \\
1260.42 \\
1265.57 \\
1270.71\end{array}$ & $\begin{array}{l}12.388 \\
12.401 \\
12.415 \\
12.428 \\
12.442 \\
12.455 \\
12.469 \\
12.482 \\
12.495 \\
12.509\end{array}$ \\
\hline
\end{tabular}


TEMPER - SPECIFIC ENTHALPY INTERNAL ENTROPY ATURE VOLUME (BTU/LB) ENERGY (BTU/LB-R) (R) (CU FT/LB)
ENERGY
$(8 T U / L 8)$
TEMPER- SPECIFIC ENTHALPY ATURE VOLUME (8TU/L8) (R) (CU FT/L8)
INTERNAL ENTROPY $(8 T U / L 8)$

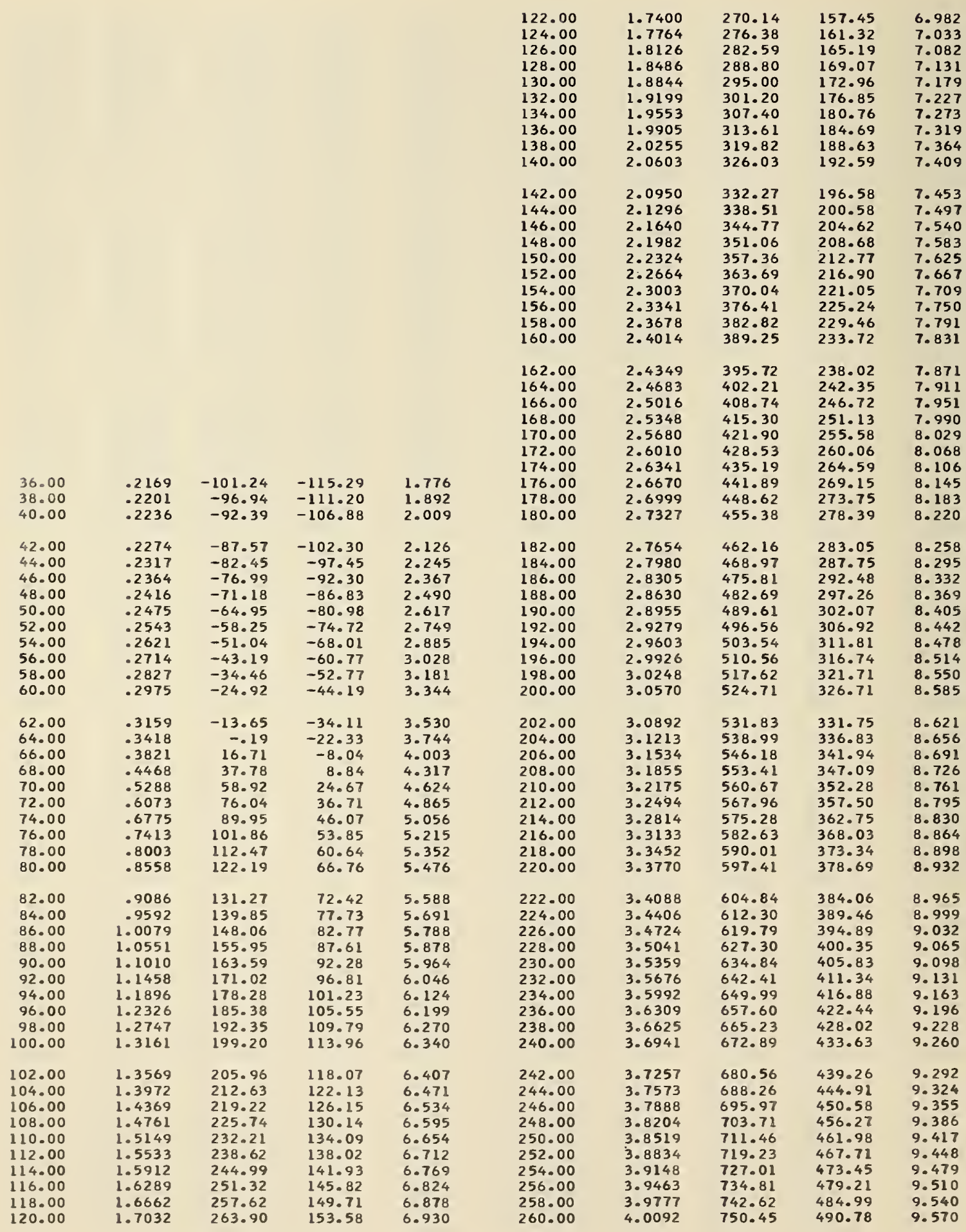


TEMPER- SPECIFIC ENTHALPY INTERNAL ENTROPY ATURE VOLUME (BTU/LB) ENERGY (BTU/LB-R) (R) (CUFT/LB)

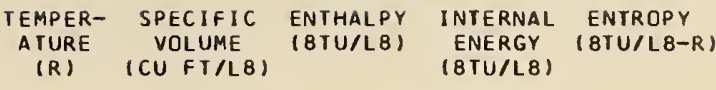

\begin{tabular}{|c|c|c|c|c|c|c|c|c|c|}
\hline $\begin{array}{l}262.00 \\
264.00 \\
266.00 \\
268.00 \\
270.00 \\
272.00 \\
274.00 \\
276.00 \\
278.00 \\
280.00\end{array}$ & $\begin{array}{l}4.0406 \\
4.0720 \\
4.1033 \\
4.1347 \\
4.1661 \\
4.1974 \\
4.2287 \\
4.2600 \\
4.2913 \\
4.3226\end{array}$ & $\begin{array}{l}758.29 \\
766.15 \\
774.01 \\
781.89 \\
789.77 \\
797.64 \\
805.52 \\
813.41 \\
821.30 \\
829.19\end{array}$ & $\begin{array}{l}496.59 \\
502.41 \\
508.24 \\
514.09 \\
519.95 \\
525.79 \\
531.64 \\
537.49 \\
543.36 \\
549.23\end{array}$ & $\begin{array}{r}9.600 \\
9.630 \\
9.660 \\
9.689 \\
9.719 \\
9.748 \\
9.777 \\
9.805 \\
9.834 \\
9.862\end{array}$ & $\begin{array}{l}402.00 \\
404.00 \\
406.00 \\
408.00 \\
410.00 \\
412.00 \\
414.00 \\
416.00 \\
418.00 \\
420.00\end{array}$ & $\begin{array}{l}6.2122 \\
6.2430 \\
6.2738 \\
6.3046 \\
6.3353 \\
6.3661 \\
6.3969 \\
6.4276 \\
6.4584 \\
6.4891\end{array}$ & $\begin{array}{l}1305.26 \\
1312.82 \\
1320.37 \\
1327.91 \\
1335.44 \\
1342.96 \\
1350.48 \\
1357.99 \\
1365.49 \\
1372.98\end{array}$ & $\begin{array}{l}902.90 \\
908.47 \\
914.02 \\
919.57 \\
925.11 \\
930.64 \\
936.16 \\
941.68 \\
947.19 \\
952.69\end{array}$ & $\begin{array}{l}11.275 \\
11.294 \\
11.313 \\
11.331 \\
11.350 \\
11.368 \\
11.386 \\
11.404 \\
11.422 \\
11.440\end{array}$ \\
\hline $\begin{array}{l}282.00 \\
284.00 \\
286.00 \\
288.00 \\
290.00 \\
292.00 \\
294.00 \\
296.00 \\
298.00 \\
300.00\end{array}$ & $\begin{array}{l}4.3538 \\
4.3851 \\
4.4163 \\
4.4475 \\
4.4787 \\
4.5099 \\
4.5411 \\
4.5723 \\
4.6034 \\
4.6346\end{array}$ & $\begin{array}{l}837.09 \\
845.00 \\
852.91 \\
860.82 \\
868.74 \\
876.66 \\
884.58 \\
892.50 \\
900.42 \\
908.35\end{array}$ & $\begin{array}{l}555.10 \\
560.99 \\
566.87 \\
572.77 \\
578.66 \\
584.56 \\
590.46 \\
596.36 \\
602.27 \\
608.18\end{array}$ & $\begin{array}{r}9.890 \\
9.918 \\
9.946 \\
9.974 \\
10.001 \\
10.028 \\
10.055 \\
10.082 \\
10.109 \\
10.135\end{array}$ & $\begin{array}{l}422.00 \\
424.00 \\
426.00 \\
428.00 \\
430.00 \\
432.00 \\
434.00 \\
436.00 \\
438.00 \\
440.00\end{array}$ & $\begin{array}{l}6.5199 \\
6.5506 \\
6.5814 \\
6.6121 \\
6.6428 \\
6.6735 \\
6.7043 \\
6.7350 \\
6.7657 \\
6.7964\end{array}$ & $\begin{array}{l}1380.46 \\
1387.93 \\
1395.39 \\
1402.85 \\
1410.29 \\
1417.73 \\
1425.16 \\
1432.58 \\
1439.99 \\
1447.39\end{array}$ & $\begin{array}{r}958.18 \\
963.66 \\
969.13 \\
974.59 \\
980.05 \\
985.50 \\
990.93 \\
996.36 \\
1001.79 \\
1007.20\end{array}$ & $\begin{array}{l}11.458 \\
11.476 \\
11.493 \\
11.511 \\
11.528 \\
11.545 \\
11.562 \\
11.580 \\
11.596 \\
11.613\end{array}$ \\
\hline $\begin{array}{l}302.00 \\
304.00 \\
306.00 \\
308.00 \\
310.00 \\
312.00 \\
314.00 \\
316.00 \\
318.00 \\
320.00\end{array}$ & $\begin{array}{l}4.6657 \\
4.6968 \\
4.7279 \\
4.7590 \\
4.7901 \\
4.8212 \\
4.8522 \\
4.8833 \\
4.9143 \\
4.9454\end{array}$ & $\begin{array}{l}916.27 \\
924.19 \\
932.12 \\
940.04 \\
947.96 \\
955.87 \\
963.79 \\
971.70 \\
979.61 \\
987.51\end{array}$ & $\begin{array}{l}614.08 \\
619.99 \\
625.90 \\
631.80 \\
637.71 \\
643.61 \\
649.52 \\
655.41 \\
661.31 \\
667.21\end{array}$ & $\begin{array}{l}10.162 \\
10.188 \\
10.214 \\
10.239 \\
10.265 \\
10.291 \\
10.316 \\
10.341 \\
10.366 \\
10.391\end{array}$ & $\begin{array}{l}442.00 \\
444.00 \\
446.00 \\
448.00 \\
450.00 \\
452.00 \\
454.00 \\
456.00 \\
458.00 \\
460.00\end{array}$ & $\begin{array}{l}6.8271 \\
6.8578 \\
6.8884 \\
6.9191 \\
6.9498 \\
6.9804 \\
7.0111 \\
7.0417 \\
7.0724 \\
7.1030\end{array}$ & $\begin{array}{l}1454.78 \\
1462.17 \\
1469.54 \\
1476.91 \\
1484.27 \\
1491.64 \\
1499.00 \\
1506.35 \\
1513.69 \\
1521.03\end{array}$ & $\begin{array}{l}1012.60 \\
1018.00 \\
1023.39 \\
1028.77 \\
1034.14 \\
1039.52 \\
1044.90 \\
1050.26 \\
1055.62 \\
1060.97\end{array}$ & $\begin{array}{l}11.630 \\
11.647 \\
11.663 \\
11.680 \\
11.696 \\
11.713 \\
11.729 \\
11.745 \\
11.761 \\
11.777\end{array}$ \\
\hline $\begin{array}{l}322.00 \\
324.00 \\
326.00 \\
328.00 \\
330.00 \\
332.00 \\
334.00 \\
336.00 \\
338.00 \\
340.00\end{array}$ & $\begin{array}{l}4.9764 \\
5.0074 \\
5.0384 \\
5.0694 \\
5.1004 \\
5.1314 \\
5.1624 \\
5.1934 \\
5.2244 \\
5.2553\end{array}$ & $\begin{array}{r}995.41 \\
1003.31 \\
1011.20 \\
1019.08 \\
1026.96 \\
1034.84 \\
1042.71 \\
1050.57 \\
1058.43 \\
1066.28\end{array}$ & $\begin{array}{l}673.10 \\
678.98 \\
684.87 \\
690.74 \\
696.62 \\
702.48 \\
708.35 \\
714.20 \\
720.05 \\
725.90\end{array}$ & $\begin{array}{l}10.415 \\
10.440 \\
10.464 \\
10.488 \\
10.512 \\
10.536 \\
10.559 \\
10.583 \\
10.606 \\
10.629\end{array}$ & $\begin{array}{l}462.00 \\
464.00 \\
466.00 \\
468.00 \\
470.00 \\
472.00 \\
474.00 \\
476.00 \\
478.00 \\
480.00\end{array}$ & $\begin{array}{l}7.1337 \\
7.1643 \\
7.1949 \\
7.2255 \\
7.2562 \\
7.2868 \\
7.3174 \\
7.3480 \\
7.3786 \\
7.4092\end{array}$ & $\begin{array}{l}1528.35 \\
1535.68 \\
1542.99 \\
1550.29 \\
1557.59 \\
1564.88 \\
1572.16 \\
1579.44 \\
1586.71 \\
1593.97\end{array}$ & $\begin{array}{l}1066.32 \\
1071.65 \\
1076.98 \\
1082.31 \\
1087.62 \\
1092.93 \\
1098.23 \\
1103.52 \\
1108.81 \\
1114.09\end{array}$ & $\begin{array}{l}11.793 \\
11.809 \\
11.824 \\
11.840 \\
11.856 \\
11.871 \\
11.887 \\
11.902 \\
11.917 \\
11.932\end{array}$ \\
\hline $\begin{array}{l}342.00 \\
344.00 \\
346.00 \\
348.00 \\
350.00 \\
352.00 \\
354.00 \\
356.00 \\
358.00 \\
360.00\end{array}$ & $\begin{array}{l}5.2863 \\
5.3172 \\
5.3482 \\
5.3791 \\
5.4100 \\
5.4410 \\
5.4719 \\
5.5028 \\
5.55337 \\
5.5646\end{array}$ & $\begin{array}{l}1074.12 \\
1081.95 \\
1089.78 \\
1097.60 \\
1105.41 \\
111.3 .21 \\
1121.01 \\
1128.80 \\
1136.57 \\
1144.34\end{array}$ & $\begin{array}{l}731.73 \\
737.56 \\
743.39 \\
749.20 \\
755.01 \\
760.81 \\
766.60 \\
772.39 \\
778.16 \\
783.93\end{array}$ & $\begin{array}{l}10.652 \\
10.675 \\
10.698 \\
10.720 \\
10.743 \\
10.765 \\
10.787 \\
10.809 \\
10.831 \\
10.853\end{array}$ & $\begin{array}{l}482.00 \\
484.00 \\
486.00 \\
488.00 \\
490.00 \\
492.00 \\
494.00 \\
496.00 \\
498.00 \\
500.00\end{array}$ & $\begin{array}{l}7.4398 \\
7.4704 \\
7.5010 \\
7.5316 \\
7.5622 \\
7.5928 \\
7.6234 \\
7.6540 \\
7.6846 \\
7.7152\end{array}$ & $\begin{array}{l}1601.23 \\
1608.48 \\
1615.73 \\
1622.96 \\
1630.20 \\
1637.42 \\
1644.65 \\
1651.86 \\
1659.07 \\
1666.28\end{array}$ & $\begin{array}{l}1119.37 \\
1124.63 \\
1129.90 \\
1135.15 \\
1140.40 \\
1145.65 \\
1150.89 \\
1156.12 \\
1161.35 \\
1166.58\end{array}$ & $\begin{array}{l}11.947 \\
11.962 \\
11.977 \\
11.992 \\
12.007 \\
12.022 \\
12.036 \\
12.051 \\
12.065 \\
12.080\end{array}$ \\
\hline $\begin{array}{l}362.00 \\
364.00 \\
366.00 \\
368.00 \\
370.00 \\
372.00 \\
374.00 \\
376.00 \\
378.00 \\
380.00\end{array}$ & $\begin{array}{l}5.5955 \\
5.6264 \\
5.6573 \\
5.6881 \\
5.7190 \\
5.7499 \\
5.7807 \\
5.8116 \\
5.8424 \\
5.8732\end{array}$ & $\begin{array}{l}1152.10 \\
1159.84 \\
1167.58 \\
1175.31 \\
1183.03 \\
1190.74 \\
1198.44 \\
1206.13 \\
1213.81 \\
1221.48\end{array}$ & $\begin{array}{l}789.69 \\
795.43 \\
801.17 \\
806.90 \\
812.62 \\
818.33 \\
824.03 \\
829.72 \\
835.41 \\
841.08\end{array}$ & $\begin{array}{l}10.874 \\
10.895 \\
10.917 \\
10.938 \\
10.959 \\
10.979 \\
11.000 \\
11.020 \\
11.041 \\
11.061\end{array}$ & $\begin{array}{l}502.00 \\
504.00 \\
506.00 \\
508.00 \\
510.00 \\
512.00 \\
514.00 \\
516.00 \\
518.00 \\
520.00\end{array}$ & $\begin{array}{l}7.7459 \\
7.7765 \\
7.8071 \\
7.8377 \\
7.8683 \\
7.8990 \\
7.9296 \\
7.9603 \\
7.9909 \\
8.0216\end{array}$ & $\begin{array}{l}1673.48 \\
1680.68 \\
1687.87 \\
1695.06 \\
1702.25 \\
1709.43 \\
1716.61 \\
1723.78 \\
1730.95 \\
1738.12\end{array}$ & $\begin{array}{l}1171.80 \\
1177.01 \\
1182.22 \\
1187.43 \\
1192.63 \\
1197.82 \\
1203.02 \\
1208.21 \\
1213.39 \\
1218.57\end{array}$ & $\begin{array}{l}12.094 \\
12.108 \\
12.123 \\
12.137 \\
12.151 \\
12.165 \\
12.179 \\
12.193 \\
12.207 \\
12.221\end{array}$ \\
\hline $\begin{array}{l}382.00 \\
384.00 \\
386.00 \\
388.00 \\
390.00 \\
392.00 \\
394.00 \\
396.00 \\
398.00 \\
400.00\end{array}$ & $\begin{array}{l}5.9041 \\
5.9349 \\
5.9657 \\
5.9966 \\
6.0274 \\
6.0582 \\
6.0890 \\
6.1198 \\
6.1506 \\
6.1814\end{array}$ & $\begin{array}{l}1229.14 \\
1236.80 \\
1244.44 \\
1252.08 \\
1259.70 \\
1267.32 \\
1274.92 \\
1282.52 \\
1290.11 \\
1297.69\end{array}$ & $\begin{array}{l}46.75 \\
852.40 \\
858.05 \\
863.69 \\
869.32 \\
874.94 \\
880.55 \\
886.15 \\
891.74 \\
897.33\end{array}$ & $\begin{array}{l}11.081 \\
11.101 \\
11.121 \\
11.141 \\
11.160 \\
11.180 \\
11.199 \\
11.218 \\
11.238 \\
11.257\end{array}$ & $\begin{array}{l}522.00 \\
524.00 \\
526.00 \\
528.00 \\
530.00 \\
532.00 \\
534.00 \\
536.00 \\
538.00 \\
540.00\end{array}$ & $\begin{array}{l}8.0522 \\
8.0829 \\
8.1135 \\
8.1442 \\
8.1749 \\
8.2055 \\
8.2362 \\
8.2669 \\
8.2976 \\
8.3282\end{array}$ & $\begin{array}{l}1745.28 \\
1752.44 \\
1759.59 \\
1766.75 \\
1773.89 \\
1781.04 \\
1788.18 \\
1795.32 \\
1802.46 \\
1809.59\end{array}$ & $\begin{array}{l}1223.75 \\
1228.92 \\
1234.09 \\
1239.26 \\
1244.42 \\
1249.58 \\
1254.73 \\
1259.89 \\
1265.04 \\
1270.18\end{array}$ & $\begin{array}{l}12.234 \\
12.248 \\
12.262 \\
12.275 \\
12.289 \\
12.302 \\
12.316 \\
12.329 \\
12.342 \\
12.356\end{array}$ \\
\hline
\end{tabular}


TEMPER- SPECIFIC ENTHALPY INTERNAL ENTROPY ATURE VOLUME (8TU/L8) ENERGY (8TU/L8-R) (R) (CU FT/L8)

\section{(8TU/L8)}

TEMPER- SPECIFIC ENTHALPY ATURE VOLUME (R) (CU FT/L8)
INTERNAL ENTROPY ENERGY
$(8 T U / L 8)$

\begin{tabular}{|c|c|c|c|c|c|c|c|c|c|}
\hline & & & & & $\begin{array}{l}122.00 \\
124.00 \\
126.00 \\
128.00 \\
130.00 \\
132.00 \\
134.00 \\
136.00 \\
138.00 \\
140.00\end{array}$ & $\begin{array}{l}1.5119 \\
1.5444 \\
1.5767 \\
1.6088 \\
1.6407 \\
1.6724 \\
1.7038 \\
1.7351 \\
1.7663 \\
1.7972\end{array}$ & $\begin{array}{l}265.64 \\
272.01 \\
278.37 \\
284.71 \\
291.04 \\
297.36 \\
303.67 \\
309.98 \\
316.30 \\
322.62\end{array}$ & $\begin{array}{l}153.73 \\
157.69 \\
161.66 \\
165.62 \\
169.59 \\
173.57 \\
177.55 \\
181.55 \\
185.56 \\
189.59\end{array}$ & $\begin{array}{l}6.822 \\
6.874 \\
6.925 \\
6.975 \\
7.024 \\
7.072 \\
7.119 \\
7.166 \\
7.212 \\
7.258\end{array}$ \\
\hline & & & & & $\begin{array}{l}142.00 \\
144.00 \\
146.00 \\
148.00 \\
150.00 \\
152.00 \\
154.00 \\
156.00 \\
158.00 \\
160.00\end{array}$ & $\begin{array}{l}1.8280 \\
1.8587 \\
1.8892 \\
1.9196 \\
1.9499 \\
1.9800 \\
2.0101 \\
2.0400 \\
2.0698 \\
2.0996\end{array}$ & $\begin{array}{l}328.95 \\
335.29 \\
341.64 \\
348.01 \\
354.40 \\
360.81 \\
367.24 \\
373.69 \\
380.17 \\
386.68\end{array}$ & $\begin{array}{l}193.64 \\
197.71 \\
201.80 \\
205.92 \\
210.07 \\
214.24 \\
218.45 \\
222.69 \\
226.96 \\
231.26\end{array}$ & $\begin{array}{l}7.303 \\
7.347 \\
7.391 \\
7.434 \\
7.477 \\
7.519 \\
7.561 \\
7.603 \\
7.644 \\
7.685\end{array}$ \\
\hline 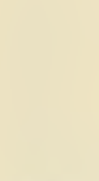 & & & & 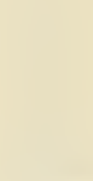 & $\begin{array}{l}162.00 \\
164.00 \\
166.00 \\
168.00 \\
170.00 \\
172.00 \\
174.00\end{array}$ & $\begin{array}{l}2.1292 \\
2.1588 \\
2.1882 \\
2.2176 \\
2.2469 \\
2.2761 \\
2.3053\end{array}$ & $\begin{array}{l}393.21 \\
399.77 \\
406.36 \\
412.99 \\
419.64 \\
426.33 \\
433.05\end{array}$ & $\begin{array}{l}235.60 \\
239.98 \\
244.39 \\
248.84 \\
253.33 \\
257.85 \\
262.41\end{array}$ & $\begin{array}{l}7.726 \\
7.766 \\
7.806 \\
7.846 \\
7.885 \\
7.924 \\
7.963\end{array}$ \\
\hline $\begin{array}{l}36.00 \\
38.00 \\
40.00\end{array}$ & $\begin{array}{r}.2159 \\
.2190 \\
.2223\end{array}$ & $\begin{array}{l}-99.74 \\
-95.49 \\
-90.99\end{array}$ & $\begin{array}{l}-115.72 \\
-111.70 \\
-107.45\end{array}$ & $\begin{array}{l}1.762 \\
1.877 \\
1.992\end{array}$ & $\begin{array}{l}176.00 \\
178.00 \\
180.00\end{array}$ & $\begin{array}{l}2.3344 \\
2.3634 \\
2.3924\end{array}$ & $\begin{array}{l}439.80 \\
446.58 \\
453.40\end{array}$ & $\begin{array}{l}267.01 \\
271.64 \\
276.31\end{array}$ & $\begin{array}{l}8.002 \\
8.040 \\
8.078\end{array}$ \\
\hline $\begin{array}{l}42.00 \\
44.00 \\
46.00 \\
48.00 \\
50.00 \\
52.00 \\
54.00 \\
56.00 \\
58.00 \\
60.00\end{array}$ & $\begin{array}{l}.2260 \\
.2300 \\
.2345 \\
.2394 \\
.2449 \\
.2511 \\
.2582 \\
.2664 \\
.2762 \\
.2888\end{array}$ & $\begin{array}{l}-86.24 \\
-81.19 \\
-75.84 \\
-70.15 \\
-64.07 \\
-57.58 \\
-50.64 \\
-43.16 \\
-34.97 \\
-26.16\end{array}$ & $\begin{array}{r}-102.96 \\
-98.22 \\
-93.19 \\
-87.87 \\
-82.20 \\
-76.17 \\
-69.75 \\
-62.89 \\
-55.41 \\
-47.54\end{array}$ & $\begin{array}{l}2.108 \\
2.225 \\
2.344 \\
2.465 \\
2.589 \\
2.717 \\
2.848 \\
2.984 \\
3.128 \\
3.278\end{array}$ & $\begin{array}{l}182.00 \\
184.00 \\
186.00 \\
188.00 \\
190.00 \\
192.00 \\
194.00 \\
196.00 \\
198.00 \\
200.00\end{array}$ & $\begin{array}{l}2.4212 \\
2.4500 \\
2.4787 \\
2.5074 \\
2.5360 \\
2.5646 \\
2.5931 \\
2.6216 \\
2.6500 \\
2.6784\end{array}$ & $\begin{array}{l}460.23 \\
467.09 \\
473.98 \\
480.90 \\
487.86 \\
494.85 \\
501.88 \\
508.94 \\
516.04 \\
523.17\end{array}$ & $\begin{array}{l}281.01 \\
285.74 \\
290.50 \\
295.30 \\
300.14 \\
305.02 \\
309.94 \\
314.89 \\
319.88 \\
324.91\end{array}$ & $\begin{array}{l}8.116 \\
8.153 \\
8.190 \\
8.227 \\
8.264 \\
8.301 \\
8.337 \\
8.374 \\
8.410 \\
8.445\end{array}$ \\
\hline $\begin{array}{l}62.00 \\
64.00 \\
66.00 \\
68.00 \\
70.00 \\
72.00 \\
74.00 \\
76.00 \\
78.00 \\
80.00\end{array}$ & $\begin{array}{r}.3033 \\
.3220 \\
.3471 \\
.3822 \\
.4304 \\
.4886 \\
.5486 \\
.6061 \\
.6603 \\
.7114\end{array}$ & $\begin{array}{r}-16.12 \\
-4.76 \\
8.38 \\
23.73 \\
41.05 \\
58.35 \\
73.88 \\
87.41 \\
99.38 \\
110.20\end{array}$ & $\begin{array}{r}-38.57 \\
-28.59 \\
-17.31 \\
-4.57 \\
9.19 \\
22.18 \\
33.27 \\
42.55 \\
50.50 \\
57.54\end{array}$ & $\begin{array}{l}3.444 \\
3.625 \\
3.826 \\
4.055 \\
4.306 \\
4.550 \\
4.762 \\
4.943 \\
5.098 \\
5.235\end{array}$ & $\begin{array}{l}202.00 \\
204.00 \\
206.00 \\
208.00 \\
210.00 \\
212.00 \\
214.00 \\
216.00 \\
218.00 \\
220.00\end{array}$ & $\begin{array}{l}2.7067 \\
2.7350 \\
2.7633 \\
2.7915 \\
2.8197 \\
2.8478 \\
2.8759 \\
2.9040 \\
2.9321 \\
2.9601\end{array}$ & $\begin{array}{l}530.33 \\
537.52 \\
544.75 \\
552.01 \\
559.30 \\
566.62 \\
573.97 \\
581.36 \\
588.76 \\
596.20\end{array}$ & $\begin{array}{l}329.97 \\
335.07 \\
340.21 \\
345.38 \\
350.58 \\
355.82 \\
361.09 \\
366.40 \\
371.73 \\
377.08\end{array}$ & $\begin{array}{l}8.481 \\
8.516 \\
8.552 \\
8.587 \\
8.622 \\
8.656 \\
8.691 \\
8.725 \\
8.759 \\
8.793\end{array}$ \\
\hline $\begin{array}{r}82.00 \\
84.00 \\
86.00 \\
88.00 \\
90.00 \\
92.00 \\
94.00 \\
96.00 \\
98.00 \\
100.00\end{array}$ & $\begin{array}{r}.7599 \\
.8064 \\
.8510 \\
.8942 \\
.9361 \\
.9769 \\
1.0167 \\
1.0556 \\
1.0938 \\
1.1313\end{array}$ & $\begin{array}{l}120.18 \\
129.52 \\
138.37 \\
146.83 \\
154.97 \\
162.84 \\
170.49 \\
177.96 \\
185.26 \\
192.42\end{array}$ & $\begin{array}{r}63.93 \\
69.83 \\
75.38 \\
80.64 \\
85.68 \\
90.53 \\
95.24 \\
99.82 \\
104.29 \\
108.68\end{array}$ & $\begin{array}{l}5.359 \\
5.471 \\
5.575 \\
5.673 \\
5.764 \\
5.851 \\
5.933 \\
6.011 \\
6.087 \\
6.159\end{array}$ & $\begin{array}{l}222.00 \\
224.00 \\
226.00 \\
228.00 \\
230.00 \\
232.00 \\
234.00 \\
236.00 \\
238.00 \\
240.00\end{array}$ & $\begin{array}{l}2.9881 \\
3.0161 \\
3.0440 \\
3.0719 \\
3.0998 \\
3.1277 \\
3.1556 \\
3.1834 \\
3.2112 \\
3.2390\end{array}$ & $\begin{array}{l}603.66 \\
611.14 \\
618.66 \\
626.20 \\
633.76 \\
641.35 \\
648.96 \\
656.60 \\
664.25 \\
671.93\end{array}$ & $\begin{array}{l}382.47 \\
387.89 \\
393.34 \\
398.81 \\
404.31 \\
409.83 \\
415.38 \\
420.96 \\
426.56 \\
432.18\end{array}$ & $\begin{array}{l}8.827 \\
8.861 \\
8.894 \\
8.927 \\
8.960 \\
8.993 \\
9.026 \\
9.058 \\
9.091 \\
9.123\end{array}$ \\
\hline $\begin{array}{l}102.00 \\
104.00 \\
106.00 \\
108.00 \\
110.00 \\
112.00 \\
114.00 \\
116.00 \\
118.00 \\
120.00\end{array}$ & $\begin{array}{l}1.1681 \\
1.2044 \\
1.2402 \\
1.2755 \\
1.3103 \\
1.3447 \\
1.3788 \\
1.4125 \\
1.4459 \\
1.4790\end{array}$ & $\begin{array}{l}199.45 \\
206.38 \\
213.22 \\
219.97 \\
226.65 \\
233.26 \\
239.82 \\
246.33 \\
252.80 \\
259.23\end{array}$ & $\begin{array}{l}112.99 \\
117.23 \\
121.42 \\
125.56 \\
129.66 \\
133.72 \\
137.76 \\
141.77 \\
145.77 \\
149.75\end{array}$ & $\begin{array}{l}6.229 \\
6.296 \\
6.361 \\
6.424 \\
6.485 \\
6.545 \\
6.603 \\
6.660 \\
6.715 \\
6.769\end{array}$ & $\begin{array}{l}242.00 \\
244.00 \\
246.00 \\
248.00 \\
250.00 \\
252.00 \\
254.00 \\
256.00 \\
258.00 \\
260.00\end{array}$ & $\begin{array}{l}3.2667 \\
3.2945 \\
3.3222 \\
3.3499 \\
3.3776 \\
3.4053 \\
3.4330 \\
3.4606 \\
3.4882 \\
3.5158\end{array}$ & $\begin{array}{l}679.63 \\
687.34 \\
695.08 \\
702.84 \\
710.61 \\
718.40 \\
726.20 \\
734.02 \\
741.86 \\
749.70\end{array}$ & $\begin{array}{l}437.82 \\
443.48 \\
449.17 \\
454.87 \\
460.59 \\
466.33 \\
472.09 \\
477.86 \\
483.65 \\
489.46\end{array}$ & $\begin{array}{l}9.155 \\
9.186 \\
9.218 \\
9.249 \\
9.281 \\
9.312 \\
9.342 \\
9.373 \\
9.404 \\
9.434\end{array}$ \\
\hline
\end{tabular}


TEMPER- SPECIFIC ENTHALPY INTERNAL ENTROPY ATURE VOLUME (BTU/L8) ENERGY (8TU/L8-R) (R) (CU FT/LB)
( 8 TU/L8)
TEMPER- SPECIFIC ENTHALPY ATURE VOLUME (8TU/L8) (R) (CU FT/L8)
INTERNAL ENTROPY

ENERGY (BTU/L8-R) (8TU/L8)

\begin{tabular}{|c|c|c|c|c|c|c|c|c|c|}
\hline $\begin{array}{l}262.00 \\
264.00 \\
266.00 \\
268.00 \\
270.00 \\
272.00 \\
274.00 \\
276.00 \\
278.00 \\
280.00\end{array}$ & $\begin{array}{l}3.5434 \\
3.5710 \\
3.5986 \\
3.6261 \\
3.6536 \\
3.6812 \\
3.7087 \\
3.7362 \\
3.7636 \\
3.7911\end{array}$ & $\begin{array}{l}757.56 \\
765.44 \\
773.32 \\
781.22 \\
789.12 \\
797.01 \\
804.91 \\
812.81 \\
820.72 \\
828.63\end{array}$ & $\begin{array}{l}495.28 \\
501.11 \\
506.95 \\
512.81 \\
518.67 \\
524.53 \\
530.39 \\
536.25 \\
542.13 \\
548.01\end{array}$ & $\begin{array}{l}9.464 \\
9.494 \\
9.524 \\
9.553 \\
9.583 \\
9.612 \\
9.641 \\
9.669 \\
9.698 \\
9.726\end{array}$ & $\begin{array}{l}402.00 \\
404.00 \\
406.00 \\
408.00 \\
410.00 \\
412.00 \\
414.00 \\
416.00 \\
418.00 \\
420.00\end{array}$ & $\begin{array}{l}5.4483 \\
5.4753 \\
5.5023 \\
5.5292 \\
5.5562 \\
5.5832 \\
5.6101 \\
5.6371 \\
5.6640 \\
5.6910\end{array}$ & $\begin{array}{l}1305.43 \\
1313.00 \\
1320.56 \\
1328.11 \\
1335.65 \\
1343.18 \\
1350.70 \\
1358.22 \\
1365.73 \\
1373.22\end{array}$ & $\begin{array}{l}902.14 \\
907.71 \\
913.27 \\
918.83 \\
924.37 \\
929.91 \\
935.43 \\
940.96 \\
946.47 \\
951.97\end{array}$ & $\begin{array}{l}11.142 \\
11.161 \\
11.179 \\
11.198 \\
11.216 \\
11.235 \\
11.253 \\
11.271 \\
11.289 \\
11.307\end{array}$ \\
\hline $\begin{array}{l}282.00 \\
284.00 \\
286.00 \\
288.00 \\
290.00 \\
292.00 \\
294.00 \\
296.00 \\
298.00 \\
300.00\end{array}$ & $\begin{array}{l}3.8185 \\
3.8460 \\
3.8734 \\
3.9008 \\
3.9282 \\
3.9556 \\
3.9829 \\
4.0103 \\
4.0376 \\
4.0649\end{array}$ & $\begin{array}{l}836.55 \\
844.47 \\
852.40 \\
860.33 \\
868.26 \\
876.19 \\
884.13 \\
892.07 \\
900.01 \\
907.95\end{array}$ & $\begin{array}{l}553.90 \\
559.79 \\
565.69 \\
571.59 \\
577.49 \\
583.40 \\
589.31 \\
595.22 \\
601.14 \\
607.05\end{array}$ & $\begin{array}{r}9.755 \\
9.783 \\
9.810 \\
9.838 \\
9.865 \\
9.893 \\
9.920 \\
9.947 \\
9.973 \\
10.000\end{array}$ & $\begin{array}{l}422.00 \\
424.00 \\
426.00 \\
428.00 \\
430.00 \\
432.00 \\
434.00 \\
436.00 \\
438.00 \\
440.00\end{array}$ & $\begin{array}{l}5.7179 \\
5.7448 \\
5.7718 \\
5.7987 \\
5.8256 \\
5.8525 \\
5.8794 \\
5.9063 \\
5.9332 \\
5.9601\end{array}$ & $\begin{array}{l}1380.71 \\
1388.19 \\
1395.67 \\
1403.13 \\
1410.58 \\
1418.03 \\
1425.46 \\
1432.89 \\
1440.30 \\
1447.71\end{array}$ & $\begin{array}{r}957.47 \\
962.95 \\
968.43 \\
973.90 \\
979.36 \\
984.81 \\
990.26 \\
995.69 \\
1001.12 \\
1006.54\end{array}$ & $\begin{array}{l}11.325 \\
11.342 \\
11.360 \\
11.377 \\
11.395 \\
11.412 \\
11.429 \\
11.446 \\
11.463 \\
11.480\end{array}$ \\
\hline $\begin{array}{l}302.00 \\
304.00 \\
306.00 \\
308.00 \\
310.00 \\
312.00 \\
314.00 \\
316.00 \\
318.00 \\
320.00\end{array}$ & $\begin{array}{l}4.0923 \\
4.1196 \\
4.1469 \\
4.1741 \\
4.2014 \\
4.2287 \\
4.2559 \\
4.2832 \\
4.3104 \\
4.3376\end{array}$ & $\begin{array}{l}915.88 \\
923.82 \\
931.76 \\
939.69 \\
947.63 \\
955.56 \\
963.49 \\
971.41 \\
979.33 \\
987.25\end{array}$ & $\begin{array}{l}612.97 \\
618.89 \\
624.80 \\
630.72 \\
636.63 \\
642.55 \\
648.46 \\
654.36 \\
660.27 \\
666.17\end{array}$ & $\begin{array}{l}10.026 \\
10.053 \\
10.079 \\
10.104 \\
10.130 \\
10.156 \\
10.181 \\
10.206 \\
10.231 \\
10.256\end{array}$ & $\begin{array}{l}442.00 \\
444.00 \\
446.00 \\
448.00 \\
450.00 \\
452.00 \\
454.00 \\
456.00 \\
458.00 \\
460.00\end{array}$ & $\begin{array}{l}5.9870 \\
6.0139 \\
6.0408 \\
6.0677 \\
6.0945 \\
6.1214 \\
6.1482 \\
6.1751 \\
6.2019 \\
6.2288\end{array}$ & $\begin{array}{l}1455.11 \\
1462.51 \\
1469.89 \\
1477.26 \\
1484.63 \\
1492.00 \\
1499.37 \\
1506.73 \\
1514.08 \\
1521.42\end{array}$ & $\begin{array}{l}1011.95 \\
1017.35 \\
1022.74 \\
1028.13 \\
1033.51 \\
1038.89 \\
1044.27 \\
1049.64 \\
1055.00 \\
1060.36\end{array}$ & $\begin{array}{l}11.497 \\
11.514 \\
11.530 \\
11.547 \\
11.563 \\
11.579 \\
11.596 \\
11.612 \\
11.628 \\
11.644\end{array}$ \\
\hline $\begin{array}{l}322.00 \\
324.00 \\
326.00 \\
328.00 \\
330.00 \\
332.00 \\
334.00 \\
336.00 \\
338.00 \\
340.00\end{array}$ & $\begin{array}{l}4.3649 \\
4.3921 \\
4.4193 \\
4.4465 \\
4.4737 \\
4.5008 \\
4.5280 \\
4.5552 \\
4.5823 \\
4.6095\end{array}$ & $\begin{array}{r}995.16 \\
1003.07 \\
1010.98 \\
1018.87 \\
1026.77 \\
1034.65 \\
1042.54 \\
1050.41 \\
1058.28 \\
1066.14\end{array}$ & $\begin{array}{l}672.07 \\
677.97 \\
683.86 \\
689.74 \\
695.62 \\
701.50 \\
707.37 \\
713.23 \\
719.09 \\
724.94\end{array}$ & $\begin{array}{l}10.281 \\
10.305 \\
10.329 \\
10.353 \\
10.377 \\
10.401 \\
10.425 \\
10.448 \\
10.472 \\
10.495\end{array}$ & $\begin{array}{l}462.00 \\
464.00 \\
466.00 \\
468.00 \\
470.00 \\
472.00 \\
474.00 \\
476.00 \\
478.00 \\
480.00\end{array}$ & $\begin{array}{l}6.2556 \\
6.2825 \\
6.3093 \\
6.3361 \\
6.3629 \\
6.3898 \\
6.4166 \\
6.4434 \\
6.4702 \\
6.4970\end{array}$ & $\begin{array}{l}1528.76 \\
1536.08 \\
1543.40 \\
1550.71 \\
1558.02 \\
1565.31 \\
1572.60 \\
1579.89 \\
1587.16 \\
1594.43\end{array}$ & $\begin{array}{l}1065.71 \\
1071.05 \\
1076.38 \\
1081.71 \\
1087.03 \\
1092.34 \\
1097.64 \\
1102.94 \\
1108.23 \\
1113.51\end{array}$ & $\begin{array}{l}11.660 \\
11.676 \\
11.691 \\
11.707 \\
11.723 \\
11.738 \\
11.754 \\
11.769 \\
11.784 \\
11.799\end{array}$ \\
\hline $\begin{array}{l}342.00 \\
344.00 \\
346.00 \\
348.00 \\
350.00 \\
352.00 \\
354.00 \\
356.00 \\
358.00 \\
360.00\end{array}$ & $\begin{array}{l}4.6366 \\
4.6638 \\
4.6909 \\
4.7180 \\
4.7452 \\
4.7723 \\
4.7994 \\
4.8265 \\
4.8536 \\
4.8807\end{array}$ & $\begin{array}{l}1073.99 \\
1081.84 \\
1089.68 \\
1097.51 \\
1105.33 \\
1113.14 \\
1120.95 \\
1128.75 \\
1136.54 \\
1144.32\end{array}$ & $\begin{array}{l}730.78 \\
736.62 \\
742.45 \\
748.27 \\
754.09 \\
759.90 \\
765.69 \\
771.49 \\
777.27 \\
783.04\end{array}$ & $\begin{array}{l}10.518 \\
10.541 \\
10.564 \\
10.586 \\
10.609 \\
10.631 \\
10.653 \\
10.675 \\
10.697 \\
10.718\end{array}$ & $\begin{array}{l}482.00 \\
484.00 \\
486.00 \\
488.00 \\
490.00 \\
492.00 \\
494.00 \\
496.00 \\
498.00 \\
500.00\end{array}$ & $\begin{array}{l}6.5238 \\
6.5506 \\
6.5774 \\
6.6042 \\
6.6310 \\
6.6579 \\
6.6847 \\
6.7115 \\
6.7383 \\
6.7 .651\end{array}$ & $\begin{array}{l}1601.69 \\
1608.95 \\
1616.20 \\
1623.44 \\
1630.68 \\
1637.91 \\
1645.14 \\
1652.36 \\
1659.58 \\
1666.79\end{array}$ & $\begin{array}{l}1118.79 \\
1124.06 \\
1129.33 \\
1134.59 \\
1139.84 \\
1145.09 \\
1150.33 \\
1155.57 \\
1160.80 \\
1166.03\end{array}$ & $\begin{array}{l}11.814 \\
11.829 \\
11.844 \\
11.859 \\
11.874 \\
11.889 \\
11.903 \\
11.918 \\
11.933 \\
11.947\end{array}$ \\
\hline $\begin{array}{l}362.00 \\
364.00 \\
366.00 \\
368.00 \\
370.00 \\
372.00 \\
374.00 \\
376.00 \\
378.00 \\
380.00\end{array}$ & $\begin{array}{l}4.9078 \\
4.9348 \\
4.9619 \\
4.9890 \\
5.0160 \\
5.0431 \\
5.0701 \\
5.0972 \\
5.1242 \\
5.1512\end{array}$ & $\begin{array}{l}1152.08 \\
1159.84 \\
1167.59 \\
1175.32 \\
1183.05 \\
1190.77 \\
1198.48 \\
1206.18 \\
1213.87 \\
1221.56\end{array}$ & $\begin{array}{l}788.80 \\
794.56 \\
800.30 \\
806.04 \\
811.76 \\
817.48 \\
823.19 \\
828.88 \\
834.57 \\
840.26\end{array}$ & $\begin{array}{l}10.740 \\
10.761 \\
10.783 \\
10.804 \\
10.825 \\
10.845 \\
10.866 \\
10.887 \\
10.907 \\
10.927\end{array}$ & $\begin{array}{l}502.00 \\
504.00 \\
506.00 \\
508.00 \\
510.00 \\
512.00 \\
514.00 \\
516.00 \\
518.00 \\
520.00\end{array}$ & $\begin{array}{l}6.7919 \\
6.8187 \\
6.8455 \\
6.8723 \\
6.8992 \\
6.9260 \\
6.9528 \\
6.9797 \\
7.0065 \\
7.0333\end{array}$ & $\begin{array}{l}1674.00 \\
1681.20 \\
1688.40 \\
1695.59 \\
1702.78 \\
1709.96 \\
1717.14 \\
1724.32 \\
1731.50 \\
1738.67\end{array}$ & $\begin{array}{l}1171.25 \\
1176.47 \\
1181.68 \\
1186.89 \\
1192.09 \\
1197.29 \\
1202.49 \\
1207.68 \\
1212.87 \\
1218.05\end{array}$ & $\begin{array}{l}11.961 \\
11.976 \\
11.990 \\
12.004 \\
12.018 \\
12.032 \\
12.046 \\
12.060 \\
12.074 \\
12.088\end{array}$ \\
\hline $\begin{array}{l}382.00 \\
384.00 \\
386.00 \\
388.00 \\
390.00 \\
392.00 \\
394.00 \\
396.00 \\
398.00 \\
400.00\end{array}$ & $\begin{array}{l}5.1783 \\
5.2053 \\
5.2323 \\
5.2593 \\
5.2863 \\
5.3133 \\
5.3403 \\
5.3673 \\
5.3943 \\
5.4213\end{array}$ & $\begin{array}{l}1229.23 \\
1236.89 \\
1244.54 \\
1252.19 \\
1259.82 \\
1267.45 \\
1275.06 \\
1282.67 \\
1290.26 \\
1297.85\end{array}$ & $\begin{array}{l}845.93 \\
851.59 \\
857.24 \\
862.89 \\
868.52 \\
874.15 \\
879.76 \\
885.37 \\
890.97 \\
896.56\end{array}$ & $\begin{array}{l}10.947 \\
10.967 \\
10.987 \\
11.007 \\
11.027 \\
11.046 \\
11.066 \\
11.085 \\
11.104 \\
11.123\end{array}$ & $\begin{array}{l}522.00 \\
524.00 \\
526.00 \\
528.00 \\
530.00 \\
532.00 \\
534.00 \\
536.00 \\
538.00 \\
540.00\end{array}$ & $\begin{array}{l}7.0602 \\
7.0870 \\
7.1138 \\
7.1407 \\
7.1675 \\
7.1944 \\
7.2212 \\
7.2481 \\
7.2749 \\
7.3018\end{array}$ & $\begin{array}{l}1745.83 \\
1752.99 \\
1760.15 \\
1767.31 \\
1774.46 \\
1781.61 \\
1788.76 \\
1795.90 \\
1803.04 \\
1810.17\end{array}$ & $\begin{array}{l}1223.23 \\
1228.41 \\
1233.58 \\
1238.75 \\
1243.91 \\
1249.07 \\
1254.23 \\
1259.39 \\
1264.54 \\
1269.68\end{array}$ & $\begin{array}{l}12.102 \\
12.115 \\
12.129 \\
12.143 \\
12.156 \\
12.170 \\
12.183 \\
12.196 \\
12.210 \\
12.223\end{array}$ \\
\hline
\end{tabular}




\begin{tabular}{|c|c|c|c|c|c|c|c|c|c|}
\hline $\begin{array}{l}\text { TEMPER- } \\
\text { ATURE } \\
\text { (R) }\end{array}$ & $\begin{array}{c}\text { SPECIFIC } \\
\text { VOLUME } \\
\text { (CU FT/LB) }\end{array}$ & $\begin{array}{l}\text { ENTHALPY } \\
(8 T U / L 8)\end{array}$ & $\begin{array}{l}\text { INTERNAL } \\
\text { ENERGY } \\
\text { (8TU/L8) }\end{array}$ & $\begin{array}{l}\text { ENTROPY } \\
(8 \mathrm{TU} / L 8-R)\end{array}$ & $\begin{array}{l}\text { TEMPER- } \\
\text { ATURE } \\
\text { (R) }\end{array}$ & $\begin{array}{l}\text { SPECIFIC } \\
\text { VOLUME } \\
\text { (CU FT/L8) }\end{array}$ & $\begin{array}{l}\text { ENTHALPY } \\
\text { ( } 8 T U / L B)\end{array}$ & $\begin{array}{l}\text { INTERNAL } \\
\text { ENERGY } \\
\text { (8TU/L8) }\end{array}$ & $\begin{array}{l}\text { ENTROPY } \\
(8 \mathrm{TU} / L 8-\mathrm{R})\end{array}$ \\
\hline
\end{tabular}

\begin{tabular}{|c|c|c|c|c|c|c|c|c|c|}
\hline & & & & & $\begin{array}{l}122.00 \\
124.00 \\
126.00 \\
128.00 \\
130.00 \\
132.00 \\
134.00 \\
136.00 \\
138.00 \\
140.00\end{array}$ & $\begin{array}{l}1.3355 \\
1.3650 \\
1.3943 \\
1.4233 \\
1.4521 \\
1.4807 \\
1.5091 \\
1.5374 \\
1.5654 \\
1.5934\end{array}$ & $\begin{array}{l}261.23 \\
267.75 \\
274.24 \\
280.71 \\
287.16 \\
293.60 \\
300.02 \\
306.45 \\
312.87 \\
319.29\end{array}$ & $\begin{array}{l}150.01 \\
154.08 \\
158.13 \\
162.19 \\
166.24 \\
170.29 \\
174.35 \\
178.43 \\
182.51 \\
186.60\end{array}$ & $\begin{array}{l}6.678 \\
6.731 \\
6.783 \\
6.834 \\
6.884 \\
6.933 \\
6.982 \\
7.029 \\
7.076 \\
7.122\end{array}$ \\
\hline 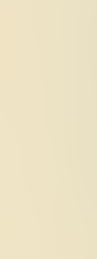 & & & & & $\begin{array}{l}142.00 \\
144.00 \\
146.00 \\
148.00 \\
150.00 \\
152.00 \\
154.00 \\
156.00 \\
158.00 \\
160.00\end{array}$ & $\begin{array}{l}1.6211 \\
1.6488 \\
1.6762 \\
1.7036 \\
1.7309 \\
1.7580 \\
1.7850 \\
1.8119 \\
1.8387 \\
1.8654\end{array}$ & $\begin{array}{l}325.71 \\
332.15 \\
338.59 \\
345.04 \\
351.51 \\
358.00 \\
364.51 \\
371.04 \\
377.59 \\
384.16\end{array}$ & $\begin{array}{l}190.72 \\
194.85 \\
199.00 \\
203.18 \\
207.38 \\
211.61 \\
215.87 \\
220.15 \\
224.47 \\
228.82\end{array}$ & $\begin{array}{l}7.168 \\
7.213 \\
7.257 \\
7.301 \\
7.344 \\
7.387 \\
7.430 \\
7.472 \\
7.514 \\
7.555\end{array}$ \\
\hline $\begin{array}{l}36.00 \\
38.00 \\
40.00\end{array}$ & $\begin{array}{r}.2149 \\
.2179 \\
.2211\end{array}$ & $\begin{array}{l}-98.23 \\
-94.02 \\
-89.58\end{array}$ & $\begin{array}{l}-116.12 \\
-112.16 \\
-107.99\end{array}$ & $\begin{array}{l}1.749 \\
1.862 \\
1.976\end{array}$ & $\begin{array}{l}162.00 \\
164.00 \\
166.00 \\
168.00 \\
170.00 \\
172.00 \\
174.00 \\
176.00 \\
178.00 \\
180.00\end{array}$ & $\begin{array}{l}1.8921 \\
1.9186 \\
1.9450 \\
1.9714 \\
1.9977 \\
2.0239 \\
2.0501 \\
2.0762 \\
2.1022 \\
2.1281\end{array}$ & $\begin{array}{l}390.76 \\
397.39 \\
404.05 \\
410.73 \\
417.45 \\
424.19 \\
430.97 \\
437.77 \\
444.61 \\
451.47\end{array}$ & $\begin{array}{l}233.20 \\
237.62 \\
242.08 \\
246.56 \\
251.09 \\
255.65 \\
260.25 \\
264.88 \\
269.55 \\
274.25\end{array}$ & $\begin{array}{l}7.596 \\
7.637 \\
7.677 \\
7.717 \\
7.757 \\
7.796 \\
7.836 \\
7.874 \\
7.913 \\
7.951\end{array}$ \\
\hline $\begin{array}{l}42.00 \\
44.00 \\
46.00 \\
48.00 \\
50.00 \\
52.00 \\
54.00 \\
56.00 \\
58.00 \\
60.00\end{array}$ & $\begin{array}{l}.2246 \\
.2285 \\
.2327 \\
.2373 \\
.2425 \\
.2483 \\
.2548 \\
.2622 \\
.2708 \\
.2820\end{array}$ & $\begin{array}{l}-84.88 \\
-79.91 \\
-74.65 \\
-69.06 \\
-63.12 \\
-56.80 \\
-50.08 \\
-42.90 \\
-35.11 \\
-26.82\end{array}$ & $\begin{array}{r}-103.59 \\
-98.94 \\
-94.02 \\
-88.83 \\
-83.32 \\
-77.48 \\
-71.29 \\
-64.73 \\
-57.66 \\
-50.30\end{array}$ & $\begin{array}{l}2.091 \\
2.206 \\
2.323 \\
2.442 \\
2.563 \\
2.687 \\
2.814 \\
2.945 \\
3.082 \\
3.223\end{array}$ & $\begin{array}{l}182.00 \\
184.00 \\
186.00 \\
188.00 \\
190.00 \\
192.00 \\
194.00 \\
196.00 \\
198.00 \\
200.00\end{array}$ & $\begin{array}{l}2.1540 \\
2.1798 \\
2.2055 \\
2.2312 \\
2.2569 \\
2.2824 \\
2.3080 \\
2.3334 \\
2.3589 \\
2.3843\end{array}$ & $\begin{array}{l}458.36 \\
465.26 \\
472.20 \\
479.17 \\
486.17 \\
493.20 \\
500.27 \\
507.37 \\
514.50 \\
521.67\end{array}$ & $\begin{array}{l}278.98 \\
283.74 \\
288.53 \\
293.36 \\
298.23 \\
303.14 \\
308.08 \\
313.06 \\
318.07 \\
323.12\end{array}$ & $\begin{array}{l}7.989 \\
8.027 \\
8.065 \\
8.102 \\
8.139 \\
8.176 \\
8.212 \\
8.249 \\
8.285 \\
8.321\end{array}$ \\
\hline $\begin{array}{l}62.00 \\
64.00 \\
66.00 \\
68.00 \\
70.00 \\
72.00 \\
74.00 \\
76.00 \\
78.00 \\
80.00\end{array}$ & $\begin{array}{l}.2940 \\
.3088 \\
.3274 \\
.3512 \\
.3822 \\
.4209 \\
.4657 \\
.5129 \\
.5600 \\
.6057\end{array}$ & $\begin{array}{r}-17.57 \\
-7.38 \\
3.95 \\
16.62 \\
30.68 \\
45.54 \\
60.37 \\
74.32 \\
87.08 \\
98.73\end{array}$ & $\begin{array}{r}-42.05 \\
-33.10 \\
-23.31 \\
-12.63 \\
-1.15 \\
10.49 \\
21.60 \\
31.61 \\
40.45 \\
48.30\end{array}$ & $\begin{array}{l}3.376 \\
3.538 \\
3.712 \\
3.901 \\
4.104 \\
4.314 \\
4.517 \\
4.703 \\
4.869 \\
5.016\end{array}$ & $\begin{array}{l}202.00 \\
204.00 \\
206.00 \\
208.00 \\
210.00 \\
212.00 \\
214.00 \\
216.00 \\
218.00 \\
220.00\end{array}$ & $\begin{array}{l}2.4096 \\
2.4349 \\
2.4602 \\
2.4854 \\
2.5106 \\
2.5358 \\
2.5609 \\
2.5860 \\
2.6111 \\
2.6361\end{array}$ & $\begin{array}{l}528.87 \\
536.09 \\
543.35 \\
550.65 \\
557.97 \\
565.32 \\
572.70 \\
580.11 \\
587.54 \\
595.00\end{array}$ & $\begin{array}{l}328.21 \\
333.33 \\
338.48 \\
343.67 \\
348.90 \\
354.15 \\
359.44 \\
364.76 \\
370.11 \\
375.48\end{array}$ & $\begin{array}{l}8.357 \\
8.392 \\
8.428 \\
8.463 \\
8.498 \\
8.533 \\
8.568 \\
8.602 \\
8.636 \\
8.670\end{array}$ \\
\hline $\begin{array}{r}82.00 \\
84.00 \\
86.00 \\
88.00 \\
90.00 \\
92.00 \\
94.00 \\
96.00 \\
98.00 \\
100.00\end{array}$ & $\begin{array}{l}.6497 \\
.6920 \\
.7329 \\
.7725 \\
.8109 \\
.8482 \\
.8847 \\
.9203 \\
.9552 \\
.9895\end{array}$ & $\begin{array}{l}109.48 \\
119.50 \\
128.95 \\
137.94 \\
146.55 \\
154.85 \\
162.89 \\
170.70 \\
178.33 \\
185.78\end{array}$ & $\begin{array}{r}55.38 \\
61.88 \\
67.92 \\
73.62 \\
79.03 \\
84.22 \\
89.22 \\
94.07 \\
98.78 \\
103.38\end{array}$ & $\begin{array}{l}5.149 \\
5.270 \\
5.381 \\
5.484 \\
5.581 \\
5.672 \\
5.759 \\
5.841 \\
5.920 \\
5.995\end{array}$ & $\begin{array}{l}222.00 \\
224.00 \\
226.00 \\
228.00 \\
230.00 \\
232.00 \\
234.00 \\
236.00 \\
238.00 \\
240.00\end{array}$ & $\begin{array}{l}2.6612 \\
2.6861 \\
2.7111 \\
2.7361 \\
2.7610 \\
2.7859 \\
2.8107 \\
2.8356 \\
2.8604 \\
2.8852\end{array}$ & $\begin{array}{l}602.49 \\
610.01 \\
617.55 \\
625.11 \\
632.70 \\
640.31 \\
647.95 \\
655.60 \\
663.28 \\
670.98\end{array}$ & $\begin{array}{l}380.89 \\
386.32 \\
391.78 \\
397.27 \\
402.78 \\
408.32 \\
413.89 \\
419.47 \\
425.09 \\
430.72\end{array}$ & $\begin{array}{l}8.704 \\
8.738 \\
8.771 \\
8.805 \\
8.838 \\
8.871 \\
8.904 \\
8.936 \\
8.969 \\
9.001\end{array}$ \\
\hline $\begin{array}{l}102.00 \\
104.00 \\
106.00 \\
108.00 \\
110.00 \\
112.00 \\
114.00 \\
116.00 \\
118.00 \\
120.00\end{array}$ & $\begin{array}{l}1.0231 \\
1.0562 \\
1.0888 \\
1.1209 \\
1.1526 \\
1.1839 \\
1.2149 \\
1.2455 \\
1.2758 \\
1.3058\end{array}$ & $\begin{array}{l}193.09 \\
200.27 \\
207.35 \\
214.32 \\
221.21 \\
228.02 \\
234.76 \\
241.45 \\
248.09 \\
254.68\end{array}$ & $\begin{array}{l}107.89 \\
112.32 \\
116.68 \\
120.98 \\
125.23 \\
129.43 \\
133.60 \\
137.73 \\
141.85 \\
145.94\end{array}$ & $\begin{array}{l}6.067 \\
6.137 \\
6.204 \\
6.270 \\
6.333 \\
6.394 \\
6.454 \\
6.512 \\
6.569 \\
6.624\end{array}$ & $\begin{array}{l}242.00 \\
244.00 \\
246.00 \\
248.00 \\
250.00 \\
252.00 \\
254.00 \\
256.00 \\
258.00 \\
260.00\end{array}$ & $\begin{array}{l}2.9100 \\
2.9348 \\
2.9595 \\
2.9843 \\
3.0090 \\
3.0337 \\
3.0584 \\
3.0831 \\
3.1077 \\
3.1324\end{array}$ & $\begin{array}{l}678.70 \\
686.44 \\
694.20 \\
701.98 \\
709.77 \\
717.58 \\
725.41 \\
733.25 \\
741.10 \\
748.97\end{array}$ & $\begin{array}{l}436.38 \\
442.05 \\
447.75 \\
453.47 \\
459.20 \\
464.95 \\
470.72 \\
476.51 \\
482.31 \\
488.13\end{array}$ & $\begin{array}{l}9.033 \\
9.065 \\
9.096 \\
9.128 \\
9.159 \\
9.190 \\
9.221 \\
9.252 \\
9.283 \\
9.313\end{array}$ \\
\hline
\end{tabular}


TEMPER- SPECIFIC ENTHALPY INTERNAL ENTROPY ATURE VOLUME (BTU/L8) ENERGY (BTU/L8-R) (R) (CU FT/LB)
TEMPER- SPECIFIC ENTHALPY ATURE VOLUME (BTU/L8) (8TU/L8)
(R) (CU FT/LB)
INTERNAL ENTROPY

ENERGY (BTU/L8-R) ( $8 \mathrm{TU} / \mathrm{LB}$ )

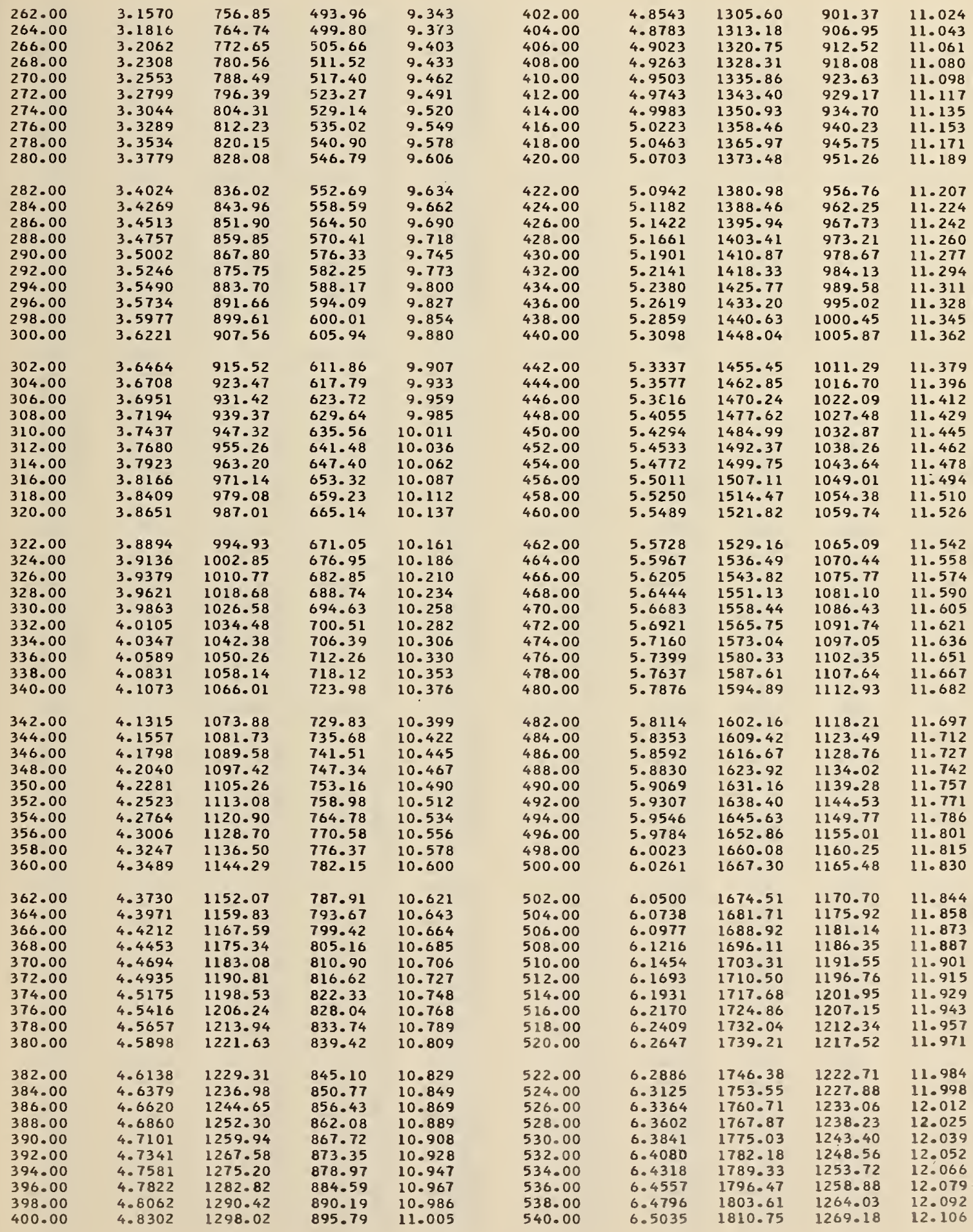


$\begin{array}{ccccc}\text { TEMPER- } & \text { SPECIFIC } & \text { ENTHALPY } & \text { INTERNAL } & \text { ENTROPY } \\ \text { ATURE } & \text { VOLUME } & \text { (8TU/L8) } & \text { ENERGY } & \text { (8TU/L8-R) }\end{array}$ (R) (CU FT/LB) (BTU/LB)
TEMPER ATURE

(R)
SPECIFIC VOLUME ( $C U$ FT/LB)
ENTHALPY )
INTERNAL ENTROPY ENERGY (BTU/L8-R) (8TU/L8)

\begin{tabular}{|c|c|c|c|c|c|c|c|c|c|}
\hline & & & & & $\begin{array}{l}122.00 \\
124.00 \\
126.00 \\
128.00 \\
130.00 \\
132.00 \\
134.00 \\
136.00 \\
138.00 \\
140.00\end{array}$ & $\begin{array}{l}1.1955 \\
1.2225 \\
1.2492 \\
1.2757 \\
1.3021 \\
1.3282 \\
1.3541 \\
1.3799 \\
1.4055 \\
1.4310\end{array}$ & $\begin{array}{l}256.93 \\
263.59 \\
270.22 \\
276.81 \\
283.38 \\
289.93 \\
296.47 \\
303.00 \\
309.52 \\
316.04\end{array}$ & $\begin{array}{l}146.32 \\
150.48 \\
154.63 \\
158.77 \\
162.91 \\
167.04 \\
171.18 \\
175.32 \\
179.48 \\
183.64\end{array}$ & $\begin{array}{l}6.547 \\
6.601 \\
6.654 \\
6.706 \\
6.757 \\
6.807 \\
6.856 \\
6.905 \\
6.952 \\
6.999\end{array}$ \\
\hline & & & & & $\begin{array}{l}142.00 \\
144.00 \\
146.00 \\
148.00 \\
150.00 \\
152.00 \\
154.00 \\
156.00 \\
158.00 \\
160.00\end{array}$ & $\begin{array}{l}1.4563 \\
1.4815 \\
1.5065 \\
1.5314 \\
1.5562 \\
1.5809 \\
1.6055 \\
1.6300 \\
1.6544 \\
1.6787\end{array}$ & $\begin{array}{l}322.56 \\
329.08 \\
335.61 \\
342.15 \\
348.70 \\
355.27 \\
361.85 \\
368.45 \\
375.07 \\
381.72\end{array}$ & $\begin{array}{l}187.82 \\
192.01 \\
196.22 \\
200.46 \\
204.71 \\
208.99 \\
213.30 \\
217.64 \\
222.00 \\
226.40\end{array}$ & $\begin{array}{l}7.045 \\
7.091 \\
7.136 \\
7.181 \\
7.225 \\
7.268 \\
7.311 \\
7.354 \\
7.396 \\
7.438\end{array}$ \\
\hline & 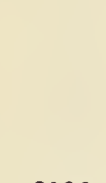 & 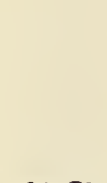 & 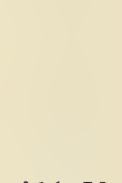 & 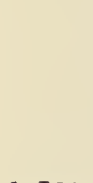 & $\begin{array}{l}162.00 \\
164.00 \\
166.00 \\
168.00 \\
170.00 \\
172.00 \\
174.00\end{array}$ & $\begin{array}{l}1.7028 \\
1.7270 \\
1.7510 \\
1.7749 \\
1.7988 \\
1.8226 \\
1.8464\end{array}$ & $\begin{array}{l}388.38 \\
395.07 \\
401.79 \\
408.54 \\
415.31 \\
422.11 \\
428.94\end{array}$ & $\begin{array}{l}230.82 \\
235.28 \\
239.78 \\
244.31 \\
248.87 \\
253.47 \\
258.10\end{array}$ & $\begin{array}{l}7.479 \\
7.520 \\
7.561 \\
7.601 \\
7.641 \\
7.681 \\
7.720\end{array}$ \\
\hline $\begin{array}{l}36.00 \\
38.00 \\
40.00\end{array}$ & $\begin{array}{r}.2139 \\
.2168 \\
.2199\end{array}$ & $\begin{array}{l}-96.71 \\
-92.55 \\
-88.15\end{array}$ & $\begin{array}{l}-116.50 \\
-112.61 \\
-108.50\end{array}$ & $\begin{array}{l}1.736 \\
1.848 \\
1.961\end{array}$ & $\begin{array}{l}176.00 \\
178.00 \\
180.00\end{array}$ & $\begin{array}{l}1.8700 \\
1.8936 \\
1.9172\end{array}$ & $\begin{array}{l}435.80 \\
442.68 \\
449.60\end{array}$ & $\begin{array}{l}262.77 \\
267.47 \\
272.21\end{array}$ & $\begin{array}{l}7.760 \\
7.799 \\
7.837\end{array}$ \\
\hline $\begin{array}{l}42.00 \\
44.00 \\
46.00 \\
48.00 \\
50.00 \\
52.00 \\
54.00 \\
56.00 \\
58.00 \\
60.00\end{array}$ & $\begin{array}{l}.2233 \\
.2270 \\
.2310 \\
.2354 \\
.2403 \\
.2457 \\
.2517 \\
.2585 \\
.2663 \\
.2762\end{array}$ & $\begin{array}{l}-83.51 \\
-78.61 \\
-73.42 \\
-67.93 \\
-62.11 \\
-55.93 \\
-49.39 \\
-42.45 \\
-34.97 \\
-27.09\end{array}$ & $\begin{array}{r}-104.17 \\
-99.61 \\
-94.80 \\
-89.72 \\
-84.35 \\
-78.67 \\
-72.69 \\
-66.37 \\
-59.61 \\
-52.65\end{array}$ & $\begin{array}{l}2.074 \\
2.188 \\
2.303 \\
2.420 \\
2.539 \\
2.660 \\
2.783 \\
2.910 \\
3.041 \\
3.176\end{array}$ & $\begin{array}{l}182.00 \\
184.00 \\
186.00 \\
188.00 \\
190.00 \\
192.00 \\
194.00 \\
196.00 \\
198.00 \\
200.00\end{array}$ & $\begin{array}{l}1.9407 \\
1.9640 \\
1.9874 \\
2.0107 \\
2.0339 \\
2.0571 \\
2.0802 \\
2.1033 \\
2.1263 \\
2.1493\end{array}$ & $\begin{array}{l}456.54 \\
463.49 \\
470.47 \\
477.48 \\
484.52 \\
491.60 \\
498.70 \\
505.84 \\
513.01 \\
520.21\end{array}$ & $\begin{array}{l}276.97 \\
281.76 \\
286.58 \\
291.44 \\
296.33 \\
301.26 \\
306.23 \\
311.23 \\
316.26 \\
321.34\end{array}$ & $\begin{array}{l}7.876 \\
7.914 \\
7.951 \\
7.989 \\
8.026 \\
8.063 \\
8.100 \\
8.136 \\
8.173 \\
8.209\end{array}$ \\
\hline $\begin{array}{l}62.00 \\
64.00 \\
66.00 \\
68.00 \\
70.00 \\
72.00 \\
74.00 \\
76.00 \\
78.00 \\
80.00\end{array}$ & $\begin{array}{l}.2866 \\
.2990 \\
.3139 \\
.3321 \\
.3546 \\
.3820 \\
.4146 \\
.4511 \\
.4897 \\
.5289\end{array}$ & $\begin{array}{r}-18.39 \\
-8.98 \\
1.28 \\
12.45 \\
24.57 \\
37.36 \\
50.58 \\
63.77 \\
76.46 \\
88.41\end{array}$ & $\begin{array}{r}-44.91 \\
-36.64 \\
-27.76 \\
-18.28 \\
-8.24 \\
2.01 \\
12.23 \\
22.04 \\
31.15 \\
39.48\end{array}$ & $\begin{array}{l}3.319 \\
3.469 \\
3.627 \\
3.793 \\
3.969 \\
4.149 \\
4.330 \\
4.506 \\
4.671 \\
4.822\end{array}$ & $\begin{array}{l}202.00 \\
204.00 \\
206.00 \\
208.00 \\
210.00 \\
212.00 \\
214.00 \\
216.00 \\
218.00 \\
220.00\end{array}$ & $\begin{array}{l}2.1723 \\
2.1952 \\
2.2181 \\
2.2409 \\
2.2637 \\
2.2865 \\
2.3092 \\
2.3320 \\
2.3546 \\
2.3773\end{array}$ & $\begin{array}{l}527.44 \\
534.70 \\
541.99 \\
549.31 \\
556.66 \\
564.04 \\
571.45 \\
578.89 \\
586.35 \\
593.84\end{array}$ & $\begin{array}{l}326.44 \\
331.58 \\
336.76 \\
341.96 \\
347.21 \\
352.48 \\
357.78 \\
363.12 \\
368.48 \\
373.87\end{array}$ & $\begin{array}{l}8.245 \\
8.281 \\
8.316 \\
8.352 \\
8.387 \\
8.422 \\
8.457 \\
8.491 \\
8.526 \\
8.560\end{array}$ \\
\hline $\begin{array}{r}82.00 \\
84.00 \\
86.00 \\
88.00 \\
90.00 \\
92.00 \\
94.00 \\
96.00 \\
98.00 \\
100.00\end{array}$ & $\begin{array}{l}.5677 \\
.6057 \\
.6427 \\
.6788 \\
.7139 \\
.7482 \\
.7816 \\
.8144 \\
.8465 \\
.8780\end{array}$ & $\begin{array}{r}99.60 \\
110.11 \\
120.03 \\
129.47 \\
138.49 \\
147.17 \\
155.56 \\
163.70 \\
171.62 \\
179.35\end{array}$ & $\begin{array}{l}47.08 \\
54.07 \\
60.57 \\
66.66 \\
72.44 \\
77.95 \\
83.24 \\
88.34 \\
93.29 \\
98.12\end{array}$ & $\begin{array}{l}4.960 \\
5.087 \\
5.203 \\
5.312 \\
5.413 \\
5.509 \\
5.599 \\
5.685 \\
5.766 \\
5.844\end{array}$ & $\begin{array}{l}222.00 \\
224.00 \\
226.00 \\
228.00 \\
230.00 \\
232.00 \\
234.00 \\
236.00 \\
238.00 \\
240.00\end{array}$ & $\begin{array}{l}2.3999 \\
2.4225 \\
2.4451 \\
2.4677 \\
2.4902 \\
2.5127 \\
2.5352 \\
2.5577 \\
2.5801 \\
2.6025\end{array}$ & $\begin{array}{l}601.35 \\
608.89 \\
616.45 \\
624.04 \\
631.66 \\
639.29 \\
646.95 \\
654.63 \\
662.34 \\
670.06\end{array}$ & $\begin{array}{l}379.29 \\
384.74 \\
390.22 \\
395.72 \\
401.25 \\
406.80 \\
412.38 \\
417.98 \\
423.61 \\
429.26\end{array}$ & $\begin{array}{l}8.594 \\
8.628 \\
8.661 \\
8.695 \\
8.728 \\
8.761 \\
8.794 \\
8.827 \\
8.859 \\
8.891\end{array}$ \\
\hline $\begin{array}{l}102.00 \\
104.00 \\
106.00 \\
108.00 \\
110.00 \\
112.00 \\
114.00 \\
116.00 \\
118.00 \\
120.00\end{array}$ & $\begin{array}{r}.9089 \\
.9393 \\
.9692 \\
.9988 \\
1.0279 \\
1.0566 \\
1.0850 \\
1.1130 \\
1.1408 \\
1.1683\end{array}$ & $\begin{array}{l}186.92 \\
194.35 \\
201.64 \\
208.83 \\
215.92 \\
222.92 \\
229.84 \\
236.70 \\
243.50 \\
250.24\end{array}$ & $\begin{array}{l}102.82 \\
107.44 \\
111.96 \\
116.42 \\
120.82 \\
125.16 \\
129.46 \\
133.71 \\
137.94 \\
142.14\end{array}$ & $\begin{array}{l}5.919 \\
5.991 \\
6.061 \\
6.128 \\
6.193 \\
6.256 \\
6.317 \\
6.377 \\
6.435 \\
6.492\end{array}$ & $\begin{array}{l}242.00 \\
244.00 \\
246.00 \\
248.00 \\
250.00 \\
252.00 \\
254.00 \\
256.00 \\
258.00 \\
260.00\end{array}$ & $\begin{array}{l}2.6249 \\
2.6473 \\
2.6697 \\
2.6921 \\
2.7144 \\
2.7367 \\
2.7590 \\
2.7813 \\
2.8036 \\
2.8258\end{array}$ & $\begin{array}{l}677.80 \\
685.56 \\
693.34 \\
701.14 \\
708.96 \\
716.79 \\
724.63 \\
732.50 \\
740.37 \\
748.26\end{array}$ & $\begin{array}{l}434.92 \\
440.61 \\
446.33 \\
452.05 \\
457.80 \\
463.57 \\
469.35 \\
475.15 \\
480.96 \\
486.79\end{array}$ & $\begin{array}{l}8.923 \\
8.955 \\
8.987 \\
9.019 \\
9.050 \\
9.081 \\
9.112 \\
9.143 \\
9.174 \\
9.204\end{array}$ \\
\hline
\end{tabular}


TEMPER- SPECIFIC ENTHALPY INTERNAL ENTROPY

ATURE VOLUME (8TU/L8) ENERGY (8TU/LB-R) (R) $(C U F T / L B)$
TEMPER(8TU/L8)
ATURE VOLUME (BTU/LB) VOLUME
INTERNAL ENTROPY

ENERGY (BTU/LB-R) (8TU/L8)

\begin{tabular}{|c|c|c|c|c|c|c|c|c|c|}
\hline $\begin{array}{l}262.00 \\
264.00 \\
266.00 \\
268.00 \\
270.00 \\
272.00 \\
274.00 \\
276.00 \\
278.00 \\
280.00\end{array}$ & $\begin{array}{l}2.8481 \\
2.8703 \\
2.8925 \\
2.9147 \\
2.9369 \\
2.9591 \\
2.9812 \\
3.0034 \\
3.0255 \\
3.0476\end{array}$ & $\begin{array}{l}756.16 \\
764.07 \\
771.99 \\
779.93 \\
787.87 \\
795.80 \\
803.73 \\
811.67 \\
819.61 \\
827.56\end{array}$ & $\begin{array}{l}492.64 \\
498.49 \\
504.36 \\
510.24 \\
516.13 \\
522.00 \\
527.89 \\
533.78 \\
539.67 \\
545.57\end{array}$ & $\begin{array}{l}9.235 \\
9.265 \\
9.295 \\
9.324 \\
9.354 \\
9.383 \\
9.412 \\
9.441 \\
9.470 \\
9.498\end{array}$ & $\begin{array}{l}402.00 \\
404.00 \\
406.00 \\
408.00 \\
410.00 \\
412.00 \\
414.00 \\
416.00 \\
418.00 \\
420.00\end{array}$ & $\begin{array}{l}4.3792 \\
4.4008 \\
4.4225 \\
4.4441 \\
4.4657 \\
4.4874 \\
4.5090 \\
4.5306 \\
4.5522 \\
4.5738\end{array}$ & $\begin{array}{l}1.305 .78 \\
1313.37 \\
1320.95 \\
1328.52 \\
1336.07 \\
1343.62 \\
1351.16 \\
1358.70 \\
1366.22 \\
1373.74\end{array}$ & $\begin{array}{l}900.59 \\
906.18 \\
911.75 \\
917.32 \\
922.88 \\
928.42 \\
933.96 \\
939.49 \\
945.02 \\
950.53\end{array}$ & $\begin{array}{l}10.918 \\
10.937 \\
10.955 \\
10.974 \\
10.992 \\
11.011 \\
11.029 \\
11.047 \\
11.065 \\
11.083\end{array}$ \\
\hline $\begin{array}{l}282.00 \\
284.00 \\
286.00 \\
288.00 \\
290.00 \\
292.00 \\
294.00 \\
296.00 \\
298.00 \\
300.00\end{array}$ & $\begin{array}{l}3.0697 \\
3.0918 \\
3.1139 \\
3.1359 \\
3.1580 \\
3.1800 \\
3.2020 \\
3.2240 \\
3.2460 \\
3.2680\end{array}$ & $\begin{array}{l}835.51 \\
843.47 \\
851.43 \\
859.39 \\
867.36 \\
875.32 \\
883.29 \\
891.26 \\
899.23 \\
907.20\end{array}$ & $\begin{array}{l}551.48 \\
557.40 \\
563.31 \\
569.24 \\
575.16 \\
581.09 \\
587.02 \\
592.95 \\
598.88 \\
604.82\end{array}$ & $\begin{array}{l}9.526 \\
9.555 \\
9.582 \\
9.610 \\
9.638 \\
9.665 \\
9.692 \\
9.719 \\
9.746 \\
9.773\end{array}$ & $\begin{array}{l}422.00 \\
424.00 \\
426.00 \\
428.00 \\
430.00 \\
432.00 \\
434.00 \\
436.00 \\
438.00 \\
440.00\end{array}$ & $\begin{array}{l}4.5954 \\
4.6170 \\
4.6386 \\
4.6602 \\
4.6818 \\
4.7034 \\
4.7250 \\
4.7466 \\
4.7681 \\
4.7897\end{array}$ & $\begin{array}{l}1381.24 \\
1388.74 \\
1396.22 \\
1403.70 \\
1411.17 \\
1418.63 \\
1426.08 \\
1433.52 \\
1440.95 \\
1448.37\end{array}$ & $\begin{array}{r}956.04 \\
961.54 \\
967.03 \\
972.51 \\
977.98 \\
983.44 \\
988.89 \\
994.34 \\
999.77 \\
1005.20\end{array}$ & $\begin{array}{l}11.101 \\
11.119 \\
11.136 \\
11.154 \\
11.171 \\
11.189 \\
11.206 \\
11.223 \\
11.240 \\
11.257\end{array}$ \\
\hline $\begin{array}{l}302.00 \\
304.00 \\
306.00 \\
308.00 \\
310.00 \\
312.00 \\
314.00 \\
316.00 \\
318.00 \\
320.00\end{array}$ & $\begin{array}{l}3.2900 \\
3.3120 \\
3.3339 \\
3.3559 \\
3.3778 \\
3.3997 \\
3.4216 \\
3.4435 \\
3.4654 \\
3.4873\end{array}$ & $\begin{array}{l}915.16 \\
923.13 \\
931.10 \\
939.06 \\
947.02 \\
954.98 \\
962.93 \\
970.88 \\
978.83 \\
986.77\end{array}$ & $\begin{array}{l}610.75 \\
616.69 \\
622.62 \\
628.55 \\
634.48 \\
640.41 \\
646.34 \\
652.26 \\
658.18 \\
664.10\end{array}$ & $\begin{array}{r}9.799 \\
9.826 \\
9.852 \\
9.878 \\
9.903 \\
9.929 \\
9.954 \\
9.980 \\
10.005 \\
10.030\end{array}$ & $\begin{array}{l}442.00 \\
444.00 \\
446.00 \\
448.00 \\
450.00 \\
452.00 \\
454.00 \\
456.00 \\
458.00 \\
460.00\end{array}$ & $\begin{array}{l}4.8113 \\
4.8328 \\
4.8544 \\
4.8759 \\
4.8975 \\
4.9190 \\
4.9405 \\
4.9621 \\
4.9836 \\
5.0051\end{array}$ & $\begin{array}{l}1455.79 \\
1463.19 \\
1470.39 \\
1477.98 \\
1485.36 \\
1492.75 \\
1500.13 \\
1507.50 \\
1514.86 \\
1522.22\end{array}$ & $\begin{array}{l}1010.62 \\
1016.03 \\
1021.43 \\
1026.83 \\
1032.22 \\
1037.61 \\
1043.00 \\
1048.38 \\
1053.75 \\
1059.11\end{array}$ & $\begin{array}{l}11.274 \\
11.290 \\
11.307 \\
11.324 \\
11.340 \\
11.356 \\
11.373 \\
11.389 \\
11.405 \\
11.421\end{array}$ \\
\hline $\begin{array}{l}322.00 \\
324.00 \\
326.00 \\
328.00 \\
330.00 \\
332.00 \\
334.00 \\
336.00 \\
338.00 \\
340.00\end{array}$ & $\begin{array}{l}3.5092 \\
3.5311 \\
3.5529 \\
3.5748 \\
3.5966 \\
3.6185 \\
3.6403 \\
3.6621 \\
3.6839 \\
3.7058\end{array}$ & $\begin{array}{r}994.71 \\
1002.64 \\
1010.57 \\
1018.49 \\
1026.40 \\
1034.31 \\
1042.22 \\
1050.11 \\
1058.00 \\
1065.88\end{array}$ & $\begin{array}{l}670.01 \\
675.92 \\
681.83 \\
687.73 \\
693.62 \\
699.51 \\
705.39 \\
711.27 \\
717.14 \\
723.00\end{array}$ & $\begin{array}{l}10.054 \\
10.079 \\
10.103 \\
10.127 \\
10.152 \\
10.175 \\
10.199 \\
10.223 \\
10.246 \\
10.269\end{array}$ & $\begin{array}{l}462.00 \\
464.00 \\
466.00 \\
468.00 \\
470.00 \\
472.00 \\
474.00 \\
476.00 \\
478.00 \\
480.00\end{array}$ & $\begin{array}{l}5.0266 \\
5.0481 \\
5.0697 \\
5.0912 \\
5.1127 \\
5.1342 \\
5.1557 \\
5.1772 \\
5.1987 \\
5.2202\end{array}$ & $\begin{array}{l}1529.56 \\
1536.90 \\
1544.23 \\
1551.56 \\
1558.87 \\
1566.18 \\
1573.48 \\
1580.78 \\
1588.06 \\
1595.34\end{array}$ & $\begin{array}{l}1064.47 \\
1069.81 \\
1075.16 \\
1080.49 \\
1085.81 \\
1091.13 \\
1096.45 \\
1101.75 \\
1107.05 \\
1112.34\end{array}$ & $\begin{array}{l}11.437 \\
11.453 \\
11.469 \\
11.484 \\
11.500 \\
11.515 \\
11.531 \\
11.546 \\
11.561 \\
11.577\end{array}$ \\
\hline $\begin{array}{l}342.00 \\
344.00 \\
346.00 \\
348.00 \\
350.00 \\
352.00 \\
354.00 \\
356.00 \\
358.00 \\
360.00\end{array}$ & $\begin{array}{l}3.7276 \\
3.7494 \\
3.7712 \\
3.7930 \\
3.8147 \\
3.8365 \\
3.8583 \\
3.8801 \\
3.9018 \\
3.9236\end{array}$ & $\begin{array}{l}1073.76 \\
1081.63 \\
1089.49 \\
1097.34 \\
1105.18 \\
1113.01 \\
1120.84 \\
1128.66 \\
1136.47 \\
1144.26\end{array}$ & $\begin{array}{l}728.86 \\
734.71 \\
740.55 \\
746.39 \\
752.22 \\
758.04 \\
763.85 \\
769.65 \\
775.44 \\
781.23\end{array}$ & $\begin{array}{l}10.292 \\
10.315 \\
10.338 \\
10.361 \\
10.383 \\
10.406 \\
10.428 \\
10.450 \\
10.472 \\
10.493\end{array}$ & $\begin{array}{l}482.00 \\
484.00 \\
486.00 \\
488.00 \\
490.00 \\
492.00 \\
494.00 \\
496.00 \\
498.00 \\
500.00\end{array}$ & $\begin{array}{l}5.2417 \\
5.2632 \\
5.2847 \\
5.3062 \\
5.3276 \\
5.3491 \\
5.3706 \\
5.3921 \\
5.4136 \\
5.4351\end{array}$ & $\begin{array}{l}1602.62 \\
1609.88 \\
1617.14 \\
1624.40 \\
1631.65 \\
1638.89 \\
1646.13 \\
1653.36 \\
1660.58 \\
1667.80\end{array}$ & $\begin{array}{l}1117.62 \\
1122.90 \\
1128.17 \\
1133.44 \\
1138.70 \\
1143.95 \\
1149.20 \\
1154.45 \\
1159.68 \\
1164.92\end{array}$ & $\begin{array}{l}11.592 \\
11.607 \\
11.622 \\
11.637 \\
11.651 \\
11.666 \\
11.681 \\
11.696 \\
11.710 \\
11.725\end{array}$ \\
\hline $\begin{array}{l}362.00 \\
364.00 \\
366.00 \\
368.00 \\
370.00 \\
372.00 \\
374.00 \\
376.00 \\
378.00 \\
380.00\end{array}$ & $\begin{array}{l}3.9453 \\
3.9671 \\
3.9888 \\
4.0105 \\
4.0322 \\
4.0540 \\
4.0757 \\
4.0974 \\
4.1191 \\
4.1408\end{array}$ & $\begin{array}{l}1152.05 \\
1159.83 \\
1167.60 \\
1175.35 \\
1183.10 \\
1190.84 \\
1198.57 \\
1206.29 \\
1214.00 \\
1221.70\end{array}$ & $\begin{array}{l}787.00 \\
792.77 \\
798.53 \\
804.27 \\
810.01 \\
815.74 \\
821.46 \\
827.18 \\
832.88 \\
838.57\end{array}$ & $\begin{array}{l}10.515 \\
10.536 \\
10.558 \\
10.579 \\
10.600 \\
10.621 \\
10.641 \\
10.662 \\
10.682 \\
10.703\end{array}$ & $\begin{array}{l}502.00 \\
504.00 \\
506.00 \\
508.00 \\
510.00 \\
512.00 \\
514.00 \\
516.00 \\
518.00 \\
520.00\end{array}$ & $\begin{array}{l}5.4566 \\
5.4781 \\
5.4996 \\
5.5210 \\
5.5425 \\
5.5640 \\
5.5855 \\
5.6070 \\
5.6285 \\
5.6500\end{array}$ & $\begin{array}{l}1675.02 \\
1682.23 \\
1689.44 \\
1696.64 \\
1703.84 \\
1711.03 \\
1718.22 \\
1725.40 \\
1732.58 \\
1739.76\end{array}$ & $\begin{array}{l}1170.14 \\
1175.37 \\
1180.58 \\
1185.80 \\
1191.01 \\
1196.21 \\
1201.41 \\
1206.61 \\
1211.80 \\
1216.99\end{array}$ & $\begin{array}{l}11.739 \\
11.753 \\
11.768 \\
11.782 \\
11.796 \\
11.810 \\
11.824 \\
11.838 \\
11.852 \\
11.866\end{array}$ \\
\hline $\begin{array}{l}382.00 \\
384.00 \\
386.00 \\
388.00 \\
390.00 \\
392.00 \\
394.00 \\
396.00 \\
398.00 \\
400.00\end{array}$ & $\begin{array}{l}4.1625 \\
4.1842 \\
4.2058 \\
4.2275 \\
4.2492 \\
4.2709 \\
4.2925 \\
4.3142 \\
4.3359 \\
4.3575\end{array}$ & $\begin{array}{l}1229.40 \\
1237.08 \\
1244.75 \\
1252.41 \\
1260.07 \\
1267.71 \\
1275.34 \\
1282.97 \\
1290.58 \\
1298.19\end{array}$ & $\begin{array}{l}844.26 \\
849.93 \\
855.60 \\
861.25 \\
866.90 \\
872.54 \\
878.17 \\
883.79 \\
889.40 \\
895.00\end{array}$ & $\begin{array}{l}10.723 \\
10.743 \\
10.763 \\
10.783 \\
10.802 \\
10.822 \\
10.841 \\
10.861 \\
10.880 \\
10.899\end{array}$ & $\begin{array}{l}522.00 \\
524.00 \\
526.00 \\
528.00 \\
530.00 \\
532.00 \\
534.00 \\
536.00 \\
538.00 \\
540.00\end{array}$ & $\begin{array}{l}5.6715 \\
5.6930 \\
5.7145 \\
5.7360 \\
5.7575 \\
5.7790 \\
5.8005 \\
5.8219 \\
5.8434 \\
5.8649\end{array}$ & $\begin{array}{l}1746.93 \\
1754.10 \\
1761.27 \\
1768.43 \\
1775.59 \\
1782.75 \\
1789.90 \\
1797.05 \\
1804.19 \\
1811.33\end{array}$ & $\begin{array}{l}1222.17 \\
1227.35 \\
1232.53 \\
1237.70 \\
1242.87 \\
1248.04 \\
1253.20 \\
1258.36 \\
1263.52 \\
1268.67\end{array}$ & $\begin{array}{l}11.879 \\
11.893 \\
11.907 \\
11.920 \\
11.934 \\
11.947 \\
11.961 \\
11.974 \\
11.987 \\
12.001\end{array}$ \\
\hline
\end{tabular}


$\begin{array}{ccccc}\text { TEMPER- } & \text { SPECIFIC } & \text { ENTHALPY } & \text { INTERNAL } & \text { ENTROPY } \\ \text { ATURE } & \text { VOLUME } & (8 T U / L 8) & \text { ENERGY } & (B T U / L 8-R)\end{array}$ (R) (CU FT/LB)
(8TU/L8)
TEMPER- SPECIFIC ENTHALPY ATURE (R)
VOLUME (CU FT/L8)
INTERNAL ENTROPY (BTU/L8)

\begin{tabular}{|c|c|c|c|c|c|c|c|c|c|}
\hline & & & & & $\begin{array}{l}122.00 \\
124.00 \\
126.00 \\
128.00 \\
130.00 \\
132.00 \\
134.00 \\
136.00 \\
138.00 \\
140.00\end{array}$ & $\begin{array}{r}.9881 \\
1.0112 \\
1.0341 \\
1.0568 \\
1.0793 \\
1.1016 \\
1.1237 \\
1.1457 \\
1.1676 \\
1.1893\end{array}$ & $\begin{array}{l}248.75 \\
255.66 \\
262.53 \\
269.37 \\
276.17 \\
282.94 \\
289.68 \\
296.41 \\
303.13 \\
309.83\end{array}$ & $\begin{array}{l}139.04 \\
143.39 \\
147.72 \\
152.03 \\
156.33 \\
160.63 \\
164.92 \\
169.20 \\
173.49 \\
177.79\end{array}$ & $\begin{array}{l}6.315 \\
6.372 \\
6.427 \\
6.480 \\
6.533 \\
6.585 \\
6.636 \\
6.685 \\
6.734 \\
6.783\end{array}$ \\
\hline & & & & & $\begin{array}{l}142.00 \\
144.00 \\
146.00 \\
148.00 \\
150.00 \\
152.00 \\
154.00 \\
156.00 \\
158.00 \\
160.00\end{array}$ & $\begin{array}{l}1.2108 \\
1.2323 \\
1.2536 \\
1.2748 \\
1.2959 \\
1.3168 \\
1.3377 \\
1.3585 \\
1.3792 \\
1.3998\end{array}$ & $\begin{array}{l}316.53 \\
323.22 \\
329.92 \\
336.62 \\
343.32 \\
350.04 \\
356.77 \\
363.51 \\
370.26 \\
377.03\end{array}$ & $\begin{array}{l}182.09 \\
186.41 \\
190.73 \\
195.08 \\
199.44 \\
203.83 \\
208.24 \\
212.67 \\
217.13 \\
221.61\end{array}$ & $\begin{array}{l}6.830 \\
6.877 \\
6.923 \\
6.969 \\
7.014 \\
7.058 \\
7.102 \\
7.146 \\
7.189 \\
7.231\end{array}$ \\
\hline $\begin{array}{l}36.00 \\
38.00 \\
40.00\end{array}$ & $\begin{array}{l}.2121 \\
.2148 \\
.2177\end{array}$ & $\begin{array}{l}-93.66 \\
-89.57 \\
-85.26\end{array}$ & $\begin{array}{l}-117.21 \\
-113.42 \\
-109.44\end{array}$ & $\begin{array}{l}1.711 \\
1.821 \\
1.932\end{array}$ & $\begin{array}{l}162.00 \\
164.00 \\
166.00 \\
168.00 \\
170.00 \\
172.00 \\
174.00 \\
176.00 \\
178.00 \\
180.00\end{array}$ & $\begin{array}{l}1.4203 \\
1.4408 \\
1.4611 \\
1.4814 \\
1.5016 \\
1.5218 \\
1.5419 \\
1.5619 \\
1.5819 \\
1.6018\end{array}$ & $\begin{array}{l}383.83 \\
390.64 \\
397.48 \\
404.34 \\
411.22 \\
418.13 \\
425.07 \\
432.03 \\
439.01 \\
446.02\end{array}$ & $\begin{array}{l}226.13 \\
230.67 \\
235.25 \\
239.85 \\
244.49 \\
249.17 \\
253.87 \\
258.61 \\
263.38 \\
268.18\end{array}$ & $\begin{array}{l}7.273 \\
7.315 \\
7.357 \\
7.398 \\
7.439 \\
7.479 \\
7.519 \\
7.559 \\
7.598 \\
7.637\end{array}$ \\
\hline $\begin{array}{l}42.00 \\
44.00 \\
46.00 \\
48.00 \\
50.00 \\
52.00 \\
54.00 \\
56.00 \\
58.00 \\
60.00\end{array}$ & $\begin{array}{l}.2209 \\
.2243 \\
.2280 \\
.2320 \\
.2364 \\
.2412 \\
.2465 \\
.2523 \\
.2588 \\
.2671\end{array}$ & $\begin{array}{l}-80.72 \\
-75.94 \\
-70.89 \\
-65.56 \\
-59.94 \\
-54.00 \\
-47.76 \\
-41.18 \\
-34.17 \\
-26.84\end{array}$ & $\begin{array}{r}-105.25 \\
-100.84 \\
-96.20 \\
-91.32 \\
-86.19 \\
-80.78 \\
-75.12 \\
-69.20 \\
-62.91 \\
-56.50\end{array}$ & $\begin{array}{l}2.043 \\
2.154 \\
2.266 \\
2.379 \\
2.494 \\
2.610 \\
2.728 \\
2.848 \\
2.971 \\
3.096\end{array}$ & $\begin{array}{l}182.00 \\
184.00 \\
186.00 \\
188.00 \\
190.00 \\
192.00 \\
194.00 \\
196.00 \\
198.00 \\
200.00\end{array}$ & $\begin{array}{l}1.6216 \\
1.6413 \\
1.6611 \\
1.6807 \\
1.7003 \\
1.7199 \\
1.7394 \\
1.7589 \\
1.7783 \\
1.7977\end{array}$ & $\begin{array}{l}453.05 \\
460.08 \\
467.14 \\
474.23 \\
481.35 \\
488.49 \\
495.67 \\
502.88 \\
510.11 \\
517.38\end{array}$ & $\begin{array}{l}273.00 \\
277.84 \\
282.71 \\
287.62 \\
292.56 \\
297.53 \\
302.54 \\
307.58 \\
312.66 \\
317.78\end{array}$ & $\begin{array}{l}7.676 \\
7.715 \\
7.753 \\
7.791 \\
7.828 \\
7.866 \\
7.903 \\
7.940 \\
7.977 \\
8.013\end{array}$ \\
\hline $\begin{array}{l}62.00 \\
64.00 \\
66.00 \\
68.00 \\
70.00 \\
72.00 \\
74.00 \\
76.00 \\
78.00 \\
80.00\end{array}$ & $\begin{array}{l}.2754 \\
.2848 \\
.2956 \\
.3082 \\
.3228 \\
.3398 \\
.3594 \\
.3818 \\
.4067 \\
.4335\end{array}$ & $\begin{array}{r}-18.90 \\
-10.46 \\
-1.48 \\
8.05 \\
18.11 \\
28.48 \\
39.22 \\
50.27 \\
61.48 \\
72.65\end{array}$ & $\begin{array}{r}-49.48 \\
-42.08 \\
-34.30 \\
-26.17 \\
-17.73 \\
-9.25 \\
-.69 \\
7.87 \\
16.32 \\
24.52\end{array}$ & $\begin{array}{l}3.227 \\
3.362 \\
3.500 \\
3.641 \\
3.787 \\
3.933 \\
4.080 \\
4.228 \\
4.373 \\
4.515\end{array}$ & $\begin{array}{l}202.00 \\
204.00 \\
206.00 \\
208.00 \\
210.00 \\
212.00 \\
214.00 \\
216.00 \\
218.00 \\
220.00\end{array}$ & $\begin{array}{l}1.8171 \\
1.8364 \\
1.8557 \\
1.8749 \\
1.8941 \\
1.9133 \\
1.9324 \\
1.9516 \\
1.9707 \\
1.9897\end{array}$ & $\begin{array}{l}524.68 \\
532.00 \\
539.36 \\
546.74 \\
554.15 \\
561.59 \\
569.06 \\
576.56 \\
584.08 \\
591.62\end{array}$ & $\begin{array}{l}322.92 \\
328.10 \\
333.32 \\
338.57 \\
343.84 \\
349.16 \\
354.50 \\
359.87 \\
365.27 \\
370.70\end{array}$ & $\begin{array}{l}8.050 \\
8.086 \\
8.121 \\
8.157 \\
8.193 \\
8.228 \\
8.263 \\
8.298 \\
8.332 \\
8.367\end{array}$ \\
\hline $\begin{array}{r}82.00 \\
84.00 \\
86.00 \\
88.00 \\
90.00 \\
92.00 \\
94.00 \\
96.00 \\
98.00 \\
100.00\end{array}$ & $\begin{array}{r}.4617 \\
.4907 \\
.5198 \\
.5490 \\
.5778 \\
.6064 \\
.6345 \\
.6622 \\
.6894 \\
.7162\end{array}$ & $\begin{array}{r}83.62 \\
94.27 \\
104.54 \\
114.44 \\
123.97 \\
133.18 \\
142.08 \\
150.72 \\
159.13 \\
167.33\end{array}$ & $\begin{array}{l}32.35 \\
39.79 \\
46.83 \\
53.49 \\
59.81 \\
65.85 \\
71.63 \\
77.20 \\
82.59 \\
87.81\end{array}$ & $\begin{array}{l}4.650 \\
4.779 \\
4.899 \\
5.013 \\
5.120 \\
5.221 \\
5.317 \\
5.408 \\
5.495 \\
5.578\end{array}$ & $\begin{array}{l}222.00 \\
224.00 \\
226.00 \\
228.00 \\
230.00 \\
232.00 \\
234.00 \\
236.00 \\
238.00 \\
240.00\end{array}$ & $\begin{array}{l}2.0088 \\
2.0278 \\
2.0468 \\
2.0657 \\
2.0847 \\
2.1036 \\
2.1225 \\
2.1414 \\
2.1603 \\
2.1791\end{array}$ & $\begin{array}{l}599.19 \\
606.78 \\
614.40 \\
622.05 \\
629.71 \\
637.40 \\
645.11 \\
652.84 \\
660.59 \\
668.36\end{array}$ & $\begin{array}{l}376.15 \\
381.64 \\
387.15 \\
392.68 \\
398.24 \\
403.83 \\
409.44 \\
415.08 \\
420.73 \\
426.41\end{array}$ & $\begin{array}{l}8.401 \\
8.435 \\
8.469 \\
8.503 \\
8.536 \\
8.569 \\
8.603 \\
8.635 \\
8.668 \\
8.701\end{array}$ \\
\hline $\begin{array}{l}102.00 \\
104.00 \\
106.00 \\
108.00 \\
110.00 \\
112.00 \\
114.00 \\
116.00 \\
118.00 \\
120.00\end{array}$ & $\begin{array}{r}.7426 \\
.7686 \\
.7943 \\
.8195 \\
.8445 \\
.8691 \\
.8934 \\
.9175 \\
.9413 \\
.9648\end{array}$ & $\begin{array}{l}175.34 \\
183.19 \\
190.89 \\
198.45 \\
205.90 \\
213.25 \\
220.50 \\
227.66 \\
234.76 \\
241.78\end{array}$ & $\begin{array}{r}92.89 \\
97.85 \\
102.70 \\
107.46 \\
112.14 \\
116.75 \\
121.30 \\
125.79 \\
130.24 \\
134.66\end{array}$ & $\begin{array}{l}5.657 \\
5.733 \\
5.807 \\
5.877 \\
5.946 \\
6.012 \\
6.076 \\
6.138 \\
6.199 \\
6.258\end{array}$ & $\begin{array}{l}242.00 \\
244.00 \\
246.00 \\
248.00 \\
250.00 \\
252.00 \\
254.00 \\
256.00 \\
258.00 \\
260.00\end{array}$ & $\begin{array}{l}2.1979 \\
2.2167 \\
2.2355 \\
2.2543 \\
2.2731 \\
2.2918 \\
2.3105 \\
2.3293 \\
2.3480 \\
2.3666\end{array}$ & $\begin{array}{l}676.15 \\
683.96 \\
691.78 \\
699.62 \\
707.48 \\
715.36 \\
723.25 \\
731.15 \\
739.06 \\
746.99\end{array}$ & $\begin{array}{l}432.11 \\
437.83 \\
443.57 \\
449.32 \\
455.10 \\
460.89 \\
466.70 \\
472.53 \\
478.37 \\
484.22\end{array}$ & $\begin{array}{l}8.733 \\
8.765 \\
8.797 \\
8.829 \\
8.860 \\
8.892 \\
8.923 \\
8.954 \\
8.985 \\
9.015\end{array}$ \\
\hline
\end{tabular}


TEMPER- SPECIFIC ENTHALPY INTERNAL ENTROPY

ATURE VOLUME (8TU/LB) ENERGY (8TU/L8-R) (R) (CU FT/L8)
TEMPER- SPECIFIC ENTHALPY ATURE VOLUME (BTU/L8) (BTU/L8)
(R) (CU FT/LB)
INTERNAL ENTROPY ( 8 TU/LB)

\begin{tabular}{|c|c|c|c|c|c|c|c|c|c|}
\hline $\begin{array}{l}262.00 \\
264.00 \\
266.00 \\
268.00 \\
270.00 \\
272.00 \\
274.00 \\
276.00 \\
278.00 \\
280.00\end{array}$ & $\begin{array}{l}2.3853 \\
2.4040 \\
2.4226 \\
2.4412 \\
2.4598 \\
2.4784 \\
2.4970 \\
2.5156 \\
2.5341 \\
2.5526\end{array}$ & $\begin{array}{l}754.93 \\
762.88 \\
770.84 \\
778.81 \\
786.79 \\
794.75 \\
802.71 \\
810.69 \\
818.66 \\
826.64\end{array}$ & $\begin{array}{l}490.09 \\
495.96 \\
501.86 \\
507.76 \\
513.67 \\
519.57 \\
525.47 \\
531.38 \\
537.30 \\
543.22\end{array}$ & $\begin{array}{l}9.046 \\
9.076 \\
9.106 \\
9.136 \\
9.166 \\
9.195 \\
9.224 \\
9.253 \\
9.282 \\
9.310\end{array}$ & $\begin{array}{l}402.00 \\
404.00 \\
406.00 \\
408.00 \\
410.00 \\
412.00 \\
414.00 \\
416.00 \\
418.00 \\
420.00\end{array}$ & $\begin{array}{l}3.6668 \\
3.6849 \\
3.7030 \\
3.7211 \\
3.7391 \\
3.7572 \\
3.7753 \\
3.7933 \\
3.8114 \\
3.8294\end{array}$ & $\begin{array}{l}1306.23 \\
1313.83 \\
1321.43 \\
1329.01 \\
1336.58 \\
1344.15 \\
1351.71 \\
1359.25 \\
1366.79 \\
1374.32\end{array}$ & $\begin{array}{l}899.10 \\
904.69 \\
910.28 \\
915.86 \\
921.42 \\
926.98 \\
932.53 \\
938.07 \\
943.61 \\
949.13\end{array}$ & $\begin{array}{l}10.734 \\
10.753 \\
10.772 \\
10.791 \\
10.809 \\
10.828 \\
10.846 \\
10.864 \\
10.882 \\
10.900\end{array}$ \\
\hline $\begin{array}{l}282.00 \\
284.00 \\
286.00 \\
288.00 \\
290.00 \\
292.00 \\
294.00 \\
296.00 \\
298.00 \\
300.00\end{array}$ & $\begin{array}{l}2.5712 \\
2.5897 \\
2.6082 \\
2.6267 \\
2.6452 \\
2.6636 \\
2.6821 \\
2.7005 \\
2.7189 \\
2.7374\end{array}$ & $\begin{array}{l}834.63 \\
842.62 \\
850.61 \\
858.60 \\
866.60 \\
874.59 \\
882.59 \\
890.59 \\
898.58 \\
906.58\end{array}$ & $\begin{array}{l}549.15 \\
555.08 \\
561.02 \\
566.96 \\
572.90 \\
578.85 \\
584.79 \\
590.74 \\
596.69 \\
602.65\end{array}$ & $\begin{array}{l}9.339 \\
9.367 \\
9.395 \\
9.423 \\
9.451 \\
9.478 \\
9.505 \\
9.533 \\
9.559 \\
9.586\end{array}$ & $\begin{array}{l}422.00 \\
424.00 \\
426.00 \\
428.00 \\
430.00 \\
432.00 \\
434.00 \\
436.00 \\
438.00 \\
440.00\end{array}$ & $\begin{array}{l}3.8475 \\
3.8655 \\
3.8836 \\
3.9016 \\
3.9196 \\
3.9377 \\
3.9557 \\
3.9737 \\
3.9917 \\
4.0097\end{array}$ & $\begin{array}{l}1381.84 \\
1389.35 \\
1396.86 \\
1404.35 \\
1411.83 \\
1419.30 \\
1426.77 \\
1434.23 \\
1441.67 \\
1449.11\end{array}$ & $\begin{array}{r}954.65 \\
960.16 \\
965.66 \\
971.15 \\
976.63 \\
982.10 \\
987.56 \\
993.02 \\
998.46 \\
1003.90\end{array}$ & $\begin{array}{l}10.918 \\
10.936 \\
10.953 \\
10.971 \\
10.988 \\
11.006 \\
11.023 \\
11.040 \\
11.057 \\
11.074\end{array}$ \\
\hline $\begin{array}{l}302.00 \\
304.00 \\
306.00 \\
308.00 \\
310.00 \\
312.00 \\
314.00 \\
316.00 \\
318.00 \\
320.00\end{array}$ & $\begin{array}{l}2.7558 \\
2.7742 \\
2.7925 \\
2.8109 \\
2.8293 \\
2.8476 \\
2.8660 \\
2.8843 \\
2.9026 \\
2.9210\end{array}$ & $\begin{array}{l}914.57 \\
922.57 \\
930.56 \\
938.55 \\
946.53 \\
954.52 \\
962.50 \\
970.47 \\
978.44 \\
986.41\end{array}$ & $\begin{array}{l}608.60 \\
614.55 \\
620.50 \\
626.45 \\
632.39 \\
638.34 \\
644.28 \\
650.22 \\
656.16 \\
662.09\end{array}$ & $\begin{array}{l}9.613 \\
9.639 \\
9.665 \\
9.691 \\
9.717 \\
9.743 \\
9.768 \\
9.794 \\
9.819 \\
9.844\end{array}$ & $\begin{array}{l}442.00 \\
444.00 \\
446.00 \\
448.00 \\
450.00 \\
452.00 \\
454.00 \\
456.00 \\
458.00 \\
460.00\end{array}$ & $\begin{array}{l}4.0277 \\
4.0457 \\
4.0637 \\
4.0817 \\
4.0997 \\
4.1177 \\
4.1357 \\
4.1537 \\
4.1117 \\
4.11896\end{array}$ & $\begin{array}{l}1456.54 \\
1463.96 \\
1471.37 \\
1478.77 \\
1486.16 \\
1493.56 \\
1500.96 \\
1508.34 \\
1515.72 \\
1523.09\end{array}$ & $\begin{array}{l}1009.33 \\
1014.75 \\
1020.16 \\
1025.57 \\
1030.96 \\
1036.37 \\
1041.76 \\
1047.15 \\
1052.53 \\
1057.90\end{array}$ & $\begin{array}{l}11.091 \\
11.108 \\
11.124 \\
11.141 \\
11.157 \\
11.174 \\
11.190 \\
11.206 \\
11.222 \\
11.238\end{array}$ \\
\hline $\begin{array}{l}322.00 \\
324.00 \\
326.00 \\
328.00 \\
330.00 \\
332.00 \\
334.00 \\
336.00 \\
338.00 \\
340.00\end{array}$ & $\begin{array}{l}2.9393 \\
2.9576 \\
2.9759 \\
2.9942 \\
3.0125 \\
3.0307 \\
3.0490 \\
3.0673 \\
3.0855 \\
3.1038\end{array}$ & $\begin{array}{r}994.37 \\
1002.33 \\
1010.28 \\
1018.22 \\
1026.16 \\
1034.10 \\
1042.02 \\
1049.94 \\
1057.85 \\
1065.76\end{array}$ & $\begin{array}{l}668.02 \\
673.94 \\
679.86 \\
685.78 \\
691.68 \\
697.59 \\
703.49 \\
709.38 \\
715.26 \\
721.14\end{array}$ & $\begin{array}{r}9.869 \\
9.893 \\
9.918 \\
9.942 \\
9.966 \\
9.990 \\
10.014 \\
10.038 \\
10.061 \\
10.084\end{array}$ & $\begin{array}{l}462.00 \\
464.00 \\
466.00 \\
468.00 \\
470.00 \\
472.00 \\
474.00 \\
476.00 \\
478.00 \\
480.00\end{array}$ & $\begin{array}{l}4.2076 \\
4.2256 \\
4.2436 \\
4.2615 \\
4.2795 \\
4.2974 \\
4.3154 \\
4.3333 \\
4.3513 \\
4.3693\end{array}$ & $\begin{array}{l}1530.44 \\
1537.80 \\
1545.14 \\
1552.48 \\
1559.80 \\
1567.12 \\
1574.44 \\
1581.74 \\
1589.04 \\
1596.33\end{array}$ & $\begin{array}{l}1063.27 \\
1068.62 \\
1073.97 \\
1079.31 \\
1084.65 \\
1089.97 \\
1095.29 \\
1100.60 \\
1105.91 \\
1111.20\end{array}$ & $\begin{array}{l}11.254 \\
11.270 \\
11.286 \\
11.302 \\
11.317 \\
11.333 \\
11.348 \\
11.364 \\
11.379 \\
11.394\end{array}$ \\
\hline $\begin{array}{l}342.00 \\
344.00 \\
346.00 \\
348.00 \\
350.00 \\
352.00 \\
354.00 \\
35.6 .00 \\
358.00 \\
360.00\end{array}$ & $\begin{array}{l}3.1220 \\
3.1403 \\
3.1585 \\
3.1767 \\
3.1949 \\
3.2132 \\
3.2314 \\
3.2496 \\
3.2678 \\
3.2860\end{array}$ & $\begin{array}{l}1073.65 \\
1081.54 \\
1089.42 \\
1097.30 \\
1105.16 \\
1113.02 \\
1120.86 \\
1128.70 \\
1136.53 \\
1144.35\end{array}$ & $\begin{array}{l}727.01 \\
732: 87 \\
738.73 \\
744.58 \\
750.42 \\
756.25 \\
762.08 \\
767.89 \\
773.70 \\
779.50\end{array}$ & $\begin{array}{l}10.108 \\
10.131 \\
10.153 \\
10.176 \\
10.199 \\
10.221 \\
10.243 \\
10.265 \\
10.287 \\
10.309\end{array}$ & $\begin{array}{l}482.00 \\
484.00 \\
486.00 \\
488.00 \\
490.00 \\
492.00 \\
494.00 \\
496.00 \\
498.00 \\
500.00\end{array}$ & $\begin{array}{l}4.3872 \\
4.4051 \\
4.4231 \\
4.4410 \\
4.4590 \\
4.4769 \\
4.4949 \\
4.5128 \\
4.5307 \\
4.5487\end{array}$ & $\begin{array}{l}1603.61 \\
1610.89 \\
1618.16 \\
1625.43 \\
1632.69 \\
1639.94 \\
1647.18 \\
1654.42 \\
1661.66 \\
1668.89\end{array}$ & $\begin{array}{l}1116.50 \\
1121.78 \\
1127.06 \\
1132.33 \\
1137.60 \\
1142.86 \\
1148.11 \\
1153.36 \\
1158.60 \\
1163.84\end{array}$ & $\begin{array}{l}11.410 \\
11.425 \\
11.440 \\
11.454 \\
11.469 \\
11.484 \\
11.499 \\
11.513 \\
11.528 \\
11.542\end{array}$ \\
\hline $\begin{array}{l}362.00 \\
364.00 \\
366.00 \\
368.00 \\
370.00 \\
372.00 \\
374.00 \\
376.00 \\
378.00 \\
380.00\end{array}$ & $\begin{array}{l}3.3041 \\
3.3223 \\
3.3405 \\
3.3587 \\
3.3768 \\
3.3950 \\
3.4131 \\
3.4313 \\
3.4494 \\
3.4676\end{array}$ & $\begin{array}{l}1152.15 \\
1159.95 \\
1167.73 \\
1175.51 \\
1183.28 \\
1191.04 \\
1198.78 \\
1206.52 \\
1214.25 \\
1221.97\end{array}$ & $\begin{array}{l}785.29 \\
791.07 \\
796.83 \\
802.59 \\
808.34 \\
814.09 \\
819.82 \\
825.54 \\
831.26 \\
836.96\end{array}$ & $\begin{array}{l}10.331 \\
10.352 \\
10.373 \\
10.395 \\
10.416 \\
10.437 \\
10.457 \\
10.478 \\
10.498 \\
10.519\end{array}$ & $\begin{array}{l}502.00 \\
504.00 \\
506.00 \\
508.00 \\
510.00 \\
512.00 \\
514.00 \\
516.00 \\
518.00 \\
520.00\end{array}$ & $\begin{array}{l}4.5666 \\
4.5845 \\
4.6025 \\
4.6204 \\
4.6384 \\
4.6563 \\
4.6742 \\
4.6922 \\
4.7101 \\
4.7280\end{array}$ & $\begin{array}{l}1676.11 \\
1683.33 \\
1690.55 \\
1697.76 \\
1704.96 \\
1712.16 \\
1719.36 \\
1726.55 \\
1733.74 \\
1740.93\end{array}$ & $\begin{array}{l}1169.08 \\
1174.30 \\
1179.53 \\
1184.75 \\
1189.96 \\
1115.17 \\
1200.37 \\
1205.58 \\
1210.77 \\
1215.97\end{array}$ & $\begin{array}{l}11.557 \\
11.571 \\
11.586 \\
11.600 \\
11.614 \\
11.628 \\
11.642 \\
11.656 \\
11.670 \\
11.684\end{array}$ \\
\hline $\begin{array}{l}382.00 \\
384.00 \\
386.00 \\
388.00 \\
390.00 \\
392.00 \\
394.00 \\
396.00 \\
398.00 \\
400.00\end{array}$ & $\begin{array}{l}3.4857 \\
3.5038 \\
3.5219 \\
3.5401 \\
3.5582 \\
3.5763 \\
3.5944 \\
3.6125 \\
3.6306 \\
3.6487\end{array}$ & $\begin{array}{l}1229.68 \\
1237.38 \\
1245.07 \\
1252.75 \\
1260.42 \\
1268.08 \\
1275.73 \\
1283.37 \\
1291.00 \\
1298.62\end{array}$ & $\begin{array}{l}842.66 \\
848.34 \\
854.02 \\
859.69 \\
865.34 \\
870.99 \\
876.63 \\
882.26 \\
887.88 \\
893.50\end{array}$ & $\begin{array}{l}10.539 \\
10.559 \\
10.579 \\
10.599 \\
10.619 \\
10.638 \\
10.658 \\
10.677 \\
10.696 \\
10.715\end{array}$ & $\begin{array}{l}522.00 \\
524.00 \\
526.00 \\
528.00 \\
530.00 \\
532.00 \\
534.00 \\
536.00 \\
538.00 \\
540.00\end{array}$ & $\begin{array}{l}4.7459 \\
4.7639 \\
4.7818 \\
4.7997 \\
4.8177 \\
4.8356 \\
4.8535 \\
4.8714 \\
4.8894 \\
4.9073\end{array}$ & $\begin{array}{l}1748.11 \\
1755.28 \\
1762.46 \\
1769.63 \\
1776.79 \\
1783.96 \\
1791.12 \\
1798.27 \\
1805.42 \\
1812.57\end{array}$ & $\begin{array}{l}1221.16 \\
1226.34 \\
1231.53 \\
1236.70 \\
1241.88 \\
1247.05 \\
1252.22 \\
1257.39 \\
1262.55 \\
1267.71\end{array}$ & $\begin{array}{l}11.698 \\
11.711 \\
11.725 \\
11.739 \\
11.752 \\
11.766 \\
11.779 \\
11.792 \\
11.806 \\
11.819\end{array}$ \\
\hline
\end{tabular}


$\begin{array}{ccccc}\text { TEMPER- } & \text { SPECIFIC } & \text { ENTHALPY } & \text { INTERNAL ENTROPY } \\ \text { ATURE } & \text { VOLUME } & \text { (BTU/L8) } & \text { ENERGY } & \text { (8TU/L8-R) }\end{array}$ (R) (CU FT/LB) ( $8 T U / L 8)$
TEMPER- SPECIFIC ENTHALPY ATURE VOLUME (BTU/LB) (R) (CU FT/LB)
INTERNAL ENTROPY

ENERGY (BTU/LB-R) (BTU/LB)

\begin{tabular}{|c|c|c|c|c|c|c|c|c|c|}
\hline & & & & & $\begin{array}{l}122.00 \\
124.00 \\
126.00 \\
128.00 \\
130.00 \\
132.00 \\
134.00 \\
136.00 \\
138.00 \\
140.00\end{array}$ & $\begin{array}{r}.8432 \\
.8633 \\
.8833 \\
.9031 \\
.9227 \\
.9422 \\
.9615 \\
.9807 \\
.9998 \\
1.0187\end{array}$ & $\begin{array}{l}241.17 \\
248.31 \\
255.40 \\
262.45 \\
269.45 \\
276.41 \\
283.35 \\
290.26 \\
297.15 \\
304.03\end{array}$ & $\begin{array}{l}131.95 \\
136.49 \\
140.99 \\
145.47 \\
149.92 \\
154.37 \\
158.80 \\
163.22 \\
167.65 \\
172.07\end{array}$ & $\begin{array}{l}6.115 \\
6.173 \\
6.230 \\
6.285 \\
6.340 \\
6.393 \\
6.445 \\
6.496 \\
6.546 \\
6.596\end{array}$ \\
\hline & & & & & $\begin{array}{l}142.00 \\
144.00 \\
146.00 \\
148.00 \\
150.00 \\
152.00 \\
154.00 \\
156.00 \\
158.00 \\
160.00\end{array}$ & $\begin{array}{l}1.0375 \\
1.0562 \\
1.0748 \\
1.0933 \\
1.1116 \\
1.1299 \\
1.1481 \\
1.1662 \\
1.1842 \\
1.2021\end{array}$ & $\begin{array}{l}310.89 \\
317.74 \\
324.59 \\
331.44 \\
338.29 \\
345.14 \\
352.00 \\
358.87 \\
365.75 \\
372.65\end{array}$ & $\begin{array}{l}176.49 \\
180.93 \\
185.37 \\
189.82 \\
194.29 \\
198.78 \\
203.28 \\
207.81 \\
212.35 \\
216.93\end{array}$ & $\begin{array}{l}6.645 \\
6.692 \\
6.740 \\
6.786 \\
6.831 \\
6.878 \\
6.922 \\
6.967 \\
7.011 \\
7.054\end{array}$ \\
\hline $\begin{array}{l}36.00 \\
38.00 \\
40.00\end{array}$ & $\begin{array}{l}.2104 \\
.2130 \\
.2157\end{array}$ & $\begin{array}{l}-90.59 \\
-86.57 \\
-82.34\end{array}$ & $\begin{array}{l}-117.85 \\
-114.16 \\
-110.28\end{array}$ & $\begin{array}{l}1.687 \\
1.796 \\
1.905\end{array}$ & $\begin{array}{l}162.00 \\
164.00 \\
166.00 \\
168.00 \\
170.00 \\
172.00 \\
174.00 \\
176.00 \\
178.00 \\
180.00\end{array}$ & $\begin{array}{l}1.2200 \\
1.2378 \\
1.2555 \\
1.2731 \\
1.2907 \\
1.3082 \\
1.3256 \\
1.3430 \\
1.3603 \\
1.3776\end{array}$ & $\begin{array}{l}379.56 \\
386.49 \\
393.43 \\
400.40 \\
407.39 \\
414.40 \\
421.44 \\
428.49 \\
435.57 \\
442.68\end{array}$ & $\begin{array}{l}221.53 \\
226.15 \\
230.81 \\
235.49 \\
240.20 \\
244.95 \\
249.72 \\
254.53 \\
259.36 \\
264.23\end{array}$ & $\begin{array}{l}7.097 \\
7.139 \\
7.182 \\
7.223 \\
7.265 \\
7.306 \\
7.346 \\
7.387 \\
7.427 \\
7.466\end{array}$ \\
\hline $\begin{array}{l}42.00 \\
44.00 \\
46.00 \\
48.00 \\
50.00 \\
52.00 \\
54.00 \\
56.00 \\
58.00 \\
60.00\end{array}$ & $\begin{array}{l}.2187 \\
.2219 \\
.2253 \\
.2290 \\
.2330 \\
.2373 \\
.2420 \\
.2472 \\
.2529 \\
.2599\end{array}$ & $\begin{array}{l}-77.88 \\
-73.20 \\
-68.27 \\
-63.08 \\
-57.62 \\
-51.87 \\
-45.85 \\
-39.54 \\
-32.86 \\
-25.93\end{array}$ & $\begin{array}{r}-106.21 \\
-101.94 \\
-97.45 \\
-92.74 \\
-87.79 \\
-82.61 \\
-77.20 \\
-71.57 \\
-65.62 \\
-59.60\end{array}$ & $\begin{array}{l}2.013 \\
2.122 \\
2.232 \\
2.342 \\
2.454 \\
2.566 \\
2.680 \\
2.795 \\
2.912 \\
3.030\end{array}$ & $\begin{array}{l}182.00 \\
184.00 \\
186.00 \\
188.00 \\
190.00 \\
192.00 \\
194.00 \\
196.00 \\
198.00 \\
200.00\end{array}$ & $\begin{array}{l}1.3948 \\
1.4120 \\
1.4291 \\
1.4461 \\
1.4631 \\
1.4801 \\
1.4970 \\
1.5139 \\
1.5307 \\
1.5475\end{array}$ & $\begin{array}{l}449.78 \\
456.89 \\
464.03 \\
471.19 \\
478.38 \\
485.61 \\
492.85 \\
500.13 \\
507.44 \\
514.78\end{array}$ & $\begin{array}{l}269.10 \\
273.99 \\
278.91 \\
283.87 \\
288.86 \\
293.88 \\
298.94 \\
304.03 \\
309.16 \\
314.32\end{array}$ & $\begin{array}{l}7.506 \\
7.544 \\
7.583 \\
7.621 \\
7.659 \\
7.697 \\
7.735 \\
7.772 \\
7.809 \\
7.846\end{array}$ \\
\hline $\begin{array}{l}62.00 \\
64.00 \\
66.00 \\
68.00 \\
70.00 \\
72.00 \\
74.00 \\
76.00 \\
78.00 \\
80.00\end{array}$ & $\begin{array}{r}.2669 \\
.2746 \\
.2832 \\
.2929 \\
.3039 \\
.3162 \\
.3301 \\
.3457 \\
.3629 \\
.3818\end{array}$ & $\begin{array}{r}-18.48 \\
-10.65 \\
-2.41 \\
6.21 \\
15.17 \\
24.27 \\
33.59 \\
43.15 \\
52.95 \\
62.91\end{array}$ & $\begin{array}{r}-53.05 \\
-46.22 \\
-39.10 \\
-31.74 \\
-24.19 \\
-16.69 \\
-9.17 \\
-1.62 \\
5.94 \\
13.46\end{array}$ & $\begin{array}{l}3.153 \\
3.278 \\
3.405 \\
3.533 \\
3.663 \\
3.791 \\
3.918 \\
4.046 \\
4.173 \\
4.299\end{array}$ & $\begin{array}{l}202.00 \\
204.00 \\
206.00 \\
208.00 \\
210.00 \\
212.00 \\
214.00 \\
216.00 \\
218.00 \\
220.00\end{array}$ & $\begin{array}{l}1.5643 \\
1.5810 \\
1.5977 \\
1.6143 \\
1.6310 \\
1.6476 \\
1.6641 \\
1.6807 \\
1.6972 \\
1.7137\end{array}$ & $\begin{array}{l}522.14 \\
529.54 \\
536.96 \\
544.41 \\
551.89 \\
559.39 \\
566.92 \\
574.48 \\
582.06 \\
589.66\end{array}$ & $\begin{array}{l}319.51 \\
324.74 \\
330.00 \\
335.29 \\
340.62 \\
345.97 \\
351.36 \\
356.77 \\
362.21 \\
367.68\end{array}$ & $\begin{array}{l}7.883 \\
7.919 \\
7.955 \\
7.991 \\
8.027 \\
8.063 \\
8.098 \\
8.133 \\
8.168 \\
8.203\end{array}$ \\
\hline $\begin{array}{r}82.00 \\
84.00 \\
86.00 \\
88.00 \\
90.00 \\
92.00 \\
94.00 \\
96.00 \\
98.00 \\
100.00\end{array}$ & $\begin{array}{r}.4020 \\
.4234 \\
.4457 \\
.4685 \\
.4917 \\
.5150 \\
.5383 \\
.5615 \\
.5845 \\
.6074\end{array}$ & $\begin{array}{r}72.95 \\
82.98 \\
92.91 \\
102.66 \\
112.22 \\
121.55 \\
130.67 \\
139.56 \\
148.24 \\
156.73\end{array}$ & $\begin{array}{l}20.88 \\
28.13 \\
35.17 \\
41.97 \\
48.53 \\
54.84 \\
60.94 \\
66.82 \\
72.52 \\
78.05\end{array}$ & $\begin{array}{l}4.423 \\
4.544 \\
4.661 \\
4.773 \\
4.880 \\
4.983 \\
5.081 \\
5.175 \\
5.264 \\
5.350\end{array}$ & $\begin{array}{l}222.00 \\
224.00 \\
226.00 \\
228.00 \\
230.00 \\
232.00 \\
234.00 \\
236.00 \\
238.00 \\
240.00\end{array}$ & $\begin{array}{l}1.7301 \\
1.7466 \\
1.7630 \\
1.7794 \\
1.7957 \\
1.8121 \\
1.8284 \\
1.8447 \\
1.8610 \\
1.8773\end{array}$ & $\begin{array}{l}597.28 \\
604.93 \\
612.61 \\
620.30 \\
628.02 \\
635.76 \\
643.51 \\
651.29 \\
659.09 \\
666.90\end{array}$ & $\begin{array}{l}373.17 \\
378.69 \\
384.24 \\
389.81 \\
395.40 \\
401.02 \\
406.66 \\
412.33 \\
418.01 \\
423.72\end{array}$ & $\begin{array}{l}8.237 \\
8.272 \\
8.306 \\
8.340 \\
8.373 \\
8.407 \\
8.440 \\
8.473 \\
8.506 \\
8.539\end{array}$ \\
\hline $\begin{array}{l}102.00 \\
104.00 \\
106.00 \\
108.00 \\
110.00 \\
112.00 \\
114.00 \\
116.00 \\
118.00 \\
120.00\end{array}$ & $\begin{array}{r}.6300 \\
.6524 \\
.6745 \\
.6964 \\
.7180 \\
.7394 \\
.7606 \\
.7815 \\
.8023 \\
.8228\end{array}$ & $\begin{array}{l}165.04 \\
173.19 \\
181.19 \\
189.05 \\
196.79 \\
204.41 \\
211.93 \\
219.36 \\
226.70 \\
233.97\end{array}$ & $\begin{array}{r}83.44 \\
88.69 \\
93.82 \\
98.84 \\
103.77 \\
108.63 \\
113.40 \\
118.12 \\
122.78 \\
127.39\end{array}$ & $\begin{array}{l}5.432 \\
5.511 \\
5.587 \\
5.661 \\
5.732 \\
5.801 \\
5.867 \\
5.932 \\
5.994 \\
6.056\end{array}$ & $\begin{array}{l}242.00 \\
244.00 \\
246.00 \\
248.00 \\
250.00 \\
252.00 \\
254.00 \\
256.00 \\
258.00 \\
260.00\end{array}$ & $\begin{array}{l}1.8936 \\
1.9098 \\
1.9260 \\
1.9423 \\
1.9585 \\
1.9746 \\
1.9908 \\
2.0069 \\
2.0231 \\
2.0392\end{array}$ & $\begin{array}{l}674.73 \\
682.58 \\
690.45 \\
698.33 \\
706.23 \\
714.14 \\
722.06 \\
730.00 \\
737.95 \\
745.91\end{array}$ & $\begin{array}{l}429.45 \\
435.19 \\
440.95 \\
446.74 \\
452.54 \\
458.35 \\
464.18 \\
470.03 \\
475.89 \\
481.76\end{array}$ & $\begin{array}{l}8.571 \\
8.604 \\
8.636 \\
8.668 \\
8.699 \\
8.731 \\
8.762 \\
8.793 \\
8.824 \\
8.855\end{array}$ \\
\hline
\end{tabular}


TEMPER- SPECIFIC ENTHALPY INTERNAL ENTROPY ATURE VOLUME (8TU/L8) ENERGY (8TU/L8-R) (R) (CU FT/L8)
(BTU/L8)
TEMPER- SPECIFIC ENTHALPY INTERNAL ENTROPY ATURE VOLUME (8TU/L8) ENERGY (8TU/LB-R) (R) (CU FT/L8) $(8 T U / L 8)$
(C)

\begin{tabular}{|c|c|c|c|c|c|c|c|c|c|}
\hline $\begin{array}{l}262.00 \\
264.00 \\
266.00 \\
268.00 \\
270.00 \\
272.00 \\
274.00 \\
276.00 \\
278.00 \\
280.00\end{array}$ & $\begin{array}{l}2.0553 \\
2.0714 \\
2.0875 \\
2.1035 \\
2.1196 \\
2.1356 \\
2.1516 \\
2.1676 \\
2.1836 \\
2.1996\end{array}$ & $\begin{array}{l}753.88 \\
761.87 \\
769.86 \\
777.86 \\
785.87 \\
793.86 \\
801.86 \\
809.86 \\
817.87 \\
825.88\end{array}$ & $\begin{array}{l}487.65 \\
493.55 \\
499.46 \\
505.38 \\
511.31 \\
517.22 \\
523.14 \\
529.07 \\
535.01 \\
540.95\end{array}$ & $\begin{array}{l}8.885 \\
8.916 \\
8.946 \\
8.976 \\
9.006 \\
9.035 \\
9.064 \\
9.094 \\
9.122 \\
9.151\end{array}$ & $\begin{array}{l}402.00 \\
404.00 \\
406.00 \\
408.00 \\
410.00 \\
412.00 \\
414.00 \\
416.00 \\
418.00 \\
420.00\end{array}$ & $\begin{array}{l}3.1582 \\
3.1738 \\
3.1893 \\
3.2048 \\
3.2204 \\
3.2359 \\
3.2514 \\
3.2669 \\
3.2824 \\
3.2979\end{array}$ & $\begin{array}{l}1306.86 \\
1314.47 \\
1322.07 \\
1329.67 \\
1337.25 \\
1344.82 \\
1352.39 \\
1359.95 \\
1367.50 \\
1375.04\end{array}$ & $\begin{array}{l}897.75 \\
903.35 \\
908.94 \\
914.52 \\
920.09 \\
925.66 \\
931.21 \\
936.76 \\
942.30 \\
947.84\end{array}$ & $\begin{array}{l}10.579 \\
10.598 \\
10.617 \\
10.636 \\
10.654 \\
10.673 \\
10.691 \\
10.709 \\
10.727 \\
10.745\end{array}$ \\
\hline $\begin{array}{l}282.00 \\
284.00 \\
286.00 \\
288.00 \\
290.00 \\
292.00 \\
294.00 \\
296.00 \\
298.00 \\
300.00\end{array}$ & $\begin{array}{l}2.2156 \\
2.2315 \\
2.2475 \\
2.2634 \\
2.2793 \\
2.2952 \\
2.3111 \\
2.3270 \\
2.3429 \\
2.3587\end{array}$ & $\begin{array}{l}833.89 \\
841.91 \\
849.93 \\
857.96 \\
865.98 \\
874.01 \\
882.03 \\
890.06 \\
898.09 \\
906.12\end{array}$ & $\begin{array}{l}546.90 \\
552.85 \\
558.80 \\
564.76 \\
570.72 \\
576.69 \\
582.66 \\
588.63 \\
594.60 \\
600.57\end{array}$ & $\begin{array}{l}9.180 \\
9.208 \\
9.236 \\
9.264 \\
9.292 \\
9.320 \\
9.347 \\
9.374 \\
9.401 \\
9.428\end{array}$ & $\begin{array}{l}422.00 \\
424.00 \\
426.00 \\
428.00 \\
430.00 \\
432.00 \\
434.00 \\
436.00 \\
438.00 \\
440.00\end{array}$ & $\begin{array}{l}3.3135 \\
3.3290 \\
3.3445 \\
3.3599 \\
3.3754 \\
3.3909 \\
3.4064 \\
3.4219 \\
3.4374 \\
3.4528\end{array}$ & $\begin{array}{l}1382.57 \\
1390.10 \\
1397.61 \\
1405.12 \\
1412.62 \\
1420.10 \\
1427.58 \\
1435.05 \\
1442.51 \\
1449.96\end{array}$ & $\begin{array}{r}953.36 \\
958.88 \\
964.38 \\
969.88 \\
975.37 \\
980.85 \\
986.33 \\
991.79 \\
997.25 \\
1002.69\end{array}$ & $\begin{array}{l}10.763 \\
10.781 \\
10.799 \\
10.816 \\
10.834 \\
10.851 \\
10.868 \\
10.886 \\
10.903 \\
10.920\end{array}$ \\
\hline $\begin{array}{l}302.00 \\
304.00 \\
306.00 \\
308.00 \\
310.00 \\
312.00 \\
314.00 \\
316.00 \\
318.00 \\
320.00\end{array}$ & $\begin{array}{l}2.3746 \\
2.3904 \\
2.4063 \\
2.4221 \\
2.4379 \\
2.4537 \\
2.4695 \\
2.4853 \\
2.5011 \\
2.5168\end{array}$ & $\begin{array}{l}914.14 \\
922.17 \\
930.19 \\
938.21 \\
946.23 \\
954.24 \\
962.25 \\
970.26 \\
978.26 \\
986.26\end{array}$ & $\begin{array}{l}606.54 \\
612.52 \\
618.49 \\
624.46 \\
630.43 \\
636.40 \\
642.36 \\
648.32 \\
654.28 \\
660.24\end{array}$ & $\begin{array}{l}9.455 \\
9.481 \\
9.507 \\
9.534 \\
9.560 \\
9.585 \\
9.611 \\
9.636 \\
9.662 \\
9.687\end{array}$ & $\begin{array}{l}442.00 \\
444.00 \\
446.00 \\
448.00 \\
450.00 \\
452.00 \\
454.00 \\
456.00 \\
458.00 \\
460.00\end{array}$ & $\begin{array}{l}3.4683 \\
3.4838 \\
3.4992 \\
3.5147 \\
3.5301 \\
3.5456 \\
3.5610 \\
3.5765 \\
3.5919 \\
3.6074\end{array}$ & $\begin{array}{l}1457.41 \\
1464.84 \\
1472.26 \\
1479.68 \\
1487.09 \\
1494.50 \\
1501.91 \\
1509.31 \\
1516.70 \\
1524.08\end{array}$ & $\begin{array}{l}1008.13 \\
1013.56 \\
1018.99 \\
1024.40 \\
1029.81 \\
1035.22 \\
1040.62 \\
1046.02 \\
1051.41 \\
1056.79\end{array}$ & $\begin{array}{l}10.936 \\
10.953 \\
10.970 \\
10.987 \\
11.003 \\
11.019 \\
11.036 \\
11.052 \\
11.068 \\
11.084\end{array}$ \\
\hline $\begin{array}{l}322.00 \\
324.00 \\
326.00 \\
328.00 \\
330.00 \\
332.00 \\
334.00 \\
336.00 \\
338.00 \\
340.00\end{array}$ & $\begin{array}{l}2.5326 \\
2.5483 \\
2.5641 \\
2.5798 \\
2.5956 \\
2.6113 \\
2.6270 \\
2.6427 \\
2.6584 \\
2.6741\end{array}$ & $\begin{array}{r}994.25 \\
1002.24 \\
1010.22 \\
1018.20 \\
1026.17 \\
1034.13 \\
1042.09 \\
1050.04 \\
1057.98 \\
1065.91\end{array}$ & $\begin{array}{l}666.19 \\
672.14 \\
678.08 \\
684.02 \\
689.95 \\
695.87 \\
701.79 \\
707.71 \\
713.61 \\
719.51\end{array}$ & $\begin{array}{l}9.712 \\
9.736 \\
9.761 \\
9.785 \\
9.809 \\
9.833 \\
9.857 \\
9.881 \\
9.905 \\
9.928\end{array}$ & $\begin{array}{l}462.00 \\
464.00 \\
466.00 \\
468.00 \\
470.00 \\
472.00 \\
474.00 \\
476.00 \\
478.00 \\
480.00\end{array}$ & $\begin{array}{l}3.6228 \\
3.6382 \\
3.6537 \\
3.6691 \\
3.6845 \\
3.6999 \\
3.7154 \\
3.7308 \\
3.7462 \\
3.7616\end{array}$ & $\begin{array}{l}1531.45 \\
1538.82 \\
1546.17 \\
1553.52 \\
1560.86 \\
1568.20 \\
1575.52 \\
1582.84 \\
1590.15 \\
1597.45\end{array}$ & $\begin{array}{l}1062.17 \\
1067.53 \\
1072.89 \\
1078.24 \\
1083.58 \\
1088.92 \\
1094.24 \\
1099.56 \\
1104.87 \\
1110.18\end{array}$ & $\begin{array}{l}11.100 \\
11.116 \\
11.132 \\
11.148 \\
11.163 \\
11.179 \\
11.195 \\
11.210 \\
11.225 \\
11.240\end{array}$ \\
\hline $\begin{array}{l}342.00 \\
344.00 \\
346.00 \\
348.00 \\
350.00 \\
352.00 \\
354.00 \\
356.00 \\
358.00 \\
360.00\end{array}$ & $\begin{array}{l}2.6898 \\
2.7055 \\
2.7212 \\
2.7369 \\
2.7525 \\
2.7682 \\
2.7839 \\
2.7995 \\
2.8152 \\
2.8308\end{array}$ & $\begin{array}{l}1073.83 \\
1081.75 \\
1089.66 \\
1097.56 \\
1105.44 \\
1113.32 \\
1121.19 \\
1129.05 \\
1136.90 \\
1144.74\end{array}$ & $\begin{array}{l}725.40 \\
731.29 \\
737.16 \\
743.03 \\
748.89 \\
754.74 \\
760.58 \\
766.41 \\
772.23 \\
778.05\end{array}$ & $\begin{array}{r}9.951 \\
9.974 \\
9.997 \\
10.020 \\
10.043 \\
10.065 \\
10.087 \\
10.110 \\
10.132 \\
10.153\end{array}$ & $\begin{array}{l}482.00 \\
484.00 \\
486.00 \\
488.00 \\
490.00 \\
492.00 \\
494.00 \\
496.00 \\
498.00 \\
500.00\end{array}$ & $\begin{array}{l}3.7770 \\
3.7924 \\
3.8079 \\
3.8233 \\
3.8387 \\
3.8541 \\
3.8695 \\
3.8849 \\
3.9003 \\
3.9157\end{array}$ & $\begin{array}{l}1604.74 \\
1612.03 \\
1619.31 \\
1626.59 \\
1633.85 \\
1641.12 \\
1648.37 \\
1655.62 \\
1662.86 \\
1670.10\end{array}$ & $\begin{array}{l}1115.48 \\
1120.77 \\
1126.05 \\
1131.33 \\
1136.60 \\
1141.87 \\
1147.13 \\
1152.38 \\
1157.63 \\
1162.88\end{array}$ & $\begin{array}{l}11.256 \\
11.271 \\
11.286 \\
11.301 \\
11.316 \\
11.330 \\
11.345 \\
11.360 \\
11.374 \\
11.389\end{array}$ \\
\hline $\begin{array}{l}362.00 \\
364.00 \\
366.00 \\
368.00 \\
370.00 \\
372.00 \\
374.00 \\
376.00 \\
378.00 \\
380.00\end{array}$ & $\begin{array}{l}2.8465 \\
2.8621 \\
2.8777 \\
2.8933 \\
2.9090 \\
2.9246 \\
2.9402 \\
2.9558 \\
2.9714 \\
2.9870\end{array}$ & $\begin{array}{l}1152.57 \\
1160.38 \\
1168.18 \\
1175.97 \\
1183.75 \\
1191.52 \\
1199.28 \\
1207.03 \\
1214.77 \\
1222.50\end{array}$ & $\begin{array}{l}783.84 \\
789.63 \\
795.41 \\
801.18 \\
806.93 \\
812.68 \\
818.42 \\
824.15 \\
829.87 \\
835.58\end{array}$ & $\begin{array}{l}10.175 \\
10.197 \\
10.218 \\
10.239 \\
10.260 \\
10.281 \\
10.302 \\
10.323 \\
10.343 \\
10.364\end{array}$ & $\begin{array}{l}502.00 \\
504.00 \\
506.00 \\
508.00 \\
510.00 \\
512.00 \\
514.00 \\
516.00 \\
518.00 \\
520.00\end{array}$ & $\begin{array}{l}3.9311 \\
3.9465 \\
3.9619 \\
3.9773 \\
3.9927 \\
4.0080 \\
4.0234 \\
4.0388 \\
4.0542 \\
4.0696\end{array}$ & $\begin{array}{l}1677.33 \\
1684.56 \\
1691.78 \\
1699.00 \\
1706.21 \\
1713.42 \\
1720.63 \\
1727.83 \\
1735.02 \\
1742.22\end{array}$ & $\begin{array}{l}1168.11 \\
1173.35 \\
1178.58 \\
1183.80 \\
1189.02 \\
1194.23 \\
1199.44 \\
1204.65 \\
1209.85 \\
1215.05\end{array}$ & $\begin{array}{l}11.403 \\
11.418 \\
11.432 \\
11.446 \\
11.460 \\
11.474 \\
11.488 \\
11.502 \\
11.516 \\
11.530\end{array}$ \\
\hline $\begin{array}{l}382.00 \\
384.00 \\
386.00 \\
388.00 \\
390.00 \\
392.00 \\
394.00 \\
396.00 \\
398.00 \\
400.00\end{array}$ & $\begin{array}{l}3.0026 \\
3.0181 \\
3.0337 \\
3.0493 \\
3.0649 \\
3.0804 \\
3.0960 \\
3.1116 \\
3.1271 \\
3.1427\end{array}$ & $\begin{array}{l}1230.22 \\
1237.93 \\
1245.63 \\
1253.32 \\
1260.99 \\
1268.66 \\
1276.32 \\
1283.97 \\
1291.61 \\
1299.24\end{array}$ & $\begin{array}{l}841.28 \\
846.97 \\
852.65 \\
858.32 \\
863.98 \\
869.63 \\
875.27 \\
880.91 \\
886.53 \\
892.15\end{array}$ & $\begin{array}{l}10.384 \\
10.404 \\
10.424 \\
10.444 \\
10.464 \\
10.483 \\
10.503 \\
10.522 \\
10.541 \\
10.560\end{array}$ & $\begin{array}{l}522.00 \\
524.00 \\
526.00 \\
528.00 \\
530.00 \\
532.00 \\
534.00 \\
536.00 \\
538.00 \\
540.00\end{array}$ & $\begin{array}{l}4.0850 \\
4.1004 \\
4.1158 \\
4.1311 \\
4.1465 \\
4.1619 \\
4.1773 \\
4.1927 \\
4.2080 \\
4.2234\end{array}$ & $\begin{array}{l}1749.40 \\
1756.59 \\
1763.77 \\
1770.95 \\
1778.12 \\
1785.29 \\
1792.46 \\
1799.62 \\
1806.78 \\
1813.94\end{array}$ & $\begin{array}{l}1220.25 \\
1225.44 \\
1230.62 \\
1235.81 \\
1240.99 \\
1246.17 \\
1251.34 \\
1256.51 \\
1261.68 \\
1266.85\end{array}$ & $\begin{array}{l}11.544 \\
11.558 \\
11.571 \\
11.585 \\
11.599 \\
11.612 \\
11.626 \\
11.639 \\
11.652 \\
11.666\end{array}$ \\
\hline
\end{tabular}


TEMPER- SPECIFIC ENTHALPY INTERNAL ENTROPY ATURE VOLUME (BTU/LB) ENERGY (BTU/L8-R) (R) (CUFT/LB) (8TU/L8)
TEMPER - SPECIFIC ENTHALPY ATURE VOLUME (BTU/L8) (R) (CU FT/LB)
INTERNAL ENTROPY ( $8 \mathrm{TU} / \mathrm{LB}$ )
(BTU/LB-R)

\begin{tabular}{|c|c|c|c|c|}
\hline $\begin{array}{l}122.00 \\
124.00 \\
126.00 \\
128.00 \\
130.00 \\
132.00 \\
134.00 \\
136.00 \\
138.00 \\
140.00\end{array}$ & $\begin{array}{r}.7372 \\
.7550 \\
.7727 \\
.7902 \\
.8076 \\
.8248 \\
.8420 \\
.8590 \\
.8759 \\
.8926\end{array}$ & $\begin{array}{l}234.28 \\
241.61 \\
248.88 \\
256.10 \\
263.28 \\
270.41 \\
277.52 \\
284.59 \\
291.63 \\
298.66\end{array}$ & $\begin{array}{l}125.14 \\
129.83 \\
134.49 \\
139.12 \\
143.72 \\
148.31 \\
152.87 \\
157.42 \\
161.97 \\
166.51\end{array}$ & $\begin{array}{l}5.939 \\
5.999 \\
6.057 \\
6.114 \\
6.169 \\
6.224 \\
6.277 \\
6.330 \\
6.381 \\
6.432\end{array}$ \\
\hline $\begin{array}{l}42.00 \\
44.00 \\
46.00 \\
48.00 \\
50.00 \\
52.00 \\
54.00 \\
56.00 \\
58.00 \\
60.00\end{array}$ & $\begin{array}{r}.9093 \\
.9259 \\
.9423 \\
.9587 \\
.9750 \\
.9912 \\
1.0073 \\
1.0233 \\
1.0393 \\
1.0552\end{array}$ & $\begin{array}{l}305.67 \\
312.66 \\
319.65 \\
326.63 \\
333.61 \\
340.59 \\
347.57 \\
354.56 \\
361.56 \\
368.56\end{array}$ & $\begin{array}{l}171.05 \\
175.60 \\
180.14 \\
184.70 \\
189.27 \\
193.85 \\
198.45 \\
203.06 \\
207.70 \\
212.35\end{array}$ & $\begin{array}{l}6.481 \\
6.530 \\
6.578 \\
6.626 \\
6.673 \\
6.719 \\
6.765 \\
6.810 \\
6.854 \\
6.898\end{array}$ \\
\hline $\begin{array}{l}62.00 \\
64.00 \\
66.00 \\
68.00 \\
70.00 \\
72.00 \\
74.00 \\
76.00 \\
78.00 \\
80.00\end{array}$ & $\begin{array}{l}1.0710 \\
1.0867 \\
1.1024 \\
1.1180 \\
1.1335 \\
1.1490 \\
1.1645 \\
1.1798 \\
1.1952 \\
1.2105\end{array}$ & $\begin{array}{l}375.58 \\
382.62 \\
389.67 \\
396.74 \\
403.82 \\
410.93 \\
418.06 \\
425.20 \\
432.37 \\
439.56\end{array}$ & $\begin{array}{l}217.03 \\
221.74 \\
226.47 \\
231.23 \\
236.01 \\
240.82 \\
245.67 \\
250.54 \\
255.43 \\
260.36\end{array}$ & $\begin{array}{l}6.942 \\
6.985 \\
7.028 \\
7.070 \\
7.112 \\
7.154 \\
7.195 \\
7.236 \\
7.276 \\
7.316\end{array}$ \\
\hline $\begin{array}{l}82.00 \\
84.00 \\
86.00 \\
88.00 \\
90.00 \\
92.00 \\
94.00 \\
96.00 \\
98.00 \\
00.00\end{array}$ & $\begin{array}{l}1.2257 \\
1.2408 \\
1.2560 \\
1.2710 \\
1.2861 \\
1.3010 \\
1.3160 \\
1.3309 \\
1.3458 \\
1.3606\end{array}$ & $\begin{array}{l}446.75 \\
453.94 \\
461.16 \\
468.41 \\
475.68 \\
482.98 \\
490.30 \\
497.65 \\
505.03 \\
512.44\end{array}$ & $\begin{array}{l}265.30 \\
270.25 \\
275.23 \\
280.24 \\
285.29 \\
290.37 \\
295.48 \\
300.62 \\
305.80 \\
311.01\end{array}$ & $\begin{array}{l}7.356 \\
7.395 \\
7.434 \\
7.473 \\
7.512 \\
7.550 \\
7.588 \\
7.625 \\
7.663 \\
7.700\end{array}$ \\
\hline $\begin{array}{l}02.00 \\
04.00 \\
06.00 \\
08.00 \\
10.00 \\
12.00 \\
14.00 \\
16.00 \\
18.00 \\
20.00\end{array}$ & $\begin{array}{l}1.3754 \\
1.3902 \\
1.4049 \\
1.4196 \\
1.4343 \\
1.4489 \\
1.4636 \\
1.4782 \\
1.4927 \\
1.5073\end{array}$ & $\begin{array}{l}519.87 \\
527.33 \\
534.82 \\
542.33 \\
549.87 \\
557.43 \\
565.02 \\
572.63 \\
580.26 \\
587.91\end{array}$ & $\begin{array}{l}316.25 \\
321.53 \\
326.83 \\
332.17 \\
337.53 \\
342.93 \\
348.35 \\
353.80 \\
359.27 \\
364.77\end{array}$ & $\begin{array}{l}7.737 \\
7.774 \\
7.810 \\
7.847 \\
7.883 \\
7.919 \\
7.954 \\
7.990 \\
8.025 \\
8.060\end{array}$ \\
\hline $\begin{array}{l}22.00 \\
24.00 \\
26.00 \\
28.00 \\
30.00 \\
32.00 \\
34.00 \\
36.00 \\
38.00 \\
40.00\end{array}$ & $\begin{array}{l}1.5218 \\
1.5363 \\
1.5508 \\
1.5652 \\
1.5796 \\
1.5941 \\
1.6084 \\
1.6228 \\
1.6372 \\
1.6515\end{array}$ & $\begin{array}{l}595.59 \\
603.28 \\
611.00 \\
618.74 \\
626.50 \\
634.27 \\
642.07 \\
649.88 \\
657.71 \\
665.56\end{array}$ & $\begin{array}{l}370.30 \\
375.85 \\
381.42 \\
387.02 \\
392.64 \\
398.29 \\
403.95 \\
409.64 \\
415.34 \\
421.07\end{array}$ & $\begin{array}{l}8.094 \\
8.129 \\
8.163 \\
8.197 \\
8.231 \\
8.265 \\
8.298 \\
8.332 \\
8.365 \\
8.397\end{array}$ \\
\hline $\begin{array}{l}2.00 \\
4.00 \\
6.00 \\
8.00 \\
0.00 \\
2.00 \\
4.00 \\
6.00 \\
8.00 \\
0.00\end{array}$ & $\begin{array}{l}1.6659 \\
1.6802 \\
1.6945 \\
1.7087 \\
1.7230 \\
1.7372 \\
1.7515 \\
1.7657 \\
1.7799 \\
1.7941\end{array}$ & $\begin{array}{l}673.43 \\
681.31 \\
689.20 \\
697.11 \\
705.04 \\
712.98 \\
720.93 \\
728.89 \\
736.87 \\
744.86\end{array}$ & $\begin{array}{l}426.81 \\
432.57 \\
438.35 \\
444.15 \\
449.96 \\
455.79 \\
461.64 \\
467.50 \\
473.37 \\
479.26\end{array}$ & $\begin{array}{l}8.430 \\
8.463 \\
8.495 \\
8.527 \\
8.559 \\
8.590 \\
8.622 \\
8.653 \\
8.684 \\
8.715\end{array}$ \\
\hline
\end{tabular}


TEMPER- SPECIFIC ENTHALPY INTERNAL ENTROPY ATURE VDLUME (BTU/L8) ENERGY (BTU/L8-R) (R) (CU FT/LB) $\begin{array}{ccccc}\text { TEMPER- } & \text { SPECIFIC } & \text { ENTHALPY } & \text { INTERNAL } & \text { ENTROPY } \\ \text { ATURE } & \text { VOLUME } & (8 T U / L 8) & \text { ENERGY } & \text { (8TU/L8-R) } \\ (R) & (C U F T / L 8) & & \text { (BTU/L8) } & \end{array}$

\begin{tabular}{|c|c|c|c|c|c|c|c|c|c|}
\hline $\begin{array}{l}262.00 \\
264.00 \\
266.00 \\
268.00 \\
270.00 \\
272.00 \\
274.00 \\
276.00 \\
278.00 \\
280.00\end{array}$ & $\begin{array}{l}1.8083 \\
1.8224 \\
1.8366 \\
1.8507 \\
1.8648 \\
1.8789 \\
1.8930 \\
1.9071 \\
1.9212 \\
1.9352\end{array}$ & $\begin{array}{l}752.85 \\
760.86 \\
768.88 \\
776.91 \\
784.94 \\
792.96 \\
800.99 \\
809.02 \\
817.05 \\
825.09\end{array}$ & $\begin{array}{l}485.16 \\
491.07 \\
496.99 \\
502.93 \\
508.87 \\
514.80 \\
520.74 \\
526.68 \\
532.64 \\
538.60\end{array}$ & $\begin{array}{l}8.745 \\
8.776 \\
8.806 \\
8.836 \\
8.866 \\
8.896 \\
8.925 \\
8.954 \\
8.983 \\
9.012\end{array}$ & $\begin{array}{l}402.00 \\
404.00 \\
406.00 \\
408.00 \\
410.00 \\
412.00 \\
414.00 \\
416.00 \\
418.00 \\
420.00\end{array}$ & $\begin{array}{l}2.7770 \\
2.7906 \\
2.8042 \\
2.8179 \\
2.8315 \\
2.8451 \\
2.8587 \\
2.8723 \\
2.8859 \\
2.8995\end{array}$ & $\begin{array}{l}1307.51 \\
1315.12 \\
1322.73 \\
1330.32 \\
1337.91 \\
1345.48 \\
1353.05 \\
1360.62 \\
1368.18 \\
1375.73\end{array}$ & $\begin{array}{l}896.40 \\
902.00 \\
907.58 \\
913.16 \\
918.73 \\
924.29 \\
929.84 \\
935.40 \\
940.94 \\
946.48\end{array}$ & $\begin{array}{l}10.445 \\
10.464 \\
10.482 \\
10.501 \\
10.520 \\
10.538 \\
10.556 \\
10.575 \\
10.593 \\
10.611\end{array}$ \\
\hline $\begin{array}{l}282.00 \\
284.00 \\
286.00 \\
288.00 \\
290.00 \\
292.00 \\
294.00 \\
296.00 \\
298.00 \\
300.00\end{array}$ & $\begin{array}{l}1.9493 \\
1.9633 \\
1.9773 \\
1.9913 \\
2.0053 \\
2.0193 \\
2.0333 \\
2.0472 \\
2.0612 \\
2.0751\end{array}$ & $\begin{array}{l}833.14 \\
841.18 \\
849.24 \\
857.29 \\
865.35 \\
873.41 \\
881.47 \\
889.53 \\
897.60 \\
905.66\end{array}$ & $\begin{array}{l}544.56 \\
550.53 \\
556.51 \\
562.49 \\
568.48 \\
574.47 \\
580.46 \\
586.45 \\
592.45 \\
598.45\end{array}$ & $\begin{array}{l}9.041 \\
9.069 \\
9.097 \\
9.125 \\
9.153 \\
9.181 \\
9.209 \\
9.236 \\
9.263 \\
9.290\end{array}$ & $\begin{array}{l}422.00 \\
424.00 \\
426.00 \\
428.00 \\
430.00 \\
432.00 \\
434.00 \\
436.00 \\
438.00 \\
440.00\end{array}$ & $\begin{array}{l}2.9131 \\
2.9267 \\
2.9403 \\
2.9539 \\
2.9675 \\
2.9810 \\
2.9946 \\
3.0082 \\
3.0218 \\
3.0353\end{array}$ & $\begin{array}{l}1383.28 \\
1390.81 \\
1398.34 \\
1405.86 \\
1413.37 \\
1420.87 \\
1428.36 \\
1435.84 \\
1443.32 \\
1450.78\end{array}$ & $\begin{array}{r}952.01 \\
957.53 \\
963.05 \\
968.56 \\
974.05 \\
979.54 \\
985.03 \\
990.50 \\
995.97 \\
1001.43\end{array}$ & $\begin{array}{l}10.629 \\
10.647 \\
10.664 \\
10.682 \\
10.699 \\
10.717 \\
10.734 \\
10.751 \\
10.768 \\
10.785\end{array}$ \\
\hline $\begin{array}{l}302.00 \\
304.00 \\
306.00 \\
308.00 \\
310.00 \\
312.00 \\
314.00 \\
316.00 \\
318.00 \\
320.00\end{array}$ & $\begin{array}{l}2.0891 \\
2.1030 \\
2.1169 \\
2.1308 \\
2.1447 \\
2.1586 \\
2.1725 \\
2.1863 \\
2.2002 \\
2.2140\end{array}$ & $\begin{array}{l}913.72 \\
921.78 \\
929.84 \\
937.90 \\
945.96 \\
954.02 \\
962.07 \\
970.11 \\
978.16 \\
986.20\end{array}$ & $\begin{array}{l}604.45 \\
610.45 \\
616.45 \\
622.46 \\
628.46 \\
634.45 \\
640.45 \\
646.45 \\
652.44 \\
658.42\end{array}$ & $\begin{array}{l}9.317 \\
9.343 \\
9.370 \\
9.396 \\
9.422 \\
9.448 \\
9.474 \\
9.499 \\
9.525 \\
9.550\end{array}$ & $\begin{array}{l}442.00 \\
444.00 \\
446.00 \\
448.00 \\
450.00 \\
452.00 \\
454.00 \\
456.00 \\
458.00 \\
460.00\end{array}$ & $\begin{array}{l}3.0489 \\
3.0625 \\
3.0760 \\
3.0896 \\
3.1031 \\
3.1167 \\
3.1302 \\
3.1437 \\
3.1573 \\
3.1708\end{array}$ & $\begin{array}{l}1458.24 \\
1465.69 \\
1473.13 \\
1480.56 \\
1487.99 \\
1495.42 \\
1502.84 \\
1510.25 \\
1517.66 \\
1525.05\end{array}$ & $\begin{array}{l}1006.88 \\
1012.32 \\
1017.75 \\
1023.18 \\
1028.59 \\
1034.02 \\
1039.44 \\
1044.85 \\
1050.25 \\
1055.64\end{array}$ & $\begin{array}{l}10.802 \\
10.819 \\
10.836 \\
10.852 \\
10.869 \\
10.885 \\
10.902 \\
10.918 \\
10.934 \\
10.950\end{array}$ \\
\hline $\begin{array}{l}322.00 \\
324.00 \\
326.00 \\
328.00 \\
330.00 \\
332.00 \\
334.00 \\
336.00 \\
338.00 \\
340.00\end{array}$ & $\begin{array}{l}2.2279 \\
2.2417 \\
2.2555 \\
2.2694 \\
2.2832 \\
2.2970 \\
2.3108 \\
2.3246 \\
2.3384 \\
2.3522\end{array}$ & $\begin{array}{r}994.23 \\
1002.26 \\
1010.28 \\
1018.29 \\
1026.30 \\
1034.30 \\
1042.30 \\
1050.28 \\
1058.26 \\
1066.22\end{array}$ & $\begin{array}{l}664.41 \\
670.39 \\
676.36 \\
682.33 \\
688.29 \\
694.25 \\
700.20 \\
706.14 \\
712.08 \\
718.00\end{array}$ & $\begin{array}{l}9.575 \\
9.600 \\
9.624 \\
9.649 \\
9.673 \\
9.697 \\
9.721 \\
9.745 \\
9.769 \\
9.793\end{array}$ & $\begin{array}{l}462.00 \\
464.00 \\
466.00 \\
468.00 \\
470.00 \\
472.00 \\
474.00 \\
476.00 \\
478.00 \\
480.00\end{array}$ & $\begin{array}{l}3.1843 \\
3.1979 \\
3.2114 \\
3.2249 \\
3.2385 \\
3.2520 \\
3.2655 \\
3.2790 \\
3.2925 \\
3.3060\end{array}$ & $\begin{array}{l}1532.44 \\
1539.82 \\
1547.19 \\
1554.55 \\
1561.90 \\
1569.25 \\
1576.58 \\
1583.91 \\
1591.23 \\
1598.55\end{array}$ & $\begin{array}{l}1061.02 \\
1066.40 \\
1071.77 \\
1077.12 \\
1082.48 \\
1087.82 \\
1093.15 \\
1098.48 \\
1103.80 \\
1109.11\end{array}$ & $\begin{array}{l}10.967 \\
10.982 \\
10.998 \\
11.014 \\
11.030 \\
11.045 \\
11.061 \\
11.076 \\
11.092 \\
11.107\end{array}$ \\
\hline $\begin{array}{l}342.00 \\
344.00 \\
346.00 \\
348.00 \\
350.00 \\
352.00 \\
354.00 \\
356.00 \\
358.00 \\
360.00\end{array}$ & $\begin{array}{l}2.3660 \\
2.3797 \\
2.3935 \\
2.4073 \\
2.4210 \\
2.4348 \\
2.4485 \\
2.4623 \\
2.4760 \\
2.4897\end{array}$ & $\begin{array}{l}1074.18 \\
1082.13 \\
1090.07 \\
1098.00 \\
1105.91 \\
1113.82 \\
1121.71 \\
1129.60 \\
1137.47 \\
1145.33\end{array}$ & $\begin{array}{l}723.92 \\
729.83 \\
735.73 \\
741.62 \\
747.50 \\
753.37 \\
759.23 \\
765.08 \\
770.92 \\
776.74\end{array}$ & $\begin{array}{r}9.816 \\
9.839 \\
9.862 \\
9.885 \\
9.908 \\
9.930 \\
9.952 \\
9.975 \\
9.997 \\
10.019\end{array}$ & $\begin{array}{l}482.00 \\
484.00 \\
486.00 \\
488.00 \\
490.00 \\
492.00 \\
494.00 \\
496.00 \\
498.00 \\
500.00\end{array}$ & $\begin{array}{l}3.3195 \\
3.3331 \\
3.3466 \\
3.3601 \\
3.3736 \\
3.3871 \\
3.4006 \\
3.4141 \\
3.4276 \\
3.4411\end{array}$ & $\begin{array}{l}1605.85 \\
1613.15 \\
1620.44 \\
1627.72 \\
1635.00 \\
1642.26 \\
1649.53 \\
1656.78 \\
1664.03 \\
1671.28\end{array}$ & $\begin{array}{l}1114.42 \\
1119.71 \\
1125.00 \\
1130.29 \\
1135.56 \\
1140.83 \\
1146.10 \\
1151.36 \\
1156.61 \\
1161.86\end{array}$ & $\begin{array}{l}11.122 \\
11.137 \\
11.152 \\
11.167 \\
11.182 \\
11.197 \\
11.212 \\
11.226 \\
11.241 \\
11.255\end{array}$ \\
\hline $\begin{array}{l}362.00 \\
364.00 \\
366.00 \\
368.00 \\
370.00 \\
372.00 \\
374.00 \\
376.00 \\
378.00 \\
380.00\end{array}$ & $\begin{array}{l}2.5035 \\
2.5172 \\
2.5309 \\
2.5446 \\
2.5583 \\
2.5720 \\
2.5857 \\
2.5994 \\
2.6131 \\
2.6267\end{array}$ & $\begin{array}{l}1153.17 \\
1160.99 \\
1168.80 \\
1176.60 \\
1184.39 \\
1192.17 \\
1199.93 \\
1207.69 \\
1215.43 \\
1223.16\end{array}$ & $\begin{array}{l}782.55 \\
788.34 \\
794.12 \\
799.89 \\
805.65 \\
811.40 \\
817.14 \\
822.87 \\
828.58 \\
834.29\end{array}$ & $\begin{array}{l}10.040 \\
10.062 \\
10.083 \\
10.105 \\
10.126 \\
10.147 \\
10.167 \\
10.188 \\
10.209 \\
10.229\end{array}$ & $\begin{array}{l}502.00 \\
504.00 \\
506.00 \\
508.00 \\
510.00 \\
512.00 \\
514.00 \\
516.00 \\
518.00 \\
520.00\end{array}$ & $\begin{array}{l}3.4545 \\
3.4680 \\
3.4815 \\
3.4950 \\
3.5085 \\
3.5220 \\
3.5355 \\
3.5490 \\
3.5624 \\
3.5759\end{array}$ & $\begin{array}{l}1678.52 \\
1685.75 \\
1692.98 \\
1700.20 \\
1707.42 \\
1714.64 \\
1721.85 \\
1729.05 \\
1736.26 \\
1743.46\end{array}$ & $\begin{array}{l}1167.10 \\
1172.33 \\
1177.57 \\
1182.79 \\
1188.02 \\
1193.23 \\
1198.45 \\
1203.66 \\
1208.87 \\
1214.07\end{array}$ & $\begin{array}{l}11.270 \\
11.284 \\
11.298 \\
11.313 \\
11.327 \\
11.341 \\
11.355 \\
11.369 \\
11.383 \\
11.397\end{array}$ \\
\hline $\begin{array}{l}382.00 \\
384.00 \\
386.00 \\
388.00 \\
390.00 \\
392.00 \\
394.00 \\
396.00 \\
398.00 \\
400.00\end{array}$ & $\begin{array}{l}2.6404 \\
2.6541 \\
2.6678 \\
2.6814 \\
2.6951 \\
2.7087 \\
2.7224 \\
2.7361 \\
2.7497 \\
2.7633\end{array}$ & $\begin{array}{l}1230.88 \\
1238.59 \\
1246.29 \\
1253.97 \\
1261.65 \\
1269.32 \\
1276.98 \\
1284.62 \\
1292.26 \\
1299.89\end{array}$ & $\begin{array}{l}839.98 \\
845.67 \\
851.34 \\
857.01 \\
862.66 \\
868.31 \\
873.95 \\
879.57 \\
885.19 \\
890.80\end{array}$ & $\begin{array}{l}10.249 \\
10.269 \\
10.289 \\
10.309 \\
10.329 \\
10.349 \\
10.368 \\
10.387 \\
10.407 \\
10.426\end{array}$ & $\begin{array}{l}522.00 \\
524.00 \\
526.00 \\
528.00 \\
530.00 \\
532.00 \\
534.00 \\
536.00 \\
538.00 \\
540.00\end{array}$ & $\begin{array}{l}3.5894 \\
3.6029 \\
3.6163 \\
3.6298 \\
3.6433 \\
3.6568 \\
3.6702 \\
3.6837 \\
3.6972 \\
3.7106\end{array}$ & $\begin{array}{l}1750.65 \\
1757.84 \\
1765.03 \\
1772.22 \\
1779.40 \\
1786.58 \\
1793.75 \\
1800.93 \\
1808.10 \\
1815.26\end{array}$ & $\begin{array}{l}1219.27 \\
1224.47 \\
1229.66 \\
1234.85 \\
1240.04 \\
1245.22 \\
1250.40 \\
1255.58 \\
1260.76 \\
1265.93\end{array}$ & $\begin{array}{l}11.411 \\
11.424 \\
11.438 \\
11.452 \\
11.465 \\
11.479 \\
11.492 \\
11.506 \\
11.519 \\
11.532\end{array}$ \\
\hline
\end{tabular}


TEMPER- SPECIFIC ENTHALPY INTERNAL ENTROPY ENERGY (BTU/LB-RI (R) (CU FT/LB) (BTU/L8)
TEMPERATURE (R)
SPECIFIC VOLUME (CU FT/LB)
ENTHALPY $(8 T U / L 8)$
INTERNAL ENTROPY ENERGY (BTU/LB-R) (8TU/L8)

\begin{tabular}{|c|c|c|c|c|}
\hline $\begin{array}{l}36.00 \\
38.00 \\
40.00\end{array}$ & $\begin{array}{l}.2074 \\
.2097 \\
.2121\end{array}$ & $\begin{array}{l}-84.42 \\
-80.51 \\
-76.40\end{array}$ & $\begin{array}{r}-118.96 \\
-115.43 \\
-111.73\end{array}$ & $\begin{array}{l}1.644 \\
1.750 \\
1.855\end{array}$ \\
\hline $\begin{array}{l}42.00 \\
44.00 \\
46.00 \\
48.00 \\
50.00 \\
52.00 \\
54.00 \\
56.00 \\
58.00 \\
60.00\end{array}$ & $\begin{array}{l}.2148 \\
.2176 \\
.2206 \\
.2238 \\
.2272 \\
.2308 \\
.2348 \\
.2390 \\
.2436 \\
.2491\end{array}$ & $\begin{array}{l}-72.10 \\
-67.58 \\
-62.84 \\
-57.87 \\
-52.65 \\
-47.19 \\
-41.50 \\
-35.58 \\
-29.36 \\
-22.91\end{array}$ & $\begin{array}{r}-107.87 \\
-103.81 \\
-99.57 \\
-95.13 \\
-90.49 \\
-85.63 \\
-80.60 \\
-75.39 \\
-69.93 \\
-64.40\end{array}$ & $\begin{array}{l}1.960 \\
2.065 \\
2.170 \\
2.276 \\
2.383 \\
2.490 \\
2.597 \\
2.705 \\
2.814 \\
2.924\end{array}$ \\
\hline $\begin{array}{l}62.00 \\
64.00 \\
66.00 \\
68.00 \\
70.00 \\
72.00 \\
74.00 \\
76.00 \\
78.00 \\
80.00\end{array}$ & $\begin{array}{l}.2544 \\
.2601 \\
.2664 \\
.2732 \\
.2807 \\
.2888 \\
.2976 \\
.3073 \\
.3177 \\
.3289\end{array}$ & $\begin{array}{r}-16.11 \\
-9.02 \\
-1.65 \\
5.96 \\
13.75 \\
21.52 \\
29.36 \\
37.31 \\
45.42 \\
53.70\end{array}$ & $\begin{array}{l}-58.47 \\
-52.35 \\
-46.02 \\
-39.55 \\
-32.99 \\
-26.57 \\
-20.21 \\
-13.86 \\
-7.49 \\
-1.09\end{array}$ & $\begin{array}{l}3.036 \\
3.149 \\
3.262 \\
3.375 \\
3.488 \\
3.598 \\
3.705 \\
3.811 \\
3.916 \\
4.021\end{array}$ \\
\hline $\begin{array}{l}82.00 \\
84.00 \\
86.00 \\
88.00 \\
90.00 \\
92.00 \\
94.00 \\
96.00 \\
98.00 \\
100.00\end{array}$ & $\begin{array}{l}.3410 \\
.3539 \\
.3676 \\
.3819 \\
.3968 \\
.4123 \\
.4281 \\
.4442 \\
.4605 \\
.4770\end{array}$ & $\begin{array}{r}62.14 \\
70.72 \\
79.42 \\
88.20 \\
97.01 \\
105.82 \\
114.60 \\
123.31 \\
131.96 \\
140.51\end{array}$ & $\begin{array}{l}5.34 \\
11.78 \\
18.20 \\
24.59 \\
30.92 \\
37.16 \\
43.30 \\
49.34 \\
55.26 \\
61.06\end{array}$ & $\begin{array}{l}4.125 \\
4.229 \\
4.331 \\
4.432 \\
4.531 \\
4.628 \\
4.722 \\
4.814 \\
4.903 \\
4.990\end{array}$ \\
\hline $\begin{array}{l}102.00 \\
104.00 \\
106.00 \\
108.00 \\
110.00 \\
112.00 \\
114.00 \\
116.00 \\
118.00 \\
120.00\end{array}$ & $\begin{array}{l}.4936 \\
.5102 \\
.5268 \\
.5434 \\
.5599 \\
.5764 \\
.5927 \\
.6090 \\
.6252 \\
.6413\end{array}$ & $\begin{array}{l}148.96 \\
157.30 \\
165.55 \\
173.68 \\
181.72 \\
189.66 \\
197.52 \\
205.28 \\
212.96 \\
220.57\end{array}$ & $\begin{array}{r}66.75 \\
72.33 \\
77.81 \\
83.19 \\
88.47 \\
93.67 \\
98.80 \\
103.85 \\
108.84 \\
113.77\end{array}$ & $\begin{array}{l}5.073 \\
5.154 \\
5.233 \\
5.309 \\
5.383 \\
5.454 \\
5.524 \\
5.591 \\
5.657 \\
5.721\end{array}$ \\
\hline
\end{tabular}

\begin{tabular}{|c|c|c|c|c|}
\hline $\begin{array}{l}122.00 \\
124.00 \\
126.00 \\
128.00 \\
130.00 \\
132.00 \\
134.00 \\
136.00 \\
138.00 \\
140.00\end{array}$ & $\begin{array}{l}.6572 \\
.6731 \\
.6888 \\
.7045 \\
.7200 \\
.7354 \\
.7508 \\
.7660 \\
.7811 \\
.7962\end{array}$ & $\begin{array}{l}228.11 \\
235.59 \\
243.00 \\
250.37 \\
257.69 \\
264.96 \\
272.20 \\
279.41 \\
286.59 \\
293.74\end{array}$ & $\begin{array}{l}118.65 \\
123.48 \\
128.28 \\
133.04 \\
137.77 \\
142.48 \\
147.16 \\
151.83 \\
156.49 \\
161.14\end{array}$ & $\begin{array}{l}5.783 \\
5.844 \\
5.903 \\
5.961 \\
6.018 \\
6.073 \\
6.128 \\
6.181 \\
6.234 \\
6.285\end{array}$ \\
\hline $\begin{array}{l}142.00 \\
144.00 \\
146.00 \\
148.00 \\
150.00 \\
152.00 \\
154.00 \\
156.00 \\
158.00 \\
160.00\end{array}$ & $\begin{array}{r}.8111 \\
.8260 \\
.8407 \\
.8554 \\
.8700 \\
.8846 \\
.8990 \\
.9134 \\
.9277 \\
.9420\end{array}$ & $\begin{array}{l}300.88 \\
308.00 \\
315.11 \\
322.21 \\
329.30 \\
336.39 \\
343.48 \\
350.58 \\
357.68 \\
364.79\end{array}$ & $\begin{array}{l}165.79 \\
170.44 \\
175.08 \\
179.74 \\
184.40 \\
189.07 \\
193.75 \\
198.45 \\
203.17 \\
207.91\end{array}$ & $\begin{array}{l}6.336 \\
6.386 \\
6.435 \\
6.483 \\
6.530 \\
6.577 \\
6.624 \\
6.670 \\
6.715 \\
6.760\end{array}$ \\
\hline $\begin{array}{l}162.00 \\
164.00 \\
166.00 \\
168.00 \\
170.00 \\
172.00 \\
174.00 \\
176.00 \\
178.00 \\
180.00\end{array}$ & $\begin{array}{r}.9562 \\
.9703 \\
.9843 \\
.9984 \\
1.0123 \\
1.0262 \\
1.0400 \\
1.0538 \\
1.0676 \\
1.0813\end{array}$ & $\begin{array}{l}371.91 \\
379.04 \\
386.19 \\
393.35 \\
400.52 \\
407.72 \\
414.93 \\
422.16 \\
429.40 \\
436.67\end{array}$ & $\begin{array}{l}212.66 \\
217.44 \\
222.25 \\
227.07 \\
231.93 \\
236.80 \\
241.71 \\
246.64 \\
251.60 \\
256.59\end{array}$ & $\begin{array}{l}6.804 \\
6.848 \\
6.891 \\
6.934 \\
6.976 \\
7.018 \\
7.060 \\
7.101 \\
7.142 \\
7.183\end{array}$ \\
\hline $\begin{array}{l}182.00 \\
184.00 \\
186.00 \\
188.00 \\
190.00 \\
192.00 \\
194.00 \\
196.00 \\
198.00 \\
200.00\end{array}$ & $\begin{array}{l}1.0949 \\
1.1085 \\
1.1221 \\
1.1356 \\
1.1491 \\
1.1625 \\
1.1759 \\
1.1893 \\
1.2026 \\
1.2159\end{array}$ & $\begin{array}{l}443.94 \\
451.19 \\
458.47 \\
465.78 \\
473.11 \\
480.47 \\
487.85 \\
495.26 \\
502.70 \\
510.16\end{array}$ & $\begin{array}{l}261.58 \\
266.57 \\
271.59 \\
276.65 \\
281.73 \\
286.85 \\
292.00 \\
297.19 \\
302.40 \\
307.65\end{array}$ & $\begin{array}{l}7.223 \\
7.263 \\
7.302 \\
7.341 \\
7.380 \\
7.418 \\
7.457 \\
7.495 \\
7.532 \\
7.570\end{array}$ \\
\hline $\begin{array}{l}202.00 \\
204.00 \\
206.00 \\
208.00 \\
210.00 \\
212.00 \\
214.00 \\
216.00 \\
218.00 \\
220.00\end{array}$ & $\begin{array}{l}1.2292 \\
1.2424 \\
1.2556 \\
1.2688 \\
1.2819 \\
1.2951 \\
1.3081 \\
1.3212 \\
1.3343 \\
1.3473\end{array}$ & $\begin{array}{l}517.65 \\
525.17 \\
532.71 \\
540.27 \\
547.87 \\
555.48 \\
563.12 \\
570.79 \\
578.47 \\
586.17\end{array}$ & $\begin{array}{l}312.93 \\
318.24 \\
323.59 \\
328.96 \\
334.36 \\
339.80 \\
345.26 \\
350.74 \\
356.25 \\
361.79\end{array}$ & $\begin{array}{l}7.607 \\
7.644 \\
7.681 \\
7.717 \\
7.754 \\
7.790 \\
7.826 \\
7.861 \\
7.897 \\
7.932\end{array}$ \\
\hline $\begin{array}{l}222.00 \\
224.00 \\
226.00 \\
228.00 \\
230.00 \\
232.00 \\
234.00 \\
236.00 \\
238.00 \\
240.00\end{array}$ & $\begin{array}{l}1.3603 \\
1.3732 \\
1.3862 \\
1.3991 \\
1.4121 \\
1.4250 \\
1.4378 \\
1.4507 \\
1.4635 \\
1.4764\end{array}$ & $\begin{array}{l}593.90 \\
601.64 \\
609.41 \\
617.19 \\
625.00 \\
632.82 \\
640.65 \\
648.51 \\
656.38 \\
664.27\end{array}$ & $\begin{array}{l}367.35 \\
372.93 \\
378.54 \\
384.17 \\
389.82 \\
395.49 \\
401.19 \\
406.90 \\
412.63 \\
418.38\end{array}$ & $\begin{array}{l}7.967 \\
8.002 \\
8.036 \\
8.070 \\
8.104 \\
8.138 \\
8.172 \\
8.205 \\
8.239 \\
8.272\end{array}$ \\
\hline $\begin{array}{l}242.00 \\
244.00 \\
246.00 \\
248.00 \\
250.00 \\
252.00 \\
254.00 \\
256.00 \\
258.00 \\
260.00\end{array}$ & $\begin{array}{l}1.4892 \\
1.5020 \\
1.5148 \\
1.5275 \\
1.5403 \\
1.5530 \\
1.5657 \\
1.5784 \\
1.5911 \\
1.6038\end{array}$ & $\begin{array}{l}672.17 \\
680.09 \\
688.02 \\
695.97 \\
703.93 \\
711.90 \\
719.88 \\
727.88 \\
735.89 \\
743.91\end{array}$ & $\begin{array}{l}424.15 \\
429.94 \\
435.74 \\
441.56 \\
447.40 \\
453.25 \\
459.11 \\
464.99 \\
470.89 \\
476.79\end{array}$ & $\begin{array}{l}8.304 \\
8.337 \\
8.369 \\
8.402 \\
8.434 \\
8.465 \\
8.497 \\
8.528 \\
8.559 \\
8.590\end{array}$ \\
\hline
\end{tabular}


TEMPER- SPECIFIC ENTHALPY INTERNAL ENTROPY ATURE VOLUME (BTU/LB) ENERGY (8TU/L8-R) (R) (CU FT/LB)
(BTU/LB)
TEMPER- SPECIFIC ENTHALPY INTERNAL ENTROPY ATURE VOLUME (8TU/L8) ENERGY (8TU/L8-R) (R) $(C U F T / L B)$
$(8 T U / L 8)$

\begin{tabular}{|c|c|c|c|c|c|c|c|c|c|}
\hline $\begin{array}{l}262.00 \\
264.00 \\
266.00 \\
268.00 \\
270.00 \\
272.00 \\
274.00 \\
276.00 \\
278.00 \\
280.00\end{array}$ & $\begin{array}{l}1.6165 \\
1.6291 \\
1.6418 \\
1.6544 \\
1.6670 \\
1.6796 \\
1.6922 \\
1.7048 \\
1.7174 \\
1.7299\end{array}$ & $\begin{array}{l}751.93 \\
759.97 \\
768.02 \\
776.07 \\
784.14 \\
792.19 \\
800.24 \\
808.30 \\
816.36 \\
824.43\end{array}$ & $\begin{array}{l}482.71 \\
488.64 \\
494.58 \\
500.54 \\
506.50 \\
512.45 \\
518.40 \\
524.37 \\
530.34 \\
536.31\end{array}$ & $\begin{array}{l}8.621 \\
8.652 \\
8.682 \\
8.712 \\
8.742 \\
8.772 \\
8.801 \\
8.831 \\
8.860 \\
8.889\end{array}$ & $\begin{array}{l}402.00 \\
404.00 \\
406.00 \\
408.00 \\
410.00 \\
412.00 \\
414.00 \\
416.00 \\
418.00 \\
420.00\end{array}$ & $\begin{array}{l}2.4806 \\
2.4928 \\
2.5049 \\
2.5170 \\
2.5292 \\
2.5413 \\
2.5534 \\
2.5656 \\
2.5777 \\
2.5898\end{array}$ & $\begin{array}{l}1308.17 \\
1315.78 \\
1323.39 \\
1330.99 \\
1338.58 \\
1346.16 \\
1353.74 \\
1361.31 \\
1368.88 \\
1376.45\end{array}$ & $\begin{array}{l}895.03 \\
900.62 \\
906.20 \\
911.78 \\
917.35 \\
922.91 \\
928.47 \\
934.03 \\
939.58 \\
945.12\end{array}$ & $\begin{array}{l}10.325 \\
10.344 \\
10.363 \\
10.382 \\
10.400 \\
10.419 \\
10.437 \\
10.455 \\
10.474 \\
10.492\end{array}$ \\
\hline $\begin{array}{l}282.00 \\
284.00 \\
286.00 \\
288.00 \\
290.00 \\
292.00 \\
294.00 \\
296.00 \\
298.00 \\
300.00\end{array}$ & $\begin{array}{l}1.7425 \\
1.7550 \\
1.7675 \\
1.7800 \\
1.7925 \\
1.8050 \\
1.8175 \\
1.8299 \\
1.8424 \\
1.8548\end{array}$ & $\begin{array}{l}832.50 \\
840.58 \\
848.66 \\
856.74 \\
864.83 \\
872.92 \\
881.01 \\
889.11 \\
897.20 \\
905.29\end{array}$ & $\begin{array}{l}542.30 \\
548.29 \\
554.28 \\
560.29 \\
566.29 \\
572.30 \\
578.32 \\
584.33 \\
590.35 \\
596.37\end{array}$ & $\begin{array}{l}8.917 \\
8.946 \\
8.974 \\
9.002 \\
9.030 \\
9.058 \\
9.086 \\
9.113 \\
9.141 \\
9.168\end{array}$ & $\begin{array}{l}422.00 \\
424.00 \\
426.00 \\
428.00 \\
430.00 \\
432.00 \\
434.00 \\
436.00 \\
438.00 \\
440.00\end{array}$ & $\begin{array}{l}2.6019 \\
2.6140 \\
2.6261 \\
2.6382 \\
2.6503 \\
2.6624 \\
2.6745 \\
2.6866 \\
2.6987 \\
2.7107\end{array}$ & $\begin{array}{l}1384.00 \\
1391.55 \\
1399.09 \\
1406.62 \\
1414.14 \\
1421.65 \\
1429.16 \\
1436.66 \\
1444.15 \\
1451.63\end{array}$ & $\begin{array}{r}950.66 \\
956.19 \\
961.71 \\
967.23 \\
972.74 \\
978.24 \\
983.73 \\
989.21 \\
994.69 \\
1000.16\end{array}$ & $\begin{array}{l}10.510 \\
10.527 \\
10.545 \\
10.563 \\
10.580 \\
10.598 \\
10.615 \\
10.632 \\
10.649 \\
10.667\end{array}$ \\
\hline $\begin{array}{l}302.00 \\
304.00 \\
306.00 \\
308.00 \\
310.00 \\
312.00 \\
314.00 \\
316.00 \\
318.00 \\
320.00\end{array}$ & $\begin{array}{l}1.8673 \\
1.8797 \\
1.8921 \\
1.9045 \\
1.9169 \\
1.9293 \\
1.9417 \\
1.9541 \\
1.9664 \\
1.9788\end{array}$ & $\begin{array}{l}913.39 \\
921.48 \\
929.58 \\
937.67 \\
945.76 \\
953.84 \\
961.93 \\
970.01 \\
978.09 \\
986.16\end{array}$ & $\begin{array}{l}602.40 \\
608.42 \\
614.45 \\
620.47 \\
626.50 \\
632.52 \\
638.54 \\
644.56 \\
650.58 \\
656.59\end{array}$ & $\begin{array}{l}9.195 \\
9.221 \\
9.248 \\
9.274 \\
9.300 \\
9.326 \\
9.352 \\
9.378 \\
9.403 \\
9.429\end{array}$ & $\begin{array}{l}442.00 \\
444.00 \\
446.00 \\
448.00 \\
450.00 \\
452.00 \\
454.00 \\
456.00 \\
458.00 \\
460.00\end{array}$ & $\begin{array}{l}2.7228 \\
2.7349 \\
2.7470 \\
2.7590 \\
2.7711 \\
2.7832 \\
2.7952 \\
2.8073 \\
2.8193 \\
2.8314\end{array}$ & $\begin{array}{l}1459.10 \\
1466.56 \\
1474.02 \\
1481.46 \\
1488.90 \\
1496.35 \\
1503.78 \\
1511.21 \\
1518.63 \\
1526.04\end{array}$ & $\begin{array}{l}1005.62 \\
1011.07 \\
1016.52 \\
1021.95 \\
1027.38 \\
1032.82 \\
1038.24 \\
1043.66 \\
1049.07 \\
1054.47\end{array}$ & $\begin{array}{l}10.683 \\
10.700 \\
10.717 \\
10.734 \\
10.750 \\
10.767 \\
10.783 \\
10.800 \\
10.816 \\
10.832\end{array}$ \\
\hline $\begin{array}{l}322.00 \\
324.00 \\
326.00 \\
328.00 \\
330.00 \\
332.00 \\
334.00 \\
336.00 \\
338.00 \\
340.00\end{array}$ & $\begin{array}{l}1.9911 \\
2.0035 \\
2.0158 \\
2.0281 \\
2.0405 \\
2.0528 \\
2.0651 \\
2.0774 \\
2.0897 \\
2.1020\end{array}$ & $\begin{array}{r}994.22 \\
1002.29 \\
1010.34 \\
1018.39 \\
1026.43 \\
1034.46 \\
1042.49 \\
1050.50 \\
1058.51 \\
1066.51\end{array}$ & $\begin{array}{l}662.60 \\
668.61 \\
674.61 \\
680.60 \\
686.59 \\
692.58 \\
698.55 \\
704.52 \\
710.47 \\
716.42\end{array}$ & $\begin{array}{l}9.454 \\
9.479 \\
9.503 \\
9.528 \\
9.552 \\
9.577 \\
9.601 \\
9.625 \\
9.649 \\
9.672\end{array}$ & $\begin{array}{l}462.00 \\
464.00 \\
466.00 \\
468.00 \\
470.00 \\
472.00 \\
474.00 \\
476.00 \\
478.00 \\
480.00\end{array}$ & $\begin{array}{l}2.8434 \\
2.8555 \\
2.8675 \\
2.8796 \\
2.8916 \\
2.9037 \\
2.9157 \\
2.9277 \\
2.9398 \\
2.9518\end{array}$ & $\begin{array}{l}1533.44 \\
1540.83 \\
1548.21 \\
1555.58 \\
1562.95 \\
1570.31 \\
1577.65 \\
1584.99 \\
1592.32 \\
1599.64\end{array}$ & $\begin{array}{l}1059.87 \\
1065.25 \\
1070.63 \\
1076.00 \\
1081.36 \\
1086.71 \\
1092.05 \\
1097.38 \\
1102.71 \\
1108.03\end{array}$ & $\begin{array}{l}10.848 \\
10.864 \\
10.880 \\
10.896 \\
10.911 \\
10.927 \\
10.942 \\
10.958 \\
10.973 \\
10.989\end{array}$ \\
\hline $\begin{array}{l}342.00 \\
344.00 \\
346.00 \\
348.00 \\
350.00 \\
352.00 \\
354.00 \\
356.00 \\
358.00 \\
360.00\end{array}$ & $\begin{array}{l}2.1143 \\
2.1266 \\
2.1388 \\
2.1511 \\
2.1634 \\
2.1756 \\
2.1879 \\
2.2002 \\
2.2124 \\
2.2246\end{array}$ & $\begin{array}{l}1074.49 \\
1082.47 \\
1090.44 \\
1098.39 \\
1106.33 \\
1114.26 \\
1122.18 \\
1130.09 \\
1137.98 \\
1145.86\end{array}$ & $\begin{array}{l}722.37 \\
728.30 \\
734.22 \\
740.13 \\
746.03 \\
751.91 \\
757.79 \\
763.66 \\
769.51 \\
775.35\end{array}$ & $\begin{array}{l}9.696 \\
9.719 \\
9.742 \\
9.765 \\
9.788 \\
9.810 \\
9.833 \\
9.855 \\
9.877 \\
9.899\end{array}$ & $\begin{array}{l}482.00 \\
484.00 \\
486.00 \\
488.00 \\
490.00 \\
492.00 \\
494.00 \\
496.00 \\
498.00 \\
500.00\end{array}$ & $\begin{array}{l}2.9638 \\
2.9758 \\
2.9879 \\
2.9999 \\
3.0119 \\
3.0239 \\
3.0360 \\
3.0480 \\
3.0600 \\
3.0720\end{array}$ & $\begin{array}{l}1606.96 \\
1614.26 \\
1621.56 \\
1628.85 \\
1636.14 \\
1643.41 \\
1650.68 \\
1657.95 \\
1665.20 \\
1672.46\end{array}$ & $\begin{array}{l}1113.34 \\
1118.64 \\
1123.94 \\
1129.23 \\
1134.51 \\
1139.78 \\
1145.05 \\
1150.32 \\
1155.57 \\
1160.82\end{array}$ & $\begin{array}{l}11.004 \\
11.019 \\
11.034 \\
11.049 \\
11.064 \\
11.079 \\
11.093 \\
11.108 \\
11.123 \\
11.137\end{array}$ \\
\hline $\begin{array}{l}362.00 \\
364.00 \\
366.00 \\
368.00 \\
370.00 \\
372.00 \\
374.00 \\
376.00 \\
378.00 \\
380.00\end{array}$ & $\begin{array}{l}2.2369 \\
2.2491 \\
2.2613 \\
2.2735 \\
2.2858 \\
2.2980 \\
2.3102 \\
2.3224 \\
2.3346 \\
2.3468\end{array}$ & $\begin{array}{l}1153.71 \\
1161.54 \\
1169.37 \\
1177.18 \\
1184.98 \\
1192.76 \\
1200.53 \\
1208.29 \\
1216.04 \\
1223.78\end{array}$ & $\begin{array}{l}781.16 \\
786.96 \\
792.75 \\
798.53 \\
804.29 \\
810.04 \\
815.78 \\
821.51 \\
827.23 \\
832.93\end{array}$ & $\begin{array}{r}9.921 \\
9.942 \\
9.964 \\
9.985 \\
10.006 \\
10.027 \\
10.048 \\
10.069 \\
10.089 \\
10.110\end{array}$ & $\begin{array}{l}502.00 \\
504.00 \\
506.00 \\
508.00 \\
510.00 \\
512.00 \\
514.00 \\
516.00 \\
518.00 \\
520.00\end{array}$ & $\begin{array}{l}3.0840 \\
3.0960 \\
3.1080 \\
3.1200 \\
3.1320 \\
3.1440 \\
3.1560 \\
3.1680 \\
3.1800 \\
3.1920\end{array}$ & $\begin{array}{l}1679.70 \\
1686.94 \\
1694.18 \\
1701.41 \\
1708.63 \\
1715.85 \\
1723.07 \\
1730.28 \\
1737.49 \\
1744.70\end{array}$ & $\begin{array}{l}1166.07 \\
1171.31 \\
1176.54 \\
1181.77 \\
1187.00 \\
1192.22 \\
1197.44 \\
1202.66 \\
1207.87 \\
1213.08\end{array}$ & $\begin{array}{l}11.152 \\
11.166 \\
11.180 \\
11.195 \\
11.209 \\
11.223 \\
11.237 \\
11.251 \\
11.265 \\
11.279\end{array}$ \\
\hline $\begin{array}{l}382.00 \\
384.00 \\
386.00 \\
388.00 \\
390.00 \\
392.00 \\
394.00 \\
396.00 \\
398.00 \\
400.00\end{array}$ & $\begin{array}{l}2.3589 \\
2.3711 \\
2.3833 \\
2.3955 \\
2.4077 \\
2.4198 \\
2.4320 \\
2.4442 \\
2.4563 \\
2.4685\end{array}$ & $\begin{array}{l}1231.50 \\
1239.21 \\
1246.92 \\
1254.61 \\
1262.29 \\
1269.96 \\
1277.62 \\
1285.27 \\
1292.91 \\
1300.54\end{array}$ & $\begin{array}{l}838.63 \\
844.31 \\
849.98 \\
855.65 \\
861.30 \\
866.94 \\
872.58 \\
878.20 \\
883.82 \\
889.43\end{array}$ & $\begin{array}{l}10.130 \\
10.150 \\
10.170 \\
10.190 \\
10.210 \\
10.229 \\
10.249 \\
10.268 \\
10.287 \\
10.306\end{array}$ & $\begin{array}{l}522.00 \\
524.00 \\
526.00 \\
528.00 \\
530.00 \\
532.00 \\
534.00 \\
536.00 \\
538.00 \\
540.00\end{array}$ & $\begin{array}{l}3.2040 \\
3.2160 \\
3.2280 \\
3.2400 \\
3.2520 \\
3.2640 \\
3.2759 \\
3.2879 \\
3.2999 \\
3.3119 .\end{array}$ & $\begin{array}{l}1751.90 \\
1759.10 \\
1766.30 \\
1773.49 \\
1780.68 \\
1787.87 \\
1795.05 \\
1802.23 \\
1809.41 \\
1816.59\end{array}$ & $\begin{array}{l}1218.28 \\
1223.48 \\
1228.68 \\
1233.88 \\
1239.07 \\
1244.26 \\
1249.45 \\
1254.64 \\
1259.82 \\
1265.00\end{array}$ & $\begin{array}{l}11.293 \\
11.306 \\
11.320 \\
11.334 \\
11.347 \\
11.361 \\
11.374 \\
11.388 \\
11.401 \\
11.415\end{array}$ \\
\hline
\end{tabular}


$\begin{array}{ccccc}\text { TEMPER- } & \text { SPECIFIC } & \text { ENTHALPY } & \text { INTERNAL } & \text { ENTROPY } \\ \text { ATURE } & \text { VOLUME } & (8 T U / L B) & \text { ENERGY } & \text { (BTU/LB-R) }\end{array}$

(R) (CU FT/LB) (BTU/LB)
TEMPER- SPECIFIC ENTHALPY ATURE VOLUME (CU FT/LB)
INTERNAL ENTROPY (BTU/LB)

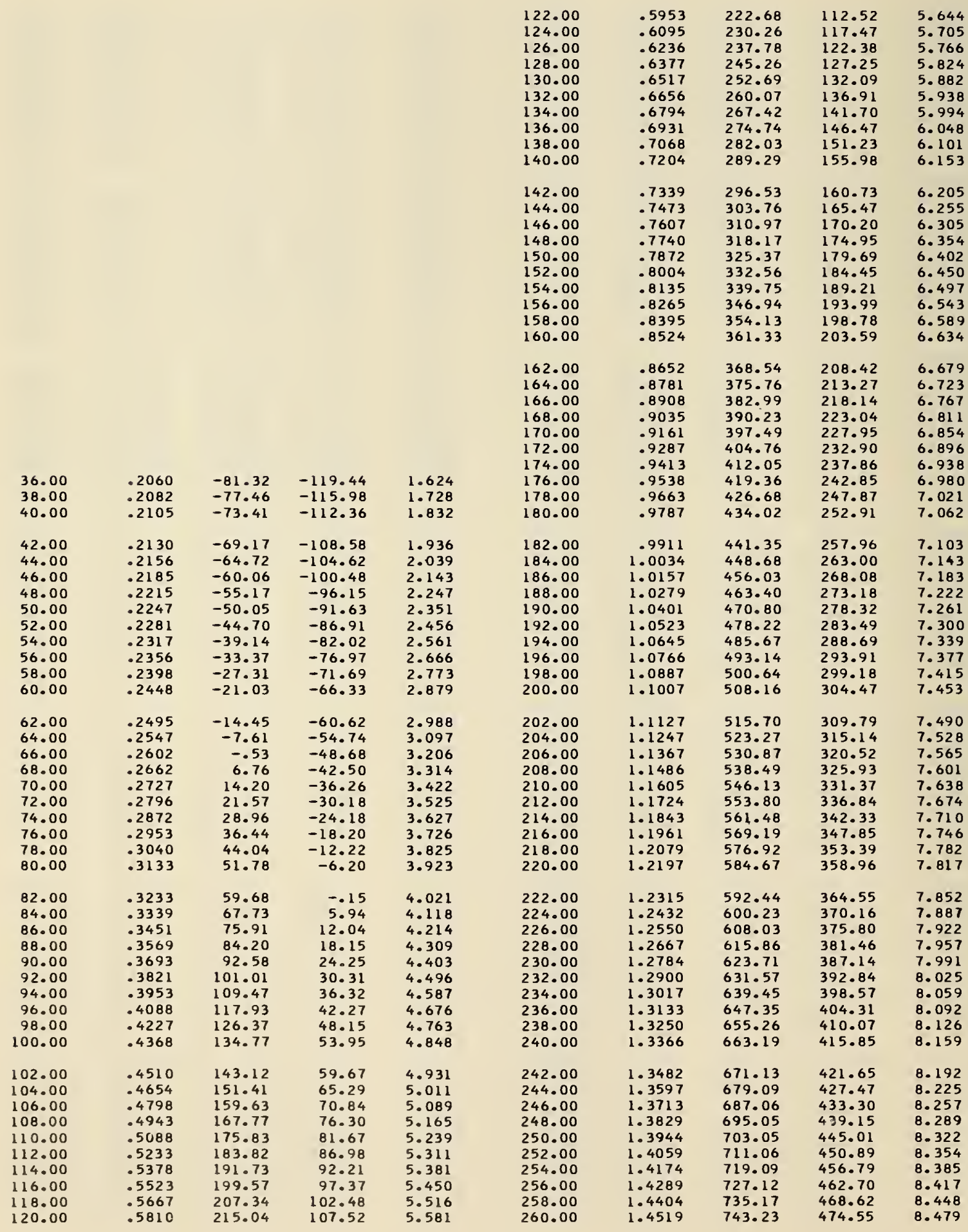


TEMPER- SPECIFIC ENTHALPY INTERNAL ENTROPY ATURE VOLUME (8TU/L8) ENERGY (8TU/L8-R) (R) (CU FT/L8) $\begin{array}{ccccc}\text { TEMPER- } & \text { SPECIFIC } & \text { ENTHALPY } & \text { INTERNAL } & \text { ENTROPY } \\ \text { ATURE } & \text { VOLUME } & \text { (8TU/L8) } & \text { ENERGY } & \text { (8TU/LL-R) } \\ \text { (R) } & \text { (CU FT/L8) } & & \text { (8TU/LB) } & \end{array}$

\begin{tabular}{|c|c|c|c|c|c|c|c|c|c|}
\hline $\begin{array}{l}262.00 \\
264.00 \\
266.00 \\
268.00 \\
270.00 \\
272.00 \\
274.00 \\
276.00 \\
278.00 \\
280.00\end{array}$ & $\begin{array}{l}1.4633 \\
1.4748 \\
1.4862 \\
1.4976 \\
1.5090 \\
1.5204 \\
1.5318 \\
1.5432 \\
1.5545 \\
1.5659\end{array}$ & $\begin{array}{l}751.29 \\
759.37 \\
767.45 \\
775.55 \\
783.65 \\
791.73 \\
799.82 \\
807.91 \\
816.01 \\
824.11\end{array}$ & $\begin{array}{l}480.50 \\
486.46 \\
492.43 \\
498.41 \\
504.40 \\
510.37 \\
516.35 \\
522.34 \\
528.33 \\
534.33\end{array}$ & $\begin{array}{l}8.510 \\
8.541 \\
8.571 \\
8.602 \\
8.632 \\
8.662 \\
8.691 \\
8.721 \\
8.750 \\
8.779\end{array}$ & $\begin{array}{l}402.00 \\
404.00 \\
406.00 \\
408.00 \\
410.00 \\
412.00 \\
414.00 \\
416.00 \\
418.00 \\
420.00\end{array}$ & $\begin{array}{l}2.2436 \\
2.2545 \\
2.2655 \\
2.2764 \\
2.2874 \\
2.2983 \\
2.3093 \\
2.3202 \\
2.3311 \\
2.3420\end{array}$ & $\begin{array}{l}1308.93 \\
1316.57 \\
1324.20 \\
1331.82 \\
1339.43 \\
1347.04 \\
1354.64 \\
1362.23 \\
1369.82 \\
1377.40\end{array}$ & $\begin{array}{l}893.75 \\
899.36 \\
904.96 \\
910.56 \\
916.15 \\
921.73 \\
927.30 \\
932.87 \\
938.44 \\
943.99\end{array}$ & $\begin{array}{l}10.219 \\
10.238 \\
10.257 \\
10.275 \\
10.294 \\
10.312 \\
10.331 \\
10.349 \\
10.367 \\
10.385\end{array}$ \\
\hline $\begin{array}{l}282.00 \\
284.00 \\
286.00 \\
288.00 \\
290.00 \\
292.00 \\
294.00 \\
296.00 \\
298.00 \\
300.00\end{array}$ & $\begin{array}{l}1.5772 \\
1.5886 \\
1.5999 \\
1.6112 \\
1.6225 \\
1.6338 \\
1.6450 \\
1.6563 \\
1.6675 \\
1.6788\end{array}$ & $\begin{array}{l}832.21 \\
840.32 \\
848.43 \\
856.54 \\
864.65 \\
872.77 \\
880.88 \\
889.00 \\
897.12 \\
905.23\end{array}$ & $\begin{array}{l}540.34 \\
546.35 \\
552.37 \\
558.39 \\
564.41 \\
570.44 \\
576.47 \\
582.50 \\
588.53 \\
594.57\end{array}$ & $\begin{array}{l}8.808 \\
8.836 \\
8.865 \\
8.893 \\
8.921 \\
8.949 \\
8.977 \\
9.004 \\
9.032 \\
9.059\end{array}$ & $\begin{array}{l}422.00 \\
424.00 \\
426.00 \\
428.00 \\
430.00 \\
432.00 \\
434.00 \\
436.00 \\
438.00 \\
440.00\end{array}$ & $\begin{array}{l}2.3530 \\
2.3639 \\
2.3748 \\
2.3857 \\
2.3966 \\
2.4075 \\
2.4184 \\
2.4293 \\
2.4402 \\
2.4511\end{array}$ & $\begin{array}{l}1384.97 \\
1392.53 \\
1400.08 \\
1407.62 \\
1415.16 \\
1422.69 \\
1430.20 \\
1437.71 \\
1445.21 \\
1452.70\end{array}$ & $\begin{array}{l}949.54 \\
955.08 \\
960.62 \\
966.14 \\
971.66 \\
977.17 \\
982.67 \\
988.16 \\
993.64 \\
999.12\end{array}$ & $\begin{array}{l}10.403 \\
10.421 \\
10.439 \\
10.457 \\
10.474 \\
10.492 \\
10.509 \\
10.526 \\
10.544 \\
10.561\end{array}$ \\
\hline $\begin{array}{l}302.00 \\
304.00 \\
306.00 \\
308.00 \\
310.00 \\
312.00 \\
314.00 \\
316.00 \\
318.00 \\
320.00\end{array}$ & $\begin{array}{l}1.6900 \\
1.7012 \\
1.7125 \\
1.7237 \\
1.7349 \\
1.7461 \\
1.7572 \\
1.7684 \\
1.7796 \\
1.7907\end{array}$ & $\begin{array}{l}913.34 \\
921.46 \\
929.57 \\
937.67 \\
945.78 \\
953.88 \\
961.98 \\
970.07 \\
978.16 \\
986.24\end{array}$ & $\begin{array}{l}600.60 \\
606.64 \\
612.67 \\
618.70 \\
624.74 \\
630.77 \\
636.80 \\
642.82 \\
648.84 \\
654.86\end{array}$ & $\begin{array}{l}9.086 \\
9.112 \\
9.139 \\
9.165 \\
9.192 \\
9.218 \\
9.244 \\
9.269 \\
9.295 \\
9.320\end{array}$ & $\begin{array}{l}442.00 \\
444.00 \\
446.00 \\
448.00 \\
450.00 \\
452.00 \\
454.00 \\
456.00 \\
458.00 \\
460.00\end{array}$ & $\begin{array}{l}2.4620 \\
2.4729 \\
2.4838 \\
2.4947 \\
2.5055 \\
2.5164 \\
2.5273 \\
2.5382 \\
2.5490 \\
2.5599\end{array}$ & $\begin{array}{l}1460.19 \\
1467.66 \\
1475.12 \\
1482.58 \\
1490.02 \\
1497.48 \\
1504.92 \\
1512.36 \\
1519.79 \\
1527.21\end{array}$ & $\begin{array}{l}1004.58 \\
1010.04 \\
1015.49 \\
1020.94 \\
1026.37 \\
1031.81 \\
1037.24 \\
1042.67 \\
1048.08 \\
1053.49\end{array}$ & $\begin{array}{l}10.578 \\
10.594 \\
10.611 \\
10.628 \\
10.645 \\
10.661 \\
10.677 \\
10.694 \\
10.710 \\
10.726\end{array}$ \\
\hline $\begin{array}{l}322.00 \\
324.00 \\
326.00 \\
328.00 \\
330.00 \\
332.00 \\
334.00 \\
336.00 \\
338.00 \\
340.00\end{array}$ & $\begin{array}{l}1.8019 \\
1.8130 \\
1.8242 \\
1.8353 \\
1.8464 \\
1.8575 \\
1.8687 \\
1.8798 \\
1.8909 \\
1.9020\end{array}$ & $\begin{array}{r}994.32 \\
1002.39 \\
1010.45 \\
1018.50 \\
1026.55 \\
1034.59 \\
1042.63 \\
1050.65 \\
1058.66 \\
1066.67\end{array}$ & $\begin{array}{l}660.87 \\
666.88 \\
672.88 \\
678.88 \\
684.87 \\
690.85 \\
696.83 \\
702.79 \\
708.75 \\
714.70\end{array}$ & $\begin{array}{l}9.345 \\
9.370 \\
9.395 \\
9.420 \\
9.444 \\
9.468 \\
9.493 \\
9.517 \\
9.540 \\
9.564\end{array}$ & $\begin{array}{l}462.00 \\
464.00 \\
466.00 \\
468.00 \\
470.00 \\
472.00 \\
474.00 \\
476.00 \\
478.00 \\
480.00\end{array}$ & $\begin{array}{l}2.5707 \\
2.5816 \\
2.5925 \\
2.6033 \\
2.6142 \\
2.6250 \\
2.6359 \\
2.6467 \\
2.6576 \\
2.6684\end{array}$ & $\begin{array}{l}1534.62 \\
1542.02 \\
1549.41 \\
1556.79 \\
1564.16 \\
1571.53 \\
1578.89 \\
1586.23 \\
1593.57 \\
1600.91\end{array}$ & $\begin{array}{l}1058.89 \\
1064.28 \\
1069.66 \\
1075.04 \\
1080.40 \\
1085.76 \\
1091.11 \\
1096.45 \\
1101.78 \\
1107.11\end{array}$ & $\begin{array}{l}10.742 \\
10.758 \\
10.774 \\
10.790 \\
10.806 \\
10.821 \\
10.837 \\
10.852 \\
10.868 \\
10.883\end{array}$ \\
\hline $\begin{array}{l}342.00 \\
344.00 \\
346.00 \\
348.00 \\
350.00 \\
352.00 \\
354.00 \\
356.00 \\
358.00 \\
360.00\end{array}$ & $\begin{array}{l}1.9131 \\
1.9241 \\
1.9352 \\
1.9463 \\
1.9574 \\
1.9684 \\
1.9795 \\
1.9905 \\
2.0016 \\
2.0126\end{array}$ & $\begin{array}{l}1074.66 \\
1082.65 \\
1090.62 \\
1098.58 \\
1106.54 \\
1114.48 \\
1122.40 \\
1130.32 \\
1138.23 \\
1146.12\end{array}$ & $\begin{array}{l}720.65 \\
726.58 \\
732.50 \\
738.42 \\
744.32 \\
750.21 \\
756.09 \\
761.96 \\
767.82 \\
773.67\end{array}$ & $\begin{array}{l}9.587 \\
9.611 \\
9.634 \\
9.657 \\
9.679 \\
9.702 \\
9.725 \\
9.747 \\
9.769 \\
9.791\end{array}$ & $\begin{array}{l}482.00 \\
484.00 \\
486.00 \\
488.00 \\
490.00 \\
492.00 \\
494.00 \\
496.00 \\
498.00 \\
500.00\end{array}$ & $\begin{array}{l}2.6793 \\
2.6901 \\
2.7009 \\
2.7118 \\
2.7226 \\
2.7334 \\
2.7443 \\
2.7551 \\
2.7659 \\
2.7767\end{array}$ & $\begin{array}{l}1608.23 \\
1615.55 \\
1622.86 \\
1630.16 \\
1637.45 \\
1644.74 \\
1652.02 \\
1659.29 \\
1666.56 \\
1673.82\end{array}$ & $\begin{array}{l}1112.43 \\
1117.74 \\
1123.04 \\
1128.34 \\
1133.63 \\
1138.91 \\
1144.19 \\
1149.46 \\
1154.72 \\
1159.98\end{array}$ & $\begin{array}{l}10.898 \\
10.913 \\
10.929 \\
10.944 \\
10.958 \\
10.973 \\
10.988 \\
11.003 \\
11.017 \\
11.032\end{array}$ \\
\hline $\begin{array}{l}362.00 \\
364.00 \\
366.00 \\
368.00 \\
370.00 \\
372.00 \\
374.00 \\
376.00 \\
378.00 \\
380.00\end{array}$ & $\begin{array}{l}2.0237 \\
2.0347 \\
2.0457 \\
2.0568 \\
2.0678 \\
2.0788 \\
2.0898 \\
2.1008 \\
2.1118 \\
2.1228\end{array}$ & $\begin{array}{l}1153.99 \\
1161.84 \\
1169.69 \\
1177.52 \\
1185.34 \\
1193.15 \\
1200.94 \\
1208.73 \\
1216.50 \\
1224.26\end{array}$ & $\begin{array}{l}779.50 \\
785.31 \\
791.12 \\
796.91 \\
802.69 \\
808.46 \\
814.22 \\
819.96 \\
825.70 \\
831.42\end{array}$ & $\begin{array}{r}9.813 \\
9.834 \\
9.856 \\
9.877 \\
9.898 \\
9.919 \\
9.940 \\
9.961 \\
9.982 \\
10.002\end{array}$ & $\begin{array}{l}502.00 \\
504.00 \\
506.00 \\
508.00 \\
510.00 \\
512.00 \\
514.00 \\
516.00 \\
518.00 \\
520.00\end{array}$ & $\begin{array}{l}2.7876 \\
2.7984 \\
2.8092 \\
2.8200 \\
2.8308 \\
2.8417 \\
2.8525 \\
2.8633 \\
2.8741 \\
2.8849\end{array}$ & $\begin{array}{l}1681.08 \\
1688.33 \\
1695.57 \\
1702.81 \\
1710.05 \\
1717.28 \\
1724.51 \\
1731.73 \\
1738.95 \\
1746.16\end{array}$ & $\begin{array}{l}1165.23 \\
1170.48 \\
1175.72 \\
1180.96 \\
1186.19 \\
1191.42 \\
1196.65 \\
1201.87 \\
1207.09 \\
1212.30\end{array}$ & $\begin{array}{l}11.046 \\
11.061 \\
11.075 \\
11.089 \\
11.104 \\
11.118 \\
11.132 \\
11.146 \\
11.160 \\
11.174\end{array}$ \\
\hline $\begin{array}{l}382.00 \\
384.00 \\
386.00 \\
388.00 \\
390.00 \\
392.00 \\
394.00 \\
396.00 \\
398.00 \\
400.00\end{array}$ & $\begin{array}{l}2.1338 \\
2.1448 \\
2.1558 \\
2.1668 \\
2.1778 \\
2.1887 \\
2.1997 \\
2.2107 \\
2.2217 \\
2.2326\end{array}$ & $\begin{array}{l}1232.01 \\
1239.75 \\
1247.48 \\
1255.19 \\
1262.90 \\
1270.60 \\
1278.28 \\
1285.96 \\
1293.63 \\
1301.28\end{array}$ & $\begin{array}{l}837.14 \\
842.84 \\
848.54 \\
854.22 \\
859.90 \\
865.56 \\
871.22 \\
876.86 \\
882.50 \\
888.13\end{array}$ & $\begin{array}{l}10.023 \\
10.043 \\
10.063 \\
10.083 \\
10.103 \\
10.122 \\
10.142 \\
10.161 \\
10.181 \\
10.200\end{array}$ & $\begin{array}{l}522.00 \\
524.00 \\
526.00 \\
528.00 \\
530.00 \\
532.00 \\
534.00 \\
536.00 \\
538.00 \\
540.00\end{array}$ & $\begin{array}{l}2.8957 \\
2.9065 \\
2.9173 \\
2.9281 \\
2.9389 \\
2.9497 \\
2.9605 \\
2.9713 \\
2.9821 \\
2.9929\end{array}$ & $\begin{array}{l}1753.37 \\
1760.58 \\
1767.78 \\
1774.98 \\
1782.18 \\
1789.37 \\
1796.56 \\
1803.75 \\
1810.93 \\
1818.11\end{array}$ & $\begin{array}{l}1217.51 \\
1222.72 \\
1227.92 \\
1233.12 \\
1238.32 \\
1243.52 \\
1248.71 \\
1253.90 \\
1259.08 \\
1264.27\end{array}$ & $\begin{array}{l}11.188 \\
11.201 \\
11.215 \\
11.229 \\
11.242 \\
11.256 \\
11.269 \\
11.283 \\
11.296 \\
11.310\end{array}$ \\
\hline
\end{tabular}


TEMPER- SPECIFIC ENTHALPY INTERNAL ENTROPY ATURE VOLUME (BTU/LB) ENERGY (BTU/L8-R) (R) (CU FT/LB)
TEMPER- SPECIFIC ENTHALPY (R) VOLUME ( CU FT/LB)
INTERNAL ENTROPY ENERGY (BTU/LB-R) (BTU/LB)

\begin{tabular}{|c|c|c|c|c|c|c|c|c|c|}
\hline & & & & & $\begin{array}{l}122.00 \\
124.00 \\
126.00 \\
128.00 \\
130.00 \\
132.00 \\
134.00 \\
136.00 \\
138.00 \\
140.00\end{array}$ & $\begin{array}{l}.4900 \\
.5010 \\
.5120 \\
.5230 \\
.5339 \\
.5449 \\
.5558 \\
.5667 \\
.5775 \\
.5883\end{array}$ & $\begin{array}{l}212.22 \\
219.89 \\
227.53 \\
235.14 \\
242.71 \\
250.26 \\
257.77 \\
265.26 \\
272.72 \\
280.17\end{array}$ & $\begin{array}{r}98.88 \\
104.01 \\
109.10 \\
114.17 \\
119.21 \\
124.22 \\
129.21 \\
134.18 \\
139.13 \\
144.08\end{array}$ & $\begin{array}{l}5.354 \\
5.416 \\
5.478 \\
5.537 \\
5.596 \\
5.654 \\
5.710 \\
5.766 \\
5.820 \\
5.874\end{array}$ \\
\hline & & & & & $\begin{array}{l}142.00 \\
144.00 \\
146.00 \\
148.00 \\
150.00 \\
152.00 \\
154.00 \\
156.00 \\
158.00 \\
160.00\end{array}$ & $\begin{array}{l}.5991 \\
.6099 \\
.6205 \\
.6312 \\
.6418 \\
.6524 \\
.6629 \\
.6734 \\
.6839 \\
.6943\end{array}$ & $\begin{array}{l}287.59 \\
295.00 \\
302.40 \\
309.79 \\
317.17 \\
324.54 \\
331.92 \\
339.29 \\
346.66 \\
354.04\end{array}$ & $\begin{array}{l}149.01 \\
153.94 \\
158.86 \\
163.78 \\
168.71 \\
173.64 \\
178.57 \\
183.52 \\
188.48 \\
193.45\end{array}$ & $\begin{array}{l}5.926 \\
5.978 \\
6.029 \\
6.079 \\
6.129 \\
6.178 \\
6.226 \\
6.274 \\
6.321 \\
6.367\end{array}$ \\
\hline $\begin{array}{l}36.00 \\
38.00 \\
40.00\end{array}$ & $\begin{array}{l}.2028 \\
.2047 \\
.2068\end{array}$ & $\begin{array}{l}-73.55 \\
-69.79 \\
-65.86\end{array}$ & $\begin{array}{l}-120.45 \\
-117.15 \\
-113.70\end{array}$ & $\begin{array}{l}1.577 \\
1.679 \\
1.780\end{array}$ & $\begin{array}{l}162.00 \\
164.00 \\
166.00 \\
168.00 \\
170.00 \\
172.00 \\
174.00 \\
176.00 \\
178.00 \\
180.00\end{array}$ & $\begin{array}{l}.7046 \\
.7150 \\
.7253 \\
.7355 \\
.7458 \\
.7559 \\
.7661 \\
.7762 \\
.7863 \\
.7963\end{array}$ & $\begin{array}{l}361.43 \\
368.82 \\
376.22 \\
383.63 \\
391.06 \\
398.50 \\
405.95 \\
413.41 \\
420.89 \\
428.38\end{array}$ & $\begin{array}{l}198.43 \\
203.44 \\
208.46 \\
213.50 \\
218.55 \\
223.64 \\
228.74 \\
233.86 \\
239.01 \\
244.17\end{array}$ & $\begin{array}{l}6.413 \\
6.458 \\
6.503 \\
6.547 \\
6.591 \\
6.635 \\
6.678 \\
6.721 \\
6.763 \\
6.805\end{array}$ \\
\hline $\begin{array}{l}42.00 \\
44.00 \\
46.00 \\
48.00 \\
50.00 \\
52.00 \\
54.00 \\
56.00 \\
58.00 \\
60.00\end{array}$ & $\begin{array}{l}.2090 \\
.2114 \\
.2139 \\
.2165 \\
.2193 \\
.2222 \\
.2253 \\
.2285 \\
.2320 \\
.2360\end{array}$ & $\begin{array}{l}-61.75 \\
-57.45 \\
-52.95 \\
-48.25 \\
-43.33 \\
-38.20 \\
-32.89 \\
-27.40 \\
-21.65 \\
-15.68\end{array}$ & $\begin{array}{r}-110.11 \\
-106.35 \\
-102.42 \\
-98.32 \\
-94.05 \\
-89.60 \\
-85.00 \\
-80.26 \\
-75.32 \\
-70.26\end{array}$ & $\begin{array}{l}1.880 \\
1.980 \\
2.080 \\
2.180 \\
2.280 \\
2.381 \\
2.481 \\
2.581 \\
2.682 \\
2.783\end{array}$ & $\begin{array}{l}182.00 \\
184.00 \\
186.00 \\
188.00 \\
190.00 \\
192.00 \\
194.00 \\
196.00 \\
198.00 \\
200.00\end{array}$ & $\begin{array}{l}.8064 \\
.8163 \\
.8263 \\
.8362 \\
.8461 \\
.8560 \\
.8658 \\
.8756 \\
.8854 \\
.8952\end{array}$ & $\begin{array}{l}435.87 \\
443.37 \\
450.88 \\
458.41 \\
465.96 \\
473.53 \\
481.12 \\
488.73 \\
496.35 \\
504.00\end{array}$ & $\begin{array}{l}249.35 \\
254.53 \\
259.74 \\
264.98 \\
270.24 \\
275.53 \\
280.84 \\
286.18 \\
291.54 \\
296.93\end{array}$ & $\begin{array}{l}6.846 \\
6.887 \\
6.928 \\
6.968 \\
7.008 \\
7.048 \\
7.087 \\
7.126 \\
7.165 \\
7.203\end{array}$ \\
\hline $\begin{array}{l}62.00 \\
64.00 \\
66.00 \\
68.00 \\
70.00 \\
72.00 \\
74.00 \\
76.00 \\
78.00 \\
80.00\end{array}$ & $\begin{array}{l}.2398 \\
.2439 \\
.2483 \\
.2529 \\
.2578 \\
.2631 \\
.2686 \\
.2745 \\
.2807 \\
.2873\end{array}$ & $\begin{array}{r}-9.50 \\
-3.11 \\
3.48 \\
10.21 \\
17.04 \\
23.75 \\
30.42 \\
37.12 \\
43.89 \\
50.76\end{array}$ & $\begin{array}{l}-64.97 \\
-59.53 \\
-53.95 \\
-48.29 \\
-42.60 \\
-37.10 \\
-31.71 \\
-26.37 \\
-21.04 \\
-15.69\end{array}$ & $\begin{array}{l}2.885 \\
2.987 \\
3.088 \\
3.188 \\
3.287 \\
3.382 \\
3.473 \\
3.562 \\
3.650 \\
3.737\end{array}$ & $\begin{array}{l}202.00 \\
204.00 \\
206.00 \\
208.00 \\
210.00 \\
212.00 \\
214.00 \\
216.00 \\
218.00 \\
220.00\end{array}$ & $\begin{array}{l}.9049 \\
.9146 \\
.9243 \\
.9340 \\
.9436 \\
.9533 \\
.9629 \\
.9725 \\
.9820 \\
.9916\end{array}$ & $\begin{array}{l}511.67 \\
519.35 \\
527.05 \\
534.78 \\
542.52 \\
550.29 \\
558.07 \\
565.87 \\
573.69 \\
581.52\end{array}$ & $\begin{array}{l}302.34 \\
307.78 \\
313.24 \\
318.73 \\
324.24 \\
329.78 \\
335.34 \\
340.93 \\
346.53 \\
352.16\end{array}$ & $\begin{array}{l}7.241 \\
7.279 \\
7.317 \\
7.354 \\
7.391 \\
7.428 \\
7.464 \\
7.501 \\
7.537 \\
7.572\end{array}$ \\
\hline $\begin{array}{r}82.00 \\
84.00 \\
86.00 \\
88.00 \\
90.00 \\
92.00 \\
94.00 \\
96.00 \\
98.00 \\
100.00\end{array}$ & $\begin{array}{r}.2942 \\
.3015 \\
.3092 \\
.3172 \\
.3256 \\
.3344 \\
.3434 \\
.3528 \\
.3624 \\
.3723\end{array}$ & $\begin{array}{r}57.76 \\
64.88 \\
72.13 \\
79.52 \\
87.02 \\
94.63 \\
102.33 \\
110.10 \\
117.94 \\
125.82\end{array}$ & $\begin{array}{r}-10.30 \\
-4.87 \\
.61 \\
6.13 \\
11.69 \\
17.28 \\
22.89 \\
28.50 \\
34.11 \\
39.70\end{array}$ & $\begin{array}{l}3.824 \\
3.910 \\
3.995 \\
4.080 \\
4.164 \\
4.248 \\
4.330 \\
4.412 \\
4.493 \\
4.573\end{array}$ & $\begin{array}{l}222.00 \\
224.00 \\
226.00 \\
228.00 \\
230.00 \\
232.00 \\
234.00 \\
236.00 \\
238.00 \\
240.00\end{array}$ & $\begin{array}{l}1.0011 \\
1.0106 \\
1.0201 \\
1.0296 \\
1.0390 \\
1.0485 \\
1.0579 \\
1.0673 \\
1.0767 \\
1.0861\end{array}$ & $\begin{array}{l}589.37 \\
597.25 \\
605.14 \\
613.05 \\
620.97 \\
628.92 \\
636.88 \\
644.86 \\
652.85 \\
660.86\end{array}$ & $\begin{array}{l}357.81 \\
363.48 \\
369.17 \\
374.89 \\
380.63 \\
386.39 \\
392.17 \\
397.97 \\
403.79 \\
409.63\end{array}$ & $\begin{array}{l}7.608 \\
7.643 \\
7.678 \\
7.713 \\
7.748 \\
7.782 \\
7.816 \\
7.850 \\
7.884 \\
7.917\end{array}$ \\
\hline $\begin{array}{l}102.00 \\
104.00 \\
106.00 \\
108.00 \\
110.00 \\
112.00 \\
114.00 \\
116.00 \\
118.00 \\
120.00\end{array}$ & $\begin{array}{l}.3824 \\
.3927 \\
.4031 \\
.4137 \\
.4244 \\
.4352 \\
.4461 \\
.4570 \\
.4680 \\
.4790\end{array}$ & $\begin{array}{l}133.73 \\
141.65 \\
149.58 \\
157.50 \\
165.41 \\
173.29 \\
181.14 \\
188.96 \\
196.75 \\
204.50\end{array}$ & $\begin{array}{l}45.28 \\
50.82 \\
56.33 \\
61.80 \\
67.23 \\
72.61 \\
77.95 \\
83.25 \\
88.50 \\
93.71\end{array}$ & $\begin{array}{l}4.651 \\
4.728 \\
4.803 \\
4.877 \\
4.950 \\
5.021 \\
5.091 \\
5.159 \\
5.225 \\
5.290\end{array}$ & $\begin{array}{l}242.00 \\
244.00 \\
246.00 \\
248.00 \\
250.00 \\
252.00 \\
254.00 \\
256.00 \\
258.00 \\
260.00\end{array}$ & $\begin{array}{l}1.0955 \\
1.1049 \\
1.1142 \\
1.1235 \\
1.1329 \\
1.1422 \\
1.1515 \\
1.1608 \\
1.1701 \\
1.1793\end{array}$ & $\begin{array}{l}668.89 \\
676.93 \\
684.99 \\
693.06 \\
701.15 \\
709.24 \\
717.35 \\
725.48 \\
733.61 \\
741.75\end{array}$ & $\begin{array}{l}415.49 \\
421.36 \\
427.26 \\
433.17 \\
439.10 \\
445.04 \\
451.00 \\
456.97 \\
462.96 \\
468.96\end{array}$ & $\begin{array}{l}7.951 \\
7.984 \\
8.017 \\
8.049 \\
8.082 \\
8.114 \\
8.146 \\
8.178 \\
8.210 \\
8.241\end{array}$ \\
\hline
\end{tabular}


TEMPER- SPECIFIC ENTHALPY INTERNAL ENTROPY ATURE VOLUME (BTU/L8) ENERGY (8TU/L8-R) (R) (CU FT/L8) $\{8 \mathrm{TU} / \mathrm{L} 8\}$
TEMPERATURE (R)
SPECIFIC ENTHALPY VOLUME
INTERNAL ENTROPY

ENERGY (BTU/LB-R) $(8 \mathrm{TU} / 28)$

\begin{tabular}{|c|c|c|c|c|c|c|c|c|c|}
\hline $\begin{array}{l}262.00 \\
264.00 \\
266.00 \\
268.00 \\
270.00 \\
272.00 \\
274.00 \\
276.00 \\
278.00 \\
280.00\end{array}$ & $\begin{array}{l}1.1886 \\
1.1978 \\
1.2071 \\
1.2163 \\
1.2255 \\
1.2347 \\
1.2439 \\
1.2531 \\
1.2623 \\
1.2714\end{array}$ & $\begin{array}{l}749.91 \\
758.07 \\
766.24 \\
774.41 \\
782.60 \\
790.76 \\
798.93 \\
807.10 \\
815.28 \\
823.46\end{array}$ & $\begin{array}{l}474.97 \\
480.99 \\
487.02 \\
493.07 \\
499.12 \\
505.16 \\
511.20 \\
517.25 \\
523.30 \\
529.36\end{array}$ & $\begin{array}{l}8.272 \\
8.303 \\
8.334 \\
8.365 \\
8.395 \\
8.425 \\
8.455 \\
8.485 \\
8.515 \\
8.544\end{array}$ & $\begin{array}{l}402.00 \\
404.00 \\
406.00 \\
408.00 \\
410.00 \\
412.00 \\
414.00 \\
416.00 \\
418.00 \\
420.00\end{array}$ & $\begin{array}{l}1.8172 \\
1.8260 \\
1.8348 \\
1.8436 \\
1.8524 \\
1.8612 \\
1.8700 \\
1.8788 \\
1.8876 \\
1.8964\end{array}$ & $\begin{array}{l}1310.86 \\
1318.56 \\
1326.24 \\
1333.91 \\
1341.58 \\
1349.23 \\
1356.87 \\
1364.51 \\
1372.13 \\
1379.75\end{array}$ & $\begin{array}{l}890.51 \\
896.16 \\
901.81 \\
907.45 \\
913.08 \\
918.70 \\
924.31 \\
929.91 \\
935.50 \\
941.09\end{array}$ & $\begin{array}{r}9.992 \\
10.011 \\
10.030 \\
10.049 \\
10.067 \\
10.086 \\
10.104 \\
10.123 \\
10.141 \\
10.159\end{array}$ \\
\hline $\begin{array}{l}282.00 \\
284.00 \\
286.00 \\
288.00 \\
290.00 \\
292.00 \\
294.00 \\
296.00 \\
298.00 \\
300.00\end{array}$ & $\begin{array}{l}1.2806 \\
1.2897 \\
1.2989 \\
1.3080 \\
1.3171 \\
1.3262 \\
1.3353 \\
1.3444 \\
1.3535 \\
1.3625\end{array}$ & $\begin{array}{l}831.64 \\
839.81 \\
847.99 \\
856.17 \\
864.35 \\
872.53 \\
880.71 \\
888.88 \\
897.05 \\
905.22\end{array}$ & $\begin{array}{l}535.42 \\
541.48 \\
547.55 \\
553.62 \\
559.69 \\
565.76 \\
571.83 \\
577.90 \\
583.97 \\
590.04\end{array}$ & $\begin{array}{l}8.573 \\
8.602 \\
8.631 \\
8.659 \\
8.687 \\
8.716 \\
8.743 \\
8.771 \\
8.799 \\
8.826\end{array}$ & $\begin{array}{l}422.00 \\
424.00 \\
426.00 \\
428.00 \\
430.00 \\
432.00 \\
434.00 \\
436.00 \\
438.00 \\
440.00\end{array}$ & $\begin{array}{l}1.9051 \\
1.9139 \\
1.9227 \\
1.9315 \\
1.9402 \\
1.9490 \\
1.9577 \\
1.9665 \\
1.9752 \\
1.9840\end{array}$ & $\begin{array}{l}1387.35 \\
1394.95 \\
1402.53 \\
1410.11 \\
1417.67 \\
1425.23 \\
1432.77 \\
1440.31 \\
1447.83 \\
1455.35\end{array}$ & $\begin{array}{l}946.66 \\
952.23 \\
957.78 \\
963.33 \\
968.87 \\
974.40 \\
979.91 \\
985.42 \\
990.92 \\
996.42\end{array}$ & $\begin{array}{l}10.177 \\
10.195 \\
10.213 \\
10.231 \\
10.248 \\
10.266 \\
10.283 \\
10.301 \\
10.318 \\
10.335\end{array}$ \\
\hline $\begin{array}{l}302.00 \\
304.00 \\
306.00 \\
308.00 \\
310.00 \\
312.00 \\
314.00 \\
316.00 \\
318.00 \\
320.00\end{array}$ & $\begin{array}{l}1.3716 \\
1.3807 \\
1.3897 \\
1.3988 \\
1.4078 \\
1.4168 \\
1.4258 \\
1.4348 \\
1.4438 \\
1.4528\end{array}$ & $\begin{array}{l}913.38 \\
921.54 \\
929.69 \\
937.84 \\
945.98 \\
954.12 \\
962.25 \\
970.37 \\
978.49 \\
986.60\end{array}$ & $\begin{array}{l}596.10 \\
602.17 \\
608.23 \\
614.28 \\
620.34 \\
626.39 \\
632.43 \\
638.47 \\
644.51 \\
650.53\end{array}$ & $\begin{array}{l}8.853 \\
8.880 \\
8.907 \\
8.933 \\
8.960 \\
8.986 \\
9.012 \\
9.038 \\
9.063 \\
9.089\end{array}$ & $\begin{array}{l}442.00 \\
444.00 \\
446.00 \\
448.00 \\
450.00 \\
452.00 \\
454.00 \\
456.00 \\
458.00 \\
460.00\end{array}$ & $\begin{array}{l}1.9927 \\
2.0015 \\
2.0102 \\
2.0190 \\
2.0277 \\
2.0364 \\
2.0452 \\
2.0539 \\
2.0626 \\
2.0713\end{array}$ & $\begin{array}{l}1462.85 \\
1470.35 \\
1477.83 \\
1485.31 \\
1492.78 \\
1500.26 \\
1507.72 \\
1515.18 \\
1522.63 \\
1530.07\end{array}$ & $\begin{array}{l}1001.90 \\
1007.37 \\
1012.84 \\
1018.29 \\
1023.74 \\
1029.20 \\
1034.64 \\
1040.08 \\
1045.51 \\
1050.93\end{array}$ & $\begin{array}{l}10.352 \\
10.369 \\
10.386 \\
10.403 \\
10.419 \\
10.436 \\
10.452 \\
10.469 \\
10.485 \\
10.501\end{array}$ \\
\hline $\begin{array}{l}322.00 \\
324.00 \\
326.00 \\
328.00 \\
330.00 \\
332.00 \\
334.00 \\
336.00 \\
338.00 \\
340.00\end{array}$ & $\begin{array}{l}1.4618 \\
1.4708 \\
1.4798 \\
1.4887 \\
1.4977 \\
1.5067 \\
1.5156 \\
1.5246 \\
1.5335 \\
1.5424\end{array}$ & $\begin{array}{r}994.70 \\
1002.79 \\
1010.88 \\
1018.95 \\
1027.02 \\
1035.08 \\
1043.13 \\
1051.17 \\
1059.20 \\
1067.23\end{array}$ & $\begin{array}{l}656.56 \\
662.57 \\
668.58 \\
674.58 \\
680.58 \\
686.57 \\
692.55 \\
698.52 \\
704.48 \\
710.44\end{array}$ & $\begin{array}{l}9.114 \\
9.139 \\
9.164 \\
9.188 \\
9.213 \\
9.237 \\
9.261 \\
9.285 \\
9.309 \\
9.333\end{array}$ & $\begin{array}{l}462.00 \\
464.00 \\
466.00 \\
468.00 \\
470.00 \\
472.00 \\
474.00 \\
476.00 \\
478.00 \\
480.00\end{array}$ & $\begin{array}{l}2.0801 \\
2.0888 \\
2.0975 \\
2.1062 \\
2.1149 \\
2.1236 \\
2.1324 \\
2.1411 \\
2.1498 \\
2.1585\end{array}$ & $\begin{array}{l}1537.50 \\
1544.92 \\
1552.33 \\
1559.73 \\
1567.13 \\
1574.52 \\
1581.90 \\
1589.27 \\
1596.63 \\
1603.99\end{array}$ & $\begin{array}{l}1056.34 \\
1061.75 \\
1067.14 \\
1072.53 \\
1077.91 \\
1083.28 \\
1088.65 \\
1094.01 \\
1099.36 \\
1104.70\end{array}$ & $\begin{array}{l}10.517 \\
10.533 \\
10.549 \\
10.565 \\
10.581 \\
10.597 \\
10.612 \\
10.628 \\
10.643 \\
10.658\end{array}$ \\
\hline $\begin{array}{l}342.00 \\
344.00 \\
346.00 \\
348.00 \\
350.00 \\
352.00 \\
354.00 \\
356.00 \\
358.00 \\
360.00\end{array}$ & $\begin{array}{l}1.5514 \\
1.5603 \\
1.5692 \\
1.5781 \\
1.5870 \\
1.5959 \\
1.6048 \\
1.6137 \\
1.6226 \\
1.6315\end{array}$ & $\begin{array}{l}1075.24 \\
1083.24 \\
1091.23 \\
1099.22 \\
1107.19 \\
1115.15 \\
1123.10 \\
1131.05 \\
1138.98 \\
1146.90\end{array}$ & $\begin{array}{l}716.39 \\
722.32 \\
728.25 \\
734.17 \\
740.09 \\
745.99 \\
751.88 \\
757.77 \\
763.64 \\
769.51\end{array}$ & $\begin{array}{l}9.356 \\
9.380 \\
9.403 \\
9.426 \\
9.449 \\
9.472 \\
9.494 \\
9.516 \\
9.539 \\
9.561\end{array}$ & $\begin{array}{l}482.00 \\
484.00 \\
486.00 \\
488.00 \\
490.00 \\
492.00 \\
494.00 \\
496.00 \\
498.00 \\
500.00\end{array}$ & $\begin{array}{l}2.1672 \\
2.1759 \\
2.1846 \\
2.1933 \\
2.2020 \\
2.2107 \\
2.2193 \\
2.2280 \\
2.2367 \\
2.2454\end{array}$ & $\begin{array}{l}1611.33 \\
1618.67 \\
1626.01 \\
1633.33 \\
1640.65 \\
1647.96 \\
1655.27 \\
1662.57 \\
1669.86 \\
1677.14\end{array}$ & $\begin{array}{l}1110.03 \\
1115.36 \\
1120.68 \\
1125.99 \\
1131.30 \\
1136.60 \\
1141.90 \\
1147.19 \\
1152.47 \\
1157.75\end{array}$ & $\begin{array}{l}10.674 \\
10.689 \\
10.704 \\
10.719 \\
10.734 \\
10.749 \\
10.764 \\
10.779 \\
10.793 \\
10.808\end{array}$ \\
\hline $\begin{array}{l}362.00 \\
364.00 \\
366.00 \\
368.00 \\
370.00 \\
372.00 \\
374.00 \\
376.00 \\
378.00 \\
380.00\end{array}$ & $\begin{array}{l}1.6404 \\
1.6493 \\
1.6581 \\
1.6670 \\
1.6759 \\
1.6847 \\
1.6936 \\
1.7024 \\
1.7113 \\
1.7201\end{array}$ & $\begin{array}{l}1154.81 \\
1162.71 \\
1170.60 \\
1178.48 \\
1186.35 \\
1194.21 \\
1202.06 \\
1209.90 \\
1217.73 \\
1225.55\end{array}$ & $\begin{array}{l}775.37 \\
781.21 \\
787.05 \\
792.88 \\
798.70 \\
804.51 \\
810.31 \\
816.10 \\
821.88 \\
827.65\end{array}$ & $\begin{array}{l}9.583 \\
9.604 \\
9.626 \\
9.647 \\
9.669 \\
9.690 \\
9.711 \\
9.732 \\
9.753 \\
9.773\end{array}$ & $\begin{array}{l}502.00 \\
504.00 \\
506.00 \\
508.00 \\
510.00 \\
512.00 \\
514.00 \\
516.00 \\
518.00 \\
520.00\end{array}$ & $\begin{array}{l}2.2541 \\
2.2628 \\
2.2715 \\
2.2801 \\
2.2888 \\
2.2975 \\
2.3062 \\
2.3148 \\
2.3235 \\
2.3322\end{array}$ & $\begin{array}{l}1684.42 \\
1691.70 \\
1698.97 \\
1706.23 \\
1713.49 \\
1720.74 \\
1727.99 \\
1735.23 \\
1742.47 \\
1749.71\end{array}$ & $\begin{array}{l}1163.02 \\
1168.28 \\
1173.54 \\
1178.80 \\
1184.05 \\
1189.30 \\
1194.54 \\
1199.77 \\
1205.01 \\
1210.23\end{array}$ & $\begin{array}{l}10.822 \\
10.837 \\
10.851 \\
10.866 \\
10.880 \\
10.894 \\
10.908 \\
10.922 \\
10.936 \\
10.950\end{array}$ \\
\hline $\begin{array}{l}382.00 \\
384.00 \\
386.00 \\
388.00 \\
390.00 \\
392.00 \\
394.00 \\
396.00 \\
398.00 \\
400.00\end{array}$ & $\begin{array}{l}1.7290 \\
1.7378 \\
1.7467 \\
1.7555 \\
1.7643 \\
1.7731 \\
1.7820 \\
1.7908 \\
1.7996 \\
1.8084\end{array}$ & $\begin{array}{l}1233.35 \\
1241.15 \\
1248.94 \\
1256.71 \\
1264.48 \\
1272.24 \\
1279.98 \\
1287.72 \\
1295.44 \\
1303.16\end{array}$ & $\begin{array}{l}833.41 \\
839.17 \\
844.91 \\
850.64 \\
856.37 \\
862.08 \\
867.78 \\
873.48 \\
879.16 \\
884.84\end{array}$ & $\begin{array}{l}9.794 \\
9.814 \\
9.834 \\
9.854 \\
9.874 \\
9.894 \\
9.914 \\
9.934 \\
9.953 \\
9.972\end{array}$ & $\begin{array}{l}522.00 \\
524.00 \\
526.00 \\
528.00 \\
530.00 \\
532.00 \\
534.00 \\
536.00 \\
538.00 \\
540.00\end{array}$ & $\begin{array}{l}2.3408 \\
2.3495 \\
2.3582 \\
2.3668 \\
2.3755 \\
2.3842 \\
2.3928 \\
2.4015 \\
2.4101 \\
2.4188\end{array}$ & $\begin{array}{l}1756.93 \\
1764.16 \\
1771.38 \\
1778.60 \\
1785.81 \\
1793.02 \\
1800.22 \\
1807.42 \\
1814.62 \\
1821.81\end{array}$ & $\begin{array}{l}1215.46 \\
1220.68 \\
1225.90 \\
1231.11 \\
1236.32 \\
1241.52 \\
1246.72 \\
1251.92 \\
1257.11 \\
1262.31\end{array}$ & $\begin{array}{l}10.964 \\
10.978 \\
10.992 \\
11.005 \\
11.019 \\
11.032 \\
11.046 \\
11.059 \\
11.073 \\
11.086\end{array}$ \\
\hline
\end{tabular}


TEMPER - SPECIFIC ENTHALPY INTERNAL ENTROPY

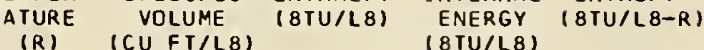
(R)
TEMPER

ATURE
SPECIFIC ENTHALPY VOLUME (CU FT/L8)
(8TU/L8)

$$
1
$$

INTERNAL ENTROPY ENERGY (BTU/LB-R) $(8 \mathrm{TU} / \mathrm{LB})$

\begin{tabular}{|c|c|c|c|c|c|c|c|c|c|}
\hline & & & & & $\begin{array}{l}122.00 \\
124.00 \\
126.00 \\
128.00 \\
130.00 \\
132.00 \\
134.00 \\
136.00 \\
138.00 \\
140.00\end{array}$ & $\begin{array}{l}.4254 \\
.4342 \\
.4429 \\
.4517 \\
.4605 \\
.4694 \\
.4782 \\
.4871 \\
.4959 \\
.5047\end{array}$ & $\begin{array}{l}205.63 \\
213.25 \\
220.85 \\
228.45 \\
236.03 \\
243.59 \\
251.14 \\
258.68 \\
266.20 \\
273.72\end{array}$ & $\begin{array}{r}87.54 \\
92.74 \\
97.91 \\
103.06 \\
108.19 \\
113.30 \\
118.40 \\
123.48 \\
128.56 \\
133.62\end{array}$ & $\begin{array}{l}5.127 \\
5.189 \\
5.250 \\
5.310 \\
5.369 \\
5.426 \\
5.483 \\
5.539 \\
5.594 \\
5.648\end{array}$ \\
\hline & & & & & $\begin{array}{l}142.00 \\
144.00 \\
146.00 \\
148.00 \\
150.00 \\
152.00 \\
154.00 \\
156.00 \\
158.00 \\
160.00\end{array}$ & $\begin{array}{l}.5135 \\
.5223 \\
.5311 \\
.5399 \\
.5487 \\
.5574 \\
.5661 \\
.5748 \\
.5835 \\
.5921\end{array}$ & $\begin{array}{l}281.22 \\
288.72 \\
296.21 \\
303.69 \\
311.18 \\
318.66 \\
326.14 \\
333.62 \\
341.11 \\
348.61\end{array}$ & $\begin{array}{l}138.68 \\
143.73 \\
148.78 \\
153.83 \\
158.88 \\
163.93 \\
169.00 \\
174.07 \\
179.15 \\
184.24\end{array}$ & $\begin{array}{l}5.701 \\
5.754 \\
5.805 \\
5.856 \\
5.906 \\
5.956 \\
6.005 \\
6.053 \\
6.101 \\
6.148\end{array}$ \\
\hline $\begin{array}{l}36.00 \\
38.00 \\
40.00\end{array}$ & $\begin{array}{l}.1999 \\
.2017 \\
.2036\end{array}$ & $\begin{array}{l}-65.76 \\
-62.09 \\
-58.26\end{array}$ & $\begin{array}{l}-121.25 \\
-118.08 \\
-114.78\end{array}$ & $\begin{array}{l}1.535 \\
1.634 \\
1.732\end{array}$ & $\begin{array}{l}162.00 \\
164.00 \\
166.00 \\
168.00 \\
170.00 \\
172.00 \\
174.00 \\
176.00 \\
178.00 \\
180.00\end{array}$ & $\begin{array}{l}.6007 \\
.6093 \\
.6179 \\
.6265 \\
.6350 \\
.6435 \\
.6520 \\
.6604 \\
.6689 \\
.6773\end{array}$ & $\begin{array}{l}356.11 \\
363.62 \\
371.13 \\
378.66 \\
386.20 \\
393.76 \\
401.32 \\
408.90 \\
416.50 \\
424.10\end{array}$ & $\begin{array}{l}189.35 \\
194.47 \\
199.61 \\
204.77 \\
209.94 \\
215.13 \\
220.35 \\
225.58 \\
230.83 \\
236.11\end{array}$ & $\begin{array}{l}6.195 \\
6.241 \\
6.286 \\
6.331 \\
6.376 \\
6.420 \\
6.464 \\
6.507 \\
6.550 \\
6.593\end{array}$ \\
\hline $\begin{array}{l}42.00 \\
44.00 \\
46.00 \\
48.00 \\
50.00 \\
52.00 \\
54.00 \\
56.00 \\
58.00 \\
60.00\end{array}$ & $\begin{array}{l}.2056 \\
.2077 \\
.2099 \\
.2123 \\
.2147 \\
.2173 \\
.2200 \\
.2228 \\
.2258 \\
.2291\end{array}$ & $\begin{array}{r}-54.26 \\
-50.08 \\
-45.71 \\
-41.14 \\
-36.38 \\
-31.42 \\
-26.30 \\
-21.00 \\
-15.48 \\
-9.71\end{array}$ & $\begin{array}{l}-111.33 \\
-107.73 \\
-103.98 \\
-100.06 \\
-95.98 \\
-91.74 \\
-87.36 \\
-82.86 \\
-78.16 \\
-73.30\end{array}$ & $\begin{array}{l}1.830 \\
1.927 \\
2.024 \\
2.121 \\
2.219 \\
2.316 \\
2.412 \\
2.509 \\
2.606 \\
2.703\end{array}$ & $\begin{array}{l}182.00 \\
184.00 \\
186.00 \\
188.00 \\
190.00 \\
192.00 \\
194.00 \\
196.00 \\
198.00 \\
200.00\end{array}$ & $\begin{array}{l}.6857 \\
.6940 \\
.7024 \\
.7107 \\
.7190 \\
.7273 \\
.7355 \\
.7438 \\
.7520 \\
.7602\end{array}$ & $\begin{array}{l}431.71 \\
439.33 \\
446.96 \\
454.61 \\
462.27 \\
469.96 \\
477.66 \\
485.37 \\
493.10 \\
500.85\end{array}$ & $\begin{array}{l}241.39 \\
246.68 \\
252.00 \\
257.34 \\
262.70 \\
268.08 \\
273.49 \\
278.92 \\
284.37 \\
289.85\end{array}$ & $\begin{array}{l}6.635 \\
6.676 \\
6.717 \\
6.758 \\
6.799 \\
6.839 \\
6.879 \\
6.919 \\
6.958 \\
6.997\end{array}$ \\
\hline $\begin{array}{l}62.00 \\
64.00 \\
66.00 \\
68.00 \\
70.00 \\
72.00 \\
74.00 \\
76.00 \\
78.00 \\
80.00\end{array}$ & $\begin{array}{l}.2323 \\
.2358 \\
.2394 \\
.2432 \\
.2472 \\
.2515 \\
.2559 \\
.2606 \\
.2654 \\
.2705\end{array}$ & $\begin{array}{r}-3.81 \\
2.27 \\
8.52 \\
14.89 \\
21.33 \\
27.62 \\
33.84 \\
40.07 \\
46.33 \\
52.67\end{array}$ & $\begin{array}{l}-68.31 \\
-63.18 \\
-57.93 \\
-52.62 \\
-47.30 \\
-42.18 \\
-37.19 \\
-32.26 \\
-27.35 \\
-22.42\end{array}$ & $\begin{array}{l}2.801 \\
2.898 \\
2.994 \\
3.088 \\
3.182 \\
3.270 \\
3.356 \\
3.439 \\
3.520 \\
3.600\end{array}$ & $\begin{array}{l}202.00 \\
204.00 \\
206.00 \\
208.00 \\
210.00 \\
212.00 \\
214.00 \\
216.00 \\
218.00 \\
220.00\end{array}$ & $\begin{array}{l}.7683 \\
.7765 \\
.7846 \\
.7927 \\
.8008 \\
.8089 \\
.8170 \\
.8250 \\
.8331 \\
.8411\end{array}$ & $\begin{array}{l}508.62 \\
516.40 \\
524.20 \\
532.01 \\
539.85 \\
547.70 \\
555.56 \\
563.45 \\
571.34 \\
579.26\end{array}$ & $\begin{array}{l}295.34 \\
300.86 \\
306.40 \\
311.96 \\
317.55 \\
323.15 \\
328.78 \\
334.43 \\
340.10 \\
345.79\end{array}$ & $\begin{array}{l}7.035 \\
7.074 \\
7.112 \\
7.150 \\
7.187 \\
7.224 \\
7.261 \\
7.298 \\
7.334 \\
7.370\end{array}$ \\
\hline $\begin{array}{r}82.00 \\
84.00 \\
86.00 \\
88.00 \\
90.00 \\
92.00 \\
94.00 \\
96.00 \\
98.00 \\
100.00\end{array}$ & $\begin{array}{l}.2759 \\
.2815 \\
.2873 \\
.2934 \\
.2997 \\
.3063 \\
.3130 \\
.3200 \\
.3272 \\
.3347\end{array}$ & $\begin{array}{r}59.12 \\
65.67 \\
72.34 \\
79.13 \\
86.03 \\
93.05 \\
100.18 \\
107.41 \\
114.72 \\
122.10\end{array}$ & $\begin{array}{r}-17.47 \\
-12.47 \\
-7.42 \\
-2.31 \\
2.84 \\
8.05 \\
13.29 \\
18.57 \\
23.88 \\
29.21\end{array}$ & $\begin{array}{l}3.680 \\
3.759 \\
3.837 \\
3.915 \\
3.993 \\
4.070 \\
4.147 \\
4.223 \\
4.298 \\
4.373\end{array}$ & $\begin{array}{l}222.00 \\
224.00 \\
226.00 \\
228.00 \\
230.00 \\
232.00 \\
234.00 \\
236.00 \\
238.00 \\
240.00\end{array}$ & $\begin{array}{l}.8491 \\
.8571 \\
.8650 \\
.8730 \\
.8809 \\
.8889 \\
.8968 \\
.9047 \\
.9126 \\
.9205\end{array}$ & $\begin{array}{l}587.19 \\
595.13 \\
603.10 \\
611.08 \\
619.08 \\
627.09 \\
635.12 \\
643.17 \\
651.23 \\
659.31\end{array}$ & $\begin{array}{l}351.50 \\
357.23 \\
362.98 \\
368.75 \\
374.54 \\
380.36 \\
386.19 \\
392.05 \\
397.92 \\
403.81\end{array}$ & $\begin{array}{l}7.406 \\
7.442 \\
7.477 \\
7.512 \\
7.547 \\
7.582 \\
7.617 \\
7.651 \\
7.685 \\
7.719\end{array}$ \\
\hline $\begin{array}{l}102.00 \\
104.00 \\
106.00 \\
108.00 \\
110.00 \\
112.00 \\
114.00 \\
116.00 \\
118.00 \\
120.00\end{array}$ & $\begin{array}{l}.3423 \\
.3500 \\
.3580 \\
.3660 \\
.3742 \\
.3826 \\
.3910 \\
.3995 \\
.4081 \\
.4167\end{array}$ & $\begin{array}{l}129.56 \\
137.06 \\
144.61 \\
152.20 \\
159.81 \\
167.44 \\
175.08 \\
182.72 \\
190.36 \\
198.00\end{array}$ & $\begin{array}{l}34.55 \\
39.90 \\
45.25 \\
50.60 \\
55.93 \\
61.25 \\
66.55 \\
71.83 \\
77.09 \\
82.33\end{array}$ & $\begin{array}{l}4.446 \\
4.519 \\
4.591 \\
4.662 \\
4.732 \\
4.801 \\
4.868 \\
4.935 \\
5.000 \\
5.064\end{array}$ & $\begin{array}{l}242.00 \\
244.00 \\
246.00 \\
248.00 \\
250.00 \\
252.00 \\
254.00 \\
256.00 \\
258.00 \\
260.00\end{array}$ & $\begin{array}{l}.9283 \\
.9362 \\
.9440 \\
.9519 \\
.9597 \\
.9675 \\
.9753 \\
.9831 \\
.9909 \\
.9987\end{array}$ & $\begin{array}{l}667.41 \\
675.52 \\
683.64 \\
691.78 \\
699.93 \\
708.09 \\
716.27 \\
724.46 \\
732.66 \\
740.87\end{array}$ & $\begin{array}{l}409.72 \\
415.65 \\
421.60 \\
427.56 \\
433.54 \\
439.54 \\
445.55 \\
451.57 \\
457.61 \\
463.66\end{array}$ & $\begin{array}{l}7.752 \\
7.786 \\
7.819 \\
7.852 \\
7.884 \\
7.917 \\
7.949 \\
7.981 \\
8.013 \\
8.045\end{array}$ \\
\hline
\end{tabular}


TEMPER- SPECIFIC ENTHALPY INTERNAL ENTROPY ATURE VOLUME (8TU/LB) ENERGY (8TU/L8-R) (R) (CU FT/LB)
(BTU/LB) $\begin{array}{ccccc}\text { TEMPER- } & \text { SPECIFIC } & \text { ENTHALPY } & \text { INTERNAL } & \text { ENTROPY } \\ \text { ATURE } & \text { VOLUME } & \text { (BTU/LB) } & \text { ENERGY } & \text { (8TU/L8-R) } \\ \text { (R) } & \text { (CUFT/LB) } & & \text { (8TU/L8) } & \end{array}$

\begin{tabular}{|c|c|c|c|c|c|c|c|c|c|}
\hline $\begin{array}{l}262.00 \\
264.00 \\
266.00 \\
268.00 \\
270.00 \\
272.00 \\
274.00 \\
276.00 \\
278.00 \\
280.00\end{array}$ & $\begin{array}{l}1.0064 \\
1.0142 \\
1.0219 \\
1.0297 \\
1.0374 \\
1.0451 \\
1.0529 \\
1.0606 \\
1.0683 \\
1.0760\end{array}$ & $\begin{array}{l}749.09 \\
757.32 \\
765.55 \\
773.80 \\
782.05 \\
790.28 \\
798.51 \\
806.75 \\
814.99 \\
823.23\end{array}$ & $\begin{array}{l}469.72 \\
475.80 \\
481.88 \\
487.98 \\
494.08 \\
500.17 \\
506.26 \\
512.36 \\
518.46 \\
524.56\end{array}$ & $\begin{array}{l}8.076 \\
8.108 \\
8.139 \\
8.170 \\
8.200 \\
8.231 \\
8.261 \\
8.291 \\
8.321 \\
8.350\end{array}$ & $\begin{array}{l}402.00 \\
404.00 \\
406.00 \\
408.00 \\
410.00 \\
412.00 \\
414.00 \\
416.00 \\
418.00 \\
420.00\end{array}$ & $\begin{array}{l}1.5333 \\
1.5407 \\
1.5481 \\
1.5554 \\
1.5628 \\
1.5702 \\
1.5775 \\
1.5849 \\
1.5922 \\
1.5996\end{array}$ & $\begin{array}{l}1313.19 \\
1320.91 \\
1328.62 \\
1336.32 \\
1344.02 \\
1351.70 \\
1359.37 \\
1367.03 \\
1374.68 \\
1382.32\end{array}$ & $\begin{array}{l}887.56 \\
893.24 \\
898.91 \\
904.57 \\
910.21 \\
915.85 \\
921.48 \\
927.10 \\
932.71 \\
938.31\end{array}$ & $\begin{array}{l}9.806 \\
9.825 \\
9.844 \\
9.863 \\
9.882 \\
9.900 \\
9.919 \\
9.937 \\
9.956 \\
9.974\end{array}$ \\
\hline $\begin{array}{l}282.00 \\
284.00 \\
286.00 \\
288.00 \\
290.00 \\
292.00 \\
294.00 \\
296.00 \\
298.00 \\
300.00\end{array}$ & $\begin{array}{l}1.0837 \\
1.0913 \\
1.0990 \\
1.1067 \\
1.1143 \\
1.1220 \\
1.1296 \\
1.1372 \\
1.1449 \\
1.1525\end{array}$ & $\begin{array}{l}831.47 \\
839.72 \\
847.96 \\
856.20 \\
864.44 \\
872.68 \\
880.91 \\
889.14 \\
897.37 \\
905.60\end{array}$ & $\begin{array}{l}530.67 \\
536.78 \\
542.90 \\
549.01 \\
555.13 \\
561.24 \\
567.36 \\
573.47 \\
579.58 \\
585.69\end{array}$ & $\begin{array}{l}8.379 \\
8.409 \\
8.438 \\
8.466 \\
8.495 \\
8.523 \\
8.551 \\
8.579 \\
8.607 \\
8.634\end{array}$ & $\begin{array}{l}422.00 \\
424.00 \\
426.00 \\
428.00 \\
430.00 \\
432.00 \\
434.00 \\
436.00 \\
438.00 \\
440.00\end{array}$ & $\begin{array}{l}1.6069 \\
1.6142 \\
1.6216 \\
1.6289 \\
1.6362 \\
1.6435 \\
1.6509 \\
1.6582 \\
1.6655 \\
1.6728\end{array}$ & $\begin{array}{l}1389.95 \\
1397.57 \\
1405.17 \\
1412.77 \\
1420.36 \\
1427.94 \\
1435.50 \\
1443.06 \\
1450.61 \\
1458.15\end{array}$ & $\begin{array}{l}943.91 \\
949.49 \\
955.06 \\
960.62 \\
966.18 \\
971.72 \\
977.26 \\
982.78 \\
988.30 \\
993.81\end{array}$ & $\begin{array}{r}9.992 \\
10.010 \\
10.028 \\
10.046 \\
10.063 \\
10.081 \\
10.098 \\
10.116 \\
10.133 \\
10.150\end{array}$ \\
\hline $\begin{array}{l}302.00 \\
304.00 \\
306.00 \\
308.00 \\
310.00 \\
312.00 \\
314.00 \\
316.00 \\
318.00 \\
320.00\end{array}$ & $\begin{array}{l}1.1601 \\
1.1677 \\
1.1753 \\
1.1829 \\
1.1904 \\
1.1980 \\
1.2056 \\
1.2131 \\
1.2207 \\
1.2282\end{array}$ & $\begin{array}{l}913.81 \\
922.03 \\
930.24 \\
938.44 \\
946.63 \\
954.82 \\
963.00 \\
971.17 \\
979.33 \\
987.49\end{array}$ & $\begin{array}{l}591.80 \\
597.90 \\
604.00 \\
610.10 \\
616.19 \\
622.28 \\
628.36 \\
634.43 \\
640.50 \\
646.56\end{array}$ & $\begin{array}{l}8.662 \\
8.689 \\
8.716 \\
8.742 \\
8.769 \\
8.795 \\
8.821 \\
8.847 \\
8.873 \\
8.899\end{array}$ & $\begin{array}{l}442.00 \\
444.00 \\
446.00 \\
448.00 \\
450.00 \\
452.00 \\
454.00 \\
456.00 \\
458.00 \\
460.00\end{array}$ & $\begin{array}{l}1.6801 \\
1.6874 \\
1.6948 \\
1.7021 \\
1.7094 \\
1.7167 \\
1.7240 \\
1.7313 \\
1.7386 \\
1.7458\end{array}$ & $\begin{array}{l}1465.68 \\
1473.19 \\
1480.70 \\
1488.20 \\
1495.69 \\
1503.19 \\
1510.68 \\
1518.16 \\
1525.62 \\
1533.09\end{array}$ & $\begin{array}{r}999.30 \\
1004.79 \\
1010.27 \\
1015.75 \\
1021.21 \\
1026.68 \\
1032.14 \\
1037.59 \\
1043.04 \\
1048.48\end{array}$ & $\begin{array}{l}10.167 \\
10.184 \\
10.201 \\
10.218 \\
10.235 \\
10.251 \\
10.268 \\
10.284 \\
10.301 \\
10.317\end{array}$ \\
\hline $\begin{array}{l}322.00 \\
324.00 \\
326.00 \\
328.00 \\
330.00 \\
332.00 \\
334.00 \\
336.00 \\
338.00 \\
340.00\end{array}$ & $\begin{array}{l}1.2358 \\
1.2433 \\
1.2508 \\
1.2583 \\
1.2658 \\
1.2734 \\
1.2808 \\
1.2883 \\
1.2958 \\
1.3033\end{array}$ & $\begin{array}{r}995.64 \\
1003.77 \\
1011.90 \\
1020.02 \\
1028.13 \\
1036.23 \\
1044.32 \\
1052.40 \\
1060.47 \\
1068.53\end{array}$ & $\begin{array}{l}652.61 \\
658.66 \\
664.70 \\
670.73 \\
676.76 \\
682.77 \\
688.78 \\
694.78 \\
700.77 \\
706.76\end{array}$ & $\begin{array}{l}8.924 \\
8.949 \\
8.974 \\
8.999 \\
9.024 \\
9.048 \\
9.072 \\
9.097 \\
9.120 \\
9.144\end{array}$ & $\begin{array}{l}462.00 \\
464.00 \\
466.00 \\
468.00 \\
470.00 \\
472.00 \\
474.00 \\
476.00 \\
478.00 \\
480.00\end{array}$ & $\begin{array}{l}1.7531 \\
1.7604 \\
1.7677 \\
1.7750 \\
1.7823 \\
1.7896 \\
1.7968 \\
1.8041 \\
1.8114 \\
1.8187\end{array}$ & $\begin{array}{l}1540.54 \\
1547.98 \\
1555.42 \\
1562.84 . \\
1570.26 \\
1577.67 \\
1585.07 \\
1592.46 \\
1599.85 \\
1607.22\end{array}$ & $\begin{array}{l}1053.90 \\
1059.32 \\
1064.74 \\
1070.14 \\
1075.54 \\
1080.92 \\
1086.30 \\
1091.68 \\
1097.04 \\
1102.40\end{array}$ & $\begin{array}{l}10.333 \\
10.349 \\
10.365 \\
10.381 \\
10.397 \\
10.412 \\
10.428 \\
10.444 \\
10.459 \\
10.475\end{array}$ \\
\hline $\begin{array}{l}342.00 \\
344.00 \\
346.00 \\
348.00 \\
350.00 \\
352.00 \\
354.00 \\
356.00 \\
358.00 \\
360.00\end{array}$ & $\begin{array}{l}1.3108 \\
1.3183 \\
1.3257 \\
1.3332 \\
1.3406 \\
1.3481 \\
1.3555 \\
1.3630 \\
1.3704 \\
1.3779\end{array}$ & $\begin{array}{l}1076.58 \\
1084.61 \\
1092.64 \\
1100.66 \\
1108.67 \\
1116.66 \\
1124.65 \\
1132.62 \\
1140.59 \\
1148.54\end{array}$ & $\begin{array}{l}712.73 \\
718.69 \\
724.65 \\
730.59 \\
736.53 \\
742.46 \\
748.38 \\
754.29 \\
760.19 \\
766.08\end{array}$ & $\begin{array}{l}9.168 \\
9.191 \\
9.215 \\
9.238 \\
9.261 \\
9.283 \\
9.306 \\
9.328 \\
9.351 \\
9.373\end{array}$ & $\begin{array}{l}482.00 \\
484.00 \\
486.00 \\
488.00 \\
490.00 \\
492.00 \\
494.00 \\
496.00 \\
498.00 \\
500.00\end{array}$ & $\begin{array}{l}1.8259 \\
1.8332 \\
1.8405 \\
1.8477 \\
1.8550 \\
1.8623 \\
1.8695 \\
1.8768 \\
1.8841 \\
1.8913\end{array}$ & $\begin{array}{l}1614.59 \\
1621.95 \\
1629.31 \\
1636.66 \\
1644.00 \\
1651.33 \\
1658.66 \\
1665.98 \\
1673.29 \\
1680.60\end{array}$ & $\begin{array}{l}1107.75 \\
1113.09 \\
1118.43 \\
1123.76 \\
1129.08 \\
1134.40 \\
1139.71 \\
1145.01 \\
1150.31 \\
1155.60\end{array}$ & $\begin{array}{l}10.490 \\
10.505 \\
10.520 \\
10.535 \\
10.550 \\
10.565 \\
10.580 \\
10.595 \\
10.610 \\
10.624\end{array}$ \\
\hline $\begin{array}{l}362.00 \\
364.00 \\
366.00 \\
368.00 \\
370.00 \\
372.00 \\
374.00 \\
376.00 \\
378.00 \\
380.00\end{array}$ & $\begin{array}{l}1.3853 \\
1.3927 \\
1.4001 \\
1.4076 \\
1.4150 \\
1.4224 \\
1.4298 \\
1.4372 \\
1.4447 \\
1.4521\end{array}$ & $\begin{array}{l}1156.49 \\
1164.42 \\
1172.35 \\
1180.26 \\
1188.17 \\
1196.06 \\
1203.94 \\
1211.81 \\
1219.68 \\
1227.53\end{array}$ & $\begin{array}{l}771.96 \\
777.83 \\
783.69 \\
789.55 \\
795.39 \\
801.23 \\
807.05 \\
812.86 \\
818.67 \\
824.47\end{array}$ & $\begin{array}{l}9.395 \\
9.417 \\
9.438 \\
9.460 \\
9.481 \\
9.503 \\
9.524 \\
9.545 \\
9.566 \\
9.586\end{array}$ & $\begin{array}{l}502.00 \\
504.00 \\
506.00 \\
508.00 \\
510.00 \\
512.00 \\
514.00 \\
516.00 \\
518.00 \\
520.00\end{array}$ & $\begin{array}{l}1.9986 \\
1.9058 \\
1.9131 \\
1.9203 \\
1.9276 \\
1.9348 \\
1.9421 \\
1.9493 \\
1.9566 \\
1.9638\end{array}$ & $\begin{array}{l}1687.90 \\
1695.19 \\
1702.48 \\
1709.76 \\
1717.04 \\
1724.31 \\
1731.58 \\
1738.84 \\
1746.10 \\
1753.35\end{array}$ & $\begin{array}{l}1160.89 \\
1166.17 \\
1171.45 \\
1176.72 \\
1181.98 \\
1187.24 \\
1192.50 \\
1197.75 \\
1202.99 \\
1208.24\end{array}$ & $\begin{array}{l}10.639 \\
10.653 \\
10.668 \\
10.682 \\
10.696 \\
10.711 \\
10.725 \\
10.739 \\
10.753 \\
10.767\end{array}$ \\
\hline $\begin{array}{l}382.00 \\
384.00 \\
386.00 \\
388.00 \\
390.00 \\
392.00 \\
394.00 \\
396.00 \\
398.00 \\
400.00\end{array}$ & $\begin{array}{l}1.4595 \\
1.4669 \\
1.4743 \\
1.4817 \\
1.4890 \\
1.4964 \\
1.5038 \\
1.5112 \\
1.5186 \\
1.5260\end{array}$ & $\begin{array}{l}1235.37 \\
1243.20 \\
1251.02 \\
1258.82 \\
1266.62 \\
1274.41 \\
1282.19 \\
1289.95 \\
1297.71 \\
1305.45\end{array}$ & $\begin{array}{l}830.25 \\
836.03 \\
841.79 \\
847.55 \\
853.29 \\
859.03 \\
864.76 \\
870.47 \\
876.18 \\
881.88\end{array}$ & $\begin{array}{l}9.607 \\
9.627 \\
9.648 \\
9.668 \\
9.688 \\
9.708 \\
9.728 \\
9.747 \\
9.767 \\
9.786\end{array}$ & $\begin{array}{l}522.00 \\
524.00 \\
526.00 \\
528.00 \\
530.00 \\
532.00 \\
534.00 \\
536.00 \\
538.00 \\
540.00\end{array}$ & $\begin{array}{l}1.9711 \\
1.9783 \\
1.9855 \\
1.9928 \\
2.0000 \\
2.0072 \\
2.0145 \\
2.0217 \\
2.0289 \\
2.0362\end{array}$ & $\begin{array}{l}1760.60 \\
1767.84 \\
1775.08 \\
1782.31 \\
1789.54 \\
1796.77 \\
1803.98 \\
1811.20 \\
1818.41 \\
1825.62\end{array}$ & $\begin{array}{l}1213.47 \\
1218.71 \\
1223.93 \\
1229.16 \\
1234.38 \\
1239.60 \\
1244.81 \\
1250.02 \\
1255.22 \\
1260.42\end{array}$ & $\begin{array}{l}10.781 \\
10.795 \\
10.809 \\
10.822 \\
10.836 \\
10.850 \\
10.863 \\
10.877 \\
10.890 \\
10.903\end{array}$ \\
\hline
\end{tabular}


TEMPER- SPECIFIC ENTHALPY INTERNAL ENTROPY ATURE VOLUME (BTU/L8) ENERGY (BTU/L8-R)

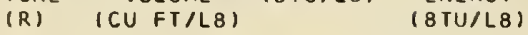

TEMPER- SPECIFIC ENTHALPY INTERNAL ENTROPY

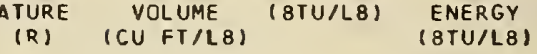

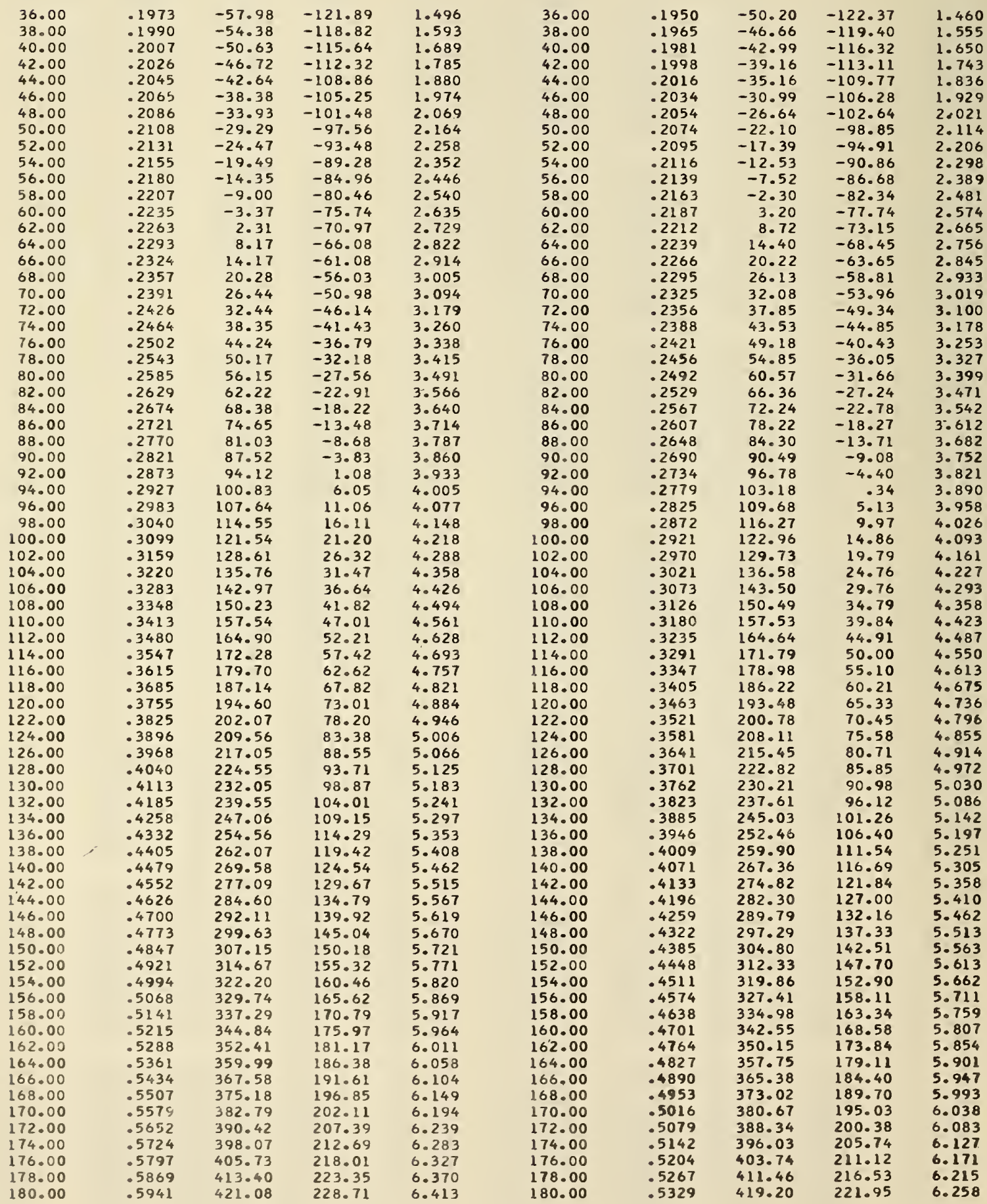


TEMPER- SPECIFIC ENTHALPY INTERNAL ENTROPY

ATURE VOLUME (BTU/LB) ENERGY (8TU/LB-R) (R) (CU FT/LB)
( 8 TU/L 8 )
TEMPER- SPECIFIC ENTHALPY INTERNAL ENTROPY

ATURE VOLUME (8TU/L8) ENERGY (8TU/L8-R)

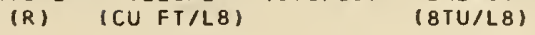

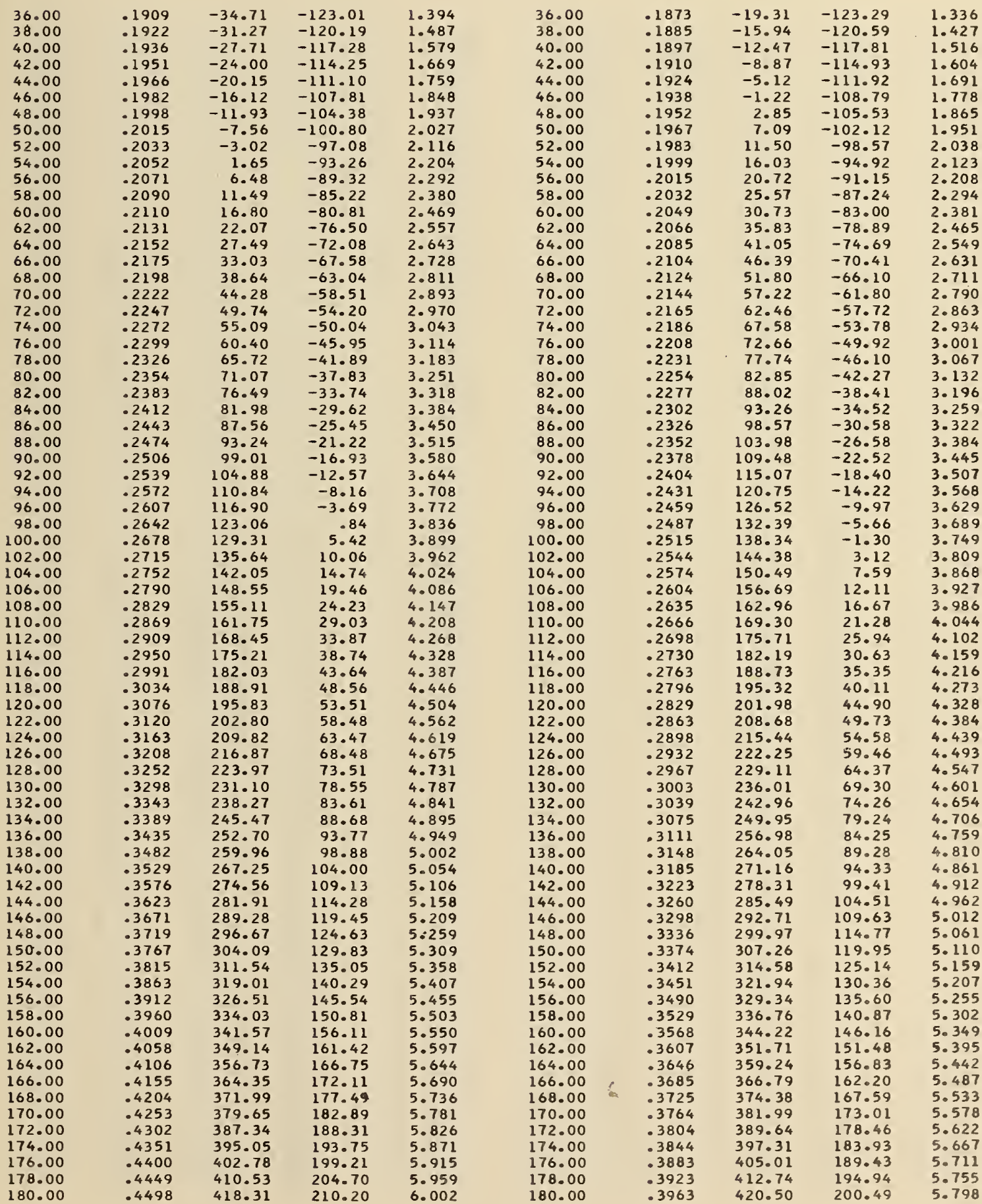


TEMPER- SPECIFIC ENTHALPY INTERNAL ENTROPY ATURE VOLUME (8TU/LB) ENERGY (BTU/L8-R) (R) (CU FT/L8)
(BTU/LB)
TEMPER- SPECIFIC ENTHALPY INTERNAL ENTROPY ATURE VOLUME (BTU/LB) ENERGY (BTU/LB-R) (R) $\quad(C U \mathrm{FT} / L 8) \quad$ (BTU/LB)
(B)

\begin{tabular}{|c|c|c|c|}
\hline $\begin{array}{l}38.00 \\
40.00\end{array}$ & $\begin{array}{r}1852 \\
.1864\end{array}$ & $\begin{array}{r}-.71 \\
2.69\end{array}$ & $\begin{array}{l}-120.68 \\
-118.01\end{array}$ \\
\hline 42.00 & $\begin{array}{r}1875 \\
.1187\end{array}$ & 6.21 & -115.25 \\
\hline 44.00 & .1887 & 9.87 & -112.37 \\
\hline 46.00 & .1900 & 13.67 & -109.37 \\
\hline 48.00 & .1913 & 17.64 & -106.24 \\
\hline 50.00 & .1926 & 21.78 & -102.97 \\
\hline 52.00 & .1940 & 26.08 & -99.56 \\
\hline 54.00 & .1954 & 30.50 & -96.05 \\
\hline 56.00 & .1969 & 35.08 & -92.43 \\
\hline 58.00 & .1984 & 39.81 & -88.66 \\
\hline 60.00 & .1998 & 44.82 & -84.59 \\
\hline 62.00 & .2014 & 49.78 & -80.64 \\
\hline 64.00 & .2030 & 54.87 & -76.61 \\
\hline 66.00 & .2047 & 60.06 & -72.49 \\
\hline 68.00 & .2064 & 65.30 & $-68 \cdot 36$ \\
\hline 70.00 & .2081 & 70.57 & -64.24 \\
\hline 72.00 & .2099 & 75.64 & -60.34 \\
\hline 74.00 & .2118 & 80.60 & -56.58 \\
\hline 76.00 & .2137 & 85.51 & -52.89 \\
\hline 78.00 & .2156 & 90.41 & -49.24 \\
\hline 80.00 & .2176 & 95.35 & -45.59 \\
\hline 82.00 & .2196 & 100.33 & -41.91 \\
\hline 84.00 & .2217 & 105.38 & -38.19 \\
\hline 86.00 & .2238 & 110.51 & -34.42 \\
\hline 88.00 & .2259 & 115.73 & -30.59 \\
\hline 90.00 & .2281 & 121.03 & -26.71 \\
\hline 92.00 & .2303 & 126.42 & -22.76 \\
\hline 94.00 & .2326 & 131.90 & -18.74 \\
\hline 96.00 & .2349 & 137.47 & -14.66 \\
\hline 98.00 & .2372 & 143.13 & -10.52 \\
\hline 100.00 & .2396 & 148.87 & -6.33 \\
\hline 102.00 & .2420 & 154.69 & -2.07 \\
\hline 104.00 & .2445 & 160.60 & 2.24 \\
\hline 106.00 & .2470 & 166.58 & 6.60 \\
\hline 108.00 & .2495 & 172.63 & 11.01 \\
\hline 110.00 & .2521 & 178.76 & 15.47 \\
\hline 112.00 & .2547 & 184.96 & 19.98 \\
\hline 114.00 & .2574 & 191.22 & 24.52 \\
\hline 116.00 & .2601 & 197.54 & 29.11 \\
\hline 118.00 & .2628 & 203.93 & 33.73 \\
\hline 120.00 & .2655 & 210.37 & 38.40 \\
\hline 122.00 & .2683 & 216.87 & 43.09 \\
\hline 124.00 & .2711 & 223.43 & 47.83 \\
\hline 126.00 & .2740 & 230.04 & 52.59 \\
\hline 128.00 & .2768 & 236.70 & 57.39 \\
\hline 130.00 & .2798 & 243.41 & 62.22 \\
\hline 132.00 & .2827 & 250.17 & 67.08 \\
\hline 134.00 & .2857 & 256.98 & 71.96 \\
\hline 136.00 & .2886 & 263.83 & 76.88 \\
\hline 138.00 & .2917 & 270.73 & 81.83 \\
\hline 140.00 & .2947 & 277.68 & 86.81 \\
\hline 142.00 & .2978 & 284.67 & 91.81 \\
\hline 144.00 & .3008 & 291.70 & 96.85 \\
\hline 146.00 & .3040 & 298.78 & 101.91 \\
\hline 148.00 & .3071 & 305.89 & 107.00 \\
\hline 150.00 & .3102 & 313.05 & 112.12 \\
\hline 152.00 & .3134 & 320.25 & 117.27 \\
\hline 154.00 & .3166 & 327.49 & 122.45 \\
\hline 156.00 & .3198 & 334.77 & 127.66 \\
\hline 158.00 & .3230 & 342.09 & 132.90 \\
\hline 160.00 & .3262 & 349.45 & 138.17 \\
\hline 162.00 & .3294 & 356.84 & 143.46 \\
\hline 164.00 & .3327 & 364.28 & 148.79 \\
\hline 166.00 & .3360 & 371.75 & 154.15 \\
\hline 168.00 & .3392 & 379.25 & 159.53 \\
\hline 170.00 & .3425 & 386.80 & 164.95 \\
\hline 172.00 & .3458 & 394.38 & 170.39 \\
\hline 174.00 & .3491 & 401.99 & 175.86 \\
\hline 176.00 & .3524 & 409.63 & 181.36 \\
\hline 178.00 & .3558 & 417.31 & 186.88 \\
\hline 180.00 & .3591 & 425.02 & 192.43 \\
\hline
\end{tabular}

1.373

1.546

1.631

1.800

1.885

1.969

2.053

2.136

2.219

2.304

2.386

2.468

2.547

2.625

2.701

2.773

2.841

2.906

2.970

3.032

3.094

3.155

3.215

3.275

3.335

3.394

3.453

3.511

3.570

3.628

3.685

3.743

3.800

3.856

3.912

3.968

4.024

4.079

4.133

4.187

4.241

4.294

4.347

4.400

4.452

4.503

4.555

4.605

4.656

4.706

4.755

4.804

4.853

4.902

4.950

4.997

5.045

5.092

5.138

5.184

5.230

5.276

5.321

5.366

5.411

5.455

5.499

5.543

5.586

5.629

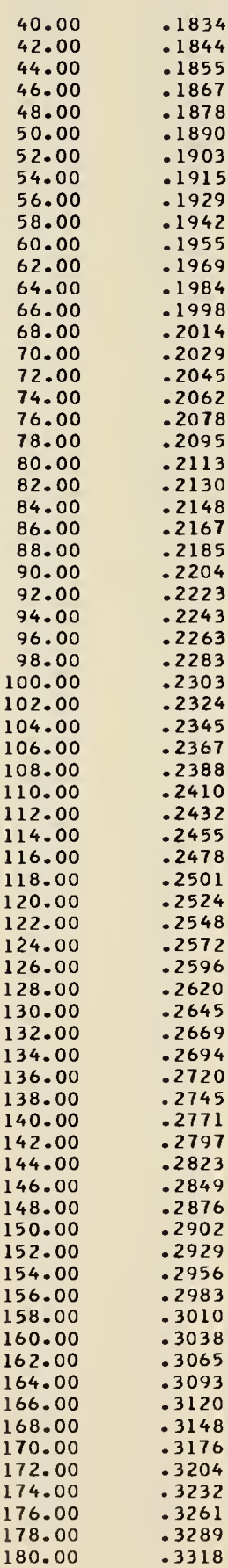

17.77
21.22
24.80
28.52
32.41
36.46
40.66

$-117.95$

1.410

$\begin{array}{ll}-109.64 & 1.577 \\ -106.62 & 1.660\end{array}$

$-106.62 \quad 1.743$

$-103.46 \quad 1.825$

$\begin{array}{ll}-100.17 & 1.908 \\ -96.78 & 1.990\end{array}$

$\begin{array}{lll}49.48 & -93.27 & 2.071\end{array}$

$\begin{array}{lll}54.12 & -89.62 & 2.153\end{array}$

$\begin{array}{lll}58.99 & -85.73 & 2.235\end{array}$

$63.85 \quad-81.91 \quad 2.316$

$68.82 \quad-78.01 \quad 2.396$

$\begin{array}{lll}73.89 & -74.04 & 2.473\end{array}$

$\begin{array}{lll}79.01 & -70.05 & 2.549\end{array}$

$84.14 \quad-66.07 \quad 2.624$

$89.08 \quad-62.31 \quad 2.693$

$\begin{array}{lll}93.91 & -58.69 & 2.759\end{array}$

$\begin{array}{lll}98.69 & -55.15 & 2.823\end{array}$

$\begin{array}{lll}103.47 & -51.64 & 2.885\end{array}$

$\begin{array}{lll}108.26 & -48.12 & 2.946\end{array}$

$113.11 \quad-44.58 \quad 3.006$

$118.03 \quad-41.00 \quad 3.065$

$\begin{array}{lll}123.01 & -37.36 & 3.124\end{array}$

$128.08 \quad-33.67 \quad 3.182$

$\begin{array}{lll}133.24 & -29.92 & 3.240\end{array}$

$\begin{array}{lll}138.48 & -26.10 & 3.297\end{array}$

$\begin{array}{lll}143.81 & -22.22 & 3.355\end{array}$

$149.23 \quad-18.27 \quad 3.412$

$\begin{array}{lll}154.73 & -14.26 & 3.468\end{array}$

$\begin{array}{lll}160.31 & -10.19 & 3.525\end{array}$

$\begin{array}{lll}165.98 & -6.07 & 3.581\end{array}$

$\begin{array}{lll}171.72 & -1.88 & 3.637\end{array}$

$\begin{array}{lll}177.54 & 2.35 & 3.692\end{array}$

$\begin{array}{lll}183.43 & 6.64 & 3.747\end{array}$

$\begin{array}{lll}189.39 & 10.98 & 3.802\end{array}$

$195.42 \quad 15.37 \quad 3.856$

$201.52 \quad 19.80 \quad 3.910$

$\begin{array}{lll}207.68 & 24.27 & 3.964\end{array}$

$213.90 \quad 28.78 \quad 4.017$

$\begin{array}{lll}220.18 & 33.34 & 4.070\end{array}$

$226.51 \quad 37.93 \quad 4.122$

$232.91 \quad 42.56 \quad 4.174$

$239.36 \quad 47.22 \quad 4.226$

$\begin{array}{lll}245.86 & 51.93 & 4.277\end{array}$

$\begin{array}{lll}252.42 & 56.66 & 4.328\end{array}$

$\begin{array}{lll}259.02 & 61.43 & 4.378\end{array}$

$\begin{array}{lll}265.68 & 66.23 & 4.428\end{array}$

$272.39 \quad 71.07 \quad 4.478$

$\begin{array}{lll}279.14 & 75.94 & 4.527\end{array}$

$\begin{array}{lll}285.95 & 80.84 & 4.576\end{array}$

$292.80 \quad 85.78 \quad 4.625$

$299.69 \quad 90.75 \quad 4.673$

$\begin{array}{lll}306.64 & 95.75 & 4.721\end{array}$

$313.63 \quad 100.78 \quad 4.768$

$\begin{array}{lll}320.66 & 105.85 & 4.815\end{array}$

$\begin{array}{lll}327.74 & 110.94 & 4.862\end{array}$

$334.87 \quad 116.07 \quad 4.909$

$\begin{array}{lll}342.04 & 121.24 & 4.955\end{array}$

$349.25 \quad 126.44 \quad 5.001$

$\begin{array}{lll}356.51 & 131.67 & 5.047\end{array}$

$363.80 \quad 136.93 \quad 5.092$

$\begin{array}{lll}371.15 & 142.22 & 5.137\end{array}$

$\begin{array}{lll}378.53 & 147.55 & 5.182\end{array}$

$385.95 \quad 152.91 \quad 5.226$

$393.41 \quad 158.30 \quad 5.270$

$\begin{array}{lll}400.91 & 163.73 & 5.314\end{array}$

$408.45 \quad 169.18 \quad 5.358$

$\begin{array}{lll}416.03 & 174.67 & 5.401\end{array}$

$\begin{array}{lll}423.64 & 180.18 & 5.444 \\ 431.29 & 185.72 & 5.487\end{array}$ 
TEMPER- SPECIFIC ENTHALPY INTERNAL ENTROPY

ATURE VOLUME (BTU/LB) ENERGY (BTU/LB-R) (R) (CU FT/L8)
(8TU/LB)
TEMPER- SPECIFIC ENTHALPY INTERNAL ENTROPY

ATURE VOLUME (BTU/L8) ENERGY (8TU/LB-R) (R) (CUFT/LB) (BTU/L8)
(B)

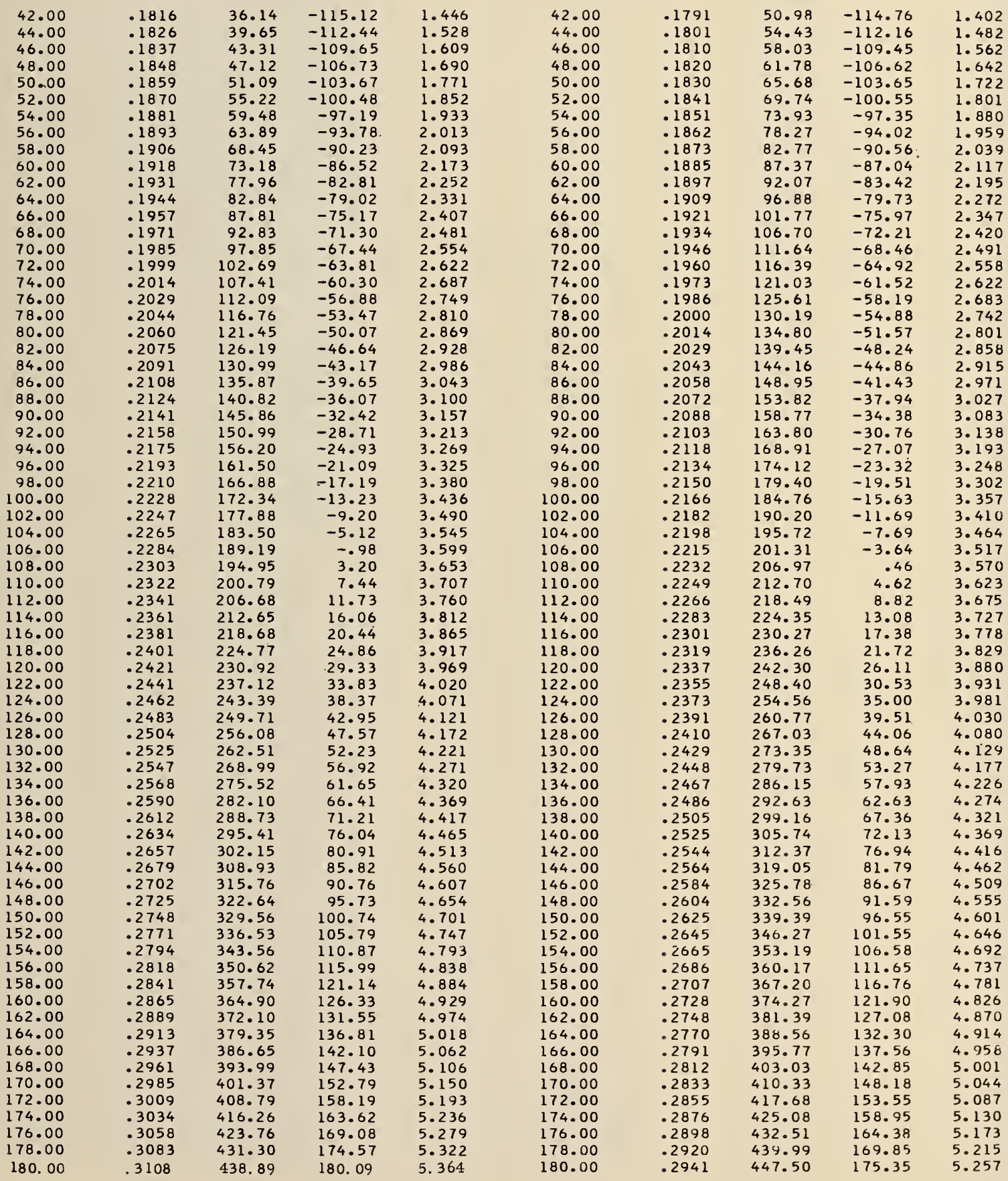





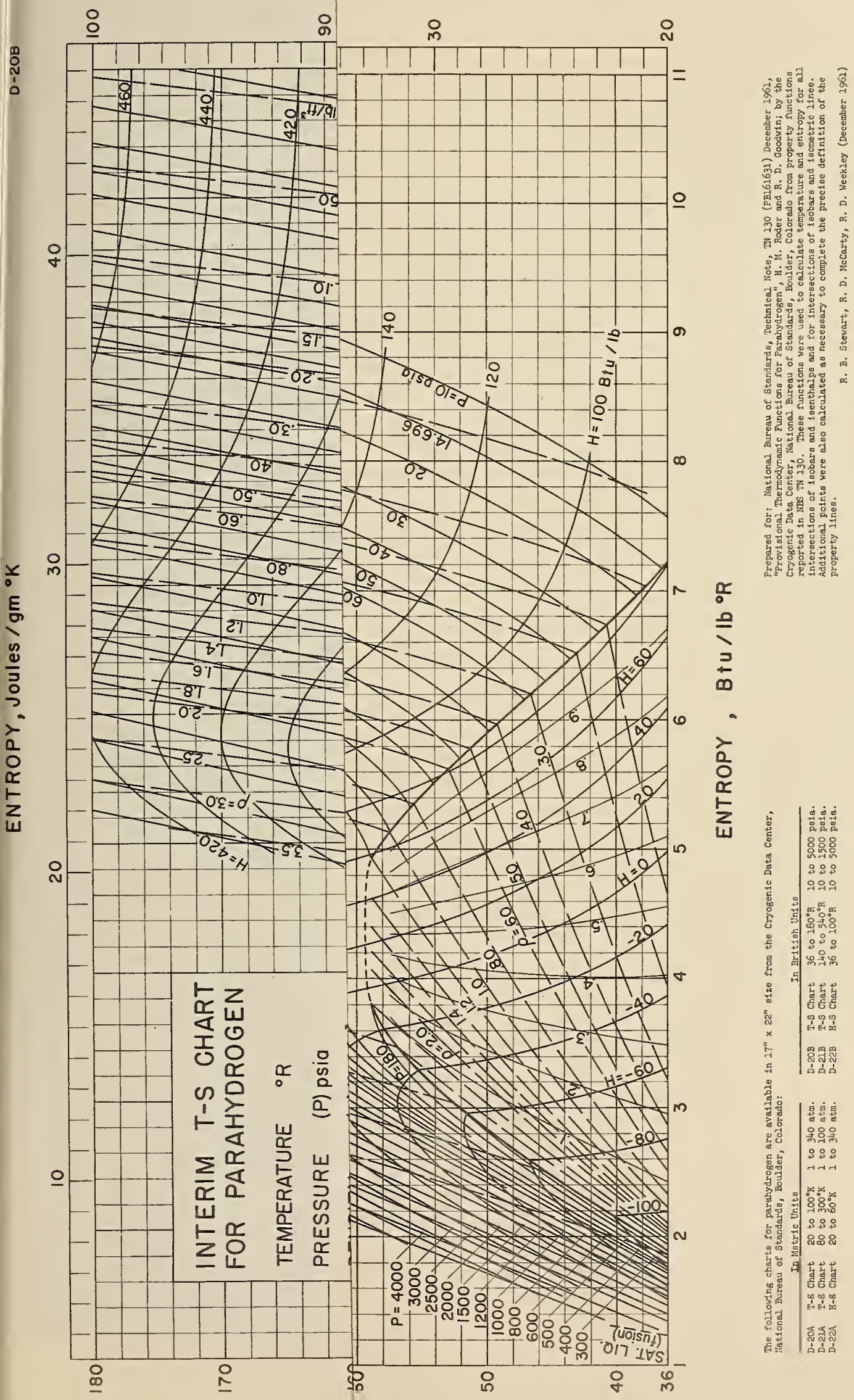




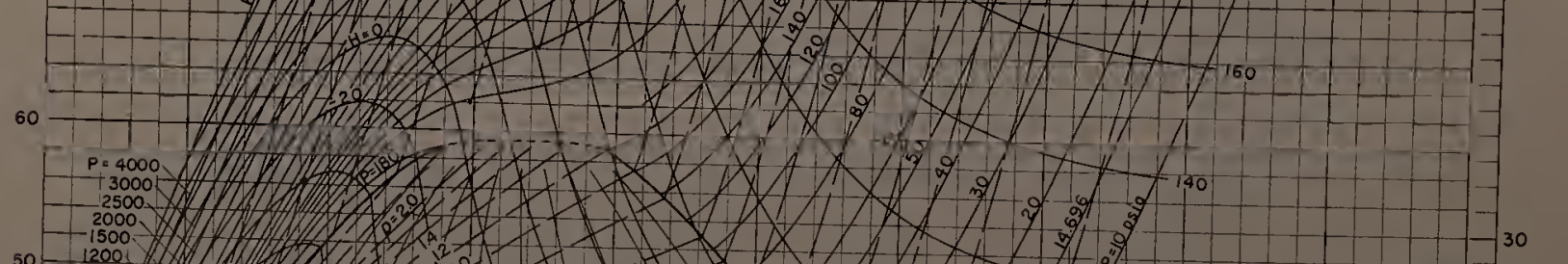

$$
90
$$
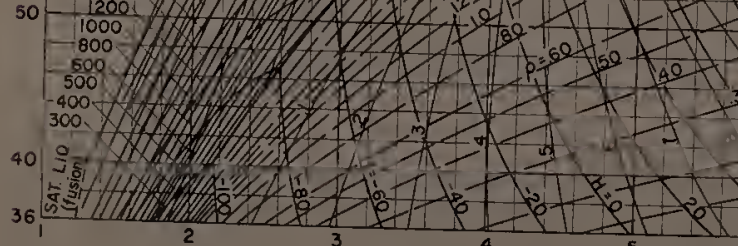

ENTROPY , BTU//b ${ }^{\circ} R$ 





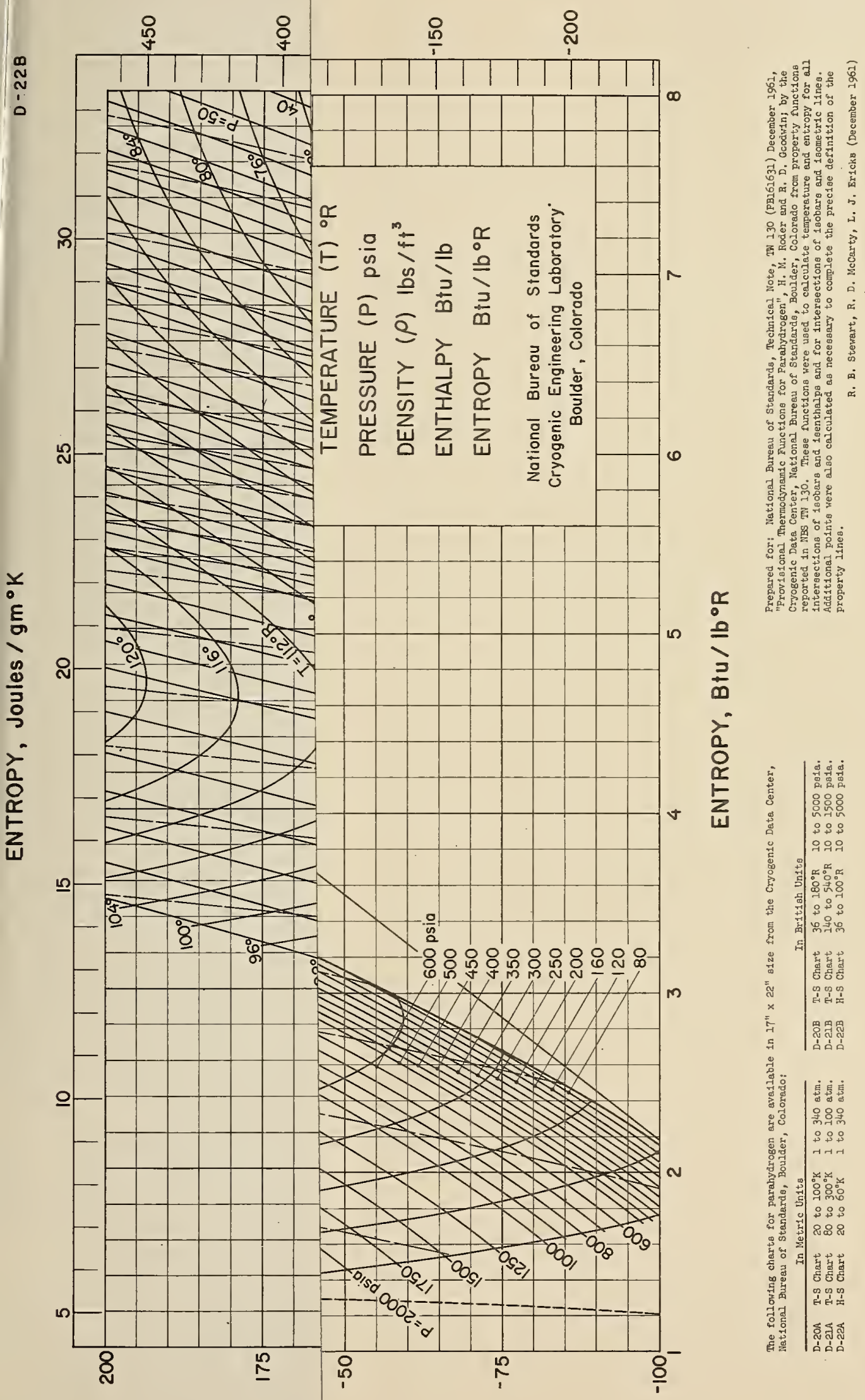



20 $-25, T+1,1+30$

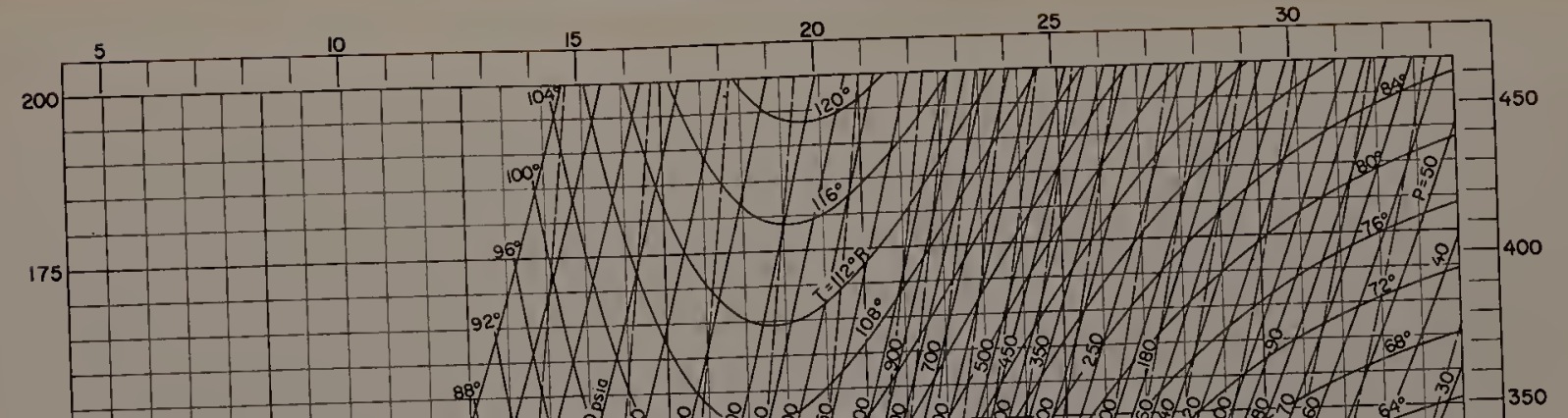

150 14 (1) $T=80^{\circ} R$
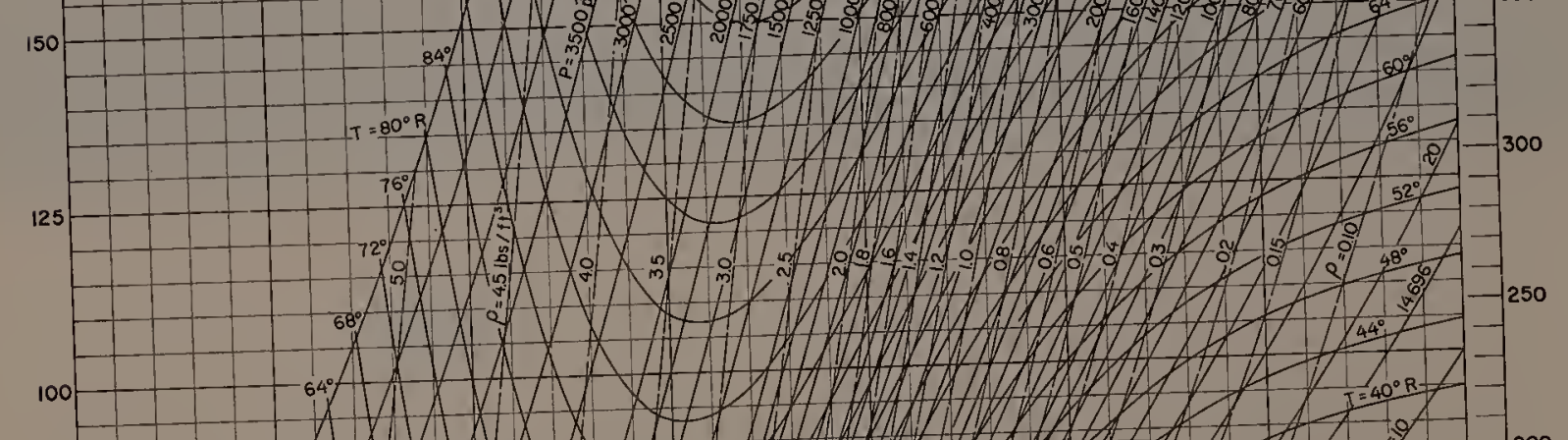

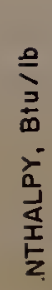
$60^{\circ} \sqrt{A}$

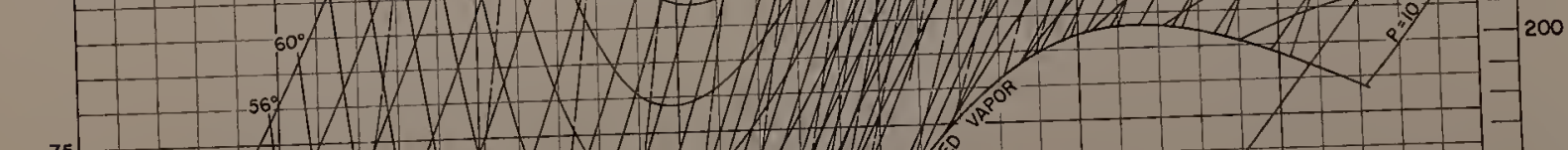

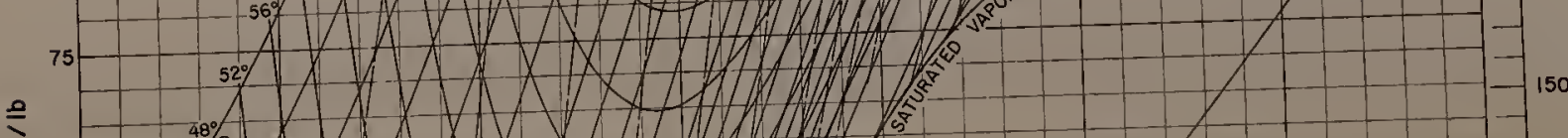

50

$44^{\circ} 2^{\circ}$

$1 \sqrt{ } \times \sqrt{ }{ }^{2}$

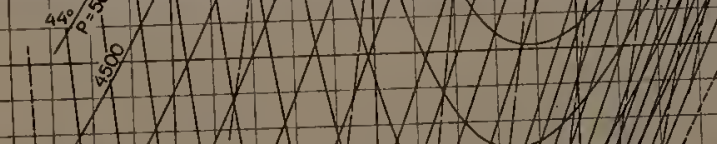

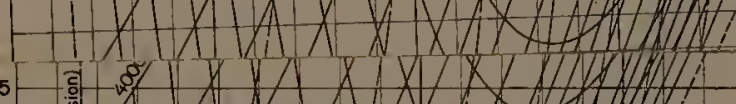

$1+10 \%$

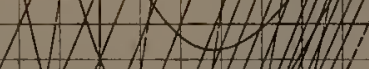

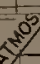

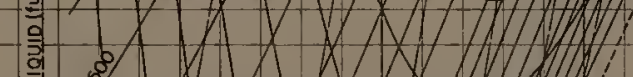

吾

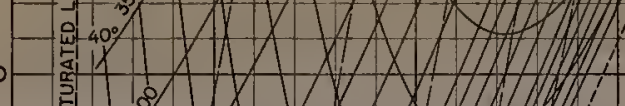

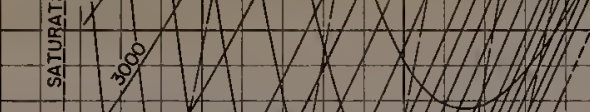
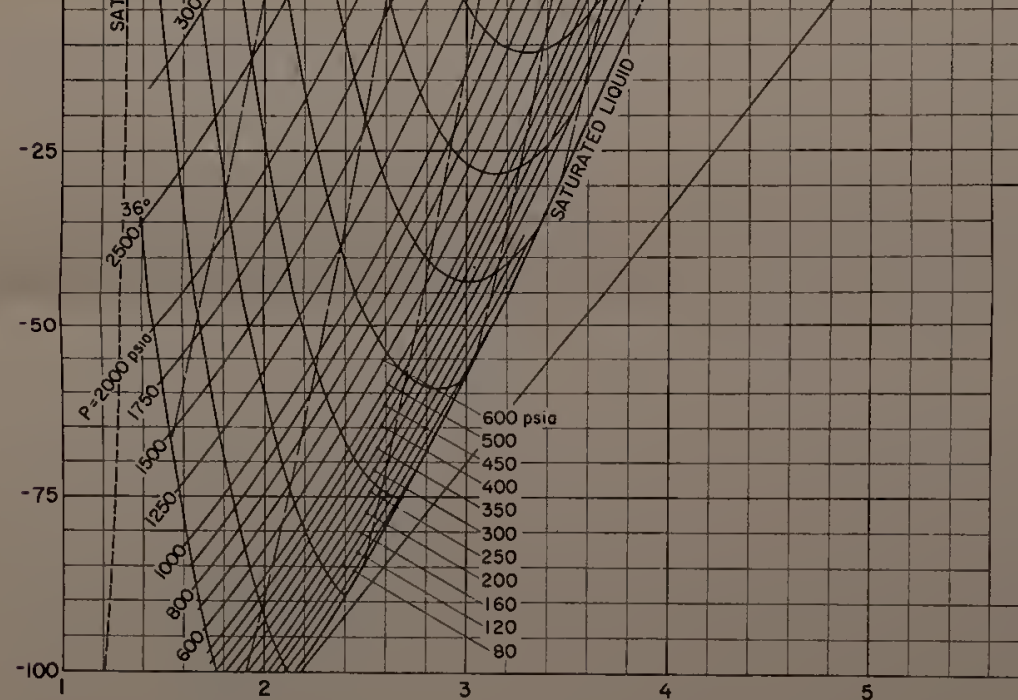

\section{INTERIM H-S CHART FOR PARAHYDROGEN}

TEMPERATURE $(T)^{\circ} R$ PRESSURE $(P)$ PSIa DENSITY $(\rho) \mathrm{lbs} / \mathrm{ft}^{3}$

ENTHALPY BtU/Ib ENTROPY BIU/Ib ${ }^{\circ}$

Notional Bureau of Standords

Cryogenic Engineering Loboralory Boulder, Colorado

ENTROPY, BIU/ Ib ${ }^{\circ} R$
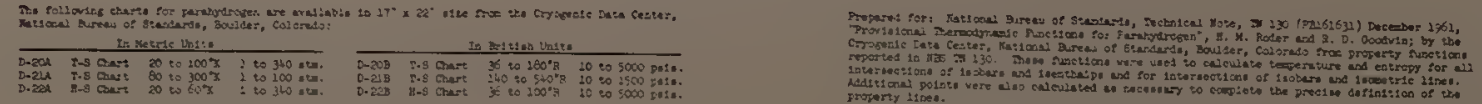

U. S. DEPARTMENT OF COMMERCE

Luther H. Hodges, Secretary

NATIONAL BUREAU OF STANDARDS

A. V. Astin, Director

\section{THE NATIONAL BUREAU OF STANDARDS}

The scope of activities of the National Bureau of Standards at its major laboratories in Washington, U.C., and Boulder, Colorado, is suggested in the following listing of the divisions and sections engaged in technical work. In general, each section carries out specialized research, development, and engineering in the field indicated by its title. A brief deseription of the activities, and of the resultant publications, appears on the inside of the front cover.

\section{WASHINGTON, D.C.}

Electricity. Resistance and Reactance. Electrochemistry. Electrical lnstruments. Magnetic Measurements. Dielectrics. High Voltage.

Metrology. Photometry and Colorimetry. Refractometry. Photographic Research. Length. Engineering Metrology. Mass and Scale. Volumetry and Densimetry.

leat. Temperature Physics. Heat Measurements. Cryogenic Physics. Equation of State. Statistical Physics. Radiation Physics. X-ray. Radioactivity. Radiation Theory. High Energy Radiation. Radiological Equipment. Nucleonic lnstrumentation. Neutron Physics.

Analytical and Inorganic Chemistry. Pure Substances. Spectrochemistry. Solution Chemistry. Standard Reference Materials. Applied Analytical Research.

Mechanics. Sound. Pressure and Vacuum. Fluid Mechanics. Engineering Mechanics. Rheology. Combustion Controls.

Organic and Fibrous Materials. Rubber. Textiles. Paper. Leather. Testing and Specifications. Polymer Structure. Plastics. Dental Research.

Metallurgy. Thermal Metallurgy. Chemical Metallurgy. Mechanical Metallurgy. Corrosion. Metal Physics. Electrolysis and Metal Deposition.

Mineral Products. Engineering Ceramics. Glass. Refractories. Enameled Metals. Crystal Growth. Physical Properties. Constitution and Microstructure.

Building Research. Structural Engineering. Fire Research. Mechanical Systems. Organic Building Materials. Codes and Safety Standards. Heat Transfer. Inorganic Building Materials.

Applied Mathematics. Numerical Analysis. Computation. Statistical Engineering. Mathematical Physics. Operations Research.

Data Processing Systems. Components and Techniques. Computer Teohnology. Measurements Automation. Engineering Applications. Systems Analysis.

Atomic Physics. Spectroscopy. Infrared Spectroscopy. Solid State Physics. Electron Physics. Atomic Physics. Instrumentation. Engineering Electronics. Electron Devices. Electronic Instrumentation. Mechanical Instruments. Basic lnstrumentation.

Physical Chemistry. Thermochemistry. Surface Chemistry. Organic Chemistry. Molecular Spectroscopy. Molecular Kinetics. Mass Spectrometry.

Office of Weights and Measures.

\section{BOULDER, COLO.}

Cryogenic Engineering. Cryogenic Equipment. Cryogenic Processes. Properties of Materials. Cryogenic Technical Services.

Ionosphere Research and Propagation. Low Frequency and Very Low Frequencv Research. Ionosphere Research. Prediction Services. Sun-Eartb Relationships. Field Engineering. Radio Warning Services. Vertical Soundings Research.

Radio Propagation Engineering. Data Reduction Instrumentation. Radio. Noise. Tropospheric Measurements. Tropospheric Analysis. Propagation-Terrain Effects. Radio-Neteorology. Lower Atmosphere Physics.

Radio Standards. High Frequency Electrical Standards. Radio Broadcast Service. Radio and Microwave Materials. Atomic Frequency and Time Interval Standards. Electronic Calibration Center. Millimeter-Wave Research. Microwave Circuit Standards.

Radio Systems. Applied Electromagnetic Theory. High Frequency and Very High Frequency Research. Modulation Research. Antenna Research. Navigation Systems.

Upper Atmosphere and Space Physics. Upper Atmosphere and Plasma Physics. lonosphere and Exosphere Scatter. Airglow and Aurora. Ionospheric Radio Astronomy. 


\footnotetext{
NBS

$\langle$ NBS $>$
}

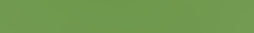

$-$ 



, 